\title{
Personalized treatment strategies for depression
}

\author{
Citation for published version (APA):
}

van Bronswijk, S. C. (2020). Personalized treatment strategies for depression. [Doctoral Thesis, Maastricht University]. Drukkerij Walters. https://doi.org/10.26481/dis.20201211sb

Document status and date:

Published: 01/01/2020

DOI:

10.26481/dis.20201211sb

Document Version:

Publisher's PDF, also known as Version of record

\section{Please check the document version of this publication:}

- A submitted manuscript is the version of the article upon submission and before peer-review. There can be important differences between the submitted version and the official published version of record.

People interested in the research are advised to contact the author for the final version of the publication, or visit the DOI to the publisher's website.

- The final author version and the galley proof are versions of the publication after peer review.

- The final published version features the final layout of the paper including the volume, issue and page numbers.

Link to publication

\footnotetext{
General rights rights.

- You may freely distribute the URL identifying the publication in the public portal. please follow below link for the End User Agreement:

www.umlib.nl/taverne-license

Take down policy

If you believe that this document breaches copyright please contact us at:

repository@maastrichtuniversity.nl

providing details and we will investigate your claim.
}

Copyright and moral rights for the publications made accessible in the public portal are retained by the authors and/or other copyright owners and it is a condition of accessing publications that users recognise and abide by the legal requirements associated with these

- Users may download and print one copy of any publication from the public portal for the purpose of private study or research.

- You may not further distribute the material or use it for any profit-making activity or commercial gain

If the publication is distributed under the terms of Article $25 \mathrm{fa}$ of the Dutch Copyright Act, indicated by the "Taverne" license above, 
Personalized treatment strategies for depression 
Cover / design: Studio Noto (Ivo Straetmans)

Lay out: Tiny Wouters

Printed by: Drukkerij Walters

ISBN: 978-94-6380-781-4

(C) Suzanne van Bronswijk, Maastricht 2020

All rights reserved. No part of this book may be reproduced or transmitted in any form or by any means without permission in writing by the author, or when appropriate, by the publishers of the publication. 


\section{Personalized treatment strategies for depression}

\section{PROEFSCHRIFT}

ter verkrijging van de graad van doctor aan de Universiteit Maastricht, op gezag van de Rector Magnificus, Prof. dr. Rianne M. Letschert, volgens het besluit van het College van Decanen,

in het openbaar te verdedigen

op vrijdag 11 december 2020 om 12:00 uur

door

Suzanne Catharina van Bronswijk 


\section{Promotores}

Prof. dr. F. P. M. L. Peeters

Prof. dr. M. J. H. Huibers (Vrije Universiteit Amsterdam)

\section{Copromotor}

Dr. L. H. J. M. Lemmens

\section{Beoordelingscommissie}

Prof. dr. R. W. H. M. Ponds (voorzitter)

Prof. dr. A. T. F. Beekman (Amsterdam Universitair Medische Centra)

Dr. E. Driessen (Radboud Universiteit Nijmegen)

Prof. dr. P. Muris

Prof. dr. J. J. M. H. Strik

The research presented in this thesis was performed at the Department of Psychiatry and Neuropsychology, School for Mental Health and Neuroscience (MHeNS), Maastricht University, Maastricht, the Netherlands. Part of the research was also performed at the Department of Psychology, University of Pennsylvania, Philadelphia, USA.

Part of the research in this thesis has received funding from the research Institute of Experimental Psychopathology (EPP), the Netherlands, and the Academic Community Mental Health Centre (RIAGG) in Maastricht, the Netherlands. The dissertation was printed with financial support kindly provided by Maastricht University. 



\section{Contents}

$\begin{array}{lll}\text { Chapter } 1 & \text { General introduction } & 9\end{array}$

$\begin{array}{lll}\text { PART } 1 \quad \text { Depression treatment and comorbidity } & 29\end{array}$

Chapter 2 The influence of comorbid anxiety on the effectiveness 31 of cognitive therapy and interpersonal psychotherapy for major depressive disorder

Chapter 3 The impact of personality disorder pathology on the effectiveness of cognitive therapy and interpersonal psychotherapy for major depressive disorder

Chapter 4 Effectiveness of acute-phase treatment of depression is not influenced by comorbid personality disorders: results from a meta-analysis and meta-regression

Chapter 5 The impact of comorbid personality disorders on received outpatient treatment for depression: do we treat these patients differently?

\section{PART 2 Treatment planning for depression}

Chapter 6 Long-term outcomes of acute treatment with cognitive therapy versus interpersonal psychotherapy for adult depression: follow-up of a randomized controlled trial

Chapter 7 A prognostic index for long-term outcome after successful acute phase cognitive therapy and Interpersonal psychotherapy for major depressive disorder

Chapter 8 Precision medicine for long-term depression outcomes using the Personalized Advantage Index approach: cognitive therapy or interpersonal psychotherapy?

Chapter 9 Effectiveness of psychotherapy for treatment-resistant depression: a meta-analysis and meta-regression 
Chapter 10 Selecting the optimal treatment for a depressed individual: clinical judgment or statistical prediction?

Chapter 11 Cross-trial prediction in psychotherapy: external validation of the Personalized Advantage Index using machine learning in two Dutch randomized trials comparing cognitive behavioral therapy versus interpersonal psychotherapy for depression

Chapter 12 Bayesian estimation of treatment response subgroups in major depressive disorder

Chapter 13 General discussion

Addendum Summary

Samenvatting 335

Valorization 349

Dankwoord / Acknowledgements 355

Curriculum vitae 361 
Chapter

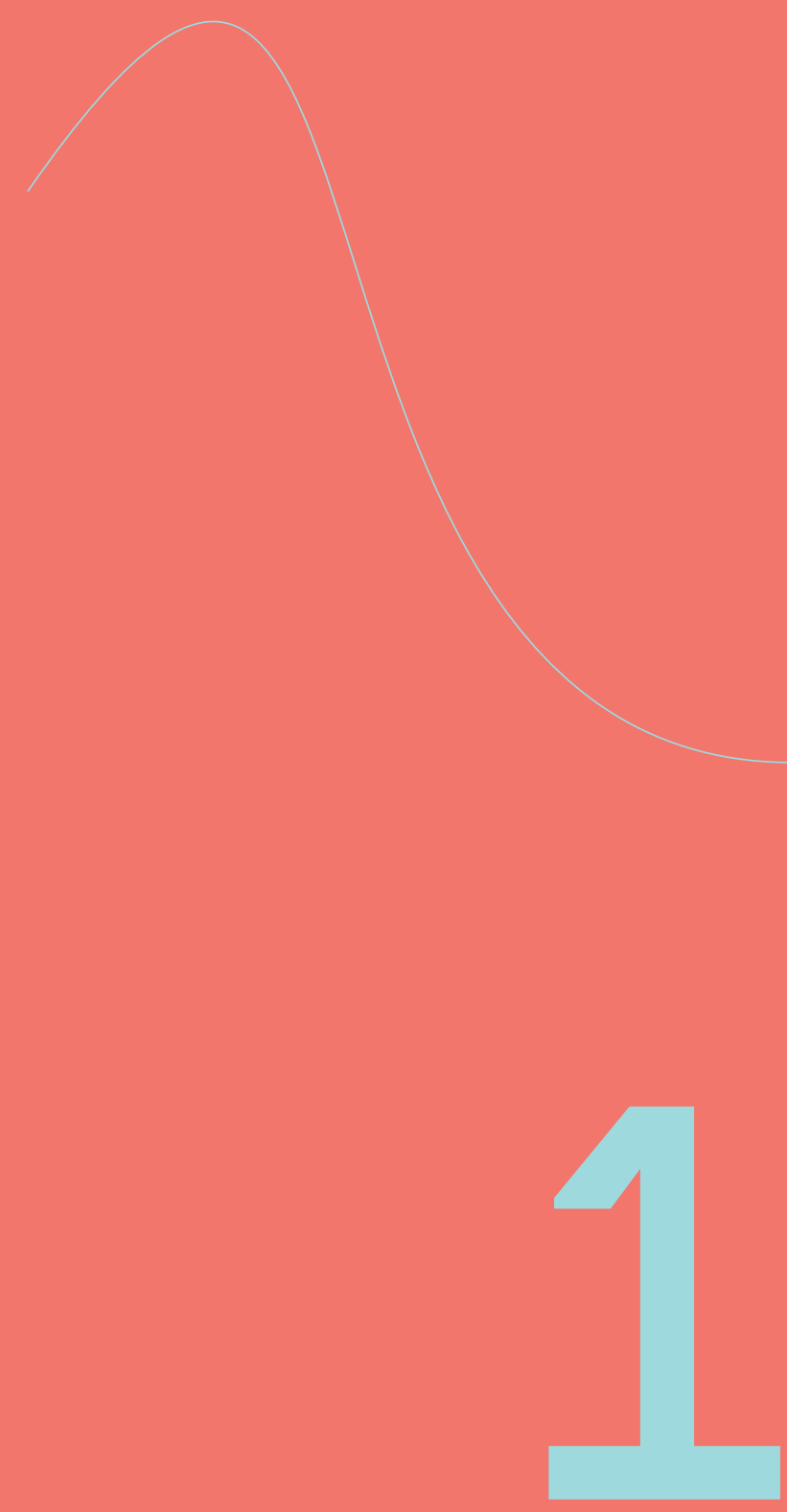




\section{GENERAL INTRODUCTION}



The subject of this thesis is "personalized treatment strategies for depression". This refers to strategies that aim to find the optimal depression treatment for a specific individual at a specific point in time. In this context, optimal treatment is defined as a treatment that results in high depressive symptom reduction in the acute phase and sustained depressive symptom reduction in the long term. Efforts to match individuals to their most optimal treatment are also referred to as personalized or precision medicine. This chapter first introduces the criteria for, and prevalence and heterogeneity of, a depression diagnosis. Second, current treatments for depression are described, in particular focusing on the acute and enduring effects of the main treatments examined in this thesis: cognitive therapy and interpersonal psychotherapy. In addition, the so-called "dodo bird verdict" and "trial and error approach" that are associated with current treatment strategies are discussed. Next, two potential ways to optimize treatment strategies are described. One strategy is to focus on the impact of comorbid disorders and symptoms, and adjust treatment plans accordingly. A second strategy is the use of clinical judgement or statistical prediction (i.e., prediction models) based on multiple (complex) aspects of an individual. Finally, the aims and the outline of this thesis will be described.

\section{Depression}

\subsection{Diagnosis}

What if an individual is diagnosed with major depressive disorder (MDD) or more simply with "depression"? What does this diagnosis exactly mean? These days, most clinicians and researchers classify mental disorders using the Diagnostic and Statistical Manual of Mental Disorders (DSM-5; American Psychiatric Association, 2013). With this classification system, diagnoses are based on specific symptom descriptions and criteria. By doing so, the DSM-5 provides a standard language by which clinicians, researchers, policy makers, and health insurance companies communicate about mental disorders.

The DSM-5 language for an MDD diagnosis involves the following criteria (APA, 2013): an individual has to have five or more symptoms in (at least) a two-week period (nearly) every day:

1. Depressed mood.

2. Diminished interest or pleasure in (almost) all activities.

3. Significant weight loss (when not dieting) or significant weight gain, or a decreased or increased appetite.

4. Insomnia or hypersomnia.

5. Psychomotor agitation or retardation.

6. Fatigue or loss of energy. 
7. Feelings of worthlessness or inappropriate guilt.

8. Diminished ability to think or concentrate, or indecisiveness.

9. Recurrent thoughts of death, recurrent suicidal ideation, a specific plan for committing suicide, or a suicide attempt.

Of the five or more symptoms, at least one of these symptoms should be (1) depressed mood or (2) diminished interest or pleasure in (almost) all activities. In addition, these symptoms must cause significant distress or impairment in social, occupational or other important areas of functioning.

\subsection{Prevalence}

MDD is highly prevalent. Worldwide, around 300 million individuals meet criteria for an MDD diagnosis (WHO, 2017). In the Netherlands, estimates indicate that around 500000 individuals have an MDD diagnosis every year (Nemesis-2 study, De Graaf, Ten Have, \& van Dorsselaer, 2010). Information on life time prevalence (i.e., the proportion of individuals in a population that will experience a MDD at some point in their life) varies according to the applied study design (Moffitt et al., 2010). While epidemiological studies with retrospective assessments have more conservative estimates ranging from 15 to 20\% (De Graaf, Ten Have, Van Gool, \& Van Dorsselaer, 2012; Kessler et al., 2003); prospective studies report a higher cumulative lifetime prevalence around 30-50\% (Angst et al., 2016; Moffitt et al., 2010; Rohde, Lewinsohn, Klein, Seeley, \& Gau, 2013; Wilhelm et al., 2006). These different findings could be explained by memory problems ("recall bias"), however, such differences are not found in studies on physical problems such as diabetes, hypertension or arthritis (Takayanagi et al., 2014). A more plausible explanation for this discrepancy could be that many individuals experience depressive symptoms as "adaptive sadness", and do not report them as a pathological episode in a psychiatric history assessment (Lorenzo-Luaces, 2015).

\subsection{Heterogeneity}

DSM-5 classifications are highly heterogeneous (Wardenaar \& de Jonge, 2013). When considering the nine MDD criteria described above, all criteria except depressed mood consist of at least two sub-symptoms (e.g., feelings of worthlessness or inappropriate guilt, insomnia or hypersomnia). Theoretically, this means that it is possible that two individuals with an MDD diagnosis have completely different symptom profiles with no single symptom in common. In fact, there are 227 hypothetical symptoms profiles based on the 9 criteria, and 16400 possibilities considering the sub-symptoms as well (Fried \& Nesse, 2015). This striking heterogeneity in symptom clusters is not only hypothetical: in 3703 participants of the STAR*D study, a total of 1030 unique symptoms profiles were identified (Fried \& Nesse, 2015). 
The heterogeneity of an MDD diagnosis goes beyond symptom variation. The course and prognosis of MDD is also highly heterogeneous. While about half of the MDD index episodes are of short duration and unlikely to recur (a "common cold"), the other half of these index episodes is chronic or recurrent (a "chronic disease"; Eaton et al., 2008). Since these remarkable differences are highly unpredictable, matching treatment intensity levels to individual prognoses is extremely difficult, and under- and overtreatment of individuals with an MDD diagnosis is therefore common (Lorenzo-Luaces, 2015; Thornicroft et al., 2017).

\section{Current treatment strategies}

\subsection{Depression treatments}

Current clinical practice guidelines prescribe antidepressant medication, psychotherapy, and their combination, among the first choices of treatment for MDD (APA, 2015; NICE, 2010; Spijker et al., 2013). The effectiveness of these treatments is based on a large body of scientific evidence; and there are multiple options available. Antidepressant medications include Selective Serotonin Reuptake Inhibitors (SSRI's), Selective Serotonin and Norepinephrine Reuptake Inhibitors (SNRI's), Tricyclic Antidepressants (TCA's), mirtazapine, bupropion, lithium and certain types of antipsychotics (Spijker et al., 2013). For psychotherapy, there are various types available as well, of which cognitive therapy (CT) and interpersonal psychotherapy (IPT) are the most well-studied and frequently applied (Cuijpers et al., 2013a; Cuijpers et al., 2011a; Lemmens et al., 2015). CT and IPT are both structured and time-limited psychotherapies. CT derives from Beck's cognitive theory, which perceives MDD as a result of maladaptive information processing and dysfunctional beliefs (Beck, 1967, 1979). IPT was developed by Klerman, Weissman, Rounsaville, and Chevron (1984), and links MDD to distressing interpersonal life events and lack of social support (Klerman, Weissman, Rounsaville, \& Chevron, 1984). Both CT and IPT have shown to be effective MDD treatments in the acute phase with initial response rates up to $60 \%$ (Barth et al., 2016; Cuijpers, Andersson, Donker, \& van Straten, 2011b; Cuijpers et al., 2013a; Lemmens et al., 2015). However, even when treated effectively in the acute phase, MDD can be highly recurrent (Burcusa \& lacono, 2007; Eaton et al., 2008; Solomon et al., 2000). CT has consistently been shown to have enduring effects that last beyond the end of treatment, with survival rates higher than those associated with (prior) antidepressant medication (Cuijpers et al., 2013b; Vittengl, Clark, Dunn, \& Jarrett, 2007). Research in IPT is less extensive. Even though IPT has shown to prevent relapse and recurrence when continued or maintained (Cuijpers, Donker, Weissman, Ravitz, \& Cristea, 2016), only one older study with small sample sizes has examined potential prophylactic effects after treatment termination. In this study comparable 
rates of relapse between prior CT, IPT and antidepressant medications were found, indicating possible enduring effects of IPT as well (Shea et al., 1992).

\subsection{Dodo bird verdict}

The term "dodo bird verdict" was first introduced by Saul Rosenzweig in 1936 to illustrate the notion that all psychotherapies are equally effective. Quoting the Dodo Bird in Lewis Carroll's Alice in Wonderland, he concluded that 'everyone has won and all must have prizes' (Rosenzweig, 1936). This controversy gained more attention after the publication of a qualitative review on psychotherapy research by Luborsky et al. (1975). In this review it was concluded that there was no clear support for the superiority of any type of psychological therapy over any other. Decades later, research consistently shows that different types of psychotherapy, and also pharmacotherapy, are equally effective on average in MDD (Cipriani et al., 2009; Cuijpers et al., 2011b; Gartlehner et al., 2008). However, the fact that these therapies are equally effective on average, does not imply that treatment selection is not important (Kravitz, Duan, \& Braslow, 2004; Simon \& Perlis, 2010). Since an individual's response to different therapies may vary greatly, different treatments may not be equally effective for each specific individual. This is illustrated in Figure 1.1 with data from a randomized trial comparing CT and IPT for depression (Lemmens et al., 2015). In Figure 1.1a, the comparable average effectiveness of the two therapies is shown (i.e., the dodo bird verdict). However, when examining spaghetti plots of the raw depression severity scores in CT (Figure 1.1b) and IPT (Figure 1.1c), individual trajectories appear to be highly heterogeneous, and completely different from the average effectiveness plot.

\subsection{The trial-and-error approach}

Not only do individual responses to MDD treatments vary widely; individual acute and long-term treatment outcomes are highly unpredictable. In clinical practice, the mean comparable effectiveness of different treatment options and the unpredictability of individual responses, often lead to the "trial-and-error approach", in which consecutive treatments are being offered to determine what works best for the given individual (Rush et al., 2006). This trial-and-error approach has negative consequences. Since this approach is inefficient, it may lead to unnecessary long treatment trajectories that are accompanied with high societal costs. These long trajectories with failed treatment attempts can in turn increase the burden and demoralization of individuals with MDD and their relatives. In addition, by offering treatments that do not work for a specific individual, risks of treatment dropout are high (+/- 20\%; Warden et al., 2009). In other words, this approach and its 
consequences all add to the known over- and under treatment of MDD (LorenzoLuaces, 2015; Thornicroft et al., 2017).
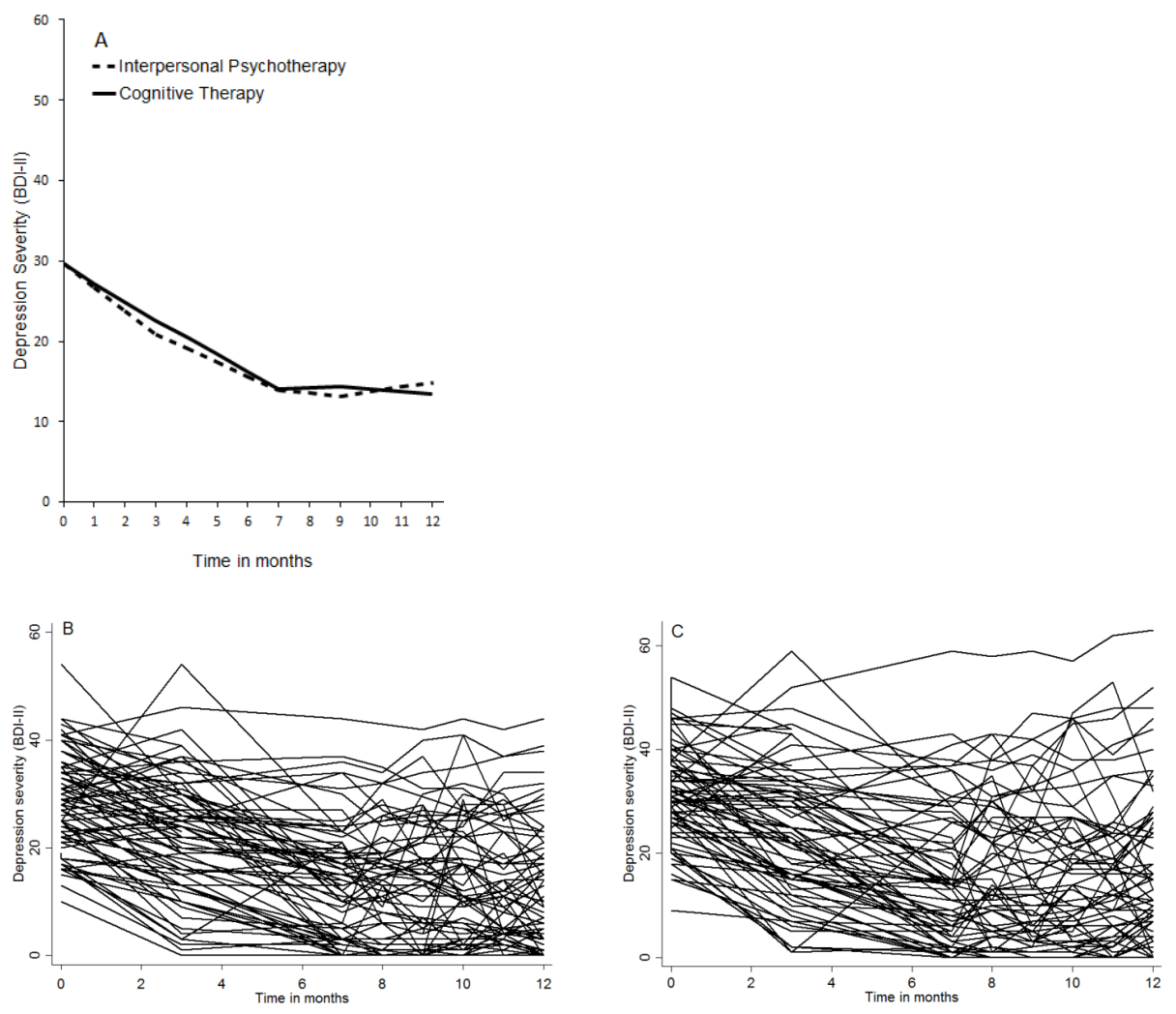

Figure 1.1: Heterogeneity in treatment responses between individuals beyond average treatment effects. These are data from a randomized trial comparing CT $(n=75)$ and IPT $(n=76)$ for depression (Lemmens et al., 2015). In A the mean comparable effectiveness of both therapies is illustrated. In B the raw depression severity scores of individuals randomized to $\mathrm{CT}$ are plotted, and in $\mathrm{C}$ the raw BDI-II scores of individuals randomized to IPT are plotted. BDI-II = Beck Depression Inventory, second edition.

\section{Optimizing treatment strategies}

An alternative to the trial-and-error approach is to optimize treatment selection (Cohen \& DeRubeis, 2018). Treatment selection aims to move beyond average effectiveness and focuses on the question "What works for whom?". Matching individuals with specific treatments is also referred to as personalized or precision medicine (Cohen \& DeRubeis, 2018; Katsnelson, 2013; Simon \& Perlis, 2010). To 
answer the question "What works for whom?", individual characteristics that reliably predict (differential) acute and long-term treatment outcomes need to be identified. In this context, two types of characteristics can be discerned: predictors and moderators (Cohen \& DeRubeis, 2018). Predictors (i.e., prognostic variables) are associated with acute or long-term treatment outcomes, irrespective of the type of treatment. Moderators (i.e., prescriptive variables) predict acute and long-term treatment outcomes as a function of treatment type (Kraemer, 2013).

One approach of studying potential predictors and moderators of treatment outcome, is by focusing on specific symptoms clusters that occur in MDD. This can either be done by investigating the impact of different symptom combinations within the MDD diagnosis (see paragraph about heterogeneity); or by examining the influence of comorbid diagnoses and symptoms. A second approach to find answers on how to optimize treatment strategies is by examining and evaluating multiple predictors and moderators at once. This more holistic approach is a method that some may consider the core business of clinical practice: fitting all pieces together using clinical judgement (Tomba \& Fava, 2012). Others may argue that the best way to integrate such complex information is by using statistical prediction (e.g., prediction models, Cohen \& DeRubeis, 2018).

\subsection{Comorbid diagnosis and symptoms}

In MDD, the level of comorbidity is very high. Two of the most prevalent DSM-5 comorbid diagnoses are anxiety disorders and personality disorders.

The prevalence of comorbid anxiety disorders and anxiety symptoms in MDD varies between $40 \%$ and 60\% (Fava et al., 2004; Fava et al., 2000; Kessler et al., 2003). Comorbid anxiety in MDD, also referred to as anxious depression, is considered to be an important subtype (American Psychiatric Association, 2013; Rao \& Zisook, 2009; Ten Have et al., 2016). Anxious depression has been associated with a more severe clinical picture compared to MDD without comorbid anxiety, including more severe depressive symptoms, more functional impairment and intensified suicidal thoughts and behavior (Fava et al., 2004; Fava et al., 2000; Pfeiffer, Ganoczy, Ilgen, Zivin, \& Valenstein, 2009). However, findings on the impact of comorbid anxiety on MDD treatment outcome are equivocal; worse outcomes (Frank et al., 2011; Frank et al., 2000; Young, Mufson, \& Davies, 2006), similar outcomes (Forand \& Derubeis, 2013; Fournier et al., 2009; McEvoy \& Nathan, 2007; Smits, Minhajuddin, \& Jarrett, 2009), and a more accelerated depressive symptom improvement have been reported (de Azevedo Cardoso et al., 2014; Forand, Gunthert, Cohen, Butler, \& Beck, 2011; Kashdan \& Roberts, 2011; Smits, Minhajuddin, Thase, \& Jarrett, 2012). A possible explanation for these mixed results is the use of different definitions to classify anxious depression (lonescu, Niciu, Henter, \& Zarate, 2013). This concern is illustrated by a recent study that showed that the syndromal and dimensional definitions of anxious depression 
classify two different groups of individuals under the same "anxious depression" label (MDD with a comorbid anxiety disorder versus MDD with comorbid anxiety symptoms; van der Veen, Comijs, van Zelst, Schoevers, \& Oude Voshaar, 2014). These different conceptualizations of anxious depression are a major concern for integrating the outcomes of previous studies into broader knowledge and translating findings to clinical practice. In addition, mixed results could be explained by small study samples and the use of different instruments for the measurement of anxiety disorders and anxiety symptoms between studies.

The prevalence of comorbid personality disorders in MDD is about $45 \%$, and in particular personality disorders grouped in cluster $\mathrm{C}$ are prevalent: obsessive compulsive personality disorder, dependent personality disorder and avoidant personality disorder (Friborg et al., 2014). As with co-morbid anxiety, the presence of comorbid personality pathology is associated with a more severe clinical picture prior to treatment, including higher depression severity and a lower age of onset (Mulder, 2002). Despite extensive research spanning several decades, findings regarding the impact of comorbid personality disorders on MDD treatment outcome are inconsistent as well. Results from previous studies vary from a negative association between the presence of a comorbid personality disorder and clinical outcome (Newton-Howes et al., 2014) to the absence of any difference between outcome in individuals with and without comorbid personality disorders (Kool et al., 2005; Mulder, 2002). These inconsistencies across studies are probably best explained by methodological problems. Evidence is often biased by unstructured assessment of personality disorder and MDD, naturalistic treatment allocation, a lack of control for significant confounders (e.g., earlier onset, childhood adversity, and episode duration), and no consideration of baseline depression severity differences between individuals with and without comorbid personality disorders (Mulder, 2002).

With these inconsistent results regarding the impact of comorbid anxiety and personality pathology on MDD treatment outcome, there has been little agreement on tailored treatment strategies for individuals with these comorbidities.

\subsection{Clinical judgment}

In clinical psychology and psychiatry, the consideration of multiple aspects of an individual with MDD to optimize treatment selection is everyday practice. Generating treatment strategies heavily depends on clinical judgment of expected responses to available treatments. Clinical judgment is the result of informal or intuitive processes, and treatment recommendations are often formulated by an individual clinician or during multidisciplinary staff meetings. These recommendations are based on the clinicians' experience with similar patients, the clinicians' training background, the patient's preference and the clinicians' beliefs, theoretical perspective, and familiarity with empirical literature and (inter)national guidelines (Delgadillo, Gellatly, \& 
Stephenson-Bellwood, 2015; Lorenzo Lorenzo-Luaces, DeRubeis, \& Bennett, 2015). Despite the potential flexibility of clinical judgment, it is susceptible to errors. Sources of errors include low inter- and intra-rater reliability, limited information processing abilities, an overreliance on the clinical interview, representative biases (i.e., law of small numbers), lack of adequate feedback, anchoring biases (i.e., depending heavily on an initial piece of information), and confirmation bias based on theoretical frameworks, hypothesis and decisions of others (Bell \& Mellor, 2009; Grove, Zald, Lebow, Snitz, \& Nelson, 2000).

\subsection{Statistical prediction}

One way to overcome the errors of human judgment is to use statistical or actuarial predictions that are based on reproducible algorithms. The accuracy of these predictions, relative to predictions based on clinical judgment, has been a topic of debate since the publication of Paul Meehl's "disturbing little book" (Meehl, 1954, 1986). Here, he showed that in 19 out of 20 studies statistical predictions were as accurate as or more accurate than predictions based on clinical judgment. Since then, research has consistently shown that statistical prediction works at least as well as predictions based on clinical judgment, with on average $10 \%$ to $13 \%$ more accuracy for statistical predictions (Ægisdóttir et al., 2006; Grove et al., 2000). Despite the evidence that statistical prediction is at least as accurate as predictions based on clinical judgment, its use in clinical practice is still limited (Bell \& Mellor, 2009). However, the potential of novel statistical approaches (e.g., machine learning) and the availability of big data (e.g., smartphone and biosensor data) are leading us to an renewed attention for and rethinking of the potential of statistical predictions for personalized treatment recommendations (Perna, Grassi, Caldirola, \& Nemeroff, 2018).

In the context of treatment selection for MDD, this development is illustrated by the use of novel multivariate models (or prediction algorithms) that promise to generate powerful predictions (Cohen \& DeRubeis, 2018). Two types of multivariate models can be distinguished: prognostic models and prescriptive models. Like in the case of a prognostic variable (predictor), a prognostic model predicts acute and longterm outcome irrespective of the type of treatment. Prognostic models generate personalized predictions that determine the optimal level of treatment intensity (i.e., scores on a prognostic index; Delgadillo, Moreea, \& Lutz, 2016; Lorenzo LorenzoLuaces, DeRubeis, van Straten, \& Tiemens, 2017). Previous research has studied prognostic models to determine acute phase outcome (Chekroud et al., 2016; Uher et al., 2012), and long-term prognosis of MDD (van Loo, Aggen, Gardner, \& Kendler, 2015; Wang et al., 2014). A prescriptive model, like a prescriptive variable (moderator), predicts acute and long-term treatment outcomes as a function of treatment type. Prescriptive models aim to convert the predictive information of 
multiple moderators into actionable recommendations to guide treatment selection. Examples of these multivariate models are the "matching factor" (Barber \& Muenz, 1996), the "nearest-neighbors" approach (Lutz et al., 2006), and the $M^{*}$ approach (Kraemer, 2013; Niles et al., 2017a; Niles, Wolitzky-Taylor, Arch, \& Craske, 2017b; Smagula et al., 2016; Wallace, Frank, \& Kraemer, 2013). Another promising prescriptive modelling approach to guide treatment selection between two or more treatments is the Personalized Advantage Index (PAl; DeRubeis et al., 2014). This method not only provides an individual treatment recommendation, it also delivers a quantitative estimate of the predicted advantage of the indicated treatment over the non-indicated treatment(s). These recommendations are based on the difference between predicted outcomes of two or more treatments using a model that includes multiple predictors and moderators. DeRubeis et al. (2014) developed and introduced this approach by predicting outcomes of acute-phase CT versus pharmacotherapy. Since then, the PAI approach has been replicated and extended to acute phase CT versus IPT for MDD (Huibers et al., 2015), continuation CT versus fluoxetine for recurrent MDD (Vittengl, Clark, Thase, \& Jarrett, 2017), sertraline versus placebo for MDD (Webb et al., 2018), cognitive behavioral therapy (CBT) versus psychodynamic treatment for MDD (Cohen, Kim, Van, Dekker, \& Driessen, 2019), CBT versus a positive psychology intervention for MDD (Lopez-Gomez et al., 2019), CBT versus CBT with integrated exposure and emotion-focused elements for MDD (Friedl, Berger, Krieger, Caspar, \& Grosse Holtforth, 2019), trauma-focused cognitive behavioral therapy and eye movement desensitization for posttraumatic stress disorder (PTSD) (Deisenhofer et al., 2018), and dropout in MDD (Zilcha-Mano et al., 2016) and PTSD (Keefe et al., 2018).

\section{Aims of this thesis}

The aim of this thesis is to investigate ways to personalize treatment strategies for MDD. Personalized treatment strategies refer to strategies that search for the optimal depression treatment for a specific individual at a specific point in time. To examine these potential strategies, three objectives can be discerned. The first objective is to examine the impact of comorbid anxiety and personality pathology on acute and longterm treatment outcome in MDD. The question is whether anxiety and personality pathology act as predictors or moderators of treatment outcome, and are therefore useful in guiding treatment strategies. The second objective is to investigate potential ways of treatment planning that lead to better treatment outcomes for MDD in the long term. More specifically, the enduring effects of CT and IPT for MDD are investigated, and the potential of prognostic and prescriptive prediction models in estimating long-term effects are examined. In addition, the role of psychotherapy in 
treating individuals that are not responsive to other treatments is evaluated. The third objective is to provide insight into possibilities and challenges of future personalized treatment strategies for MDD. The historical "clinical judgment vs. statistical prediction" debate is entered with new types of prediction models, and the obstacles that prevent implementation of new prediction models are evaluated. In addition, a critical review of these prediction models is included as well; in particular on the subject of external validation. Finally, a new methodology is presented that can aid subgroup modelling in MDD, which is one of the current and promising types of predictive modelling (Wardenaar \& de Jonge, 2013).

\section{Outline of this thesis}

This thesis is divided into three parts that correspond to the three objectives that are described in the previous paragraph. The first part of this thesis is labeled "Depression treatment and comorbidity" and comprises four chapters (chapter 2 - 5). Chapter 2 describes the results of an empirical study that examined the (differential) effects of comorbid anxiety on the effectiveness and treatment continuation of CT and IPT for MDD during treatment and across five months of follow-up. In order to confirm the hypothesis that different definitions of anxious depression lead to the mixed results on this topic, both the impact of anxiety disorders and anxiety symptoms are examined separately. Chapter 3 reports the results of an empirical study aimed at investigating the impact of personality disorder diagnoses and cluster $\mathrm{C}$ personality features on the effectiveness and treatment continuation of CT and IPT for MDD in the acute phase and throughout five months of follow-up. In Chapter 4, a metaanalysis and meta-regression is presented on impact of comorbid personality disorders on acute-phase treatment outcome for MDD. As mentioned earlier, it was hypothesized that the inconsistent results regarding the impact of personality disorders on acute phase treatment outcome in MDD, were due to methodological problems. To avoid these problems, only trials in which treatment was controlled for and that used (semi-)structured interviews for diagnosis of both MDD and personality disorders were included. Additionally, the primary outcome was depression severity change during treatment, thereby avoiding bias following from baseline severity differences between individuals with and without comorbid personality disorders. In Chapter 5, results of an observational study are presented. The aim of this study was to examine whether and to what extend the presence of co-morbid personality pathology affects received MDD treatment in the context of naturalistic outpatient care. This study answers the question whether clinicians apply different treatment strategies for individuals with and without personality pathology, even though this is 
not recommended by clinical practice guidelines (APA, 2015; NICE, 2010; Spijker et al., 2013).

The second part of this thesis is labeled "Treatment planning for depression" and comprises four chapters (chapter 6 -9). Chapter 6 describes the results of an empirical study into the enduring effects of acute-phase CT and IPT for MDD throughout 17 months of follow-up. As mentioned previously, the enduring effects of $\mathrm{CT}$ are well established, while there is sparse evidence for the long-lasting effects of acute phase IPT. Chapter 7 involves a study on a prognostic model (i.e., a Prognostic Index) for long-term outcome after successful acute phase CT and IPT for MDD. If replicated, this model aims to identify individuals with unfavorable long-term depression severity, which enables selection of appropriate candidates for maintenance therapy. In Chapter 8, a study on a prescriptive model, the Personalized Advantage Index is presented that provides treatment recommendations of CT or IPT prior to treatment, based on long-term outcome predictions. The aim of this prescriptive model is to guide treatment selection by focusing on keeping a (formerly depressed) individual well over the long term. In Chapter 9, a meta-analysis and metaregression is presented, about the effectiveness of psychotherapy for treatmentresistant depression. This study tries to answer the question whether psychotherapy is an (add-on) treatment option if other treatment options for MDD have failed.

The third and final part of this thesis is labeled "Future strategies" and comprises four chapters (chapter 10 - 13). Chapter 10 describes a direct comparison of clinical judgment and statistical prediction (with the Personalized Advantage Index) for treatment selection for MDD. Since the Personalized Advantage Index is a product of recent technological advances, the contribution of these new techniques to the longstanding clinical judgment versus statistical prediction debate was examined. This chapter also describes several challenges that need to be faced in achieving implementation of prediction models in clinical practice. In Chapter 11, cross-trial predictions to externally validate the Personalized Advantage Index are presented, using data of two Dutch randomized trials comparing CT and IPT for MDD. This chapter focusses on challenges of external validation of prediction models, including heterogeneity in data collection, statistical methods, study populations, and treatments. Chapter 12 introduces a new Bayesian methodology to estimate multimodal density features in psychological data, such as treatment outcome studies for MDD. The aim of this method is to provide a descriptive procedure that precedes and strengthens latent subgroup modeling that is a promising approach in prediction research. In Chapter 13, findings of the studies presented in this thesis are summarized and evaluated, and future directions of personalized treatment strategies for depression are discussed in more detail.

The addendum comprises of an English and a Dutch summary, and a chapter on valorization. 


\section{References}

ÆEgisdóttir, S., White, M. J., Spengler, P. M., Maugherman, A. S., Anderson, L. A., Cook, R. S., . . Cohen, G. (2006). The meta-analysis of clinical judgment project: Fifty-six years of accumulated research on clinical versus statistical prediction. The Counseling Psychologist, 34(3), 341-382.

American Psychiatric Association. (2013). Diagnostic and statistical manual of mental disorders (5th ed.). Washington DC.

Angst, J., Paksarian, D., Cui, L., Merikangas, K., Hengartner, M. P., Ajdacic-Gross, V., \& Rössler, W. (2016). The epidemiology of common mental disorders from age 20 to 50: results from the prospective Zurich cohort Study. Epidemiology and Psychiatric Sciences, 25(1), 24-32.

Association, A. P. (2015). Practice Guidelines for the Treatment of Patients With Major Depressive Disorder, Third Edition.

Barber, J. P., \& Muenz, L. R. (1996). The role of avoidance and obsessiveness in matching patients to cognitive and interpersonal psychotherapy: empirical findings from the treatment for depression collaborative research program. J Consult Clin Psychol, 64(5), 951-958. Retrieved from http://www.ncbi.nIm.nih.gov/entrez/query.fcgi?cmd=Retrieve\&db=PubMed\&dopt=Citation\&list_uids $=8916624$.

Barth, J., Munder, T., Gerger, H., Nüesch, E., Trelle, S., Znoj, H., . . Cuijpers, P. (2016). Comparative efficacy of seven psychotherapeutic interventions for patients with depression: a network meta-analysis. Focus, 14(2), 229-243.

Beck, A. T. (1967). Depression: Clinical, experimental, and theoretical aspects: University of Pennsylvania Press.

Beck, A. T. (1979). Cognitive therapy of depression: Guilford press.

Bell, I., \& Mellor, D. (2009). Clinical judgements: Research and practice. Australian Psychologist, 44(2), 112121.

Burcusa, S. L., \& lacono, W. G. (2007). Risk for recurrence in depression. Clinical psychology review, 27(8), 959-985.

Chekroud, A. M., Zotti, R. J., Shehzad, Z., Gueorguieva, R., Johnson, M. K., Trivedi, M. H., . . Corlett, P. R. (2016). Cross-trial prediction of treatment outcome in depression: a machine learning approach. The Lancet Psychiatry, 3(3), 243-250.

Cipriani, A., Furukawa, T. A., Salanti, G., Geddes, J. R., Higgins, J. P., Churchill, R., . . McGuire, H. (2009). Comparative efficacy and acceptability of 12 new-generation antidepressants: a multiple-treatments meta-analysis. The lancet, 373(9665), 746-758.

Cohen, Z. D., \& DeRubeis, R. J. (2018). Treatment selection in depression. Annual review of clinical psychology, 14, 209-236.

Cohen, Z. D., Kim, T. T., Van, H. L., Dekker, J. J., \& Driessen, E. (2019). A demonstration of a multi-method variable selection approach for treatment selection: Recommending cognitive-behavioral versus psychodynamic therapy for mild to moderate adult depression. Psychotherapy Research, 1-14.

Cuijpers, P., Andersson, G., Donker, T., \& van Straten, A. (2011b). Psychological treatment of depression: results of a series of meta-analyses. Nordic journal of psychiatry, 65(6), 354-364.

Cuijpers, P., Berking, M., Andersson, G., Quigley, L., Kleiboer, A., \& Dobson, K. S. (2013a). A meta-analysis of cognitive-behavioural therapy for adult depression, alone and in comparison with other treatments. The Canadian Journal of Psychiatry, 58(7), 376-385.

Cuijpers, P., Donker, T., Weissman, M. M., Ravitz, P., \& Cristea, I. A. (2016). Interpersonal psychotherapy for mental health problems: a comprehensive meta-analysis. American Journal of Psychiatry, 173(7), 680687.

Cuijpers, P., Geraedts, A. S., van Oppen, P., Andersson, G., Markowitz, J. C., \& van Straten, A. (2011a). Interpersonal psychotherapy for depression: a meta-analysis. American Journal of Psychiatry, 168(6), 581-592.

Cuijpers, P., Hollon, S. D., van Straten, A., Bockting, C., Berking, M., \& Andersson, G. (2013b). Does cognitive behaviour therapy have an enduring effect that is superior to keeping patients on continuation pharmacotherapy? A meta-analysis. BMJ open, 3(4), e002542. 
de Azevedo Cardoso, T., Mondin, T. C., Spessato, B. C., de Avila Quevedo, L., de Mattos Souza, L. D., da Silva, R. A., \& Jansen, K. (2014). The impact of anxious symptoms in the remission of depressive symptoms in a clinical trial for depression: follow-up of six months. J Affect Disord, 168, 331-336. Retrieved from http://www.ncbi.nlm.nih.gov/pubmed/25089513. doi:10.1016/j.jad.2014.03.034

De Graaf, R., Ten Have, M., \& van Dorsselaer, S. (2010). De psychische gezondheid van de Nederlandse bevolking. Nemesis-2: Opzet en eerste resultaten, Trimbos-Instituut, Utrecht.

De Graaf, R., Ten Have, M., Van Gool, C., \& Van Dorsselaer, S. (2012). Prevalentie van psychische aandoeningen en trends van 1996 tot 2009; resultaten van NEMESIS-2. Tijdschrift voor psychiatrie, 54(1), 27.

Deisenhofer, A. K., Delgadillo, J., Rubel, J. A., Böhnke, J. R., Zimmermann, D., Schwartz, B., \& Lutz, W. (2018). Individual treatment selection for patients with posttraumatic stress disorder. Depression and anxiety, 35(6), 541-550.

Delgadillo, J., Gellatly, J., \& Stephenson-Bellwood, S. (2015). Decision making in stepped care: how do therapists decide whether to prolong treatment or not? Behavioural and cognitive psychotherapy, 43(3), 328-341.

Delgadillo, J., Moreea, O., \& Lutz, W. (2016). Different people respond differently to therapy: A demonstration using patient profiling and risk stratification. Behaviour Research and Therapy, 79, 1522.

DeRubeis, R. J., Cohen, Z. D., Forand, N. R., Fournier, J. C., Gelfand, L. A., \& Lorenzo-Luaces, L. (2014). The Personalized Advantage Index: translating research on prediction into individualized treatment recommendations. A demonstration. PloS one, 9(1), e83875.

Eaton, W. W., Shao, H., Nestadt, G., Lee, B. H., Bienvenu, O. J., \& Zandi, P. (2008). Population-based study of first onset and chronicity in major depressive disorder. Archives of general psychiatry, 65(5), 513-520.

Fava, M., Alpert, J. E., Carmin, C. N., Wisniewski, S. R., Trivedi, M. H., Biggs, M. M., . . Rush, A. J. (2004). Clinical correlates and symptom patterns of anxious depression among patients with major depressive disorder in STAR*D. Psychol Med, 34(7), 1299-1308. Retrieved from http://www.ncbi.nlm.nih.gov/pubmed/15697056.

Fava, M., Rankin, M. A., Wright, E. C., Alpert, J. E., Nierenberg, A. A., Pava, J., \& Rosenbaum, J. F. (2000). Anxiety disorders in major depression. Compr Psychiatry, 41(2), 97-102. Retrieved from http://www.ncbi.nlm.nih.gov/pubmed/10741886.

Forand, N. R., \& Derubeis, R. J. (2013). Pretreatment anxiety predicts patterns of change in cognitive behavioral therapy and medications for depression. J Consult Clin Psychol, 81(5), 774-782. Retrieved from http://www.ncbi.nlm.nih.gov/pubmed/23647285. doi:10.1037/a0032985

Forand, N. R., Gunthert, K. C., Cohen, L. H., Butler, A. C., \& Beck, J. S. (2011). Preliminary Evidence that Anxiety is Associated with Accelerated Response in Cognitive Therapy for Depression. Cognitive Therapy and Research, 35(2), 151-160.

Fournier, J. C., DeRubeis, R. J., Shelton, R. C., Hollon, S. D., Amsterdam, J. D., \& Gallop, R. (2009). Prediction of response to medication and cognitive therapy in the treatment of moderate to severe depression. $J$ Consult Clin Psychol, 77(4), 775-787. Retrieved from http://www.ncbi.nlm.nih.gov/pubmed/ 19634969. doi:10.1037/a0015401

Frank, E., Cassano, G. B., Rucci, P., Thompson, W. K., Kraemer, H. C., Fagiolini, A., . . Forgione, R. N. (2011). Predictors and moderators of time to remission of major depression with interpersonal psychotherapy and SSRI pharmacotherapy. Psychol Med, 41(1), 151-162. Retrieved from http://www.ncbi.nlm.nih.gov/pubmed/20380782. doi:10.1017/S0033291710000553

Frank, E., Shear, M. K., Rucci, P., Cyranowski, J. M., Endicott, J., Fagiolini, A., . . Cassano, G. B. (2000). Influence of panic-agoraphobic spectrum symptoms on treatment response in patients with recurrent major depression. Am J Psychiatry, 157(7), 1101-1107. Retrieved from http://www.ncbi.nlm.nih.gov/ pubmed/10873918. doi:10.1176/appi.ajp.157.7.1101

Friborg, O., Martinsen, E. W., Martinussen, M., Kaiser, S., Overgard, K. T., \& Rosenvinge, J. H. (2014). Comorbidity of personality disorders in mood disorders: a meta-analytic review of 122 studies from 1988 to 2010. Journal of affective disorders, 152-154, 1-11. Retrieved from http://www.ncbi.nlm.nih.gov/pubmed/24120406. doi:10.1016/j.jad.2013.08.023 
Fried, E. I., \& Nesse, R. M. (2015). Depression is not a consistent syndrome: an investigation of unique symptom patterns in the STAR* D study. Journal of affective disorders, 172, 96-102.

Friedl, N., Berger, T., Krieger, T., Caspar, F., \& Grosse Holtforth, M. (2019). Using the Personalized Advantage Index for individual treatment allocation to cognitive behavioral therapy (CBT) or a CBT with integrated exposure and emotion-focused elements (CBT-EE). Psychotherapy Research, 1-13.

Gartlehner, G., Gaynes, B. N., Hansen, R. A., Thieda, P., DeVeaugh-Geiss, A., Krebs, E. E., . . . Lohr, K. N. (2008). Comparative benefits and harms of second-generation antidepressants: background paper for the American College of Physicians. Annals of internal medicine, 149(10), 734-750.

Grove, W. M., Zald, D. H., Lebow, B. S., Snitz, B. E., \& Nelson, C. (2000). Clinical versus mechanical prediction: a meta-analysis. Psychological assessment, 12(1), 19.

Health, N. C. C. f. M. (2010). Depression: the treatment and management of depression in adults (updated edition).

Huibers, M. J., Cohen, Z. D., Lemmens, L. H., Arntz, A., Peeters, F. P., Cuijpers, P., \& DeRubeis, R. J. (2015). Predicting Optimal Outcomes in Cognitive Therapy or Interpersonal Psychotherapy for Depressed Individuals Using the Personalized Advantage Index Approach. PloS one, 10(11), e0140771. Retrieved from http://www.ncbi.nlm.nih.gov/pubmed/26554707. doi:10.1371/journal.pone.0140771

Ionescu, D. F., Niciu, M. J., Henter, I. D., \& Zarate, C. A. (2013). Defining anxious depression: a review of the literature. CNS Spectr, 18(5), 252-260. Retrieved from http://www.ncbi.nlm.nih.gov/pubmed/ 23507190. doi:10.1017/S1092852913000114

Kashdan, T. B., \& Roberts, J. E. (2011). Comorbid social anxiety disorder in clients with depressive disorders: predicting changes in depressive symptoms, therapeutic relationships, and focus of attention in group treatment. Behav Res Ther, 49(12), 875-884. Retrieved from http://www.ncbi.nlm.nih.gov/ pubmed/22018535. doi:10.1016/j.brat.2011.10.002

Katsnelson, A. (2013). Momentum grows to make'personalized'medicine more'precise'. In: Nature Publishing Group.

Keefe, J. R., Wiltsey Stirman, S., Cohen, Z. D., DeRubeis, R. J., Smith, B. N., \& Resick, P. A. (2018). In rape trauma PTSD, patient characteristics indicate which trauma-focused treatment they are most likely to complete. Depression and anxiety, 35(4), 330-338.

Kessler, R. C., Berglund, P., Demler, O., Jin, R., Koretz, D., Merikangas, K. R., . . Wang, P. S. (2003). The epidemiology of major depressive disorder: results from the National Comorbidity Survey Replication (NCS-R). Jama, 289(23), 3095-3105.

Klerman, G. L., Weissman, M. M., Rounsaville, B. J., \& Chevron, E. S. (1984). Interpersonal psychotherapy for depression. New York: Basis Books.

Kool, S., Schoevers, R., de Maat, S., Van, R., Molenaar, P., Vink, A., \& Dekker, J. (2005). Efficacy of pharmacotherapy in depressed patients with and without personality disorders: a systematic review and meta-analysis. J Affect Disord, 88(3), 269-278. Retrieved from http://www.ncbi.nlm.nih.gov/pubmed/16165217. doi:10.1016/j.jad.2005.05.017

Kraemer, H. C. (2013). Discovering, comparing, and combining moderators of treatment on outcome after randomized clinical trials: a parametric approach. Statistics in medicine, 32(11), 1964-1973.

Kravitz, R. L., Duan, N., \& Braslow, J. (2004). Evidence-based medicine, heterogeneity of treatment effects, and the trouble with averages. The Milbank Quarterly, 82(4), 661-687.

Lemmens, L., Arntz, A., Peeters, F., Hollon, S., Roefs, A., \& Huibers, M. (2015). Clinical effectiveness of cognitive therapy $v$. interpersonal psychotherapy for depression: results of a randomized controlled trial. Psychological medicine, 45(10), 2095-2110.

Lopez-Gomez, I., Lorenzo-Luaces, L., Chaves, C., Hervas, G., DeRubeis, R. J., \& Vázquez, C. (2019). Predicting optimal interventions for clinical depression: Moderators of outcomes in a positive psychological intervention vs. cognitive-behavioral therapy. General Hospital Psychiatry.

Lorenzo-Luaces, L. (2015). Heterogeneity in the prognosis of major depression: from the common cold to a highly debilitating and recurrent illness. Epidemiology and Psychiatric Sciences, 24(6), 466-472.

Lorenzo-Luaces, L., DeRubeis, R. J., \& Bennett, I. M. (2015). Primary care physicians' selection of lowintensity treatments for patients with depression. Fam Med, 47(7), 511-516. 
Lorenzo-Luaces, L., DeRubeis, R. J., van Straten, A., \& Tiemens, B. (2017). A prognostic index (PI) as a moderator of outcomes in the treatment of depression: A proof of concept combining multiple variables to inform risk-stratified stepped care models. Journal of affective disorders, 213, 78-85.

Luborsky, L., Singer, B., \& Luborsky, L. (1975). Comparative studies of psychotherapies: is it true that everyone has won and all must have prizes? Archives of general psychiatry, 32(8), 995-1008.

Lutz, W., Saunders, S. M., Leon, S. C., Martinovich, Z., Kosfelder, J., Schulte, D., . . . Tholen, S. (2006). Empirically and clinically useful decision making in psychotherapy: Differential predictions with treatment response models. Psychological assessment, 18(2), 133.

McEvoy, P. M., \& Nathan, P. (2007). Effectiveness of cognitive behavior therapy for diagnostically heterogeneous groups: a benchmarking study. J Consult Clin Psychol, 75(2), 344-350. Retrieved from http://www.ncbi.nlm.nih.gov/pubmed/17469892. doi:10.1037/0022-006X.75.2.344

Meehl, P. E. (1954). Clinical versus statistical prediction: A theoretical analysis and a review of the evidence.

Meehl, P. E. (1986). Causes and effects of my disturbing little book. Journal of personality assessment, 50(3), 370-375.

Moffitt, T. E., Caspi, A., Taylor, A., Kokaua, J., Milne, B. J., Polanczyk, G., \& Poulton, R. (2010). How common are common mental disorders? Evidence that lifetime prevalence rates are doubled by prospective versus retrospective ascertainment. Psychological medicine, 40(6), 899-909.

Mulder, R. T. (2002). Personality pathology and treatment outcome in major depression: a review. American Journal of Psychiatry, 159(3), 359-371.

Newton-Howes, G., Tyrer, P., Johnson, T., Mulder, R., Kool, S., Dekker, J., \& Schoevers, R. (2014). Influence of personality on the outcome of treatment in depression: systematic review and meta-analysis. Journal of Personality Disorders, 28(4), 577-593.

Niles, A. N., Loerinc, A. G., Krull, J. L., Roy-Byrne, P., Sullivan, G., Sherbourne, C. D., . . . Craske, M. G. (2017a). Advancing personalized medicine: application of a novel statistical method to identify treatment moderators in the coordinated anxiety learning and management study. Behavior therapy, $48(4), 490-500$.

Niles, A. N., Wolitzky-Taylor, K. B., Arch, J. J., \& Craske, M. G. (2017b). Applying a novel statistical method to advance the personalized treatment of anxiety disorders: A composite moderator of comparative drop-out from CBT and ACT. Behaviour Research and Therapy, 91, 13-23.

Perna, G., Grassi, M., Caldirola, D., \& Nemeroff, C. (2018). The revolution of personalized psychiatry: will technology make it happen sooner? Psychological medicine, 48(5), 705-713.

Pfeiffer, P. N., Ganoczy, D., Ilgen, M., Zivin, K., \& Valenstein, M. (2009). Comorbid anxiety as a suicide risk factor among depressed veterans. Depress Anxiety, 26(8), 752-757. Retrieved from http://www.ncbi.nlm.nih.gov/pubmed/19544314. doi:10.1002/da.20583

Rao, S., \& Zisook, S. (2009). Anxious depression: clinical features and treatment. Curr Psychiatry Rep, 11(6), 429-436. Retrieved from http://www.ncbi.nlm.nih.gov/pubmed/19909663.

Rohde, P., Lewinsohn, P. M., Klein, D. N., Seeley, J. R., \& Gau, J. M. (2013). Key characteristics of major depressive disorder occurring in childhood, adolescence, emerging adulthood, and adulthood. Clinical Psychological Science, 1(1), 41-53.

Rosenzweig, S. (1936). Some implicit common factors in diverse methods of psychotherapy. American journal of Orthopsychiatry, 6(3), 412-415.

Rush, A. J., Trivedi, M. H., Wisniewski, S. R., Nierenberg, A. A., Stewart, J. W., Warden, D., . . Lebowitz, B. D. (2006). Acute and longer-term outcomes in depressed outpatients requiring one or several treatment steps: a STAR* D report. American Journal of Psychiatry, 163(11), 1905-1917.

Shea, M. T., Elkin, I., Imber, S. D., Sotsky, S. M., Watkins, J. T., Collins, J. F., . . Dolan, R. T. (1992). Course of depressive symptoms over follow-up: findings from the National Institute of Mental Health Treatment of Depression Collaborative Research Program. Archives of general psychiatry, 49(10), 782-787.

Simon, G. E., \& Perlis, R. H. (2010). Personalized medicine for depression: can we match patients with treatments? American Journal of Psychiatry, 167(12), 1445-1455.

Smagula, S. F., Wallace, M. L., Anderson, S. J., Karp, J. F., Lenze, E. J., Mulsant, B. H., . . Lotrich, F. E. (2016). Combining moderators to identify clinical profiles of patients who will, and will not, benefit from aripiprazole augmentation for treatment resistant late-life major depressive disorder. Journal of psychiatric research, 81, 112-118. 
Smits, J. A., Minhajuddin, A., \& Jarrett, R. B. (2009). Cognitive therapy for depressed adults with comorbid social phobia. J Affect Disord, 114(1-3), 271-278. Retrieved from http://www.ncbi.nlm.nih.gov/ pubmed/18804285. doi:10.1016/j.jad.2008.08.008

Smits, J. A., Minhajuddin, A., Thase, M. E., \& Jarrett, R. B. (2012). Outcomes of acute phase cognitive therapy in outpatients with anxious versus nonanxious depression. Psychother Psychosom, 81(3), 153160. Retrieved from http://www.ncbi.nlm.nih.gov/pubmed/22398963. doi:10.1159/000334909

Solomon, D. A., Keller, M. B., Leon, A. C., Mueller, T. I., Lavori, P. W., Shea, M. T., . . Maser, J. D. (2000). Multiple recurrences of major depressive disorder. American Journal of Psychiatry, 157(2), 229-233.

Spijker, J., Bockting, C., Meeuwissen, J., Van Vliet, I., Emmelkamp, P., Hermens, M., \& van Balkom, A. (2013). Multidisciplinaire richtlijn Depressie (Derde revisie): Richtlijn voor de diagnostiek, behandeling en begeleiding van volwassen patiënten met een depressieve stoornis. Trimbos Instituut: Utrecht.

Takayanagi, Y., Spira, A. P., Roth, K. B., Gallo, J. J., Eaton, W. W., \& Mojtabai, R. (2014). Accuracy of reports of lifetime mental and physical disorders: results from the Baltimore Epidemiological Catchment Area study. JAMA psychiatry, 71(3), 273-280.

Ten Have, M., Lamers, F., Wardenaar, K., Beekman, A., de Jonge, P., van Dorsselaer, S., . . . de Graaf, R. (2016). The identification of symptom-based subtypes of depression: A nationally representative cohort study. I Affect Disord, 190, 395-406. Retrieved from http://www.ncbi.nlm.nih.gov/ pubmed/26546775. doi:10.1016/j.jad.2015.10.040

Thornicroft, G., Chatterji, S., Evans-Lacko, S., Gruber, M., Sampson, N., Aguilar-Gaxiola, S., . . Borges, G. (2017). Undertreatment of people with major depressive disorder in 21 countries. The British Journal of Psychiatry, 210(2), 119-124.

Tomba, E., \& Fava, G. A. (2012). Treatment selection in depression: the role of clinical judgment. Psychiatric Clinics, 35(1), 87-98.

Uher, R., Perlis, R., Henigsberg, N., Zobel, A., Rietschel, M., Mors, O., . . . Bajs, M. (2012). Depression symptom dimensions as predictors of antidepressant treatment outcome: replicable evidence for interest-activity symptoms. Psychological medicine, 42(5), 967-980.

van der Veen, D. C., Comijs, H. C., van Zelst, W. H., Schoevers, R. A., \& Oude Voshaar, R. C. (2014). Defining anxious depression in later life: a scaring heterogeneity in results. Am J Geriatr Psychiatry, 22(11), 1375-1378. Retrieved from http://www.ncbi.nlm.nih.gov/pubmed/24703569. doi:10.1016/ j.jagp.2014.02.012

van Loo, H. M., Aggen, S. H., Gardner, C. O., \& Kendler, K. S. (2015). Multiple risk factors predict recurrence of major depressive disorder in women. J Affect Disord, 180, 52-61. Retrieved from http://www.ncbi.nlm.nih.gov/pubmed/25881281. doi:10.1016/j.jad.2015.03.045

Vittengl, J. R., Clark, L. A., Dunn, T. W., \& Jarrett, R. B. (2007). Reducing relapse and recurrence in unipolar depression: a comparative meta-analysis of cognitive-behavioral therapy's effects. Journal of consulting and clinical psychology, 75(3), 475.

Vittengl, J. R., Clark, L. A., Thase, M. E., \& Jarrett, R. B. (2017). Initial Steps to inform selection of continuation cognitive therapy or fluoxetine for higher risk responders to cognitive therapy for recurrent major depressive disorder. Psychiatry research, 253, 174-181.

Wallace, M. L., Frank, E., \& Kraemer, H. C. (2013). A novel approach for developing and interpreting treatment moderator profiles in randomized clinical trials. JAMA psychiatry, 70(11), 1241-1247.

Wang, J., Sareen, J., Patten, S., Bolton, J., Schmitz, N., \& Birney, A. (2014). A prediction algorithm for first onset of major depression in the general population: development and validation. J Epidemiol Community Health, 68(5), 418-424. Retrieved from http://www.ncbi.nlm.nih.gov/pubmed/24391206. doi:10.1136/jech-2013-202845

Warden, D., Rush, A. J., Wisniewski, S. R., Lesser, I. M., Kornstein, S. G., Balasubramani, G., . . Young, E. A. (2009). What predicts attrition in second step medication treatments for depression?: a STAR* D Report. International Journal of Neuropsychopharmacology, 12(4), 459-473.

Wardenaar, K. J., \& de Jonge, P. (2013). Diagnostic heterogeneity in psychiatry: towards an empirical solution. BMC medicine, 11(1), 201.

Webb, C. A., Trivedi, M. H., Cohen, Z. D., Dillon, D. G., Fournier, J. C., Goer, F., . . . Parsey, R. (2018). Personalized prediction of antidepressant v. placebo response: evidence from the EMBARC study. Psychological medicine, 1-10. 
WHO. (2017). Other Common Mental Disorders: Global Health Estimates. Geneva: World Health Organization, 1-24.

Wilhelm, K., Mitchell, P. B., Niven, H., Finch, A., Wedgwood, L., Scimone, A., . . Schofield, P. R. (2006). Life events, first depression onset and the serotonin transporter gene. The British Journal of Psychiatry, 188(3), 210-215.

Young, J. F., Mufson, L., \& Davies, M. (2006). Impact of comorbid anxiety in an effectiveness study of interpersonal psychotherapy for depressed adolescents. J Am Acad Child Adolesc Psychiatry, 45(8), 904-912. Retrieved from http://www.ncbi.nlm.nih.gov/pubmed/16865032. doi:10.1097/01.chi. $0000222791.23927 .5 f$

Zilcha-Mano, S., Keefe, J. R., Chui, H., Rubin, A., Barrett, M. S., \& Barber, J. P. (2016). Reducing Dropout in Treatment for Depression: Translating Dropout Predictors Into Individualized Treatment Recommendations. The Journal of clinical psychiatry, 77(12), e1584-e1590. 
Part

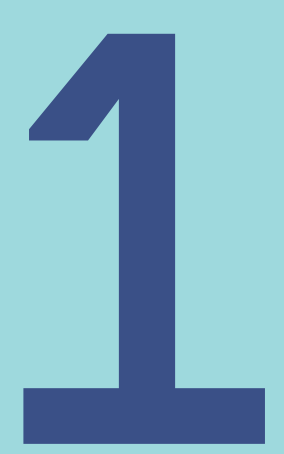




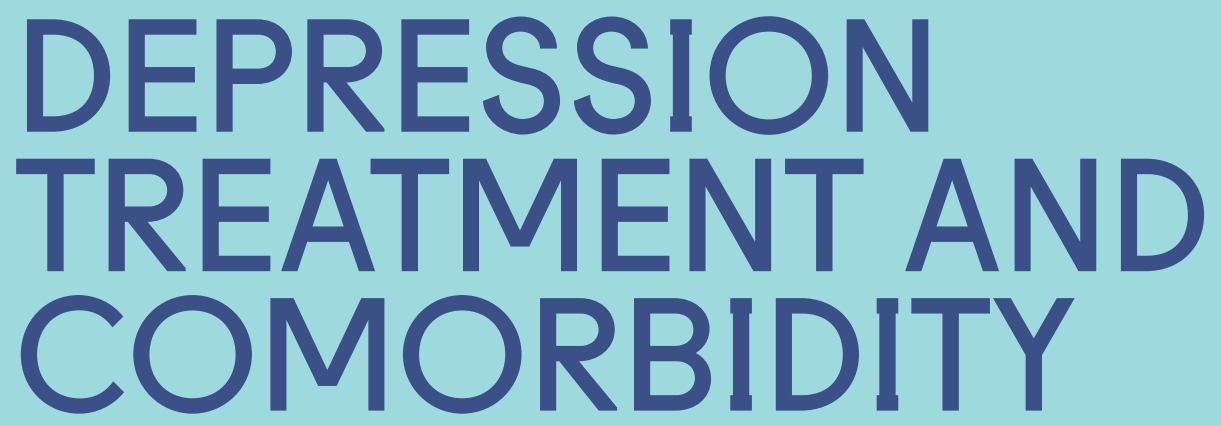


S. van Bronswijk, L. Lemmens, M. Huibers, A. Arntz, F. Peeters

- Journal of Affective Disorders 2018,232,52-60

\section{Chapter}

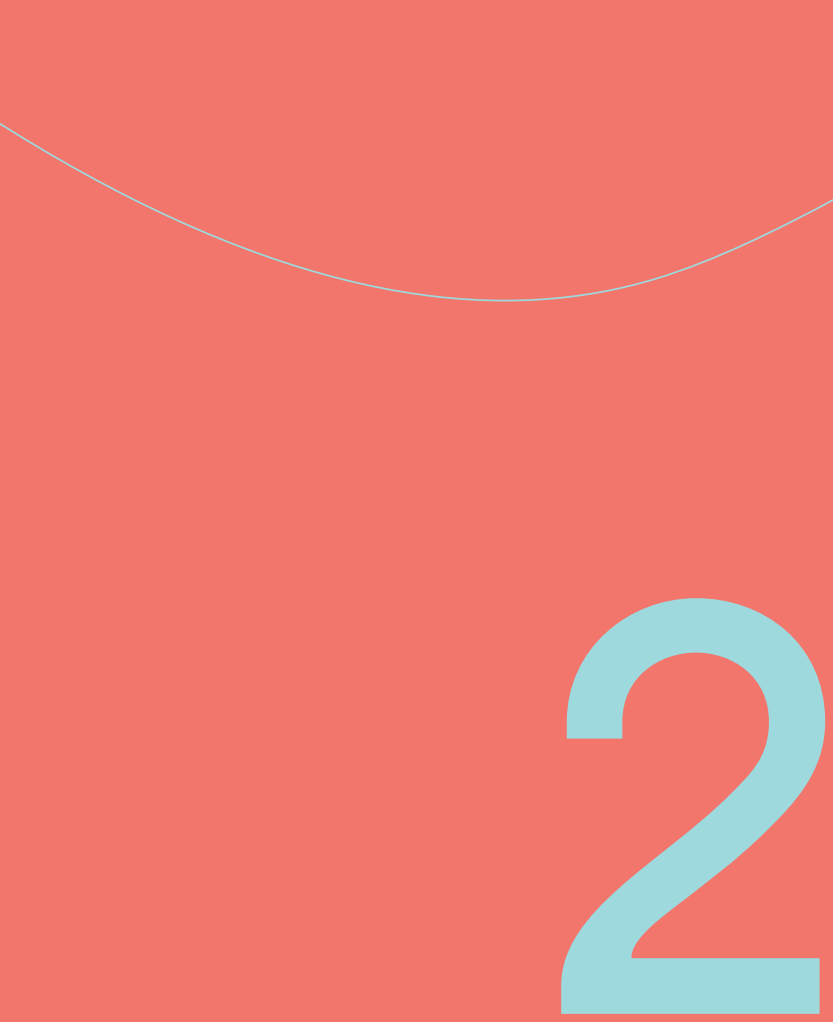




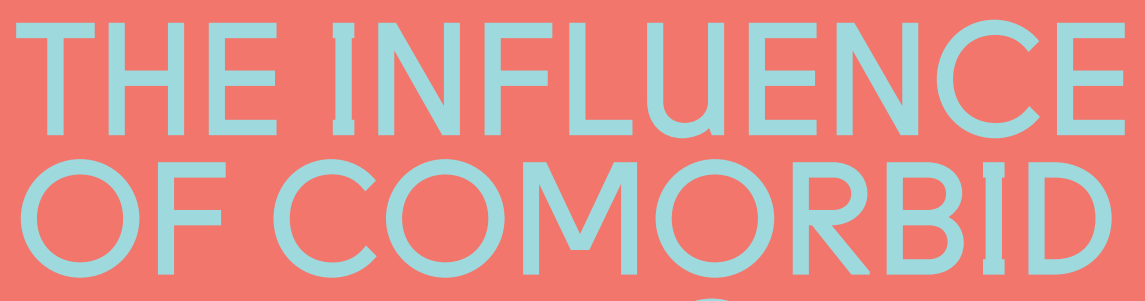

ANXIETY ON THE

EFFECTIVENESS

OF COGNITIVE

THERAPY AND

INTERPERSONAL

PSYCHOTHERAPY

FOR MAJOR

DEPRESSIVE

DISORDER 


\section{Abstract}

\section{Background}

Anxious depression is an important subtype of major depressive disorder (MDD) defined by both syndromal (anxiety disorders) and dimensional (anxiety symptoms) criteria. A debated question is how anxiety affects MDD treatment. This study examined the impact of comorbid anxiety disorders and symptoms on the effectiveness of and dropout during cognitive therapy (CT) and interpersonal psychotherapy (IPT) for MDD.

\section{Methods}

Depressed individuals were randomized to CT $(n=76)$ or IPT $(n=75)$. Outcome was depression severity measured with the Beck Depression Inventory-II (BDI-II) at the start of each therapy session, post treatment, and monthly up to five months follow-up. Anxiety disorders were assessed with the Structured Clinical Interview for DSM-IV Axis I disorders, (phobic) anxiety symptoms were assessed with Brief Symptom Inventory subscales.

\section{Results}

Approximately one third of participants had a comorbid anxiety disorder. Comorbid anxiety disorders and anxiety symptoms were associated with less favorable depression change during IPT as compared to CT in the treatment phase, but not in the trial follow-up phase. Individuals with a comorbid anxiety disorder had significantly higher treatment dropout during both treatments.

\section{Conclusions}

A preference for CT over IPT for MDD is justifiable when comorbid anxiety is present, although long-term differences are not established and replication of this finding is needed. Clinicians should be aware of the risk of dropout for depressed individuals with an anxiety disorder. 


\section{Introduction}

Given high comorbid anxiety in major depressive disorder (MDD), anxious depression is considered to be an important subtype (American Psychiatric Association, 2013; Rao, 2009; Ten Have et al., 2016). This subtype can be defined as MDD with a comorbid anxiety disorder (syndromal definition) or subthreshold comorbid anxiety symptoms (dimensional definition) (Ionescu, Niciu, Henter, \& Zarate, 2013; Rao, 2009). For both definitions, anxious depression has been associated with a severe clinical picture including more severe depressive symptoms (Brown, Schulberg, Madonia, Shear, \& Houck, 1996; Fava et al., 2004; Penninx et al., 2011; Smits, Minhajuddin, \& Jarrett, 2009), more functional impairment (Fava et al., 2004; Smits et al., 2009) and intensified suicidal thoughts and behavior (Brown et al., 1996; Fava et al., 2004; Fawcett, Scheftner, Fogg, Clark, \& Young, 1990; Pfeiffer, Ganoczy, Ilgen, Zivin, \& Valenstein, 2009). With these profound clinical disadvantages, one could expect an adverse effect of comorbid anxiety on MDD treatment outcome and treatment continuation. However, findings regarding the impact of comorbid anxiety on MDD treatment outcome and dropout are inconsistent for both pharmacotherapy and psychotherapy, and there has been little agreement on tailored treatment recommendations.

Studies examining the effects of antidepressants found that individuals with comorbid anxiety disorders had less favorable outcomes (Howland et al., 2009; Lee et al., 2012) and higher dropout rates (Arnow et al., 2007; Howland et al., 2009). In contrast to anxiety disorders, the impact of comorbid anxiety symptoms on MDD outcome varied from lower response and remission rates (Davidson, Meoni, Haudiquet, Cantillon, \& Hackett, 2002; Fava et al., 2008; Flint \& Rifat, 1997; Frank et al., 2011; Papakostas et al., 2008; Wiethoff et al., 2010) to no adverse effects (Forand \& DeRubeis, 2013; Fournier et al., 2009; Joffe, Bagby, \& Levitt, 1993; Nelson, 2010; Russell et al., 2001; Tollefson, Holman, Sayler, \& Potvin, 1994). In addition, anxiety symptoms were not associated with dropout in other antidepressant trials (Flint \& Rifat, 1997; Fournier et al., 2009; Russell et al., 2001; Tollefson et al., 1994; Wiethoff et al., 2010).

In psychotherapy research, findings on the influence of comorbid anxiety on treatment outcome and continuation are even more inconclusive. Two effective and frequently applied psychotherapies for MDD are cognitive therapy (CT) and interpersonal psychotherapy (IPT). Most of the psychotherapy studies that examined the impact of comorbid anxiety on MDD outcome focused on CT. Overall, CT studies did not show an adverse effect of anxiety on MDD outcome. Several studies showed similar treatment outcomes for individuals with and without anxiety disorders or with and without anxiety symptoms (Brent et al., 1998; Forand \& DeRubeis, 2013; Fournier et al., 2009; McEvoy \& Nathan, 2007; Persons, Roberts, Zalecki, \& Brechwald, 2006; Smits et al., 2009). Surprisingly, other CT studies reported a more accelerated 
depressive symptom improvement when comorbid anxiety disorders or symptoms were present (de Azevedo Cardoso et al., 2014; Forand, Gunthert, Cohen, Butler, \& Beck, 2011; Kashdan \& Roberts, 2011; Rohde, Clarke, Lewinsohn, Seeley, \& Kaufman, 2001; Smits, Minhajuddin, Thase, \& Jarrett, 2012). With regard to treatment continuation, in the majority of CT studies, anxiety disorders (Forand et al., 2011; Kashdan \& Roberts, 2011; Rohde et al., 2001; Schindler, Hiller, \& Witthöft, 2013; Smits et al., 2009) and anxiety symptoms (Forand et al., 2011; Fournier et al., 2009; McEvoy \& Nathan, 2007; Smits et al., 2012) did not affect treatment dropout, although one study reported higher dropout rates for individuals with a comorbid anxiety disorder (Arnow et al., 2007). For IPT, fewer findings on the impact of comorbid anxiety on MDD treatment outcome and completion are available. In contrast to $\mathrm{CT}$, findings of these studies suggest an adverse effect of (lifetime) anxiety disorders and anxiety symptoms on MDD outcome (Brown et al., 1996; Frank et al., 2011; Frank et al., 2000; Young, Mufson, \& Davies, 2006). In addition, one study reported higher dropout rates for individuals with lifetime anxiety disorders as compared to individuals without lifetime anxiety disorders (Brown et al., 1996). To date, there are no head-to-head comparisons of CT and IPT focusing on anxious depression, although one study showed that axis I disorders in general did not predict (differential) MDD treatment outcomes for CT and IPT (Carter et al., 2011).

A possible explanation for these mixed results is the use of different definitions to classify anxious depression (Ionescu et al., 2013). This concern is illustrated by a recent study that showed that the syndromal and dimensional definitions of anxious depression classify two different groups of individuals under the same "anxious depression" label (van der Veen, Comijs, van Zelst, Schoevers, \& Oude Voshaar, 2014). This lack of overlap is not surprising; the syndromal definition refers to two distinct disorders, while the dimensional criteria define a certain subtype of depression. These different conceptualizations of anxious depression are however a major concern for integrating the outcomes of previous studies into broader knowledge and translating findings to clinical practice. In addition, mixed results could be explained by small study samples and the different measures of anxiety disorders and anxiety symptoms. Anxiety disorders diagnoses are measured both lifetime and current, with different types of instruments. Furthermore, diagnosing a distinct anxiety disorder from an acute phase depression can be challenging. For anxiety symptoms, some studies used specific anxiety oriented instruments while others used the Anxiety/ Somatization Factor of the Hamilton Rating Scale for Depression (Fava et al., 2000).

The aim of this study was to examine the (differential) effect of comorbid anxiety on the effectiveness and treatment continuation of CT and IPT for MDD in a head-tohead comparison. In order to confirm the assumption that the syndromal definition of anxious depression is different from the dimensional one, the impact of anxiety disorders, anxiety symptoms (i.e. generalized anxiety symptoms), and phobic anxiety symptoms (i.e. agoraphobic symptoms) was examined separately. Based on previous 
studies, we expected no adverse effect of comorbid anxiety on MDD treatment outcomes and completion for CT. Based on the limited research available for IPT, one could expect less favorable outcomes and higher dropout rates for individuals with comorbid anxiety disorders or symptoms treated with IPT.

\section{Methods}

\subsection{Study design}

Data were collected in the context of a randomized controlled trial examining the effectiveness of CT and IPT for MDD. Individuals with a primary MDD diagnosis as confirmed with the Structured Clinical Interview for DSM-IV Axis I disorders (SCID-I, First, Spitzer, Gibbon, \& Williams, 1995) by trained clinicians were recruited from the mood disorders unit of the Maastricht Outpatient Mental Health Center (RIAGG Maastricht). Additional inclusion criteria were: internet access, an email address, and sufficient knowledge of the Dutch language. Exclusion criteria were a bipolar or chronic depression (current episode $>5$ years), elevated acute suicide risk, concomitant pharmacological or psychological treatment, drugs and alcohol abuse/dependence, and mental retardation $(\mathrm{IQ}<80)$. A total of 1562 individuals were screened for eligibility. Of these 1562 individuals, 1191 did not meet the inclusion criteria (main reasons were the use of antidepressant medication ( $n=362$ ) or MDD not being the primary diagnosis $(n=434), 78$ patients met inclusion criteria but refused to participate, and 111 were excluded for other reasons. A total of 182 depressed outpatients were randomly allocated to CT $(n=76)$, IPT $(n=75)$, or a 2-month waitinglist control condition followed by treatment of choice $(n=31)$. For the current analyses, we limited the sample to data of individuals randomized to the active conditions CT and IPT ( $n=151)$ in the acute phase of therapy (month $0-7)$ and a trial follow-up phase (month 7-12). The study was approved by the Medical Ethics Committee of Maastricht University Medical Center, written informed consent was obtained, and the study was registered at isrctn.com (identifier: ISRCTN 67561918). A detailed description about the study design and main outcomes are provided elsewhere (Lemmens et al., 2015; Lemmens et al., 2011).

\subsection{Treatments and therapists}

CT and IPT were carried out using the guidelines by Beck et al. (1979) for CT, and the guidelines by Klerman et al. (1984) for IPT. Participants received 16-20 sessions of 45 min (17 sessions on average, SD=2.9) by ten experienced licensed psychologist, psychotherapists, and psychiatrists (9.1 years of clinical experience on average, $\mathrm{SD}=5.4$, range $4-21$ years). Therapists were already trained in the treatment modality 
that they delivered in the study, but received additional training $(2 \times 8 \mathrm{~h})$ by Steven Hollon (CT) and John Markowitz (IPT) prior to the start of the study, and delivered exclusively CT or IPT. Sessions were scheduled weekly and allowed to be less frequent planned towards the end of therapy. All sessions were videotaped and a random selection of 106 tapes were examined on treatment competence (i.e. quality of treatment) and adherence (i.e. therapy-specific behavior) by independent raters. Treatment competence was rated good to excellent using the Cognitive Therapy Scale for CT (Dobson, Shaw, \& Vallis, 1985, mean=3.31, SD=0.93) and the short version of the IPT Adherence and Quality Scale for IPT (Stuart, 2011, mean=3.50, SD=0.70). The Collaborative Study Psychotherapy Rating Scale version 6 (Hollon et al., 1988; Hollon, Evans, Elkin, \& Lowery, 1984) indicated significant differences in therapy-specific behavior between CT and IPT with higher CT-specific behavior in CT as compared to IPT (mean=80.80, SD=25.64 vs. mean=52.42, SD =13.00 $\mathrm{t}=7.23, \mathrm{p}<0.001$ ), and higher IPT-specific behavior in IPT as compared to CT (mean=85.75, SD=23.22 vs. mean=44.57, $S D=15.06, t=10.79, p<0.001$ ) (Lemmens et al., 2015). Anxiety disorders, phobic anxiety symptoms and anxiety symptoms did not change quality of treatment or therapy-specific behavior of the therapists (results not shown, all $p$-values>0.3).

\subsection{Measures}

\subsubsection{Primary outcome}

The primary outcome, depression severity was measured with the Beck Depression Inventory, second edition (Beck, Steer, \& Brown, 1996), a 21-item self-report instrument. The total score was obtained by summing up the items, ranging between 0 and 63 , with a higher score indicated more severe depressive symptoms. The BDI-II has shown to be a strong screening measure for depression with high reliability and improved concurrent, content, and structural validity (Wang \& Gorenstein, 2013). The $\mathrm{BDI}-\mathrm{II}$ was repeatedly assessed across the treatment phase (BDI-II measures at the start of each therapy session), and the subsequent trial follow-up phase (BDI-II measures at 7, 8, 9, 10, 11 and 12 months).

\subsubsection{Comorbid anxiety}

Anxiety disorders were assessed at baseline using the results of the SCID-I (First et al., 1995). Prior to treatment, anxiety symptoms and phobic anxiety symptoms were measured with the Brief Symptom Inventory (BSI) anxiety subscale and the phobic anxiety subscale representing generalized anxiety symptoms and agoraphobic symptoms respectively. Higher scores indicated more severe symptoms (De Beurs, 2009; Derogatis \& Melisaratos, 1983). The BSI is a 53-item self-report instrument that derived from the Symptom Checklist 90 Revised and has demonstrated to have good psychometric properties (De Beurs \& Zitman, 2005; Derogatis \& Melisaratos, 1983; 
Khalil, Hall, Moser, Lennie, \& Frazier, 2011). Therapists and participants were blind to the results of the BSI (anxiety symptoms), but not to the outcomes of the SCID-I assessment (anxiety disorders).

\subsection{Statistical analysis}

To examine pre-treatment characteristics, cutoffs for low and high (phobic) anxiety symptoms were used based on the Dutch outpatients population (De Beurs, 2009). Pre-treatment characteristics and (study and treatment ${ }^{1}$ ) dropout rates of individuals with and without comorbid anxiety disorders, with low and high anxiety symptoms, and with low and high phobic anxiety symptoms were examined with t-tests and $\chi^{2}$ tests where appropriate. To examine the differential impact of anxiety disorders, anxiety symptoms, and phobic anxiety symptoms on study and treatment dropout for CT and IPT, logistic regression models were applied. To confirm that the syndromal definition of anxious depression differs from the dimensional one, anxiety symptoms (standardized continuous score of the BSI anxiety subscale) and phobic anxiety symptoms (standardized continuous score of the BSI phobic anxiety subscale) were compared between individuals with and without an anxiety disorder (t-test with Cohen's d). In addition, subgroups of individuals who met the criteria for the syndromal and dimensional definition were identified, and overlap between these groups was examined with a kappa statistics ${ }^{2}$.

To analyze the impact of anxiety disorders, anxiety symptoms (standardized continuous score of the BSI anxiety subscale) and phobic anxiety symptoms (standardized continuous score of the BSI phobic anxiety subscale) on BDI-II change across treatment (measured at the start of each therapy session) and follow-up (measured at 7, 8, 9, 10, 11 and 12 months), mixed-effects models were used (Diggle et al., 2002). Analyses were intention-to-treat, using all available data of the 151 study participants. Since sessions were allowed to be scheduled in a flexible manner, for some participants, there was a slight overlap between treatment and trial follow-up phase ( 1 or 2 sessions). Due to this overlap, BDI-II change was modeled separately for each phase. The following fixed effects were included in the models: standardized BDI-II baseline scores (to adjust for different BDI-II scores prior to treatment (Lemmens et al., 2015)), time (treatment phase model: number of sessions; trial follow-up phase model: time in months), treatment (centered at $\mathrm{CT}=-0.5$ or IPT=0.5),

1 Treatment dropout was defined as discontinuation of treatment; study dropout was defined as discontinuation of study participation.

2 For the dimensional criteria (MDD plus subthreshold anxiety symptoms) the BSI anxiety subscale was used and not the BSI phobic anxiety subscale. We choose this subscale since these items overlapped most with the somatic and physic anxiety subscales of the Hamilton Depression Rating Scale and Hamilton Anxiety Rating Scale that are often used to assess the dimensional criteria. Since guidelines for the appropriate thresholds of the BSI anxiety subscale for the dimensional definition were lacking, the analyses were repeated with different cutoffs based on a Dutch outpatient population (de Beurs, 2009). 
and a time-by-treatment interaction (difference between CT and IPT over time). Anxiety disorder diagnosis (centered at no diagnosis $=-0.5$ or one or more $=0.5$ ), anxiety symptoms (standardized continuous score of the BSI anxiety subscale) and phobic anxiety symptoms (standardized continuous score of the BSI phobic anxiety subscale) were subsequently added to these models together with their interactions with treatment, time and treatment-by time. Since previous studies showed that anxiety could affect different types of depression change (i.e. early rapid change instead of overall change) (Forand \& DeRubeis, 2013; Forand et al., 2011), different transformations of time (linear quadratic, loglinear) were assessed for each model with fit indices and visual inspection. For the acute phase model with anxiety disorder diagnosis as a predictor, a curvilinear parametrization of time (linear and quadratic slope) was considered the best fit. All other models had the best fit with only linear slopes. For these linear models, the endpoint of time was coded as zero (treatment phase model: session 20; trial follow-up phase model: month 12). To reduce multicollinearity between the linear and quadratic slopes, time was centered midway for the curvilinear model (treatment phase model: session 10) (Forand et al., 2011). For all models, intercepts and slopes were allowed to be correlated and vary randomly over individuals. An autoregressive covariance structure for the residuals was modeled, with a correction for the irregularly spaced time lags between the sessions (Jones, 1993). Backwards elimination was applied when the anxiety-by-treatment-bytime-interaction was not significant ( $p>0.05)$.

For all models, we conducted a sensitivity analysis by subsequently adding the following variables measured at baseline to the models: therapist, demographics (age, gender, employment, level of education), clinical features (first or recurrent depression) and functionality (Work and Social Adjustment Scale (W\&SAS); Mundt, Marks, Shear, \& Greist, 2002), the RAND-36 (van der Zee \& Sanderman, 1993) and the EuroQol-6D (EQ-6D; EuroQol, 1990) for the EQ-5D.

\section{Results}

\subsection{Descriptive statistics}

Pre-treatment characteristics are shown in Table 2.1. As described by Lemmens et al. (2015), the baseline EQ-5D and BDI-II were borderline significantly higher in CT as compared to IPT ( $t=2.00, d f=148, p=0.05$ and $t=1.90, d f=149, p=0.06)$ for the complete sample. There were no significant differences between the two conditions in treatment ( $n=36$, CT n=20, IPT $n=16)$ and study ( $n=23$, CT $n=20$, IPT $n=16)$ dropout. 


\subsubsection{Anxiety disorders}

Forty-three out of 151 individuals were diagnosed with one ( $n=35 ; 23.2 \% ; C T=19$, IPT=16) or two ( $n=8 ; 5.3 \% ; C T=5, I P T=3$ ) anxiety disorders. The number of individuals with anxiety disorders did not differ between the two conditions ( $C T=24, I P T=19$, $\left.\chi^{2}=0.72, d f=1, p=0.40\right)$. The most prevalent anxiety disorder was panic disorder $(n=18)$, followed by social phobia $(n=16)$, specific phobia $(n=7)$, post-traumatic stress disorder $(n=6)$, generalized anxiety disorder $(n=2)$, agoraphobia without panic disorder $(n=1)$, obsessive compulsive disorder $(n=1)$ and anxiety disorder not otherwise specified $(n=1)$. As shown in Table 2.1, individuals with a comorbid anxiety disorder were significantly younger compared to individuals without a comorbid anxiety disorder $(t=2.62, d f=149, p=0.0098)$.

\subsubsection{Anxiety symptoms and phobic anxiety symptoms}

Thirty-six of 151 individuals scored above the 'high anxiety symptoms cutoff' (De Beurs, 2009) on the BSI anxiety subscale and the number of individuals with high anxiety symptoms did not significantly differ between CT and IPT (CT=17, IPT=19, $\chi^{2}=0.18, d f=1, p=0.67$ ). For the BSI phobic anxiety subscale, 26 of 151 individuals scored above the 'high phobic anxiety symptoms cutoff' (De Beurs, 2009) and appeared to be equally divided between CT and IPT as well (CT=13, IPT=13, $\chi^{2}=0.0014$, $\mathrm{df}=1, \mathrm{p}=0.970$ ). As presented in Table 2.1, participants with high anxiety symptoms and high phobic anxiety symptoms were significantly younger $(t=2.30, d f=149, p=0.03$ and $t=2.59, d f=149, p=0.01)$, had higher baseline depression severity $(t=-6.23, d f=149$, $p<0.001$ and $t=-3.88, d f=149, p=0.002$ ), and lower quality of life measured with the EQ-5D ( $t=3.17, d f=148, p=0.002$ and $t=2.19, d f=148, p=0.03)$ as compared to individuals with low anxiety symptoms and low phobic anxiety symptoms respectively. 


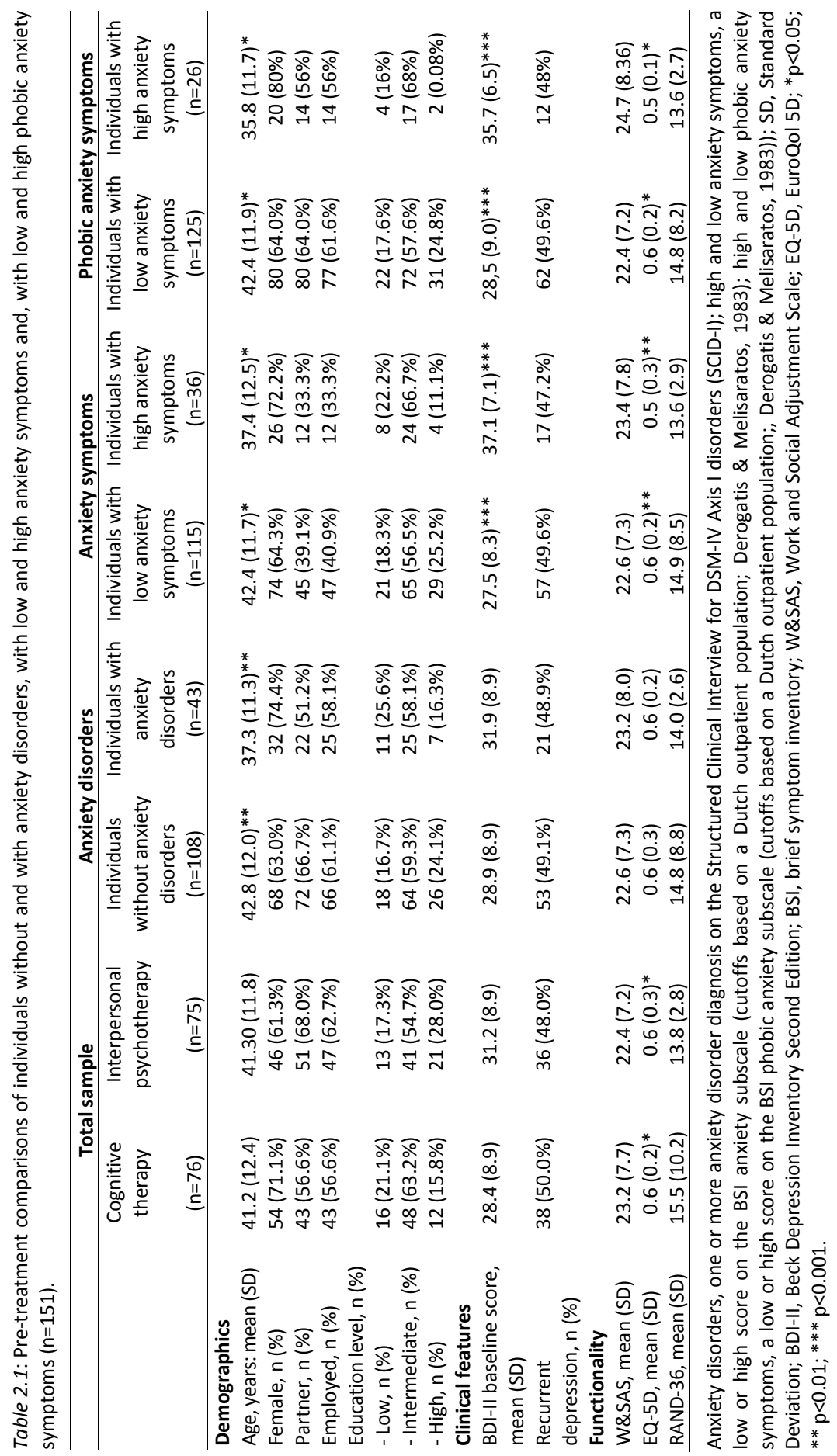




\subsection{Comparing the syndromal and dimensional definition of anxious depression}

Individuals with an anxiety disorder scored significantly higher on the BSI anxiety subscale as compared to individuals without an anxiety disorder $(M=1.58, S D=0.81$ vs. $M=1.29, S D=0.79, t=-2.0, d f=149, p=0.05$, Cohen's $d=-0.36$ ). In addition, scores on the BSI phobic anxiety subscale were higher for individuals with an anxiety disorder compared to individuals without an anxiety disorder $(M=1.36, S D=0.81$ vs. $M=0.81$, $\mathrm{SD}=0.71, \mathrm{t}=-4.13, \mathrm{df}=149, \mathrm{p}=0.0001$, Cohen's $\mathrm{d}=-0.74)$. However, when dividing the sample into subgroups of syndromal and dimensional defined anxious depression and non-anxious depression, little agreement was found between the syndromal and dimensional subgroups with kappa statistics ranging between -0.003 and 0.16 .

\subsection{The impact of anxiety disorders on BDI-II change and dropout}

Table 2.2 provides the estimates of the mixed-effect models examining the impact of anxiety disorders on BDI-II change for the treatment phase (0-7 months) and the trial follow-up phase (7-12 months). For the treatment phase model, there was a significant three way interaction between anxiety disorder status, condition and the quadratic time slope, indicating higher BDI-II scores for individuals with an anxiety disorder receiving IPT as compared to CT. This effect is illustrated by Figure 2.1, where the raw means of the BDI-II scores are plotted and show a curvilinear unfavorable course for IPT as compared to the CT group. This differential effect was not found in the trial follow-up phase. Sensitivity analyses did not change these results.

Cognitive Therapy

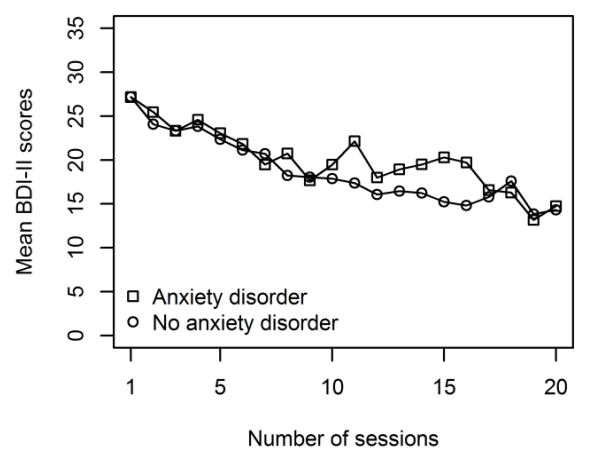

Interpersonal Psychotherapy

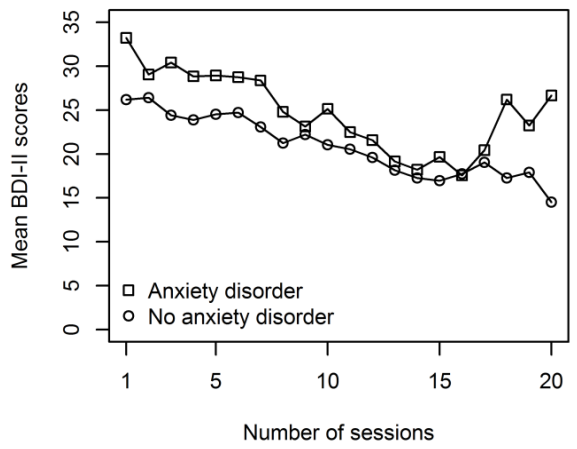

Figure 2.1: Mean Beck Depression Inventory - second edition (BDI-II) scores for individuals with and without an anxiety disorder for cognitive therapy and interpersonal psychotherapy during the treatment phase. Data were available for 149, 142, 124, 89 and 25 individuals at session 1, 5, 10, 15 and 20 respectively (partly due to treatment termination). 


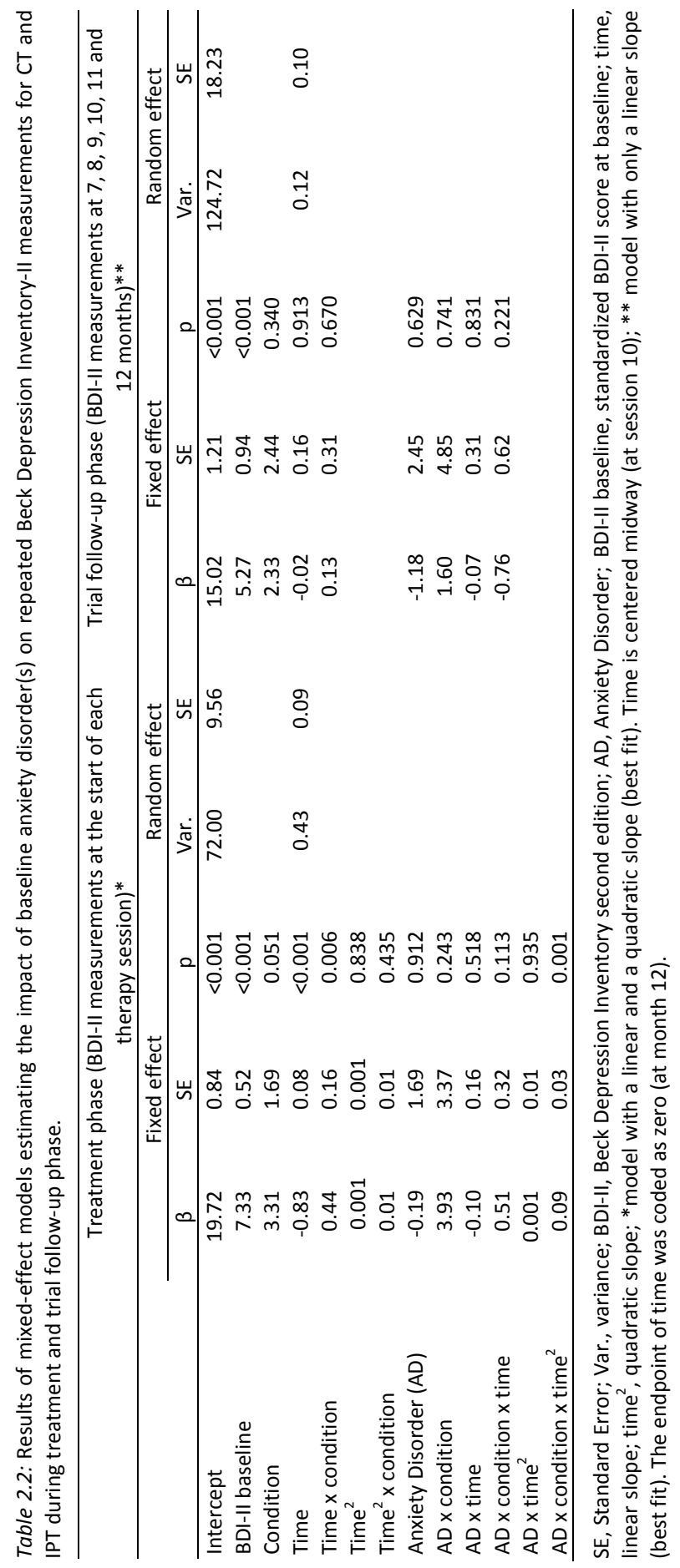


For individuals with a comorbid anxiety disorder the proportion of treatment dropouts was significantly higher $(n=17,39.5 \%)$ as compared to individuals without a comorbid anxiety disorder $\left(n=19,17.6 \%, \chi^{2}=8.16, d f=1, p=0.004\right)$, although this difference was not found for study dropouts $\left(\chi^{2}=1.51, d f=1, p=0.219\right)$. As indicated by the non-significant treatment by anxiety disorder interactions in the logistic regression models, no differential effects between CT and IPT were found for both treatment ( $\beta=0.67, p=0.409)$ and study $(\beta=-0.23, p=0.807)$ dropout.

\subsection{The impact of anxiety symptoms and phobic anxiety symptoms on BDI-Il change and dropout}

Table 2.3 summarizes the effects of anxiety symptoms (standardized continuous score of the BSI anxiety subscale) and phobic anxiety symptoms (standardized continuous score of the BSI phobic anxiety subscale) on BDI-II change as estimated with separate linear mixed-effects models. Initially, there was no (differential) effect of anxiety symptoms on BDI-II change in both conditions during treatment and trial follow-up phase. However, after eliminating the anxiety symptoms $x$ time $x$ condition interaction from the treatment phase model, a significant anxiety symptoms $\mathrm{x}$ condition interaction appeared, indicating overall higher BDI-II scores for IPT compared to CT. Figure 2.2 illustrates this lower order effect with mean BDI-II scores during the treatment phase.

Cognitive Therapy

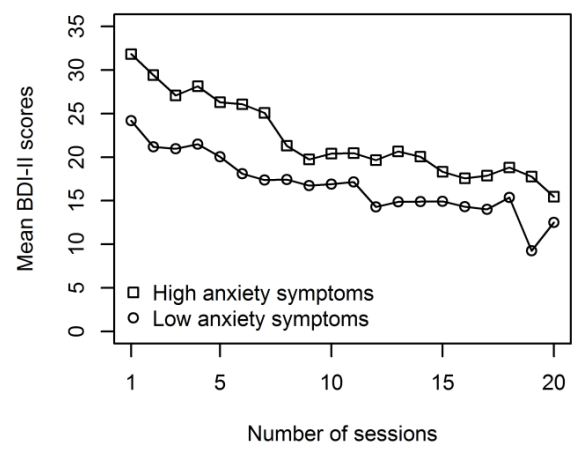

Interpersonal Psychotherapy

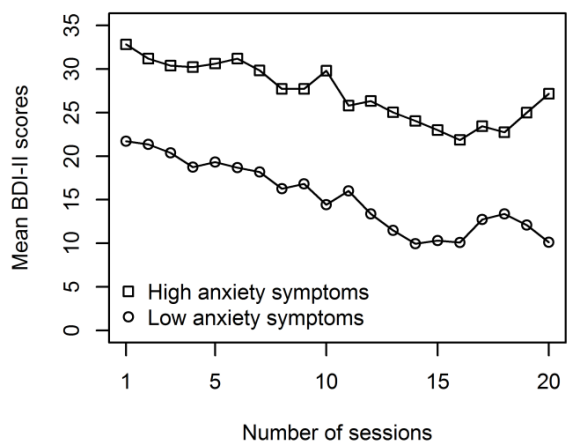

Figure 2.2: Mean Beck Depression Inventory - second edition (BDI-II) scores for individuals with low and high anxiety symptoms for cognitive therapy and interpersonal psychotherapy during the treatment phase. Data were available for 149, 142, 124, 89 and 25 individuals at session 1, 5, 10, 15 and 20 respectively (partly due to treatment termination). 


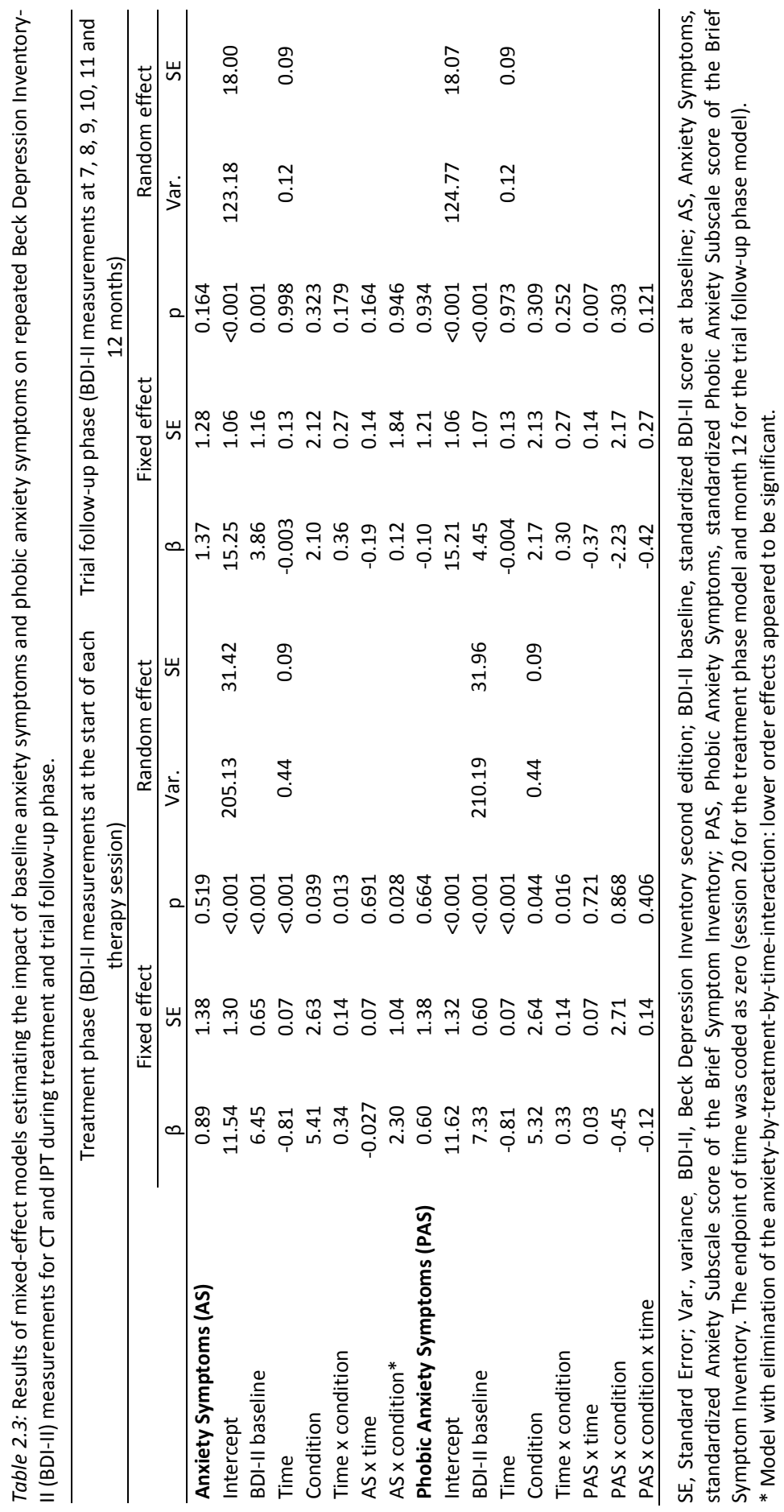


This effect was not found in the trial follow-up phase. Phobic anxiety did not affect BDI-II change and backwards elimination did not alter the lower order effects significantly. Sensitivity analyses did not change the results. Treatment dropout rates did not differ between individuals with low and high anxiety symptoms $\left(\chi^{2}=0.04, d f=1\right.$, $\mathrm{p}=0.852$ ). However, there were significantly higher study dropout rates for individuals with high anxiety symptoms $(27.8 \%)$ as compared to low anxiety symptoms $(11.3 \%$, $\left.\chi^{2}=5.76, d f=1, p=0.02\right)$. No differences in treatment and study dropout rates were found between individuals with low and high phobic anxiety symptoms $\left(\chi^{2}=2.01, d f=1\right.$, $p=0.16$ and $\chi^{2}=1.50, d f=1, p=0.22$ ). As indicated by the non-significant treatment by anxiety symptoms interactions in the logistic regression models, no differential effects between CT and IPT were found for both treatment $(\beta=0.06, p=0.474)$ and study $(\beta=0.02, p=0.798)$ dropout. For phobic anxiety symptoms, nonsignificant interactions for treatment $(\beta=0.02, p=0.837)$ and study $(\beta=-0.07, p=0.549)$ dropout were found as well.

\section{Discussion}

The main goal of the current study was to determine the influence of comorbid anxiety on the effectiveness and treatment completion of CT and IPT for MDD. Our most important findings were that, in the treatment phase, anxiety disorders and anxiety symptoms were associated with better depression change in CT as compared to IPT, and that individuals with anxiety disorders (but not anxiety symptoms) were more likely to dropout during both treatments.

The finding that anxiety disorders and anxiety symptoms had a less favorable impact on depression change for IPT as compared to CT in the treatment phase, was not completely unexpected. Although no previous head-to-head comparisons were available, comorbid anxiety have been found to negatively impact MDD outcome for IPT (Brown et al., 1996; Frank et al., 2011; Frank et al., 2000; Young et al., 2006), in contrast to the absence of adverse effects of anxiety for CT (Brent et al., 1998; de Azevedo Cardoso et al., 2014; Forand \& DeRubeis, 2013; Forand et al., 2011; Fournier et al., 2009; Kashdan \& Roberts, 2011; McEvoy \& Nathan, 2007; Persons et al., 2006; Rohde et al., 2001; Smits et al., 2009). In addition, there is evidence that CT outperformed IPT in the treatment of panic disorder (Vos, Huibers, Diels, \& Arntz, 2012) and social anxiety disorder (Stangier, Schramm, Heidenreich, Berger, \& Clark, 2011). A possible explanation for these differences is the focus on exposure in CT through "in vivo" homework assignments. The moderating effect of anxiety however was not found across five months follow-up indicating no differential effects in the long term. A possible interpretation of these follow-up findings could be that the impact of IPT took longer to manifest as compared to $\mathrm{CT}$, but is equally effective for 
anxious depression at the longer term. Similar temporal patterns and differences between CT and IPT were also found in two randomized controlled trials on bulimia nervosa. In these trials, CT appeared to be more effective than IPT in reducing symptoms at the end of therapy. However, these differences were not found during follow-up (Agras, Walsh, Fairburn, Wilson, \& Kraemer, 2000; Fairburn, Jones, Peveler, Hope, \& O'Connor, 1993). Possibly, these temporal differences could reflect specific mechanisms of change of CT and IPT. To better understand the discrepancies between our treatment and follow-up phase outcomes, further CT versus IPT comparisons on anxious depression are needed with longer follow-up durations and the inclusion of possible mediating variables that could explain different mechanism of change.

The higher treatment dropout rates for individuals with comorbid anxiety disorders have been found in some (Arnow et al., 2007; Brown et al., 1996; Howland et al., 2009), but not all (Forand et al., 2011; Kashdan \& Roberts, 2011; Rohde et al., 2001; Schindler et al., 2013; Smits et al., 2009) previous studies. One could explain these higher dropout rates as a form of avoidance behavior, a key feature of anxiety disorders (American Psychiatric Association, 2013). In contrast to our hypothesis, no differential effects of anxiety symptoms on dropout rates were found between CT and IPT.

In our study, we also found that the two different (syndromal and dimensional) definitions for anxious depression define two different groups of individuals. This is line with the results of a previous study (van der Veen et al., 2014) and further supported by the differences we found for pre-treatment depression severity and quality of life with a more severe clinical picture for individuals with the dimensional definition of anxious depression. As mentioned before, a plausible explanation for these differences is that the syndromal criteria identify two distinct disorders (MDD and anxiety disorder), while the dimensional criteria identify a more severe (anxious) subtype of MDD. Logically, one of the exclusion criteria for a DSM anxiety disorder is "not better accounted for by another mental disorder", so the anxiety cannot be part of the depression symptomatology. With the central position of anxiety symptoms in MDD (Ten Have et al., 2016), one could argue that anxious depression should only be defined with dimensional criteria (Ionescu et al., 2013; Silverstone \& von Studnitz, 2003), which is also in accordance to the newly proposed DSM-5 'anxious distress specifier' for the diagnosis of MDD (American Psychiatric Association, 2013).

To our knowledge this is the first study that examined anxious depression determined by both syndromal and dimensional criteria in a head-to-head comparison of CT and IPT. Other strengths of our study are the random allocation, the relative large sample size, and the multiple assessments of depressive symptoms that were analyzed with state of the art mixed models (Lemmens et al., 2015). The current study also has some limitations. First, no inter-reliability data for the SCID-I was collected and patients and therapist were not blind for the results of the SCID-I. Therapists were blind for the results of the BSI anxiety subscale and phobic anxiety subscale. Although 
therapists were not instructed to adapt treatments if comorbid anxiety was present, this knowledge could have altered treatment strategies. However, our competence and adherence check revealed no impact of comorbid anxiety on quality of treatment or therapy specific behavior. Second, our treatment and the trial follow-up phase models slightly overlap since a few participants had one or two therapy session during the trial follow-up phase. Third, although the overall dropout rates of the current study were low, individuals with anxiety disorders had relatively high dropout rates. Due to the use of mixed models, this is unlikely to affect our findings drastically. However, we think that larger sample sizes of individuals with comorbid anxiety disorders are warranted. In addition, the presence of these high dropout rates is an informative and clinically relevant finding.

The findings of this study have significant clinical implications. Most importantly, the presence of anxiety disorders and anxiety symptoms should be considered when selecting an effective psychotherapy for a depressed individual. Based on these results, a preference to CT over IPT is justifiable. Selecting the best treatment option for a given individual, is in line with the "personalized medicine" movement in health care research and practice today (Simon \& Perlis, 2010). If IPT is the first choice of treatment for other reasons, clinicians could also consider to use a modified version of IPT that includes cognitive-behavioral strategies to target symptoms of panic, anxiety and avoidance that interfere with interpersonal problem solving (Cyranowski et al., 2005). Another important clinical implication is that the patient's adherence to therapy should be carefully monitored when a comorbid anxiety disorder is diagnosed. With the assessment of other predictors of treatment drop-out (e.g., ethnic minority status, younger age, lower income, low motivation for change, poor therapeutic alliance, low treatment credibility and failure to improve early in treatment (Arnow et al., 2007; Cooper \& Conklin, 2015; Schindler et al., 2013; Taylor, Abramowitz, \& McKay, 2012), individual risks for dropout should be estimated, and specific interventions to prevent dropout can be considered, for example motivational interviewing techniques (Miller \& Rollnick, 2002). Another issue is that based on our finding that anxiety symptoms are associated with more severe depressive symptomatology and lower quality of life prior to treatment, a clinician can be confronted with significant levels of distress when anxiety symptoms are present. To deal with this more severe clinical picture, combination therapy (psychotherapy and pharmacotherapy) can be considered, since it has been formulated as the treatment of choice for severe depression (NICE, 2009). Finally, when depression and anxiety cooccur, transdiagnostic approaches for both CT and IPT focussing both on depressive and anxiety symptoms show promising results (Newby, McKinnon, Kuyken, Gilbody, \& Dalgleish, 2015; Wright, Hallquist, Swartz, Frank, \& Cyranowski, 2014). 


\section{References}

Agras, W. S., Walsh, B. T., Fairburn, C. G., Wilson, G. T., \& Kraemer, H. C. (2000). A multicenter comparison of cognitive-behavioral therapy and interpersonal psychotherapy for bulimia nervosa. Archives of general psychiatry, 57(5), 459-466.

American Psychiatric Association. (2013). Diagnostic and statistical manual of mental disorders (5th ed.). Washington DC.

Arnow, B. A., Blasey, C., Manber, R., Constantino, M. J., Markowitz, J. C., Klein, D. N., . . Rush, A. J. (2007). Dropouts versus completers among chronically depressed outpatients. Journal of affective disorders, 97(1-3), 197-202.

Beck, A. T., Rush, A. J., Shaw, B. F., \& Emery, G. (1979). Cognitive therapy of depression. New York: Guilford Press.

Beck, A. T., Steer, R., \& Brown, G. K. (1996). Beck Depression Inventory II: Manual. Boston: Harcourt Brace.

Brent, D. A., Kolko, D. J., Birmaher, B., Baugher, M., Bridge, J., Roth, C., \& Holder, D. (1998). Predictors of treatment efficacy in a clinical trial of three psychosocial treatments for adolescent depression. Journal of the American Academy of Child \& Adolescent Psychiatry, 37(9), 906-914.

Brown, C., Schulberg, H. C., Madonia, M. J., Shear, M. K., \& Houck, P. R. (1996). Treatment outcomes for primary care patients with major depression and lifetime anxiety disorders. The American Journal of Psychiatry.

Carter, J. D., Luty, S. E., McKenzie, J. M., Mulder, R. T., Frampton, C. M., \& Joyce, P. R. (2011). Patient predictors of response to cognitive behaviour therapy and interpersonal psychotherapy in a randomised clinical trial for depression. Journal of affective disorders, 128(3), 252-261.

Cooper, A. A., \& Conklin, L. R. (2015). Dropout from individual psychotherapy for major depression: A metaanalysis of randomized clinical trials. Clinical psychology review, 40, 57-65.

Cyranowski, J. M., Frank, E., Shear, M. K., Swartz, H., Fagiolini, A., Scott, J., \& Kupfer, D. J. (2005). Interpersonal psychotherapy for depression with panic spectrum symptoms: a pilot study. Depression and anxiety, 21(3), 140-142.

Davidson, J. R., Meoni, P., Haudiquet, V., Cantillon, M., \& Hackett, D. (2002). Achieving remission with venlafaxine and fluoxetine in major depression: its relationship to anxiety symptoms. Depression and anxiety, 16(1), 4-13.

de Azevedo Cardoso, T., Mondin, T. C., Spessato, B. C., de Avila Quevedo, L., de Mattos Souza, L. D., da Silva, R. A., \& Jansen, K. (2014). The impact of anxious symptoms in the remission of depressive symptoms in a clinical trial for depression: Follow-up of six months. Journal of affective disorders, 168, 331-336.

De Beurs, E. (2009). Brief symptom inventory, handleiding addendum. Leiden, The Netherlands: PITS BV.

De Beurs, E., \& Zitman, F. (2005). De brief symptom inventory (BSI). De betrouwbaarheid en validiteit van een handzaam alternatief voor de SCL-90. Leiden: Leids universitair medisch centrum.

Derogatis, L. R., \& Melisaratos, N. (1983). The brief symptom inventory: an introductory report. Psychological Medicine, 13(3), 595-605.

Diggle, P., Diggle, P. J., Heagerty, P., Heagerty, P. J., Liang, K.-Y., \& Zeger, S. (2002). Analysis of longitudinal data: Oxford University Press.

Dobson, K. S., Shaw, B. F., \& Vallis, T. M. (1985). Reliability of a measure of the quality of cognitive therapy. British Journal of Clinical Psychology, 24(4), 295-300.

EuroQol, G. (1990). EuroQol--a new facility for the measurement of health-related quality of life. Health Policy, 16(3), 199-208.

Fairburn, C. G., Jones, R., Peveler, R. C., Hope, R., \& O'Connor, M. (1993). Psychotherapy and bulimia nervosa: Longer-term effects of interpersonal psychotherapy, behavior therapy, and cognitive behavior therapy. Archives of general psychiatry, 50(6), 419-428.

Fava, M., Alpert, J. E., Carmin, C. N., Wisniewski, S. R., Trivedi, M. H., Biggs, M. M., . . Balasubramani, G. (2004). Clinical correlates and symptom patterns of anxious depression among patients with major depressive disorder in STAR* D. Psychological Medicine, 34(7), 1299-1308. 
Fava, M., Rosenbaum, J. F., Hoog, S. L., Tepner, R. G., Kopp, J. B., \& Nilsson, M. E. (2000). Fluoxetine versus sertraline and paroxetine in major depression: tolerability and efficacy in anxious depression. Journal of affective disorders, 59(2), 119-126.

Fava, M., Rush, A. J., Alpert, J. E., Balasubramani, G., Wisniewski, S. R., Carmin, C. N., . . Howland, R. (2008). Difference in treatment outcome in outpatients with anxious versus nonanxious depression: a STAR* D report. American Journal of Psychiatry, 165(3), 342-351.

Fawcett, J., Scheftner, W. A., Fogg, L., Clark, D. C., \& Young, M. A. (1990). Time-related predictors of suicide in major affective disorder. The American Journal of Psychiatry, 147(9), 1189.

First, M. B., Spitzer, R. L., Gibbon, M., \& Williams, J. B. W. (1995). Structured Clinical Interview for DSM-IV Axis I Disorders (SCID-I). New York: Biometrics Research Department New York State Psychiatric Institute.

Flint, A. J., \& Rifat, S. L. (1997). Anxious depression in elderly patients: response to antidepressant treatment. The American Journal of Geriatric Psychiatry, 5(2), 107-115.

Forand, N. R., \& DeRubeis, R. J. (2013). Pretreatment anxiety predicts patterns of change in cognitive behavioral therapy and medications for depression. Journal of Consulting and Clinical Psychology, 81(5), 774.

Forand, N. R., Gunthert, K. C., Cohen, L. H., Butler, A. C., \& Beck, J. S. (2011). Preliminary evidence that anxiety is associated with accelerated response in cognitive therapy for depression. Cognitive Therapy and Research, 35(2), 151-160.

Fournier, J. C., DeRubeis, R. J., Shelton, R. C., Hollon, S. D., Amsterdam, J. D., \& Gallop, R. (2009). Prediction of response to medication and cognitive therapy in the treatment of moderate to severe depression. Journal of Consulting and Clinical Psychology, 77(4), 775.

Frank, E., Cassano, G. B., Rucci, P., Thompson, W. K., Kraemer, H. C., Fagiolini, A., . . Houck, P. R. (2011). Predictors and moderators of time to remission of major depression with interpersonal psychotherapy and SSRI pharmacotherapy. Psychological Medicine, 41(1), 151-162.

Frank, E., Shear, M. K., Rucci, P., Cyranowski, J. M., Endicott, J., Fagiolini, A., . . . Maser, J. D. (2000). Influence of panic-agoraphobic spectrum symptoms on treatment response in patients with recurrent major depression. American Journal of Psychiatry, 157(7), 1101-1107.

Hollon, S., Evans, M., Auerbach, A., DeRubeis, R., Elkin, I., Lowery, A., . . Piasecki, J. (1988). Development of a system for rating therapies for depression: Differentiating cognitive therapy, interpersonal psychotherapy, and clinical management pharmacotherapy. Unpublished manuscript.

Hollon, S., Evans, M., Elkin, I., \& Lowery, A. (1984). System for rating therapies for depression. Paper presented at the 92nd Annual Convention of the American Psychological Association, Toronto, Ontario, Canada.

Howland, R. H., Rush, A. J., Wisniewski, S. R., Trivedi, M. H., Warden, D., Fava, M., . . Berman, S. R. (2009). Concurrent anxiety and substance use disorders among outpatients with major depression: clinical features and effect on treatment outcome. Drug and alcohol dependence, 99(1-3), 248-260.

Ionescu, D. F., Niciu, M. J., Henter, I. D., \& Zarate, C. A. (2013). Defining anxious depression: a review of the literature. CNS spectrums, 18(5), 252-260.

Joffe, R. T., Bagby, R. M., \& Levitt, A. (1993). Anxious and nonanxious depression. The American Journal of Psychiatry, 150(8), 1257-1258.

Jones, R. H. (1993). Longitudinal data with serial correlation: a state-space approach: Chapman and Hall/CRC.

Kashdan, T. B., \& Roberts, J. E. (2011). Comorbid social anxiety disorder in clients with depressive disorders: Predicting changes in depressive symptoms, therapeutic relationships, and focus of attention in group treatment. Behaviour research and therapy, 49(12), 875-884.

Khalil, A. A., Hall, L. A., Moser, D. K., Lennie, T. A., \& Frazier, S. K. (2011). The psychometric properties of the Brief Symptom Inventory depression and anxiety subscales in patients with heart failure and with or without renal dysfunction. Archives of psychiatric nursing, 25(6), 419-429.

Klerman, G. L., Weissman, M. M., Rounsaville, B. J., \& Chevron, E. S. (1984). Interpersonal psychotherapy for depression. New York: Basis Books. 
Lee, T.-S., Assam, P. N., Gersing, K. R., Chan, E., Burchett, B. M., Sim, K., . . . Rush, A. J. (2012). The Effectiveness of antidepressant monotherapy in a naturalistic outpatient setting. The primary care companion for CNS disorders, 14(5).

Lemmens, L. H., Arntz, A., Peeters, F., Hollon, S. D., Roefs, A., \& Huibers, M. J. (2015). Clinical effectiveness of cognitive therapy $v$. interpersonal psychotherapy for depression: results of a randomized controlled trial. Psychological Medicine, 1-16.

Lemmens, L. H., Arntz, A., Peeters, F. P., Hollon, S. D., Roefs, A., \& Huibers, M. J. (2011). Effectiveness, relapse prevention and mechanisms of change of cognitive therapy vs. interpersonal therapy for depression: Study protocol for a randomised controlled trial. Trials, 12, 150.

McEvoy, P. M., \& Nathan, P. (2007). Effectiveness of cognitive behavior therapy for diagnostically heterogeneous groups: A benchmarking study. Journal of Consulting and Clinical Psychology, 75(2), 344.

Miller, W. R., \& Rollnick, S. (2002). Motivational interviewing: preparing people for change (2nd ed.) New York: Guilford.

Mundt, J. C., Marks, I. M., Shear, M. K., \& Greist, J. H. (2002). The Work and Social Adjustment Scale: a simple measure of impairment in functioning. Br J Psychiatry, 180, 461-464.

National Institute for Health and Care Excellence. (2009). Depression in adults: recognition and management.

Nelson, J. C. (2010). Anxiety does not predict response to duloxetine in major depression: results of a pooled analysis of individual patient data from 11 placebo-controlled trials. Depression and anxiety, 27(1), 12-18.

Newby, J. M., McKinnon, A., Kuyken, W., Gilbody, S., \& Dalgleish, T. (2015). Systematic review and metaanalysis of transdiagnostic psychological treatments for anxiety and depressive disorders in adulthood. Clinical psychology review, 40, 91-110.

Papakostas, G. I., McGrath, P., Stewart, J., Charles, D., Chen, Y., Mischoulon, D., . . Fava, M. (2008). Psychic and somatic anxiety symptoms as predictors of response to fluoxetine in major depressive disorder. Psychiatry Research, 161(1), 116-120.

Penninx, B. W., Nolen, W. A., Lamers, F., Zitman, F. G., Smit, J. H., Spinhoven, P., . . van der Meer, K. (2011). Two-year course of depressive and anxiety disorders: results from the Netherlands Study of Depression and Anxiety (NESDA). Journal of affective disorders, 133(1-2), 76-85.

Persons, J. B., Roberts, N. A., Zalecki, C. A., \& Brechwald, W. A. (2006). Naturalistic outcome of case formulation-driven cognitive-behavior therapy for anxious depressed outpatients. Behaviour research and therapy, 44(7), 1041-1051.

Pfeiffer, P. N., Ganoczy, D., Ilgen, M., Zivin, K., \& Valenstein, M. (2009). Comorbid anxiety as a suicide risk factor among depressed veterans. Depression and anxiety, 26(8), 752-757.

Rao, S. (2009). Anxious depression: clinical features and treatment. Current psychiatry reports, 11(6), 429436.

Rohde, P., Clarke, G. N., Lewinsohn, P. M., Seeley, J. R., \& Kaufman, N. K. (2001). Impact of comorbidity on a cognitive-behavioral group treatment for adolescent depression. Journal of the American Academy of Child \& Adolescent Psychiatry, 40(7), 795-802.

Russell, J. M., Koran, L. M., Rush, J., Hirschfeld, R. M., Harrison, W., Friedman, E. S., . . Keller, M. (2001). Effect of concurrent anxiety on response to sertraline and imipramine in patients with chronic depression. Depression and anxiety, 13(1), 18-27.

Schindler, A., Hiller, W., \& Witthöft, M. (2013). What predicts outcome, response, and drop-out in CBT of depressive adults? A naturalistic study. Behavioural and Cognitive Psychotherapy, 41(3), 365-370.

Silverstone, P. H., \& von Studnitz, E. (2003). Defining anxious depression: going beyond comorbidity. The Canadian Journal of Psychiatry, 48(10), 675-680.

Simon, G. E., \& Perlis, R. H. (2010). Personalized medicine for depression: can we match patients with treatments? American Journal of Psychiatry, 167(12), 1445-1455.

Smits, J. A., Minhajuddin, A., \& Jarrett, R. B. (2009). Cognitive therapy for depressed adults with comorbid social phobia. Journal of affective disorders, 114(1-3), 271-278. 
Smits, J. A., Minhajuddin, A., Thase, M. E., \& Jarrett, R. B. (2012). Outcomes of acute phase cognitive therapy in outpatients with anxious versus nonanxious depression. Psychotherapy and psychosomatics, 81(3), 153-160.

Stangier, U., Schramm, E., Heidenreich, T., Berger, M., \& Clark, D. M. (2011). Cognitive therapy vs interpersonal psychotherapy in social anxiety disorder: a randomized controlled trial. Archives of general psychiatry, 68(7), 692-700.

Stuart, S. (2011). IPT Adherence and Quality Scale. Interpersonal Psychotherapy Institute, lowa (unpublished manuscript).

Taylor, S., Abramowitz, J. S., \& McKay, D. (2012). Non-adherence and non-response in the treatment of anxiety disorders. Journal of anxiety disorders, 26(5), 583-589.

Ten Have, M., Lamers, F., Wardenaar, K., Beekman, A., de Jonge, P., van Dorsselaer, S., . . . de Graaf, R. (2016). The identification of symptom-based subtypes of depression: A nationally representative cohort study. Journal of affective disorders, 190, 395-406.

Tollefson, G. D., Holman, S. L., Sayler, M. E., \& Potvin, J. H. (1994). Fluoxetine, placebo, and tricyclic antidepressants in major depression with and without anxious features. The Journal of clinical psychiatry.

van der Veen, D. C., Comijs, H. C., van Zelst, W. H., Schoevers, R. A., \& Oude Voshaar, R. C. (2014). Defining anxious depression in later life: a scaring heterogeneity in results. The American Journal of Geriatric Psychiatry, 22(11), 1375-1378.

van der Zee, K. I., \& Sanderman, R. (1993). Het meten van de algemene gezondheidstoestand met de RAND36: een handleiding. Groningen: Noordelijk Centrum voor Gezondheidsvraagstukken.

Vos, S., Huibers, M., Diels, L., \& Arntz, A. (2012). A randomized clinical trial of cognitive behavioral therapy and interpersonal psychotherapy for panic disorder with agoraphobia. Psychological Medicine, 42(12), 2661-2672.

Wang, Y.-P., \& Gorenstein, C. (2013). Psychometric properties of the Beck Depression Inventory-II: a comprehensive review. Brazilian Journal of Psychiatry, 35(4), 416-431.

Wiethoff, K., Bauer, M., Baghai, T. C., Möller, H.-J., Fisher, R., Hollinde, D., . . Cordes, J. (2010). Prevalence and treatment outcome in anxious versus nonanxious depression: results from the German Algorithm Project. The Journal of clinical psychiatry, 71(8), 1047-1054.

Wright, A. G., Hallquist, M. N., Swartz, H. A., Frank, E., \& Cyranowski, J. M. (2014). Treating co-occurring depression and anxiety: Modeling the dynamics of psychopathology and psychotherapy using the time-varying effect model. Journal of Consulting and Clinical Psychology, 82(5), 839.

Young, J. F., Mufson, L., \& Davies, M. (2006). Impact of comorbid anxiety in an effectiveness study of interpersonal psychotherapy for depressed adolescents. Journal of the American Academy of Child \& Adolescent Psychiatry, 45(8), 904-912. 
S. van Bronswijk, L. Lemmens, W. Viechtbauer, M. Huibers, A. Arntz, F. Peeters - Journal of Affective Disorders 2018, 225, 530-538

\section{Chapter}

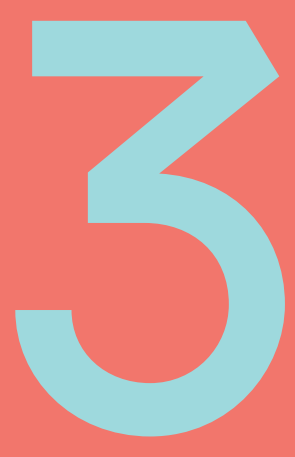




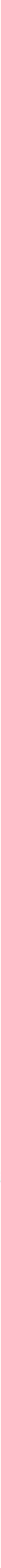




\section{Abstract}

\section{Background}

Despite extensive research, there is no consensus how personality disorders (PD) and PD features affect outcome for major depressive disorder (MDD). The present study evaluated the effects of PD (features) on treatment continuation and effectiveness in cognitive therapy (CT) and interpersonal psychotherapy (IPT) for MDD.

Methods

Depressed outpatients were randomized to CT $(n=72)$ and IPT $(n=74)$. Primary outcome was depression severity measured repeatedly with the Beck Depression Inventory-II (BDI-II) at baseline, three months, at the start of each therapy session, at post-treatment and monthly during five months follow-up.

\section{Results}

Comorbid PD and PD features did not affect dropout. Multilevel and Cox regression models indicated no negative effect of PD on BDI-II change and remission rates during treatment and follow-up, irrespective of the treatment received. For both therapies, higher dependent PD features predicted overall lower BDI-II scores during treatment, however this effect did not sustain through follow-up. Cluster A PD features moderated treatment outcome during treatment and follow-up: individuals with high cluster A PD features had greater BD-II reductions over time in CT as compared to IPT

\section{Conclusions}

We found no negative impact of PD on the effectiveness and treatment retention of CT and IPT for MDD during treatment and follow-up. If replicated, cluster A PD features can be used to optimize treatment selection. 


\section{Introduction}

Individuals with major depressive disorder (MDD) often meet criteria for DSM defined comorbid personality disorders (PD), in particular PD's grouped in cluster C: obsessive compulsive PD, dependent PD and avoidant PD (Friborg et al., 2014). Despite extensive research spanning several decades, results from research into the impact of comorbid PD on the effectiveness of acute phase treatment for MDD is equivocal; results vary from a negative association between the presence of a comorbid PD and clinical outcome (Hardy et al., 1995; Newton-Howes et al., 2014; Reich \& Vasile, 1993; Sato, Sakado, Sato, \& Morikawa, 1994) to the absence of any difference between outcome in individuals with and without comorbid PD (De Bolle et al., 2011; Kool et al., 2005; Moradveisi, Huibers, Renner, Arasteh, \& Arntz, 2013; Mulder, 2002). These inconsistencies across studies are probably best explained by methodological problems. Uncontrolled study designs are common, allowing selection bias based on clinicians' decision-making. Nevertheless, the studies that used a randomized design also reported inconsistent findings varying from no differences between individuals with and without a comorbid PD (Hirschfeld et al., 1998; Maddux et al., 2009) to a negative effect of PD on MDD outcome (DeRubeis et al., 2014; Fournier et al., 2008). Another concern is that only a few studies provide data on the relationship between PD and dropout (Kool et al., 2005), which can lead to biased or inaccurate conclusions. The few studies that reported drop-out rates have mixed results: some showed no difference between individuals with and without PD (Fournier et al., 2008; Kool, Dekker, Duijsens, de Jonghe, \& Puite, 2003; Sullivan, Joyce, \& Mulder, 1994), while others reported higher drop-out rates for individuals with PD (Moradveisi et al., 2013). In addition, despite substantial differences (e.g., depression severity) between individuals with and without PD, multivariable models controlling for significant confounders are rarely used (Casey et al., 2004; Mulder, 2002). Finally, inconsistent findings could also be explained by different outcomes on self-report versus clinicianrated measures of personality pathology and depression outcome (Mulder, 2002; Stanley \& Wilson, 2006; Unger, Hoffmann, Köhler, Mackert, \& Fydrich, 2013).

Concerning the various treatment options for MDD, researchers and clinicians alike have come to realize that a 'one size fits all' approach is not very effective. A major challenge in health care research today is to select the best treatment option for a given individual, a concept referred to as personalized medicine (Simon \& Perlis, 2010). Therefore, it is highly relevant to examine whether depressed individuals with comorbid PD respond differentially to evidence-based psychotherapies for MDD. At present, both cognitive therapy (CT) and interpersonal psychotherapy (IPT) are recommended as first choice evidence-based psychotherapies for MDD (Cuijpers et al., 2014). A recent trial sequential analysis showed that both treatments do not differ in effectiveness for MDD when a difference of 4 points on the Beck Depression Inventory, second edition (BDI-II) is taken as futile (Lemmens et al., 2015). 
Previous research comparing CT and IPT head-to-head suggests specific PD features as potential treatment moderators. The National Institute of Mental Health Treatment of Depression Collaborative Program found that depressed individuals with obsessive-compulsive PD features were more likely to respond to IPT, while depressed participants with more avoidant PD features responded better to CT (Barber \& Muenz, 1996). These results were partially replicated by the Christchurch Psychotherapy for Depression Study, where individuals with MDD and comorbid PD features responded less well to IPT compared to CT (Carter et al., 2011) particularly in individuals with avoidant PD features (Joyce et al., 2007). In another study, depressive PD features predicted better outcome in CT compared to IPT, while other PD features did not predict differential treatment outcome (Ryder, Quilty, Vachon, \& Bagby, 2010). These inconsistencies might be explained by the use of different PD assessments, which complicates comparison of these findings. Moreover, most studies use simple linear regression models, while multilevel modeling is rarely used. The latter can explore the dynamic and individual course of depression more accurately (De Bolle et al., 2011).

The overall aim of the current study was to determine whether the presence of a DSM PD diagnosis, affected depressive symptom change and treatment retention in CT and IPT during treatment and follow-up. In addition, the effect of PD on remission rates was evaluated, since achieving remission is an evident treatment goal in CT and IPT. Potential differences in these effects between CT and IPT were examined. To acquire a more detailed understanding about cluster C PD features, dimensional scores of obsessive-compulsive, avoidant and dependent PD features as defined by the DSM were calculated, together with cluster A and cluster B PD feature scores. With these scores, general and differential effects of specific PD features on depressive symptom change, treatment retention and remission were examined in CT and IPT. Based on previous studies, we were unsure how PD affected depression outcomes and dropout in CT and IPT during treatment and follow-up. Based on the previous comparisons between CT and IPT we expected cluster C features, in particular obsessive-compulsive and avoidant PD features to moderate treatment outcomes.

\section{Methods}

\subsection{Design and participants}

Data were collected in the context of a large randomized clinical trial. A detailed description about sample characteristics, study design, interventions, and main treatment outcome findings is provided elsewhere (Lemmens et al., 2015; Lemmens et al., 2011). The study was conducted at the mood disorders unit of the Maastricht Community Mental Health Centre (RIAGG Maastricht) and included 182 outpatients, 
18-65 years of age, with a primary diagnosis of MDD (as confirmed with the Structured Clinical Interview for DSM-IV Axis I disorders (SCID-I; First, Spitzer, Gibbon, \& Williams, 1995)). Other inclusion criteria were: internet access, an email address, and sufficient knowledge of the Dutch language. Exclusion criteria were a bipolar or chronic (current episode $>5$ years) depression, high acute suicide risk, concomitant pharmacological or psychological treatment, drugs and alcohol abuse/dependence, and mental retardation $(\mathrm{IQ}<80)$.

Participants were randomly allocated to CT $(n=76)$, IPT $(n=75)$, or a Waiting List Condition (WLC; $n=31$ ). For the current analyses, we limited the sample to data of individuals randomized to CT and IPT $(n=151)$. This sample participated in a treatment phase (0-7 months) and a subsequent trial follow-up phase (7-12 months). Five participants ( 4 in the CT group and 1 in the IPT group) were excluded in the current study, because PD assessments were incomplete or missing. All participants provided written informed consent and the study was approved by the Medical Ethics Committee of Maastricht University Medical Center. The study is registered at The Netherlands Trial Register, part of the Dutch Cochrane Centre (ISRCTN 67561918).

\subsection{Measures}

\subsubsection{Primary outcome}

Primary outcome, depressive symptom severity, was assessed with the BDI-II (Beck, Steer, \& Brown, 1996). BDI-II measurements included in this study were collected at baseline, at the start of each therapy session and at 3, 7, 8, 9, 10, 11, and 12 months.

\subsubsection{Personality measures}

PD diagnoses were administered prior to treatment using the Structured Clinical Interview for DSM-IV Axis II (SCID-II; First, Gibbon, Spitzer, Williams, \& Benjamin, 1997) by well-trained therapists that were participating in the study. In addition, the results of the SCID-II assessment were available in the patient record during treatment. Therefore, therapists and participants were not blind to the results of the SCID-II assessment. Passive-Aggressive PD and Depressive PD were excluded from the analyses, given their position in the section of criteria sets for further study (American Psychiatric Association, 2000). Dimensional PD scores were calculated by summing up the items answered with 'present' for each PD criterion, dismissing items that were scored 'uncertain' or 'absent'. For the cluster C PD features, we calculated individual avoidant PD features scores (range 0-7), dependent PD features scores (range 0-8), and obsessive-compulsive PD features scores (range $0-8$ ). We calculated individual cluster A PD features (range 0-23) and cluster B PD features (range 0-41) total scores providing two reference groups of other, non-Cluster $\mathrm{C}$, PD features. 


\subsection{Treatments and therapists}

Both interventions consisted of 16-20 sessions of $45 \mathrm{~min}$, depending on the participants' improvement, with an average of 17 sessions per person (SD=2.9; Lemmens et al., 2015). Sessions were planned weekly and allowed to be less frequently scheduled towards the end of therapy. Interventions were described in CT and IPT protocols following guidelines by Beck et al. (1979) for CT, and guidelines by Klerman et al. (1984) for IPT. Treatments were performed by ten licensed psychologist, psychotherapists, and psychiatrists (five in each condition) with on average 9.1 years $(S D=5.4)$ of clinical experience (range 4-21 years). To avoid treatment contamination, therapist delivered exclusively CT or IPT. All sessions were videotaped. The quality of treatment was rated as good to excellent by independent assessors using the Cognitive Therapy Scale for CT (M=3.31, SD=0.93; Dobson, Shaw, \& Vallis, 1985) and the short version of the IPT Adherence and Quality Scale for IPT ( $M=3.50, S D=0.70 ;$ Stuart, 2011). There were significant differences in therapy-specific behavior between conditions as indicated by the Collaborative Study Psychotherapy Rating Scale (Hollon et al., 1988; Hollon, Evans, Elkin, \& Lowery, 1984) with higher CTspecific behavior in CT as compared to IPT ( $M=80.80$ versus $M=52.42, t_{79.21}=7.23$, $\mathrm{p}<0.001)$, and higher IPT specific behavior in IPT as compared to $C T$ ( $M=85.75$ versus $M=44.57, t_{86.96}=10.79, p<0.001$; Lemmens et al., 2015). The presence or absence of a comorbid PD diagnosis did not change therapy-specific behavior of the therapists; adherence remained equally well (results not shown, all $p$-values $>0.3$ ).

\subsection{Statistical analysis}

A detailed description of statistical analyses is presented in online Data Supplement I. Differences in pretreatment (clinical) characteristics and observed mean depression outcomes were examined for individuals with and without PD using descriptive statistics. Study dropout (loss to follow-up) and treatment dropout (discontinuation of the treatment intervention) were compared between individuals with and without PD $\left(\chi^{2}\right)$ and treatment dropout was further investigated with a Cox regression analysis.

Linear (2-level) mixed-effects models were used to examine the effects of PD and PD features on BDI-II change for the treatment phase (BDI-II scores measured at the start of each therapy session) and the follow-up phase models (BDI-II scores at 7, 8, 9, 10, 11 and 12 months) separately. All linear mixed-effects models included the following fixed effects: BDI-II baseline score (mean centered), a BDI-II baseline score $x$ time interaction, time, treatment (centered at -0.5 for $\mathrm{CT},+0.5$ for IPT) and a time $x$ treatment interaction. For the time variable, the endpoint was coded as zero (treatment phase model: session 20; trial follow-up phase model: month 12). The effect of PD was analyzed, by adding a predictor of PD (centered at $-0.5=$ no PD, $+0.5=$ one or more PD's) to the treatment and trial follow-up phase models, with PD $\times$ time, PD $\times$ condition and PD $\times$ time $\times$ condition interactions. To analyze the effect of 
PD features, mean centered scores of avoidant PD features, dependent PD features, obsessive-compulsive PD features, cluster A and cluster B PD features were added simultaneously as continuous predictors to the treatment and trial follow-up phase models with $\times$ time, $\times$ condition and time $\times$ condition interactions. To determine the pretreatment associations between PD features and BD-II baseline scores, Pearson correlation coefficients were computed.

To examine effects of PD and PD features on remission, we applied two Cox regression models, with for each a different definition of remission based on the frequency of the BDI-II measurements. For the first model, referred to as the treatment phase Cox regression model, BDI-II scores at the start of each weekly therapy session were used and remission was defined as a BDI-II score of $<10$ for three consecutive weeks. For the second model, referred to as the combined Cox regression model, BDI-II measures at 3, 7, 8, 9, 10, 11 and 12 months across the treatment and trial follow-up phase were used and remission was defined as a BDI-II score $<10$ at one of the assessment points. Dropouts were censored after the last available observation. All models included "condition" as a predictor. The effect of PD was examined by adding PD as a dichotomous predictor (yes/no) to the models, along with a PD $\times$ condition interaction. The effect of PD features was examined by adding avoidant PD features, dependent PD features, obsessive-compulsive PD features, cluster A PD features, cluster B PD features and their interactions with condition simultaneously to the models.

All outcomes were tested at $\alpha=0.05$ (two-tailed). For all models, we conducted a sensitivity analyses by consecutively adding the following baseline measures to the models: therapist, demographics (age, gender, employment, level of education), clinical features (first or recurrent depression, general psychological distress (BSI; Derogatis \& Melisaratos, 1983), axis I comorbidity (SCID-I; First et al., 1995)) and functionality (Work and Social Adjustment Scale (W\&SAS; Mundt, Marks, Shear, \& Greist, 2002), the RAND-36 (van der Zee \& Sanderman, 1993) and the EuroQol-6D (EQ-6D; EuroQol, 1990) for the EQ-5D).

\section{Results}

\subsection{Descriptive statistics}

In our sample of 146 participants, 49 individuals were diagnosed with one ( $n=37$; $C T=19, I P T=18$ ) or multiple PDs ( $n=12 ; C T=10, I P T=2)$. The number of individuals with a $P D$ diagnosis did not significantly differ between the two treatment groups ( $C T=29$, IPT=20, $\left.\chi^{2}=2.87, p=0.09\right)$. The most prevalent PD in this sample was an avoidant PD (46.0\%), followed by obsessive-compulsive PD (31.7\%), paranoid PD (6.3\%), borderline PD (6.3\%), dependent PD (3.2\%), schizotypal PD (3.2\%), schizoid PD (1.6\%), and PD not 
otherwise specified (1.6\%). Examining dimensional PD features, participants had on average 1.8 avoidant PD features, 0.9 dependent PD features, 1.5 obsessivecompulsive PD features, 1.3 cluster A PD features and 1.3 cluster B PD features. These PD features did not appear to significantly differ between the two treatments (all p's $>0.05$ ).

Table 3.1 provides the differences in baseline demographics, clinical characteristics, functionality measures, treatment compliance, and observed mean treatment outcomes between participants with and without PD in each treatment condition. Baseline BDI-II scores were significantly higher in participants with PD compared to participants without PD $(\mathrm{t}(144)=2.28, \mathrm{p}=0.02)$. In addition, higher levels of general psychological distress (as measured with the $\mathrm{BSI}$ ) and more axis I comorbidity were found in participants with PD compared to participants without PD $\left(t(144)=2.61, p=0.01\right.$ and $\left.\chi^{2}(1)=3.73, p=0.053\right)$.

Table 3.1: Sample description: demographics, clinical characteristics, quality of life, treatment compliance and depression outcome at 6 and 12 months.

\begin{tabular}{|c|c|c|c|c|}
\hline & \multicolumn{2}{|c|}{$\begin{array}{l}\text { Cognitive therapy } \\
\qquad(n=72)\end{array}$} & \multicolumn{2}{|c|}{$\begin{array}{l}\text { Interpersonal psychotherapy } \\
\qquad(\mathrm{n}=74)\end{array}$} \\
\hline & $\begin{array}{l}\text { Participants } \\
\text { without PD } \\
(n=43)\end{array}$ & $\begin{array}{c}\text { Participants } \\
\text { with PD } \\
(n=29)\end{array}$ & $\begin{array}{l}\text { Participants } \\
\text { without PD } \\
(n=54)\end{array}$ & $\begin{array}{c}\text { Participants } \\
\text { with PD } \\
(n=20)\end{array}$ \\
\hline \multicolumn{5}{|l|}{ Demographics } \\
\hline Age, years: mean (SD) & $41.7(12.9)$ & $41.2(12.1)$ & $41.5(12.2)$ & $41.0(11.2)$ \\
\hline Female, $\mathrm{n}(\%)$ & $32(74.4)$ & $20(69.0)$ & $35(64.8)$ & $11(55.0)$ \\
\hline Partner, n (\%) & $23(53.5)$ & $17(58.6)$ & $34(63.0)$ & $16(80.0)$ \\
\hline Employed, n (\%) & $27(62.8)$ & $16(55.2)$ & $31(57.4)$ & $16(80.0)$ \\
\hline \multicolumn{5}{|l|}{ Education, n (\%) } \\
\hline - Low, n (\%) & $8(18.2)$ & $7(25.0)$ & $7(13.0)$ & $6(30.0)$ \\
\hline - Medium, n (\%) & $29(67.4)$ & $16(55.2)$ & $30(55.6)$ & $10(50.0)$ \\
\hline - High, n (\%) & $6(13.6)$ & $6(21.4)$ & $17(31.5)$ & $4(20.0)$ \\
\hline \multicolumn{5}{|l|}{ Clinical characteristics } \\
\hline BDI-II baseline score, mean (SD)* & $25.9(8.0)^{* *}$ & $32.0(8.7)^{* *}$ & $30.9(9.3)$ & $32.4(7.7)$ \\
\hline Recurrent depression, $\mathrm{n}(\%)$ & 18 (41.9) & $16(55.2)$ & $24(44.4)$ & $11(55.0)$ \\
\hline Axis I comorbidity, $\mathrm{n}(\%)$ & 15 (34.9) & $17(58.6)$ & $26(48.2)$ & $12(60.0)$ \\
\hline $\mathrm{BSI}$, mean $(\mathrm{SD})^{*}$ & $56.3(24.8)^{* *}$ & $78.6(30.6)^{* *}$ & $70.3(27.5)$ & $75.2(32.6)$ \\
\hline \multicolumn{5}{|l|}{ Functionality } \\
\hline WSAS, mean (SD) & $22.1(8.1)$ & $24.4(7.0)$ & $23.1(7.0)$ & $20.8(7.7)$ \\
\hline EQ-5D, mean (SD) & $0.6(0.2)$ & $0.6(0.2)$ & $0.6(0.3)$ & $0.6(0.3)$ \\
\hline RAND-36, mean (SD) & $46.7(18.2)$ & $46.8(13.6)$ & $44.3(14.7)$ & $43.8(11.7)$ \\
\hline \multicolumn{5}{|l|}{ Treatment retention } \\
\hline Dropouts, n (\%) & $12(27.9)$ & $5(17.2)$ & $13(24.1)$ & $2(10.0)$ \\
\hline \multicolumn{5}{|l|}{ Depression outcome: at 7 months } \\
\hline $\mathrm{BDI}-\mathrm{II}$, mean (SD) & $13.6(9.4)$ & $13.3(11.3)$ & $17.1(14.1)$ & $14.3(11.7)$ \\
\hline \multicolumn{5}{|l|}{ Depression outcome: at 12 months } \\
\hline $\mathrm{BDI}-\mathrm{II}$, mean (SD) & $13.1(11.3)$ & $11.2(11.0)$ & $17.9(16.1)$ & $16.3(12.4)$ \\
\hline
\end{tabular}

$\mathrm{PD}=$ personality disorder; BDI-II=Beck Depression Inventory, second edition; BSI=Brief Symptom Inventory; WSAS=Work and Social Adjustment Scale; EQ-5D=EuroQol-5D; SD=Standard Deviation. ${ }^{*} \mathrm{p}<0.05$ differences between participants with PD and participants without PD; ** $p<0.05$ differences between participants with PD and participants without PD within treatment group. 


\subsection{The effect of PD and PD features on study and treatment dropout}

Participants with and without PD did not differ significantly in proportion of study and treatment dropouts $\left(\chi^{2}(1)=0.27, p=0.60\right.$ and $\left.\chi^{2}(1)=2.51, p=0.113\right)$. When further examining treatment dropout with a Cox regression model, no significant effect of PD was found ( $H R=0.59, p=0.33$ ) and there was no significant $P D \times$ treatment interaction ( $H R=0.61, p=0.59)$. Obsessive-compulsive PD features, dependent PD features, avoidant PD features, cluster A PD features and cluster B PD features did not significantly affect treatment dropout, and their interactions with treatment were not significant (all p's $>0.3$ ).

\subsection{The effect of PD on BDI-II change and remission}

Table 3.2 summarizes the results of the linear mixed-effects models estimating the effects of PD, PD $\times$ time, $P D \times$ condition, and PD $\times$ time $\times$ condition on BDI-II change in the treatment and trial follow-up phase. There was no significant effect of PD on BDI-II change during treatment and trial follow-up phase. Additionally, PD $\times$ condition and $P D \times$ time $\times$ condition were not significant, indicating no differential effect of PD on depressive symptom change between the two interventions. During the treatment phase, 46 participants achieved remission including 21 participants with PD $(43.75 \%$ of all individuals with PD) and 25 participants without PD (25.51\% of all individuals without $P D)$. No significant effect of $P D(H R=1.5 ; p=0.33)$ or the $P D \times$ condition interaction $(H R=1.3 ; p=0.63)$ was found with the treatment phase Cox regression model, indicating no (differential) effect of PD on time to remission. In the treatment and trial follow-up phase combined, 74 participants achieved remission, 28 with PD (57.1\% of all individuals with PD) and 46 without PD (47.4\% of all individuals without $\mathrm{PD})$. The combined Cox regression model did not indicate a significant effect of PD on time to remission ( $H R=1.1 ; p=0.69)$, and no significant effect of condition $P D \times$ condition ( $H R=1.2, p=0.66)$ as well. Sensitivity analyses did not change these results.

\subsection{The effect of PD features on BDI-II change and remission}

Table 3.3 provides estimates of the effects of avoidant PD features, dependent PD features, obsessive-compulsive PD features, cluster A PD features and cluster B PD features and their interactions with $\times$ time, $\times$ condition, and $\times$ time $\times$ condition on BDI-II change during treatment and follow-up phase. During the treatment phase a significant dependent PD features $\times$ time interaction was found, Indicating that higher dependent PD features predicted overall lower BD-II scores across the treatment phase. 


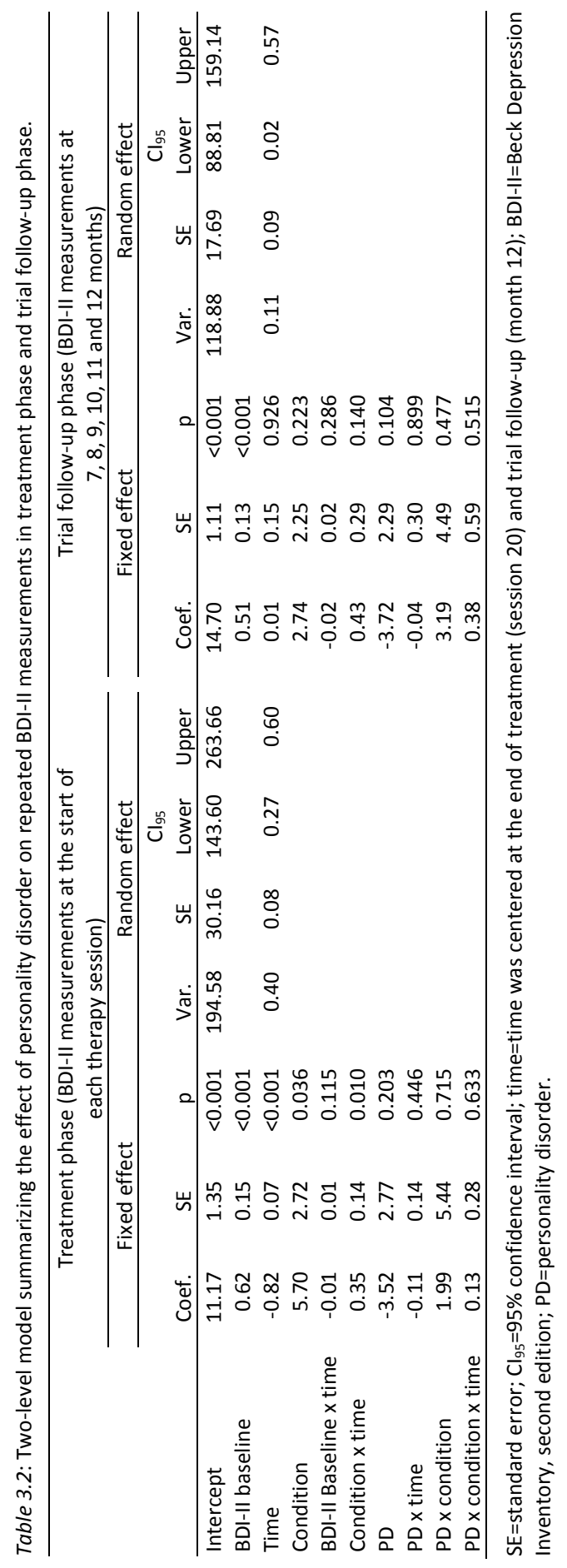


This effect is illustrated in Figure 3.1 with plots of the mean BDI-II scores for individuals with high and low dependent features (groups created via a median split). The dependent PD features $\times$ time interaction was no longer significant in the trail follow-up phase. Significant cluster A PD features $\times$ time $\times$ condition interactions were found in both the treatment and trial follow-up phase. For IPT, individuals with high cluster A PD features had less BDI-II decrease over time as compared to individuals with low cluster A PD features, while cluster A PD features did not affect BDI-Il change in CT. This moderating effect is illustrated with plots of mean BDI-Il scores for participants with high and low cluster A PD features (groups created via a medium split) in the treatment phase (Figure 3.2) and trial follow-up phase (Figure 3.3). Obsessive-compulsive PD features, avoidant PD features and cluster B PD features did not show significant (interaction) effects in any of the models. Results were not affected during sensitivity analyses.

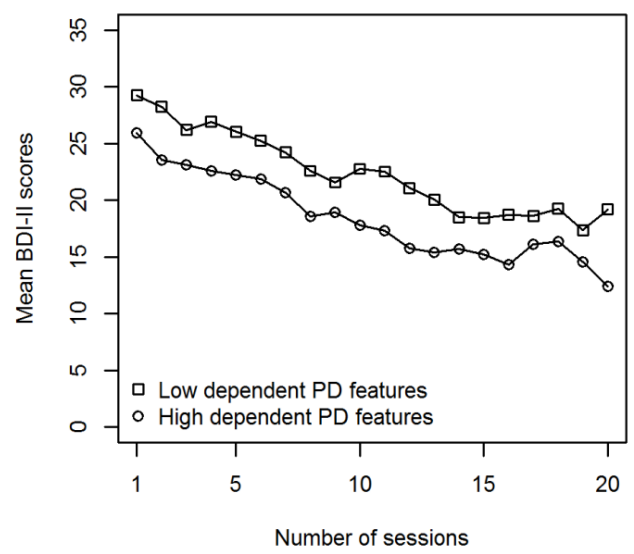

Figure 3.1: Mean Beck Depression Inventory-II (BDI-II) scores for individuals with low and high dependent personality disorder features in the treatment phase.

Note: PD: personality disorder; Low and high dependent PD features: subgroups of individuals with low and high dependent PD features were created via a medium split.

The treatment phase Cox regression model indicated that obsessive-compulsive PD features did significantly affect time to remission during the treatment phase $(H R=1.3 ; p=0.006)$ with more OCPD features predicting faster remission for both treatments (no differential effect). However, this effect was not significant in the combined Cox regression model covering both the treatment and trial follow-up phase. Avoidant, dependent, cluster A and cluster B PD features did not affect time to remission in both the treatment phase and the combined Cox regression models and no significant $\times$ condition interactions with these feature scores were found. Sensitivity analyses did not change these results. 
a) Cognitive Therapy

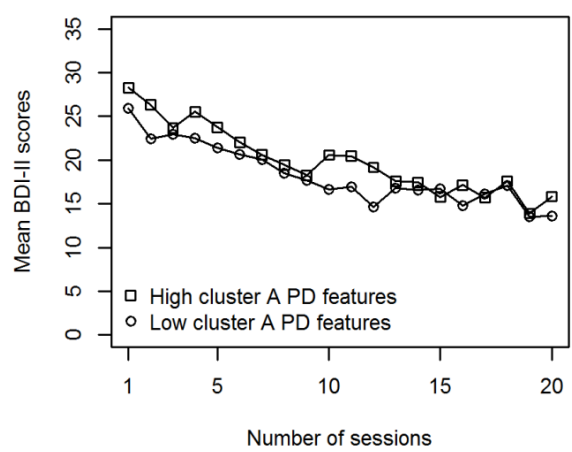

b) Interpersonal Psychotherapy

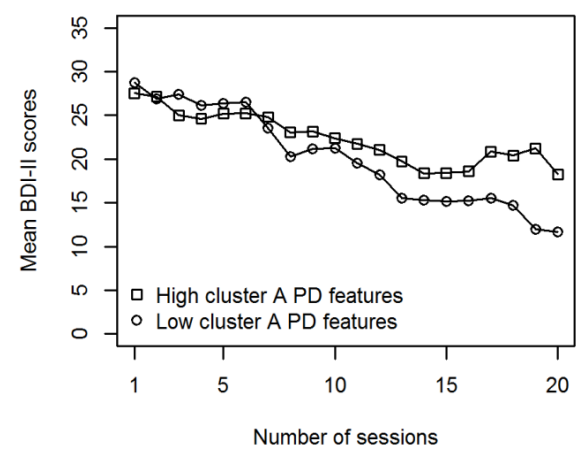

Figure 3.2: Mean Beck Depression Inventory-II (BDI-II) scores for individuals with low and high cluster A personality disorder features for a) cognitive therapy and b) interpersonal psychotherapy in the treatment phase.

Note: PD: personality disorder; Low and high cluster A PD features: subgroups of individuals with low and high cluster A PD features were created via a medium split.

a) Cognitive Therapy

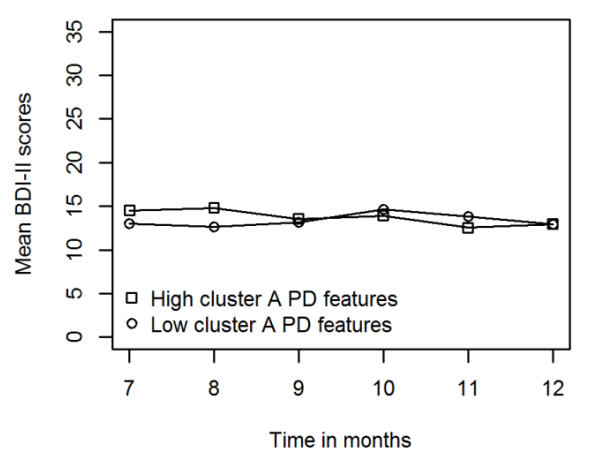

b) Interpersonal Psychotherapy

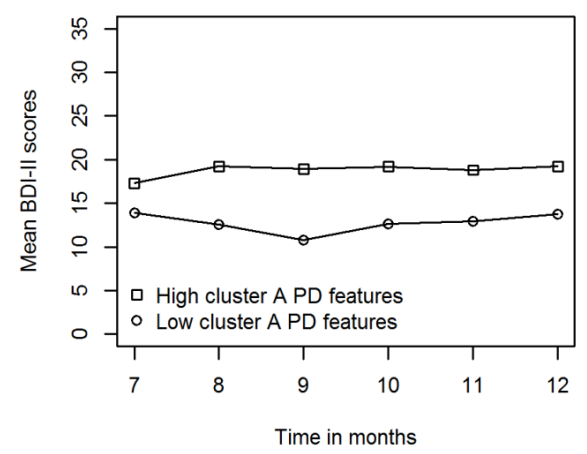

Figure 3.3: Mean Beck Depression Inventory-II (BDI-II) scores for individuals with low and high cluster A personality disorder features for a) cognitive therapy and b) interpersonal psychotherapy in the trial followup phase.

Note: PD: personality pisorder; Low and high cluster A PD features: subgroups of individuals with low and high cluster A PD features were created via a medium split.

Pearson correlation coefficients between BDI-II baseline scores and PD features were as follows: avoidant PD features $(r=0.26)$, dependent PD features $(r=0.14)$, obsessive-compulsive PD features $(r=0.10)$, cluster A PD features $(r=0.18)$ and cluster B PD features $(r=0.08)$. Given these weak correlations, it is unlikely that the described PD features effects on BDI-II change were due to pretreatment associations between PD features and depression severity. 


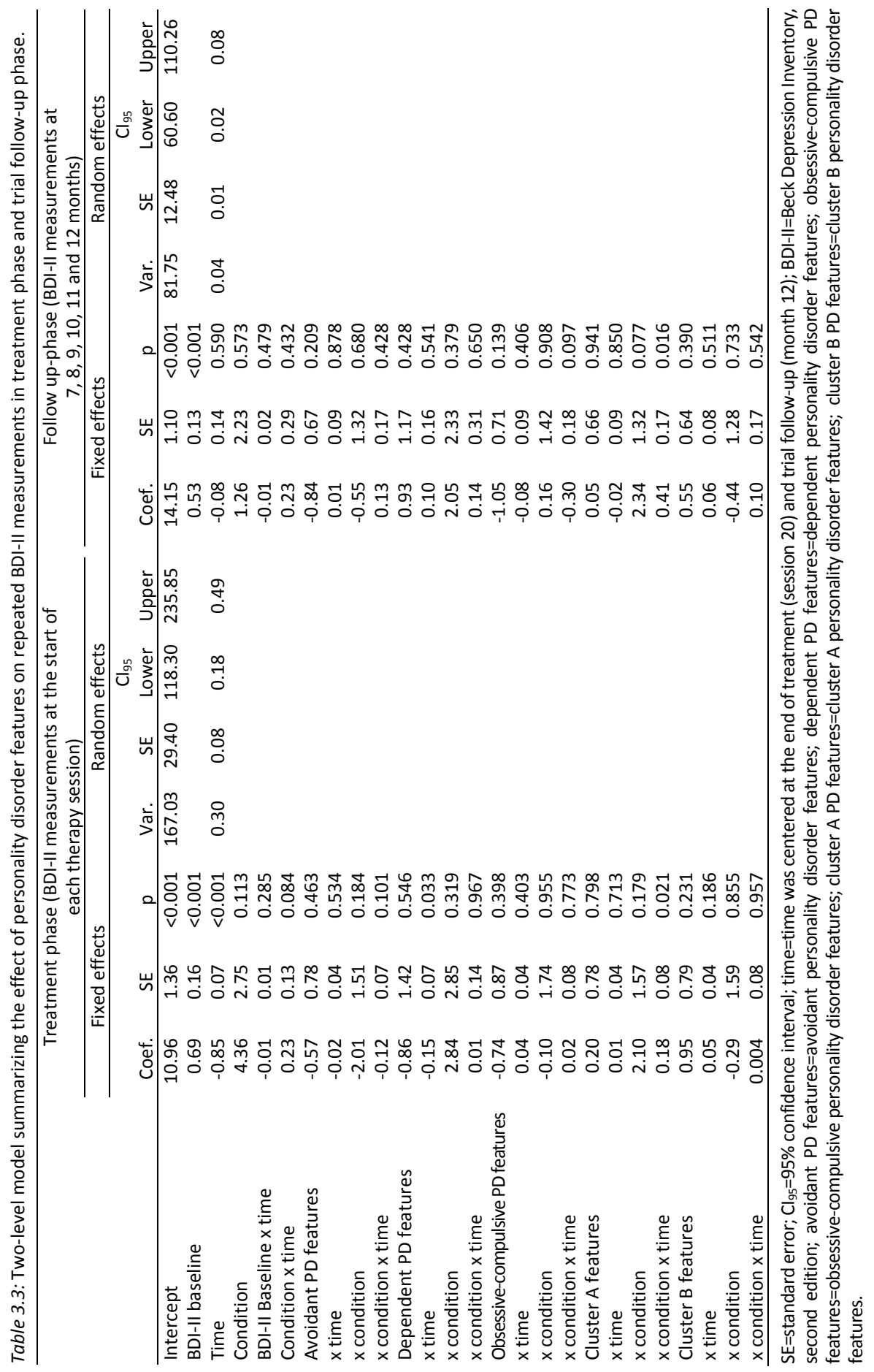




\section{Discussion}

The current study examined the impact of PD and PD features on treatment dropout, depressive symptom change and remission in CT and IPT during treatment and a five month follow-up phase. We found that individuals with PD were more severely depressed prior to treatment compared to individuals without PD. Despite these baseline differences, PD (features) did not affect dropout, remission rates and depression severity change for CT and IPT. In addition, more subtle effects were found regarding specific PD features.

As often found in previous studies (Mulder, 2002), individuals with a PD had a more complex clinical picture compared to individuals without a PD prior to treatment, with higher depression severity, higher general psychological distress and more axis I comorbidity. However, PD did not negatively impacted depression severity change and remission rates during treatment and follow-up. Moreover, the percentage of individuals achieving remission was non-significantly higher for participants with PD, and individuals with and without PD had comparable mean posttreatment BDI-II scores, indicative of no adverse effect of PD.

Contrary to what is often assumed, no adverse effects of PD (features) on treatment retention in both CT and IPT were found. This is consistent with most (Fournier et al., 2008; Kool et al., 2003; Sullivan et al., 1994), but not all previous literature: one study reported that PD was associated with higher dropout; however these findings may be somewhat limited, since only a small proportion of the participants had a PD (Moradveisi et al., 2013). Regarding MDD outcome, the current study did not find a (negative) impact of PD on depressive symptom change and remission. With respect to previous head-to-head comparisons of CT and IPT, one study found similar results (Shea et al., 1990), while another study reported an adverse effect of PD on MDD outcome in IPT as compared to CT (Joyce et al., 2007). However, comparing these findings is difficult since one study used another PD assessment instrument and both studies compared pre- and posttreatment depression scores instead of our repeated measurements approach. Based on our findings we suggest that CT and IPT can both attain clinical significant depressive change, irrespective of a more complex clinical presentation at the start of treatment. With regard to the effect of specific PD features, our findings deviate from previous research. Our finding that dependent PD features predicted a BDI-II decrease over time across the treatment phase has not been reported previously. A possible explanation could be that individuals with higher dependent PD features respond initially better to contact with a therapist, but that this effect is lost towards treatment termination. The finding that participants with high cluster A PD features fared better in CT compared to IPT has been previously reported for schizoid symptoms (Joyce et al., 2007). In addition, in a comparison of CT with pharmacotherapy and behavioral therapy, cluster A PD features were associated with 
better outcomes in CT for the long-term (Moradveisi et al., 2013). However, these effects were absent in the acute phase of MDD. Our findings might be explained by the fact that individuals with higher cluster A PD features respond better to a structured therapy in which the therapists takes a more directive role. In addition, one could argue that focusing on interpersonal problems is quite complex for individuals with cluster A PD features. In previous research, avoidant PD features were found to predict superior treatment outcome in CT compared to IPT (Barber \& Muenz, 1996; Joyce et al., 2007), a finding that was not replicated in the current study. The absence of an effect of cluster B PD features on BDI-II change is in line with a study into MDD with comorbid borderline PD of Bellino et al. (2007), although it should be noted that numerous studies have reported otherwise (Agosti, Hellerstein, \& Stewart, 2009; Grilo et al., 2005; Gunderson et al., 2011; Levenson, Wallace, Fournier, Rucci, \& Frank, 2012; Skodol et al., 2011). A possible explanation for our finding is that we included participants who met criteria for a primary diagnosis of MDD with comorbid PD, and not participants with a primary PD diagnosis with comorbid MDD.

The finding that comorbid PD did not affect MDD outcome seems at odds with a previous paper using a subset of the same data of this study. This study reported that the presence of any PD predicted lower posttreatment BDI-II scores (Huibers et al., 2015, 2016). These findings derived from a linear regression model with 20 other variables, using a completers sample $(n=134)$, including diagnoses of PassiveAggressive and Depressive PD. We repeated our treatment phase analyses using the same covariates, data subset and PD diagnoses to explain these conflicting results, but our findings remained similar. However, after removing the random slope (and the autoregressive structure for residuals), a decreasing effect of PD on BDI-Il change became apparent but was non-significant $(\beta=-1.97, p=0.11)$. This suggests that the random effects of the multilevel model are responsible for the differences between the analyses, which is in line with our earlier statement that multivariable linear regression approaches are not perfectly suited to address the effects of PD and PD features on treatment outcome reliably. The impact of adding a random slope to our model, underlines the importance of modeling the erratic and individual symptom trajectories of depression.

Contrary to previous findings (De Bolle et al., 2011), our sensitivity analyses did not indicate an effect of therapist differences on treatment outcome. One of the possible reasons for the absence of a therapist effect, may be the (very) good to excellent quality of therapy, and the absence of therapists differences in protocol adherence (Lemmens et al., 2015).

\subsection{Clinical implications}

Translating our findings to clinical practice, a few recommendations can be considered. Firstly, depressed individuals with or without comorbid cluster C PD 
benefitted equally from both evidence-based psychotherapies and higher premature treatment discontinuation in individuals with personality pathology was not observed. This is relevant as comorbid cluster C PD is highly prevalent in MDD (Friborg et al., 2014) and research shows that depressed individuals with comorbid PD are less likely to receive adequate MDD treatment (Black, Bell, Hulbert, \& Nasrallah, 1988; Charney, Nelson, \& Quinlan, 1981; Lewis \& Appleby, 1988). To avoid undertreatment of MDD in individuals with a comorbid cluster C PD, adherence to standard clinical treatment guidelines seems mandatory. Second, based on these findings, depressed individuals with high cluster A PD features could be advised to start with CT instead of IPT, although replication of these results is needed.

\subsection{Methodological considerations}

The current study evaluated the impact of personality pathology on MDD outcome in CT and IPT using state-of-the art multilevel modeling with repeated measurement of depressive symptoms. These models were corrected for baseline BDI-II differences to allow a fair comparison between two initially different participant groups. Other important strengths of this study are the random allocation, the relative large sample size, the low and reported dropout rates and an extensive integrity check (Lemmens et al., 2015). Since these data were collected in an effectiveness study (Lemmens et al., 2015), we can translate our findings more directly to clinical practice, while benefitting from the controlled circumstances.

Our study has some limitations. First, MDD and PD diagnoses were only assessed by one rater, so inter-rater reliability could not be tested. Second, therapist and participants were not blind to the results of the SCID-II assessment. This knowledge could have affected therapeutic strategies and the participant's motivation. Although our adherence check showed no difference in therapy-specific behavior for individuals with and without PD, therapeutic interventions could have been adapted for individuals with PD (e.g., responsivity effects, Stiles, 2009). Third, dimensional scores of PD features were calculated by summing up the items answered with 'present' for each PD. It can be doubted if every point of increase on these dimensional scales corresponds to a comparable increase of PD severity. Fourth, we based our primary outcome only on one self-report instrument and we did not include a clinician-rated measure of depression severity change. The magnitude of convergence between the two types of measurement is uncertain given different results in the literature (Cuijpers, Li, Hofmann, \& Andersson, 2010; Vittengl, Clark, Thase, \& Jarrett, 2013). Fifth, since PD and PD features were only assessed at baseline, we could not determine if these PD findings are state or trait dependent. The PD assessment could be contaminated by the depressive perspective (Fava et al., 2002; Joffe \& Regan, 1988). To deal with these complex concepts, a pragmatic approach is suggested: if measures of PD (features) predict (differential) depression outcome when MDD is 
diagnosed, then they are considered to be useful (Mulder, 2002). Sixth, our primary outcome was the level of depressive symptomatology. It is uncertain if differences in other life domains (e.g., quality of interpersonal relations) would emerge between remitted individuals with our without PD after successful treatment. Finally, since appointments were allowed to be scheduled in a flexible manner some participants received one or two more therapy sessions during the trial follow-up phase, but this is unlikely to impact our outcomes drastically.

\subsection{Future directions}

More research is needed to further explore our results. Since our sample, in line with previous outpatient research (Friborg et al., 2014), consisted predominantly of individuals with a cluster $\mathrm{C}$ diagnosis, our considerations about cluster $\mathrm{A}$ and $\mathrm{B}$ PDs on MDD outcome in IPT and CT should be interpreted with caution. Although our study demonstrates promising results of cluster A PD features as potential treatment moderators, further research is needed to replicate these findings. Since cluster A PD features is a heterogeneous category, a more detailed understanding about specific cluster A PD features is warranted. Ideally, a 'what fits for whom' algorithm, based on individual personality features can be generated. While this study focused on the treatment phase and a five-month trial follow-up phase, a number of studies indicate an adverse effect of PD on long-term MDD outcomes (Grilo et al., 2010; Markowitz et al., 2007). Further research is needed to examine the enduring effects of PD and PD features on CT and IPT outcomes over time. Preferably, when examining long-term outcome effects, PD and MDD should be simultaneously and frequently assessed to determine state and trait dependent changes.

Supplementary data associated with this article can be found in the online version at http://dx.doi.org/10.1016/j.jad.2017.08.043. 


\section{References}

Agosti, V., Hellerstein, D. J., \& Stewart, J. W. (2009). Does personality disorder decrease the likelihood of remission in early-onset chronic depression? Comprehensive psychiatry, 50(6), 491-495.

American Psychiatric Association. (2000). Diagnostic and Statistical Manual of Mental Disorders (4th ed.). Washington DC.

Barber, J. P., \& Muenz, L. R. (1996). The role of avoidance and obsessiveness in matching patients to cognitive and interpersonal psychotherapy: empirical findings from the treatment for depression collaborative research program. Journal of Consulting and Clinical Psychology, 64(5), 951-958.

Beck, A. T., Rush, A. J., Shaw, B. F., \& Emery, G. (1979). Cognitive therapy of depression. New York: Guilford Press.

Beck, A. T., Steer, R., \& Brown, G. K. (1996). Beck Depression Inventory II: Manual. Boston: Harcourt Brace.

Bellino, S., Zizza, M., Rinaldi, C., \& Bogetto, F. (2007). Combined therapy of major depression with concomitant borderline personality disorder: comparison of interpersonal and cognitive psychotherapy. The Canadian Journal of Psychiatry, 52(11), 718-725.

Black, D. W., Bell, S., Hulbert, J., \& Nasrallah, A. (1988). The importance of Axis II in patients with major depression. A controlled study. Journal of affective disorders, 14(2), 115-122.

Carter, J. D., Luty, S. E., McKenzie, J. M., Mulder, R. T., Frampton, C. M., \& Joyce, P. R. (2011). Patient predictors of response to cognitive behaviour therapy and interpersonal psychotherapy in a randomised clinical trial for depression. Journal of affective disorders, 128(3), 252-261.

Casey, P., Birbeck, G., McDonagh, C., Horgan, A., Dowrick, C., Dalgard, O., . . G Group, O. (2004). Personality disorder, depression and functioning: results from the ODIN study. Journal of affective disorders, 82(2), 277-283.

Charney, D. S., Nelson, J. C., \& Quinlan, D. M. (1981). Personality traits and disorder in depression. The American Journal of Psychiatry, 138(12), 1601-1604.

Cuijpers, P., Karyotaki, E., Weitz, E., Andersson, G., Hollon, S. D., \& van Straten, A. (2014). The effects of psychotherapies for major depression in adults on remission, recovery and improvement: a metaanalysis. Journal of affective disorders, 159, 118-126.

Cuijpers, P., Li, J., Hofmann, S. G., \& Andersson, G. (2010). Self-reported versus clinician-rated symptoms of depression as outcome measures in psychotherapy research on depression: a meta-analysis. Clinical psychology review, 30(6), 768-778.

De Bolle, M., De Fruyt, F., Quilty, L. C., Rolland, J.-P., Decuyper, M., \& Bagby, R. M. (2011). Does personality disorder co-morbidity impact treatment outcome for patients with major depression?: a multi-level analysis. Journal of Personality Disorders, 25(1), 1-15.

Derogatis, L. R., \& Melisaratos, N. (1983). The Brief Symptom Inventory: an introductory report. Psychological Medicine, 13(3), 595-605.

DeRubeis, R. J., Cohen, Z. D., Forand, N. R., Fournier, J. C., Gelfand, L. A., \& Lorenzo-Luaces, L. (2014). The Personalized Advantage Index: translating research on prediction into individualized treatment recommendations. A demonstration. PLoS One, 9(1), e83875.

Dobson, K. S., Shaw, B. F., \& Vallis, T. M. (1985). Reliability of a measure of the quality of cognitive therapy. British Journal of Clinical Psychology, 24(4), 295-300.

EuroQol, G. (1990). EuroQol--a new facility for the measurement of health-related quality of life. Health Policy, 16(3), 199-208.

Fava, M., Farabaugh, A. H., Sickinger, A. H., Wright, E., Alpert, J. E., Sonawalla, S., . . Worthington, J. J., 3rd. (2002). Personality disorders and depression. Psychological Medicine, 32(6), 1049-1057.

First, M. B., Gibbon, M., Spitzer, R. L., Williams, J. B. W., \& Benjamin, L. S. (1997). Structured Clinical Interview for DSM-IV Axis II Personality Disorders (SCID-II) Washington, D.C: American Psychiatric Press.

First, M. B., Spitzer, R. L., Gibbon, M., \& Williams, J. B. W. (1995). Structured Clinical Interview for DSM-IV Axis I Disorders (SCID-I). New York: Biometrics Research Department New York State Psychiatric Institute. 
Fournier, J. C., DeRubeis, R. J., Shelton, R. C., Gallop, R., Amsterdam, J. D., \& Hollon, S. D. (2008). Antidepressant medications v. cognitive therapy in people with depression with or without personality disorder. The British Journal of Psychiatry, 192(2), 124-129.

Friborg, O., Martinsen, E. W., Martinussen, M., Kaiser, S., Overgard, K. T., \& Rosenvinge, J. H. (2014). Comorbidity of personality disorders in mood disorders: a meta-analytic review of 122 studies from 1988 to 2010. Journal of affective disorders, 152-154, 1-11.

Grilo, C. M., Sanislow, C. A., Shea, M. T., Skodol, A. E., Stout, R. L., Gunderson, J. G., . . . Zanarini, M. C. (2005). Two-year prospective naturalistic study of remission from major depressive disorder as a function of personality disorder comorbidity. Journal of Consulting and Clinical Psychology, 73(1), 78.

Grilo, C. M., Stout, R. L., Markowitz, J. C., Sanislow, C. A., Ansell, E. B., Skodol, A. E., . . McGlashan, T. H. (2010). Personality disorders predict relapse after remission from an episode of major depressive disorder: a 6-year prospective study. The Journal of clinical psychiatry, 71(12), 1629-1635.

Gunderson, J. G., Stout, R. L., McGlashan, T. H., Shea, M. T., Morey, L. C., Grilo, C. M., . . . Sanislow, C. (2011). Ten-year course of borderline personality disorder: psychopathology and function from the Collaborative Longitudinal Personality Disorders study. Archives of general psychiatry, 68(8), 827-837.

Hardy, G. E., Barkham, M., Shapiro, D. A., Stiles, W. B., Rees, A., \& Reynolds, S. (1995). Impact of Cluster C personality disorders on outcomes of contrasting brief psychotherapies for depression. Journal of Consulting and Clinical Psychology, 63(6), 997-1004.

Hirschfeld, R., Russell, J. M., Delgado, P. L., Fawcett, J., Friedman, R. A., Harrison, W. M., . . Howland, R. H. (1998). Predictors of response to acute treatment of chronic and double depression with sertraline or imipramine. The Journal of clinical psychiatry.

Hollon, S., Evans, M., Auerbach, A., DeRubeis, R., Elkin, I., Lowery, A., . . Piasecki, J. (1988). Development of a system for rating therapies for depression: Differentiating cognitive therapy, interpersonal psychotherapy, and clinical management pharmacotherapy. Unpublished manuscript.

Hollon, S., Evans, M., Elkin, I., \& Lowery, A. (1984). System for rating therapies for depression. Paper presented at the 92nd Annual Convention of the American Psychological Association, Toronto, Ontario, Canada.

Huibers, M. J., Cohen, Z. D., Lemmens, L. H., Arntz, A., Peeters, F. P., Cuijpers, P., \& DeRubeis, R. J. (2015). Predicting optimal outcomes in cognitive therapy or interpersonal psychotherapy for depressed individuals using the personalized advantage index approach. PLoS One, 10(11), e0140771.

Huibers, M. J., Cohen, Z. D., Lemmens, L. H., Arntz, A., Peeters, F. P., Cuijpers, P., \& DeRubeis, R. J. (2016). Correction: Predicting Optimal Outcomes in Cognitive Therapy or Interpersonal Psychotherapy for Depressed Individuals Using the Personalized Advantage Index Approach. PLoS One, 11(2).

Joffe, R. T., \& Regan, J. J. (1988). Personality and depression. Journal of Psychiatric Research, 22(4), 279-286.

Joyce, P. R., McKenzie, J. M., Carter, J. D., Rae, A. M., Luty, S. E., Frampton, C. M., \& Mulder, R. T. (2007). Temperament, character and personality disorders as predictors of response to interpersonal psychotherapy and cognitive-behavioural therapy for depression. The British Journal of Psychiatry, 190(6), 503-508.

Klerman, G. L., Weissman, M. M., Rounsaville, B. J., \& Chevron, E. S. (1984). Interpersonal psychotherapy for depression. New York: Basis Books.

Kool, S., Dekker, J., Duijsens, I. J., de Jonghe, F., \& Puite, B. (2003). Efficacy of combined therapy and pharmacotherapy for depressed patients with or without personality disorders. Harvard Review of Psychiatry, 11(3), 133-141.

Kool, S., Schoevers, R., de Maat, S., Van, R., Molenaar, P., Vink, A., \& Dekker, J. (2005). Efficacy of pharmacotherapy in depressed patients with and without personality disorders: a systematic review and meta-analysis. Journal of affective disorders, 88(3), 269-278.

Lemmens, L. H., Arntz, A., Peeters, F., Hollon, S. D., Roefs, A., \& Huibers, M. J. (2015). Clinical effectiveness of cognitive therapy $v$. interpersonal psychotherapy for depression: results of a randomized controlled trial. Psychological Medicine, 1-16.

Lemmens, L. H., Arntz, A., Peeters, F. P., Hollon, S. D., Roefs, A., \& Huibers, M. J. (2011). Effectiveness, relapse prevention and mechanisms of change of cognitive therapy vs. interpersonal therapy for depression: Study protocol for a randomised controlled trial. Trials, 12, 150. 
Levenson, J. C., Wallace, M. L., Fournier, J. C., Rucci, P., \& Frank, E. (2012). The role of personality pathology in depression treatment outcome with psychotherapy and pharmacotherapy. Journal of Consulting and Clinical Psychology, 80(5), 719.

Lewis, G., \& Appleby, L. (1988). Personality disorder: the patients psychiatrists dislike. The British Journal of Psychiatry, 153, 44-49.

Maddux, R. E., Riso, L. P., Klein, D. N., Markowitz, J. C., Rothbaum, B. O., Arnow, B. A., . . Thase, M. E. (2009). Select comorbid personality disorders and the treatment of chronic depression with nefazodone, targeted psychotherapy, or their combination. Journal of affective disorders, 117(3), 174179.

Markowitz, J. C., Skodol, A. E., Petkova, E., Cheng, J., Sanislow, C. A., Grilo, C. M., . . . McGlashan, T. H. (2007). Longitudinal effects of personality disorders on psychosocial functioning of patients with major depressive disorder. Journal of Clinical Psychiatry, 68(2), 186-193.

Moradveisi, L., Huibers, M. J., Renner, F., Arasteh, M., \& Arntz, A. (2013). The influence of comorbid personality disorder on the effects of behavioural activation vs. antidepressant medication for major depressive disorder: Results from a randomized trial in Iran. Behaviour research and therapy, 51(8), 499-506.

Mulder, R. T. (2002). Personality pathology and treatment outcome in major depression: a review. American Journal of Psychiatry, 159(3), 359-371.

Mundt, J. C., Marks, I. M., Shear, M. K., \& Greist, J. H. (2002). The Work and Social Adjustment Scale: a simple measure of impairment in functioning. Br J Psychiatry, 180, 461-464.

Newton-Howes, G., Tyrer, P., Johnson, T., Mulder, R., Kool, S., Dekker, J., \& Schoevers, R. (2014). Influence of personality on the outcome of treatment in depression: systematic review and meta-analysis. Journal of Personality Disorders, 28(4), 577-593.

Reich, J. H., \& Vasile, R. G. (1993). Effect of personality disorders on the treatment outcome of axis I conditions: an update. Journal of Nervous and mental Disease, 181(8), 475-484.

Ryder, A. G., Quilty, L. C., Vachon, D. D., \& Bagby, R. M. (2010). Depressive personality and treatment outcome in major depressive disorder. Journal of Personality Disorders, 24(3), 392-404.

Sato, T., Sakado, K., Sato, S., \& Morikawa, T. (1994). Cluster a personality disorder: a marker of worse treatment outcome of major depression? Psychiatry Research, 53(2), 153-159.

Shea, M. T., Pilkonis, P. A., Beckham, E., Collins, J. F., Elkin, I., Sotsky, S. M., \& Docherty, J. P. (1990). Personality disorders and treatment outcome in the NIMH Treatment of Depression Collaborative Research Program. American Journal of Psychiatry, 147(6), 711-718.

Simon, G. E., \& Perlis, R. H. (2010). Personalized medicine for depression: can we match patients with treatments? American Journal of Psychiatry, 167(12), 1445-1455.

Skodol, A. E., Grilo, C. M., Keyes, K. M., Geier, T., Grant, B. F., \& Hasin, D. S. (2011). Relationship of personality disorders to the course of major depressive disorder in a nationally representative sample. American Journal of Psychiatry, 168(3), 257-264.

Stanley, B., \& Wilson, S. T. (2006). Heightened subjective experience of depression in borderline personality disorder. Journal of Personality Disorders, 20(4), 307-318.

Stiles, W. B. (2009). Responsiveness as an obstacle for psychotherapy outcome research: It's worse than you think. Clinical Psychology: Science and Practice, 16(1), 86-91.

Stuart, S. (2011). IPT Adherence and Quality Scale. Interpersonal Psychotherapy Institute, lowa (unpublished manuscript).

Sullivan, P. F., Joyce, P. R., \& Mulder, R. T. (1994). Borderline personality disorder in major depression. Journal of Nervous and mental Disease, 182(9), 508-516.

Unger, T., Hoffmann, S., Köhler, S., Mackert, A., \& Fydrich, T. (2013). Personality disorders and outcome of inpatient treatment for depression: a 1-year prospective follow-up study. Journal of Personality Disorders, 27(5), 636-651.

van der Zee, K. I., \& Sanderman, R. (1993). Het meten van de algemene gezondheidstoestand met de RAND36: een handleiding. Groningen: Noordelijk Centrum voor Gezondheidsvraagstukken.

Vittengl, J. R., Clark, L. A., Thase, M. E., \& Jarrett, R. B. (2013). Nomothetic and idiographic symptom change trajectories in acute-phase cognitive therapy for recurrent depression. Journal of Consulting and Clinical Psychology, 81(4), 615. 
S. van Bronswijk, E. Köster, F. Peeters - Psychotherapy and Psychosomatics 2019, 1-2

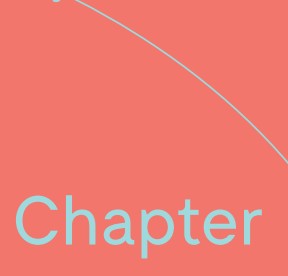




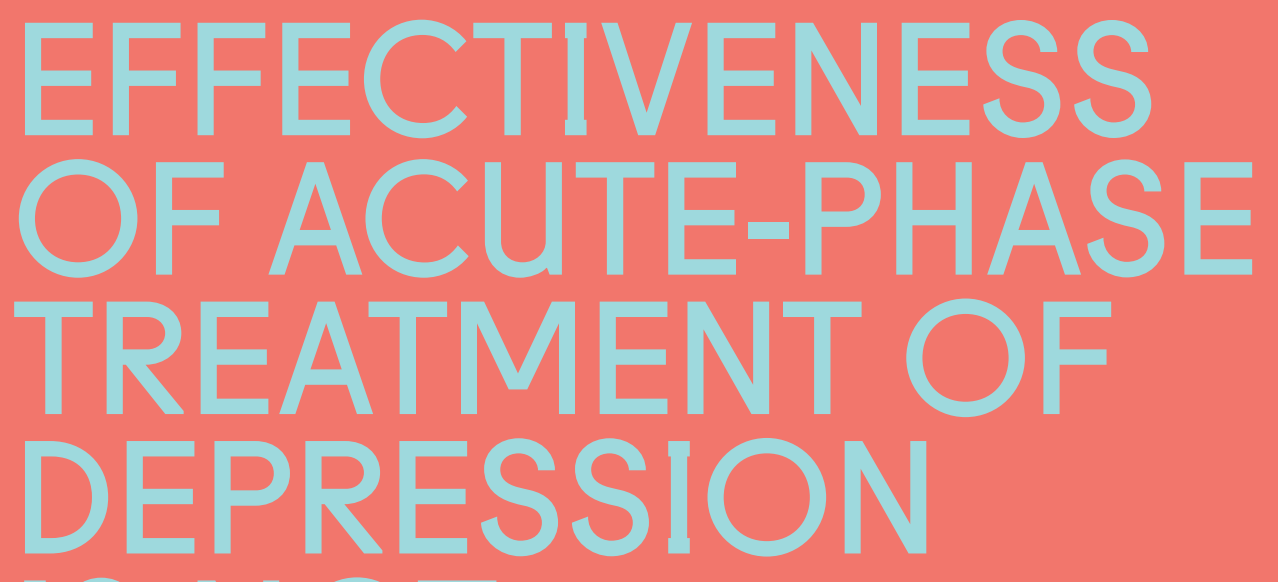

IS NOT

INFLUENCED

BY COMORBID

PERSONALITY

DISORDERS:

RESULTS

FROM A

META-ANALYSIS

AND META-

REGRESSION 


\section{Abstract}

\section{Background}

Previous meta-analyses into the impact of comorbid personality disorders (PDs) on treatment outcome for major depressive disorder (MDD) are not robust, and biased by inclusion of uncontrolled designs, and unstructured instruments. To estimate the impact of comorbid PD on acute phase treatment outcome for MDD, using only data from well-designed trials.

Methods

In this meta-analysis (PROSPERO, CRD42019120200), a database search up to April 3th 2017 was conducted in Pubmed, PsycINFO and Embase. References of selected articles, earlier reviews, and meta-analyses were searched. Treatment studies for MDD with a subset of individuals with comorbid PD were included. MDD and PD diagnoses had to be based on pretreatment structured interviews. Primary outcome was depression severity change during treatment. Data was extracted from published records, corresponding authors were contacted to provide additional information. Effect sizes were estimated with random-effects models. Study-level variables were examined using meta-regression. Included studies were evaluated with the Risk of Bias tool. Publication bias was assessed using funnel plots and Egger's test.

\section{Results}

Six studies with 942 individuals (447 with PD) were included. There was no significant difference in depression severity change between individuals with and without PD $(\mathrm{g}=0.03,95 \% \mathrm{Cl}=-0.15$ to $0.20, p=0.27$ ). Heterogeneity and risk of bias were low. The meta-regression did not indicate relevant variables. There was no indication for publication bias.

\section{Conclusions}

Findings indicate no effect of comorbid PD on acute phase treatment outcome for MDD. Depressed individuals with and without comorbid PD should receive evidence-based depression treatments alike. 


\section{Letter to the editor}

About $45 \%$ of the individuals suffering from major depressive disorder (MDD) also meet criteria for a personality disorder (PD), falling predominantly in the DSM-based cluster $\mathrm{C}$. This comorbidity is regarded clinically relevant as it is accompanied by lower levels of daily functioning, and is often considered to be a predictor of poor acutephase treatment response in MDD (Newton-Howes, Tyrer, \& Johnson, 2006; NewtonHowes et al., 2014). However, the evidence that the presence of a comorbid PD may have a negative impact on acute-phase treatment outcomes for MDD is not robust and biased by unstructured PD and MDD assessments, naturalistic treatment allocation, not controlling for significant confounders (e.g., earlier onset, childhood adversity, and episode duration), and no consideration of baseline depression severity differences between individuals with and without comorbid PD (Mulder, 2002). As the last meta-analysis (Newton-Howes et al., 2014) included only studies published before 2007, and given the methodological considerations above (Mulder, 2002), we conducted a meta-analysis and meta-regression with strict inclusion criteria to maximize validity of the results. For that purpose, we collected only well-designed controlled trials that investigated the impact of comorbid PD on acute-phase treatment outcome for MDD with the use of (semi-) structured interviews for both MDD and PD. Additionally, the primary outcome was depression severity change during treatment, thereby avoiding bias following from baseline severity differences between individuals with and without comorbid PD.

A database search up to April 3, 2017, was conducted in PubMed, PsycINFO, and Embase (study protocol: PROSPERO, CRD42019120200). References of selected articles, earlier reviews, and meta-analyses were searched. Treatment studies for MDD with a subset of individuals with comorbid PD were included. MDD and PD diagnoses had to be based on pre-treatment structured interviews. Primary outcome was depression severity change during treatment, and secondary outcomes were response and remission rates. Data was extracted from published records, and corresponding authors were contacted to provide additional information. Effect sizes (Hedges' g effect sizes, risk ratios [RRs] and odds ratios [ORs]) were estimated with random-effects models. Included studies were evaluated with the Risk of Bias tool. Study-level variables were examined using meta-regression. Publication bias was assessed using funnel plots and Egger's test.

A total of 11 studies were identified, of which 6 studies (12 comparisons) with 942 individuals ( 447 with a comorbid PD) were included in the meta-analysis (Fava et al., 2002; Fournier et al., 2008; Hardy et al., 1995; Lemmens et al., 2015; Moradveisi, Huibers, Renner, Arasteh, \& Arntz, 2013b; Papakostas et al., 2003). Trials were conducted in outpatient settings in the Netherlands $(n=1)$, Iran $(n=1)$, the United States $(n=3)$, and the United Kingdom $(n=1)$. Treatment consisted of different types of psychotherapy (cognitive [behavioral] therapy, interpersonal psychotherapy, 
behavioral activation, psychodynamic interpersonal psychotherapy; 8 conditions, $\mathrm{n}=350$ ) and antidepressant medication (sertraline, paroxetine, nortriptyline, fluoxetine; 4 conditions, $n=592$ ). Change in depression severity was measured with self-report questionnaires (Beck Depression Inventory; BDI), clinician-rated measures (Hamilton Depression Rating Scale; HDRS), or with both. Response rates were available for 4 studies (Fava et al., 2002; Fournier et al., 2008; Lemmens et al., 2015; Papakostas et al., 2003). These were defined as (i) at least $50 \%$ reduction, or (ii) a predefined minimum decrease on the severity scale, or (iii) a combination of both criteria. Remission rates were available for 4 studies (Fava et al., 2002; Fournier et al., 2008; Hardy et al., 1995; Lemmens et al., 2015) and were based on cutoff scores on depression severity scales (HDRS $\leq 7, \mathrm{BDI} \leq 9,10$ or 15 ). As shown in Figure 4.1, the mean pooled Hedges' $\mathrm{g}$ effect size was $\mathrm{g}=0.03(95 \% \mathrm{Cl}=-0.15$ to $0.20, \mathrm{p}=0.27)$, indicating no significant difference in the average depression severity change between individuals with and without a PD. The heterogeneity of the pooled effect size was low with a moderately high level of uncertainty $\left(\mathrm{I}^{2}=17.6 \%, 95 \% \mathrm{Cl}=0.00 \%\right.$ to $\left.56.95 \%\right)$. A total of 219 individuals with a PD achieved response (58.2\%), compared to 209 individuals without a PD (54.5\%); the pooled RR for response was $1.14(95 \% \mathrm{Cl}=0.99$ to $1.31, p=0.07)$, and the pooled OR was $1.32(95 \% \mathrm{Cl}=0.83$ to $2.12, p=0.24)$, indicating no significant difference between individuals with and without a PD. A total of 98 individuals with a PD met criteria for remission (44.3\%), and 125 individuals without $\mathrm{PD}$ achieved remission (47.2\%); the pooled $\mathrm{RR}$ for remission was $0.90(95 \% \mathrm{Cl}=0.74$ to $1.09, \mathrm{p}=0.27)$, and the pooled $\mathrm{OR}$ was $0.75(95 \% \mathrm{Cl}=0.47$ to $1.19, \mathrm{p}=0.23)$, indicating no significant difference between individuals with and without a PD. Risk of bias was low. The meta-regression did not indicate significant associations between specific studylevel variables and between-study heterogeneity of the results; however, these analyses are highly exploratory due to missing data and a low number of included studies. There was no indication for publication bias.

Findings indicate no significant difference between individuals with and without a comorbid PD in terms of average depression severity change, response rates, and remission rates. Our findings do not concur with results from the most recent previous meta-analyses (Newton-Howes et al., 2006; Newton-Howes et al., 2014). However, in contrast to these meta-analyses, we included only controlled studies in which diagnoses were based on (semi-) structured interviews for both mood disorder and personality to reduce heterogeneity and biases, and maximize the validity of the results. These stringent inclusion criteria did, however, result in a low number of included studies, which can be considered as a limitation. Other limitations are the lack of dimensional assessment of personality and long-term treatment outcomes.

Based on the findings from the current meta-analysis, we strongly suggest that depressed patients with comorbid PD receive evidence-based treatments following guidelines for their MDD; their acute-phase treatment outcome appears not worse than that of individuals with MDD without comorbid PD. More high-quality studies are 
needed to establish the effects of comorbid PD for specific depression treatments (differential treatment effects), different types of personality pathology, and longterm outcomes. In addition, disentangling the complex relationships between MDD and PD is and will remain a challenging task for researchers and clinicians alike: How do depression and personality relate to each other, and, to what degree are they really distinct (Klein, Kotov, \& Bufferd, 2011)? In future studies, MDD and PD should be reliably, frequently, and concurrently assessed to further understand their interrelationship, and their "state" (current illness) and "trait" (stable set of characteristics) aspects.

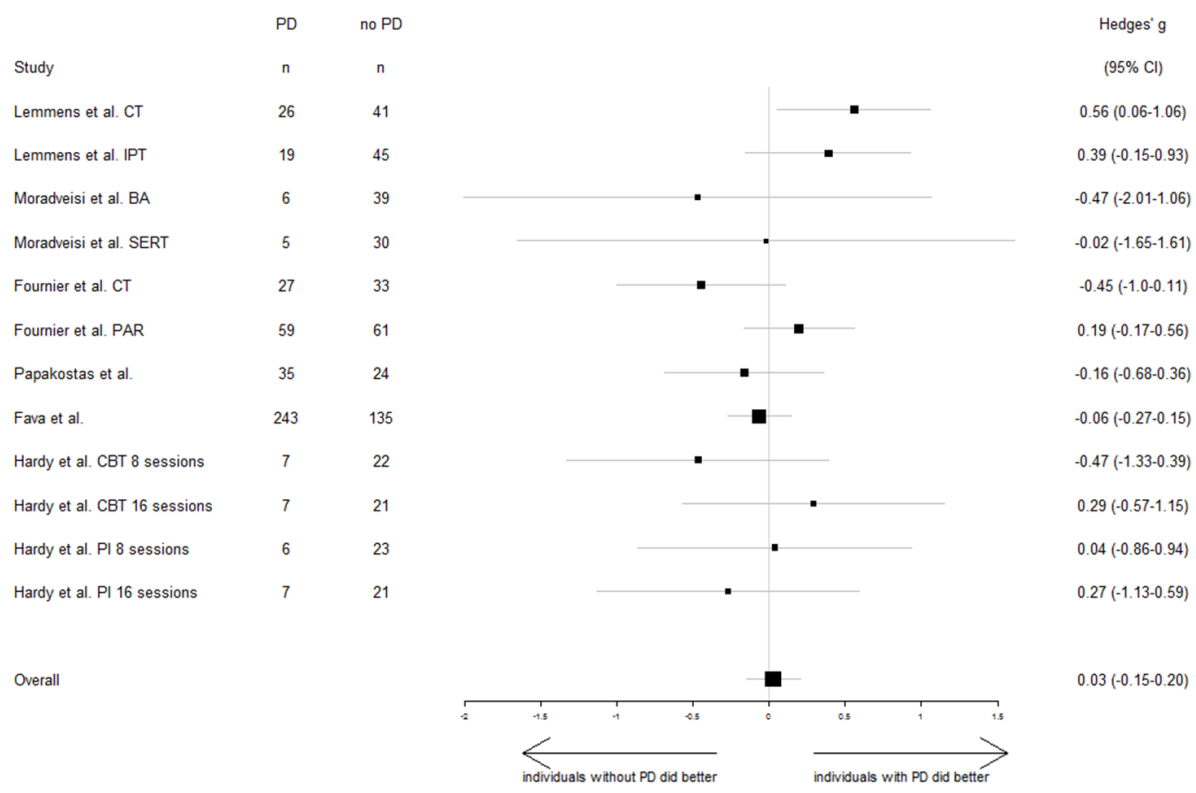

Figure 4.1: Effects of comorbid personality disorder on Hedges 'g effect sizes.

$\mathrm{PD}=$ personality disorder; $\mathrm{Cl}=$ confidence interval; $\mathrm{CT}=$ cognitive therapy; $\mathrm{IPT}=$ =interpersonal pPsychotherapy; $\mathrm{BA}=$ behavioral activation; $\mathrm{SERT}=$ sertraline; $\mathrm{PAR}=$ paroxetine; $\mathrm{CBT}=$ cognitive behavioral therapy; $\mathrm{Pl}=$ psychodynamic interpersonal psychotherapy. 


\section{Methods}

\subsection{Search strategy and study selection}

In this meta-analysis, an electronic database search was conducted in Pubmed, PsycINFO and Embase using a combination of terms regarding major depressive disorder (MDD), personality pisorder (PD) and antidepressant treatments (see Table 4.1 for specific search terms). Additional records were identified by examining the reference lists of the selected articles, and earlier reviews and meta-analyses on this topic. Studies about the effectiveness of acute phase treatment for MDD that included a subset of individuals with a comorbid personality disorder were included. Criteria for eligibility were: 1 ) adults with a primary diagnosis of MDD, 2) acute phase treatment for MDD, including all forms of talking therapy, antidepressant medication (ADM), and other biological treatments (electroconvulsive therapy and transcranial magnetic stimulation), 3) controlled treatment(s) and random treatment assignment for studies with more than one treatment condition, 4) MDD and PD diagnoses based on a structured interview derived from DSM-III, DSM-IV, DSM-5, ICD-9 or ICD-10 conducted prior to the start of treatment, 5) change of depression severity scores from baseline to post-treatment assessed with the Beck Depression inventory (BDI; Beck, Steer, \& Brown, 1996), the Hamilton Depression Rating Scale (HDRS; Hamilton, 1960), the Montgomery Asberg Depression Rating Scale (MADRS; Montgomery \& Åsberg, 1979) or the (Quick) Inventory of Depressive Symptomatology (IDS; Rush, Carmody, \& Reimitz, 2000). Studies were excluded if the primary diagnosis was not MDD, e.g., bipolar disorder, dysthymia or PD. In addition, studies with MDD and PD diagnoses based on self-report questionnaires or non-structured clinical interviews were excluded. The search was restricted to articles written in English, Dutch or German, and to a human and adult sample. Publications up to the 3rd of April 2017 were included in the search. The study protocol was registered with PROSPERO (CRD42019120200). Two reviewers (EK, FP) independently screened abstracts of studies retrieved from the initial search. Records that were selected by at least one of the reviewers were then screened full-text by three reviewers independently (EK; all records, FP first part and SB second part of all records). Conflicts over inclusion were resolved through discussion with a third independent reviewer (FP or SB depending on the record). If information on eligibility criteria remained unclear, corresponding authors were contacted with specific questions concerning these criteria. 
Table 4.1: Database search terms

\begin{tabular}{|c|c|}
\hline \multicolumn{2}{|c|}{ Search terms in PUBMED } \\
\hline & $\begin{array}{l}\text { ("depressive disorder"[MeSH Terms] OR depress*[Text Word] OR unipolar depress*[Text Word] OR major } \\
\text { depress*[Text Word]) }\end{array}$ \\
\hline AND & 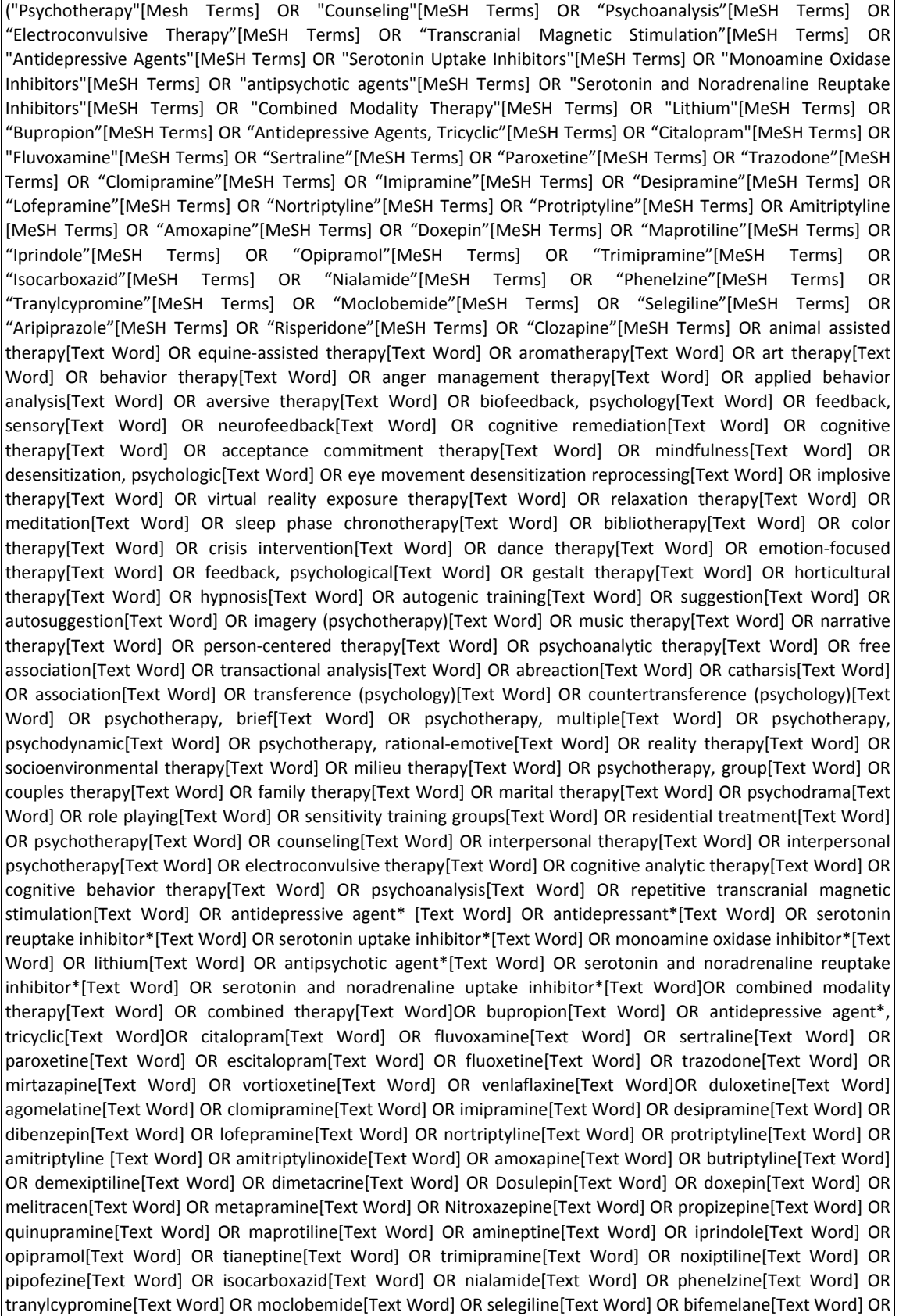 \\
\hline
\end{tabular}


\begin{tabular}{|l|l|}
\hline & $\begin{array}{l}\text { pirlindole[Text Word] OR toloxatone[Text Word] OR rasagiline[Text Word] OR hydracarbazine[Text Word] OR } \\
\text { aripiprazole[Text Word] OR risperidone[Text Word] OR quetiapine[Text Word] OR lurasidone[Text Word] OR } \\
\text { olanzapine[Text Word] OR ziprasidone[Text Word] OR clozapine[Text Word]) }\end{array}$ \\
\hline AND & $\begin{array}{l}\text { ("personality disorders"[MeSH Terms] OR personality disorder*[Text Word] antisocial personality disorder*[Text } \\
\text { Word] OR borderline personality disorder*[Text Word] OR compulsive personality disorder*[Text Word] OR } \\
\text { dependent personality disorder*[Text Word] OR histrionic personality disorder*[Text Word] OR hysteria[Text } \\
\text { Word] OR paranoid personality disorder*[Text Word] OR passive-aggressive personality disorder*[Text Word] OR } \\
\text { schizoid personality disorder*[Text Word] OR schizotypal personality disorder*[Text Word] OR Narcissistic } \\
\text { personality disorder*[Text Word] OR depressive personality disorder[Text Word] OR personality disorder* not } \\
\text { otherwise specified[Text Word]) }\end{array}$ \\
\hline Limits & English, Dutch, German, All Adults: 19+ years, Humans \\
\hline
\end{tabular} Search terms in PsycINFO

\begin{tabular}{|c|c|}
\hline & 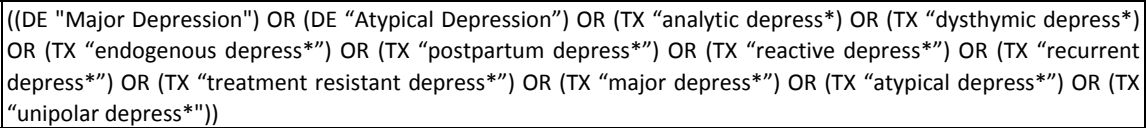 \\
\hline AND & 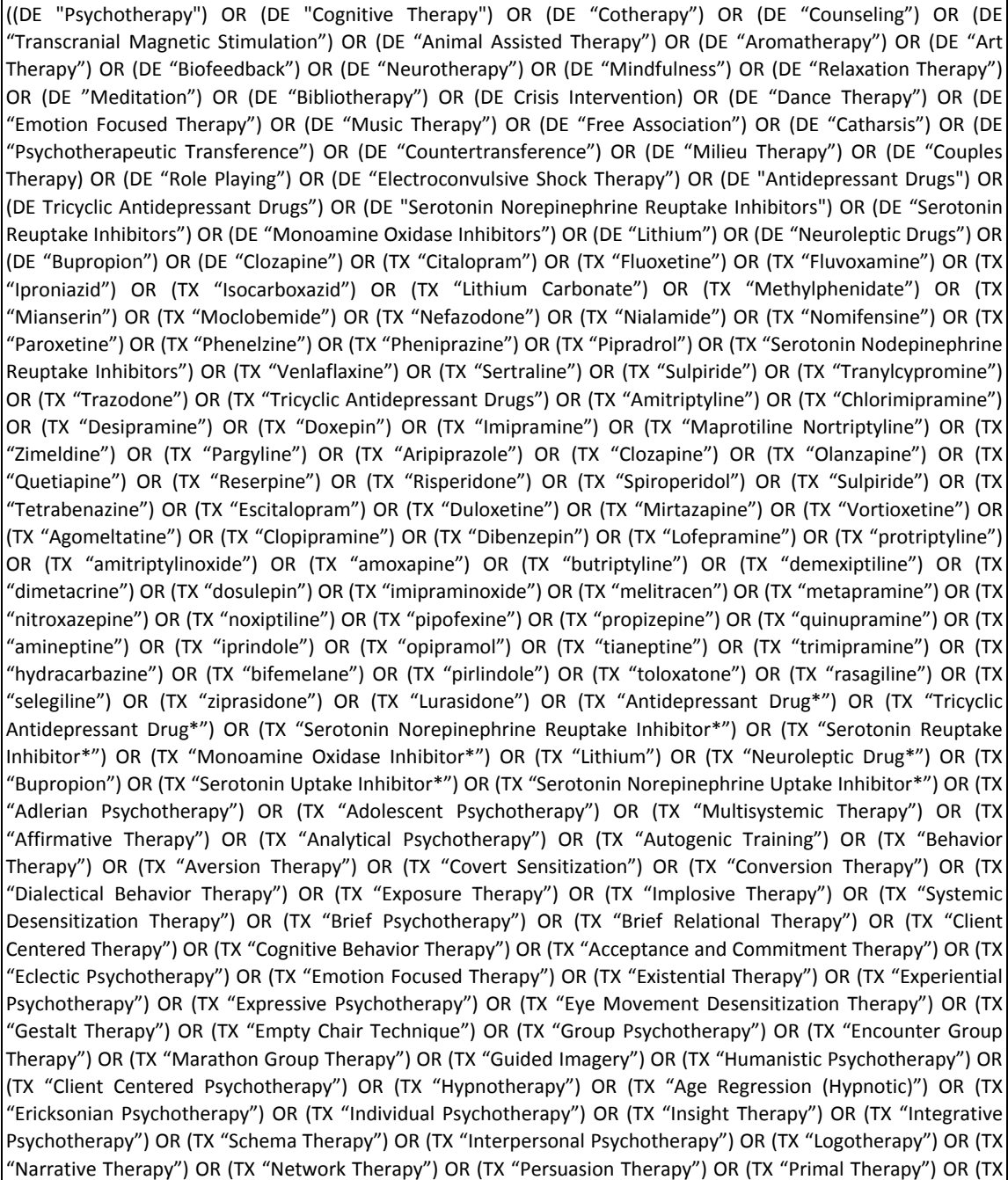 \\
\hline
\end{tabular}




\begin{tabular}{|c|c|}
\hline & 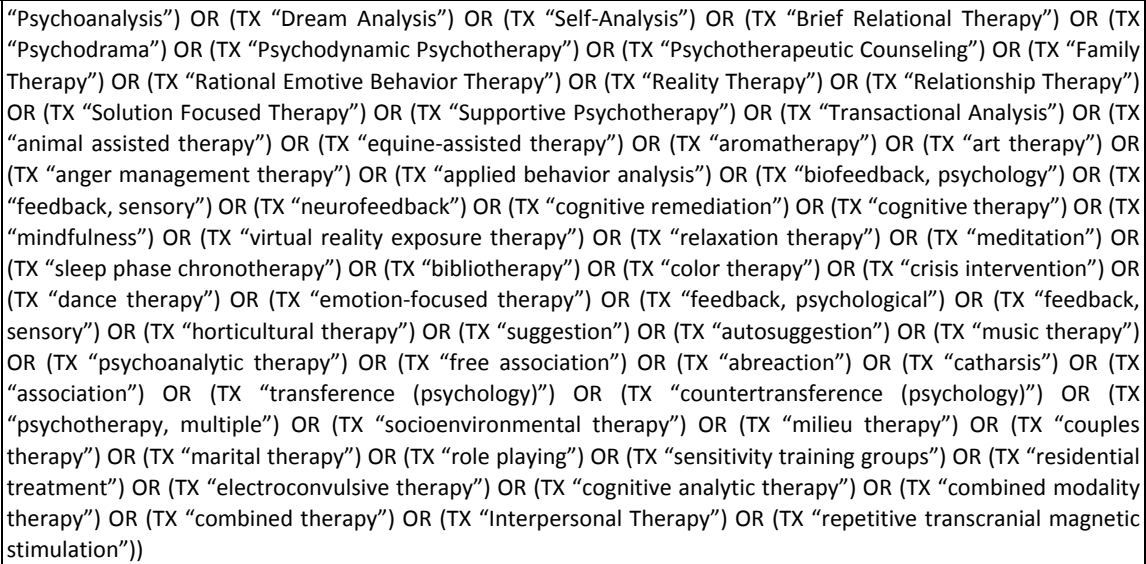 \\
\hline AND & 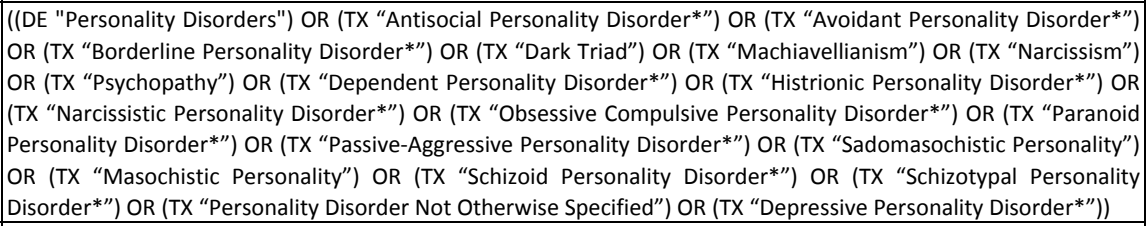 \\
\hline Limits & English, German, Dutch, adulthood (18 years and older), Inpatients, Outpatients, Human \\
\hline \multicolumn{2}{|c|}{ Search terms in Embase } \\
\hline & ((Major depression OR depressive disorder).sh. OR (depress* OR major depress* OR unipolar de \\
\hline AND & 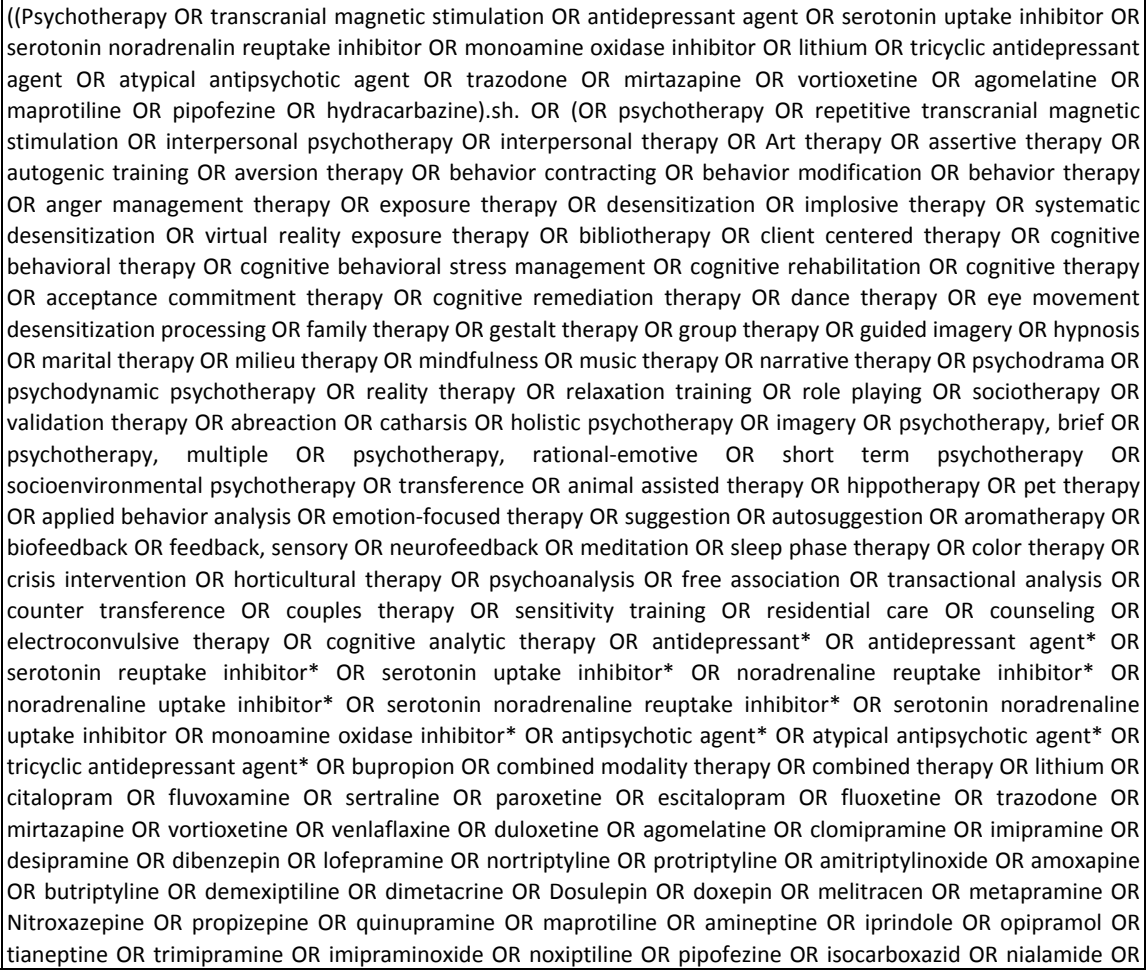 \\
\hline
\end{tabular}




\begin{tabular}{|l|l|}
\hline & $\begin{array}{l}\text { phenelzine OR tranylcypromine OR moclobemide OR selegiline OR bifemelane OR pirlindole OR toloxatone OR } \\
\text { rasagiline OR hydracarbazine OR aripiprazole OR risperidone OR quetiapine OR lurasidone OR olanzapine OR } \\
\text { ziprasidone OR clozapine).tw.) }\end{array}$ \\
\hline AND & $\begin{array}{l}\text { (Personality disorder OR narcissism).sh.) OR (personality disorder* OR antisocial personality disorder* OR } \\
\text { avoidant personality disorder* OR borderline state OR borderline personality disorder* OR character disorder* OR } \\
\text { compulsive personality disorder* OR dependent personality disorder* OR histrionic personality disorder* OR } \\
\text { narcissism OR paranoid personality disorder* OR passive-aggressive personality disorder* OR psychopathy OR } \\
\text { schizoid personality disorder OR schizotypal personality disorder* OR narcissistic personality disorder* OR } \\
\text { depressive personality disorder* OR personality disorder* not otherwise specified)tw.) }\end{array}$ \\
\hline Limits & Human, Dutch or English or German, Adult (18-64 years) \\
\hline
\end{tabular}

\subsection{Data extraction and quality assessment}

Data extraction was done independently by three reviewers (EK; all selected records, FP first part and SB second part of all selected records). Data extraction was checked by - and discrepancies were discussed with - an independent reviewer (FP or SB depending on the record). Primary outcome measures were average depression severity change scores from pre- to posttreatment and secondary outcome measures involved response and remission rates at the end of treatment. In each trial, primary and secondary outcome measures (summary estimates) were extracted for individuals with and without PD. Other extracted information included: study setting, demographics (male/female ratio, mean age of the sample), number of dropouts, time of post-treatment measurement, treatment description (type of treatment, treatment duration, dose of antidepressant medication, number psychotherapy sessions, number of electroconvulsive therapy sessions, number of transcranial magnetic stimulation sessions, integrity checks for specific psychotherapies), depression variables (type of measurement instrument, mean baseline depression severity, age of onset of the first depressive episode, number of previous depressive episodes, total illness duration and duration of the current depressive episode), and personality variables (type of measurement instrument, specific diagnoses). All corresponding authors were contacted and asked to provide missing data and to check the extracted data. Studies were included in the meta-analysis if calculation of an effect size was possible based on the extracted and received data. The validity of the included studies was evaluated by two independent reviewers (FP and SB) using the following four criteria of the 'Risk of Bias' assessment tool, developed by the Cochrane Collaboration (Higgins \& Green, 2011): 1) adequate random sequence generation, 2) allocation to treatments by an independent party, 3) blinding of the outcome assessment and 4) the quantity, nature and management of incomplete outcome data. For each study, these criteria were rated as "unclear", "low risk", or "high risk", and disagreements between reviewers were resolved through discussion. 


\subsection{Data analysis}

All analyses were done using STATA software (version 13.1). First, meta-analyses comparing individuals with and without PD were conducted for both primary (depression severity change scores) and secondary (response and remission rates) outcomes, using random-effects models with the DerSimonian and Laird method (DerSimonian \& Laird, 1986). Hedges' g effect sizes (Formula 1), risk ratios (RRs) and odds ratios (ORs) comparing individuals with and without PD were calculated for each treatment condition; if a study contained multiple treatment conditions, multiple effect sizes were calculated. Depending on data availability, we intended to calculate effect sizes with intention to treat study-level data. Effect sizes were pooled with $95 \%$ confidence intervals $(\mathrm{Cl})$. A rule of thumb for interpreting Hedges' $\mathrm{g}$ effect sizes is that 0.2 represents a small effect, 0.5 a moderate effect, and 0.8 a large effect of comorbid PD on mean depression severity change during treatment (Cohen, 2013). RRs and ORs can be interpreted as the multiplication of the risk or odds respectively of response or remission when individuals have a comorbid PD diagnosis. To test the homogeneity of these outcomes, $\mathrm{I}^{2}$ statistics were estimated with $95 \% \mathrm{Cl}$ (Ioannidis, Patsopoulos, \& Evangelou, 2007). Estimates were interpreted as $0-40 \%$ representing low heterogeneity, 30-60\% representing moderate heterogeneity, 50-90\% representing substantial heterogeneity, and $75-100 \%$ representing considerable heterogeneity (Higgins \& Green, 2011). If multiple measurement instruments were available to measure the primary outcome, the mean Hedges' $g$ effect size was calculated (Borenstein, Hedges, Higgins, \& Rothstein, 2009). If average change scores and their SD's were unavailable, they were computed using average pre- and posttreatment depression severity scores, their SD's, and the correlation coefficients between these scores (SD calculations were based on Formula 2). If these correlation coefficients were unavailable, a correlation of $r=0.5$ was assumed based on data of a recent controlled trial comparing acute phase depression treatments (Lemmens et al., 2015).

Second, multiple sensitivity analyses were conducted to test if the following changes affected the findings: 1) reducing (0.2) and increasing (0.8) the assumed correlation coefficient between mean pre- and posttreatment depression severity scores to calculate mean change score SD's, if these correlation coefficients and SD's were unavailable (this was the case for one study that included four comparisons; Hardy et al., 1995), 2) including only studies with just one (and not multiple) measurement instrument for depression severity, 3) including only studies with a lowrisk score for all four risk-of-bias criteria. Third, a meta-regression was conducted to examine the association between specific study-level variables and between-study heterogeneity of the results (Thompson \& Higgins, 2002). To this end, univariate meta-regressions were done with the following variables: male/female ratio, mean age of the sample, time of post treatment measurement, type of treatment (talking therapy, ADM, other biological treatment), treatment duration, percentage of 
dropout, mean baseline depression severity, age of onset of the first depressive episode, number of previous depressive episodes, total illness duration, duration of the current depressive episode, self report or clinician-rated depression severity scores and an intention to treat approach for the extracted outcome measures (absence or imputation of missing data). Variables with a $p$ value $<0.10$ were then included in a multivariate meta-regression. For the multivariate meta-regression, $\mathrm{p}$ values were based on a Bonferroni correction. Correlations between the variables included in the multivariate meta-regression were calculated to check for multicollinearity. Finally, potential publication bias was examined by inspecting funnel plots and applying the Egger's test (Egger, Davey Smith, Schneider, \& Minder, 1997).

\section{Formula 1: calculating Hedges' $g$}

Formula to calculate Hedges' g effect sizes. The calculation includes a includes an adjustment for small sample bias:

$$
\text { Hedges'g }=\frac{\text { Depression change }_{P D}-\text { Depression change }_{n o P D}}{S D_{\text {change }}} \times\left(1-\frac{3}{4\left(n_{P D}+n_{n o P D}\right)-9}\right)
$$

Depression change $_{\mathrm{PD}}=$ average depression severity change for individuals with comorbid PD

Depression change $_{\text {noPD }}=$ average depression severity change for individuals without comorbid PD

SD change $=$ standard deviation of depression severity change scores

Formula 2: calculating the standard deviation of depression severity change

Formula to estimate the standard deviation of the average depression severity change using the standard deviations of the pre and post intervention depression severity scores and the correlation between pre and post intervention depression severity:

$$
S D_{\text {change }}=\sqrt{S D_{\text {pre }}^{2}+S D_{\text {post }}^{2}-\left(2 R S D_{\text {pre }} S D_{\text {post }}\right)}
$$

SD change = estimated standard deviation of the average depression severity change $\mathrm{SD}_{\text {pre }}=$ standard deviation of average depression severity pre intervention $\mathrm{SD}_{\text {post }}=$ standard deviation of average depression severity post intervention $\mathrm{R}=$ correlation between average depression severity pre and post intervention 


\section{Results}

A total of 2046 citations were identified through database searching, 10 additional citations were extracted from other sources (Figure 4.2). After removing duplicates and ineligible studies based on abstract screening, a total of 206 records were screened full-text. From these records, 11 studies met the inclusion criteria; however, only six studies (12 comparisons) had sufficient data to be included in the metaanalysis. Study characteristics for all six studies are summarized in Table 4.2. The included trials comprised of 942 depressed individuals of which a subset of 447 individuals (47.5\%) had a comorbid PD. All trials were conducted in an outpatient setting in the Netherlands $(n=1)$, Iran $(n=1)$, the United States $(n=3)$ and the United Kingdom $(n=1)$. Treatment consisted of different types of psychotherapy (cognitive (behavioral) therapy, interpersonal psychotherapy, behavioral activation, psychodynamic interpersonal psychotherapy, 8 conditions, $n=350$,) and ADM (sertraline, paroxetine, nortriptyline, fluoxetine, 4 conditions, $n=592$ ). Change in depression severity scores was measured with self-report questionnaires (BDI), clinician-rated measures (HDRS) or with both. For one study, intention to treat data were available (Fournier et al., 2008). Response rates were available for four studies (Fava et al., 2002; Fournier et al., 2008; Lemmens et al., 2015; Papakostas et al., 2003). These were defined as i) at least $50 \%$ reduction ,or ii) a predefined minimum decrease of the score on the severity scale, or a combination of both criteria. Remission rates were available for four studies (Fava et al., 2002; Fournier et al., 2008; Hardy et al., 1995; Lemmens et al., 2015) and were based on cut-off scores on depression severity scales (HDRS $\leq 7, \mathrm{BDI} \leq 9,10$ or 15). One study did not report response and remission rates (Moradveisi, Huibers, Renner, Arasteh, \& Arntz, 2013a; Moradveisi et al., 2013b).

The primary outcome, depression severity change, was pooled using Hedges 'g effect sizes for six studies that included 12 comparisons. The mean pooled Hedges ' $g$ effect size was $\mathrm{g}=0.03(95 \% \mathrm{Cl}=-0.15$ to $0.20, \mathrm{p}=0.27)$ indicating no significant difference in average depression severity change between individuals with and without a PD. The heterogeneity of the pooled effect size was low with a moderately high level of uncertainty $\left(\mathrm{I}^{2}=17.6 \%, 95 \% \mathrm{Cl}=0.00 \%\right.$ to $\left.56.95 \%\right)$. The sensitivity analyses described in the data analysis section did not change these results.

Secondary outcomes, response and remission rates, were pooled using risk ratios (RRs) and odds ratios (ORs). A total of 219 individuals with a PD achieved response (58.2\%), compared to 209 individuals without a PD (54.5\%). The pooled RR for response was $1.14(95 \% \mathrm{Cl}=0.99$ to $1.31, \mathrm{p}=0.07)$, and the pooled $\mathrm{OR}$ was $1.32(95 \% \mathrm{Cl}=$ 0.83 to $2.12, p=0.24$ ), indicating no significant difference between individuals with and without a PD. Heterogeneity of these pooled estimates was low to moderate with moderate to substantial uncertainty (RR: $I^{2}=25.0 \%, 95 \% \mathrm{Cl}=0.0 \%$ to $68.0 \%$; OR: $\mathrm{I}^{2}=42.3 \%, 95 \% \mathrm{Cl}=0.0 \%$ to $77.0 \%$ ). A total of 98 individuals with a PD met criteria for 
remission (44.3\%), and 125 individuals without PD achieved remission (47.2\%). The pooled RR for remission was $0.895(95 \% \mathrm{Cl}=0.736$ to $1.089, \mathrm{p}=0.27)$, and the pooled $\mathrm{OR}$ was $0.751(95 \% \mathrm{Cl}=0.473$ to $1.194, \mathrm{p}=0.23)$, indicating no significant difference between individuals with and without a PD. These summary estimates had low to moderate heterogeneity with substantial uncertainty (RR: $I^{2}=13.7 \%, 95 \% \mathrm{Cl}=0.00 \%$ to $56.0 \%$; OR: $I^{2}=36.2 \%, 95 \% \mathrm{Cl}=0.0 \%$ to $71.0 \%$ ).
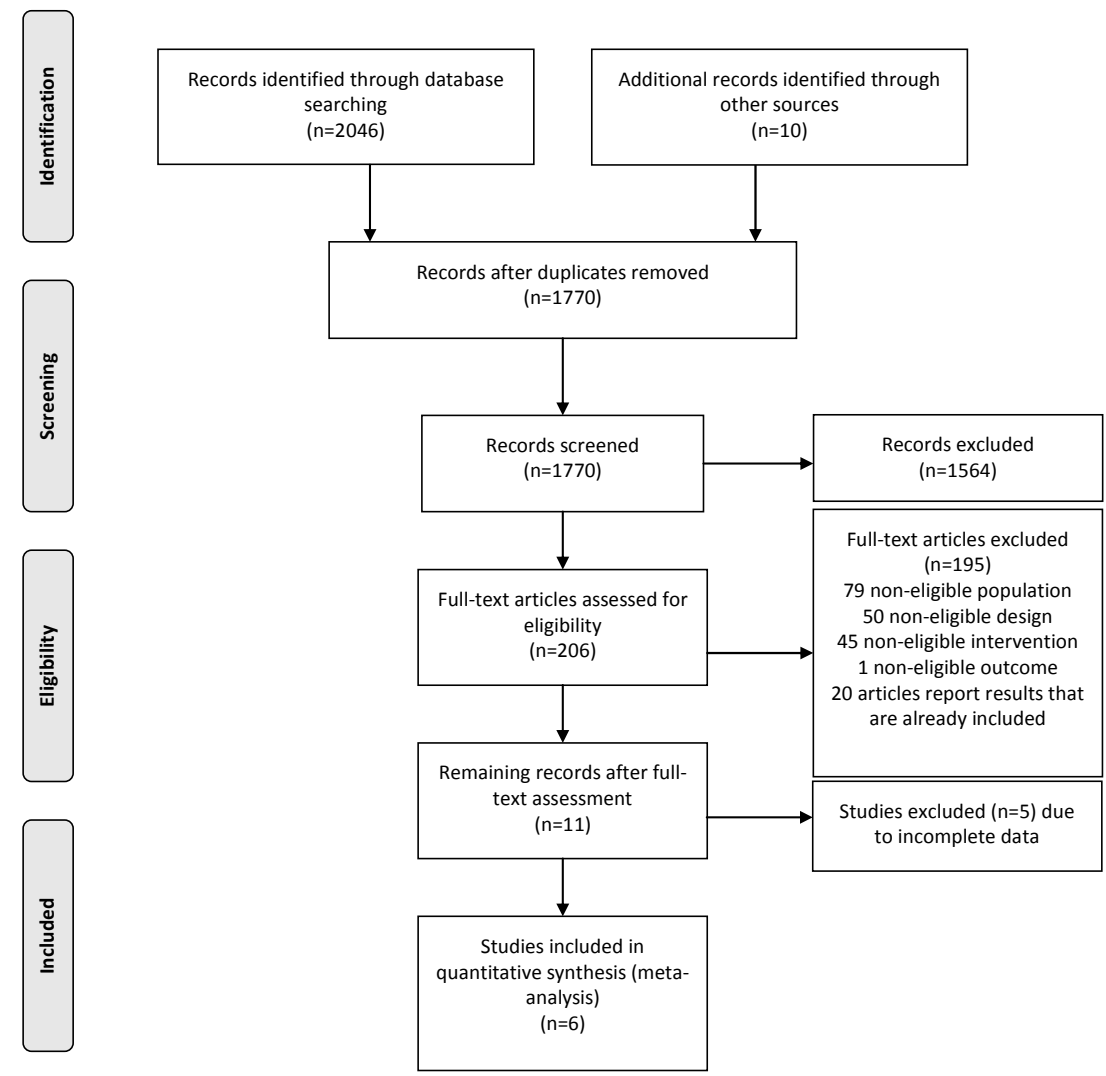

Figure 4.2: Study selection process.

The risk of bias assessment is described in Table 4.2 and summarized in Figure 4.3. A total of three studies had a "low-risk" score on all four criteria. In three studies, the randomization process was adequately executed by an independent party and there was a low risk of attrition bias based on the quantity, nature and management of missing data. In five studies, the outcome assessors were blinded to treatment allocation and/or results of earlier assessments. 


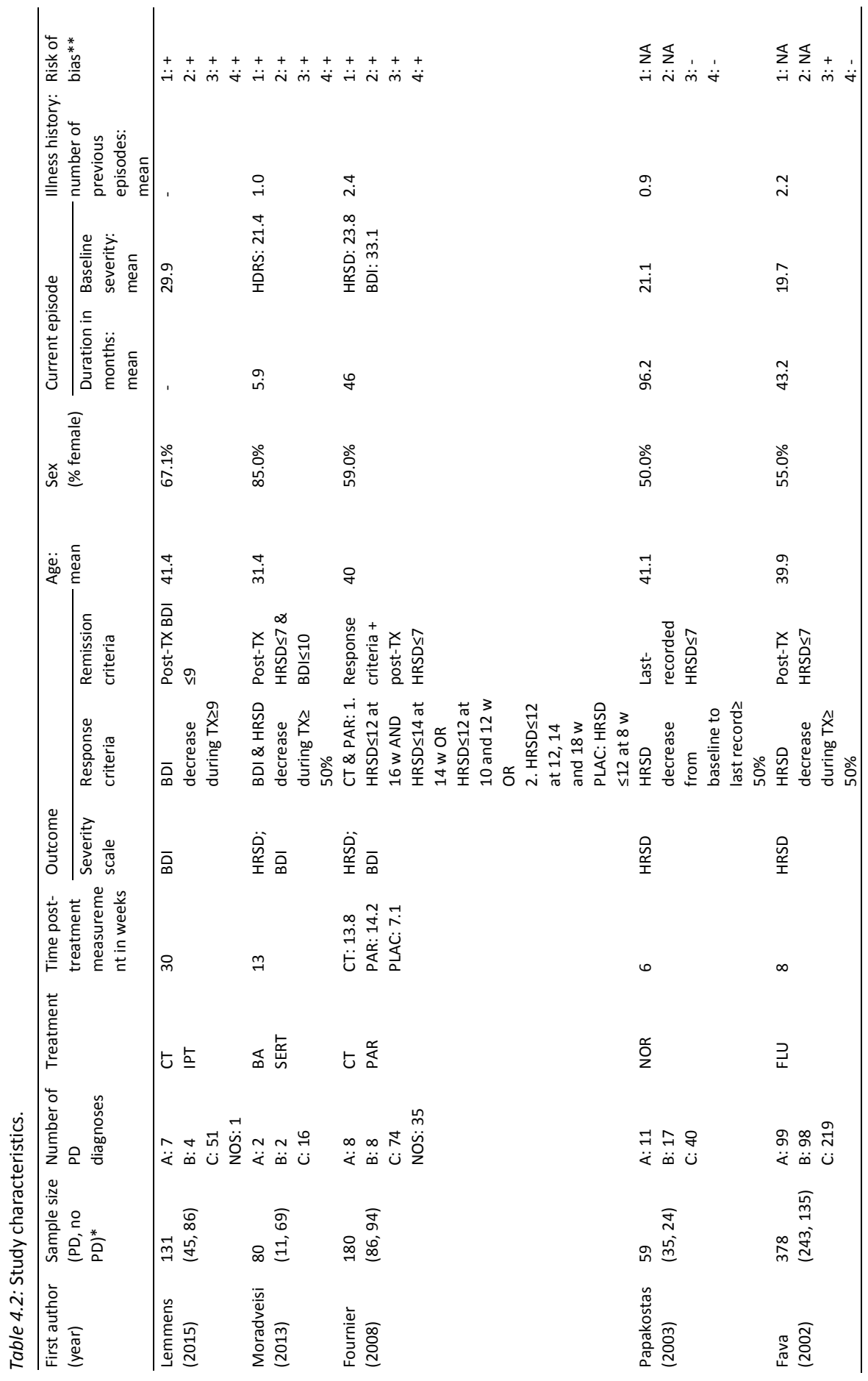




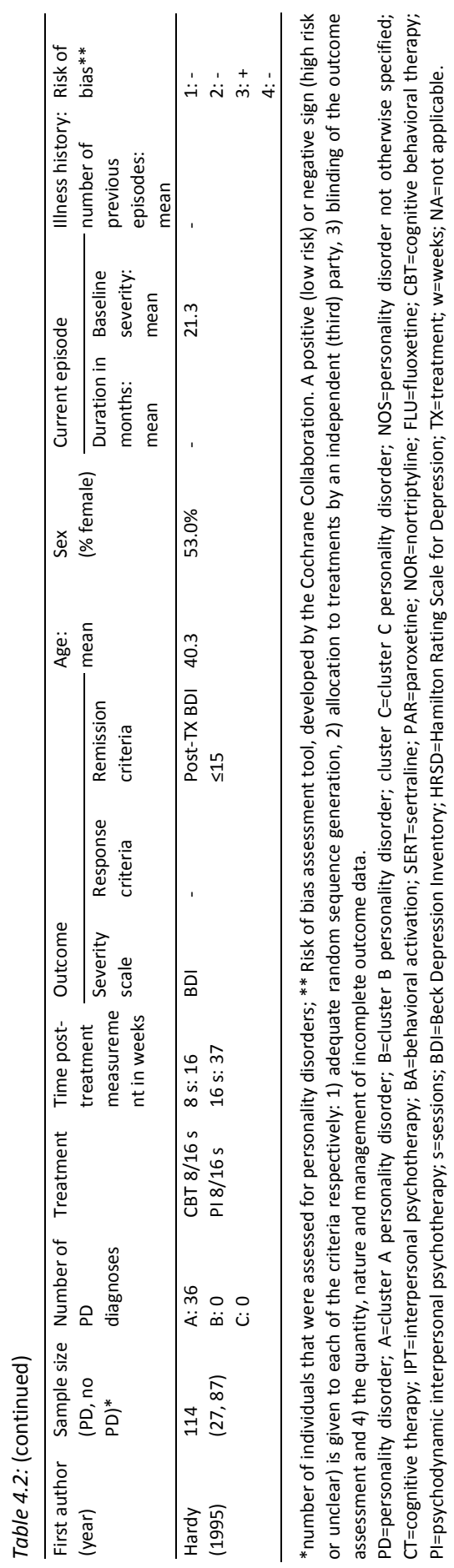


Because of missing data we were only able to compute the meta-regression for the following variables: male/female ratio, mean age of the sample, time of posttreatment measurement, type of treatment (talking therapy/ADM), treatment duration, percentage of dropout, mean baseline depression severity, number of previous depressive episodes, duration of the current depressive episode, self-report or clinician-rated depression severity scores and an intention to treat approach for the extracted outcome measures. Male/female ratio $(\beta=0.02 ; \mathrm{SE}=0.01 ; p=0.09)$ and time of posttreatment measurement $(\beta=0.02 ; \mathrm{SE}=0.01 ; p=0.07)$ met our criterion of $p<0.10$ and were included in the multivariate meta-regression. In our multivariate metaregression none of variables remained significant, and there was no indication for multicollinearity (between variables cor. $=0.1$ ).

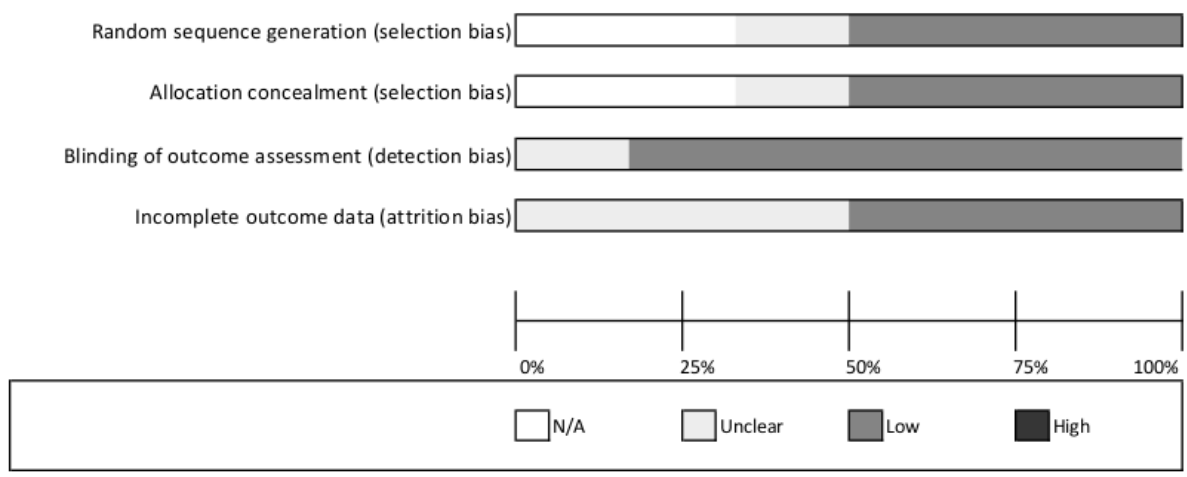

Figure 4.3: Risk of bias assessment.

Reviewers' judgement about each risk of bias item as a proportion of all included controlled trials. $\mathrm{N} / \mathrm{A}=$ Not applicable

There was no indication for publication bias based on visual inspection of the funnel plot (Figure 4.4) and the Egger's tests (intercept: $-0.10 ; 95 \% \mathrm{Cl}=-1.51$ to 1.31 ; $p=0.875)$. 


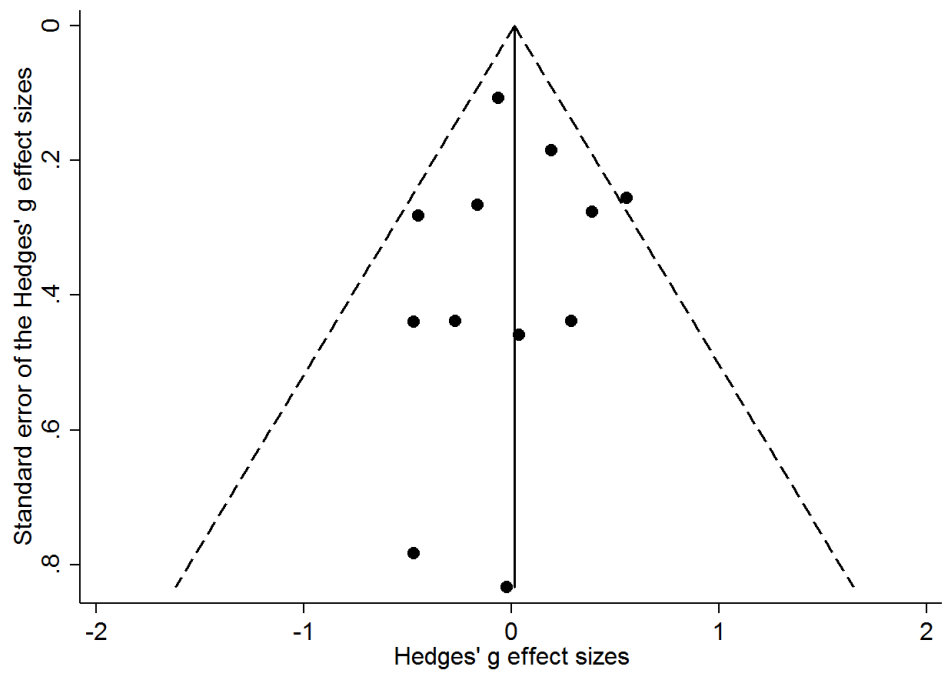

Figure 4.4: Funnel plot to examine publication bias 


\section{References}

Beck, A. T., Steer, R. A., \& Brown, G. K. (1996). Beck depression inventory-II. San Antonio, 78(2), 490-498.

Borenstein, M., Hedges, L. V., Higgins, J. P. T., \& Rothstein, H. R. (2009). Introduction to Meta-Analysis. NYC: Wiley.

Cohen, J. (2013). Statistical power analysis for the behavioral sciences: Routledge.

DerSimonian, R., \& Laird, N. (1986). Meta-analysis in clinical trials. Controlled clinical trials, 7(3), 177-188.

Egger, M., Davey Smith, G., Schneider, M., \& Minder, C. (1997). Bias in meta-analysis detected by a simple, graphical test. BMJ, 315(7109), 629-634.

Fava, M., Farabaugh, A., Sickinger, A., Wright, E., Alpert, J. E., Sonawalla, S., . . Worthington lii, J. (2002). Personality disorders and depression. Psychological Medicine, 32(6), 1049-1057.

Fournier, J. C., DeRubeis, R. J., Shelton, R. C., Gallop, R., Amsterdam, J. D., \& Hollon, S. D. (2008). Antidepressant medications v. cognitive therapy in people with depression with or without personality disorder. The British Journal of Psychiatry, 192(2), 124-129.

Hamilton, M. (1960). A rating scale for depression. Journal of neurology, neurosurgery, and psychiatry, 23(1), 56.

Hardy, G. E., Barkham, M., Shapiro, D. A., Stiles, W. B., Rees, A., \& Reynolds, S. (1995). Impact of Cluster C personality disorders on outcomes of contrasting brief psychotherapies for depression. Journal of Consulting and Clinical Psychology, 63(6), 997.

Higgins, J. P. T., \& Green, S. (Eds.). (2011). Cochrane Handbook for Systematic Reviews of Interventions Version 5.1.0 The Cochrane Collaboration.

Ioannidis, J. P., Patsopoulos, N. A., \& Evangelou, E. (2007). Uncertainty in heterogeneity estimates in metaanalyses. BMJ, 335(7626), 914-916. doi: 10.1136/bmj.39343.408449.80

Klein, D. N., Kotov, R., \& Bufferd, S. J. (2011). Personality and depression: explanatory models and review of the evidence. Annual review of clinical psychology, 7, 269-295.

Lemmens, L., Arntz, A., Peeters, F., Hollon, S., Roefs, A., \& Huibers, M. (2015). Clinical effectiveness of cognitive therapy $\mathrm{v}$. interpersonal psychotherapy for depression: results of a randomized controlled trial. Psychological Medicine, 45(10), 2095-2110.

Montgomery, S. A., \& Åsberg, M. (1979). A new depression scale designed to be sensitive to change. The British Journal of Psychiatry, 134(4), 382-389.

Moradveisi, L., Huibers, M. J., Renner, F., Arasteh, M., \& Arntz, A. (2013a). Behavioural activation v. antidepressant medication for treating depression in Iran: randomised trial. The British Journal of Psychiatry, 202(3), 204-211.

Moradveisi, L., Huibers, M. J., Renner, F., Arasteh, M., \& Arntz, A. (2013b). The influence of comorbid personality disorder on the effects of behavioural activation vs. antidepressant medication for major depressive disorder: Results from a randomized trial in Iran. Behaviour research and therapy, 51(8), 499-506.

Mulder, R. T. (2002). Personality pathology and treatment outcome in major depression: a review. American Journal of Psychiatry, 159(3), 359-371.

Newton-Howes, G., Tyrer, P., \& Johnson, T. (2006). Personality disorder and the outcome of depression: meta-analysis of published studies. The British Journal of Psychiatry, 188(1), 13-20.

Newton-Howes, G., Tyrer, P., Johnson, T., Mulder, R., Kool, S., Dekker, J., \& Schoevers, R. (2014). Influence of personality on the outcome of treatment in depression: systematic review and meta-analysis. Journal of Personality Disorders, 28(4), 577-593.

Papakostas, G. I., Petersen, T. J., Farabaugh, A. H., Murakami, J. L., Pava, J. A., Alpert, J. E., . . Nierenberg, A. A. (2003). Psychiatric comorbidity as a predictor of clinical response to nortriptyline in treatmentresistant major depressive disorder. The Journal of clinical psychiatry, 64(11), 1357-1361.

Rush, A. J., Carmody, T., \& Reimitz, P. E. (2000). The Inventory of Depressive Symptomatology (IDS): clinician (IDS-C) and self-report (IDS-SR) ratings of depressive symptoms. International Journal of Methods in Psychiatric Research, 9(2), 45-59.

Thompson, S. G., \& Higgins, J. P. (2002). How should meta-regression analyses be undertaken and interpreted? Statistics in medicine, 21(11), 1559-1573. 
S. van Bronswijk, D. van Dijk, Th. M. van den Boogaard, M. Deen, H. Ruhé, J. Spijker, F. Peeters - Submitted

\section{Chapter}

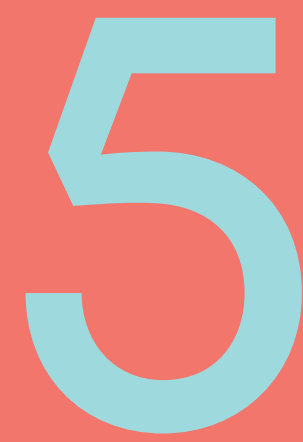




\section{Abstract}

\section{Background}

The impact of personality disorder (PD) on treatment effectiveness for major depressive disorder (MDD) has been the topic of debate with inconsistent study results. However, studies that report a negative impact of PD on MDD treatment outcomes are often characterized by uncontrolled treatment designs. Within such contexts, depressed individuals with PD are at risk to receive suboptimal treatment. The aim of this observational study was to investigate whether and to what extend comorbid PD is associated with the type and amount of received MDD treatment in routine outpatient care.

\section{Methods}

Retrospectively extracted data from electronic patient records of 1455 individuals with MDD were included. The type and number of treatment sessions and visits were analysed using regression models.

\section{Results}

Depressed individuals with comorbid PD received more psychotherapy sessions than individuals without comorbid PD, irrespective of depression severity. The number of pharmacotherapy sessions and supportive and crisis visits did not differ between individuals with and without comorbid PD.

\section{Conclusions}

Results indicate that depressed individuals with comorbid PD receive more intensive treatment than individuals without comorbid PD. This conflicts with recommendations of treatment guidelines and high quality studies, and might be indicative for overtreatment of this large group of patients. 


\section{Introduction}

Despite the many available and effective pharmacological and psychotherapeutic treatment options for major depressive disorder (MDD; Cipriani et al., 2018; Cuijpers et al., 2013), a substantial number of individuals do not achieve remission (Kolovos et al., 2017; Rush et al., 2006). Since individual treatment responses vary and are highly unpredictable, identifying risk factors associated with treatment outcome will help to personalize treatment selection and planning and subsequently improve remission rates (Cohen and DeRubeis, 2018; Simon and Perlis, 2010). In addition to well known risk factors for treatment resistance like higher baseline depression severity and chronicity of the index episode (Fekadu et al., 2009; Friedman et al., 2012), the presence of comorbid personality disorder (PD) is often put forward in the literature (Berk et al., 2018; Van and Kool, 2018). Its relevance is potentially large, because PD is a common comorbidity in MDD outpatients with incidence rates varying from $44 \%$ to more than 50\% (Friborg et al., 2014; Zimmerman et al., 2005). Two frequently cited meta-analyses reported that PD negatively affects MDD acute phase treatment outcome (Newton-Howes et al., 2006; Newton-Howes et al., 2014), therefore concluding that its presence is of high importance in the prognosis of MDD. Contrary to this evidence, a review of Mulder in 2002 (Mulder, 2002) described no negative effects of PD when focusing solely on randomized and controlled studies. According to Mulder, this could be explained by comorbid patients receiving less optimal treatment in uncontrolled studies, as these studies are likely to be biased by clinicians that regard PD as a relevant variable in treatment selection. A meta-analysis that included only controlled studies contributed to this hypothesis, indicating no significant differences between individuals with and without PD in the treatment outcome of pharmacotherapy for MDD (Kool et al., 2005). This finding was replicated and extended by a recent meta-analysis focusing on treatment outcomes of pharmacotherapy and psychotherapy for MDD using data from only well-designed controlled trials. Results showed no significant differences between individuals with and without comorbid PD in terms of average depression severity change, response rates, and remission rates (van Bronswijk et al., 2019). Since individuals with PD appear to have better treatment outcomes in studies with controlled treatment selection, the less satisfactory treatment outcomes in naturalistic designs may be incorrectly attributed to the comorbidity itself instead of insufficiently provided MDD treatment. Based on this, one could hypothesize that in care as usual, as with naturalistic studies, treatment might be less optimal for individuals with PD. However, there are only a few studies available reporting data on this issue, indicating that individuals with comorbid PD received less pharmacotherapy and electroconvulsive therapy (ECT) for MDD as compared to non-comorbid counterparts (Black et al., 1988; Charney et al., 1981). Additionally, recent studies addressing this research question are lacking. 
To gain a more precise understanding on how comorbid PD affects provided treatment in care as usual, the aim of the current study was to examine whether and to what extend the presence of comorbid PD is associated with the type and the amount of received treatment sessions in the context of naturalistic outpatient MDD care. First, we examined if individuals with comorbid PD received different types and intensities of MDD treatment according to (inter)national evidence-based guidelines (i.e. psychotherapy, pharmacotherapy, a combination of both, or ECT (American Psychiatric Association, 2009; 2010; Spijker et al., 2013). We hypothesized that depressed subjects with comorbid PD would receive less sessions of these treatments than depressed individuals without comorbid PD, irrespective of their level of depression severity. Second, we investigated if comorbid PD affected the amount of other types of received care including supportive visits and crisis visits. We hypothesized that depressed individuals with comorbid PD were more likely to receive a higher number of supportive and crisis visits than those without comorbid PD.

\section{Methods}

\subsection{Design and participants}

This is a naturalistic cohort study conducted at PsyQ, a nationwide organization in the Netherlands, providing outpatient secondary mental healthcare. Seven PsyQ locations (Zoetermeer, Zaandam, Rotterdam Kralingen, Rotterdam South, Beverwijk, Spijkenisse, and The Hague) were involved in this study. Because we only used information that was routinely collected, the Medical Research Ethics Committee of the Leiden University Medical Centre waived formal informed consent (van Dijk et al., 2019). Anonymized data were retrospectively extracted from the electronic patient record (EPR) of individuals who had a complete diagnostic work-up between June 2014 and June 2016. Adults ( $\geq 18$ years) who met the DSM-IV-TR criteria for MDD or dysthymic disorder according to their EPR were selected. Data from individuals who objected to the use of their information for scientific purposes prior or during treatment were not included. Exclusion criteria were a primary diagnosis of bipolar disorder, psychotic disorder or substance dependence (except for nicotine). In these secondary care settings, all subjects received treatment as usual which implies treatment according to the Dutch national evidence-based guideline for MDD treatment (Spijker et al., 2013). The guideline recommends evidence-based shortterm psychotherapy (e.g., interpersonal psychotherapy (IPT) or cognitive behavioral therapy (CBT)) by trained professionals (psychiatrists, psychologists and psychiatric nurses) or pharmacotherapy and their combination. 


\subsection{Measures}

\subsubsection{Outcomes}

The primary outcome of this study was the type and amount of received treatment sessions in accordance with (inter)national evidence-based guidelines for MDD treatment including pharmacotherapy, psychotherapy, a combination of both, and ECT. In addition, we defined a "treatment intensity" outcome variable that comprised of three levels: low (less than four sessions psychotherapy or pharmacotherapy), moderate (four or more psychotherapy or pharmacotherapy sessions), and high (four or more psychotherapy and pharmacotherapy sessions or ECT). The threshold of four or more sessions was based on recommendations regarding the lowest optimal "dose" for psychotherapy (Delgadillo et al., 2014; Robinson et al., 2020) and approaching the recommendation of 5-6 pharmacotherapy sessions for combination treatment in depression (pharmacotherapy and psychotherapy; Smit et al., 2016). To further examine the other types of care that depressed individuals with and without a comorbid PD received, the number of supportive visits and crisis visits were examined as secondary outcomes. Outcomes were extracted from the EPR. The EPR uses a coding-system that represents the type and amount of received treatment sessions (psychotherapy, pharmacotherapy, ECT) and visits (supportive visits and crisis visits). We used these codes for our primary and secondary outcome measure, and only included face-to-face appointments in our analyses.

\subsubsection{Comorbid personality disorder}

The presence of comorbid PD was based on the Standardized Assessment of Personality-Abbreviated Scale (SAPAS) - score that was an element of the routine diagnostic work-up and was available to the therapists. The SAPAS is a clinician rated eight-item questionnaire that is used to screen for the presence of personality disorders in routine clinical settings (Bukh et al., 2010; Moran et al., 2003). All eight questions are items from the opening section of the Standardized Assessment of Personality (SAP; Mann et al., 1981), and involve a descriptive statement about the person that can be scored 0 (not present) or 1 (present). A score of 3 or higher indicates the presence of a $P D$, and for the remainder of the paper we will refer to this as "comorbid PD". Individuals that had a missing SAPAS were excluded from this study sample.

\subsubsection{Additional pre-treatment variables}

Demographic variables (age and gender) were extracted from the EPR's. Baseline depression severity was measured with the Quick Inventory of Depressive Symptomatology-Self Report (QIDS-SR; Rush et al., 2003), a 16-item self-report depression symptom severity scale with higher scores indicating higher depression 
severity (scores range between 0 and 27). The QIDS-SR score (not included in the diagnostic work up but filled out separately online by subjects) was considered a baseline measure when it was obtained \pm 45 days from the diagnostic work-up. Individuals with missing baseline QIDS-SR scores were not included in this study sample. Furthermore, we obtained information on episode duration and severity, comorbid anxiety, previous treatment failures (including psychotherapy, pharmacotherapy, inpatient treatment, day treatment and ECT), functional impairment, the presence of childhood adversity and psychosocial stressors from the EPR.

\subsection{Statistical analyses}

First, we compared pre-treatment characteristics of individuals that were considered study dropouts (missing baseline QIDS-SR and/or SAPAS; $n=780$ ) with individuals that were included in the analyses (no missing baseline QIDS-SR and no missing SAPAS; $\mathrm{n}=1455$ ) using t-tests (with Cohen's $d$ effect sizes) and $\chi^{2}$-tests for continuous and categorical variables respectively. Second, we examined pre-treatment variables of individuals with and without comorbid PD, using t-tests (with Cohen's $d$ effect sizes) and $\chi^{2}$-tests where appropriate. Third, we tested the association between the amount of received MDD treatment in accordance with (inter)national evidence-based guidelines and the presence of comorbid PD. For this two separate negative binomial regression models were used. In the first model, the number of psychotherapy sessions was the dependent variable and in the second model the number of pharmacotherapy sessions was the dependent variable. For both models the presence of comorbid PD was included as an independent variable. To examine the potential (moderating) impact of baseline depression severity on the association between sessions and comorbid PD, QIDS-SR baseline and a QIDS-SR baseline $x$ comorbid PD interaction were added to the models as independent variables as well. After that, we examined the association between evidence-based treatment intensity and comorbid PD using a multinomial regression model with low level intensity as the reference group. In this model, treatment intensity was the dependent variable, and comorbid PD, QIDS-SR baseline and a QIDS-SR baseline $x$ comorbid PD interaction were the independent variables. Finally, to further assess other types of care that the patients received, we ran two negative binomial regression models with the two secondary outcomes (the number of supportive and the number of crisis visits) as dependent variables, and comorbid PD, QIDS-SR baseline and QIDS-SR baseline $x$ comorbid PD interaction as independent variables. For all analyses the independent variables were (mean) centered to prevent errors in statistical inference (Kraemer and Blasey, 2004). 


\section{3. $\quad$ Results}

\subsection{Pre-treatment comparisons between individuals with and without missing baseline QIDS-SR and SAPAS}

As illustrated in Figure 5.1, 1455 out of 2235 individuals completed a baseline QIDS-SR and a SAPAS interview, and were therefore included in our data analyses. Individuals with and without a completed baseline QIDS-SR and/or SAPAS interview did not significantly differ in terms of age, gender, baseline depression severity, previous treatment failures involving pharmacotherapy, psychotherapy and ECT, functional impairment, comorbid anxiety, psychosocial stressors and the presence of childhood adversity. Individuals with missing baseline QIDS-SR and/or SAPAS scores had significantly more failures of intensified treatment (i.e., inpatient treatment and day treatment) than those without complete QIDS-SR and/or SAPAS scores, however the proportion of individuals that underwent these intensified treatments were low in both groups $\left(4.2 \%\right.$ vs. $2.5 \%$, $\left.^{2} i^{2}=4.768, p=0.029\right)$.

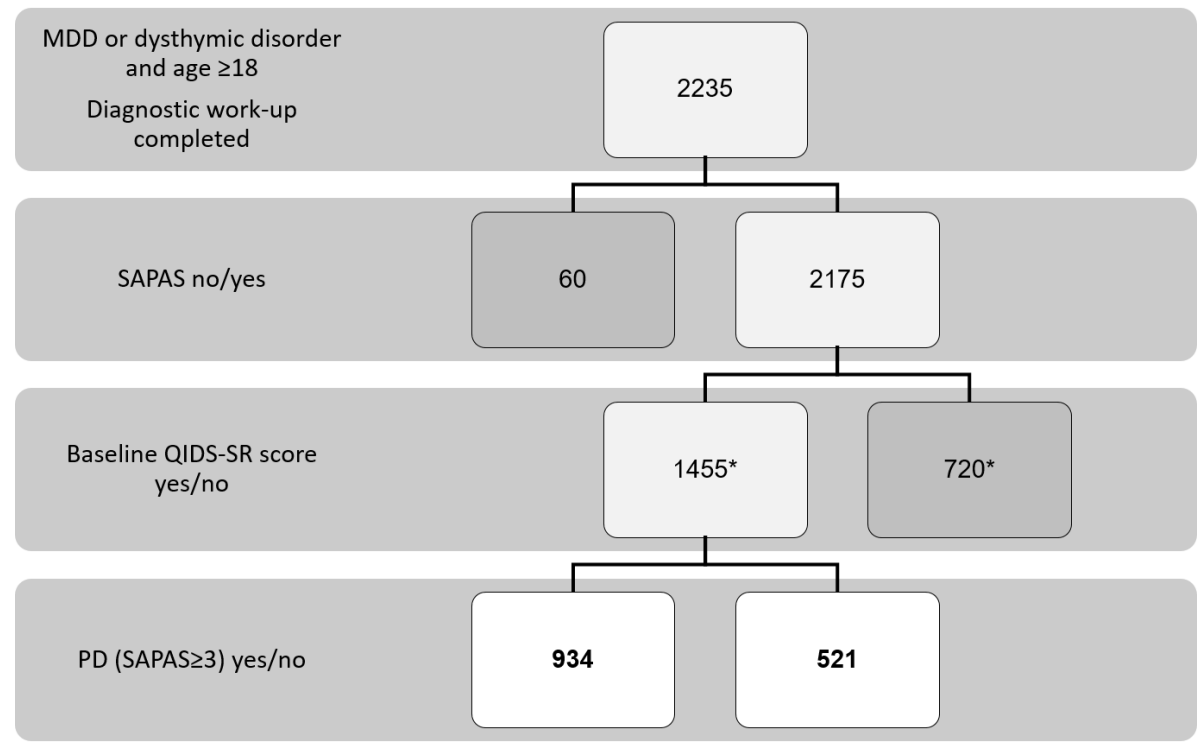

Figure 5.1: Flow-chart of the study population. MDD=major depressive disorder; SAPAS=Standardized Assessment of Personality-Abbreviated Scale; QIDS-SR=Quick Inventory of Depressive Symptomatology-Self Report; PD=personality disorder.

Note: There were no significant differences on clinical and demographical parameters between analyzed subjects ( $n=1455)$ and subjects missing a completed baseline QIDS-SR and/or SAPAS ( $n=780)$ except for previously undergone inpatient or day treatment. However, portions were small in both groups $(4.2 \%$ vs. $\left.2.5 \%, \mathrm{chi}^{2}=4.768, \mathrm{p}=0.029\right)$. 


\subsection{Pre-treatment comparisons between individuals with and without comorbid PD}

Table 5.1 provides the differences in pre-treatment characteristics between individuals with and without comorbid PD. Individuals with comorbid PD were significantly older compared to individuals without a PD $(t=2.987, d f=1453, p=0.003)$, although the effect size of this difference was small (Cohen's $d=0.163$ ). The presence of comorbid PD was associated with higher average QIDS-SR baseline scores ( $t=3.799$, $d f=1453, p<0.0001$ ), however the effect size of the difference between individuals with and without PD was small as well (Cohen's $d=0.208$ ). The index episode was significantly longer for individuals with comorbid PD compared to individuals without comorbid PD $\left(\chi^{2}=24.9, p=<0.0001\right)$. In addition, higher levels of comorbid anxiety, psychosocial stressors and childhood adversity were found in subjects with comorbid PD compared to those without PD $\left(\chi^{2}=27.1, p=<0.0001 ; \chi^{2}=4.6, p=0.033 ; \chi^{2}=46.7\right.$, $p<0.0001)$, although the effect sizes of these differences were small $(\phi=0.14, \phi=0.06$ and $\phi=0.18$ respectively).

Table 5.1: Pre-treatment characteristics of individuals with and without co-morbid personality disorders.

\begin{tabular}{|c|c|c|}
\hline & $\begin{array}{l}\text { Individuals without PD } \\
\qquad(n=521)\end{array}$ & $\begin{array}{l}\text { Individuals with PD } \\
(n=934)\end{array}$ \\
\hline Age, years mean $(S D) * *$ & $41.3(13.2)$ & $39.2(12.4)$ \\
\hline Female, n (\%) & $353(67.8 \%)$ & $611(65.4 \%)$ \\
\hline QIDS-SR score, mean (SD) $* * *$ & $16.3(4.9)$ & $17.2(4.4)$ \\
\hline \multicolumn{3}{|l|}{ Episode duration $* * *$} \\
\hline$\leq 12$ months & $286(54.9 \%)$ & $410(43.9 \%)$ \\
\hline$>12$ months $-\leq 24$ months & 117 (22.5\%) & 199 (21.3\%) \\
\hline$>24$ month & $118(22.6 \%)$ & 325 (34.8\%) \\
\hline \multicolumn{3}{|l|}{ Treatment failures } \\
\hline Antidepressants, yes n (\%) & 215 (41.3\%) & $380(40.7 \%)$ \\
\hline Psychotherapy, yes n (\%) & $213(40.9 \%)$ & 397 (42.5\%) \\
\hline Electroconvulsive therapy, yes $\mathrm{n}(\%)$ & $4(0.8 \%)$ & $4(0.4 \%)$ \\
\hline Intensified treatment, yes $\mathrm{n}(\%)$ & $13(2.5 \%)$ & $24(2.6 \%)$ \\
\hline \multicolumn{3}{|l|}{ Functional impairment } \\
\hline No impairment (GAF 90-100) & $2(0.4 \%)$ & $4(0.4 \%)$ \\
\hline Mild (GAF 60-90) & $84(16.1 \%)$ & $104(11.1 \%)$ \\
\hline Moderate (GAF 30-60) & 427 (82.0\%) & 808 (86.5\%) \\
\hline Severe (GAF 0-30) & $8(1.5 \%)$ & $18(1.9 \%)$ \\
\hline \multicolumn{3}{|l|}{ Comorbid anxiety * } \\
\hline Not present & $254(48.8 \%)$ & $330(35.3 \%)$ \\
\hline Present, but not fulfilling DSM-IV-criteria & $188(36.1 \%)$ & $394(42.2 \%)$ \\
\hline Fulfilling criteria DSM-IV & $79(15.2 \%)$ & $210(22.5 \%)$ \\
\hline Psychosocial stressors, yes n (\%) & $394(75.6 \%)$ & $751(80.4 \%)$ \\
\hline Childhood adversity, yes $\mathrm{n}(\%) * * *$ & 125 (24.0\%) & 391 (41.9\%) \\
\hline
\end{tabular}

$\mathrm{PD}=$ personality disorder; $\mathrm{SD}=$ standard deviation; intensified treatment=day patient treatment or inpatient treatment; GAF=Global Assessment of Functioning; DSM-IV=Diagnostic and Statistical Manual of Mental Disorders. * $\mathrm{p}<0.05 ; * * \mathrm{p}<0.01 ; * * * \mathrm{p}=<0.0001$. 


\subsection{Received depression treatment in accordance with (inter)national evidence-based guidelines: pharmacotherapy, psychotherapy and treatment intensity}

Table 5.2 provides the mean number of pharmacotherapy and psychotherapy sessions for subjects with and without comorbid PD. Table 5.3 summarizes the results of the negative binominal regression models on the association between comorbid PD and the amount of pharmacotherapy and psychotherapy sessions. There was no significant association between the presence of comorbid PD and the number of received pharmacotherapy sessions. However, higher baseline depression severity was associated with a higher number of pharmacotherapy sessions, but there was no significant interaction between depression severity and PD. For the number of psychotherapy sessions, individuals with comorbid PD had significantly more psychotherapy sessions than individuals without PD. No significant effect of baseline severity or of the baseline severity $x$ PD interaction on received psychotherapy sessions was found. The number of ECT sessions was not analysed as a separate outcome as only four subjects received ECT.

Table 5.2: Amount and type of received depression treatment in individuals with and without comorbid personality disorders.

\begin{tabular}{lcc}
\hline & $\begin{array}{c}\text { Individuals without PD } \\
(\mathrm{n}=521)\end{array}$ & $\begin{array}{c}\text { Individuals with PD } \\
(\mathrm{n}=934)\end{array}$ \\
\hline Number of pharmacotherapy sessions, mean (SD) & $4.44(7.35)$ & $4.40(6.01)$ \\
Number of psychotherapy sessions, mean (SD) & $17.72(23.22)$ & $25.11(34.26)$ \\
Number of supportive visits, mean (SD) & $9.12(20.51)$ & $12.00(25.86)$ \\
Number of crisis visits, mean (SD) & $0.84(7.34)$ & $0.64(4.41)$ \\
\hline
\end{tabular}

$\mathrm{PD}=$ personality disorder; $\mathrm{SD}=$ standard deviation.

Figure 5.2 illustrates the different treatment intensity levels that individuals with and without comorbid PD received. The multinomial logistic regression model indicated that subjects with comorbid PD had significantly higher rates of moderate treatment intensity (four or more psychotherapy or pharmacotherapy sessions) compared to those without PD, using low level treatment as the reference group (less than four sessions of psychotherapy or pharmacotherapy; OR=1.71; CI95\%=1.30-2.25; $p<0.0001$ ). The effects of depression severity and of the depression severity $x$ PD interaction were not significant for this comparison. For high intensity treatment (four or more psychotherapy and pharmacotherapy sessions or ECT) versus low intensity treatment comparison, depression severity and comorbid PD were significant, indicating that the presence of comorbid PD or high depression severity increased the odds of receiving high level treatment $(\mathrm{OR}=1.47 ; \mathrm{Cl} 95 \%=1.10-1.95 ; \mathrm{p}=0.009$ and $\mathrm{OR}=1.11 ; \mathrm{Cl} 95 \%=1.06-1.17 ; \mathrm{p}<0.0001)$. There was no significant interaction between depression severity and comorbid PD. 


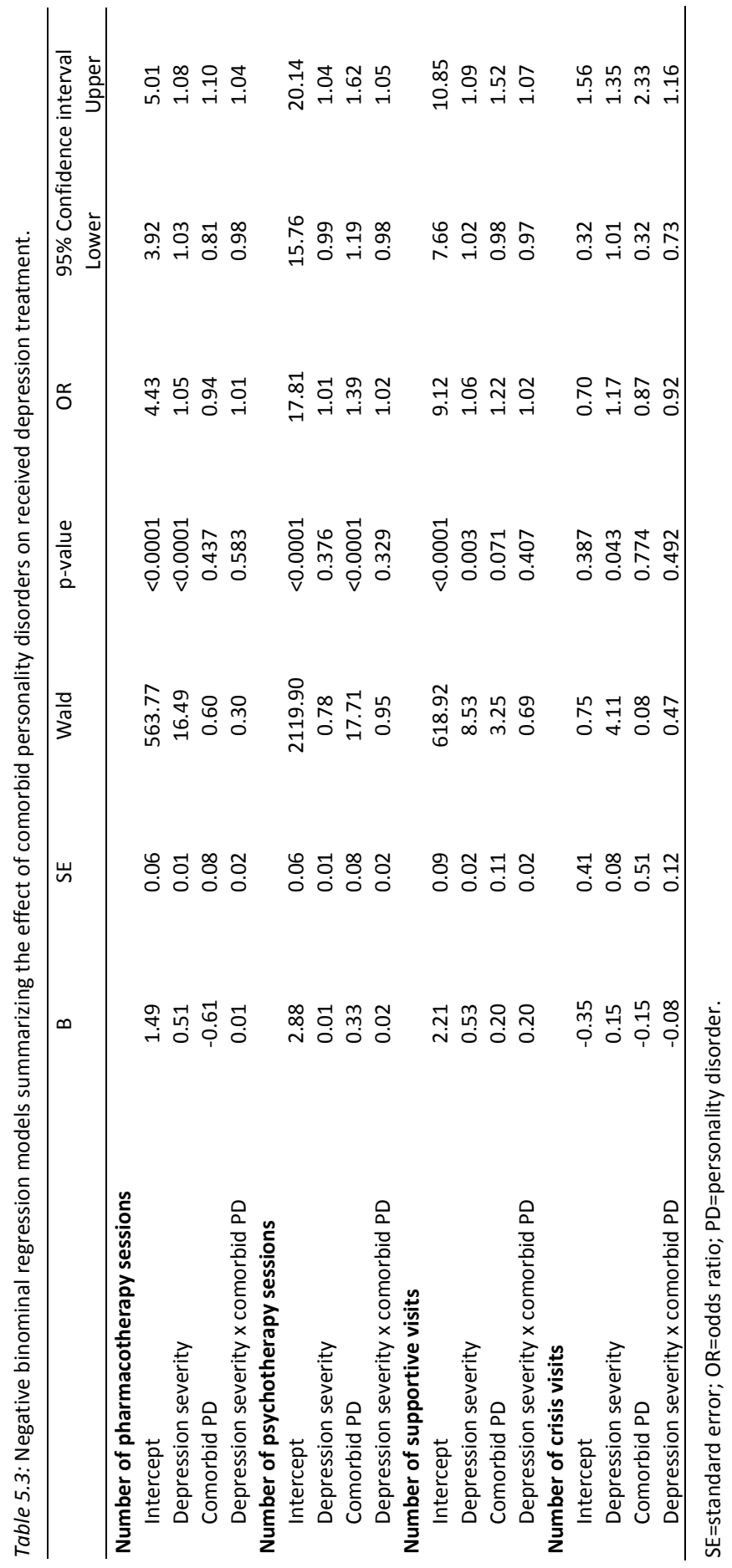




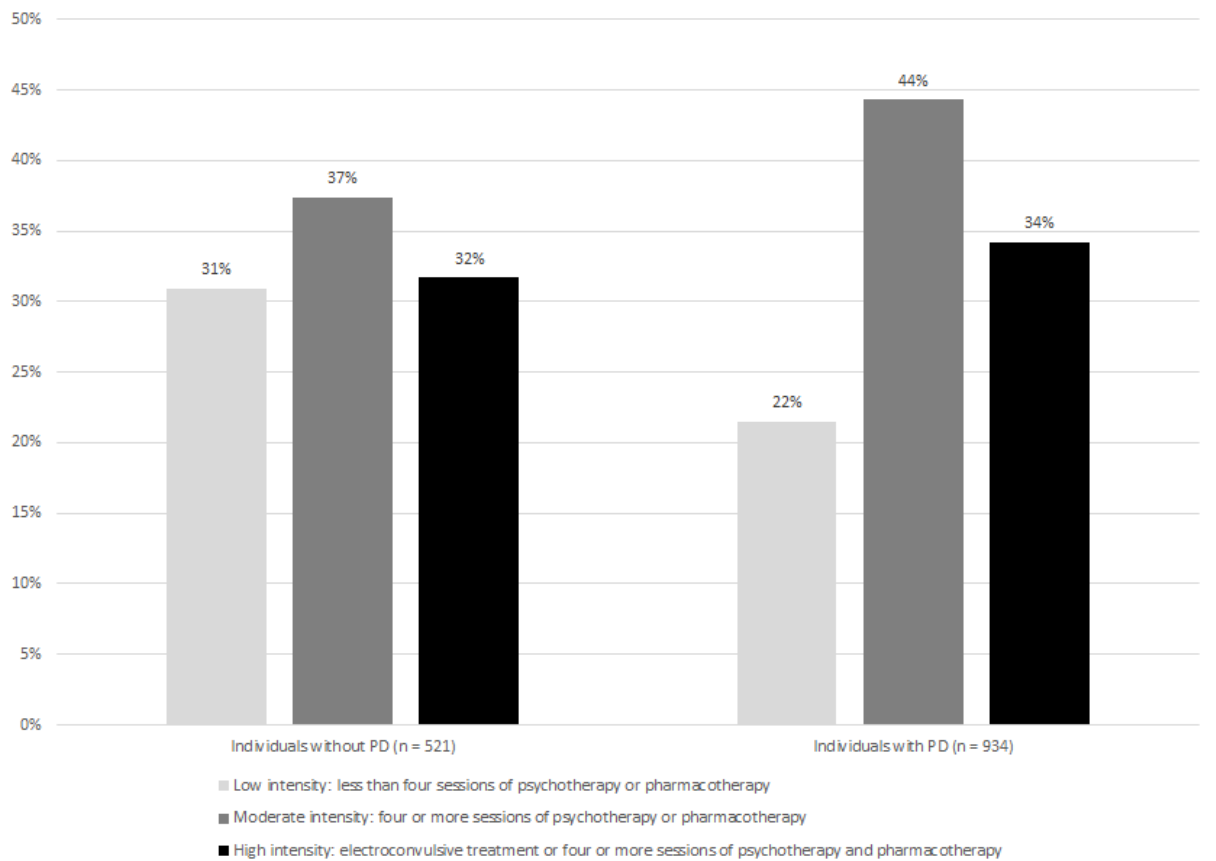

Figure 5.2: The received treatment intensity in individuals with and without a comorbid personality disorders. PD=Personality disorder.

\subsection{Received total amount of care including non-evidence-based treatments}

Table 5.2 provides the mean number of supportive and crisis visits for subjects with and without comorbid PD. Table 5.3 summarizes the estimates of the two negative binomial regression models with the secondary outcomes including the number of supportive visits and crisis visits. For both models, higher baseline depression severity was significantly associated with more supportive visits and crisis visits. In addition, the presence of comorbid PD was not significantly associated with more (or less) supportive and crisis visits, and there were no significant PD $\mathrm{x}$ depression severity interactions in both models.

\section{Discussion}

In the present observational study we investigated whether and to what extend comorbid PD was associated with the amount and type of received treatment in 1455 
depressed individuals in seven outpatient mental health care centres in The Netherlands. Our main finding was that depressed individuals with comorbid PD received a higher amount of psychotherapy sessions and therefore more intensive treatment than individuals without comorbid PD, irrespective of initial depression severity. In addition, we found that there was no difference between depressed individuals with and without comorbid PD in the number of pharmacotherapy sessions, supportive visits, and crisis visits.

The finding that depressed individuals with comorbid PD received more psychotherapy sessions for MDD is not in line with our hypothesis and with previous findings. Results of earlier studies indicated that depressed individuals with a comorbid PD were less likely to receive evidence-based treatments including ECT and antidepressant medication than individuals without comorbid PD (Black et al., 1988; Charney et al., 1981). However, recent evidence addressing this issue is lacking. The results of our study are not in line with (inter)national evidence-based guidelines as well. In these guidelines there is an overall consensus that treatment strategies for MDD should not vary based on depression subtypes or individual characteristics such as comorbid PD (American Psychiatric Association, 2009; 2010; Spijker et al., 2013). One exception is depression severity in this regard: antidepressant medication (with or without psychotherapy) is considered to be the treatment of choice for individuals with severe depression although recommendations of guidelines are not entirely consistent (Zimmerman et al., 2018). In this study, the total number of pharmacotherapy sessions was indeed related to baseline depression severity, however based on our findings there is no guarantee that these visits focussed solely on antidepressant medication.

The finding that individuals with comorbid PD did not receive more supportive and crisis visits did not align with our secondary hypothesis either. Since individuals with comorbid PD can have more interpersonal problems (Markowitz et al., 2007), lower social functioning (Newton-Howes et al., 2018), and suicidality (Oquendo et al., 2004), we expected them to receive a higher amount of supportive and crisis visits. However, one could speculate that these crisis visits were prevented by or handled within the higher number of psychotherapy sessions.

A key question underlying our findings is whether the higher number of psychotherapy sessions is either a required solution for a complex clinical problem or an unnecessary intervention that adds to overtreatment of MDD (Lorenzo-Luaces, 2015). The complexity of individuals with comorbid PD in this sample is reflected by their initial clinical presentation with a higher level of depressive and anxiety symptoms, longer episode duration and a higher incidence of childhood trauma, which has been found in previous research as well (Kelly et al., 2009; van Bronswijk et al., 2018; van den Hout et al., 2006). On could speculate that therapists feel confident that extension of treatment increases the odds of a good outcome (Delgadillo et al., 2015). Another possible explanation could be that the higher amount of 
psychotherapy sessions was an indicator for the responsiveness of therapists to the comorbid PD symptoms; possibly therapists were (unconsciously) focusing on PD symptoms. In addition, the finding could also indicate that patients with comorbid PD needed received more sessions for the process of ending the therapeutic relationship. However, despite the complex clinical problems, a significant higher number of psychotherapy sessions could be a sign of overtreatment. First of all, the association between the number of psychotherapy sessions and a favourable outcome in MDD is not significant and very small (Cuijpers et al., 2013). Moreover, findings from high quality studies with controlled treatment do not indicate that comorbid PD negatively affects acute phase depression outcome (Kool et al., 2005) and therefore the need for additional therapy remains questionable. Given the current problems in the timely availability of treatment in (Dutch) mental health care (2018; Vektis, 2019), it is of great importance not to unnecessarily extend therapy.

Although this study provides an interesting "look behind the scenes" of daily mental health care practice, some limitations apply. First of all, a substantial amount of subjects did not have a baseline QIDS-SR or SAPAS interview and had to be excluded from analyses. However, pre-treatment comparisons indicate that these individuals were not significantly different from those that were included in the analyses. Secondly, the presence of PD in our study was determined by a cut-off score on a brief standardized clinical interview (SAPAS; Bukh et al., 2010; Moran et al., 2003), and more validated methods such as the Structured Clinical Interview for DSMIV Axis II (SCID-II; First et al., 1997) might be more appropriate for studying PD. In addition, this screening instrument did not provide information on the different types of PDs. However, time and resources for the use of such instruments are typically not available in routine outpatient depression care. Taking this into consideration, we think that the SAPAS is an appropriate compromise between the need for a well validated extensive instrument and the need for representativeness of diagnostic work-ups in daily clinical practice. The third limitation concerns the lack of information on how treatment decisions were motivated; it is possible that the clinicians decided explicitly that more psychotherapy sessions were indicated given the comorbid PD, but we cannot exclude other reasons for the positive association between the comorbidity and number of psychotherapy sessions. In addition, the input of patients in these treatment decisions was not assessed. The fourth limitation involves the information on the received MDD treatment. Although we had reliable data on the type and amount of MDD treatment sessions, additional informative details were lacking, such as information on quality of psychotherapy and pharmacotherapy, or switches between different types of psychotherapy and pharmacotherapy.

To our knowledge this is the first observational study investigating the impact of comorbid PD on the type and the amount of received MDD treatment in naturalistic outpatient care. Our findings could be indicative for overtreatment of depressed individuals with comorbid PD. Further research is needed to study subgroups of 
depressed patients that are possibly undertreated and overtreated (Lorenzo-Luaces, 2015), and to identify indicators related to those subgroups. To achieve this, research should focus on types and amounts of received treatments, as well as on the specific contents and quality of these treatments. If the goal is to effectively improve MDD treatment strategies, we need to understand and evaluate our current clinical decision making more thoroughly. With this understanding, we could reflect and formulate suggestions on how to modify treatment decisions to a more evidence based practice.

In sum, this large observational study provides evidence that treatments differ to some extent between individuals with and without PD in outpatient MDD care. There were no differences in the amount of pharmacotherapy sessions, supportive visits and crisis visits between individuals with and without PD. However, individuals with comorbid PD received more psychotherapy sessions then individuals without comorbid PD. Although this could be explained by the clinical complexity of the patient and the responsiveness of the therapist, the higher number of psychotherapy sessions is not supported by (inter)national treatment guidelines and findings of high quality studies. 


\section{References}

American Psychiatric Association, 2009. Practice guideline for the treatment of patients with major depressive disorder (3rd). http://psychiatryonline. org/guidelines. aspx.

Berk, M., Boyce, P., Hamilton, A., Morris, G., Outhred, T., Das, P., Bassett, D., Baune, B.T., Lyndon, B., Mulder, R., 2018. Personality: distraction or driver in the diagnosis of depression. Personality and mental health 12, 126-130.

Black, D.W., Bell, S., Hulbert, J., Nasrallah, A., 1988. The importance of Axis II in patients with major depression: A controlled study. Journal of Affective Disorders 14, 115-122.

Bukh, J.D., Bock, C., Vinberg, M., Gether, U., Kessing, L.V., 2010. Clinical utility of Standardised Assessment of Personality-Abbreviated Scale (SAPAS) among patients with first episode depression. Journal of affective disorders 127, 199-202.

Charney, D., Nelson, J.C., Quinlan, D.M., 1981. Personality traits and disorder in depression. Am J Psychiatry 138, 1601.

Cipriani, A., Furukawa, T.A., Salanti, G., Chaimani, A., Atkinson, L.Z., Ogawa, Y., Leucht, S., Ruhe, H.G., Turner, E.H., Higgins, J.P., 2018. Comparative efficacy and acceptability of 21 antidepressant drugs for the acute treatment of adults with major depressive disorder: a systematic review and network metaanalysis. Focus 16, 420-429.

Cohen, Z.D., DeRubeis, R.J., 2018. Treatment selection in depression. Annual Review of Clinical Psychology 14.

Cuijpers, P., Sijbrandij, M., Koole, S.L., Andersson, G., Beekman, A.T., Reynolds III, C.F., 2013. The efficacy of psychotherapy and pharmacotherapy in treating depressive and anxiety disorders: A meta-analysis of direct comparisons. World Psychiatry 12, 137-148.

Delgadillo, J., Gellatly, J., Stephenson-Bellwood, S., 2015. Decision making in stepped care: how do therapists decide whether to prolong treatment or not? Behavioural and cognitive psychotherapy 43, 328-341.

Delgadillo, J., McMillan, D., Lucock, M., Leach, C., Ali, S., Gilbody, S., 2014. Early changes, attrition, and dose-response in low intensity psychological interventions. British Journal of Clinical Psychology 53, 114-130.

Fekadu, A., Wooderson, S.C., Markopoulou, K., Cleare, A.J., 2009. The Maudsley Staging Method for treatment-resistant depression: prediction of longer-term outcome and persistence of symptoms. The Journal of clinical psychiatry 70, 952-957.

First, M.B., Gibbon, M., Spitzer, R.L., Williams, J.B.W., Benjamin, L.S., 1997. Structured Clinical Interview for DSM-IV Axis II Personality Disorders (SCID-II) American Psychiatric Press, Washington, D.C.

Friborg, O., Martinsen, E.W., Martinussen, M., Kaiser, S., Øvergård, K.T., Rosenvinge, J.H., 2014. Comorbidity of personality disorders in mood disorders: a meta-analytic review of 122 studies from 1988 to 2010. Journal of affective disorders 152, 1-11.

Friedman, E.S., Davis, L.L., Zisook, S., Wisniewski, S.R., Trivedi, M.H., Fava, M., Rush, A.J., Team, C.-M.S., 2012. Baseline depression severity as a predictor of single and combination antidepressant treatment outcome: results from the CO-MED trial. European Neuropsychopharmacology 22, 183-199.

GGZNederland, 2018. Q\&A Wachttijden GGZ.

Health, N.C.C.f.M., 2010. Depression: the treatment and management of depression in adults (updated edition). British Psychological Society.

Kelly, B.D., Nur, U.A., Tyrer, P., Casey, P., 2009. Impact of severity of personality disorder on the outcome of depression. European psychiatry 24, 322-326.

Kolovos, S., van Tulder, M.W., Cuijpers, P., Prigent, A., Chevreul, K., Riper, H., Bosmans, J.E., 2017. The effect of treatment as usual on major depressive disorder: a meta-analysis. Journal of Affective Disorders 210, 72-81.

Kool, S., Schoevers, R., de Maat, S., Van, R., Molenaar, P., Vink, A., Dekker, J., 2005. Efficacy of pharmacotherapy in depressed patients with and without personality disorders: a systematic review and meta-analysis. Journal of affective disorders 88, 269-278. 
Kraemer, H.C., Blasey, C.M., 2004. Centring in regression analyses: a strategy to prevent errors in statistical inference. International journal of methods in psychiatric research 13, 141-151.

Lorenzo-Luaces, L., 2015. Heterogeneity in the prognosis of major depression: from the common cold to a highly debilitating and recurrent illness. Epidemiology and Psychiatric Sciences 24, 466-472.

Mann, A., Jenkins, R., Cutting, J., Cowen, P., 1981. The development and use of a standardized assessment of abnormal personality. Psychological Medicine 11, 839-847.

Markowitz, J.C., Skodol, A.E., Petkova, E., Cheng, J., Sanislow, C.A., Grilo, C.M., Gunderson, J.G., McGlashan, T.H., 2007. Longitudinal effects of personality disorders on psychosocial functioning of patients with major depressive disorder. Journal of Clinical Psychiatry 68, 186.

Moran, P., Leese, M., Lee, T., Walters, P., Thornicroft, G., Mann, A., 2003. Standardised Assessment of Personality-Abbreviated Scale (SAPAS): preliminary validation of a brief screen for personality disorder. The British Journal of Psychiatry 183, 228-232.

Mulder, R.T., 2002. Personality pathology and treatment outcome in major depression: a review. American Journal of Psychiatry 159, 359-371.

Newton-Howes, G., Mulder, R., Ellis, P.M., Boden, J.M., Joyce, P., 2018. Predictive Utility of Personality Disorder in Depression: Comparison of Outcomes and Taxonomic Approach. Journal of personality disorders 32, 513-526.

Newton-Howes, G., Tyrer, P., Johnson, T., 2006. Personality disorder and the outcome of depression: metaanalysis of published studies. The British Journal of Psychiatry 188, 13-20.

Newton-Howes, G., Tyrer, P., Johnson, T., Mulder, R., Kool, S., Dekker, J., Schoevers, R., 2014. Influence of personality on the outcome of treatment in depression: systematic review and meta-analysis. Journal of Personality Disorders 28, 577-593.

Oquendo, M.A., Galfalvy, H., Russo, S., Ellis, S.P., Grunebaum, M.F., Burke, A., Mann, J.J., 2004. Prospective study of clinical predictors of suicidal acts after a major depressive episode in patients with major depressive disorder or bipolar disorder. American Journal of Psychiatry 161, 1433-1441.

Robinson, L., Delgadillo, J., Kellett, S., 2020. The dose-response effect in routinely delivered psychological therapies: A systematic review. Psychotherapy Research 30, 79-96.

Rush, A.J., Trivedi, M.H., Ibrahim, H.M., Carmody, T.J., Arnow, B., Klein, D.N., Markowitz, J.C., Ninan, P.T., Kornstein, S., Manber, R., 2003. The 16-Item Quick Inventory of Depressive Symptomatology (QIDS), clinician rating (QIDS-C), and self-report (QIDS-SR): a psychometric evaluation in patients with chronic major depression. Biological psychiatry 54, 573-583.

Rush, A.J., Trivedi, M.H., Wisniewski, S.R., Nierenberg, A.A., Stewart, J.W., Warden, D., Niederehe, G., Thase, M.E., Lavori, P.W., Lebowitz, B.D., 2006. Acute and longer-term outcomes in depressed outpatients requiring one or several treatment steps: a STAR* D report. American Journal of Psychiatry 163, 19051917.

Simon, G.E., Perlis, R.H., 2010. Personalized medicine for depression: can we match patients with treatments? American Journal of Psychiatry 167, 1445-1455.

Smit, A., Schene, A., Peeters, F., Spijker, J., 2016. Tailored pharmacotherapy. Consultations about medication in a care programme for depression. Tijdschrift voor psychiatrie 58, 881-885.

Spijker, J., Bockting, C., Meeuwissen, J., Van Vliet, I., Emmelkamp, P., Hermens, M., van Balkom, A., 2013. Multidisciplinaire richtlijn Depressie (Derde revisie): Richtlijn voor de diagnostiek, behandeling en begeleiding van volwassen patiënten met een depressieve stoornis. Trimbos Instituut: Utrecht.

van Bronswijk, S.C., Köster, E.M., Peeters, F.P., 2019. Effectiveness of Acute-Phase Treatment of Depression Is Not Influenced by Comorbid Personality Disorders: Results from a Meta-Analysis and MetaRegression. Psychotherapy and psychosomatics, 1-2.

van Bronswijk, S.C., Lemmens, L.H., Viechtbauer, W., Huibers, M.J., Arntz, A., Peeters, F.P., 2018. The impact of personality disorder pathology on the effectiveness of cognitive therapy and interpersonal psychotherapy for major depressive disorder. Journal of affective disorders 225, 530-538.

van den Hout, M., Brouwers, C., Oomen, J., 2006. Clinically diagnosed axis II co-morbidity and the short term outcome of CBT for axis I disorders. Clinical Psychology \& Psychotherapy: An International Journal of Theory \& Practice 13, 56-63. 
van Dijk, D., van den Boogaard, T.M., Deen, M., Spijker, J., Ruhé, H., Peeters, F., 2019. Predicting clinical course in major depressive disorder: The association between DM-TRD score and symptom severity over time in 1115 outpatients. Depression and anxiety 36, 345-352.

Van, H.L., Kool, M., 2018. What we do, do not, and need to know about comorbid depression and personality disorders. The Lancet Psychiatry 5, 776-778.

Vektis, 2019. Kwartaalcijfers wachttijden GGZ.

Zimmerman, M., Morgan, T.A., Stanton, K., 2018. The severity of psychiatric disorders. World Psychiatry 17, 258-275.

Zimmerman, M., Rothschild, L., Chelminski, I., 2005. The prevalence of DSM-IV personality disorders in psychiatric outpatients. American Journal of Psychiatry 162, 1911-1918. 
Part

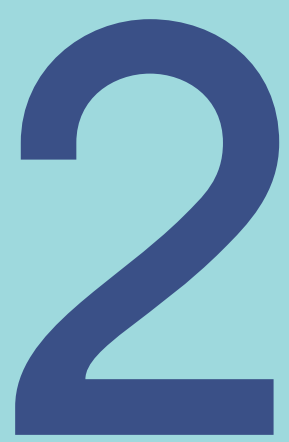




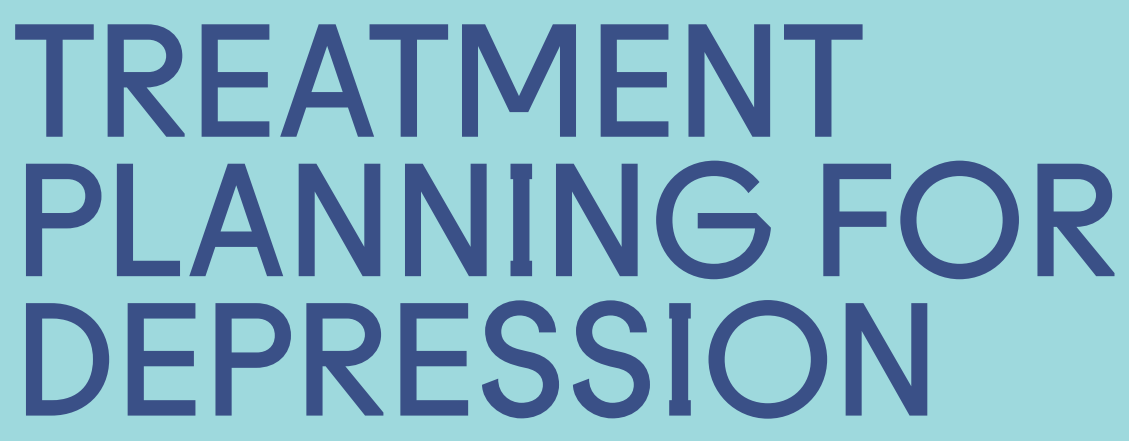


L. Lemmens, S. van Bronswijk, F. Peeters, A. Arntz, S. Hollon, M. Huibers

- Psychological Medicine 2019;49(3):465-473

\section{Chapter}

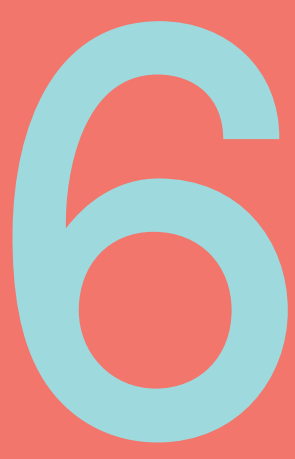




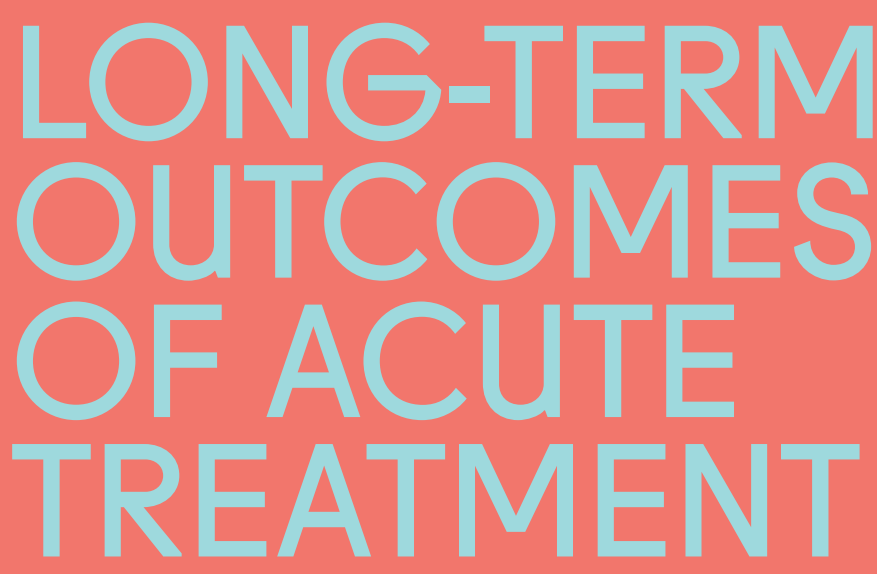

WITH COGNITIVE

THERAPY VERSUS

INTERPERSONAL

PSYCHOTHERAPY

FOR ADULT

DEPRESSION:

FOLLOW-UP OF

A RANDOMIZED

CONTROLLED

TRIAL 


\section{Abstract}

Background

Although equally efficacious in the acute phase, it is not known how cognitive therapy (CT) and interpersonal psychotherapy (IPT) for major depressive disorder (MDD) compare in the longrun. This study examined long-term outcomes of CT vs. IPT for MDD.

\section{Methods}

134 adult (18-65) depressed outpatients who were treated with CT $(n=69)$ or IPT $(n=65)$ in a large open label RCT (parallel group-design; computer-generated block-randomization) were monitored across a 17-month follow-up phase. Mixed regression was used to determine the course of self-reported depressive symptom severity (BDI-II) after treatment termination, and to test whether CT and IPT differed throughout the follow-up phase. Analyses were conducted for the total sample $(n=134)$, and for the subsample of treatment responders $(n=85)$. Furthermore, for treatment responders, rates of relapse and sustained response were examined for self-reported (BDI-II) and clinician-rated (LIFE) depression using Cox regression.

\section{Results}

On average, the symptom reduction achieved during the 7-month treatment phase was maintained across follow-up (7-24 months) for CT and IPT, both in the total sample and in the responder sample. Two thirds (67\%) of the treatment-responders did not relapse across the follow-up period on the BDI-II. Relapse rates assessed with the LIFE were somewhat lower. No differential effects between conditions were found.

\section{Conclusions}

Patients who responded to IPT were no more likely to relapse following treatment termination than patients who responded to CT. Given that CT appears to have a prophylactic effect following successful treatment, our findings suggest that IPT might have a prophylactic effect as well. 


\section{Introduction}

Cognitive therapy (CT) and interpersonal psychotherapy (IPT) - the two best studied and commonly practiced psychological interventions for the treatment of major depressive disorder (MDD) - have shown to be effective treatments for many depressed patients (Cuijpers et al. 2011, Barth et al. 2013, Cuijpers et al. 2013a, Cuijpers et al. 2016). With initial response rates up to $60 \%$, they have shown to be at least as efficacious as antidepressant medications (ADM) in the acute phase of the disorder (Cuijpers et al. 2013b, Cuijpers et al. 2013c). However, even when treated effectively in the acute phase ${ }^{1}$, depression has an unfavorable prognosis. It is estimated that at least $50 \%$ of those who recover from a first episode of MDD will have one or more additional episodes later on in life, and the risk of recurrence progressively increases with each additional episode (Solomon et al. 2000, Burcusa and lacono 2007, Eaton et al. 2008). It is therefore important that treatments do not only reduce symptoms in the acute phase, but also produce enduring effects.

CT has consistently been shown to have an enduring effect that lasts beyond the end of treatment, with survival rates higher than those associated with (prior) pharmacological treatment ${ }^{2}$ (Vittengl et al. 2007, Cuijpers et al. 2013b). Research in IPT is less extensive. Even though IPT has shown to prevent relapse and recurrence when continued or maintained (Cuijpers et al. 2016), only one older study has examined whether it has a prophylactic effect following treatment termination (Shea et al. 1992). This was the follow-up to the NIMH Treatment of Depression Collaborative Research Program (TDCRP), a placebo controlled randomized comparison among CT, IPT and ADM that found comparable rates of relapse between prior IPT and prior CT (33\% vs. 36\%) that were each non-significantly lower than prior ADM (50\%). These findings must be interpreted with caution, since sample sizes were small, ADM was continued for six months following the end of acute treatment, and the difference between prior CT and prior ADM was among the smallest reported in the literature, but they are suggestive of a possible enduring effect for prior IPT. Additional research into the extent to which the effects of IPT persist following the cessation of treatment is needed.

Recently we conducted a large randomized controlled trial (RCT) investigating the effects of individual CT and IPT for adult depression, primarily designed to compare long-term outcomes of both therapies in a research-oriented routine clinical setting (Lemmens et al. 2011, Lemmens et al. 2015, Lemmens et al. 2017). CT and IPT were both superior to a waiting-list control condition over the first two months of treatment, and did not differ from another across the rest of the seven-month treatment phase (Lemmens et al. 2015) - as has been the case in most acute phase

\footnotetext{
${ }^{1}$ Acute phase treatment is aimed at inducing remission.

${ }^{2}$ Survival is defined here as 'no relapse'.
} 
comparisons between the two modalities (Jakobsen et al. 2012). We now report on the long-term outcomes of these two interventions over the next 17 months, through the end of 24-months post-randomization. We expected depression scores to be relatively stable across the follow-up period. Furthermore, we expected relapse rates in CT to be similar to those reported in previous studies; approximately $30 \%$. Following earlier findings (Shea et al. 1992), one would not expect large differences between CT and IPT. However, since CT has a stronger tradition in focusing on relapse prevention compared to IPT, we expected that CT would do somewhat better.

\section{Methods}

\subsection{Design and participants}

Data come from a single-center RCT (parallel group design) into the clinical effects and mechanisms of change of individual CT and IPT for MDD (Lemmens et al. 2011, Lemmens et al. 2015). In this study, 182 depressed adults were randomly allocated to CT $(n=76)$, IPT $(n=75)$, or a 2-month Waiting-List Control (WLC) condition followed by treatment of choice $(n=31)$. In the present study, we only included the patients who were assigned to one of the two active conditions (CT and IPT), and who provided data at post-treatment (month 7; $n=134 ; C T=69$, IPT=65; henceforth; total sample).

Details concerning study design, participants, interventions, and acute outcomes have been fully described elsewhere (Lemmens et al. 2011, Lemmens et al. 2015), and will therefore only briefly be summarized here. Participants were adult outpatients referred to the mood disorder treatment-program of the Academic Community Mental Health Centre Maastricht. All patients had a primary diagnosis of MDD, as ascertained by the Dutch version of the Structured Clinical Interview for DSM-IV Axis I disorders (SCID-I; First et al. 1997). Further inclusion criteria were: internet access, an e-mail address, and sufficient knowledge of the Dutch language. Patients receiving ADM or other psychological treatment at baseline were excluded from the study, as were those at imminent risk for suicide. Other exclusion criteria were: bipolar or chronic depression (current episode $>5$ years), IQ lower than 80 , and substance abuse/dependence.

All participants provided informed consent. Randomization took place via computer-generated block-randomization (10:10:4) and was pre-stratified according to presence or absence of prior episodes. The random allocation sequence was generated by an independent computer scientist and was concealed from the researchers. Blinding of patients and therapists for treatment condition was not possible. As outlined elsewhere (Lemmens et al. 2011), sample size calculations were based on long-term expectations of CT vs. IPT. An a-priori power analysis indicated that 75 patients per arm in the active conditions (taking 15\% attrition into account), 
would provide $80 \%$ power (two-tailed $\alpha=.05$ ) to detect an expected $20 \%$ difference in relapse rate between CT and IPT (favoring CT) at the end of the follow-up period.

Treatment consisted of 16 to 20 individual 45-minute sessions. The CT protocol followed the guidelines laid out by Beck et al. (1979) and included homework assignments. The IPT protocol was based on the manual by Klerman et al. (1984). Therapists were uniquely assigned to one of the treatment conditions to prevent contamination. All therapists had several years of clinical experience in the field of depression and with the assigned intervention. Prior to the study, all therapists received 16 hours of additional training by experts in the field. During the study, therapists and researchers met biweekly in consultation sessions to discuss their caseloads (separate sessions for CT and IPT). The study was approved by Maastricht University's Ethical Board, and is registered at the Netherlands Trial Register, part of the Dutch Cochrane Centre (ISRCTN67561918). Patients completed an average of 17 therapy sessions $(S D=2.9)$. Independent assessors rated the quality of therapy, measured with the Cognitive Therapy Scale (Dobson et al. 1985) for CT and the Short version of the IPT Adherence and Quality Scale (Stuart 2011) for IPT, as being "(very) good" to "excellent" in both conditions (see Lemmens et al. 2015 for more details). Furthermore, significant differences in therapy specific behavior between conditions were found (as measured with the Collaborative Study Psychotherapy Rating Scale version 6; Hollon et al. 1984, Hollon et al. 1988), indicating that therapists adhered to protocol.

Both treatments led to considerable improvement in depressive symptom severity as measured with the Beck Depression Inventory II (BDI-II; Beck et al. 1996): pre-post treatment effect size $d=1.72$ in the pooled active conditions. Response to therapy exceeded response in the WLC condition. No differential effects between the active treatments were found (Lemmens et al. 2015).

\subsection{Outcomes}

\subsubsection{Self-Reported Depression Severity}

Self-reported depressive symptom severity was measured with the BDI-II. The BDI-II is a 21-item questionnaire with strong psychometric properties (Beck et al. 1996, Van der Does 2002). Items are rated on a 4-point Likert-scale (0-3), with higher scores indicating higher levels of depression severity (range 0-63).

\subsubsection{Clinician-Rated Depression Severity}

The MDD section of the Longitudinal Interval Follow-up Evaluation (LIFE; Keller et al. 1987) - a semi-structured interview for assessing the longitudinal course of psychiatric illness using a retrospective rating system - was used to obtain a clinician-rated measure of depression. The LIFE uses DSM-IV diagnostic criteria to classify depression 
retrospectively over the course of a pre-determined follow-up period (in our case 1 year; see further). Ratings are made on a six-point scale, ranging from meeting the full criteria of MDD (ratings of 5 or 6 ) to no residual symptoms (rating of 1 ). The LIFE has shown to be a reliable and valid instrument for identifying the course of several mental disorders examined retrospectively over the period of one year (Warshaw et al. 1994, Warshaw et al. 2001).

\subsection{Procedure}

BDI-II assessments were completed at post-treatment (month 7), monthly thereafter for the next five months (month 7 - 12), and then again at the end of the follow-up (month 24). All assessments were administered on a computer. The post-treatment assessment took place at the research centre (Maastricht University). All other assessments were administered online. The LIFE interview took place after the 24 month-assessment and addressed retrospectively the period between 12 and 24 months $^{3}$. A rating was made for each two week period between months 12 and 24, resulting in a total of 26 retrospective observations. A schematic overview of the study design and the data-points used in this study, can be found in Figure 6.1.

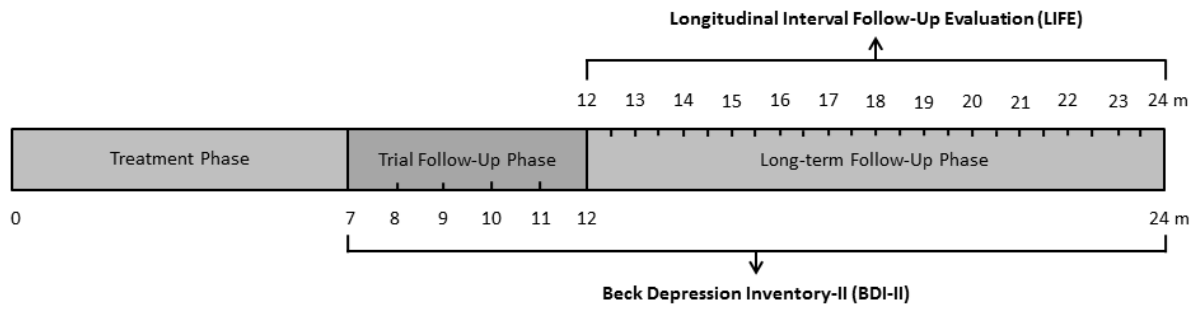

Figure 6.1: Overview of BDI-II and LIFE data-points used in the current study.

\footnotetext{
${ }^{3}$ Due to practical reasons, not all interviews could be administered immediately after study termination. In case the interview took place later on, the total period between the 12 month-assessment and the date of the interview was covered in the interview. However, only the ratings made between 12 and 24 months were used in the analyses.
} 
The majority of LIFE interviews (90\%) were administered by a clinical psychology graduate student. The remaining $10 \%$ was administered by a resident in psychiatry. Both LIFE assessors had several years of clinical experience in the field of depression. Prior to the study, assessors studied relevant literature, the original set of LIFE training materials, and the detailed instruction manual that was developed for the current study. Furthermore, they conducted several pilot interviews to familiarize themselves with the rating system. During the study, regular consensus sessions took place, in order to discuss interpretation and pitfalls. The interviewers were blind to condition, treatment adherence, - satisfaction and - outcome. Interviews took place face-to-face or by telephone ${ }^{4}$ and ratings were made after the interview. Ratings made by the psychiatric resident were discussed with the other rater until consensus was reached. Since patients were free to pursue additional treatment during the follow-up phase, we assessed whether patients received additional psychological support for MDD (conservatively defined as having one or more sessions with a general practitioner (GP) or a mental health care professional for depressive symptoms) or used ADM ( $\geq 2$ weeks) throughout the 17-month follow-up period. Information on health care status was obtained during the LIFE interview and at the 12 and 24 month assessment ${ }^{5}$ with the periodic retrospect health care consumption questionnaire (de Graaf et al. 2008).

\subsection{Definition of response and relapse}

When investigating the clinical course of a disorder after acute phase treatment, one needs to carefully consider definitions of response and relapse. Response is often conceptualized as a pre-determined change score representing a clinical significant improvement over the course of treatment (Jacobson and Truax 1991). Even though this method is useful in the majority of cases, in some cases it leads to somewhat peculiar classifications. For example, the approach excludes patients who reach remission without the necessary drop in symptoms as treatment responders (e.g., a drop from 14 to 8 on the BDI-II). Furthermore, it includes patients who do show a clinical significant improvement, but still report high depression scores at the end of

\footnotetext{
${ }^{4}$ The reason for using two methods was purely practical. At the end of the follow-up phase, all patients were invited for a face-to-face interview at the research center. For practical reasons, these interviews had to take place during office hours. Those who indicated that they could not make it to the research center (i.e. because they moved away, or because they were not available during office hours), were offered a telephone interview instead (these interviews could be scheduled in the evenings as well). Note that administering the LIFE by telephone is common practice (e.g., Meyers et al. 2002; Warshaw et al. 2001). Results of those interviewed by telephone $(47 \%$; CT $=40 \%$, IPT $=54 \%)$, did not differ from those who were interviewed at the research center. In addition, nine patients provided data via a digital version of the interview, which was rated afterwards, because they could not participate in both a face-to-face and a telephone interview.

${ }^{5}$ These assessments covered the period between 9 and 24 months. As a result, information about additional (psychological and pharmacological) support for MDD between month 7 and 9 is not available.
} 
treatment (e.g., a drop from 61 to 48), hereby indicating that treatment had some effect, but worked insufficiently to reach (partial) remission. In order to take these variations into account, we defined response to treatment as either 1) a posttreatment BDI-II score lower than 10 (the cut-off for remission in our trial determined with the method of Jacobson and Truax 1991; see Lemmens et al. 2015); or 2) an overall change of at least $9 \mathrm{BDI}-\mathrm{Il}$ points (the cut-off for reliable change in our trial determined with the method of Jacobson and Truax 1991; see Lemmens et al. 2015) and a post-treatment BDI-II score lower than 20 (the border between moderate and mild depression on the BDI-II, see ${ }^{6}$ ). Since the BDI-II and the LIFE assess different aspects of depression (depressive symptom severity vs. DSM-IV classification) we formulated two separate definitions for relapse. Relapse on the BDI-II was defined as losing $\geq 50 \%$ of the improvement that occurred over the course of treatment at any point during the follow-up $(7,8,9,10,11,12,24$ months). This was done to account for individual symptom severity change. Following Hollon et al. (2005), relapse on the LIFE occurred as soon as patients met full DSM-IV criteria for a depressive episode (rating of 5 or 6 ) on one of the 26 retrospective data-points. Definitions of response and relapse are summarized in Table 6.1.

Table 6.1: Overview of definitions of response, relapse and sustained response on the BDI-II and LIFE.

\begin{tabular}{|c|c|}
\hline \multicolumn{2}{|l|}{ BDI-II } \\
\hline Response & Post-treatment BDI-II score $<10$; or \\
\hline & $\begin{array}{l}\text { Improvement of } \geq 9 \mathrm{BDI}-\mathrm{II} \text { points during treatment and post-treatment BDI-II score } \\
<20 \text {. }\end{array}$ \\
\hline Relapse & $\begin{array}{l}\text { Losing } \geq 50 \% \text { of the improvement that occurred during treatment at any point } \\
\text { during follow-up. }\end{array}$ \\
\hline Sustained response & Response to treatment (see above) and no relapse (see above) during follow-up. \\
\hline \multicolumn{2}{|l|}{ LIFE } \\
\hline Relapse & $\begin{array}{l}\text { Meeting full DSM-IV criteria for MDD (rating of } 5 \text { or } 6 \text { ) on one of the } 26 \\
\text { retrospective data-points }\end{array}$ \\
\hline
\end{tabular}

BDI-II=Beck Depression Inventory-II; DSM-IV=Diagnostic and Statistical Manual of Mental Disorders, fourth edition; LIFE=Longitudinal Interval Follow-Up Evaluation; MDD=major depressive disorder

\subsection{Data analysis}

First - for all patients ( $n=134)$ - we mapped out study compliance (LIFE and BDI-II) across the follow-up period (7-24 months), and compared patients with and without complete data on each of the outcome measures in terms of baseline characteristics (gender, age, education level, work- and marital status, first/recurrent depression)

\footnotetext{
${ }^{6}$ The cut-off of 20 was chosen because it marks the border between mild and moderate depression on the BDI-II. Patients with mild depression only meet the minimal criteria to make the diagnosis. Clinically, this suggests that even though day to day functioning may still be hard work and might feel like a struggle, the depressive symptoms are manageable and result in only minor impairments in social and occupational functioning.
} 
and post-treatment BDI-II score. We used $\chi^{2}$ tests for categorical data and independent samples t-tests for continuous data. In addition, for each of the outcome measures (BDI-II and LIFE), BDI-II scores of patients with incomplete data were plotted to explore potential patterns in depression severity prior to drop-out. For the BDI-II, reliability at each time-point was assessed using Cronbach's alpha $(\alpha)$.

After that, we determined the course of self-reported depressive symptom severity after treatment termination, and tested whether one of the treatments was superior to the other across the follow-up period. For this, we used a linear mixedeffects (multilevel) model with repeated BDI-II scores as the dependent variable, and time, condition (CT=-0.5, IPT=0.5) and the time*condition interaction as the independent variables (Diggle et al. 2002). Because mixed regression takes the nested structure of the data into consideration and can deal with autocorrelation and missing values (see e.g., Schafer and Graham 2002, Singer and Willett 2003, Snijders and Bosker 2012), missing values were not imputed. Since CT and IPT differed in depression severity (BDI-II) and quality of life (EQ5D utility score; EuroQol-Group 1990) at baseline, albeit not significant (see Lemmens et al. 2015), we added their standardized baseline scores as covariates to the model. Visual inspection of BDI-II change scores over time showed separate linear patterns for the 7-12 and 12-24 month intervals. Therefore, for the fixed effects, the slopes were modeled separately for each interval (piecewise regression; see data-supplement I). An autoregressive covariance structure was applied to factor in the correlation between measurement points. Intercepts and slopes (for the time variable) were allowed to be correlated and to vary randomly over subjects. Robust standard errors were applied. Effect sizes Cohen's $d$ and $r$ were computed from the multilevel estimates. Withincondition change was defined as Cohen's $d=$ (post-treatment mean - mean at time i)/(pooled post-treatment standard deviation), with the estimated means derived from the mixed regression analysis. Between-group effect sizes were determined by calculating the difference between the within-condition effect sizes of CT and IPT at time i. The $r$ was defined as $V(F /(F+d)$, with $F$ and $d$ values from the fixed part of the mixed regression analysis. Effect sizes were classified as being small $( \pm 0.2)$, medium $( \pm 0.5)$ and large $( \pm 0.8$; Cohen 1988$)$.

Subsequently, we calculated response rates at 7 months, and compared pretreatment characteristics (similar to those described above) and post-treatment BDI-II scores between responders and non-responders. After that, we continued our analyses with the responder sample only (responder analysis; $n=85$ ). First, we re-ran the previously described linear mixed-effects model, and computed effect sizes $r$ and d. Second, relapse rates (separate analyses for the BDI-II and LIFE) were examined using Cox regression models with condition as independent variable and standardized baseline BDI-II and EQ5D utility scores as covariates (Cox and Oakes 1984). The proportional hazard assumption was tested with Schoenfeld Residuals Test. Betweencondition survival rates were compared using the log-rank test. Dropouts were 
censored after the last measurement. In addition, for the BDI-II, we examined the rates of sustained response: the number of patients who responded to treatment and remained well (no relapse) during the follow-up period (see Table 6.1). Following Hollon et al. (2005), percentages of sustained response rates were adjusted for missing observations and calculated from the 'baseline' sample (all patients that were initially assigned to CT or IPT, regardless of their enrollment in the current study; $\mathrm{n}=151$ ). By doing this, percentages of sustained response reflect pre-treatment probabilities of enduring treatment effects. CT/IPT differences in sustained response were examined using a $\chi^{2}$ test of independence.

Finally, sensitivity analyses were performed on all models by adding the following variables sequentially as (centered) covariates to each model: gender, work- and marital status, number of sessions received in acute phase, therapist, and additional psychological support for MDD, and/or use of antidepressant medication in the follow-up period. Multilevel analyses were carried out in SPSS (version 21). Other analyses were performed in STATA (version 13.1). All effects were tested at the $p<0.05$ level (two-tailed).

\section{Results}

\subsection{Patient flow and attrition}

Of the 134 patients that were enrolled in the current study, $119(88.8 \%)$ completed all BDI-II assessments ${ }^{7}$. Baseline characteristics of patients with incomplete data on the BDI-II ( 8 in CT and 7 in IPT: $\chi^{2}(1,134)=0.02, p=0.88$ ), were not significantly different from those with complete data (all $p^{\prime} s>.23$ ). In addition, exploration of the course of depression indicated no distinctive patterns of findings between patients with incomplete and complete BDI-II data. The depression severity pattern for patients with missing BDI-II data appeared to be random. A total of 98 LIFE interviews were administered. Among the 36 patients whose LIFE interview was missing 6 dropped out in an earlier phase of the study, 14 were unattainable and did not respond to contact requests, and 16 indicated that they no longer wanted to participate. Furthermore, LIFE data of two patients were incomplete. The conditions did not differ with respect to whether there was (complete) LIFE data (20 in CT vs. 18 in IPT: $\chi^{2}(1,134)=0.03$, $\mathrm{p}=0.87$ ). Relative to those with complete LIFE data, patients without (complete) LIFE data were significantly more likely to have an intermediate (vocational) level of education $\left(\chi^{2}(2,134)=8.62, p=0.01\right)$. Furthermore, they reported higher posttreatment $\mathrm{BDI}-\mathrm{Il}$ scores $(\mathrm{M}=18.7(\mathrm{SD}=14.7)$ vs. $\mathrm{M}=13.3 \quad(\mathrm{SD}=10.6)$; $\mathrm{t}(132)=-2.33$,

\footnotetext{
${ }^{7}$ Specific information about missing BDI-II data on each assessment point (8, 9, 10, 11, 12 and 24 months), stratified per condition, can be found in Table 6.2.
} 
$\mathrm{p}=0.02$ ). In addition, patients with incomplete LIFE data had somewhat higher BDI-II scores at all time points as compared to patients with complete LIFE data, indicating a more severe pattern of depression in general. BDI-II reliability coefficients ranged from $\alpha=0.96$ (at 7 and 8 months) to $\alpha=0.97$ (12 months). A total of 54 patients (40.3\%; $\mathrm{CT}=33, \mathrm{IPT}=21$ ) had one or more sessions with their GP or a mental health care professional during the follow-up period ${ }^{8}$. Twenty-nine patients $(21.6 \%$; CT=14, IPT=15) used ADM. As some patients received both psychological and pharmacological support, the total number of patients with some form of additional support was 63 (47.0\%; CT=36, IPT=27; $\left.\chi^{2}(1,128)=2.01, p=0.16\right)$.

\subsection{Course of depressive symptomatology in the total sample $(n=134)$}

Table 6.2 presents the observed mean BDI-II scores $(95 \% \mathrm{Cl})$ and mixed regression based estimated means $(95 \% \mathrm{Cl})$ over the course of follow-up for the total sample $(n=134)$, stratified according to treatment condition. In addition, the results of the mixed-effects model and effect sizes $r$ and $d$ are reported. As can be seen in Table 6.2, symptom scores remained stable across the follow-up for both conditions. Effect sizes were small.

\subsection{Responder analyses $(n=85)$}

At post-treatment (7-months), 85 patients (63.4\%) met criteria for response. No between-condition differences were found $\left(65.2 \%\right.$ in CT vs. $61.5 \%$ in IPT; $\chi^{2}(1$, $134)=0.20, p=0.66$ ). Responders did not differ from non-responders at baseline, but reported significantly lower $\mathrm{BDI}-\mathrm{Il}$ scores at post-treatment $(\mathrm{M}=7.5(\mathrm{SD}=6.0)$ vs. $M=27.6$ (SD=9.0); $t(132)=15.48, p<0.0001)$, and fewer received additional support for MDD throughout the follow-up (26/85 responders vs. $37 / 49$ non-responders; $\chi^{2}(1$, $128)=25.87, p<0.0001)$.

\footnotetext{
${ }^{8}$ This also includes patients whose treatments lasted longer than the expected 7-month period.
} 


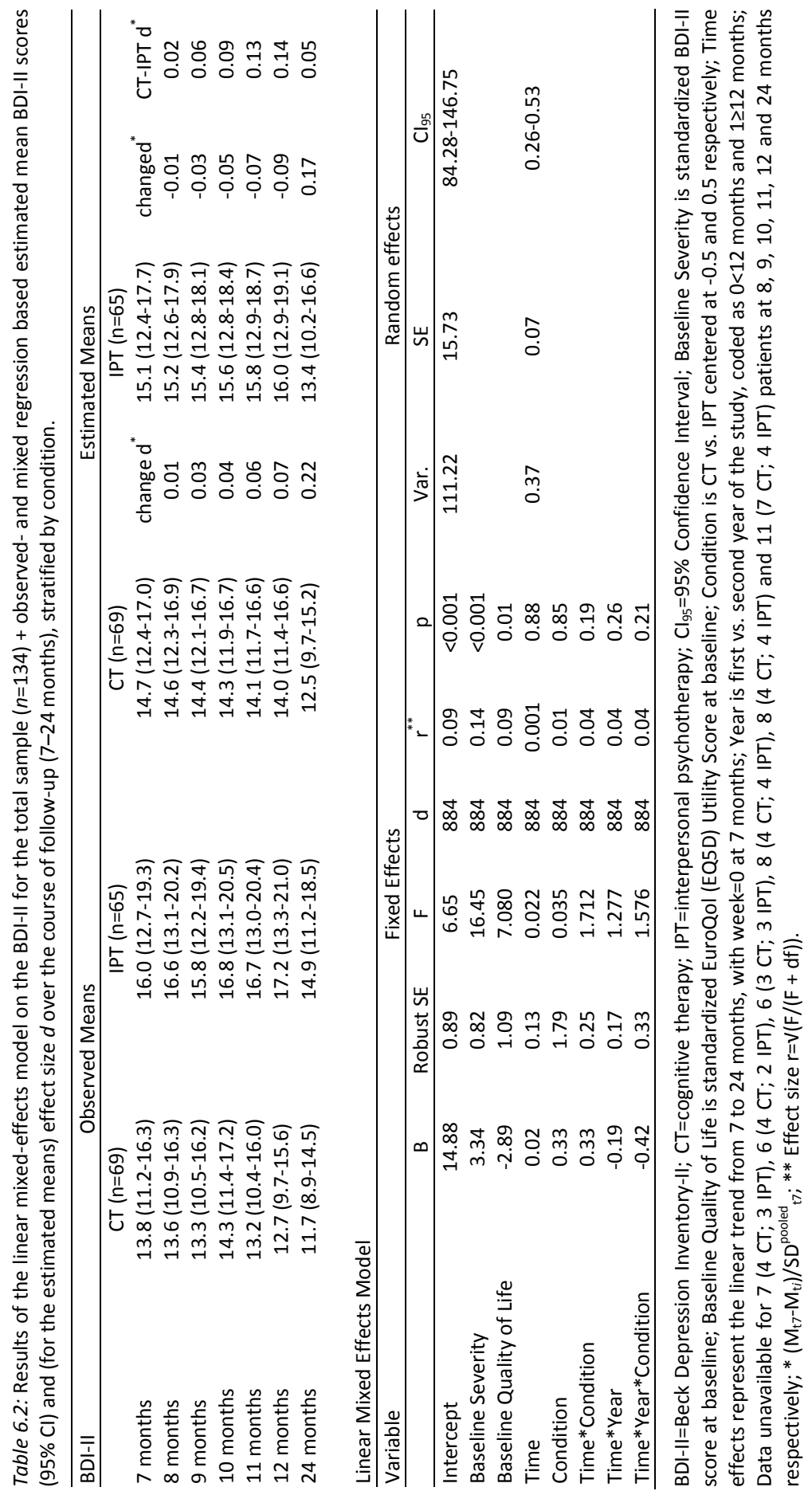




\subsubsection{Outcomes on the BDI-II}

The linear mixed-effects model on the BDI-II for the responder sample (Table 6.3) revealed significant time $x$ condition and time $x$ year $x$ condition interactions. The time $x$ condition interaction points towards more favorable outcomes for CT up to month 12 (BDI-II scores showed a slight decrease in CT, whereas they increased in IPT), and the negative time $x$ year $x$ condition interaction reflects a subsequent drop in scores for IPT in the second year. At 17 months follow-up, these opposite effects resulted in comparable overall outcomes for CT and IPT. Effect sizes of change throughout the follow-up period (7-24 months) were small for both CT and IPT. Twothirds of treatment responders $(57 / 85 ; 67.1 \%)$ completed the 17 -month follow-up phase without meeting criteria for relapse on the BDI-II. Cumulative survival rates per treatment condition are shown in Figure 6.2 (panel a). Relapse rates were 13 (28.9\%) for CT and 15 (37.5\%) for IPT. A log-rank test $\left(\chi^{2}(1,85)=0.99, p=0.32\right)$, and a Cox Regression model ( $\mathrm{HR}=1.47, \mathrm{SE}=0.57, \mathrm{p}=0.32, \mathrm{Cl} 95=0.69-3.14)$ indicated that there were no significant differences in relapse rates between CT and IPT. For those who did relapse $(n=28)$, the mean time to relapse was 61.4 weeks $(S E=5.7)$ after baseline. On average, patients in the IPT condition relapsed somewhat faster than those treated with CT (mean time to relapse of 54.1 ( $\mathrm{SE}=6.9$ ) for IPT vs. 69.8 weeks ( $\mathrm{SE}=9.2$ ) for CT). Patients were slightly more likely to show sustained response ${ }^{9}$ in CT (32 of $45=42.1 \%$ ) than in IPT ( 25 of $40=33.3 \%)$, but differences were not significant $\left(\chi^{2}(1,151)=1.24\right.$, $\mathrm{p}=0.27)$.

\subsubsection{Outcomes on the LIFE}

LIFE data was available for 65 of 85 responders (76.5\%; CT=33, IPT=32); 55 completed the follow-up phase without meeting criteria for relapse. Figure 6.2 (panel b) shows separately for CT and IPT - the cumulative proportion of treatment responders without relapse on the LIFE. Survival rates of CT and IPT were not significantly different (Log-Rank test: $\chi^{2}(1,65)=0.43, p=0.51$; Cox Regression analysis: $H R=1.57$, $\mathrm{SE}=1.03, \mathrm{p}=0.48, \mathrm{Cl} 95=0.44-5.66)$. For the patients that did show relapse on the LIFE ( $n=10 ; 4$ in CT and 6 in IPT), mean time to relapse was 75.4 weeks ( $S E=4.3$ ) after baseline (67.5 ( $\mathrm{SE}=5.4)$ and 80.7 ( $\mathrm{SE}=5.5)$ weeks in CT and IPT respectively). For all models, the proportional hazard assumption was not violated.

${ }^{9}$ Calculated as: (n responders without relapse / all responders) * (all responders / all participants); For CT $(32 / 45) *(45 / 76)=42.1 \%$; For IPT $(25 / 40) *(40 / 75)=33.3 \%$. 


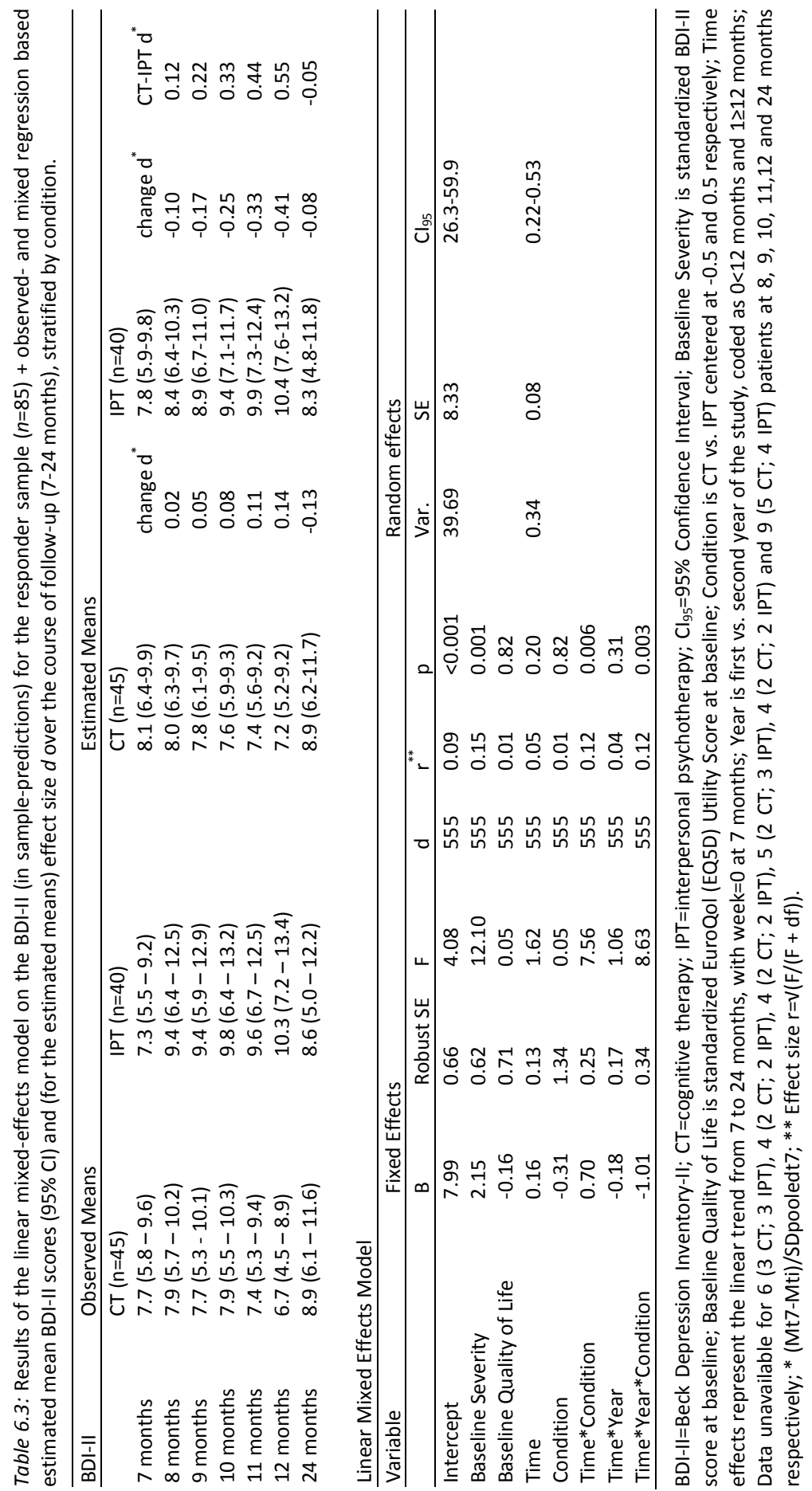


Relapse on the BDI-II

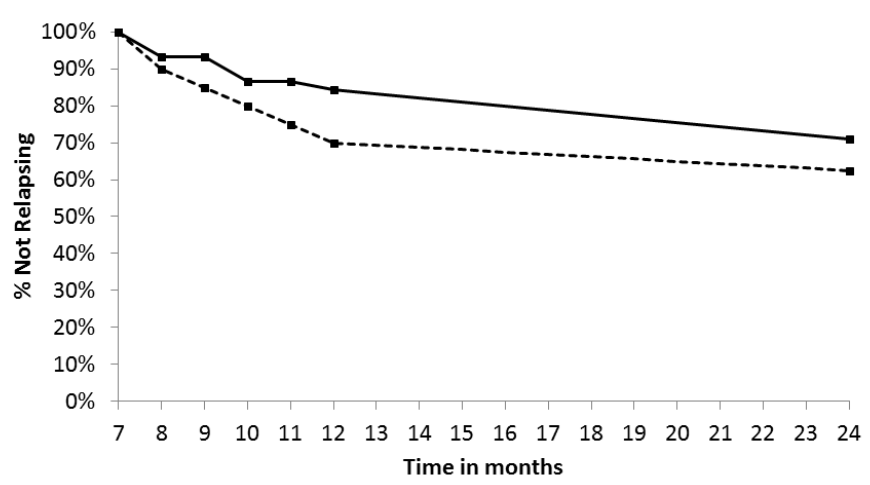

Relapse on the LIFE

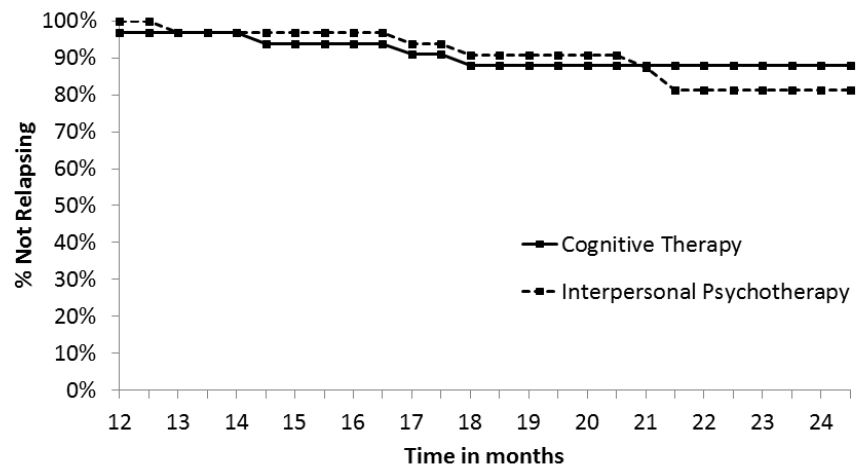

Figure 6.2: Cumulative proportion of treatment responders surviving without relapse over the course of follow-up. Separate panels for relapse on the Beck Depression Inventory-II (BDI-II; Panel a; $\mathrm{n}=85$ ) and the Longitudinal Interval Follow-Up Evaluation (LIFE; panel $b ; n=65$ ). Results stratified per condition. Time in months starts the month after baseline (baseline=month 0 ).

\subsection{Sensitivity analyses}

Sensitivity analyses indicated that total number of sessions, therapist, work- and marital status did not influence the findings. None of these covariates were significant in any of the models. Gender was a significant covariate in the survival analysis on the LIFE, but did not change the conclusions. Additional psychological and/or pharmacological support for MDD also did not change the conclusions. However, these variables were significant in some of the models as well: in both multilevel models and in the survival analysis on LIFE. Results indicated that patients who 
received psychological support for MDD and/or used ADM during the follow-up, reported higher BDI-II scores at 24 months, and were more likely to meet criteria for relapse on the LIFE than those without additional support. In the majority of cases additional support was requested after relapse occurred ( $n=6$ vs. $n=2$ for support before relapse).

\section{Discussion}

The current study evaluated the long-term outcomes of acute phase CT Vs. IPT for MDD. In the context of a large RCT, we determined the course of self-reported depressive symptom severity up to 17 months after treatment termination, and tested whether CT and IPT differed throughout the follow-up phase. Furthermore, for treatment responders, rates of relapse and sustained response were examined for self-reported (BDI-II) and clinician rated (LIFE) depression. On average, the symptom reduction achieved during the 7-month treatment phase was maintained across the follow-up period (7-24 months) for both CT and IPT. Effect sizes of change throughout the follow-up period were small. No differential effects between conditions were found. Two thirds of the treatment responders completed the follow-up phase without meeting criteria for relapse on the BDI-II. Relapse rates assessed with the LIFE were somewhat lower. Patients who responded to IPT were no more likely to experience a return of symptoms than patients who responded to CT. The betweencondition differences that were observed favored prior CT slightly but were not significant. This is important because CT has been shown to have an enduring effect that lasts beyond the end of treatment (relative to prior ADM) whereas IPT has not. Our findings are far from conclusive, but the findings from this trial suggest that IPT just might have a prophylactic effect.

One is always careful to make too much of what are essentially null findings, but there are several reasons why we think we might be justified in doing so in this instance. First, relapse rates were low in both conditions (around 33\%), and are within range of those reported in previous CT studies (see e.g. Vittengl et al. 2007 for an overview) and similar to those obtained in the previous IPT study (Shea et al. 1992). Furthermore, evidence for CT's enduring effect is relatively robust (Cuijpers et al. 2013b). While it is possible that we implemented CT in a less than adequate fashion, independent raters could tell CT from IPT in our trial and rated the quality of implementation as good (see Lemmens et al. 2015). In addition, while we relied on cross-sectional monthly assessments on the BDI over the first five months of follow-up and year-long retrospective assessments on the LIFE for the second year, it is unlikely that we missed many relapses or recurrences since the temporal intervals on the BDI were short and we detected more recurrences at the end of the interval covered by 
the LIFE than the start. The fact that CT did not outperform IPT suggests that IPT might have enduring effects as well. In the absence of a control condition that does not have an enduring effect - such as ADM - we cannot conclude that both IPT and CT were prophylactic, but it remains a real possibility.

Our study has several strengths. First of all, it was the first to examine the longterm effects of IPT since the initial study by Shea et al. (1992). Because we included a larger sample, provided high quality IPT, and used more sophisticated statistical analyses techniques, our study goes beyond the initial study. Second, our RCT design provided a unique opportunity to directly compare the long-term outcomes of IPT with those of CT. Third, because we included both the BDI-II and the LIFE, our study provided information in terms of self-reported symptom severity change and in terms of clinician-rated DSM classifications. Moreover, by examining both relapse and sustained response rates, our study does not only provide information about the prognosis after successful initial treatment (i.e., what can a treatment responder expect after treatment termination?), but also about the prognosis at the start of treatment (i.e., what are the chances for successful and stable treatment effects when patients enter the clinic?). This is valuable information for clinical practice. Other strengths include the repeated measures design, and the inclusion of a series of sensitivity analyses. In addition, by using carefully considered and rather stringent definitions for response, relapse, and treatment status, we feel confident that we have not overestimated our effects.

There were limitations as well. Inherent to conducting a study with a long followup period, we were confronted with missing data. However, dropout rates in our study were relatively low. Moreover, we accounted for missing data in our analyses by using mixed (multilevel) regression models, which are suitable to handle missing data (Schafer and Graham 2002, Singer and Willett 2003, Snijders and Bosker 2012). Another factor complicating the interpretation of results was the naturalistic setting of the follow-up phase. Even though we addressed this by controlling for additional professional support for MDD, it is impossible to exactly control for all influencing parameters. However, this approach resembles clinical practice, and hereby increases the generalisability of study findings. In addition, although the LIFE has shown to be a valid instrument to retrospectively assess depression severity up to one year, recall biases may have occurred. To conclude, our sample may have been too small to detect (smaller) differential effects between CT and IPT.

In sum, our findings suggest that IPT may have an enduring effect similar to that already established for CT. However, in order to make strong claims about the enduring effect for IPT, more powerful tests are needed. Comparisons to prior ADM are one possible option, as that is how the enduring effect for CT has been established. Furthermore, attention should be paid to predictors and moderators of relapse, as we plan to do in a follow-up publication. More insight in the (relative) longterm effects of CT and IPT, and associated factors, can provide valuable information 
about treatment options and prognosis for depressed patients, and may assist in the process of treatment selection (DeRubeis et al. 2014, Huibers et al. 2015), hereby improving everyday health care for depression.

Supplementary data associated with this article can be found in the online version at https://doi.org/10.1017/S0033291718001083. 


\section{References}

Barth J, Munder T, Gerger H, Nuësch E, Trelle S, Znoj H, Jüni P \& Cuijpers P (2013). Comparative Efficacy of Seven Psychotherapeutic Interventions for Patients with Depression: A Network Meta-Analysis. PLoS Medicine 10, e1001454.

Beck AT, Rush AJ, Shaw BF \& Emery G (1979). Cognitive therapy of depression. Guilford Press: New York. Beck AT, Steer R \& Brown GK (1996). Beck Depression Inventory II: Manual. Hartcourt Brace: Boston. Burcusa SL \& lacono WG (2007). Risk for Recurrence in Depression. Clinical Psychology Review 27, 959-985. Cohen J (1988). Statistical power analysis for the behavioral sciences. Erlbaum: Hillsdale.

Cox DR \& Oakes D (1984). Analysis of survival data. Chapman \& Hall: London.

Cuijpers P, Andersson G, Donker T \& Van Straten A (2011). Psychological treatment of depression: Results of a series of meta-analyses. Nordic Journal of Psychiatry 65, 354-364.

Cuijpers P, Berking M, Andersson G, Quigley L, Kleiboer A \& Dobson KS (2013a). A meta-analysis of cognitive behavioural therapy for adult depression, alone and in comparison with other treatments. Canadian Journal of Psychiatry 58, 376-385.

Cuijpers P, Donker T, Weissman MM, Ravitz P \& Cristea IA (2016). Interpersonal Psychotherapy for Mental Health Problems: A Comprehensive Meta-Analysis. American Journal of Psychiatry 173, 680-687.

Cuijpers P, Hollon SD, Van Straten A, Bockting C, Berking M \& Andersson G (2013b). Does cognitive behaviour therapy have an enduring effect that is superior to keeping patients on continuation pharmacotherapy? A meta-analysis. BMJ Open 3, e002542.

Cuijpers P, Sijbrandij M, Koole SL, Andersson G, Beekman AT \& Reynolds CF (2013c). The efficacy of psychotherapy and pharmacotherapy in treating depressive and anxiety disorders: a meta-analysis of direct comparisons. . World Psychiatry 12, 137-148.

De Graaf LE, Gerhards S, Evers S, Arntz A, Riper H, Severens J, Widdershoven G, Metsemakers J \& Huibers M (2008). Clinical and cost-effectiveness of computerised cognitive behavioural therapy for depression in primary care: Design of a randomised trial. BMC Public Health 8, 224.

Derubeis RJ, Cohen ZD, Forand NR, Fournier JC, Gelfand LA \& Lorenzo-Luaces L (2014). The Personalized Advantage Index: Translating Research on Prediction into Individualized Treatment Recommendations. A Demonstration. PLOS ONE 9, e83875.

Diggle P, Heagerty P, Liang K \& Zeger S (2002). Analysis of Longitudinal Data. Oxford University Press: Oxford.

Dobson KS, Shaw BF \& Vallis TM (1985). Reliability of a measure of the quality of cognitive therapy. British Journal of Clinical Psychology 24, 295-300.

Eaton WW, Shao H, Nestadt G, Lee HB, Bienvenu OJ \& Zandi P (2008). Population-based study of first onset and chronicity in major depressive disorder. Archives of General Psychiatry 65, 513-520.

Euroqol-Group (1990). EuroQol - a new facility for the measurement of health-related quality of life. Health Policy 16, 199 - 208.

First MB, Spitzer RL, Gibbon M \& Williams JBW (1997). Structured Clinical Interview for DSM-IV Axis I Disorders (SCID-I). Biometrics Research Department New York State Psychiatric Institute: New York.

Hollon SD, Derubeis RJ, Shelton RC, Amsterdam JD, Salomon RM, O'reardon JP, Lovett ML, Young PR, Haman KL, Freeman BB \& Gallop R (2005). Prevention of Relapse Following Cognitive Therapy vs Medications in Moderate to Severe Depression. Archives of General Psychiatry 62, 417-422.

Hollon SD, Evans MD, Auerbach A, Derubeis RJ, Elkin I, Lowery A, Kriss M, Grove W, Tuason VB \& Piasecki J 1988. Development of a system for rating therapies for depression: Differentiating cognitive therapy, interpersonal psychotherapy, and clinical management pharmacotherapy.

Hollon SD, Waskow IE, Evans M \& Lowery HA 1984. Systems for rating therapies for depression. Annual Convention of the American Psychiatric Association. Los Angeles, CA.

Huibers MJH, Cohen ZD, Lemmens LHJM, Arntz A, Peeters FPML, Cuijpers P \& Derubeis RJ (2015). Predicting Optimal Outcomes in Cognitive Therapy or Interpersonal Psychotherapy for Depressed Individuals Using the Personalized Advantage Index Approach. PLOS ONE 10, e0140771.

Jacobson NS \& Truax P (1991). Clinical significance: A statistical approach to define meaningful change in psychotherapy research. Journal of Consulting and Clinical Psychology 59, 12-19. 
Jakobsen JC, Hansen JL, Simonsen S, Simonsen E \& Gluud C (2012). Effects of cognitive therapy versus interpersonal psychotherapy in patients with major depressive disorder: a systematic review of randomized clinical trials with meta-analyses and trial sequential analyses. Psychological Medicine 42, 1343-1357.

Keller MB, Lavori PW, Friedman B, Nielsen E, Endicott J, Mcdonald-Scott P \& Andreasen NC (1987). The Longitudinal Interval Follow-up Evaluation: A Comprehensive Method for Assessing Outcome in Prospective Longitudinal Studies. Archives of General Psychiatry 44, 540-548.

Klerman GL, Weissman MM, Rounsaville BJ \& Chevron ES (1984). Interpersonal psychotherapy for depression. Basis Books: New York.

Lemmens LHJM, Arntz A, Peeters FPML, Hollon SD, Roefs A \& Huibers MJH (2011). Effectiveness, relapse prevention and mechanisms of change of cognitive therapy vs. interpersonal therapy for depression: study protocol for a randomised controlled trial. Trials 12, 150-162.

Lemmens LHJM, Arntz A, Peeters FPML, Hollon SD, Roefs A \& Huibers MJH (2015). Clinical Effectiveness of Cognitive Therapy vs. Interpersonal Psychotherapy for Depression: Results of a Randomized Controlled Trial. Psychological Medicine 45, 2095-2110.

Lemmens LHJM, Galindo-Garre F, Arntz A, Peeters F, Hollon SD, Derubeis RJ \& Huibers MJH (2017). Exploring Mechanisms of Change in Cognitive Therapy and Interpersonal Psychotherapy for adult Depression. Behaviour Research and Therapy 94, 81-92.

Schafer JL \& Graham JW (2002). Missing data: our view of the state of the art. Psychological methods 7, 147-177.

Shea MT, Elkin I, Imber SD, Sotsky SM, Watkins JT, Collins JF, Pilkonis PA, Beckham E, Glass DR \& Dolan RT (1992). Course of depressive symptoms over follow-up: Findings from the National Institute of Mental Health Treatment of Depression Collaborative Research Program. Archives of General Psychiatry 49, 782-787.

Singer JD \& Willett JB (2003). Applied Longitudinal Data Analysis: Modeling Change and Event Occurrence. Oxford University Press: New York.

Snijders TaB \& Bosker RJ (2012). Multilevel Analysis: An introduction to basic and advanced multilevel modeling (2nd edition). Sage: London.

Solomon DA, Keller MB, Leon AC \& Al. E (2000). Multiple Recurrences of Major Depressive Disorder. American Journal of Psychiatry 157, 229-233.

Stuart S 2011. IPT Adherence and Quality Scale. Iowa: Interpersonal Psychotherapy Institute.

Van Der Does W (2002). De Nederlandse versie van de Beck Depression Inventory - second edition (BDI-IINL). Ipskamp: Enschede.

VittengI JR, Clark LA, Dunn TW \& Jarrett RB (2007). Reducing relapse and recurrence in unipolar depression: a comparative meta-analysis of cognitive-behavioral therapy's effects. Journal of Consulting and Clinical Psychology 75, 457 - 488.

Warshaw MG, Dyck I, Allsworth J, Stout RL \& Keller MB (2001). Maintaining reliability in a long-term psychiatric study: an ongoing inter-rater reliability monitoring program using the longitudinal interval follow-up evaluation. Journal of Psychiatric Research 35, 297-305.

Warshaw MG, Keller MB \& Stout RL (1994). Reliability and Validity of the Longitudinal interval follow-up evaluation for assessing outcome of anxiety disorders. Journal of Psychiatric Research 28, 531-545. 

S. van Bronswijk, L. Lemmens, J. Keefe, M. Huibers, R. DeRubeis, F. Peeters - Depression and Anxiety 2019;36(3):252-261

\section{Chapter}

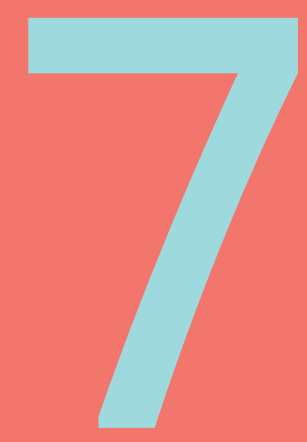


A PROGNOSTIC

INDEX FOR

LONG-TERM

OUTCOME

AFTER

SUCCESSFUL

ACUTE PHASE

COGNITIVE

THERAPY AND

INTERPERSQNA

PSYCHOTHERAPY

FOR MAJOR

DEPRESSIVE

DISORDER 


\section{Abstract}

Background

Major depressive disorder (MDD) has a highly recurrent nature. After successful treatment, it is important to identify individuals who are at risk of an unfavorable long-term course. Despite extensive research, there is no consensus yet on the clinically relevant predictors of long-term outcome in MDD, and no prediction models are implemented in clinical practice. The aim of this study was to create a prognostic index (PI) to estimate long-term depression severity after successful and high quality acute treatment for MDD.

Methods

Data come from responders to cognitive therapy (CT) and interpersonal psychotherapy (IPT) in a randomized clinical trial $(n=85 ; C T=45, I P T=40)$. Primary outcome was depression severity, assessed with the Beck Depression Inventory II, measured throughout a 17-month follow-up phase. We examined 29 variables as potential predictors, using a model-based recursive partitioning method and bootstrap resampling in conjunction with backwards elimination. The selected predictors were combined into a PI. Individual PI scores were estimated using a crossvalidation approach.

Results

A total of three post-treatment predictors were identified: depression severity, hopelessness and self-esteem. Cross-validated PI scores evidenced a strong correlation $(r=0.60)$ with followup depression severity.

\section{Conclusions}

Long-term predictions of MDD are multifactorial, involving a combination of variables that each has a small prognostic effect. If replicated and validated, the PI can be implemented to predict follow-up depression severity for each individual after acute treatment response, and to personalize long-term treatment strategies. 


\section{Introduction}

One of the major challenges in the treatment of major depressive disorder (MDD) is its highly recurrent nature, with estimated recurrence rates of up to $90 \%$ (American Psychiatric Association, 2000; Solomon et al., 2000). Two of the most well-studied and frequently applied psychotherapies for MDD are cognitive cherapy (CT) and interpersonal psychotherapy (IPT). Although the majority of the individuals who respond to acute phase CT and IPT maintain their depressive symptom reduction, approximately one third relapse within a 17-month follow-up period (Lemmens et al., 2018). For accurate clinical decision making, it is important to distinguish individuals who are at risk of an unfavorable long-term course from those who will likely maintain their improvement after terminating successful treatment. Additional monitoring and maintenance treatment should be considered for at risk individuals to prevent relapse and recurrence. Maintenance treatment options such as long-term pharmacotherapy and psychological interventions have been proven effective following acute phase treatment (Biesheuvel-Leliefeld et al., 2015; Hansen et al., 2008). However, identifying at risk individuals remains challenging, and current clinical decision-making results in both under- and overtreatment of recovered depressed individuals (Lorenzo-Luaces, 2015; Monroe \& Harkness, 2011).

In the field of medicine, it is increasingly common to improve and support clinical decision making using prediction algorithms. With these algorithms, several relevant variables, also referred to as predictors, are combined, resulting in a quantified estimate of the individual's prognosis. These estimates, also referred to as scores on a prognostic index (PI), can be used to determine the level of care that is needed in the future (Lorenzo Lorenzo-Luaces, DeRubeis, van Straten, \& Tiemens, 2017). Wellknown examples of this approach are the Framingham risk prediction algorithms for cardiovascular disease (Anderson, Wilson, Odell, \& Kannel, 1991) and prediction algorithms for cancer risk (Chen et al., 2006; Park et al., 2009).

In clinical psychology and psychiatry, research on multivariate algorithms to predict acute phase treatment outcomes in MDD is rapidly growing. Amongst others, recent studies have focused on predicting the optimal treatment for a given individual using a so-called "Personalized Advantage Index Approach" (Cohen \& DeRubeis, 2018; DeRubeis et al., 2014; Huibers et al., 2015). In addition, personalized predictions to determine the optimal level of treatment intensity have been studied (Delgadillo, Moreea, \& Lutz, 2016; Lorenzo Lorenzo-Luaces et al., 2017). So far, only two studies have investigated multivariate prediction algorithms for the long-term course of MDD (van Loo, Aggen, Gardner, \& Kendler, 2015; Wang et al., 2014). These studies - both of an epidemiological nature - have developed powerful prediction models, providing valuable insights into the multifactorial nature of depression recurrence. Unfortunately, because of their naturalistic, population-based design, these studies cannot disentangle treatment effects from natural course. 
The development of algorithms for the prediction of long-term course of MDD is impeded by several factors. First of all, evidence is inconclusive for several predictors, including stressful life events, a family history of MDD, severity of the depressive episode, and comorbidity (e.g., dysthymia and personality pathology, Burcusa \& lacono, 2007; Hardeveld, Spijker, De Graaf, Hendriks, et al., 2013; Hardeveld, Spijker, De Graaf, Nolen, \& Beekman, 2013). Only a few predictors have been repeatedly found to relate to an unfavorable long-term course of MDD, namely the number of previous episodes, residual symptoms, and childhood maltreatment (Berwian, Walter, Seifritz, \& Huys, 2017; Hardeveld, Spijker, De Graaf, Nolen, \& Beekman, 2010; Kovacs, Obrosky, \& George, 2016; Nanni, Uher, \& Danese, 2012). Second, several studies have shown that the risk of recurrence of MDD depends on the combination of multiple predictors each having a small prognostic effect (van Loo et al., 2015; Wang et al., 2014). As a consequence, slightly weaker predictors are dominated by stronger predictors. Variable selection methods that can deal with a large number of "smalleffect" variables are therefore warranted. Third, there is little knowledge how to aggregate this predictive information, especially when the directions of multiple variables conflict. For example, one individual could have several factors placing them at risk for recurrence, and other factors predicting their resilience against recurrence. This information must be combined in some way if it is to be useful clinically.

The aim of the current study was to create a multivariate prediction algorithm to estimate long-term depression severity after successful and high quality acute phase psychotherapy for MDD. With this prediction algorithm, individual scores on a prognostic index can be calculated (PI scores). Within the context of a 17 month follow-up of a recent randomized controlled trial (RCT) comparing CT and IPT (Lemmens et al., 2015; Lemmens et al., 2018), we explored various clinical and demographic predictors and generated a prediction algorithm. We expected to identify predictors that have been found repeatedly in previous research (i.e. number of previous episodes, residual depression, and childhood maltreatment). In addition, we expected that the aggregation of variables would result in a valid prediction algorithm that provides reliable prognostic information for each individual. If replicated, such an algorithm can be used to identify individuals with unfavorable long-term depression severity, which enables selection of appropriate candidates for maintenance therapy.

\section{Methods}

\subsection{Design and participants}

Data come from an RCT into the effectiveness of individual CT and IPT for MDD. A detailed description of the study design, participants, interventions and acute and 
long-term main outcomes is provided elsewhere (Lemmens et al., 2015; Lemmens et al., 2011; Lemmens et al., 2018). In brief, depressed outpatients were recruited from the mood disorders unit of the Academic Maastricht Outpatient Mental Health Centre (RIAGG Maastricht, the Netherlands). All participants had a primary diagnosis of MDD as confirmed with the Structured Clinical Interview for DSM-IV Axis I disorders (SCID-I; First, Spitzer, Gibbon, \& Williams, 1995). Other inclusions criteria were: internet access, an email address, and sufficient knowledge of the Dutch language. Exclusion criteria were bipolar disorder, highly chronic depression (current episode $>5$ years), an increased acute suicide risk, concomitant pharmacological or psychological treatment, drugs and alcohol abuse/dependence, and an IQ lower than 80. After obtaining written informed consent, a total of 182 participants were randomly allocated to either CT $(n=76)$, IPT ( $n=75)$, or a 2-month waiting-list control condition followed by treatment of choice $(n=31)$. CT and IPT were carried out following the guidelines by Beck et al. (1979) for CT, and the guidelines by Klerman et al. (1984) for IPT. Participants received 16 to 20 sessions ( $M=17$ sessions, $S D=2.9$ ), which were planned weekly and were allowed to be scheduled less frequent towards the end of treatment. The quality of therapy was rated as "(very) good" to "excellent" by independent assessors in both CT and IPT. The study was approved by the Medical Ethics Committee of Maastricht University Medical Center, and was registered at isrctn.com (identifier: ISRCTN 67561918). Based on our aims, we limited the sample for the current study to 17-month follow-up data from treatment responders to CT and IPT $(n=85 ; C T=45, I P T=40)$. Treatment responders were identified as individuals with either 1) a post-treatment Beck Depression Inventory, second edition (BDI-II) score lower than 10; or 2) an overall change of at least 9 BDI-II points and a post-treatment BDI-II score lower than 20 (Lemmens et al., 2018). Throughout the follow-up period, individuals were free to pursue additional treatment for MDD. Additional treatment included psychological support (one or more sessions with a general practitioner or a mental health care professional) or the use of antidepressant medication (ADM).

\subsection{Measures}

\subsubsection{Primary outcome}

Primary outcome was depression severity assessed with the Beck Depression Inventory, second edition (Beck, Steer, \& Brown, 1996; Lemmens et al., 2018). The BDI-II was measured at post-treatment (month 7), monthly for a 5-month period (at month $8,9,10,11,12$ ) and at the end of the follow-up phase (month 24). BDI-II scores during this 17 month period were aggregated into an Area under the Curve (AUC) to obtain an overall measure for depression severity across the follow-up phase. 


\subsubsection{Variables}

Based on previous work (Fournier et al., 2009; Huibers et al., 2015) we examined 69 variables from six domains as potential predictors: 1) depression variables, 2) demographics, 3) psychological distress, 4) general functioning 5) psychological processes and 6) life and family history. We removed redundant variables (i.e., variables that overlapped in content, e.g., subscales of one measurement instrument), by examining the correlations between all variables corrected for attenuation. As shown in Table 7.1, 29 variables were eventually included in our analyses. The variables were extracted from the following instruments: BDI-II, Beck Hopelessness Scale (BHS; Beck \& Steer, 1988), Brief Symptom Inventory (BSI; Derogatis \& Melisaratos, 1983), SCID-I; Structured Clinical Interview for DSM-IV Axis II (SCID-II; First, Gibbon, Spitzer, Williams, \& Benjamin, 1997), Work and Social Adjustment Scale (WSAS; Mundt, Marks, Shear, \& Greist, 2002), RAND-36 (van der Zee \& Sanderman, 1993), Dysfunctional Attitudes Scale (DAS; de Graaf, Roelofs, \& Huibers, 2009; Weissman \& Beck, 1978), Inventory of Interpersonal Problems (IIP; Horowitz, Rosenberg, Baer, Ureno, \& Villasenor, 1988), Self Liking and Self Competence Scale Revised (SLSC-R; Tafarodi \& Swann, 2001; Vandromme, Hermans, Spruyt, \& Eelen, 2007), Leiden Index of Depression Sensitivity (LEIDS; Van der Does, 2002), Ruminative Response Scale (RRS; Raes, Hermans, \& Eelen, 2003), and Attributional Style Questionnaire (ASQ; Cohen, Van den Bout, Kramer, \& Van Vliet, 1986; Peterson et al., 1982).

\subsection{Data analyses}

\subsubsection{Variable description and imputation of missing data}

First, we investigated the means and frequencies (where appropriate) of the 29 variables. Second, we examined the number of missing variables and BDI-II outcomes. Third, we imputed missing data with a random forest algorithm ( $R$ package "MissForest"; Stekhoven \& Bühlmann, 2012). This imputation method is considered to be highly accurate, outperforming other common imputation techniques (Stekhoven \& Bühlmann, 2012; Waljee et al., 2013). The following information was included in the imputation model: 1) all non-missing variables, 2) change during treatment of all nonmissing variables (if available), 3) change from baseline (pre-treatment) of all nonmissing BDI-II outcomes (at 7, 8, 9, 10, 11, 12 and 24 months) and 4) information about the condition (CT/IPT). Finally, we checked the accuracy of our imputation method, by applying it to artificially produced missing data in the subset of individuals with complete data. To estimate the imputation error, imputed values were compared with actual data values by calculating the normalized root mean squared error (NRMSE) for continuous data and the proportion of falsely classified entries (PFC) for categorical data using the 'prodNA' function of the R Package "MissForest". 


\subsubsection{Outcome transformation}

In order to get an indication of the 'overall' depression severity throughout the complete 17-month follow-up phase, BDI-II scores at 8, 9, 10, 11, 12 and 24 months were aggregated into an AUC using cubic splines to compute integrals. We used the square root of the AUC for further analyses, as the residuals of the AUC outcomes appeared to be non-normally distributed.

\subsubsection{Variable transformation}

Continuous variables were standardized and discrete and categorical variables were centered. For the discrete variables that had a non-normal distribution, log transformations and square root transformations were applied based on visual inspection. For each variable, we describe the applied transformation in Supplemental Methods I.

\subsubsection{Variable selection}

To construct a powerful prediction algorithm, we used two techniques to identify predictors for long-term depression severity from the 29 variables available: a modelbased recursive partitioning method followed by bootstrap resampling in conjunction with backwards elimination (R packages "mobForest" and "bootstepAIC"; Garge et al., 2013; Rizopoulos \& Rizopoulos, 2009; prevous applications of this method: Keefe et al., 2018; Zilcha-Mano et al., 2016). The model-based recursive partitioning technique is based on a random forest algorithm (Garge et al., 2013). In this method, bootstrapped samples are repeatedly split into two subgroups, forming tree-shaped structures. The splits (or nodes) of these trees depend on the variable with the strongest impact on the outcome of a predetermined model. In the current analyses, we applied a regression model with the AUC as the dependent variable. At each node of each tree, a random subset of the potential predictors was applied instead of all variables at once. By doing this, predictors with smaller effects are not eliminated by the presence of stronger predictors (Strobl, Boulesteix, Kneib, Augustin, \& Zeileis, 2008). Parameters were set as follows: 10000 trees were computed with a minimum $\alpha$ level of 0.10 for splits and a minimum node size for splitting of 15 individuals. After computing the trees, variables were ranked using a variable-importance plot to identify the predictors with the most accurate predictions. A variable importance score was calculated for each variable to indicate their importance in producing accurate predictions. Importance scores were computed for each variable by subtracting the predictive accuracy of a variable when applying the real values, from the predictive accuracy of a variable when applying randomly permutated values. This difference in accuracy before and after permutation estimates the true predictive impact of a variable. Variables were then ranked based on their importance score and 
were selected if their importance score was higher than the absolute value of the lowest ranking variable (Garge et al., 2013).

The robustness of these selected variables was then tested by applying backwards elimination on multiple bootstrapped samples (Austin \& Tu, 2004). A regression model with the AUC as the dependent variable and the variables selected at the first step as the independent variables was computed. Then, backwards elimination (with $\alpha=0.05$ ) of this model was applied to 1000 bootstrapped samples of the original data. If predictors were selected in at least $60 \%$ of the bootstrap samples, they were considered robust and subsequently used for the prediction algorithm. The $60 \%$ cut-off has been proven to select variables that construct a parsimonious model with good model fitting (Austin \& Tu, 2004). For each predictor, the direction of the estimated regression coefficients was examined by counting the number of times it showed a positive or negative value in the bootstrapped samples.

\subsubsection{Estimating Prognostic Index scores using a 5-fold cross validation}

We created a prognostic index by combining all final predictors in the following regression model with the AUC as the dependent variable: SQRT $\left(A \cup C_{8}-24\right.$ months $)=\beta 0+$ $(\beta 1$ * Predictor 1$)+(\beta 2 *$ Predictor 2$) \ldots(\beta n *$ Predictor $n)$. A 5 -fold cross validation was applied to calculate individual estimates (PI scores). In short, the sample was split into 5 equal groups and the outcomes of the individuals of each group were predicted using the regression model based on information from the other 4 groups of the sample (the "training dataset"; Picard \& Cook, 1984). This technique reduces the risk of overfitting, since the individual whose $\mathrm{PI}$ score is calculated is not included in the training dataset of the model used to calculate the score. The PI's were then compared with the actual outcomes. To facilitate interpretation of this comparison, we converted the AUC outcomes to an "average BDI-II score" across the follow-up phase by dividing the AUC by time in months. We calculated the average difference between the actual scores and the predicted scores, and examined the association between these scores using a correlation analysis. We also examined the number of individuals that had an average difference of 5 points or higher on the BDI-II between actual and observed scores, which corresponds to a clinically meaningful difference (Hiroe et al., 2005). In addition, we applied a conditional inference procedure (permutation test) to test the hypothesis of independence between the actual scores and the predicted scores (R package "coin"; Hothorn, Hornik, Van De Wiel, \& Zeileis, 2006, 2008)

\subsubsection{Additional support during follow-up}

We examined the impact of additional treatment (psychological support or antidepressant treatment) on the outcome variable (the AUC) and the PI scores. For 
this, the AUC and PI scores of individuals with and without additional support were compared using a t-test.

\subsubsection{Testing robustness of variable selection and model fitting}

Because we performed variable selection and model fitting in the same sample, it is possible that the effect size of how well the PI predicts outcomes will be inflated (Vul, Harris, Winkielman, \& Pashler, 2009), even with the statistical precautions taken above. To examine the degree to which the PI's predictive ability may be inflated, we ran a secondary analysis testing the process of both variable selection and modelfitting to predict to a 5 -fold held-out sample. This process is less clinically relevant as it produces 5 potentially different models, but it provides an estimate of how well the entire process selects variables and makes predictions that robustly predict out-ofsample.

\section{3. $\quad$ Results}

\subsection{Variable description and imputation of missing data}

Table 7.1 describes the means and frequencies (where appropriate) of the 29 variables grouped in five domains (Fournier et al., 2009; Huibers et al., 2015). Of all 29 variables, 8 values were missing $(0.003 \%)$. A total of 79 individuals $(93.0 \%)$ had no missing values for any of the variables. Of all BDI-II outcomes at 8, 9, 10, 11, 12 and 24 months, 32 outcomes were missing $(0.06 \%)$. A total of 74 individuals $(87.1 \%)$ had no missing BDI-II outcomes at any of the time points. The imputation method for the missing variables and outcomes appeared to be accurate, with an estimated normalized root mean squared error (NRMSE) of 0.36 and the estimated proportion of falsely classified entries (PFC) of 0.33 (R package "MissForest"; Stekhoven \& BühImann, 2012). 
Table 7.1: Sample description: depression variables, demographics, psychological distress variables, general functioning variables, psychological processes variables, and life and family history variables.

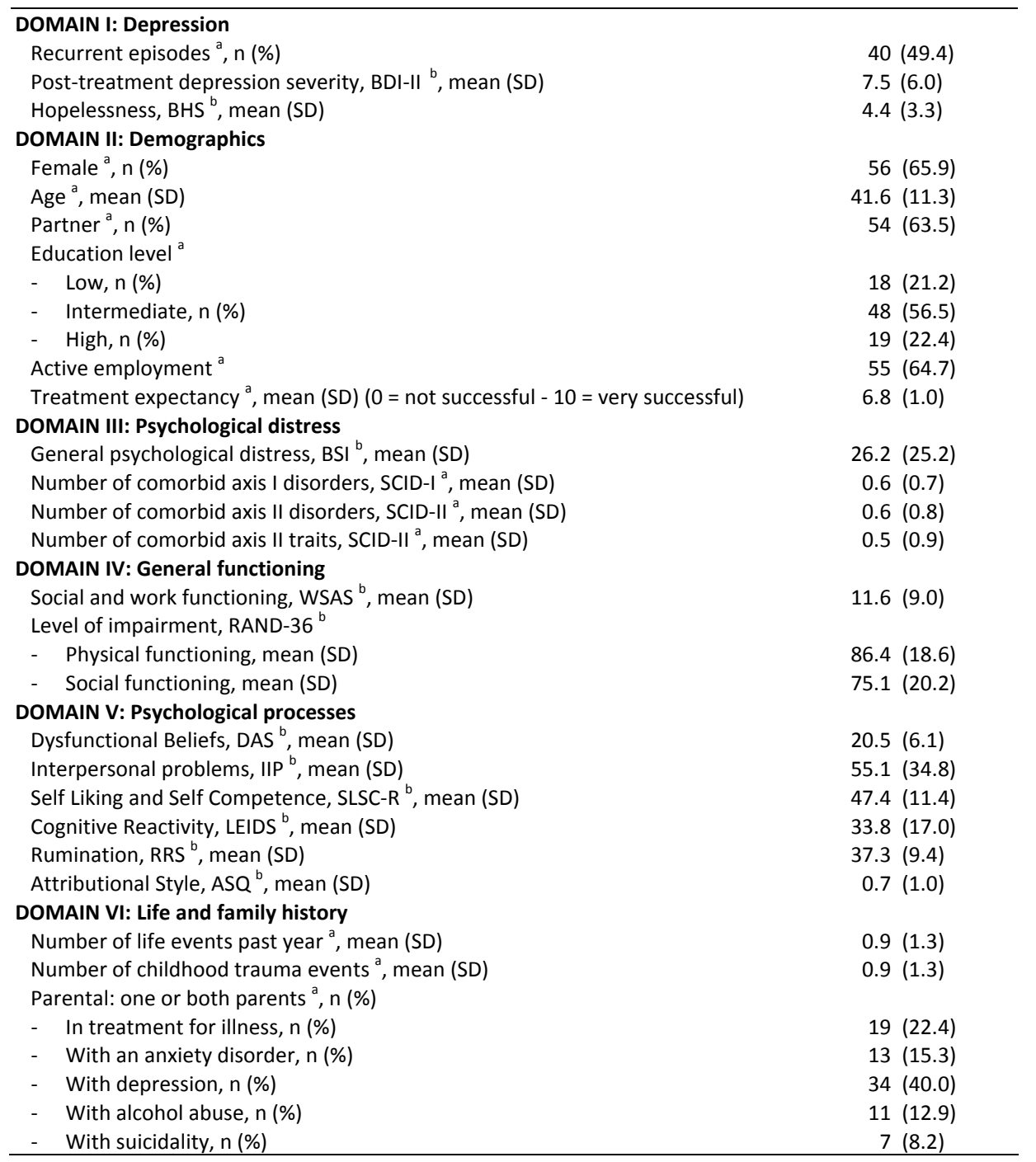

BDI-II, Beck Depression Inventory, second edition; BHS, Beck Hopelessness Scale; BSI, Brief Symptom Inventory; SCID-I, Structured Clinical Interview for DSM-IV Axis I disorders; SCID-II, Structured Clinical Interview for DSM-IV Axis II disorders; WSAS, Work and Social Adjustment Scale; DAS, Dysfunctional Attitudes Scale; IIP, Inventory of Interpersonal Problems; SLSC-R, Self Liking and Self Competence Scale Revised; LEIDS, Leiden Index of Depression Sensitivity; RRS, Ruminative Response Scale; ASQ, Attributional Style Questionnaire. ${ }^{\mathrm{a}}$ Measured pre-treatment; ${ }^{\mathrm{b}}$ Measured post-treatment. 


\subsection{Selection of predictors}

We identified 10 potential predictor variables using the model-based recursive partitioning technique. These variables are displayed in Table 7.2, ranked by their "variable importance." Of the 10 variables, 3 variables were selected in at least $60 \%$ of the bootstrap samples using the backwards elimination technique (Table 7.3). As shown in this table, for each of the 3 variables the regression coefficients across the bootstrap samples appeared to be stable: either all positive or all negative. More specifically, higher post-treatment depression severity (BDI-II) and negative beliefs about the future (Hopelessness, BHS) were associated with higher BDI-II scores across 17 months of follow-up. Higher post-treatment self-esteem (SLSC-R) was associated with lower BDI-II scores during follow-up.

Table 7.2: Predictors selected with the model-based recursive partitioning technique.

\begin{tabular}{ll}
\hline Variable importance rank (high to low) & Variable \\
\hline 1 & Depression severity, BDI-II \\
2 & Hopelessness, BHS \\
3 & Social and work functioning, WSAS \\
4 & Self Liking and Self Competence, SLSC-R \\
5 & Level of impairment: physical functioning, RAND-36 \\
6 & Dysfunctional Beliefs about achievement, DAS \\
7 & General psychological distress, BSI \\
8 & Interpersonal problems, IIP \\
9 & Level of impairment: social functioning, RAND-36 \\
10 & Number of life events past year \\
\hline
\end{tabular}

BDI-II, Beck Depression Inventory, second edition; BHS, Beck Hopelessness Scale; WSAS, Work and Social Adjustment Scale; SLSC-R, Self Liking and Self Competence Scale Revised; DAS, Dysfunctional Attitudes Scale; BSI, Brief Symptom Inventory; IIP, Inventory of Interpersonal Problems.

Table 7.3: Predictors selected with bootstrap resampling in conjunction with backwards elimination.

\begin{tabular}{lcrr}
\hline Variable & $\begin{array}{c}\text { Percentage of bootstrapped samples } \\
\text { this variable was selected in }\end{array}$ & \multicolumn{2}{c}{ Significant $\beta$} \\
\cline { 3 - 4 } & $96.0 \%$ & $100.0 \%$ & $0.0 \%$ \\
Depression severity, BDI-II & $81.5 \%$ & $0.1 \%$ & Negative \\
Self Liking and Self Competence, SLSC-R & $70.1 \%$ & $99.7 \%$ & $0.3 \%$ \\
Hopelessness, BHS & & $99.7 \%$ \\
\hline
\end{tabular}

BDI-II, Beck Depression Inventory, second edition; SLSC-R, Self Liking and Self Competence Scale Revised; BHS, Beck Hopelessness Scale.

\subsection{Estimating Prognostic Index scores using a 5-fold cross validation}

We combined the 3 selected predictors into the following regression model to create the PI: SQRT $\left(A \cup C_{8-24 \text { months }}\right)=\beta 0+\left(\beta 1 * B D I-I I_{\text {post-treatment }}\right)+\left(\beta 2 * B H S_{\text {post-treatment }}\right)+(\beta 3 *$ SLSC- $\left.R_{\text {post-treatment }}\right)$. PI scores were estimated using a 5 -fold cross validation. As shown in Figure 7.1, these predicted values (converted to average follow-up BDI-II scores) were compared with the actual average follow-up BDI-II scores. 
Chapter 7

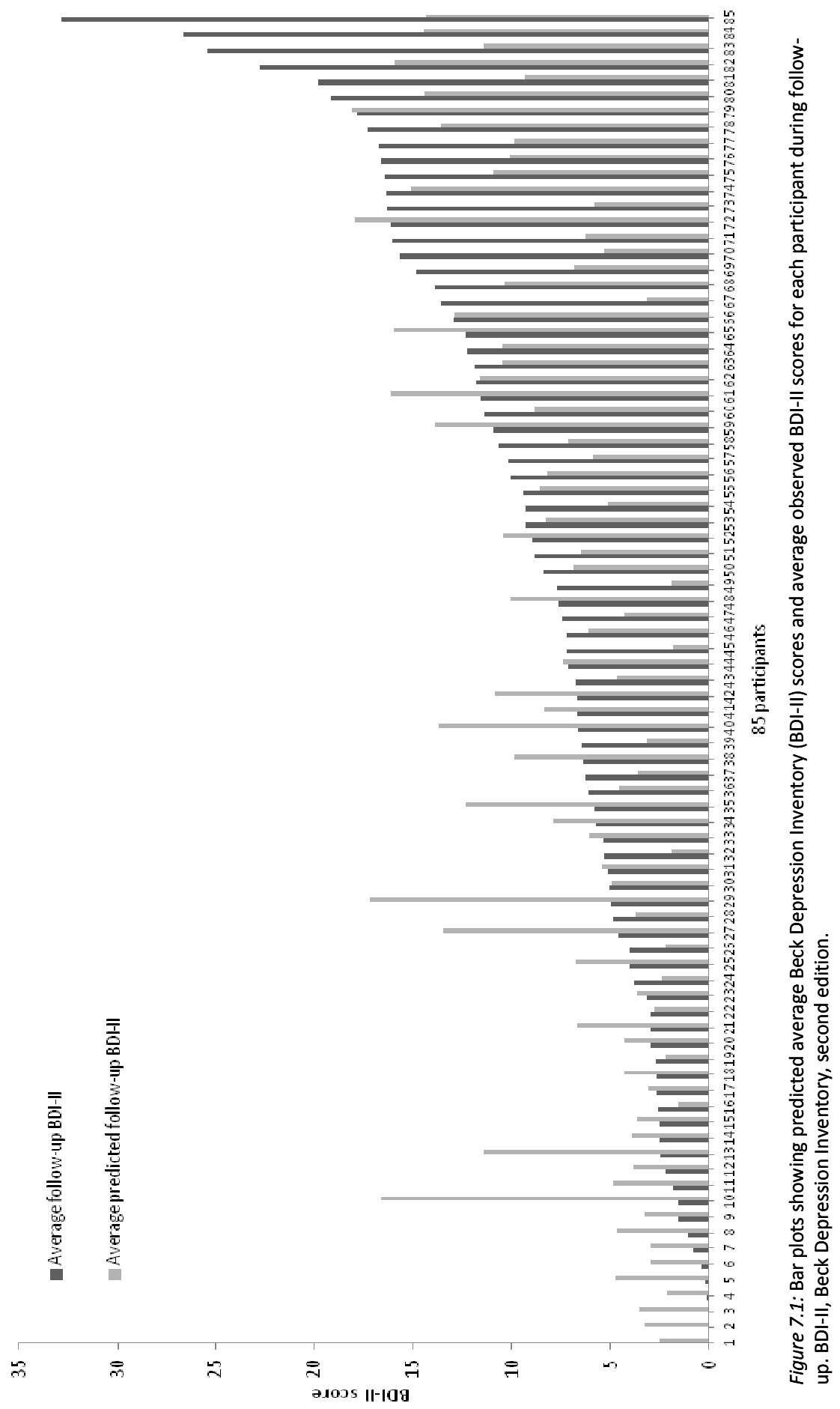


The mean difference between the actual and predicted average BDI-II scores was 0.84 $(S D=5.42)$ and the correlation between these values was significant and strong (corr. $=0.60, p<0.001$ ). For 21 of the 85 individuals, this difference was clinically significant with 5 points or higher on the BDI-II. Results of the permutation test indicated a significant association between the actual and the predicted scores $(Z=5.46, p<0.001)$. Our secondary analysis examining the estimated Pl-outcome correlation that would be obtained without "double-dipping" (i.e., performing variable selection and model fitting in the same sample) did not produce a substantively different correlation than our primary analysis (corr. $=0.62, p<0.001$ ). The five different regression models are described in Supplemental Results I.

\subsection{Additional support}

A total of 26 of the 85 responders received additional care: 23 individuals received psychological support (one or more sessions with a general practitioner or a mental health care professional) and 6 were treated with ADM. No differences between the AUC scores of responders with and without additional support were found ( $M=154.5$ $(S D=21.6)$ vs. $M=128.1(S D=109.1) ; t(79)=-1.01, p=0.31)$. However, responders with additional care had significant higher overall $\mathrm{PI}$ scores as compared to responders without additional support ( $M=151.0$ ( $S D=78.8)$ vs. $M=109.7$ ( $S D=67.9)$; $(79)=-2.4192$, $p=0.0179)$.

\section{Discussion}

The main goal of this study was to compute a prognostic index (PI) for long-term depression severity after successful psychotherapeutic treatment of MDD. In the context of a recent RCT, we explored 17-month follow-up data of 85 treatment responders to CT and IPT. From the 29 variables examined, three post-treatment predictors (depression severity, hopelessness and self-esteem) were selected and combined to create a PI. The mean difference between actual and predicted depression severity scores was 0.84 , and a total of 21 individuals had a clinically significant difference between these scores. Individual PI scores had a strong correlation with the actual follow-up depression severity, and the association between these severity scores was highly significant.

In this study we identified three post-treatment predictors for long-term depression severity: post-treatment depression severity, negative beliefs about the future (hopelessness), and lower self-esteem (self-liking and self-competence), all of which have been associated with an unfavorable course. Post-treatment depression severity, or residual depression, has been found to have a strong prognostic value for an unfavorable course of depression (Berwian et al., 2017; Fava, Ruini, \& Belaise, 
2007; Hardeveld et al., 2010; Nierenberg et al., 2010; ten Doesschate, Bockting, Koeter, \& Schene, 2010). Hopelessness as a predictor of long-term depression severity is in line with the hopelessness theory of depression (Abramson, Metalsky, \& Alloy, 1989; Liu, Kleiman, Nestor, \& Cheek, 2015). This theory hypothesizes that the interaction between negative cognitive styles and negative life events can evoke a sense of hopelessness that in turn contributes to the onset, relapse, and recurrence of depressive episodes. The importance of hopelessness is further supported by the finding that a decrease in hopelessness during various psychotherapy modalities was significantly associated with a decrease in depression severity (Cuijpers et al., 2013). In addition, other studies repeatedly reported hopelessness as a significant predictor of future suicidal behavior and ideation (Beck, Brown, Berchick, Stewart, \& Steer, 2006; Kovacs \& Garrison, 1985), an important symptom of depression. Self-esteem can be interpreted as an ability that helps individuals to maintain their depressive symptom reduction, conferring resilience. Previous studies have shown a diminished self-esteem in individuals with depression (Creemers, Scholte, Engels, Prinstein, \& Wiers, 2012; Franck, De Raedt, Dereu, \& Van den Abbeele, 2007; Phillips, Hine, \& Bhullar, 2012) and an increase of self-esteem during various treatment modalities for depression (Barkham et al., 1996; Goldman, Greenberg, \& Angus, 2006; Strunk, DeRubeis, Chiu, \& Alvarez, 2007). While one study found no predictive effect of posttreatment self-esteem on relapse one year after CT (Strunk et al., 2007), others found that an improvement in self-esteem predicted less depressive symptoms after finishing either psychodynamically-oriented therapy and cognitive-behavioral group therapy (Allart-van Dam, Hosman, Hoogduin, \& Schaap, 2003; Dinger, Ehrenthal, Nikendei, \& Schauenburg, 2017). Within an interpersonal perspective, low self-esteem and high levels of hopelessness are closely associated with loneliness and low levels of perceived social support which are well known risk factors contributing to the development of MDD (Cacioppo, Grippo, London, Goossens, \& Cacioppo, 2015; van Winkel et al., 2017). This cluster of interpersonal factors may potentially serve as targets for further psychotherapeutic or pharmacological treatment in individuals at risk for less favorable clinical course (Cacioppo et al., 2015).

Based on previous research, we expected the number of previous episodes and childhood maltreatment to be identified as predictors as well (Berwian et al., 2017; Hardeveld et al., 2010; Kovacs et al., 2016; Nanni et al., 2012). However, our selection methods did not identify these variables. Several explanations may be held responsible. First, data on previous episodes and childhood trauma are sensitive to recall-bias, which may result in inconsistent findings between studies. Second, information about previous episodes was gathered by differentiating between "first episode" and "recurrent episode," therefore lumping individuals with two or more episodes together. One could hypothesize that this categorization causes loss of potential predictive information. Third, both previous episodes and childhood maltreatment can be conceptualized as developmental risk factors that may have a 
mediating role for other predictors to trigger an unfavorable course of depression (Bockting, Spinhoven, Koeter, Wouters, \& Schene, 2006; Kendler, Thornton, \& Gardner, 2000; Nanni et al., 2012; Segal, Williams, Teasdale, \& Gemar, 1996). Following this hypothesis, these variables affect vulnerability for depression through their interaction with more proximal variables such as stressful life events (Kendler et al., 2000), and coping-related factors (Bockting et al., 2006), and were therefore not identified as independent predictors. The findings concerning self-esteem and hopelessness are in line with this, since self-esteem has been hypothesized to come from early attachment experiences (Morley \& Moran, 2011) and hopelessness has been hypothesized as a result of interactions between negative cognitive styles and negative life events (Abramson et al., 1989).

After the variable selection phase, the three post-treatment predictors were combined to create a $\mathrm{PI}$, utilizing cross-validated predictions. Individual PI scores predicted long-term depression severity, with a strong correlation with the actual depression severity scores. In addition, for a total of 64 individuals, there was no clinically significant difference between the average actual and predicted depression severity scores, and a permutation test indicated that the association between the actual and predicted scores was highly significant. Interestingly, responders with higher PI scores had more additional care throughout follow-up, with no significant association between actual depression severity and additional care. The relationship between PI scores and additional support could indicate that the PI scores are also predictive for future care seeking behavior.

We think our variable selection and subsequent PI approach has several strengths. First, instead of focusing on a single predictor, our approach combined multiple predictors and was therefore able to capture the multifactorial nature of the long-term course of MDD (Hardeveld et al., 2013; van Loo et al., 2015; Wang et al., 2014). Second, advanced variable selection methods were applied that can handle multiple predictors and prevent weaker predictors from being overshadowed by stronger ones. Third, the PI scores were calculated by combining the predictors in a meaningful way, with regression coefficients determined by a cross-validation approach. In addition, these regression coefficients were based on a sample that did not include the individual for whom the PI score was estimated for. Our findings differ from earlier studies that investigated multivariate prediction algorithms for the longterm course of MDD (van Loo et al., 2015; Wang et al., 2014). As mentioned earlier, these naturalistic studies do not disentangle treatment effects from a natural course, while this study focuses on post-treatment predictions for long-term outcome after successful controlled treatment.

To our knowledge, this is the first study creating a prognostic index for follow-up outcome after successful and high quality psychotherapy for MDD. Other strengths of this study are the broad range of potential predictors and the multiple outcome measurements throughout follow-up, avoiding recall bias. Our study also has some 
limitations. First, although we included a broad range of variables covering various domains, there is no guarantee that this is a complete selection of all relevant variables. Second, we did not validate our prediction model in an independent sample, a so-called "testing dataset." To minimize overfitting, we did apply a crossvalidation approach to our primary model. Moreover, we performed a secondary analysis running the entire process of both variable selection and model-fitting with 5-folds, and this produced an estimated PI-outcome correlation very similar to that obtained in our primary model ( 0.60 vs. 0.62 ). Third, throughout follow-up individuals were free to pursue additional treatment. However, the use of additional treatment did not affect the outcome variable and did not seem to have an impact on long-term outcome (Lemmens et al., 2018).

In the long run, PI scores could be used to predict follow-up depression severity for each individual after acute treatment response, to enable clinicians to identify atrisk individuals and personalize long-term treatment strategies. In this context, we propose that the PI would be part of a shared decision-making process, since there is evidence that this would result in higher treatment satisfaction, less dropout and better clinical outcomes (Lindhiem, Bennett, Trentacosta, \& McLear, 2014). Clinicians and patients should collaborate in aggregating and interpreting information on individual treatment goals, residual symptoms and depression severity predictions (PI scores). As a rule of thumb, a clinically significant difference between post-treatment depression severity and predicted depression severity over time (e.g., a 5 point difference on the BDI-II like in this study), may serve as a meaningful predicted negative prognosis (Hiroe et al., 2005). From a shared decision-making process, informed decisions on maintenance treatment options could be made. Previous studies have shown that psychological interventions (e.g., (mindfulness-based) CT and IPT) are suited to prevent relapse and recurrence over two years, especially when the individual received acute phase psychotherapy for MDD (Biesheuvel-Leliefeld et al., 2015). However, the PI is not ready for implementation yet, and future research is needed to develop a robust prediction model. First of all, current prediction models are predominantly composed of risk factors, but accuracy may be enhanced by the inclusion of more resilience factors such as self-esteem (Lorenzo-Luaces, 2015; Waugh \& Koster, 2015). Second, prediction models need to be validated in several distinct and related populations. Third, feasibility needs to be considered, as collecting a high number of complex variables could be problematic in routine practice. If replicated, a strength of the current PI is that the three self-report measures can be rapidly assessed in routine practice, most preferably using web-based computerized questionnaires, which enhances feasibility considerably.

Supplementary data associated with this article can be found online in the Supporting Information section at the end of the article. 


\section{References}

Abramson, L. Y., Metalsky, G. I., \& Alloy, L. B. (1989). Hopelessness depression: A theory-based subtype of depression. Psychological review, 96(2), 358.

Allart-van Dam, E., Hosman, C. M., Hoogduin, C. A., \& Schaap, C. P. (2003). The coping with depression course: Short-term outcomes and mediating effects of a randomized controlled trial in the treatment of subclinical depression. Behavior Therapy, 34(3), 381-396.

Anderson, K. M., Wilson, P. W., Odell, P. M., \& Kannel, W. B. (1991). An updated coronary risk profile. A statement for health professionals. Circulation, 83(1), 356-362. Retrieved from http://www.ncbi.nlm.nih.gov/pubmed/1984895.

Association, A. P. (2000). Diagnostic and Statistical Manual of Mental Disorders (4th ed., text rev. ed.). Washington D.C.

Austin, P. C., \& Tu, J. V. (2004). Bootstrap methods for developing predictive models. The American Statistician, 58(2), 131-137.

Barkham, M., Rees, A., Shapiro, D. A., Stiles, W. B., Agnew, R. M., Halstead, J., . . Harrington, V. M. (1996). Outcomes of time-limited psychotherapy in applied settings: replicating the Second Sheffield Psychotherapy Project. Journal of consulting and clinical psychology, 64(5), 1079.

Beck, A. T., Brown, G., Berchick, R. J., Stewart, B. L., \& Steer, R. A. (2006). Relationship between hopelessness and ultimate suicide: a replication with psychiatric outpatients. Focus.

Beck, A. T., Rush, A. J., Shaw, B. F., \& Emery, G. (1979). Cognitive therapy of depression. New York: Guilford Press.

Beck, A. T., Steer, R., \& Brown, G. K. (1996). Beck Depression Inventory II: Manual. Boston: Harcourt Brace.

Beck, A. T., \& Steer, R. A. (1988). Manual for the Beck Hopelessness Scale. San Antonio: Psychological Corporation.

Berwian, I. M., Walter, H., Seifritz, E., \& Huys, Q. J. (2017). Predicting relapse after antidepressant withdrawal - a systematic review. Psychol Med, 47(3), 426-437. Retrieved from http://www.ncbi.nIm.nih.gov/pubmed/27786144. doi:10.1017/S0033291716002580

Biesheuvel-Leliefeld, K. E., Kok, G. D., Bockting, C. L., Cuijpers, P., Hollon, S. D., van Marwijk, H. W., \& Smit, F. (2015). Effectiveness of psychological interventions in preventing recurrence of depressive disorder: meta-analysis and meta-regression. J Affect Disord, 174, 400-410. Retrieved from http://www.ncbi.nlm.nih.gov/pubmed/25553400. doi:10.1016/j.jad.2014.12.016

Bockting, C. L., Spinhoven, P., Koeter, M. W., Wouters, L. F., \& Schene, A. H. (2006). Prediction of recurrence in recurrent depression and the influence of consecutive episodes on vulnerability for depression: a 2year prospective study. The Journal of clinical psychiatry.

Burcusa, S. L., \& lacono, W. G. (2007). Risk for recurrence in depression. Clin Psychol Rev, 27(8), 959-985. Retrieved from http://www.ncbi.nlm.nih.gov/pubmed/17448579. doi:10.1016/j.cpr.2007.02.005

Cacioppo, S., Grippo, A. J., London, S., Goossens, L., \& Cacioppo, J. T. (2015). Loneliness: Clinical import and interventions. Perspectives on psychological science, 10(2), 238-249.

Chen, J., Pee, D., Ayyagari, R., Graubard, B., Schairer, C., Byrne, C., . . Gail, M. H. (2006). Projecting absolute invasive breast cancer risk in white women with a model that includes mammographic density. J Natl Cancer Inst, 98(17), 1215-1226. Retrieved from http://www.ncbi.nlm.nih.gov/pubmed/16954474. doi:10.1093/jnci/djj332

Cohen, L., Van den Bout, J., Kramer, W., \& Van Vliet, T. (1986). A Dutch attributional style questionnaire: Psychometric properties and findings of some Dutch-American differences. Cognitive therapy and research, 10(6), 665-669.

Cohen, Z. D., \& DeRubeis, R. J. (2018). Treatment selection in depression. Annual review of clinical psychology(0).

Creemers, D. H., Scholte, R. H., Engels, R. C., Prinstein, M. J., \& Wiers, R. W. (2012). Implicit and explicit selfesteem as concurrent predictors of suicidal ideation, depressive symptoms, and loneliness. Journal of behavior therapy and experimental psychiatry, 43(1), 638-646. 
Cuijpers, P., de Beurs, D. P., van Spijker, B. A., Berking, M., Andersson, G., \& Kerkhof, A. J. (2013). The effects of psychotherapy for adult depression on suicidality and hopelessness: a systematic review and metaanalysis. Journal of affective disorders, 144(3), 183-190.

de Graaf, L. E., Roelofs, J., \& Huibers, M. J. (2009). Measuring Dysfunctional Attitudes in the General Population: The Dysfunctional Attitude Scale (form A) Revised. Cognitive therapy and research, 33(4), 345-355. Retrieved from http://www.ncbi.nlm.nih.gov/pubmed/19623267. doi:10.1007/s10608-0099229-y

Delgadillo, J., Moreea, O., \& Lutz, W. (2016). Different people respond differently to therapy: A demonstration using patient profiling and risk stratification. Behaviour research and therapy, 79, 1522.

Derogatis, L. R., \& Melisaratos, N. (1983). The Brief Symptom Inventory: an introductory report. Psychological medicine, 13(3), 595-605. Retrieved from http://www.ncbi.nlm.nih.gov/pubmed/6622612.

DeRubeis, R. J., Cohen, Z. D., Forand, N. R., Fournier, J. C., Gelfand, L. A., \& Lorenzo-Luaces, L. (2014). The Personalized Advantage Index: translating research on prediction into individualized treatment recommendations. A demonstration. PLoS One, 9(1), e83875.

Dinger, U., Ehrenthal, J. C., Nikendei, C., \& Schauenburg, H. (2017). Change in self-esteem predicts depressive symptoms at follow-up after intensive multimodal psychotherapy for major depression. Clinical psychology \& psychotherapy.

Fava, G. A., Ruini, C., \& Belaise, C. (2007). The concept of recovery in major depression. Psychological medicine, 37(3), 307-317.

First, M. B., Gibbon, M., Spitzer, R. L., Williams, J. B. W., \& Benjamin, L. S. (1997). Structured Clinical Interview for DSM-IV Axis II Personality Disorders (SCID-II) Washington, D.C: American Psychiatric Press.

First, M. B., Spitzer, R. L., Gibbon, M., \& Williams, J. B. W. (1995). Structured Clinical Interview for DSM-IV Axis I Disorders (SCID-I). New York: Biometrics Research Department, New York State Psychiatric Institute.

Fournier, J. C., DeRubeis, R. J., Shelton, R. C., Hollon, S. D., Amsterdam, J. D., \& Gallop, R. (2009). Prediction of response to medication and cognitive therapy in the treatment of moderate to severe depression. Journal of consulting and clinical psychology, 77(4), 775-787. Retrieved from http://www.ncbi.nlm.nih.gov/pubmed/19634969. doi:10.1037/a0015401

Franck, E., De Raedt, R., Dereu, M., \& Van den Abbeele, D. (2007). Implicit and explicit self-esteem in currently depressed individuals with and without suicidal ideation. Journal of behavior therapy and experimental psychiatry, 38(1), 75-85.

Garge, N., Eggleston, B., Bobashev, G., \& Garge, M. N. (2013). Package 'mobForest'.

Garge, N. R., Bobashev, G., \& Eggleston, B. (2013). Random forest methodology for model-based recursive partitioning: the mobForest package for R. BMC bioinformatics, 14(1), 125.

Goldman, R. N., Greenberg, L. S., \& Angus, L. (2006). The effects of adding emotion-focused interventions to the client-centered relationship conditions in the treatment of depression. Psychotherapy Research, 16(5), 537-549.

Hansen, R., Gaynes, B., Thieda, P., Gartlehner, G., Deveaugh-Geiss, A., Krebs, E., \& Lohr, K. (2008). Metaanalysis of major depressive disorder relapse and recurrence with second-generation antidepressants. Psychiatric Services, 59(10), 1121-1130. Retrieved from http://www.ncbi.nlm.nih.gov/pubmed/18832497. doi:10.1176/appi.ps.59.10.1121

Hardeveld, F., Spijker, J., De Graaf, R., Hendriks, S. M., Licht, C. M., Nolen, W. A., . . Beekman, A. T. (2013). Recurrence of major depressive disorder across different treatment settings: results from the NESDA study. Journal of affective disorders, 147(1-3), 225-231. Retrieved from http://www.ncbi.nlm.nih.gov/pubmed/23218899. doi:10.1016/j.jad.2012.11.008

Hardeveld, F., Spijker, J., De Graaf, R., Nolen, W. A., \& Beekman, A. T. (2010). Prevalence and predictors of recurrence of major depressive disorder in the adult population. Acta Psychiatrica Scandinavica, 122(3), 184-191. Retrieved from http://www.ncbi.nlm.nih.gov/pubmed/20003092. doi:10.1111/j.1600-0447.2009.01519.x

Hardeveld, F., Spijker, J., De Graaf, R., Nolen, W. A., \& Beekman, A. T. (2013). Recurrence of major depressive disorder and its predictors in the general population: results from the Netherlands Mental 
Health Survey and Incidence Study (NEMESIS). Psychological medicine, 43(1), 39-48. Retrieved from http://www.ncbi.nlm.nih.gov/pubmed/23111147. doi:10.1017/S0033291712002395

Hiroe, T., Kojima, M., Yamamoto, I., Nojima, S., Kinoshita, Y., Hashimoto, N., . . Furukawa, T. A. (2005). Gradations of clinical severity and sensitivity to change assessed with the Beck Depression InventoryII in Japanese patients with depression. Psychiatry research, 135(3), 229-235.

Horowitz, L. M., Rosenberg, S. E., Baer, B. A., Ureno, G., \& Villasenor, V. S. (1988). Inventory of interpersonal problems: psychometric properties and clinical applications. Journal of consulting and clinical psychology, 56(6), 885-892. Retrieved from http://www.ncbi.nlm.nih.gov/pubmed/3204198.

Hothorn, T., Hornik, K., Van De Wiel, M. A., \& Zeileis, A. (2006). A Lego system for conditional inference. The American Statistician, 60(3), 257-263.

Hothorn, T., Hornik, K., Van De Wiel, M. A., \& Zeileis, A. (2008). Implementing a class of permutation pests: the coin package.

Huibers, M. J., Cohen, Z. D., Lemmens, L. H., Arntz, A., Peeters, F. P., Cuijpers, P., \& DeRubeis, R. J. (2015). Predicting Optimal Outcomes in Cognitive Therapy or Interpersonal Psychotherapy for Depressed Individuals Using the Personalized Advantage Index Approach. PLoS One, 10(11), e0140771. Retrieved from http://www.ncbi.nlm.nih.gov/pubmed/26554707. doi:10.1371/journal.pone.0140771

Keefe, J. R., Wiltsey Stirman, S., Cohen, Z. D., DeRubeis, R. J., Smith, B. N., \& Resick, P. A. (2018). In rape trauma PTSD, patient characteristics indicate which trauma-focused treatment they are most likely to complete. Depression and anxiety, 35(4), 330-338.

Kendler, K. S., Thornton, L. M., \& Gardner, C. O. (2000). Stressful life events and previous episodes in the etiology of major depression in women: an evaluation of the "kindling" hypothesis. American Journal of Psychiatry, 157(8), 1243-1251.

Klerman, G. L., Weissman, M. M., Rounsaville, B. J., \& Chevron, E. S. (1984). Interpersonal psychotherapy for depression. New York: Basis Books.

Kovacs, M., \& Garrison, B. (1985). Hopelessness and eventual suicide: a 10-year prospective study of patients hospitalized with suicidal ideation. American Journal of Psychiatry, 1(42), 559-563.

Kovacs, M., Obrosky, S., \& George, C. (2016). The course of major depressive disorder from childhood to young adulthood: Recovery and recurrence in a longitudinal observational study. Journal of affective disorders, 203, 374-381. Retrieved from http://www.ncbi.nlm.nih.gov/pubmed/27347807. doi:10.1016/j.jad.2016.05.042

Lemmens, L. H., Arntz, A., Peeters, F., Hollon, S. D., Roefs, A., \& Huibers, M. J. (2015). Clinical effectiveness of cognitive therapy $v$. interpersonal psychotherapy for depression: results of a randomized controlled trial. Psychological medicine, 45(10), 2095-2110. Retrieved from http://www.ncbi.nlm.nih.gov/pubmed/25640151. doi:10.1017/S0033291715000033

Lemmens, L. H., Arntz, A., Peeters, F. P., Hollon, S. D., Roefs, A., \& Huibers, M. J. (2011). Effectiveness, relapse prevention and mechanisms of change of cognitive therapy vs. interpersonal therapy for depression: Study protocol for a randomised controlled trial. Trials, 12, 150. Retrieved from http://www.ncbi.nlm.nih.gov/pubmed/21672217. doi:10.1186/1745-6215-12-150

Lemmens, L. H., van Bronswijk, S. C., Peeters, F., Arntz, A., Hollon, S. D., \& Huibers, M. J. (2018). Long-term outcomes of acute treatment with cognitive therapy v. interpersonal psychotherapy for adult depression: follow-up of a randomized controlled trial. Psychological medicine, 1-9.

Lindhiem, O., Bennett, C. B., Trentacosta, C. J., \& McLear, C. (2014). Client preferences affect treatment satisfaction, completion, and clinical outcome: A meta-analysis. Clinical Psychology Review, 34(6), 506-517.

Liu, R. T., Kleiman, E. M., Nestor, B. A., \& Cheek, S. M. (2015). The Hopelessness Theory of Depression: A Quarter-Century in Review. Clinical Psychology: Science and Practice, 22(4), 345-365.

Lorenzo-Luaces, L. (2015). Heterogeneity in the prognosis of major depression: from the common cold to a highly debilitating and recurrent illness. Epidemiology and Psychiatric Sciences, 24(6), 466-472. Retrieved from http://www.ncbi.nlm.nih.gov/pubmed/26081748. doi:10.1017/S2045796015000542

Lorenzo-Luaces, L., DeRubeis, R. J., van Straten, A., \& Tiemens, B. (2017). A prognostic index (PI) as a moderator of outcomes in the treatment of depression: A proof of concept combining multiple variables to inform risk-stratified stepped care models. Journal of affective disorders, 213, 78-85. 
Monroe, S. M., \& Harkness, K. L. (2011). Recurrence in major depression: a conceptual analysis. Psychological review, 118(4), 655-674. Retrieved from http://www.ncbi.nlm.nih.gov/pubmed/21895384. doi:10.1037/a0025190

Morley, T. E., \& Moran, G. (2011). The origins of cognitive vulnerability in early childhood: Mechanisms linking early attachment to later depression. Clinical Psychology Review, 31(7), 1071-1082.

Mundt, J. C., Marks, I. M., Shear, M. K., \& Greist, J. H. (2002). The Work and Social Adjustment Scale: a simple measure of impairment in functioning. British Journal of Psychiatry, 180, 461-464. Retrieved from http://www.ncbi.nlm.nih.gov/pubmed/11983645.

Nanni, V., Uher, R., \& Danese, A. (2012). Childhood maltreatment predicts unfavorable course of illness and treatment outcome in depression: a meta-analysis. American Journal of Psychiatry, 169(2), 141-151. Retrieved from http://www.ncbi.nlm.nih.gov/pubmed/22420036. doi:10.1176/appi.ajp.2011.11020335

Nierenberg, A., Husain, M., Trivedi, M., Fava, M., Warden, D., Wisniewski, S., . . Rush, A. (2010). Residual symptoms after remission of major depressive disorder with citalopram and risk of relapse: a STAR* D report. Psychological medicine, 40(1), 41-50.

Park, Y., Freedman, A. N., Gail, M. H., Pee, D., Hollenbeck, A., Schatzkin, A., \& Pfeiffer, R. M. (2009). Validation of a colorectal cancer risk prediction model among white patients age 50 years and older. Journal of Clinical Oncology, 27(5), 694-698. Retrieved from http://www.ncbi.nlm.nih.gov/pubmed/19114700. doi:10.1200/JCO.2008.17.4813

Peterson, C., Semmel, A., Von Baeyer, C., Abramson, L. Y., Metalsky, G. I., \& Seligman, M. E. (1982). The attributional style questionnaire. Cognitive therapy and research, 6(3), 287-299.

Phillips, W. J., Hine, D. W., \& Bhullar, N. (2012). A latent profile analysis of implicit and explicit cognitions associated with depression. Cognitive therapy and research, 36(5), 458-473.

Picard, R. R., \& Cook, R. D. (1984). Cross-validation of regression models. Journal of the American Statistical Association, 79(387), 575-583.

Raes, F., Hermans, D., \& Eelen, P. (2003). Kort instrumenteel De Nederlandstalige versie van de Ruminative Response Scale (RRS-NL) en de Rumination on Sadness Scale (RSS-NL). Gedragstherapie.

Rizopoulos, D., \& Rizopoulos, M. D. (2009). Package 'bootStepAIC'.

Segal, Z. V., Williams, J., Teasdale, J., \& Gemar, M. (1996). A cognitive science perspective on kindling and episode sensitization in recurrent affective disorder. Psychological medicine, 26(2), 371-380.

Solomon, D. A., Keller, M. B., Leon, A. C., Mueller, T. I., Lavori, P. W., Shea, M. T., . . Endicott, J. (2000). Multiple recurrences of major depressive disorder. American Journal of Psychiatry, 157(2), 229-233. Retrieved from http://www.ncbi.nlm.nih.gov/pubmed/10671391. doi:10.1176/appi.ajp.157.2.229

Stekhoven, D. J., \& Bühlmann, P. (2012). MissForest-non-parametric missing value imputation for mixedtype data. Bioinformatics, 28(1), 112-118.

Strobl, C., Boulesteix, A.-L., Kneib, T., Augustin, T., \& Zeileis, A. (2008). Conditional variable importance for random forests. BMC bioinformatics, 9(1), 307.

Strunk, D. R., DeRubeis, R. J., Chiu, A. W., \& Alvarez, J. (2007). Patients' competence in and performance of cognitive therapy skills: relation to the reduction of relapse risk following treatment for depression. Journal of consulting and clinical psychology, 75(4), 523.

Tafarodi, R. W., \& Swann, W. B. (2001). Two-dimensional self-esteem: Theory and measurement. Personality and individual Differences, 31(5), 653-673.

ten Doesschate, M. C., Bockting, C., Koeter, M., \& Schene, A. H. (2010). Prediction of recurrence in recurrent depression: a 5.5-year prospective study. The Journal of clinical psychiatry, 71(8), 984-991.

Van der Does, W. (2002). Cognitive reactivity to sad mood: structure and validity of a new measure. Behaviour research and therapy, 40(1), 105-119.

van der Zee, K. I., \& Sanderman, R. (1993). Het meten van de algemene gezondheidstoestand met de RAND36: een handleiding. Groningen: Noordelijk Centrum voor Gezondheidsvraagstukken.

van Loo, H. M., Aggen, S. H., Gardner, C. O., \& Kendler, K. S. (2015). Multiple risk factors predict recurrence of major depressive disorder in women. Journal of affective disorders, 180, 52-61. Retrieved from http://www.ncbi.nlm.nih.gov/pubmed/25881281. doi:10.1016/j.jad.2015.03.045

van Winkel, M., Wichers, M., Collip, D., Jacobs, N., Derom, C., Thiery, E., . . Peeters, F. (2017). Unraveling the role of loneliness in depression: the relationship between daily life experience and behavior. Psychiatry, 80(2), 104-117. 
Vandromme, H., Hermans, D., Spruyt, A., \& Eelen, P. (2007). Dutch translation of the Self-Liking/SelfCompetence Scale-Revised: A confirmatory factor analysis of the two-factor structure. Personality and individual Differences, 42(1), 157-167.

Vul, E., Harris, C., Winkielman, P., \& Pashler, H. (2009). Puzzlingly high correlations in fMRI studies of emotion, personality, and social cognition. Perspectives on psychological science, 4(3), 274-290.

Waljee, A. K., Mukherjee, A., Singal, A. G., Zhang, Y., Warren, J., Balis, U., . . . Higgins, P. D. (2013). Comparison of imputation methods for missing laboratory data in medicine. BMJ open, 3(8), e002847.

Wang, J. L., Patten, S., Sareen, J., Bolton, J., Schmitz, N., \& MacQueen, G. (2014). Development and validation of a prediction algorithm for use by health professionals in prediction of recurrence of major depression. Depression and anxiety, 31(5), 451-457. Retrieved from http://www.ncbi.nlm.nih.gov/pubmed/24877248.

Waugh, C. E., \& Koster, E. H. (2015). A resilience framework for promoting stable remission from depression. Clinical Psychology Review, 41, 49-60.

Weissman, A. N., \& Beck, A. T. (1978). Development and validation of the Dysfunctional Attitude Scale: A preliminary investigation.

Zilcha-Mano, S., Keefe, J. R., Chui, H., Rubin, A., Barrett, M. S., \& Barber, J. P. (2016). Reducing Dropout in Treatment for Depression: Translating Dropout Predictors Into Individualized Treatment Recommendations. The Journal of clinical psychiatry, 77(12), e1584-e1590. 
S. van Bronswijk, R. DeRubeis, L. Lemmens, F. Peeters, J. Keefe, Z. Cohen, M. Huibers - Psychological Medicine 2019, 1-11

\section{Chapter}

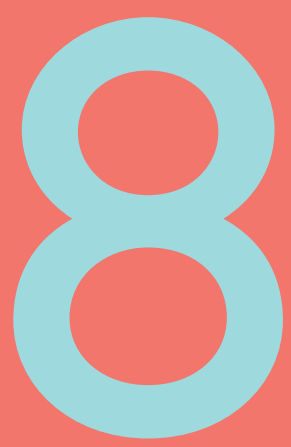




\section{Abstract}

Background

Psychotherapies for depression are equally effective on average, but individual responses vary widely. Outcomes can be improved by optimizing treatment selection using multivariate prediction models. A promising approach is the Personalized Advantage Index (PAI) that predicts the optimal treatment for a given individual and the magnitude of the advantage. The current study aimed to extend the PAI to long-term depression outcomes after acute-phase psychotherapy.

\section{Methods}

Data come from a randomized trial comparing cognitive therapy $(C T, n=76)$ and interpersonal psychotherapy (IPT, $n=75$ ) for MDD. Primary outcome was depression severity, as assessed by the BDI- II, during 17-month follow-up. First, predictors and moderators were selected from 38 pre-treatment variables using a two-step machine learning approach. Second, predictors and moderators were combined into a final model, from which PAl predictions were computed with cross-validation. Long-term PAI predictions were then compared to actual follow-up outcomes and post-treatment PAI predictions.

Results

One predictor (parental alcohol abuse) and two moderators (recent life events; childhood maltreatment) were identified. Individuals assigned to their PAI-indicated treatment had lower follow-up depression severity compared to those assigned to their PAI-non-indicated treatment. This difference was significant in two subsets of the overall sample: those whose PAI score was in the upper $60 \%$, and those whose PAI indicated CT, irrespective of magnitude. Longterm predictions did not overlap substantially with predictions for acute benefit.

\section{Conclusions}

If replicated, long-term PAI predictions could enhance precision medicine by selecting the optimal treatment for a given depressed individual over the long term. 


\section{Introduction}

Optimizing treatment selection is a promising approach to improve psychotherapy outcomes for major depressive disorder (MDD; Cohen \& DeRubeis, 2018). Although research shows that different types of psychotherapy for MDD are equally effective on average (Cuijpers, Andersson, Donker, \& van Straten, 2011), an individual's response to different therapies may vary greatly (Simon \& Perlis, 2010). In addition, treatment response is highly unpredictable; for example individuals often go through multiple antidepressant therapies before an effective regimen is identified (Rush et al., 2006). Treatment selection aims to move beyond average effectiveness and focuses on the question, "What works for whom?" Efforts to match individuals with specifics treatments are referred to as personalized or precision medicine (Cohen \& DeRubeis 2018; Katsnelson, 2013; Simon \& Perlis, 2010).

To optimize treatment selection, individual characteristics that reliably predict differential treatment outcomes, so-called moderators or prescriptive variables, need to be identified. Biomarkers (e.g., genetic or brain imaging variables), clinical features (e.g., illness severity or chronicity) and sociodemographic characteristic (e.g., gender or education level) have been the focus of efforts to identify useful moderators. However, no single moderator is likely to be robust enough, on its own, to reliably guide treatment selection in MDD (Cohen \& DeRubeis 2018; Kessler, 2018; Simon \& Perlis, 2010), and indeed none have been identified. In recent years, the development of multivariate prediction models, which aggregate multiple moderators, has shown promise as a means of producing powerful predictions (Cohen \& DeRubeis 2018). These models aim to convert the predictive information of multiple moderators into actionable recommendations to guide treatment selection. Examples of these multivariate models are the "matching factor" (Barber \& Muenz, 1996), the "nearestneighbors" approach (Lutz et al., 2006) and the $M^{*}$ approach (Kraemer, 2013; Niles et al., 2017a; Niles, Wolitzky-Taylor, Arch, \& Craske, 2017b; Smagula et al., 2016; Wallace, Frank, \& Kraemer, 2013).

Another promising multivariate approach to guide treatment selection between two or more treatments is the Personalized Advantage Index (PAI, DeRubeis et al., 2014). This method not only provides an individual treatment recommendation, it also delivers a quantitative estimate of the predicted advantage of the indicated treatment over the non-indicated treatment(s). These recommendations are based on the difference between predicted outcomes of two or more treatments using a model that includes multiple predictors and moderators. DeRubeis et al. (2014) developed and introduced this approach by predicting outcomes of acute-phase cognitive therapy (CT) and pharmacotherapy. Since then, the PAI approach has been replicated and extended to acute phase CT versus interpersonal psychotherapy (IPT) for MDD (Huibers et al., 2015), continuation CT versus fluoxetine for recurrent MDD (Vittengl, Clark, Thase, \& Jarrett, 2017), sertraline versus placebo for MDD (Webb et al., 2019), 
trauma-focused cognitive behavioral therapy and eye movement desensitization for posttraumatic stress disorder (PTSD) (Deisenhofer et al., 2018), and dropout in MDD (Zilcha-Mano et al., 2016) and PTSD (Keefe et al., 2018).

In the current study, we aim to extend the PAI approach for treatment selection to focus on longer-term depression outcomes within the context of a 17-month follow-up of a recent randomized trial comparing CT and IPT (Lemmens et al., 2015; Lemmens et al., 2019). CT and IPT are two frequently practiced psychotherapies for MDD and have been shown to be equally effective in the acute phase (Jakobsen, Hansen, Simonsen, Simonsen, \& Gluud, 2012; Lemmens et al., 2015) with comparable prophylactic effects after treatment termination (Lemmens et al., 2019). The current study extends a recently published PAl effort predicting acute treatment response (post-treatment point estimates) using a completer's subset of the same study sample (the "post-treatment" PAI, Huibers et al., 2015; Lemmens et al., 2015). In the current study a "long-term" PAI was built. First, we selected pre-treatment variables using a two-step machine learning approach, to identify reliable predictors and moderators of long-term depression outcome after CT and IPT. Second, we calculated PAI scores for individual treatment recommendations based on a final model that combined the selected predictors and moderators with a cross-validation approach. The utility of the long-term PAI recommendations was then evaluated by comparing the set of predictions with the respective observed follow-up outcomes. In addition, the longterm PAI scores per individual were compared with the post-treatment PAI scores to examine if the PAI scores for that individual overlap, and if the different intended outcomes (optimal post-treatment outcomes versus optimal long-term outcomes) led to different treatment recommendations. Finally, a secondary analyses was conducted, repeating the process of variable selection and model fitting to a five-fold held-out sample (instead of the full sample) to create five separate models. The predictions of these models were then compared to the long-term PAI predictions, to provide an insight into the method's robustness (e.g., the risk of overfitting), and its potential for out-of-sample predictions.

\section{Methods}

\subsection{Design and participants}

Data come from a randomized controlled trial into the effectiveness of individual CT and IPT for MDD. Adult outpatients (18-65 years) were recruited from the mood disorders unit of the Academic Maastricht Outpatient Mental Health Centre (RIAGG Maastricht, the Netherlands). Inclusion criteria were a primary diagnosis of MDD (confirmed with the Structured Clinical Interview for DSM-IV Axis I disorders; First, Spitzer, Gibbon, \& Williams, 1995), internet access, an email address, and sufficient 
knowledge of the Dutch language. Individuals with bipolar- or highly chronic depression (current episode $>5$ years) were excluded from the study. Other exclusion criteria were a high acute suicide risk, concomitant pharmacological or psychological treatment, drugs and alcohol abuse/dependence, and an IQ lower than 80. After providing written informed consent, a total of 182 participants were randomly assigned to CT $(n=76)$, IPT ( $n=75)$, or a 2-month waiting-list control $(n=31)$ followed by treatment of choice. For the current study, we limited our sample to the two active conditions $(n=151)$ and included pre-treatment variables and outcome data from the follow-up phase (month 7-24).

\subsection{Treatments}

Treatment consisted of 16 to 20 individual 45 -minute sessions $(M=17, S D=2.9)$ that were planned weekly and were allowed to be less frequent towards the end of therapy. CT was carried out following the guidelines by Beck et al. (1979). IPT was based on the manual by Klerman et al. (1984). Therapists were ten licensed psychologists, psychotherapists and psychiatrists with substantial clinical experience $(M=9.1$ years, $S D=5.4)$. For both $C T$ and $I P T$, treatment quality was rated by independent assessors as "(very) good" to "excellent" (Lemmens et al., 2015). During follow-up, individuals were free to seek additional treatment for MDD, including psychological support ( $n=54$, one or more sessions with a general practitioner or a mental health care professional) and antidepressant medication $(n=29)$.

\subsection{Measures}

\subsubsection{Primary outcome}

Primary outcome was depression severity measured with the Beck Depression Inventory, second edition (BDI-II, Beck, Steer, \& Brown, 1996) during follow-up at 7, 8, 9, 10, 11, 12 and 24 months. These BDI-II scores were aggregated, for each participant, into an Area under the Curve (AUC) to obtain an overall measure of depression severity across the 17-month follow-up period. The AUC can be interpreted as a summary of depressive symptom burden measured over several time points.

\subsubsection{Pre-treatment variables}

We examined 69 pre-treatment variables from six previously described domains: 1) depression variables, 2) demographics, 3) psychological distress, 4) general functioning 5) psychological processes and 6) life and family history (Fournier et al., 2009; Huibers et al., 2015). A correlation matrix corrected for attenuation was computed for all 69 variables. Variables that were highly correlated (cor. $>0.70$ ) with 
other variables were removed to prevent multicollinearity. Choices on which one of two variables should be removed depended on redundancy (e.g., multiple indicators for quality of life) and interpretability (e.g., including a total scale instead of highly correlated subscales of one measurement instrument) and were always made as a group decision of the research team. Similar pre-selection procedures have been described in previous studies (Kim et al., 2019; Lorenzo-Luaces, DeRubeis, van Straten, $\&$ Tiemens, 2017). As a result of this procedure, we removed 31 variables, and the remaining 38 pre-treatment variables were selected for further analyses (see Table 8.1). They came from the following measurement scales: Beck Hopelessness Scale (BHS, Beck \& Steer, 1988), Brief Symptom Inventory (BSI; Derogatis \& Melisaratos, 1983), Structured Clinical Interview for DSM-IV Axis I disorders (SCID-I; First et al., 1995), Structured Clinical Interview for DSM-IV Axis II disorders (SCID-II; First, Gibbon, Spitzer, Williams, \& Benjamin, 1997), Work and Social Adjustment Scale (WSAS; Mundt, Marks, Shear, \& Greist, 2002), Dysfunctional Attitudes Scale (DAS; de Graaf, Roelofs, \& Huibers, 2009; Weissman \& Beck, 1978), Inventory of Interpersonal Problems (IIP; Horowitz, Rosenberg, Baer, Ureno, \& Villasenor, 1988), Self-Liking and Self-Competence Scale Revised (SLSC; Tafarodi \& Swann, 2001; Vandromme, Hermans, Spruyt, \& Eelen, 2007), Ruminative Response Scale (RRS; Raes, Hermans, \& Eelen, 2003), and Attributional Style Questionnaire (ASQ; Cohen, Van den Bout, Kramer, \& Van Vliet, 1986; Peterson et al., 1982).

Table 8.1: Description and comparison of pre-treatment variables in cognitive cherapy versus interpersonal psychotherapy.

\begin{tabular}{lcc}
\hline & $\begin{array}{c}\text { Cognitive therapy } \\
(\mathrm{n}=76)\end{array}$ & $\begin{array}{c}\text { Interpersonal } \\
\text { psychotherapy }(\mathrm{n}=75)\end{array}$ \\
\hline $\begin{array}{l}\text { DOMAIN I: Depression } \\
\text { Recurrent depressive episodes, yes, } \mathrm{n}(\%)\end{array}$ & $36(48.0)$ \\
$\quad$ Hopelessness, BHS, mean (SD) & $11.3(4.9)$ & $12.4(4.7)$ \\
DOMAIN II: Demographics & & \\
Female sex, $\mathrm{n}(\%)$ & $54(71.1)$ & $46(61.3)$ \\
Age, mean (SD) & $41.2(12.4)$ & $41.3(11.8)$ \\
Partner, yes, $\mathrm{n}(\%)$ & $43(56.6)$ & $51(68.0)$ \\
Education level & & \\
- Low, $\mathrm{n}(\%)$ & $16(21.0)$ & $13(17.3)$ \\
- Intermediate, $\mathrm{n}(\%)$ & $48(63.2)$ & $41(54.7)$ \\
- High, $\mathrm{n}(\%)$ & $12(15.8)$ & $21(28.0)$ \\
Active employment, yes, $\mathrm{n}(\%)$ & $43(56.6)$ & $47(62.7)$ \\
Treatment expectancy, mean (SD) & $6.8(1.2)$ & $6.5(1.3)$ \\
\hline
\end{tabular}




\begin{tabular}{|c|c|c|}
\hline & $\begin{array}{c}\text { Cognitive therapy } \\
(n=76)\end{array}$ & $\begin{array}{c}\text { Interpersonal } \\
\text { psychotherapy }(n=75)\end{array}$ \\
\hline \multicolumn{3}{|l|}{ DOMAIN III: Psychological distress } \\
\hline \multicolumn{3}{|l|}{ General psychological distress, BSI } \\
\hline - Somatic complaints, mean (SD) & $7.8(5.7)$ & $7.7(4.7)$ \\
\hline - Cognitive problems, mean (SD) & $11.0(5.0)$ & $11.6(5.0)$ \\
\hline - Depression, mean (SD) & $10.7(4.7)$ & $12.1(5.1)$ \\
\hline - Anxiety, mean (SD) & $7.7(5.0)$ & $8.8(4.6)$ \\
\hline - Phobic Anxiety, mean (SD) & $4.8(4.0)$ & $4.8(3.9)$ \\
\hline - Hostility, mean (SD) & $4.4(3.3)$ & $5.1(3.9)$ \\
\hline - Paranoid Symptoms, mean (SD) & $6.6(4.4)$ & $7.2(4.1)$ \\
\hline Number of comorbid axis I disorders, SCID-I, mean (SD) & $0.6(0.8)$ & $0.7(0.7)$ \\
\hline Number of comorbid axis II disorders, SCID-II, mean (SD) & $0.7(1.0)^{*}$ & $0.4(0.7)^{*}$ \\
\hline Number of comorbid axis II traits, SCID-II, mean (SD) & $0.3(0.7)^{*}$ & $0.6(1.0)^{*}$ \\
\hline \multicolumn{3}{|l|}{ DOMAIN IV: General functioning } \\
\hline Social and work functioning, WSAS, mean (SD) & $23.2(7.7)$ & $22.4(7.2)$ \\
\hline \multicolumn{3}{|l|}{ Level of impairment, RAND-36 } \\
\hline - Physical functioning, mean (SD) & $73.7(23.0)$ & $74.1(20.5)$ \\
\hline - Social functioning, mean (SD) & $41.5(19.2)$ & $40.8(20.2)$ \\
\hline - Role limitations (physical problems), mean (SD) & $37.0(41.0)$ & $33.7(38.4)$ \\
\hline - Role limitations (emotional problems), mean (SD) & $17.8(32.1)$ & $13.3(25.1)$ \\
\hline - General health perception, mean (SD) & $46.7(16.5)$ & $43.8(14.1)$ \\
\hline - Perceived health change during past year, mean (SD) & $31.3(26.0)$ & $26.0(24.8)$ \\
\hline \multicolumn{3}{|l|}{ DOMAIN V: Psychological Processes } \\
\hline \multicolumn{3}{|l|}{ Dysfunctional Beliefs, DAS } \\
\hline - Factor 1, mean (SD) & $35.7(11.1)$ & $36.8(11.5)$ \\
\hline - Factor 2, mean (SD) & $25.4(6.4)$ & $26.1(7.1)$ \\
\hline Interpersonal problems, IIP, mean (SD) & $83.1(24.7)$ & $89.7(33.9)$ \\
\hline Self Liking and Self Competence, SLSC-R, mean (SD) & $39.3(8.6)$ & $37.6(10.7)$ \\
\hline Rumination, RRS, mean (SD) & $49.1(9.1)$ & $52.4(8.4)$ \\
\hline Attributional Style, ASQ, mean (SD) & $0.03(0.9)$ & $0.02(1.2)$ \\
\hline \multicolumn{3}{|l|}{ DOMAIN VI: Life and family history } \\
\hline Number of life events past year, mean (SD) & $1.8(1.4)$ & $1.8(1.3)$ \\
\hline Number of childhood trauma events, mean (SD) & $0.9(1.3)$ & $0.8(1.2)$ \\
\hline \multicolumn{3}{|l|}{ Parental: one or both parents } \\
\hline - $\quad$ In treatment for illness, $\mathrm{n}(\%)$ & $19(25.0)$ & $17(22.7)$ \\
\hline - With an anxiety disorder, $\mathrm{n}(\%)$ & $13(17.1)$ & $14(18.7)$ \\
\hline - With depression, $\mathrm{n}(\%)$ & $35(46.1)$ & $30(40.0)$ \\
\hline - With alcohol abuse, $\mathrm{n}(\%)$ & $9(11.8)$ & $13(17.3)$ \\
\hline - With suicidality, $\mathrm{n}(\%)$ & $6(7.9)$ & $11(14.7)$ \\
\hline
\end{tabular}

BHS, Beck Hopelessness Scale; Treatment expectancy, 0=not successful 10=very successful; BSI, Brief Symptom Inventory; SCID-I, Structured Clinical Interview for DSM-IV Axis I disorders; SCID-II, Structured Clinical Interview for DSM-IV Axis II disorders; WSAS, Work and Social Adjustment Scale; DAS, Dysfunctional Attitudes Scale; IIP, Inventory of Interpersonal Problems; SLSC-R, Self Liking and Self Competence Scale Revised; RRS, Ruminative Response Scale; ASQ, Attributional Style Questionnaire. * $p<0.05$. 


\subsection{Statistical analyses}

\subsubsection{Variable description and missing data}

Between treatment differences of the 38 variables were examined, using t-tests and

$\chi^{2}$-tests where appropriate. Missing BDI-II outcomes and variables were imputed using a non-parametric random forest approach (R package "MissForest"; Stekhoven \& Bühlmann, 2012). This imputation approach has been shown to be accurate and comparable to multiple imputation, with lower imputation errors compared to many other imputations methods (Stekhoven \& Bühlmann, 2012; Waljee et al., 2013). For the imputation model, we used the following information as input: 1) change scores from baseline of all non-missing BDI-II outcomes (at 3, 7, 8, 9, 10, 11, 12 and 24 months); 2) all scores on non-missing variables; 3) change scores from baseline to post-treatment of all non-missing variables; 4) the received treatment (CT/IPT). To test the imputation method, it was applied to the complete (non-missing) dataset with artificially produced missing data. Imputed values were then compared with actual data values by estimating the normalized root mean squared error (NRMSE) for continuous data and the proportion of falsely classified entries (PFC) for categorical data (Stekhoven \& Bühlmann, 2012).

\subsubsection{Outcome transformation}

To produce estimates of "overall' depression severity across the 17-month follow-up phase, BDI-II scores at 7, 8, 9, 10, 11, 12 and 24 months were combined into an AUC using cubic splines to compute integrals. As described elsewhere (Lemmens et al., 2015), BDI-II scores between CT and IPT differed at baseline, though the difference was a non-significant trend. To adjust for this difference, we calculated the residuals of a regression function with the AUC as the dependent variable and the BDI-II at baseline as the independent variable. We used these residuals as the outcome variable for further analyses. To avoid confusion, we will refer to these residuals as the AUC.

\subsubsection{Variable transformation}

Discrete and categorical variables were centered, and continuous variables were standardized. Discrete variables with a non-normal distribution were transformed using a log transformation or a square root transformation based on visual inspection (details about transformations can be found in Supplemental Methods I).

\subsubsection{Variable selection}

We used a two-step machine learning approach to select predictors and moderators of long-term outcome in CT and IPT, that has been employed previously (Keefe et al., 
2018; Zilcha-Mano et al., 2016). First, we applied a model-based recursive partitioning method using a random forest algorithm (Garge, Bobashev, \& Eggleston, 2013). This method splits bootstrapped samples repeatedly into two subgroups based on a predetermined model. In the current analyses, this pre-determined model was a regression model with the AUC as the dependent variable and the pre-treatment variables as interactions with treatment ( $y=x *$ treatment) to test their potential as moderators. At each potential split, a random subset of variables was available to inform the split, and the data were divided on the variable with the strongest moderator impact, to produce a tree-like structure. By repeatedly using different random subsets of variables, variables with smaller effects were less likely to be dominated by the presence of stronger variables (Strobl, Boulesteix, Kneib, Augustin, \& Zeileis, 2008). Parameters were set as follows: a total of 10000 trees were computed with a minimum $\alpha$ level of 0.10 for splitting and a minimum subgroup size for splits of 15 individuals. As an output of this method, variables were ranked based on a variable importance score indicating their predictive impact. The variable importance score was computed by subtracting the predictive accuracy of a variable when applying the real values, from the predictive accuracy of a variable when applying randomly permuted values. The higher the difference between the real and permutated values, the higher the variable importance. Variables were selected for the second step if they exceeded the threshold, which is the absolute value of the variable importance score of the lowest ranking variable. The second step involves a backward elimination approach using multiple bootstrapped samples ( $R$ package "bootstepAIC"; Austin \& Tu, 2004; Rizopoulos \& Rizopoulos, 2009). For this approach a regression model was specified with the $A U C$ as the dependent variable and the variables selected in the first variable selection step as independent variables, along with their interactions with treatment. A total of 1000 bootstrapped samples of the original data was generated, and backwards elimination (using $\alpha=0.05$ ) with the specified model was applied to each of these samples. For each variable, the number of times it was selected and had a positive or negative regression coefficient was computed. If variables were selected in at least $60 \%$ of the bootstrapped samples, they were considered robust (Austin \& Tu, 2004) and used to build the PAl. For the final moderators, the Johnson-Neyman technique was applied to examine at which value the between treatment difference was significant (Johnson \& Neyman, 1936).

\subsubsection{Building the Personalized Advantage Index (PAI)}

The PAI method was applied to generate personalized treatment recommendations based on pre-treatment predictors and moderators (DeRubeis et al., 2014). For this approach, the selected variables were combined into a regression model with the AUC as the dependent variable. The independent variables were the predictors, the moderators interacting with treatment, and main effects of the moderators. Based on 
this regression model, individual outcome predictions for each treatment were made using a fivefold cross-validation. With the five-fold cross-validation, the sample was split into five equal groups and individual outcomes of each group were predicted using the regression model with weights based on the data of the other four groups of the sample (the "training dataset"; Picard \& Cook, 1984). Applying the cross-validation approach reduces the risk of overfitting by not including the individual's data during the computation of regression parameters. For each individual two separate predictions were made: one predicted score for the treatment the individual actually received (factual) and one predicted score for the treatment the individual did not receive (counterfactual). The differences between these two predictions resulted in a positive or negative score indicating the optimal treatment: a PAI indicating CT or IPT. In addition, the magnitude of this score indicated the strength of the predicted advantage of the indicated PAI treatment, with higher scores representing a stronger need for a specific treatment.

\subsubsection{Evaluating the Personalized Advantage Index}

To test the utility of the PAI, actual follow-up outcomes (AUC's) of individuals receiving the PAl-indicated treatment were compared with those of individuals receiving the PAI non-indicated treatment, using t-tests. Following DeRubeis et al. (2014), we also compared the observed follow-up outcomes (AUC's) of those with the highest $60 \%$ (absolute values) PAI-scores. After that, we evaluated the PAI effect separately for CT and IPT. For participants whose PAI indicated CT, we compared the actual follow-up outcomes (AUC's) of those who received CT (indicated) versus those who received IPT (not-indicated). Likewise, for participants whose PAI indicated IPT, we compared actual follow-up outcomes (AUC's) of those who received IPT with those who received CT. We repeated these PAI-indicated CT and IPT comparisons in the subset of participants with the highest $60 \%$ of the PAI scores. Finally, we compared the long-term PAI score with the previously reported post-treatment PAI score for each individual, by comparing treatment recommendations ( $\chi^{2}$-test) and the magnitude of the predicted advantage (correlations). Since a completer subset of the study sample was used to build the post-treatment PAI, we limited this comparison to this smaller subset of individuals ( $n=134$, Huibers et al., 2015). For all comparisons, the follow-up AUC's were converted to "average follow-up BDI-II scores" across the 17-month period by dividing the AUC by time in months. Since the AUC and the "average BDI-II score" are interchangeably, we choose to use the latter one (labeled as "follow-up BDI-II scores/follow-up depression severity") for the remainder of this paper, to enhance interpretation and readability of the results. 


\subsubsection{Testing robustness of variable selection and model fitting}

For the two-step machine learning approach and model fitting we used the full sample. Although we applied a cross-validation method to compute the PAl scores, it is still possible that they may be inflated due to double-dipping (i.e., performing variable selection and model fitting in the same sample, Fiedler, 2011; Vul, Harris, Winkielman, \& Pashler, 2009). To examine if this affected the results, we ran secondary analyses repeating the process of variable selection and model fitting to a fivefold held-out sample creating five separate models. The predictions of these models were compared with the actual follow-up outcomes. These evaluations were then compared with the evaluations of the main method. Comparisons of these evaluations indicated the potential influence of overfitting, the method's robustness and the potential for out-of-sample predictions.

\section{Results}

\subsection{Variable description and missing data}

Table 8.1 presents the differences between treatment groups on the 38 pretreatment variables. On average, participants who received CT had a higher number of comorbid axis II disorders and a lower number of axis II traits as compared to IPT $(t=2.00, d f=144, p=0.047$ and $t=2.31, d f=144, p=0.02$ for disorders and traits respectively). The other pre-treatment variables did not differ significantly between CT and IPT. A total of 25 observations of all 38 variables were missing (0.4\%). On the $\operatorname{BDI}-I I(7,8,9,10,11,12$ and 24 months), 164 values were missing (15.5\%). Of all participants, 139 individuals (92.1\%) had no missing variables and 119 individuals (78.1\%) had no missing BDI-II scores. Imputation was proven to be accurate when applied to the complete (non-missing) data with artificially produced missing data; the estimated normalized root mean squared error (NRMSE) was 0.09 and the estimated proportion of falsely classified entries (PFC) was 0.02 .

\subsection{Variable selection}

The model-based recursive partitioning technique selected the following four variables (ranked from higher to lower variable importance): number of life events in the past year, number of traumatic events in childhood, score on the SLSC-R (a measure of self-esteem) and parental alcohol abuse (yes/no). Of these variables, three variables were selected in at least $60 \%$ of the bootstrapped samples using the backwards elimination technique: parental alcohol abuse was identified as a predictor and number of life events past year and number of childhood trauma events were selected as moderators. For parental alcohol abuse, the regression coefficients across 
the bootstrapped samples were stable with a positive value in $99.8 \%$ of the samples indicating that a history of parental alcohol abuse was associated with higher BDI-II scores during the 17-month follow-up phase. As illustrated in Figure 8.1, individuals with more recent life events were more likely to have lower overall follow-up BDI-II scores in CT as compared to IPT. Results of the Johnson-Neyman technique indicated that this between-treatment difference was significant for individuals with two or more life events. In Figure 8.2 the moderator effect of childhood trauma events is illustrated: individuals with a history of traumatic childhood events were estimated to have lower follow-up BDI-II scores in CT relatively to IPT. This difference was significant for individuals with one or more traumatic childhood events as indicated by the Johnson-Neyman findings.

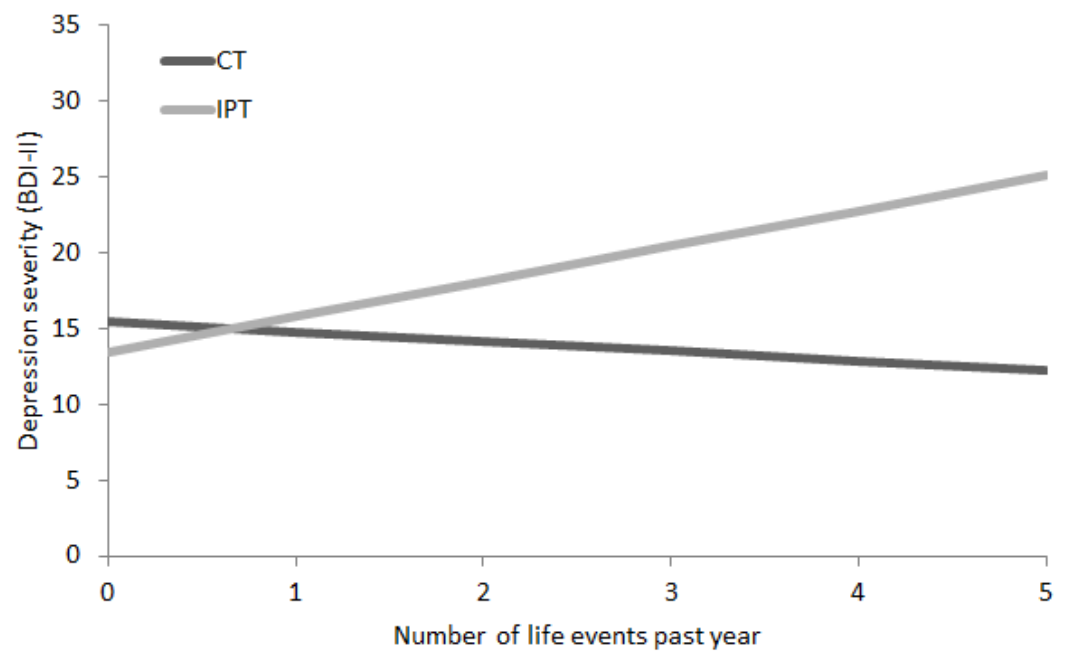

Figure 8.1: Regression-based estimated means of the average follow-up BDI-II scores (measured at 7, 8, 9, $10,11,12$ and 24 months) as a function of number of life-events.

Note: These estimates are based on the final regression model with the other model values set to sample mean. Sample description: 0 life events $(n=33), 1$ life event $(n=38), 2$ life events $(n=32), 3$ life events $(n=32)$, 4 life events $(n=11), 5$ life events $(n=4), 6$ life events $(n=1)$. BDI-II, Beck Depression Inventory, Second Edition. 


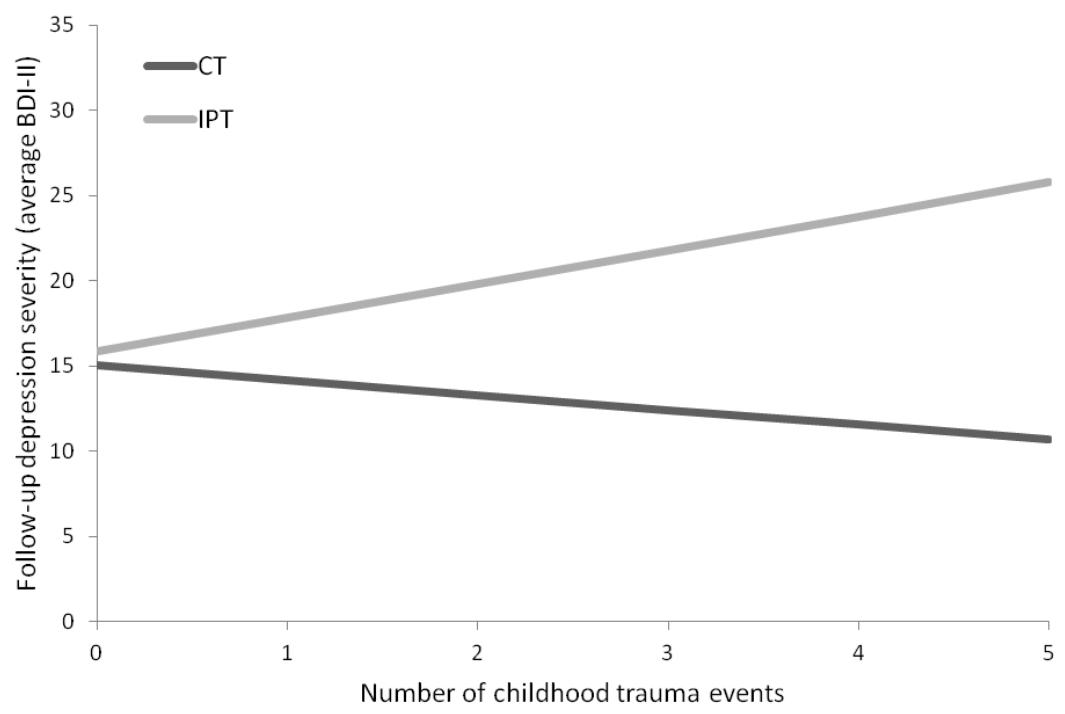

Figure 8.2: Regression-based estimated means of the average follow-up BDI-II scores as a function of number of childhood trauma events.

Note: These estimates are based on the final regression model with the other model values set to sample mean. Sample description: 0 childhood trauma events $(n=84), 1$ childhood trauma events $(n=33)$, 2 childhood trauma events ( $n=15), 3$ childhood trauma events $(n=12), 4$ childhood trauma events $(n=4)$, 5 childhood trauma events $(n=3)$. BDI-II, Beck Depression Inventory, Second Edition.

\subsection{The Personalized Advantage Index}

\subsubsection{PAl-indicated vs. PAI non-indicated treatment}

The selected variables were combined into the final regression model: $A U C_{7-24}$ months $=$ $\beta 0+(\beta 1 \times$ parental alcohol abuse $)+(\beta 2 \times$ number of life events past year $)+(\beta 3 \times$ number of childhood trauma events $)+(\beta 4 \times$ number of life events past year $x$ treatment $)+(\beta 3 \times$ number of childhood trauma events $x$ treatment). For each individual, long-term outcomes were predicted for CT and IPT using a 5-fold cross validation, and with these predictions individual PAI scores were calculated. A total of 74 individuals had been assigned, by chance, to their PAl-indicated treatment, and 77 received, by chance, their PAI non-indicated treatment. Although the average followup BDI-II scores were lower for those who received their indicated treatment, this difference was not significant (indicated treatment=14.5, non-indicated treatment=17.2, $t=1.39, d f=149, p=0.17$ ). The effect size estimate (Cohen's $d$ ) of this difference was 0.23 . Among those with the highest $60 \%$ PAl scores, 47 individuals received their $\mathrm{PAI}$-indicated treatment and 44 individuals received their PAI nonindicated treatment. Mean follow-up BDI-II scores differed significantly between 
these groups (indicated treatment=13.2, non-indicated treatment=18.2, $t=2.22, d f=89$, $p=0.03$ ), with an effect size estimate of 0.47 .

\subsubsection{Individuals with a $P A I$ indicating $C T$}

As shown in Figure 8.3, for individuals whose PAI indicated CT as the optimal treatment, those who received $\mathrm{CT}(\mathrm{n}=43)$ reported lower follow-up BDI-II scores as compared to those who were allocated to IPT ( $n=44$; indicated treatment $=14.4$, nonindicated treatment $=19.8, t=1.95, d f=85, p=0.05$, Cohen's $d=0.42$ ). As shown in Figure 8.4 , among the subset of individuals with a top $60 \%$ absolute value on the PAI, the difference in observed follow-up BDI-II scores was higher for those with a PAIindicated $\mathrm{CT}$, with lower follow-up depression severity for individuals randomized to CT $(n=25)$ as compared to those assigned to IPT $(n=22$, indicated treatment=11.1, nonindicated treatment $=22.3, t=3.56, d f=45, p<0.001$, Cohen's $d=1.04$ ).

\subsubsection{Individuals with a PAI indicating IPT}

As illustrated in Figure 8.3, for those with a PAI indicating IPT, there was no significant difference in follow-up BDI-II scores between the individuals who were randomized to IPT ( $n=31$ ) versus CT ( $n=33$; indicated treatment=14.7, non-indicated treatment $=13.7$, $t=0.43, d f=62, p=0.67$, Cohen's $d=-0.11$ ). For the IPT-indicated individuals within the top $60 \%$ of PAI values, there was no significant difference between those receiving IPT $(n=22)$ versus those receiving CT $(n=22)$ (indicated treatment $=15.6$, non-indicated treatment $=14.1, t=0.52, d f=42, p=0.61$, Cohen's $d=-0.16$ ).

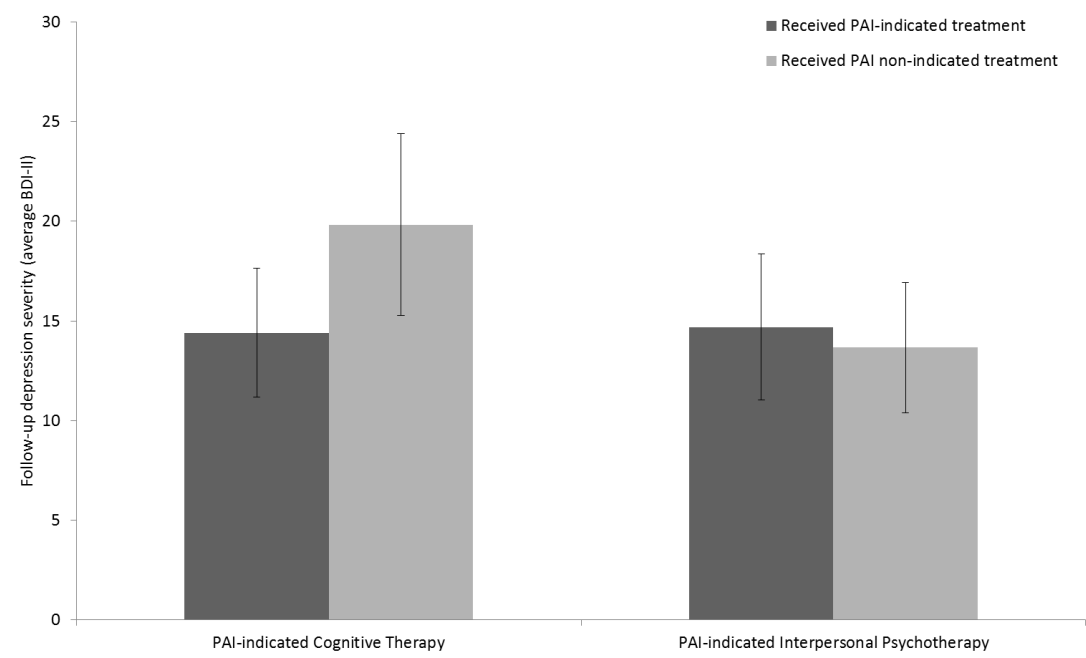

Figure 8.3: Comparison of the observed mean follow-up BDI-scores for individuals randomly assigned to their PAI-indicated optimal treatment versus their PAI-indicated non-optimal treatment. BDI-II, Beck Depression Inventory, Second Edition. 


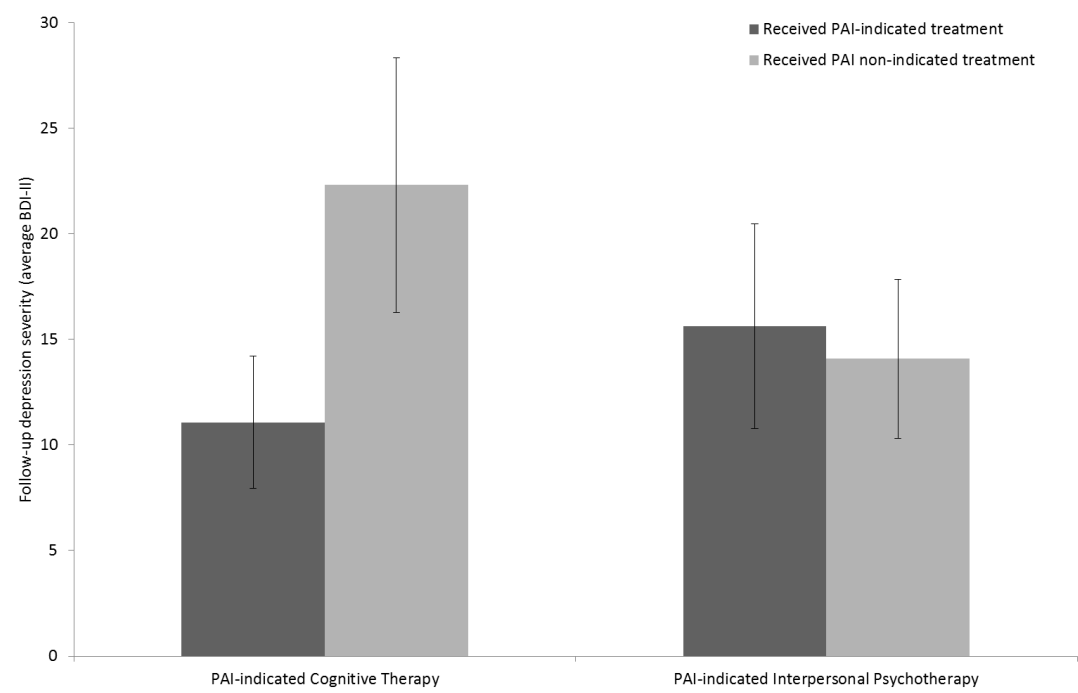

Figure 8.4: Subset of the sample with the top 60\% PAI magnitude: comparison of the observed mean followup BDI-scores for individuals randomly assigned to their PAI-indicated optimal treatment versus their PAIindicated non-optimal treatment. BDI-II, Beck Depression Inventory, Second Edition.

\subsubsection{Long-term PAl vs. post-treatment PAI}

Long-term PAI scores were then compared to post-treatment PAI scores for each individual. The magnitude of the predictive advantage was not very consistent between long-term and post-treatment PAI scores, as indicated by a weak correlation (corr. = 0.33). Of the 76 individuals with a long-term PAI indicating CT, $46(62.2 \%)$ had a post-treatment PAI indicating CT. Of the 58 individuals with a long-term PAI indicating IPT, $43(74,1 \%)$ had a post-treatment PAI indicating IPT.

\subsection{Testing robustness of variable selection and model fitting}

A secondary analysis was performed to examine the long-term PAI scores that would be obtained without "double-dipping" during the variable selection stage (i.e., performing variable selection as well as weight setting in cross-validation folds, rather than performing variable selection in the full sample followed by weight setting in cross-validation folds). This analysis yielded results that were quite similar to the primary analysis. Mean follow-up BDI-II scores for individuals with a PAI-indicated treatment $(n=75)$ versus a PAI non-indicated treatment differed at the level of a nonsignificant trend $(n=76$, indicated treatment=14.0, non-indicated treatment $=17.8$, $t=1.95, d f=149, p=0.05$ ) with an effect size of 0.32 . Similar to the primary analysis, this difference was more pronounced among those with the highest $60 \%$ PAl scores (mean 
follow-up BDI-II scores indicated treatment $(n=46)=13.7$, non-indicated treatment $(n=45)=19.9, t=2.33, d f=89, p=0.02)$, with an effect size of 0.49 .

\section{Discussion}

The aim of the current study was to replicate and extend the PAI method to long-term depression outcomes for CT and IPT for MDD. Using state-of-the art variable selection techniques, one predictor (parental alcohol abuse) and two moderators (life events past year and childhood maltreatment) for long-term depression outcome following CT and IPT were identified. PAI scores were then computed for each individual based on the final model including the selected predictor and moderators using a crossvalidation approach. PAI scores were evaluated by examining the observed follow-up depression severity scores, and by comparing the long-term PAl scores with the posttreatment PAI scores (Huibers et al., 2015). Overall, there was a small difference (2.7 points on the BDI-II) in observed depression severity for those assigned to their PAl-indicated treatment (lower follow-up depression severity) as compared to those assigned to their PAI non-indicated treatment (higher follow-up depression severity). As expected, this difference was more pronounced and statistically significant for individuals with a top $60 \%$ PAI score (5 points on the BDI-II). Notably, this difference was only present in individuals who were recommended to receive $\mathrm{CT}$, whereas no mean differences were found for individuals recommended to receive IPT. Individual treatment recommendations and predicted advantages from the long-term PAI scores and the post-treatment were correlated, but only moderately.

\subsection{Predictors and moderators}

In the current study we identified parental alcohol abuse as a predictor, and recent life events and childhood maltreatment as moderators of long-term outcome. Parental alcohol abuse was associated with an unfavorable 17-month follow-up, irrespectively of the treatment received. This finding is in line with research in adult children of alcoholics that reported an association between parental alcohol abuse and depressive mood (Kelley et al., 2010; Klostermann et al., 2011), and mood disorders (Cuijpers, Langendoen, \& Bijl, 1999), although there is evidence that this association is mediated by adverse childhood experiences (Anda et al., 2002).

An increasing number of life events in the year before the start of therapy was associated higher follow-up depression severity in IPT as compared to CT. This variable was also identified as one of the six moderators of the post-treatment PAI of the same study sample, with lower post-treatment depression severity in CT as compared to IPT (Huibers et al., 2015). In a previous study, a tendency was found for individuals with severe negative life events prior to their onset of depression to 
respond better to IPT than to CBT. However, findings of that same study indicated that response to treatment in individuals with severe negative life events prior to their depression treatment was superior in both CBT and IPT, relative to antidepressant medication (Bulmash, Harkness, Stewart, \& Bagby, 2009).

The number of childhood trauma events was associated with an unfavorable 17 month follow-up in IPT relative to CT. Differential treatment outcomes for individuals with a history of childhood maltreatment have been described in previous studies (Asarnow et al., 2009; Barbe, Bridge, Birmaher, Kolko, \& Brent, 2004; Harkness, Bagby, \& Kennedy, 2012; Lewis et al., 2010; Nemeroff et al., 2003). In line with the current findings, Harkness et al. (2012) reported lower response rates in IPT compared to cognitive behavioral therapy (CBT) and antidepressant medication for individuals with childhood trauma. However, this differential effect did not sustain throughout a 12-month follow-up phase in that sample. In addition, previous studies comparing $\mathrm{C}(\mathrm{B}) \mathrm{T}$ to systemic behavioral family therapy, nondirective supportive therapy (Barbe et al., 2004) or antidepressant medication (Asarnow et al., 2009; Lewis et al., 2010) reported relatively poorer response rates in the $C(B) T$ condition for adolescents with a history of childhood trauma.

In previous randomized trials comparing CT and IPT head-to-head, various predictors and moderators of post-treatment outcome were identified (Carter et al., 2011; Joyce et al., 2007; Luty et al., 2007; Mulder, Boden, Carter, Luty, \& Joyce, 2017; Ryder, Quilty, Vachon, \& Bagby, 2010; Sotsky et al., 1991). Only one study by Mulder et al. (2017) also identified predictors and moderators of long-term outcomes during maintenance CT and IPT following acute phase treatment. The findings of this study were not in line with our results: no significant moderators were identified, and personality variables were identified as significant predictors.

\subsection{Evaluating the long-term PAI}

After the variable selection procedure, the three variables were combined in a final model and individual PAI scores were calculated. For those assigned to their PAIindicated treatment, observed follow-up depression severity was non-significantly lower as compared to individuals randomized to their PAI non-indicated treatment. Similar to DeRubeis et al. (2014), for individuals that were estimated to have a relatively stronger need for a specific treatment (the top 60\% PAIs), the observed depression severity scores of individuals receiving their PAl-indicated treatment were significantly lower than for those that received their PAI non-indicated treatment. The mean difference of this top $60 \%$ subset was 5 points on the BDI-II, which corresponds to a clinical meaningful difference (Hiroe et al., 2005). Interestingly, further analyses showed that this difference was primarily due to the outcomes observed in individuals whose PAl indicated CT. This finding can be understood by examining the relationships obtained with the individual variables in the final PAI model. As illustrated in Figure 
8.3 and Figure 8.4, each of the two moderators produced an ordinal pattern. One can interpret these moderator effects as follows: when an individual had two or more pretreatment life events and/or one or more events of childhood maltreatment, CT would be indicated, whereas individuals with one or no life events and no childhood trauma have no indication of a meaningful difference between CT and IPT (Cohen \& DeRubeis 2018). These moderator effects and the differential performance of the PAI for CT versus IPT indicate a specific benefit of CT for a subgroup of individuals who suffered from childhood maltreatment events and recently experienced significant life events, whereas for the remainder of the individuals no differential effect was observed. In clinical words, the advantage of CT over IPT only emerges among individuals with more complex life stories. Two possible explanations for these findings are that the more complex cases require a more active and structured type of therapy in which the therapist takes a more directive role, and the pivotal role of previous life experiences in the therapeutic procedure of cognitive restructuring of thoughts and schemas that lies at the heart of CT (whereas IPT, as practiced in this trial, only focused predominantly on the present).

\subsection{Long-term PAI versus post-treatment PAI comparison}

The comparison between long-term PAI scores and post-treatment PAI scores (Huibers et al., 2015) indicated different treatment recommendations with different predicted advantages. Only the number of life events prior to treatment was a shared moderator. In addition, the final model of the post-treatment PAI included a higher number of predictors (gender, employment status, anxiety, personality disorder and quality of life) and moderators (somatic complaints, cognitive problems, paranoid symptoms, interpersonal self-sacrificing, attributional style and number of life events, Huibers et al., 2015) as compared to the model of the long-term PAI. There are several possible reasons for the lack of overlap between the post-treatment PAI and the longterm PAI. First, the post-treatment PAI and long-term PAl predicted two different types of outcomes: post-treatment depression severity versus an aggregated measure of follow-up depression severity. One could argue that these two outcomes represent two different phenomena with different combinations of moderators involved. Second, the time span between the pre-treatment variables and the predicted outcome is larger for the long-term PAI relatively to the post-treatment PAI. With this longer time period, relatively weaker variables lose their predictive power, resulting in fewer predictors and moderators for the long-term PAI. Third, for the variable selection procedure, different study samples were used for the long-term PAI ( $n=151$; intention to treat imputed dataset) and the post-treatment PAI ( $n=134$; only nonmissing post-treatment BDI-II scores). Finally, different variable selection approaches were applied: a modified domain approach for the post-treatment PAI and a two-step machine learning approach for the long-term PAI. These different variable selection 
approaches reflect the heterogeneity of statistical approaches due to rapid developments in this area of research (Cohen \& DeRubeis 2018). In sum, the fact that the short and long-term PAl advice did not overlap for each individual can be explained by a variety of reasons, and should not come as a surprise. Insofar as the inconsistency between short-and long-term indications are not an artifact but instead, reflect different influences on short and long-term outcomes, this presents a problem that would need to be resolved if such work is to inform clinical practice. In other words, if different therapies are needed for optimal outcomes at different stages of MDD (i.e., post-treatment and the longer term) for the individual patient, this poses a real dilemma in the clinician's office when selecting a treatment.

\subsection{Limitations}

The current study has limitations. First, the long-term PAI was not externally validated by applying it on an independent dataset. Although we used a cross-validation approach to compute the regression parameters of the final model, we used the full study sample for the variable selection procedure. To examine potential bias, we did a secondary analysis rerunning the complete process with 5-folds, producing five models that estimated the PAls of individuals whose data were not used in any way to develop the algorithm that yielded the PAls. This additional analysis produced very similar outcomes to those obtained in our primary analysis. Nevertheless, without external validation efforts, the degree to which this model can be generalized to new samples, populations and treatment settings is yet unknown. Second, although we began our variable selection with 69 variables, it is still possible that relevant predictors or moderators were not included in our study. Third, individuals were allowed to seek for additional treatment during follow-up. However, this did not significantly affect the long-term outcomes (Lemmens et al., 2019). Finally, our sample size of 151 individuals might be insufficient according to recent suggestions of sample size requirements for multivariate prediction models based on a single simulation study (Luedtke, Sadikova, \& Kessler, 2019), although more research in this new area is needed to reach a final conclusion on this.

\subsection{Future directions}

Despite these limitations, the current findings hold a promise for the PAI approach for longitudinal predictions for two treatments that are, on average, equally effective. Moving beyond post-treatment estimates, this type of PAl could guide treatment selection focusing on keeping a (formerly depressed) individual well over the long term. However, the long-term PAI is not ready for implementation. First of all, external validation in different populations with different treatment settings and time frames using prospective designs is needed. Second, collaboration of different 
disciplinary lines to extend the number of potential predictors and moderators is of importance, combining biomarkers, dynamic assessments, clinical-rated and selfreport measures into one algorithm. Third, consideration of cost-effectiveness and feasibility of potential predictors and moderators should be a necessary part of new study designs (Kessler, 2018). Fourth, the use pooled datasets should be considered to have adequate power to develop multivariate prescriptive prediction models (Luedtke et al., 2019). Finally, methods that combine PAl predictions prior to treatment with updated predictions during treatment need to be studied further (e.g., Lutz, Zimmermann, Müller, Deisenhofer, \& Rubel, 2017). Ultimately, these efforts will hopefully lead to guided clinical decision-making, reducing the number of treatments needed to acquire and maintain remission.

Supplementary data associated with this article can be found in the online version at https://doi.org/10.1017/S0033291719003192. 


\section{References}

Anda, R. F., Whitfield, C. L., Felitti, V. J., Chapman, D., Edwards, V. J., Dube, S. R., \& Williamson, D. F. (2002). Adverse childhood experiences, alcoholic parents, and later risk of alcoholism and depression. Psychiatric services, 53(8), 1001-1009.

Asarnow, J. R., Emslie, G., Clarke, G., Wagner, K. D., Spirito, A., Vitiello, B., . . . Birmaher, B. (2009). Treatment of selective serotonin reuptake inhibitor-Resistant depression in adolescents: Predictors and moderators of treatment response. Journal of the American Academy of Child \& Adolescent Psychiatry, 48(3), 330-339.

Austin, P. C., \& Tu, J. V. (2004). Bootstrap methods for developing predictive models. The American Statistician, 58(2), 131-137.

Barbe, R. P., Bridge, J. A., Birmaher, B., Kolko, D. J., \& Brent, D. A. (2004). Lifetime history of sexual abuse, clinical presentation, and outcome in a clinical trial for adolescent depression. The Journal of clinical psychiatry.

Barber, J. P., \& Muenz, L. R. (1996). The role of avoidance and obsessiveness in matching patients to cognitive and interpersonal psychotherapy: empirical findings from the treatment for depression collaborative research program. J Consult Clin Psychol, 64(5), 951-958. Retrieved from http://www.ncbi.nlm.nih.gov/entrez/query.fcgi?cmd=Retrieve\&db=PubMed\&dopt=Citation\&list_uids $=8916624$.

Beck, A., Rush, A., Shaw, B., \& Emery, G. (1979). Cognitive therapy of depression. 1979. New York: Guilford Press Google Scholar.

Beck, A. T., Steer, R., \& Brown, G. K. (1996). Beck Depression Inventory II: Manual. Boston: Harcourt Brace.

Beck, A. T., \& Steer, R. A. (1988). Manual for the Beck Hopelessness Scale. San Antonio: Psychological Corporation.

Bulmash, E., Harkness, K. L., Stewart, J. G., \& Bagby, R. M. (2009). Personality, stressful life events, and treatment response in major depression. Journal of consulting and clinical psychology, 77(6), 1067.

Carter, J. D., Luty, S. E., McKenzie, J. M., Mulder, R. T., Frampton, C. M., \& Joyce, P. R. (2011). Patient predictors of response to cognitive behaviour therapy and interpersonal psychotherapy in a randomised clinical trial for depression. J Affect Disord, 128(3), 252-261. Retrieved from http://www.ncbi.nlm.nih.gov/pubmed/20674982. doi:10.1016/j.jad.2010.07.002

Cohen, L., Van den Bout, J., Kramer, W., \& Van Vliet, T. (1986). A Dutch attributional style questionnaire: Psychometric properties and findings of some Dutch-American differences. Cognitive therapy and research, 10(6), 665-669.

Cohen, Z. D., \& DeRubeis, R. J. (2018). Treatment selection in depression. Annual review of clinical psychology, 14, 209-236.

Cuijpers, P., Andersson, G., Donker, T., \& van Straten, A. (2011). Psychological treatment of depression: results of a series of meta-analyses. Nordic journal of psychiatry, 65(6), 354-364.

Cuijpers, P., Langendoen, Y., \& Bijl, R. V. (1999). Psychiatric disorders in adult children of problem drinkers: prevalence, first onset and comparison with other risk factors. Addiction, 94(10), 1489-1498.

de Graaf, L. E., Roelofs, J., \& Huibers, M. J. (2009). Measuring Dysfunctional Attitudes in the General Population: The Dysfunctional Attitude Scale (form A) Revised. Cognit Ther Res, 33(4), 345-355. Retrieved from http://www.ncbi.nlm.nih.gov/pubmed/19623267. doi:10.1007/s10608-009-9229-y

Deisenhofer, A. K., Delgadillo, J., Rubel, J. A., Böhnke, J. R., Zimmermann, D., Schwartz, B., \& Lutz, W. (2018). Individual treatment selection for patients with posttraumatic stress disorder. Depression and anxiety, 35(6), 541-550.

Derogatis, L. R., \& Melisaratos, N. (1983). The Brief Symptom Inventory: an introductory report. Psychol Med, 13(3), 595-605. Retrieved from http://www.ncbi.nlm.nih.gov/pubmed/6622612.

DeRubeis, R. J., Cohen, Z. D., Forand, N. R., Fournier, J. C., Gelfand, L. A., \& Lorenzo-Luaces, L. (2014). The Personalized Advantage Index: translating research on prediction into individualized treatment recommendations. A demonstration. PLoS One, 9(1), e83875.

Fiedler, K. (2011). Voodoo correlations are everywhere-not only in neuroscience. Perspectives on psychological science, 6(2), 163-171. 
First, M., Spitzer, R., Gibbon, M., \& Williams, J. (1995). Structured Clinical Interview for DSM-IV Axis I Disorders (SCID-I). New York: Biometrics Research Department, New York State Psychiatric Institute.

First, M. B., Gibbon, M., Spitzer, R. L., Williams, J. B. W., \& Benjamin, L. S. (1997). Structured Clinical Interview for DSM-IV Axis II Personality Disorders (SCID-II) Washington, D.C: American Psychiatric Press.

Fournier, J. C., DeRubeis, R. J., Shelton, R. C., Hollon, S. D., Amsterdam, J. D., \& Gallop, R. (2009). Prediction of response to medication and cognitive therapy in the treatment of moderate to severe depression. $J$ Consult Clin Psychol, 77(4), 775-787. Retrieved from http://www.ncbi.nlm.nih.gov/pubmed/ 19634969. doi:10.1037/a0015401

Garge, N. R., Bobashev, G., \& Eggleston, B. (2013). Random forest methodology for model-based recursive partitioning: the mobForest package for R. BMC bioinformatics, 14(1), 125.

Harkness, K. L., Bagby, R. M., \& Kennedy, S. H. (2012). Childhood maltreatment and differential treatment response and recurrence in adult major depressive disorder. Journal of consulting and clinical psychology, 80(3), 342.

Hiroe, T., Kojima, M., Yamamoto, I., Nojima, S., Kinoshita, Y., Hashimoto, N., . . Furukawa, T. A. (2005). Gradations of clinical severity and sensitivity to change assessed with the Beck Depression InventoryII in Japanese patients with depression. Psychiatry research, 135(3), 229-235.

Horowitz, L. M., Rosenberg, S. E., Baer, B. A., Ureno, G., \& Villasenor, V. S. (1988). Inventory of interpersonal problems: psychometric properties and clinical applications. J Consult Clin Psychol, 56(6), 885-892. Retrieved from http://www.ncbi.nlm.nih.gov/pubmed/3204198.

Huibers, M. J., Cohen, Z. D., Lemmens, L. H., Arntz, A., Peeters, F. P., Cuijpers, P., \& DeRubeis, R. J. (2015). Predicting Optimal Outcomes in Cognitive Therapy or Interpersonal Psychotherapy for Depressed Individuals Using the Personalized Advantage Index Approach. PLoS One, 10(11), e0140771. Retrieved from http://www.ncbi.nlm.nih.gov/pubmed/26554707. doi:10.1371/journal.pone.0140771

Jakobsen, J. C., Hansen, J. L., Simonsen, S., Simonsen, E., \& Gluud, C. (2012). Effects of cognitive therapy versus interpersonal psychotherapy in patients with major depressive disorder: a systematic review of randomized clinical trials with meta-analyses and trial sequential analyses. Psychol Med, 42(7), 1343-1357. Retrieved from http://www.ncbi.nlm.nih.gov/entrez/query.fcgi?cmd=Retrieve\&db=PubMed\&dopt= Citation\&list_uids=22051174. doi:S0033291711002236 [pii] 10.1017/S0033291711002236

Johnson, P. O., \& Neyman, J. (1936). Tests of certain linear hypotheses and their application to some educational problems. Statistical research memoirs.

Joyce, P. R., McKenzie, J. M., Carter, J. D., Rae, A. M., Luty, S. E., Frampton, C. M., \& Mulder, R. T. (2007). Temperament, character and personality disorders as predictors of response to interpersonal psychotherapy and cognitive-behavioural therapy for depression. Br J Psychiatry, 190, 503-508. Retrieved from http://www.ncbi.nlm.nih.gov/pubmed/17541110. doi:10.1192/bjp.bp.106.024737

Katsnelson, A. (2013). Momentum grows to make'personalized'medicine more'precise'. In: Nature Publishing Group.

Keefe, J. R., Wiltsey Stirman, S., Cohen, Z. D., DeRubeis, R. J., Smith, B. N., \& Resick, P. A. (2018). In rape trauma PTSD, patient characteristics indicate which trauma-focused treatment they are most likely to complete. Depression and anxiety, 35(4), 330-338.

Kelley, M. L., Braitman, A., Henson, J. M., Schroeder, V., Ladage, J., \& Gumienny, L. (2010). Relationships among depressive mood symptoms and parent and peer relations in collegiate children of alcoholics. American Journal of Orthopsychiatry, 80(2), 204-212.

Kessler, R. C. (2018). The potential of predictive analytics to provide clinical decision support in depression treatment planning. Current opinion in psychiatry, 31(1), 32-39.

Kim, T. T., Dufour, S., Xu, C., Cohen, Z. D., Sylvia, L., Deckersbach, T., . . Nierenberg, A. A. (2019). Predictive modeling for response to lithium and quetiapine in bipolar disorder. Bipolar disorders.

Klerman, G. L., Weissman, M. M., Rounsaville, B. J., \& Chevron, E. S. (1984). Interpersonal psychotherapy for depression. New York: Basis Books.

Klostermann, K., Chen, R., Kelley, M. L., Schroeder, V. M., Braitman, A. L., \& Mignone, T. (2011). Coping behavior and depressive symptoms in adult children of alcoholics. Substance Use \& Misuse, 46(9), 1162-1168. 
Kraemer, H. C. (2013). Discovering, comparing, and combining moderators of treatment on outcome after randomized clinical trials: a parametric approach. Statistics in medicine, 32(11), 1964-1973.

Lemmens, L. H. J. M., Arntz, A., Peeters, F. P. M. L., Hollon, S. D., Roefs, A., \& Huibers, M. J. H. (2015). Clinical effectiveness of cognitive therapy $v$. interpersonal psychotherapy for depression: results of a randomized controlled trial. Psychological Medicine, 45(10), 2095-2110.

Lemmens, L. H. J. M., van Bronswijk, S. C., Peeters, F. P. M. L., Arntz, A., Hollon, S. D., \& Huibers, M. J. H. (2019). Long-term outcomes of acute treatment with cognitive therapy $v$. interpersonal psychotherapy for adult depression: follow-up of a randomized controlled trial. Psychological Medicine, 1-9.

Lewis, C. C., Simons, A. D., Nguyen, L. J., Murakami, J. L., Reid, M. W., Silva, S. G., \& March, J. S. (2010). Impact of childhood trauma on treatment outcome in the Treatment for Adolescents with Depression Study (TADS). Journal of the American Academy of Child \& Adolescent Psychiatry, 49(2), 132-140.

Lorenzo-Luaces, L., DeRubeis, R. J., van Straten, A., \& Tiemens, B. (2017). A prognostic index (PI) as a moderator of outcomes in the treatment of depression: A proof of concept combining multiple variables to inform risk-stratified stepped care models. Journal of affective disorders, 213, 78-85.

Luedtke, A., Sadikova, E., \& Kessler, R. C. (2019). Sample size requirements for multivariate models to predict between-patient differences in best treatments of major depressive disorder. Clinical Psychological Science, 7(3), 445-461.

Luty, S. E., Carter, J. D., McKenzie, J. M., Rae, A. M., Frampton, C. M., Mulder, R. T., \& Joyce, P. R. (2007). Randomised controlled trial of interpersonal psychotherapy and cognitive-behavioural therapy for depression. The British Journal of Psychiatry, 190(6), 496-502.

Lutz, W., Saunders, S. M., Leon, S. C., Martinovich, Z., Kosfelder, J., Schulte, D., . . . Tholen, S. (2006). Empirically and clinically useful decision making in psychotherapy: Differential predictions with treatment response models. Psychological Assessment, 18(2), 133.

Lutz, W., Zimmermann, D., Müller, V. N., Deisenhofer, A.-K., \& Rubel, J. A. (2017). Randomized controlled trial to evaluate the effects of personalized prediction and adaptation tools on treatment outcome in outpatient psychotherapy: study protocol. BMC psychiatry, 17(1), 306.

Mulder, R., Boden, J., Carter, J., Luty, S., \& Joyce, P. (2017). Ten month outcome of cognitive behavioural therapy v. interpersonal psychotherapy in patients with major depression: a randomised trial of acute and maintenance psychotherapy. Psychological Medicine, 47(14), 2540-2547.

Mundt, J. C., Marks, I. M., Shear, M. K., \& Greist, J. H. (2002). The Work and Social Adjustment Scale: a simple measure of impairment in functioning. $\mathrm{Br} J$ Psychiatry, 180, 461-464. Retrieved from http://www.ncbi.nlm.nih.gov/pubmed/11983645.

Nemeroff, C. B., Heim, C. M., Thase, M. E., Klein, D. N., Rush, A. J., Schatzberg, A. F., . . Dunner, D. L. (2003). Differential responses to psychotherapy versus pharmacotherapy in patients with chronic forms of major depression and childhood trauma. Proceedings of the National Academy of Sciences, 100(24), 14293-14296.

Niles, A. N., Loerinc, A. G., Krull, J. L., Roy-Byrne, P., Sullivan, G., Sherbourne, C. D., . . . Craske, M. G. (2017a). Advancing personalized medicine: application of a novel statistical method to identify treatment moderators in the coordinated anxiety learning and management study. Behavior therapy, 48(4), 490-500.

Niles, A. N., Wolitzky-Taylor, K. B., Arch, J. J., \& Craske, M. G. (2017b). Applying a novel statistical method to advance the personalized treatment of anxiety disorders: A composite moderator of comparative drop-out from CBT and ACT. Behaviour research and therapy, 91, 13-23.

Peterson, C., Semmel, A., Von Baeyer, C., Abramson, L. Y., Metalsky, G. I., \& Seligman, M. E. (1982). The attributional style questionnaire. Cognitive therapy and research, 6(3), 287-299.

Picard, R. R., \& Cook, R. D. (1984). Cross-validation of regression models. Journal of the American Statistical Association, 79(387), 575-583.

Raes, F., Hermans, D., \& Eelen, P. (2003). Kort instrumenteel De Nederlandstalige versie van de Ruminative Response Scale (RRS-NL) en de Rumination on Sadness Scale (RSS-NL). Gedragstherapie.

Rizopoulos, D., \& Rizopoulos, M. D. (2009). Package 'bootStepAIC'. 
Rush, A. J., Trivedi, M. H., Wisniewski, S. R., Nierenberg, A. A., Stewart, J. W., Warden, D., . . Lebowitz, B. D. (2006). Acute and longer-term outcomes in depressed outpatients requiring one or several treatment steps: a STAR* D report. American Journal of Psychiatry, 163(11), 1905-1917.

Ryder, A. G., Quilty, L. C., Vachon, D. D., \& Bagby, R. M. (2010). Depressive personality and treatment outcome in major depressive disorder. Journal of personality disorders, 24(3), 392-404.

Simon, G. E., \& Perlis, R. H. (2010). Personalized medicine for depression: can we match patients with treatments? American Journal of Psychiatry, 167(12), 1445-1455.

Smagula, S. F., Wallace, M. L., Anderson, S. J., Karp, J. F., Lenze, E. J., Mulsant, B. H., . . Lotrich, F. E. (2016). Combining moderators to identify clinical profiles of patients who will, and will not, benefit from aripiprazole augmentation for treatment resistant late-life major depressive disorder. Journal of psychiatric research, 81, 112-118.

Sotsky, S. M., Glass, D. R., Shea, M. T., Pilkonis, P. A., Collins, J. F., Elkin, I., . . Oliveri, M. E. (1991). Patients predictors of response to psychotherapy and pharmacotherapy: findings in the NIMH treatment of depression collaborative research program. American Journal of Psychiatry, 148(8), 997-1008.

Stekhoven, D. J., \& Bühlmann, P. (2012). MissForest-non-parametric missing value imputation for mixedtype data. Bioinformatics, 28(1), 112-118.

Strobl, C., Boulesteix, A.-L., Kneib, T., Augustin, T., \& Zeileis, A. (2008). Conditional variable importance for random forests. BMC bioinformatics, 9(1), 307.

Tafarodi, R. W., \& Swann, W. B. (2001). Two-dimensional self-esteem: Theory and measurement. Personality and individual Differences, 31(5), 653-673.

Vandromme, H., Hermans, D., Spruyt, A., \& Eelen, P. (2007). Dutch translation of the Self-Liking/SelfCompetence Scale-Revised: A confirmatory factor analysis of the two-factor structure. Personality and individual Differences, 42(1), 157-167.

Vittengl, J. R., Clark, L. A., Thase, M. E., \& Jarrett, R. B. (2017). Initial Steps to inform selection of continuation cognitive therapy or fluoxetine for higher risk responders to cognitive therapy for recurrent major depressive disorder. Psychiatry research, 253, 174-181.

Vul, E., Harris, C., Winkielman, P., \& Pashler, H. (2009). Puzzlingly high correlations in fMRI studies of emotion, personality, and social cognition. Perspectives on psychological science, 4(3), 274-290.

Waljee, A. K., Mukherjee, A., Singal, A. G., Zhang, Y., Warren, J., Balis, U., . . . Higgins, P. D. (2013). Comparison of imputation methods for missing laboratory data in medicine. BMJ open, 3(8), e002847.

Wallace, M. L., Frank, E., \& Kraemer, H. C. (2013). A novel approach for developing and interpreting treatment moderator profiles in randomized clinical trials. JAMA psychiatry, 70(11), 1241-1247.

Webb, C. A., Trivedi, M. H., Cohen, Z. D., Dillon, D. G., Fournier, J. C., Goer, F., . . . Parsey, R. (2019). Personalized prediction of antidepressant v. placebo response: evidence from the EMBARC study. Psychological Medicine, 1-10.

Weissman, A. N., \& Beck, A. T. (1978). Development and validation of the Dysfunctional Attitude Scale: A preliminary investigation.

Zilcha-Mano, S., Keefe, J. R., Chui, H., Rubin, A., Barrett, M. S., \& Barber, J. P. (2016). Reducing Dropout in Treatment for Depression: Translating Dropout Predictors Into Individualized Treatment Recommendations. The Journal of clinical psychiatry, 77(12), e1584-e1590. 

S. van Bronswijk, N. Moopen, L. Beijers, H. Ruhé, F. Peeters

- Psychological Medicine 2019, 49.3, 366-379

\section{Chapter}




\section{EFFECTIVENESS \\ OF \\ PSYCHOTHERAPY \\ FOR TREATMENT- \\ RESISTANT \\ DEPRESSION: \\ A META-ANALYSIS \\ AND META- \\ REGRESSION}




\section{Abstract}

Background

Despite substantial advances in treatment and management strategies for major depression, less than $50 \%$ of patients respond to first-line antidepressant treatment or psychotherapy. Given the growing number of controlled studies of psychotherapy for treatment-resistant depression (TRD) and the preference for psychotherapy of depressed subjects as a treatment option, we conducted a meta-analysis and meta-regression analysis to investigate the effectiveness of psychotherapy for TRD.

Methods

Seven different psychotherapies were studied in 21 trials that included a total of 25 comparisons.

Results

In three comparisons of psychotherapy v. treatment as usual (TAU) we found no evidence to conclude that there is a significant benefit of psychotherapy as compared with TAU. In 22 comparisons of add-on psychotherapy plus TAU vs. TAU only, we found a moderate general effect size of $0.42(95 \% \mathrm{Cl}=0.29$ to 0.54$)$ in favor of psychotherapy plus TAU. The metaregression provided evidence for a positive association between baseline severity as well as group v. individual therapy format with the treatment effect. There was no evidence for publication bias. Most frequent investigated treatments were cognitive behavior therapy, interpersonal psychotherapy, mindfulness-based cognitive therapy, and cognitive behavioral analysis system of psychotherapy.

\section{Conclusions}

Our meta-analysis provides evidence that, in addition to pharmacological and neurostimulatory treatments, the inclusion of add-on of psychotherapy to TAU in guidelines for the treatment of TRD is justified and will provide better outcomes for this difficult-to-treat population. 


\section{Introduction}

Major depressive disorder (MDD) is a highly prevalent and disabling psychiatric condition. Despite substantial advances in treatment and management strategies for MDD, less than $50 \%$ of patients respond to first-line antidepressant treatment or psychotherapy (Cuijpers et al., 2013; Kolovos et al., 2017). MDD that is treatment refractory, mostly described as treatment-resistant depression (TRD), is characterized by marked functional impairment, a large burden on patients and families, and is associated with great direct and indirect healthcare costs (Greden, 2001; Moussavi et al., 2007).

Clinical reviews suggest a prevailing inclination to manage TRD (solely) by pharmacotherapy or other somatic treatments while scarcely mentioning studies into psychotherapy for TRD with little or no discussion of the findings and prospects (e.g., Carvalho, Berk, Hyphantis, \& Mclntyre, 2014; Dold \& Kasper, 2017). This may be imprudent because (i) the likelihood of remission is considerably reduced for individuals who require third or fourth-line antidepressant treatment due to nonresponse in prior steps (Rush et al., 2006), (ii) a majority of depressed individuals seems to prefer psychotherapy over medication (McHugh, Whitton, Peckham, Welge, \& Otto, 2013), and (iii) patients receiving their preferred treatment show better outcomes (Gelhorn, Sexton, \& Classi, 2011; McHugh et al., 2013; Mergl et al., 2011; Swift, Callahan, \& Vollmer, 2011). Given these considerations, there is a need to thoroughly evaluate psychotherapy as a treatment option for TRD and accordingly formulate recommendations for clinical practice.

High-quality studies that specifically sought to examine the effectiveness of psychotherapeutic treatments for TRD are scarce and virtually absent until the beginning of this millennium. In 2002, Stimpson, Agrawal, and Lewis conducted a systematic review of randomized controlled trials (RCTs) for TRD but no psychotherapy studies met their minimal inclusion criteria at that point. Two more recent reviews, based on seven RCTs and eight uncontrolled studies, concluded that psychotherapy either as augmentation or substitute therapy could be an effective and reasonable treatment for TRD (McPherson et al., 2005; Trivedi, Nieuwsma, \& Williams, 2011). The authors emphasized the need for more high-quality, controlled trials to effectively judge the utility of psychotherapy for TRD and guide clinical practice.

Moreover, there is considerable variation in and confusion about definitions of TRD and chronic depression (cMDD) in the literature (Ruhé, van Rooijen, Spijker, Peeters, \& Schene, 2012). As a result, many studies into the psychotherapeutic treatment of CMDD appear to be carried out in clinical samples that in the majority consist of participants that already received one or more failed treatments for the index episode, thereby qualifying them, in fact, as studies of psychotherapy for TRD. 
Given the considerations above and a growing database of randomized controlled studies treating TRD with psychotherapy, we decided to conduct a metaanalysis and meta-regression. For these analyses, we collected all randomized controlled studies of psychotherapy for adult patients with TRD and/or unsuccessfully treated CMDD in which the effectiveness of psychotherapy was examined as either a substitute for or add-on to treatment as usual (TAU; routine treatments such as clinical management and/or the continuation, optimization or next step pharmacotherapy). We hypothesized (i) that switching to psychotherapy is more effective than TAU, and (ii) psychotherapy as an add-on to TAU to be more effective than TAU only.

\section{Methods}

\subsection{Search strategy and study selection}

Two authors (SB and NM) independently performed a comprehensive search through PubMed, Embase and PsychINFO electronic databases combining terms regarding psychotherapy, and TRD/cMDD (online Supplementary Methods I). Publications up until and including 19 December 2016 were reviewed. The search was limited to articles published in English, describing studies with a controlled design in samples of adult participants. References of the selected articles were checked, as well as references of earlier systematic reviews and meta-analyses (Cuijpers et al., 2010a; Kriston, Von Wolff, Westphal, Hölzel, \& Härter, 2014; McPherson et al., 2005; Negt et al., 2016; Spijker, van Straten, Bockting, Meeuwissen, \& van Balkom, 2013; Trivedi et al., 2011).

The same authors independently assessed articles for inclusion by screening titles and abstracts followed by detailed full-text evaluation if necessary. Disagreements between the reviewers were solved through discussion with a third independent reviewer (FP). We included (i) RCTs that examined the effectiveness of psychotherapy for (ii) adults ( $\geqslant 18$ years of age) with TRD by comparing (iii) psychotherapy v. TAU or (iv) add-on psychotherapy and TAU v. TAU only. For this meta-analysis, we defined TRD in line with previous reviews (McPherson et al., 2005; Stimpson et al., 2002; Trivedi et al., 2011), as an individual's failure to respond to at least one adequate trial of antidepressants for the current episode irrespective of duration of the current episode. We decided to apply this broad definition to enable comparisons with previous literature and to include a large body of evidence. A failed response was assumed when study sample descriptions mentioned the inclusion of participants not meeting the criteria for clinically significant response or remission to the previous treatment. For the current meta-analyses, we included cMDD studies if the majority of the sample fulfilled our criterion for TRD. Psychotherapy was defined as a face-to- 
face interaction with a therapist, which was allowed to be delivered either in a group or individual format in both out- and inpatient settings. TAU was defined as treatments that individuals would normally receive in routine (mental) health care, such as clinical management and pharmacotherapy. Pharmacotherapy included continuation, optimization, switching or starting of antidepressant medication. Studies were excluded if they had a maintenance treatment study design or if treatment-resistance was undefined or remained unclear based on the reported information on previously failed response to antidepressant medication.

\subsection{Quality assessment and data extraction}

Two authors (SB and FP) independently evaluated the validity of the studies eligible for the qualitative synthesis. Following recent meta-analyses (Cuijpers et al., 2015; P. Cuijpers et al., 2014), we examined all included studies on four criteria of the 'Risk of bias' assessment tool, developed by the Cochrane Collaboration (Higgins \& Green, 2011): (1) adequate random sequence generation, (2) allocation to treatments by an independent (third) party, (3) blinding of the outcome assessment and (4) the quantity, nature and management of incomplete outcome data. Disagreements between the reviewers were resolved through discussion.

Two authors (NM and LB) independently extracted the data from the included studies. Data extraction was checked and disagreements were resolved by two other reviewers (FP and SB). For the outcome variable, means and standard deviations (SD) of change in depression severity scales from pre- to post-intervention were extracted for both the treatment and TAU condition. Our research questions addressed outcomes of acute-phase treatment specifically. Therefore, the post-treatment endpoint was set a maximum of 16 weeks with a minimum session frequency of once a week. Follow-up measurements were excluded from our analyses. Although the treatment endpoint was set at 16 weeks to target the acute phase, for some studies outcome data were not available at this time point. As a result, the post-intervention depression severity score was assessed at 12.8 weeks on average, varying between 5 and 26 weeks. In addition, we extracted clinical variables, treatment variables and study variables for background information input for sensitivity analyses, and metaregressions (as described in the 'Statistical Analyses' section). Clinical variables included the male/female ratio, the mean age of the sample, mean depression severity at baseline, prior history (number of previous episodes and mean illness duration in months), mean duration of the current episode and the percentage of participants that did not respond to antidepressants or psychotherapy for the current episode prior to the study. Treatment variables included the type of psychotherapy, treatment duration (number of months and number of treatment sessions), individual or group setting, attrition rates, and treatment integrity. Study variables included the 
year the study was conducted, the clinical setting (inpatient or outpatient), and an intention to treat approach for the extracted outcome measures.

For each study, we coded the type of comparison: (1) psychotherapy v. TAU or (2) add-on psychotherapy plus TAU v. TAU. In addition, we specified the type of interventions that were considered TAU. For a few studies comparing the 'add-on psychotherapy plus TAU v. TAU', the TAU interventions were slightly different between the two groups. If so, this was coded during our data extraction.

All corresponding authors were contacted to check the data retrieved during our data extraction and were asked to provide missing data. If those attempts failed and the incomplete data made the calculation of an effect size impossible, studies were excluded from the quantitative but not the qualitative synthesis of evidence.

\subsection{Statistical analyses}

All analyses were done with Stata version 13.1. The primary outcome was the difference between the average depression severity change of treatment and control condition, calculated as Hedges' g effect sizes. If multiple instruments were available to assess symptomatic change, the mean effect size was calculated (Borenstein, Hedges, Higgins, \& Rothstein, 2009). To test whether studies with multiple depression severity scales affected the overall results of the meta-analyses, sensitivity analyses were performed with only studies using one measurement scale. Since most studies had a small sample size, effects sizes were corrected for small sample bias (Hedges' g). If average change scores were not reported, they were calculated using the average pre and post-intervention depression severity score. When unavailable, SD of the change scores were calculated using the SD of the average pre- and post-intervention depression scores and the correlation coefficient between these scores (calculations were based on the formula presented in online Supplementary Methods II). If this correlation coefficient was not reported, we assumed a correlation of $r=0.5$, which was based on data from a recent RCT comparing cognitive therapy and interpersonal psychotherapy for MDD (Lemmens et al., 2015). To examine the impact of this assumption, a sensitivity analyses were performed to test whether a reduced $(r=0.2)$ or increased ( $r=0.8$ ) correlation would change the overall results of the meta-analysis.

Summary effect sizes were pooled using a random-effects model for (i) psychotherapy v. TAU or (ii) add-on psychotherapy plus TAU v. TAU comparison. To test the homogeneity of the effect sizes, the $I^{2}$ statistic was estimated with $95 \%$ confidence intervals (Ioannidis, Patsopoulos, \& Evangelou, 2007). An I value of $>50 \%$ was assumed to be indicative of heterogeneity. When multiple comparisons of one trial were included, sensitivity analyses were performed to test whether this affected the pooled results by only including the comparison with the smallest effect size. In addition, sensitivity analyses on study quality were conducted by limiting the analyses to the studies meeting all four quality criteria with a low-risk score. For the second 
comparison, studies with slightly different TAU interventions between the two groups were excluded to see whether this affected the pooled effect size (additional sensitivity analysis). A similar sensitivity analysis was computed for studies with the inclusion of psychotherapy in the TAU interventions. We assessed publication bias by inspecting funnel plots and examining plot asymmetry using the Egger's test (Egger, Smith, Schneider, \& Minder, 1997).

A meta-regression was conducted in order to relate specific study-level variables to the statistical heterogeneity between the results of the studies (Thompson \& Higgins, 2002). First, we conducted univariate meta-regressions for each of the following a priori selected variables: mean depression severity at baseline, mean illness duration (number of months and number of previous episodes), mean duration of the current episode, the percentage of participants that did not respond to antidepressants for the current episode, mean treatment duration (number of months and number of treatment sessions), attrition rates, the clinical setting (inpatient or outpatient), individual or group format, clinician-rated or self-reported outcomes (or a combination), and whether the extracted outcomes were based on intention to treat data. When variables had at least a $p$ value $<0.10$, they were included in a multivariate meta-regression. Correlations between the variables that were included in the multivariate meta-regression were examined to check if collinearity could influence the results.

\section{Results}

\subsection{Selection and inclusion of trials}

We identified 1044 potentially relevant citations through database searching and 16 additional records through other sources. After removing duplicates and excluding non-relevant citations based on abstract examination, we retrieved 32 full-text papers for further consideration. Finally, 22 trials met our inclusion criteria for the metaanalysis, however, 1 trial was only included in the qualitative analysis as a result of reporting incomplete data. The PRISMA flow chart describing the inclusion process and exclusion reasons is presented in Figure 9.1. 
Records identified through database
searching

$(n=1044)$
Additional records identified through other sources $(n=16)$

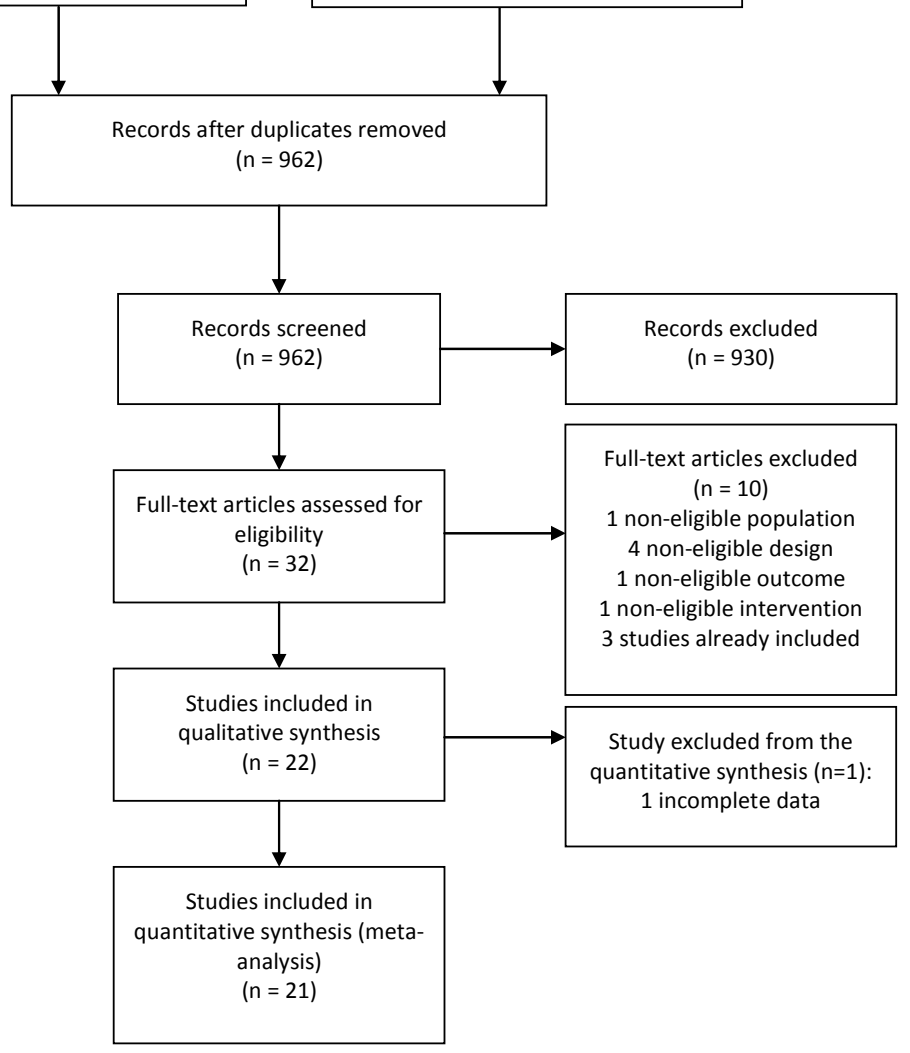

Figure 9.1: Study selection process.

\subsection{Characteristics of included trials}

The 21 trials included in the meta-analyses provided a total of 25 comparisons; three comparisons (three studies) pertained to psychotherapy v. TAU (Table 9.1), and 22 (20 studies) to a comparison of add-on psychotherapy plus TAU v. TAU only (Table 9.2). A total of 3539 patients were enrolled (293 in psychotherapy only, 1588 in addon psychotherapy plus TAU and 1638 in TAU). Sample sizes varied between 11 and 235 participants per treatment arm. 


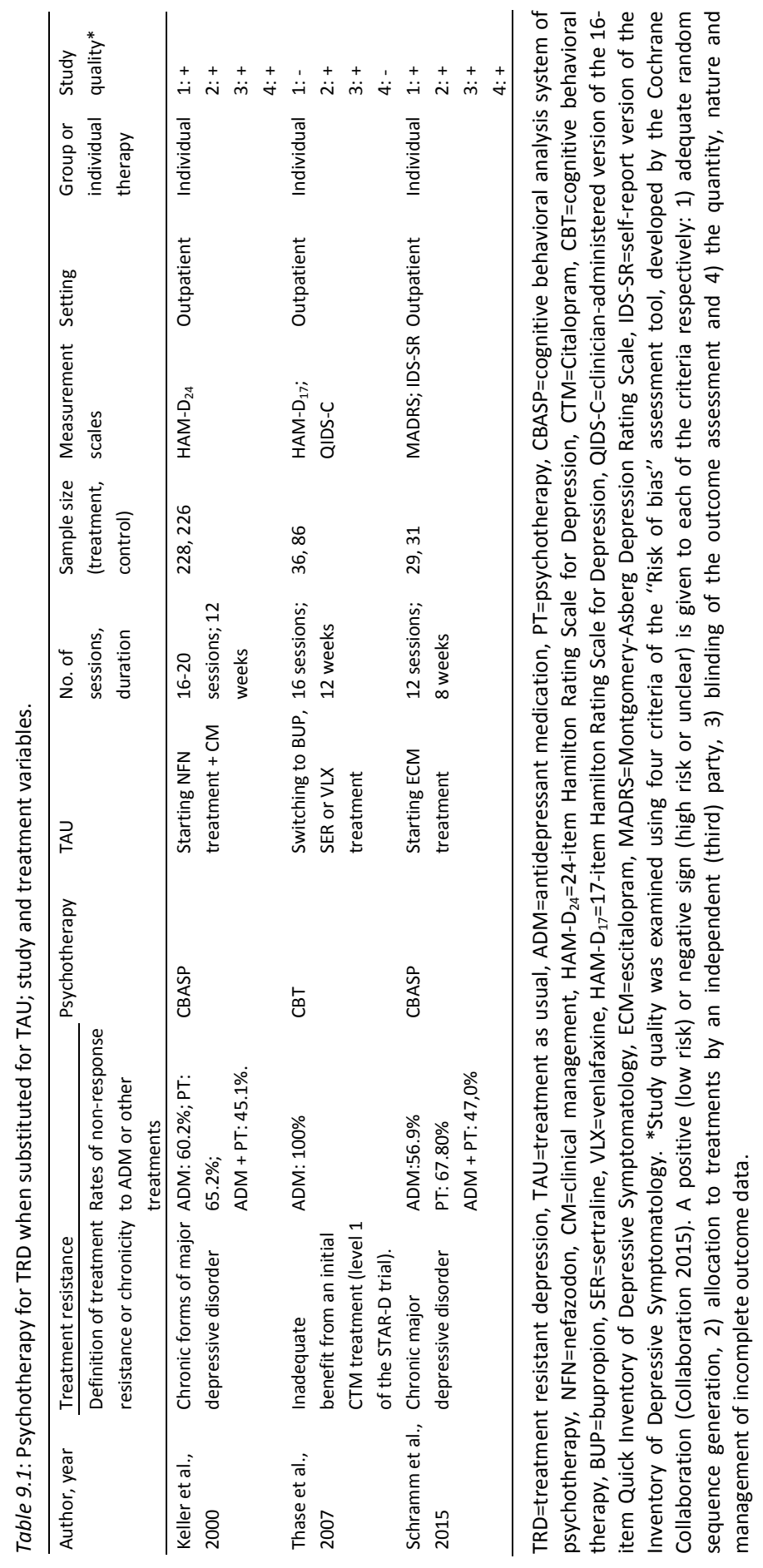




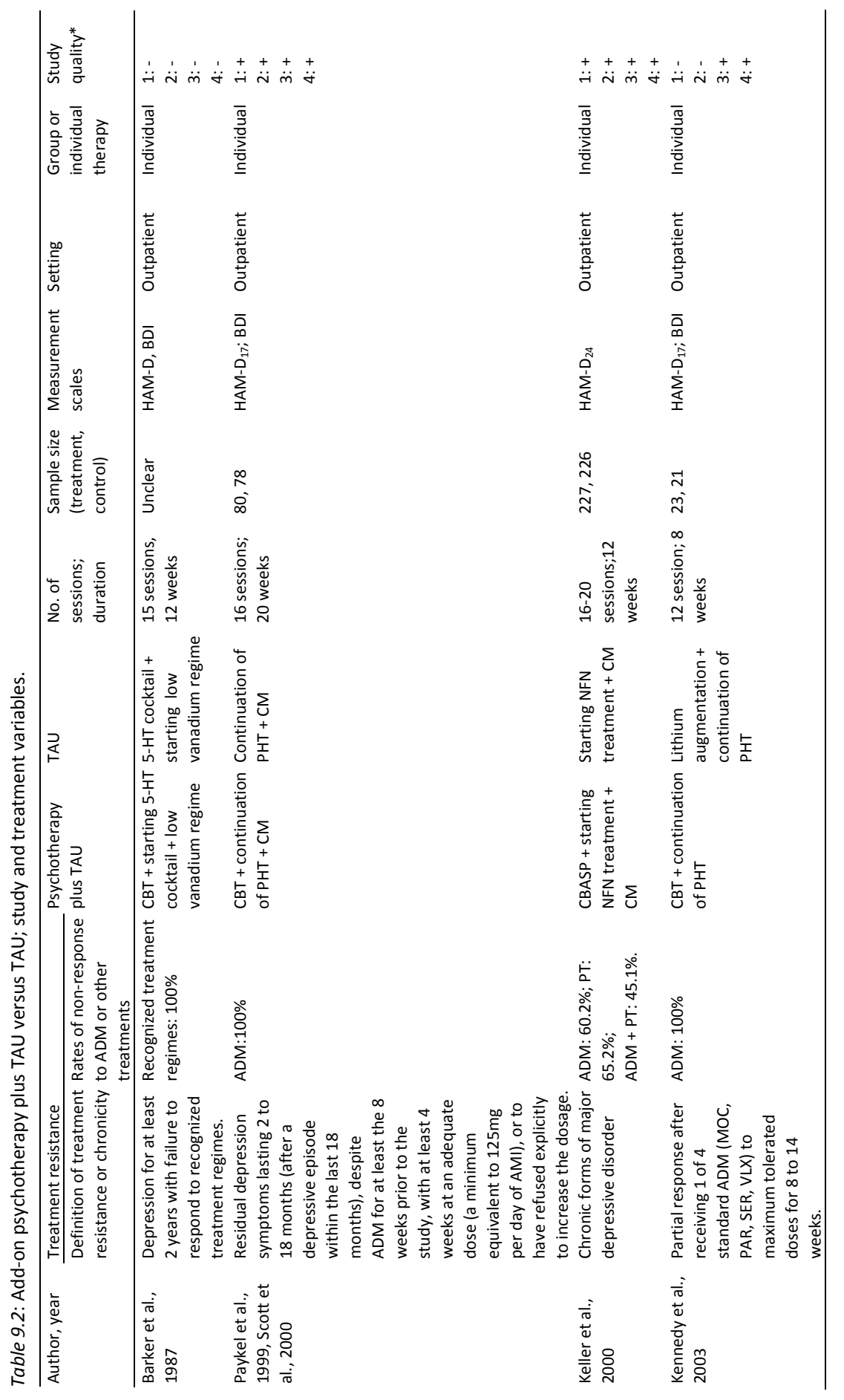




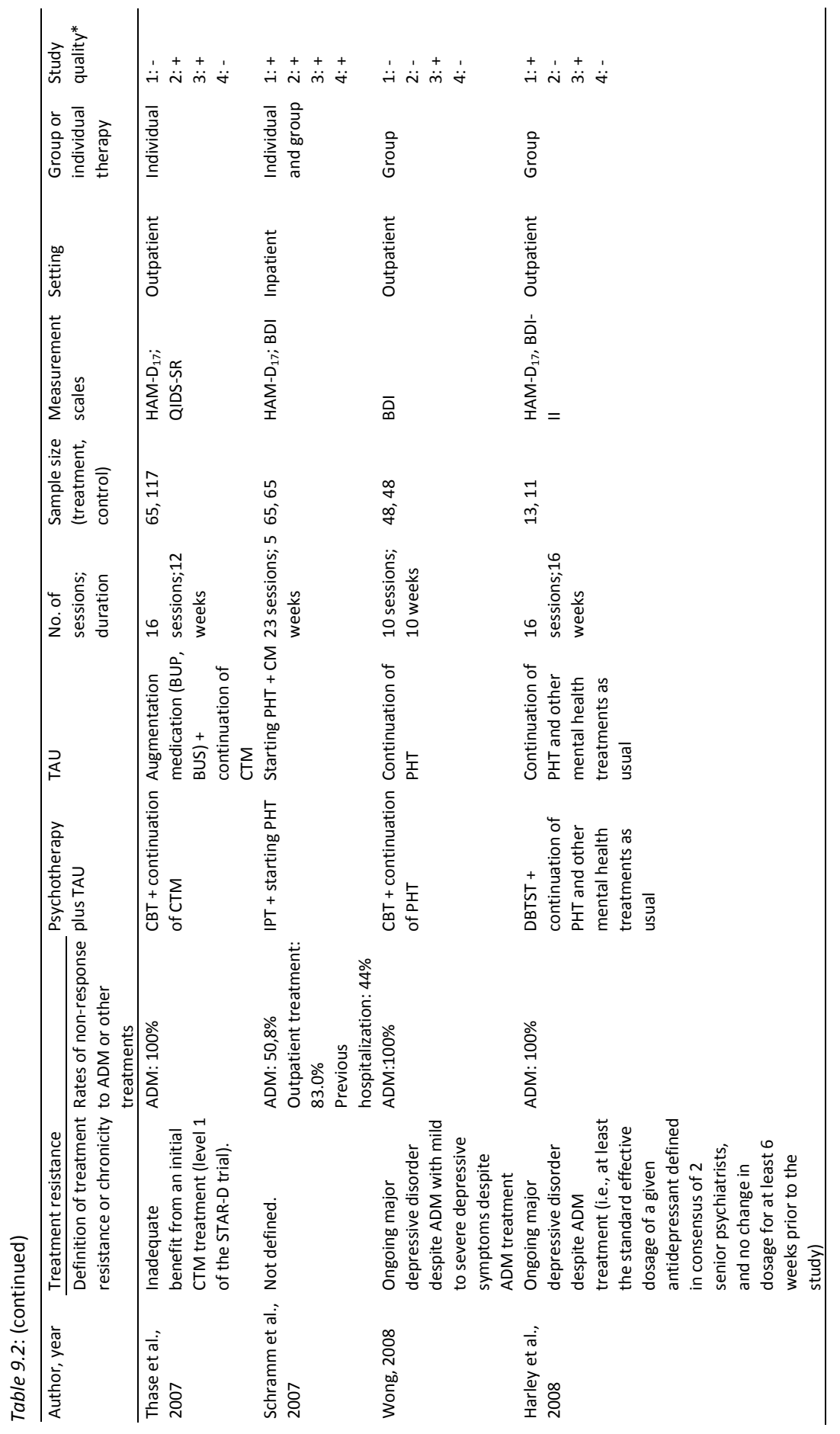




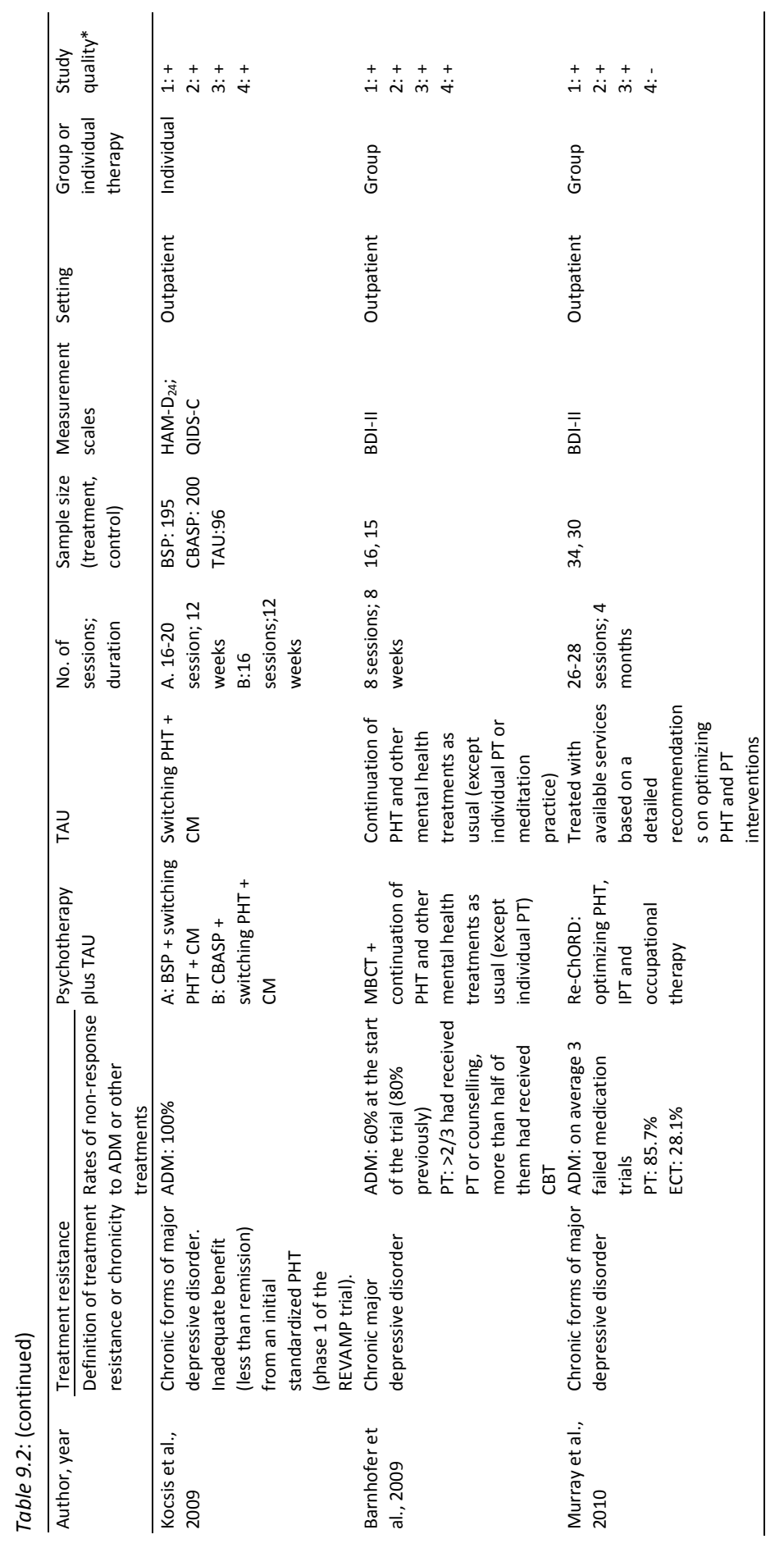




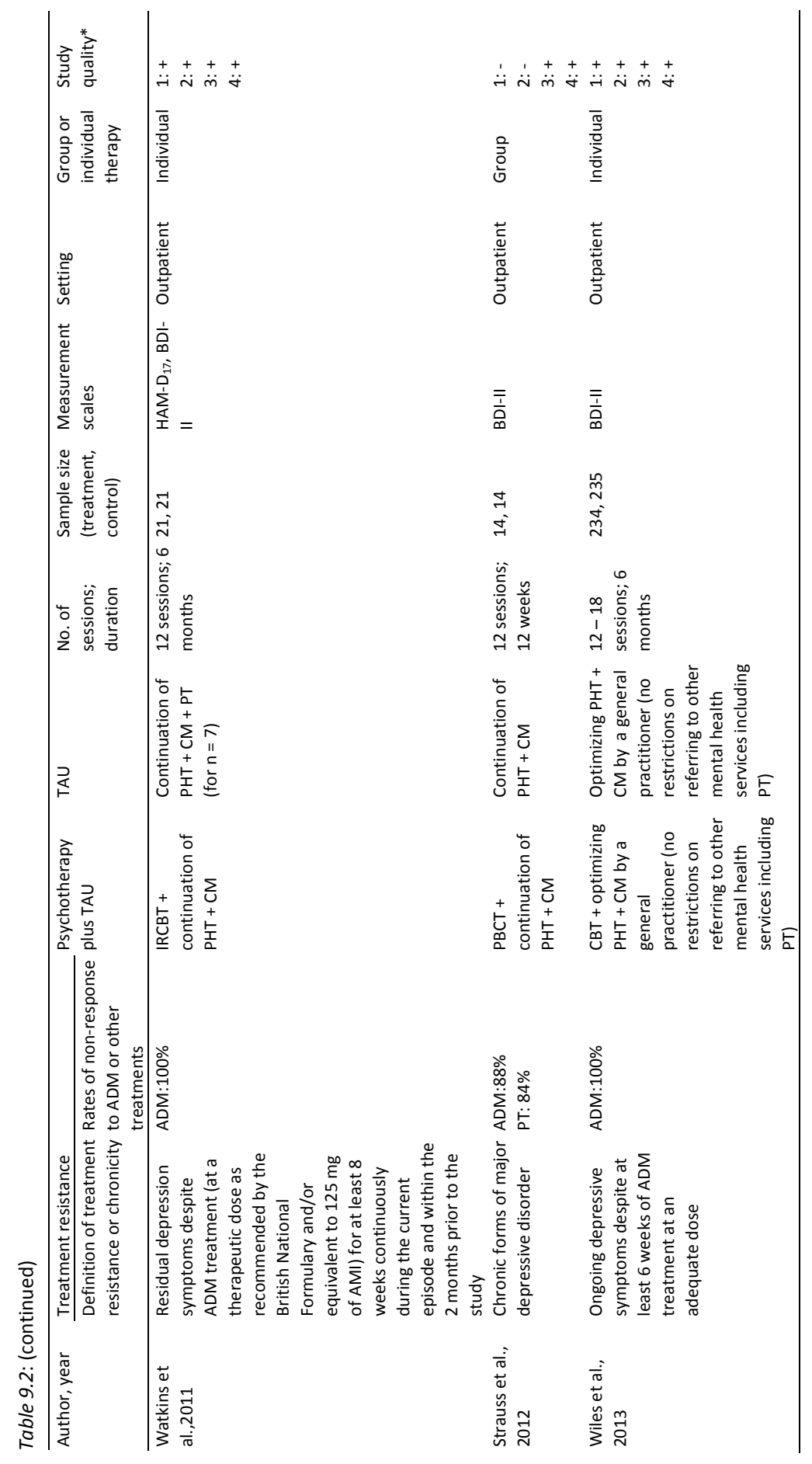




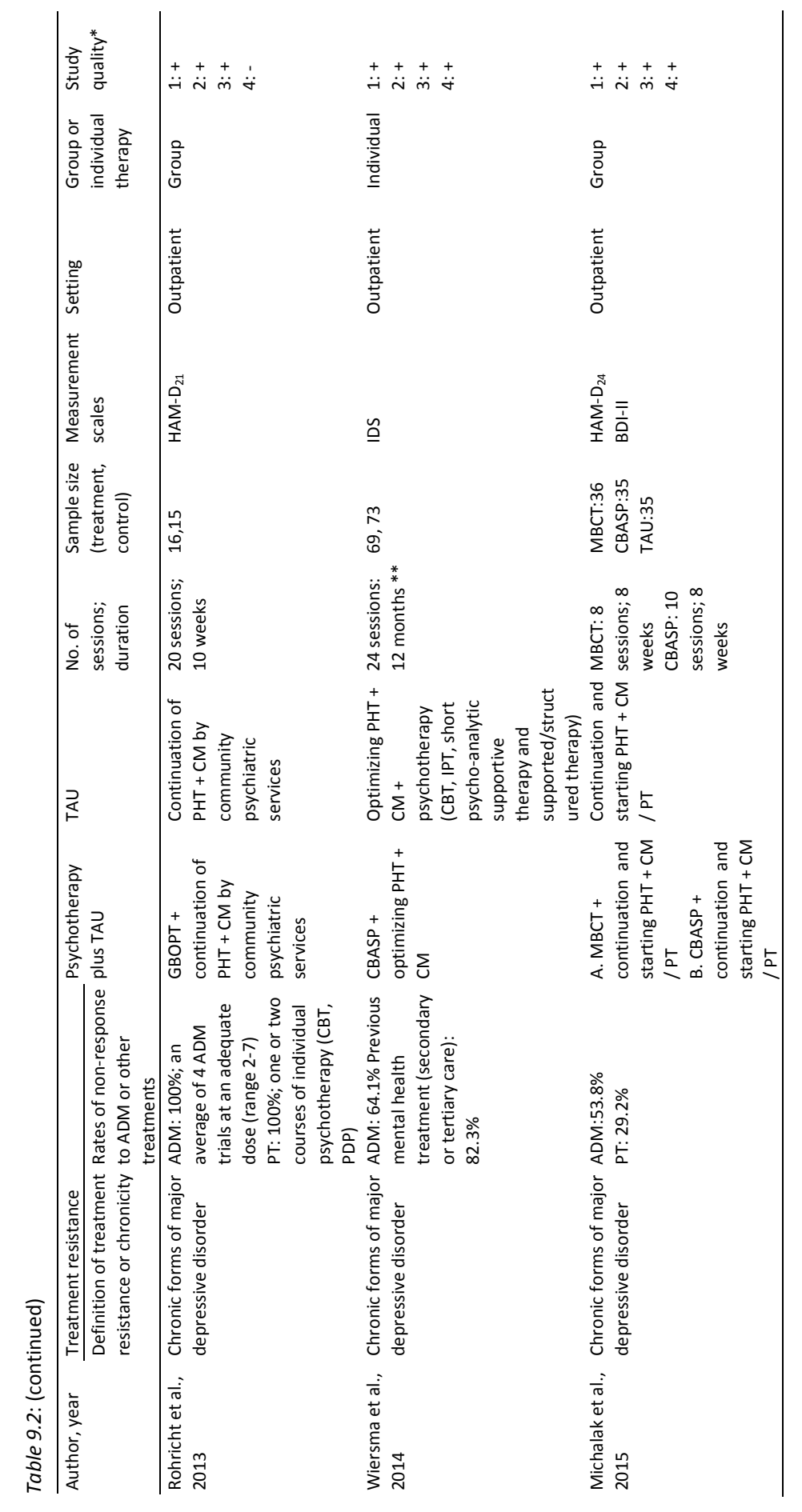




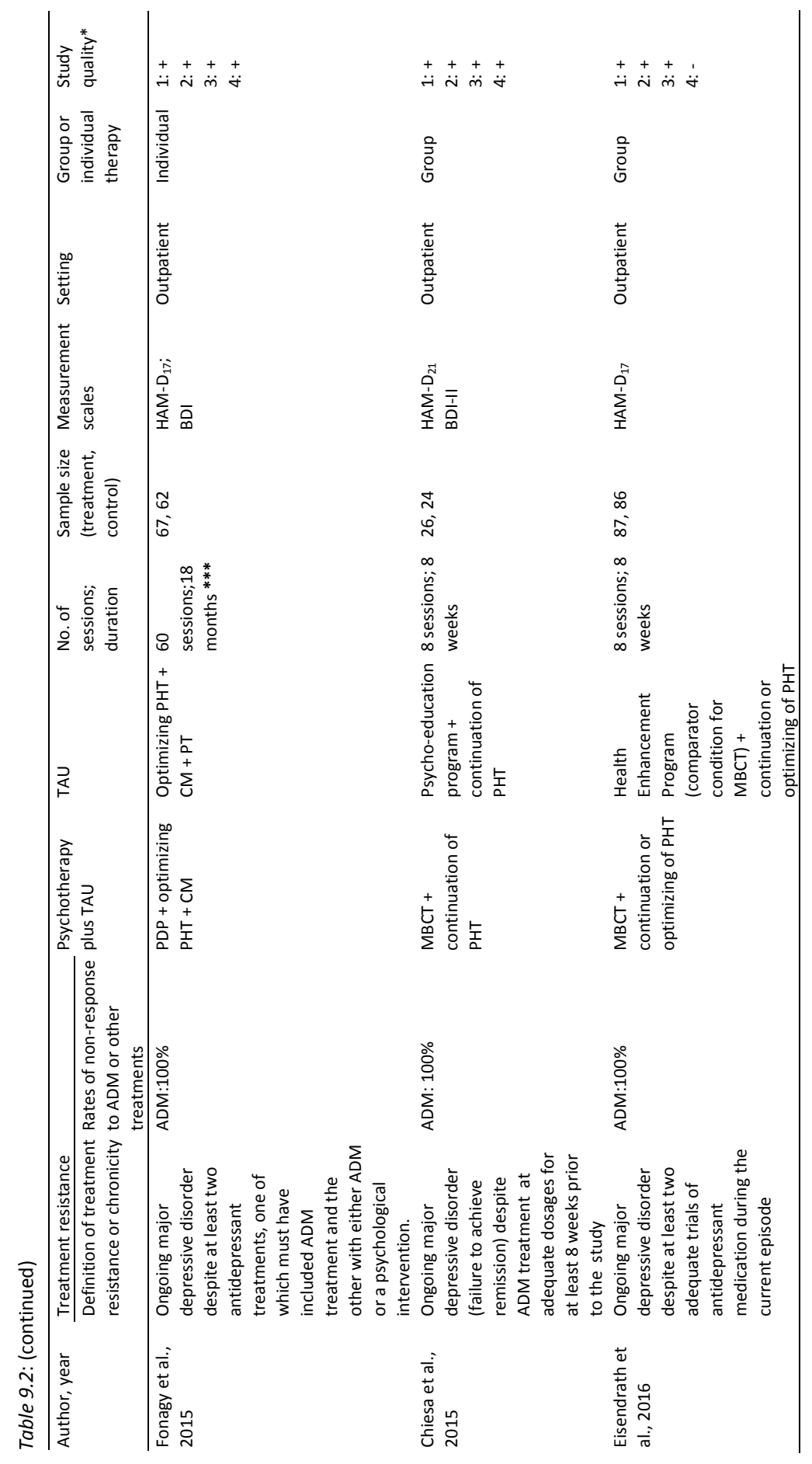




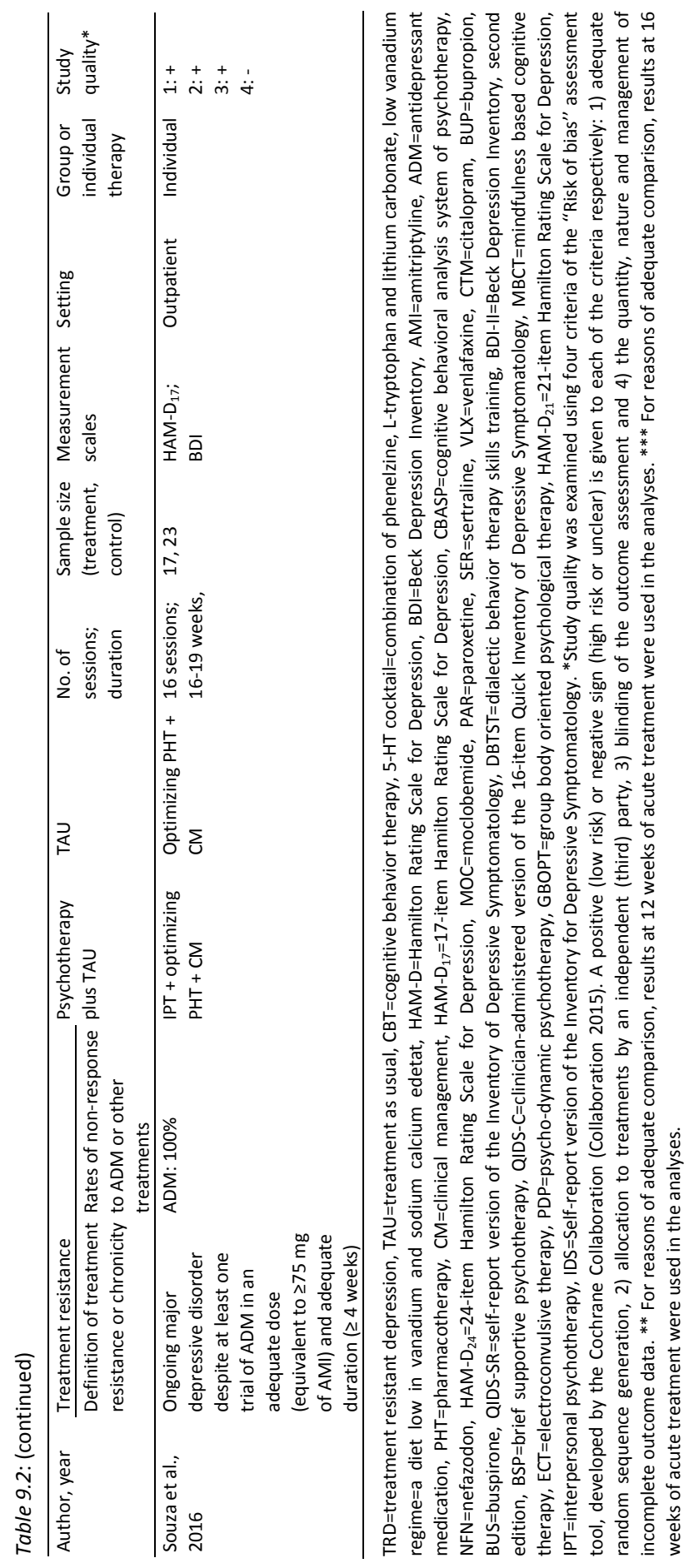


All trials ( 22 with the inclusion of the one study that was retained in the qualitative analysis only) recruited participants in secondary or tertiary care facilities except for one that was conducted in primary care (Wiles et al., 2013). Two trials were conducted in an inpatient setting. Eight trials were conducted in North- and South America, nine in the UK, four in other European countries, and one in East Asia.

In total, 11 different psychotherapeutic treatments were investigated. In the 25 comparisons, six examined cognitive behavior therapy (CBT), six cognitive behavioral-analysis system of psychotherapy (CBASP), two interpersonal psychotherapy (IPT), four mindfulness-based cognitive therapy (MBCT). The number of treatment sessions varied from 8 to 60 (although treatment endpoint for further analyses was set at 16 weeks to target the acute phase which restricted the range between treatments, see method section). Fourteen comparisons used an individual format, 10 employed a group format, and one utilized a mixed individual and group approach.

In the psychotherapy $v$. TAU comparison, TAU conditions included starting of (Keller et al., 2000; Schramm et al., 2015) and switching to (Thase et al., 2007) different types of antidepressant medication. TAU interventions in the add-on psychotherapy plus TAU v. TAU comparison included clinical management, and starting, augmenting, optimizing, and continuing pharmacotherapy. Changes in antidepressant medication (starting, augmenting and optimizing) were guided by a study protocol (Barker, Scott, \& Eccleston, 1987; Keller et al., 2000; Kennedy et al., 2003; Kocsis et al., 2009; Schramm et al., 2007; Thase et al., 2007) or left to the decision of clinicians (Eisendrath et al., 2016; Fonagy et al., 2015; Michalak, Schultze, Heidenreich, \& Schramm, 2015; Murray et al., 2010; Souza et al., 2016; Wiersma et al., 2014; Wiles et al., 2013). For a few studies comparing add-on psychotherapy plus TAU v. TAU, TAU conditions involved psychotherapy options (not for all participants) (Fonagy et al., 2015; Michalak et al., 2015; Murray et al., 2010; Watkins et al., 2011; Wiersma et al., 2014; Wiles et al., 2013), psycho-education (Chiesa et al., 2015), a health enhancement program (Eisendrath et al., 2016) or other health care services (Barnhofer et al., 2009; Harley, Sprich, Safren, Jacobo, \& Fava, 2008; Wiersma et al., 2014). In nine of 22 'psychotherapy plus TAU v. TAU' comparisons, the TAU interventions were slightly different between the two groups (Chiesa et al., 2015; Eisendrath et al., 2016; Fonagy et al., 2015; Kennedy et al., 2003; Murray et al., 2010; Schramm et al., 2007; Thase et al., 2007; Watkins et al., 2011; Wiersma et al., 2014).

Studies used various instruments to examine depression severity, including clinician-rated measures (Hamilton Rating Scale for Depression Scale, MontgomeryAsberg Depression Rating Scale, Quick Inventory of Depressive Symptomatology) and self-report measures (Inventory of Depressive Symptomatology, Beck Depression Inventory, Quick Inventory of Depressive Symptomatology). 


\subsection{Quality of included trials}

Thirteen of the 22 studies met all four quality criteria with a 'low risk' score. A total of 18 studies reported an adequate random sequence generation. In 19 studies, it was reported that the treatment allocation was done by an independent party. In 23 studies, the outcome assessors were blinded to the treatment allocation. A total of 15 studies used intention-to-treat analyses and had a balanced number of missing outcome data across interventions.

\subsection{Treatment integrity}

Two of the 22 studies did not report information on the therapist's competence in and adherence to the specific treatment type (Barker et al., 1987; Kennedy et al., 2003). Of the remaining 20 studies, seven trials provided extra training for the therapists prior to the study. Therapists received supervision (individual and/or group) in 17 studies, and sessions were video or audio recorded in 13 studies. Adherence was systematically rated in eight studies, of which two used 'checklists' and the remaining six used standardized instruments. Competence was systematically assessed in five studies with standardized instruments. Detailed information about treatment integrity for each study is provided in online Supplementary Results I.

\subsection{Psychotherapy v. TAU}

We examined three studies consisting of three comparisons (see Table 9.1). Interventions were CBT and CBASP. The TAUs as described in the trials were mainly a continuation of ongoing pharmacotherapy.

The mean pooled effect size was $\mathrm{g}=-0.13(95 \% \mathrm{Cl}=-0.30$ to 0.05$)$, heterogeneity was low with a high level of uncertainty given the wide $95 \% \mathrm{Cl}$ interval $\left(I^{2}=0.00\right.$ : $95 \% \mathrm{Cl}=0.00$ to 89.60 ). The effect sizes of the individual trials are plotted in Figure 9.2, showing that none of the interventions had significantly higher depression severity change scores on average than TAU. Given the small number of studies, we did not perform an Egger's test (Higgins \& Green, 2011). Separate sensitivity analyses (1) examining different correlations between pre- and post-intervention depression scores, (2) excluding studies that used multiple outcome measures, (3) including only the comparison with the smallest effect size, did not change the results, and (4) including only studies meeting all four quality criteria with a low risk score. Given the small number of included studies, we were not able to conduct a meta-regression. 


\begin{tabular}{|c|c|c|}
\hline Schramm, 2015 & $-0.19(-0.70,0.32)$ & 11.74 \\
\hline Thase, 2007 & $-0.18(-0.57,0.21)$ & 20.26 \\
\hline Keller, 2000 & $-0.10(-0.31,0.11)$ & 68.00 \\
\hline DL Overall $\left(I^{2}=0.0 \%\right)$ & $-0.13(-0.30,0.05)$ & 100.00 \\
\hline
\end{tabular}

Figure 9.2: Effects of psychotherapy for TRD when substituted for TAU.

TRD=treatment resistant depression, TAU=treatment as usual, $g=$ Hedges' $\mathrm{g}$ effect sizes (standardized mean difference between conditions of depression severity change scores), $95 \% \mathrm{Cl}=95 \%$ confidence interval, $\mathrm{DL}=$ DerSimonian-Laird method: between study variation was estimated using the DerSimonian-Laird method.

\subsection{Add-on psychotherapy plus TAU v. TAU}

We examined 20 studies consisting of 22 comparisons for the effectiveness of psychotherapy added to TAU v. TAU alone. The psychotherapeutic interventions were IPT, CBASP, CBT, psychodynamic therapy (PDT), body-oriented therapy (BOT), dialectic behavior therapy (DBT) and brief supportive psychotherapy (BSP). The TAUs as described in the trials were mainly a continuation of ongoing pharmacotherapy. Study characteristics are shown in Table 9.2.

The mean pooled effect size was $\mathrm{g}=0.42(95 \% \mathrm{Cl}=0.29$ to 0.54$)$, indicating that adding psychotherapy to TAU resulted in higher average depression severity change as compared with treatment with TAU alone (Figure 9.3). We found an indication for heterogeneity between studies, with again large uncertainty around this estimate $\left(\mathrm{I}^{2}=52.96 ; 95 \% \mathrm{Cl}=23.6\right.$ to 71.04$)$. When grouped together by type of treatment, IPT ( $g=0.33 ; 95 \% \mathrm{Cl}=0.02$ to 0.64$), \mathrm{CBASP}(\mathrm{g}=0.42 ; 95 \% \mathrm{Cl}=0.08$ to 0.76$), \mathrm{CBT}$ ( $\mathrm{g}=0.26$; $95 \% \mathrm{Cl}=0.01$ to 0.51$)$, and $\mathrm{MBCT}(\mathrm{g}=0.55 ; 95 \% \mathrm{Cl}=0.31$ to 0.79 ) show moderate but significant pooled effect sizes. Within the group of other therapies, BOT, PDT, individual rumination-focused cognitive behavioral therapy, and person-based cognitive therapy showed significant effect sizes (Figure 9.3). The funnel plot (Figure 9.4) did not indicate publication bias and Egger's test did not indicate asymmetry of the funnel plot (intercept: $0.78 ; 95 \% \mathrm{Cl}=-0.79$ to $2.35 ; \mathrm{p}=0.31$ ). 


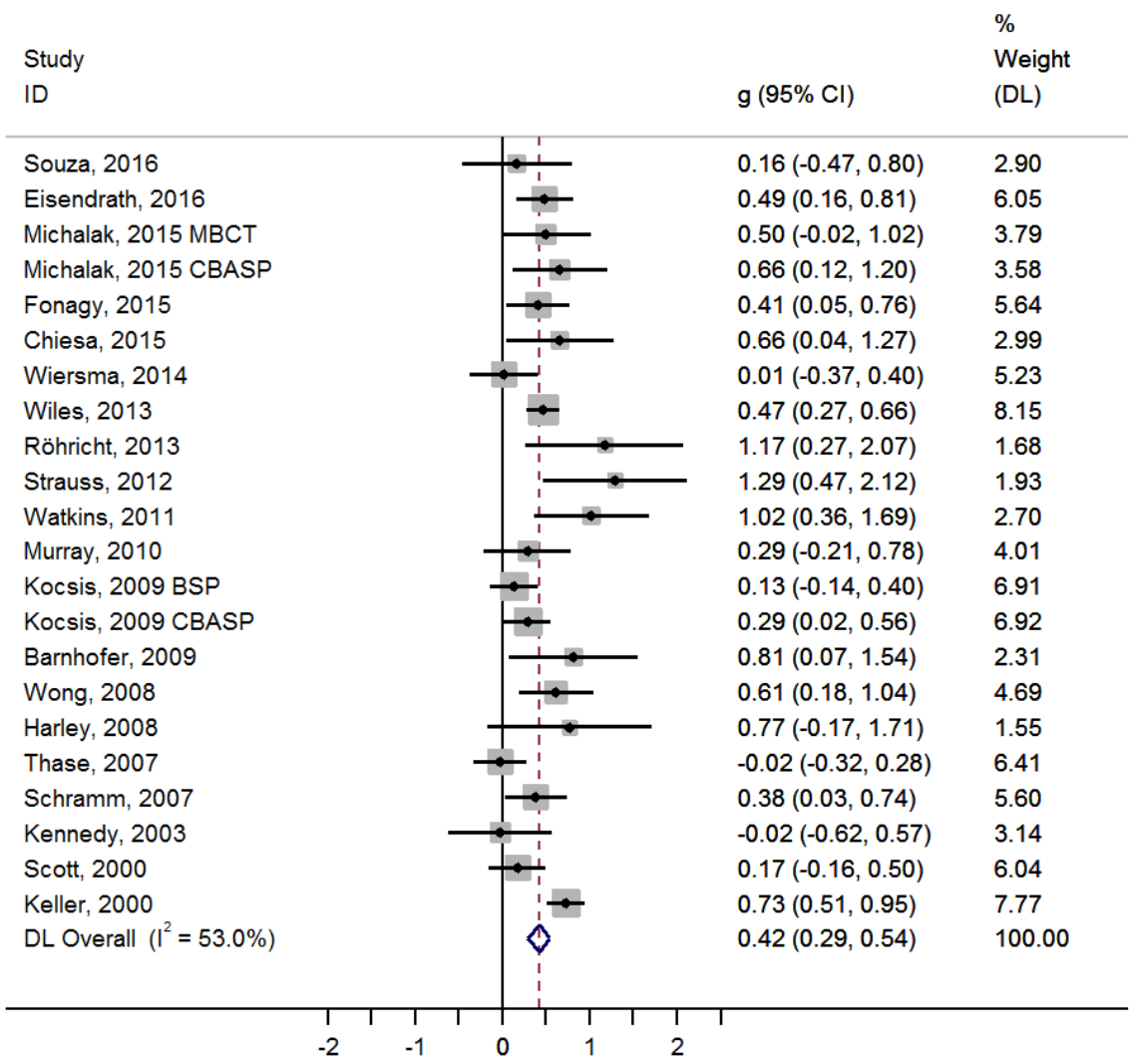

Figure 9.3: Effects of add-on psychotherapy plus TAU versus TAU for TRD.

TAU=treatment as usual, TRD=treatment resistant depression, $g=$ Hedges' $\mathrm{g}$ effect sizes (standardized mean difference between conditions of depression severity change scores), $95 \% \mathrm{Cl}=95 \%$ confidence interval, $\mathrm{DL}=$ DerSimonian-Laird method: between study variation was estimated using the DerSimonian-Laird method, $\mathrm{MBCT}=$ mindfulness based cognitive therapy, $\mathrm{CBASP}=$ cognitive behavioral analysis system of psychotherapy, $\mathrm{BSP}=$ brief supportive psychotherapy.

Separate sensitivity analyses (1) examining alternative correlations between preand post-intervention depression scores, (2) excluding studies that used multiple outcome measures, (3) including only the comparison with the smallest effect size, (4) excluding trials where the TAU in the control group was different from the TAU in the intervention arm, (5) excluding trials where TAU included psychotherapy options, and (6) including only studies meeting all four quality criteria with a low-risk score, did not change the results. 


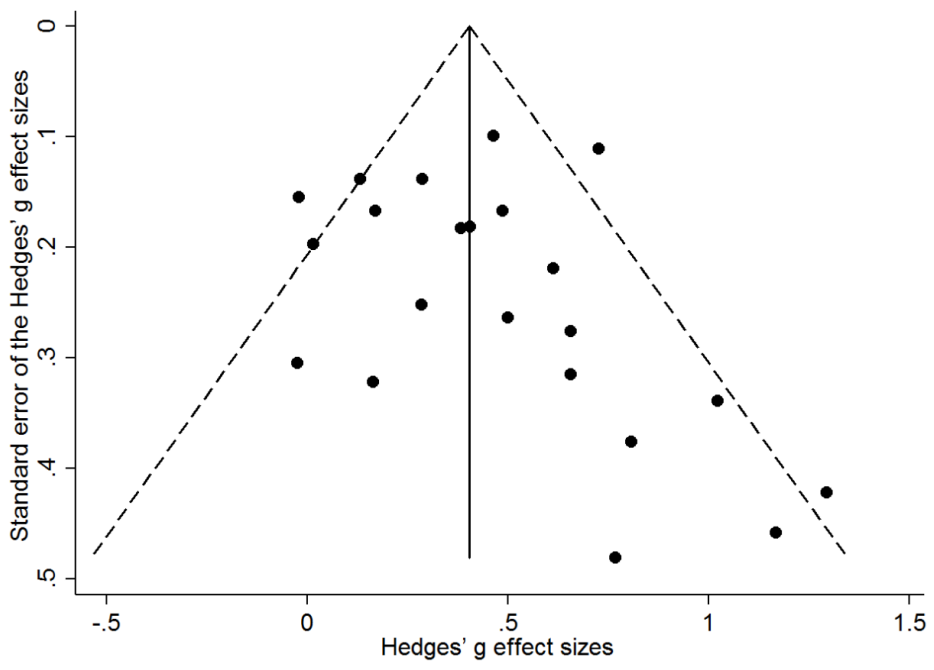

Figure 9.4: Add-on psychotherapy plus TAU versus TAU for TRD: Funnel plot TRD = treatment resistant depression, TAU = treatment as usual.

Note: The largest (and most powerful) studies have the smallest standard errors so the largest studies are placed at the top of the graph. The precision of the estimated intervention effect increases as the size of the study increases. Effect estimates of small studies will therefore scatter more widely at the bottom of the graph with the spread narrowing among larger studies. If smaller studies without statistically significant effects remain unpublished this can lead to an asymmetrical appearance of the funnel.

In the univariate meta-regression analyses regarding the set of a priori selected variables, baseline severity $(\beta=0.16 ; \mathrm{SE}=0.09 ; p=0.079)$; divided into four categories between mild and very severe (Rush, Thase, \& Dubé, 2003), number of sessions $(\beta=-0.12 ; S E=0.06 ; p=0.073)$, and individual $v$. group format $(\beta=0.32 ; S E=0.14$; $p=0.027$ ) met our criterion of $p<0.10$. In a final multivariate meta-regression higher baseline severity $(\beta=0.81 ; \mathrm{SE}=0.07 ; p=0.032)$, and group therapy-format $(\beta=0.38$; $\mathrm{SE}=0.20 ; p=0.079$ ) were associated with a larger effect size. Their correlation was 0.23 , indicative of low collinearity.

\section{Discussion}

In the present meta-analysis, we investigated the effectiveness of psychotherapy for adult patients with TRD and/or unsuccessfully treated CMDD either as a substitute or as an add-on to TAU. We identified 22 trials of which 21 could be included in the meta-analyses, yielding a total of 25 comparisons. In three comparisons of psychotherapy v. TAU we found no significant advantage of psychotherapy over TAU, 
while in 22 comparisons of add-on psychotherapy plus TAU v. TAU only, we found a significant improvement of patients due to psychotherapy with a moderate general effect size of $0.42(95 \% \mathrm{Cl}=0.29$ to 0.55$)$.

\subsection{Psychotherapy v. TAU}

Our hypothesis that for acute-phase treatment psychotherapy is more effective than TAU in TRD was not confirmed. Compared with ongoing TAU, psychotherapy, as a substitute for ongoing or recently started TAU, psychotherapy appeared not more effective in TRD (Hedges' $g=-0.02$ ). We found no indication for publication bias and the heterogeneity between studies was small although this should be interpreted with caution because $\mathrm{Cl}$ intervals were wide. In a previous meta-analysis (Cuijpers et al., 2010a), addressing the effectiveness of psychotherapy for cMDD and dysthymia, the authors reported that pharmacotherapy was more effective than psychotherapy, which seems at odds with our finding. However, it should be kept in mind that our inclusion criteria (aimed at TRD and exclusion of dysthymia) resulted in a selection of different studies. The vast majority of participants in our study selection had not responded to at least one previous trial with an antidepressant that is known to be associated with a less favorable response to subsequent treatments with pharmacotherapy (Ruhé, Huyser, Swinkels, \& Schene, 2006).

\subsection{Add-on psychotherapy plus TAU v. TAU}

We found that psychotherapy added to ongoing TAU has a moderate and significant effect size (Hedges' $g=0.42$ ) in comparison with TAU alone in TRD. Again, there was no evidence for publication bias, however there was some indication of heterogeneity between studies with wide $\mathrm{Cl}$ intervals. The results from this meta-analysis suggest that, in line with a previous meta-analysis (Cuijpers et al., 2010a) and recent clinical recommendations (Jobst et al., 2016) about the treatment of CMDD, several psychotherapeutic approaches may be of value in the treatment of TRD when added to TAU, with some evidence for more effectiveness in patients with more severe depressive symptomatology. However, some considerations may apply. First, studies of the effectiveness of CBT were done in samples with relatively low levels of TRD (mostly one unsuccessful trial with an antidepressant), which may restrict its applicability in patients with more advanced TRD. Second, some studies (e.g., Keller et al., 2000) excluded participants displaying high levels of TRD, like non-response to three previous adequate trials of different classes of antidepressants or electroconvulsive therapy, again limiting generalizability to patients with more advanced levels of TRD. Third, based on these findings one cannot rule out that TAU and psychotherapy interfere and that the combination of the two interventions leads to a greater impact than the sum of each treatment effect separately. However, a 
recent meta-analysis on combination therapy (pharmacotherapy and psychotherapy) for depression and anxiety disorders has shown equal and independent effects of pharmacotherapy and psychotherapy (Cuijpers et al., 2014). Fourth, since no active and structural equivalent placebo condition was included, the current design is insufficient to examine if the specific components of psychotherapy are responsible for the moderate effect size (Baskin, Tierney, Minami, \& Wampold, 2003).

Meta-analyses of psychotherapy for non-TRD typically report effect-sizes, that are comparable with the overall effect-size of $g=0.42$ for psychotherapy as an add-on to TAU for TRD that we have found (Cuijpers, Smit, Bohlmeijer, Hollon, \& Andersson, 2010b; Cuijpers, Van Straten, Andersson, \& Van Oppen, 2008). This is also commensurate with the results from a meta-analysis that examined the effectiveness of psychotherapy for cMDD and dysthymia (Cuijpers et al., 2010a).

In the current meta-analysis, the most frequently investigated treatments are $\mathrm{CBT}, \mathrm{CBASP}, \mathrm{MBCT}$, and to a lesser extent IPT, all with small to moderate overall effect sizes. No significant differences in the efficacy between the treatments emerged from the meta-regression. This result should be interpreted with caution since for each therapy a different number of comparisons was included (ranging between one and five studies). In addition, one could argue that aggregated results from RCTs are not suited to isolate effects of specific psychotherapies (Budd \& Hughes, 2009).

\subsection{Clinical features and study characteristics}

In our meta-regression we found no evidence, other than baseline severity and group of individual treatment format, for an association between variables such as mean duration of the current episode, mean treatment duration (number of months and number of treatment sessions), attrition rates, clinician-rated or self-reported outcomes, and an intention to treat approach for the outcome measures. The lack of an association between effect size and treatment duration or a number of sessions should be interpreted with caution since we used results from acute-phase treatment with an endpoint at approximately 16 weeks treatment for sake of comparison and absence of long-term follow-up data in many studies. Therefore, in the current study, we did not demonstrate that more treatment sessions would result in significant larger effect sizes like previously reported in a meta-analysis examining the effectiveness of psychotherapy for cMDD and dysthymia (Cuijpers et al., 2010a). However, we found comparable effects sizes between recent studies of MBCT and treatments of longer duration, suggesting that the reported association between a number of sessions and effect size may be not as strong as previously reported. It cannot be ruled out that some elements of $\mathrm{MBCT}$ (e.g., daily application of exercises after treatment termination) have enduring effects beyond the typically 8 weeks duration of training. In contrast to previous findings (Cuijpers, Li, Hofmann \& Andersson, 2010c), we did not find an effect of clinician-rated v. self-reported 
instruments on our results. This result should also be interpreted with caution since we used a combination of clinician-rated and self-reported outcomes (average effect size) for 11 of the included comparisons.

Studies that included participants with more baseline depression severity showed a larger effect-size. This is in line with previous findings that reported more efficacy of antidepressants in more severely depressed subjects (Fournier et al., 2010; Turner, Matthews, Linardatos, Tell, \& Rosenthal, 2008). An unexpected finding from the meta-regression was the larger effect-size in studies that employed a group instead of an individual treatment format. Several speculative explanations can be put forward. First, group formats consist of longer sessions than typical for individual treatment thereby increasing therapy exposure, although the literature does not support this speculation as there is no indication for greater efficacy of group format compared with individual therapy (Huntley, Araya, \& Salisbury, 2012). Additionally, one of the group therapies (MBCT) consists of daily exercises outside of the strict session context thereby increasing exposure to therapeutic interventions. Another explanation may be that the group format also provides additional peer support in subjects demoralized after unsuccessful previous treatment.

In the last decade, both the number and quality of psychotherapeutic trials in the field have increased considerably. The former is illustrated by the small number of studies that were included in previous reviews and meta-analyses (McPherson et al., 2005; Stimpson et al., 2002; Trivedi et al., 2011). Quality improved because recent studies relied on solid randomization procedures, well-described treatments, and well-trained therapists, used blinded outcome assessments, and reported intentionto-treat instead of completers-only analyses. We consider this a positive development given the preference for psychotherapy of many patients and the association between receiving a preferred treatment and clinical outcome.

Generally, one of the problems in interpreting study results of treatments for TRD is the lack of a uniform definition of TRD which may range from non-response to only one treatment trial, mostly with an antidepressant, to non-response following intensive consecutive treatments including ECT (Ruhé et al., 2012). Subsequently, nonresponse to only one treatment trial might not be considered as a relevant level of TRD in clinical practice. These different levels of treatment resistance impede interpretation of the results from this meta-analysis and its applicability in daily practice. A recommendation for future studies would be to include detailed information on previous failed treatments and include this in data-analysis. This can facilitate clinical decision-making based on the level of treatment resistance.

\subsection{Strengths and limitations}

To our knowledge, this is the largest meta-analysis to date of studies into the effectiveness of psychotherapeutic treatments when applied for the treatment of 
individuals with TRD. Although studies specifically aimed at this clinically very important population have been carried out in recent years, we were able to enlarge the database by adding studies in CMMD that in fact included a majority of patients with TRD. This enabled us to address the clinically relevant question whether psychotherapy for TRD is indeed effective. In addition, we extended this meta-analysis with a meta-regression to relate specific study-level variables to the statistical heterogeneity between the study results.

Some limitations apply to this meta-analysis. First, one of the problems in interpreting study results of treatments for TRD is the lack of a uniform definition of TRD which may range from non-response to only one treatment trial (mostly with an antidepressant) to non-response following intensive consecutive treatments including ECT (Ruhé et al., 2012). This is also illustrated by the fact that the majority of the studies included participants that were resistant to pharmacotherapy, no studies investigated specifically the efficacy of psychotherapy after previous treatment with some other form of psychotherapy. This impedes interpretation of the results from this meta-analysis and its applicability in daily practice. A recommendation for future studies would be to include more detailed information on previous failed treatments and include this in data-analysis and/or use validated tools to quantify TRD (Peeters et al., 2016). Second, although effect-sizes were roughly of equal magnitude, differences in content between experimental interventions were large, which may limit guidance for daily clinical practice; clinicians are confronted with many remaining options. Third, the impact of treatment integrity and therapists effects on the effectiveness of psychotherapy was not evaluated, since standardized instruments to assess therapy adherence and therapist's competence were often lacking and information of therapists effect was not included. Fourth, the number of studies in the comparison between psychotherapy and TAU was limited. Fifth, type and quality of the TAU conditions (mostly pharmacotherapy and clinical management) were variable, which may affect their validity as comparison intervention resulting in an overestimation of effect sizes of the experimental conditions. However, given their presumed reflection of common clinical practice in these patients, this variability might improve the generalizability of the results. Sixth, although we performed meta-regression analyses to address the impact of potentially relevant variables on outcome differences between studies, we did not find significant results apart from baseline depression severity and group/individual format. It should be noted that meta-regression is an analysis of the influence at the level of differences between and not within studies. Therefore, including variables at this level, has its limitations because only sample means are used, while ignoring the range in scores in the individual study populations (i.e., ecological bias; Thompson \& Higgins, 2002). This limits adequate subgroup analyses and better examination of heterogeneity between subjects for which individual patient data would be needed. Another limitation of our meta-regression is the missing information on some variables, limiting the power of our analyses. Eighth, 
we included studies of CMDD when a majority of the participants were reported to have failed to respond to at least one treatment trial for the current episode. This might have resulted in the inclusion of some subjects who were, in fact, true cMDD patients without qualifying for TRD, which may have influenced the results. However, it should be kept in mind that the majorities of non-responding patients in the included studies were large. Additionally, we examined this potential bias in the metaregressions; the percentage of participants that did not respond to antidepressants for the current episode was not significantly associated with effect sizes. We, therefore, feel that our results represent an accurate approximation of the effect size in TRD. Finally, as outlined earlier, we were not able to pool long-term data from the few studies that relied on treatments with longer duration and/or more treatment sessions which may obscure additional beneficial results.

\subsection{Conclusion}

Our meta-analysis provides evidence that, in addition to pharmacological and neurostimulatory treatments, add-on of psychotherapy to TAU in guidelines for the treatment of TRD is justified and will provide better outcomes for this difficult-to-treat population.

Supplementary data associated with this article can be found in the online version at https://doi.org/10.1017/S003329171800199X. 


\section{References}

Barker, W., Scott, J., \& Eccleston, D. (1987). The Newcastle Chronic Depression Study: results of a treatment regime. International Clinical Psychopharmacology, 2(3), 261-272.

Barnhofer, T., Crane, C., Hargus, E., Amarasinghe, M., Winder, R., \& Williams, J. M. G. (2009). Mindfulnessbased cognitive therapy as a treatment for chronic depression: A preliminary study. Behaviour research and therapy, 47(5), 366-373.

Baskin, T. W., Tierney, S. C., Minami, T., \& Wampold, B. E. (2003). Establishing specificity in psychotherapy: a meta-analysis of structural equivalence of placebo controls. Journal of Consulting and Clinical Psychology, 71(6), 973.

Borenstein, M., Hedges, L. V., Higgins, J. P. T., \& Rothstein, H. R. (2009). Introduction to Meta-Analysis. NYC: Wiley.

Budd, R., \& Hughes, I. (2009). The Dodo Bird Verdict-controversial, inevitable and important: a commentary on 30 years of meta-analyses. Clinical Psychology \& Psychotherapy: An International Journal of Theory \& Practice, 16(6), 510-522.

Carvalho, A. F., Berk, M., Hyphantis, T. N., \& Mclntyre, R. S. (2014). The integrative management of treatment-resistant depression: a comprehensive review and perspectives. Psychotherapy and psychosomatics, 83(2), 70-88.

Chiesa, A., Castagner, V., Andrisano, C., Serretti, A., Mandelli, L., Porcelli, S., \& Giommi, F. (2015). Mindfulness-based cognitive therapy vs. psycho-education for patients with major depression who did not achieve remission following antidepressant treatment. Psychiatry research, 226(2-3), 474-483.

Cuijpers, P., Karyotaki, E., Andersson, G., Li, J., Mergl, R., \& Hegerl, U. (2015). The effects of blinding on the outcomes of psychotherapy and pharmacotherapy for adult depression: a meta-analysis. European Psychiatry, 30(6), 685-693.

Cuijpers, P., Li, J., Hofmann, S. G., \& Andersson, G. (2010c). Self-reported versus clinician-rated symptoms of depression as outcome measures in psychotherapy research on depression: a meta-analysis. Clinical psychology review, 30(6), 768-778.

Cuijpers, P., Sijbrandij, M., Koole, S. L., Andersson, G., Beekman, A. T., \& Reynolds III, C. F. (2013). The efficacy of psychotherapy and pharmacotherapy in treating depressive and anxiety disorders: A meta-analysis of direct comparisons. World Psychiatry, 12(2), 137-148.

Cuijpers, P., Sijbrandij, M., Koole, S. L., Andersson, G., Beekman, A. T., \& Reynolds III, C. F. (2014). Adding psychotherapy to antidepressant medication in depression and anxiety disorders: a meta-analysis. Focus, 12(3), 347-358.

Cuijpers, P., Smit, F., Bohlmeijer, E., Hollon, S. D., \& Andersson, G. (2010b). Efficacy of cognitive-behavioural therapy and other psychological treatments for adult depression: meta-analytic study of publication bias. The British Journal of Psychiatry, 196(3), 173-178.

Cuijpers, P., Van Straten, A., Andersson, G., \& Van Oppen, P. (2008). Psychotherapy for depression in adults: a meta-analysis of comparative outcome studies. Journal of Consulting and Clinical Psychology, 76(6), 909.

Cuijpers, P., van Straten, A., Schuurmans, J., van Oppen, P., Hollon, S. D., \& Andersson, G. (2010a). Psychotherapy for chronic major depression and dysthymia: a meta-analysis. Clinical psychology review, 30(1), 51-62.

Dold, M., \& Kasper, S. (2017). Evidence-based pharmacotherapy of treatment-resistant unipolar depression. International journal of psychiatry in clinical practice, 21(1), 13-23.

Egger, M., Smith, G. D., Schneider, M., \& Minder, C. (1997). Bias in meta-analysis detected by a simple, graphical test. Bmj, 315(7109), 629-634.

Eisendrath, S. J., Gillung, E., Delucchi, K. L., Segal, Z. V., Nelson, J. C., McInnes, L. A., . . . Feldman, M. D. (2016). A randomized controlled trial of mindfulness-based cognitive therapy for treatment-resistant depression. Psychotherapy and psychosomatics, 85(2), 99-110.

Fonagy, P., Rost, F., Carlyle, J. a., McPherson, S., Thomas, R., Pasco Fearon, R., . . . Taylor, D. (2015). Pragmatic randomized controlled trial of long-term psychoanalytic psychotherapy for treatment-resistant depression: The Tavistock Adult Depression Study (TADS). World Psychiatry, 14(3), 312-321. 
Fournier, J. C., DeRubeis, R. J., Hollon, S. D., Dimidjian, S., Amsterdam, J. D., Shelton, R. C., \& Fawcett, J. (2010). Antidepressant drug effects and depression severity: a patient-level meta-analysis. Jama, 303(1), 47-53.

Gelhorn, H. L., Sexton, C. C., \& Classi, P. M. (2011). Patient preferences for treatment of major depressive disorder and the impact on health outcomes: a systematic review. The primary care companion to CNS disorders, 13(5).

Greden, J. F. (2001). The burden of disease for treatment-resistant depression. The Journal of clinical psychiatry, 62, 26-31.

Harley, R., Sprich, S., Safren, S., Jacobo, M., \& Fava, M. (2008). Adaptation of dialectical behavior therapy skills training group for treatment-resistant depression. The Journal of nervous and mental disease, 196(2), 136-143.

Higgins, J. P. T., \& Green, S. (Eds.). (2011). Cochrane Handbook for Systematic Reviews of Interventions (Version 5.1 .0 ed.).

Huntley, A. L., Araya, R., \& Salisbury, C. (2012). Group psychological therapies for depression in the community: systematic review and meta-analysis. The British Journal of Psychiatry, 200(3), 184-190.

Ioannidis, J. P., Patsopoulos, N. A., \& Evangelou, E. (2007). Uncertainty in heterogeneity estimates in metaanalyses. Bmj, 335(7626), 914-916.

Jobst, A., Brakemeier, E.-L., Buchheim, A., Caspar, F., Cuijpers, P., Ebmeier, K. P., . . Herpertz, S. (2016). European Psychiatric Association Guidance on psychotherapy in chronic depression across Europe. European Psychiatry, 33, 18-36.

Keller, M. B., McCullough, J. P., Klein, D. N., Arnow, B., Dunner, D. L., Gelenberg, A. J., . . . Thase, M. E. (2000). A comparison of nefazodone, the cognitive behavioral-analysis system of psychotherapy, and their combination for the treatment of chronic depression. New England Journal of Medicine, 342(20), 1462-1470.

Kennedy, S. H., Segal, Z. V., Cohen, N. L., Levitan, R. D., Gemar, M., \& Bagby, R. M. (2003). Lithium carbonate versus cognitive therapy as sequential combination treatment strategies in partial responders to antidepressant medication: an exploratory trial. The Journal of clinical psychiatry, 64(4), 439-444.

Kocsis, J. H., Gelenberg, A. J., Rothbaum, B. O., Klein, D. N., Trivedi, M. H., Manber, R., . . . Arnow, B. A. (2009). Cognitive behavioral analysis system of psychotherapy and brief supportive psychotherapy for augmentation of antidepressant nonresponse in chronic depression: the REVAMP Trial. Archives of general psychiatry, 66(11), 1178-1188.

Kolovos, S., van Tulder, M. W., Cuijpers, P., Prigent, A., Chevreul, K., Riper, H., \& Bosmans, J. E. (2017). The effect of treatment as usual on major depressive disorder: a meta-analysis. Journal of Affective Disorders, 210, 72-81.

Kriston, L., Von Wolff, A., Westphal, A., Hölzel, L. P., \& Härter, M. (2014). Efficacy and acceptability of acute treatments for persistent depressive disorder: a network meta-analysis. Depression and anxiety, 31(8), 621-630.

Lemmens, L., Arntz, A., Peeters, F., Hollon, S., Roefs, A., \& Huibers, M. (2015). Clinical effectiveness of cognitive therapy $v$. interpersonal psychotherapy for depression: results of a randomized controlled trial. Psychological Medicine, 45(10), 2095-2110.

McHugh, R. K., Whitton, S. W., Peckham, A. D., Welge, J. A., \& Otto, M. W. (2013). Patient preference for psychological vs. pharmacological treatment of psychiatric disorders: a meta-analytic review. The Journal of clinical psychiatry, 74(6), 595.

McPherson, S., Cairns, P., Carlyle, J., Shapiro, D., Richardson, P., \& Taylor, D. (2005). The effectiveness of psychological treatments for treatment-resistant depression: a systematic review. Acta Psychiatrica Scandinavica, 111(5), 331-340.

Mergl, R., Henkel, V., Allgaier, A.-K., Kramer, D., Hautzinger, M., Kohnen, R., . . . Hegerl, U. (2011). Are treatment preferences relevant in response to serotonergic antidepressants and cognitive-behavioral therapy in depressed primary care patients? Results from a randomized controlled trial including a patients' choice arm. Psychotherapy and psychosomatics, 80(1), 39-47.

Michalak, J., Schultze, M., Heidenreich, T., \& Schramm, E. (2015). A randomized controlled trial on the efficacy of mindfulness-based cognitive therapy and a group version of cognitive behavioral analysis system of psychotherapy for chronically depressed patients. Journal of Consulting and Clinical Psychology, 83(5), 951. 
Moussavi, S., Chatterji, S., Verdes, E., Tandon, A., Patel, V., \& Ustun, B. (2007). Depression, chronic diseases, and decrements in health: results from the World Health Surveys. The Lancet, 370(9590), 851-858.

Murray, G., Michalak, E. E., Axler, A., Yaxley, D., Hayashi, B., Westrin, Å., . . . Lam, R. W. (2010). Relief of Chronic or Resistant Depression (Re-ChORD): A pragmatic, randomized, open-treatment trial of an integrative program intervention for chronic depression. Journal of Affective Disorders, 123(1-3), 243248.

Negt, P., Brakemeier, E. L., Michalak, J., Winter, L., Bleich, S., \& Kahl, K. G. (2016). The treatment of chronic depression with cognitive behavioral analysis system of psychotherapy: a systematic review and meta-analysis of randomized-controlled clinical trials. Brain and behavior, 6(8), e00486.

Paykel, E. S., Scott, J., Teasdale, J. D., Johnson, A. L., Garland, A., Moore, R., . . Abbott, R. (1999). Prevention of relapse in residual depression by cognitive therapy: a controlled trial. Archives of general psychiatry, 56(9), 829-835.

Peeters, F. P., Ruhe, H. G., Wichers, M., Abidi, L., Kaub, K., van der Lande, H. J., . . Schene, A. H. (2016). The Dutch measure for quantification of treatment resistance in depression (DM-TRD): an extension of the Maudsley staging method. Journal of Affective Disorders, 205, 365-371.

Röhricht, F., Papadopoulos, N., \& Priebe, S. (2013). An exploratory randomized controlled trial of body psychotherapy for patients with chronic depression. Journal of Affective Disorders, 151(1), 85-91.

Ruhé, H. G., Huyser, J., Swinkels, J. A., \& Schene, A. H. (2006). Switching antidepressants after a first selective serotonin reuptake inhibitor in major depressive disorder: a systematic review. Journal of Clinical Psychiatry, 67(12), 1836-1855.

Ruhé, H. G., van Rooijen, G., Spijker, J., Peeters, F. P., \& Schene, A. H. (2012). Staging methods for treatment resistant depression. A systematic review. Journal of Affective Disorders, 137(1-3), 35-45.

Rush, A. J., Thase, M. E., \& Dubé, S. (2003). Research issues in the study of difficult-to-treat depression. Biological psychiatry, 53(8), 743-753.

Rush, A. J., Trivedi, M. H., Wisniewski, S. R., Nierenberg, A. A., Stewart, J. W., Warden, D., . . Lebowitz, B. D. (2006). Acute and longer-term outcomes in depressed outpatients requiring one or several treatment steps: a STAR* D report. American Journal of Psychiatry, 163(11), 1905-1917.

Schramm, E., van Calker, D., Dykierek, P., Lieb, K., Kech D Clin Psych, S., Zobel D Clin Psych, I., ... Berger, M. (2007). An intensive treatment program of interpersonal psychotherapy plus pharmacotherapy for depressed inpatients: acute and long-term results. American Journal of Psychiatry, 164(5), 768-777.

Schramm, E., Zobel, I., Schoepf, D., Fangmeier, T., Schnell, K., Walter, H., . . . Berger, M. (2015). Cognitive behavioral analysis system of psychotherapy versus escitalopram in chronic major depression. Psychotherapy and psychosomatics, 84(4), 227-240.

Souza, L. H., Salum, G. A., Mosqueiro, B. P., Caldieraro, M. A., Guerra, T. A., \& Fleck, M. P. (2016). Interpersonal psychotherapy as add-on for treatment-resistant depression: A pragmatic randomized controlled trial. Journal of Affective Disorders, 193, 373-380.

Spijker, J., van Straten, A., Bockting, C. L., Meeuwissen, J. A., \& van Balkom, A. J. (2013). Psychotherapy, antidepressants, and their combination for chronic major depressive disorder: a systematic review. The Canadian Journal of Psychiatry, 58(7), 386-392.

Stimpson, N., Agrawal, N., \& Lewis, G. (2002). Randomised controlled trials investigating pharmacological and psychological interventions for treatment-refractory depression: systematic review. The British Journal of Psychiatry, 181(4), 284-294.

Swift, J. K., Callahan, J. L., \& Vollmer, B. M. (2011). Preferences. Journal of clinical psychology, 67(2), 155165.

Thase, M. E., Friedman, E. S., Biggs, M. M., Wisniewski, S. R., Trivedi, M. H., Luther, J. F., . . W Warden, D. (2007). Cognitive therapy versus medication in augmentation and switch strategies as second-step treatments: a STAR* D report. American Journal of Psychiatry, 164(5), 739-752.

Thompson, S. G., \& Higgins, J. P. (2002). How should meta-regression analyses be undertaken and interpreted? Statistics in medicine, 21(11), 1559-1573.

Trivedi, R. B., Nieuwsma, J. A., \& Williams, J. W. (2011). Examination of the utility of psychotherapy for patients with treatment resistant depression: a systematic review. Journal of general internal medicine, 26(6), 643-650. 
Turner, E. H., Matthews, A. M., Linardatos, E., Tell, R. A., \& Rosenthal, R. (2008). Selective publication of antidepressant trials and its influence on apparent efficacy. New England Journal of Medicine, 358(3), 252-260.

Watkins, E. R., Mullan, E., Wingrove, J., Rimes, K., Steiner, H., Bathurst, N., . . Scott, J. (2011). Ruminationfocused cognitive-behavioural therapy for residual depression: Phase II randomised controlled trial. The British Journal of Psychiatry, 199(4), 317-322.

Wiersma, J. E., Van Schaik, D. J., Hoogendorn, A. W., Dekker, J. J., Van, H. L., Schoevers, R. A., . . . McCullough Jr, J. P. (2014). The effectiveness of the cognitive behavioral analysis system of psychotherapy for chronic depression: a randomized controlled trial. Psychotherapy and psychosomatics, 83(5), 263-269.

Wiles, N., Thomas, L., Abel, A., Ridgway, N., Turner, N., Campbell, J., . . . Kessler, D. (2013). Cognitive behavioural therapy as an adjunct to pharmacotherapy for primary care based patients with treatment resistant depression: results of the CoBalT randomised controlled trial. The Lancet, 381(9864), 375-384. 

Part

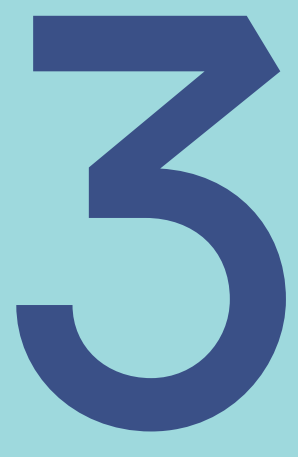




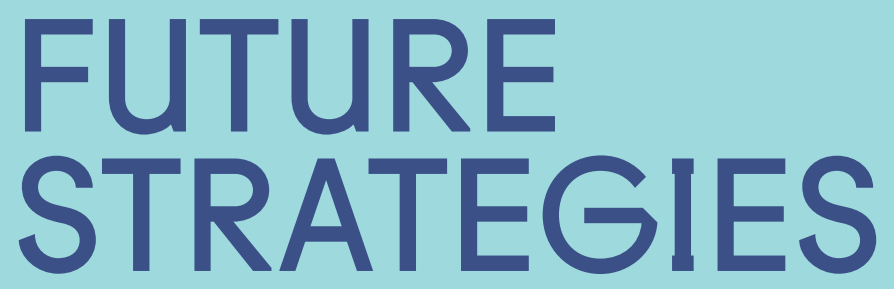


S. van Bronswijk, L. Lemmens, M. Huibers, F. Peeters

- Journal of Affective Disorders 2020

\section{Chapter}
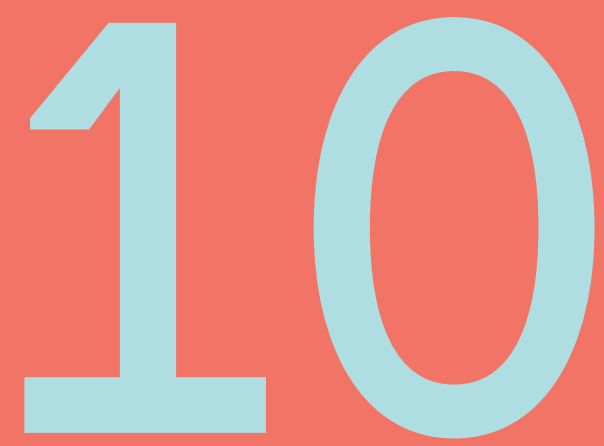


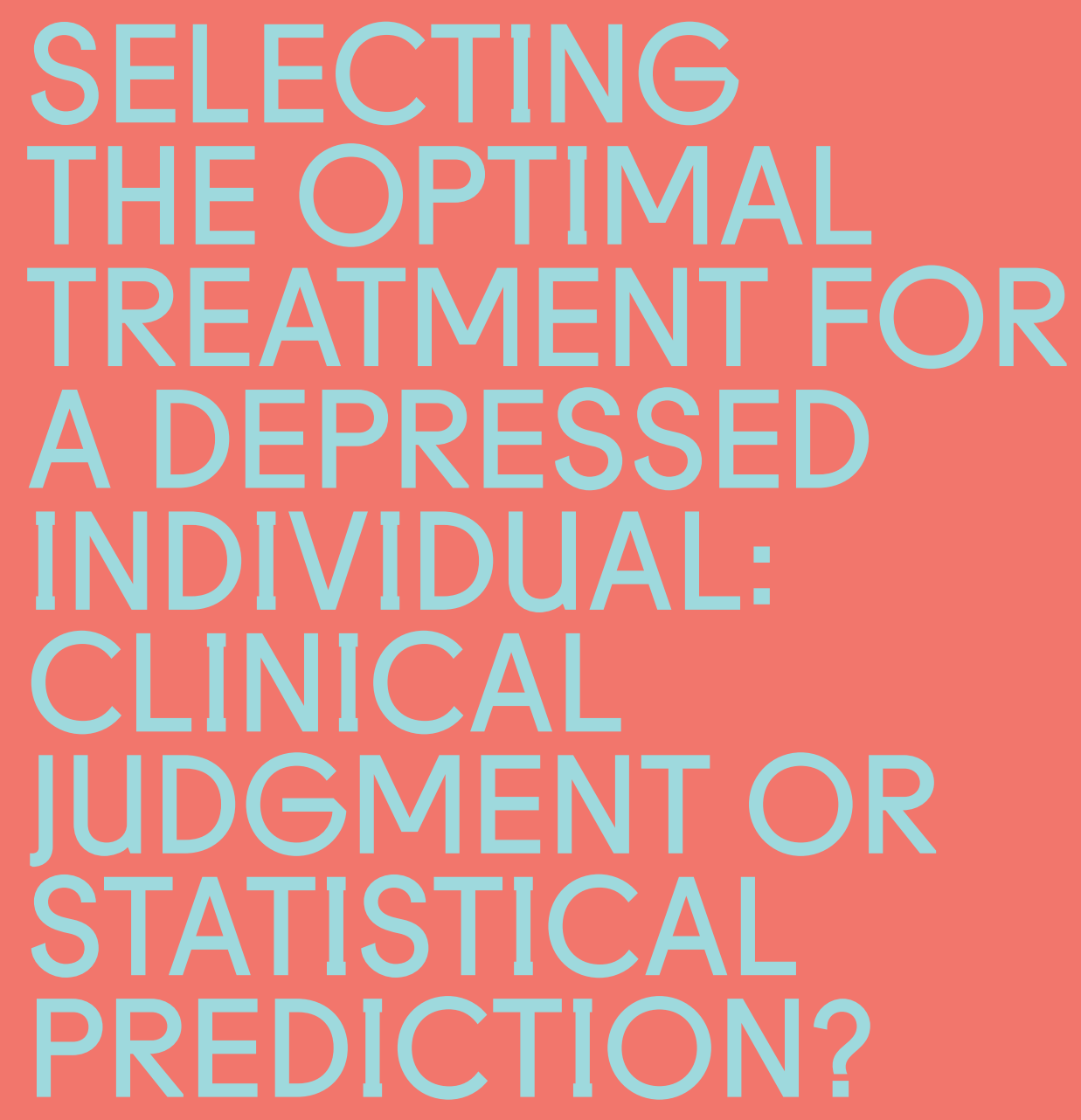




\section{Abstract}

\section{Background}

Optimizing treatment selection is a way to enhance treatment success in major depressive disorder (MDD). In clinical practice, treatment selection heavily depends on clinical judgment. However, research has consistently shown that statistical prediction is as accurate - or more accurate - than predictions based on clinical judgment. In the context of new technological developments, the current aim was to compare the accuracy of clinical judgment versus statistical predictions in selecting cognitive therapy (CT) or interpersonal psychotherapy (IPT) for MDD.

Methods

Data came from a randomized trial comparing CT $(n=76)$ with IPT $(n=75)$ for MDD. Prior to randomization, therapists' recommendations were formulated during multidisciplinary staff meetings. Statistical predictions were based on Personalized Advantage Index models. Primary outcomes were post-treatment and 17-month follow-up depression severity. Secondary outcome was treatment dropout.

Results

Individuals receiving treatment according to their statistical prediction were less depressed at post-treatment and follow-up compared to those receiving their predicted non-indicated treatment. This difference was not found for recommended versus non-recommended treatments based on clinical judgment. Moreover, for individuals with an IPT recommendation by therapists, higher post-treatment and follow-up depression severity was found for those that actually received IPT compared to those that received CT. Recommendations based on statistical prediction and clinical judgment were not associated with differences in treatment dropout.

\section{Conclusions}

Statistical prediction outperforms clinical judgment in treatment selection for MDD and has the potential to personalize treatment strategies. 


\section{Introduction}

Despite the many options available, treatments for major depressive disorder (MDD) have modest effects on symptom reduction, with overall response rates around $50 \%$ (Papakostas and Fava, 2010). Although the average efficacy of various therapies is comparable (Cuijpers et al., 2011; Cuijpers et al., 2020a; Cuijpers et al., 2020b), individual treatment responses vary greatly (Simon and Perlis, 2010). As a result, depressed individuals may receive non-effective treatments before the right match is found (Rush et al., 2006). In addition, by offering treatments that do not work for a specific individual, risks of treatment dropout in MDD are high (Cooper and Conklin, 2015; Warden et al., 2014). To enhance treatment success, a straightforward solution is to optimize treatment selection for a given individual (what works best for whom?), moving beyond the one size fits all and trial and error approach towards personalized or precision strategies (Cohen and DeRubeis, 2018; DeRubeis, 2019).

In clinical psychology and psychiatry, attempts to match individuals to the most optimal treatment are everyday practice. Treatment selection heavily depends on clinical judgment of expected responses to available treatments. Clinical judgment is the result of informal or intuitive processes, and treatment recommendations are often formulated by an individual clinician or during multidisciplinary staff meetings. These recommendations are based on the clinicians' experience with similar patients, the clinicians' training background, the patient's preference and the clinicians' beliefs, theoretical perspective, and familiarity with empirical literature and (inter)national guidelines (Delgadillo et al., 2015; Lorenzo-Luaces et al., 2015).

Despite the potential flexibility of clinical judgment, human judgment is susceptible for many errors (Bell and Mellor, 2009; Garb, 2005; Grove et al., 2000; Richards et al., 2015). First of all, clinical judgment has shown to have low intra- and inter-rater reliability; clinical judgment is inconsistent for a given clinician over time, and agreement on the same case between clinicians is low (Bell and Mellor, 2009). Second, a source of error that contributes to the low inter-rater reliability is that clinicians use different theoretical frameworks that mediate their clinical judgement (Bell and Mellor, 2009). For example, a clinician with a behaviorist background will base clinical judgment on predominantly cognitive and behavioral factors, while an interpersonal oriented therapist will likely emphasize relational and attachment-style determinants. In addition, by basing predictions on causal theories, these theories need to be comprehensive and well-supported by evidence, and all variables that are relevant for these theories need to be available and measurable with accurate instruments (Grove and Meehl, 1996). No theory has yet met all of these conditions, for example illustrated by the lack of an exact understanding on working mechanisms of psychological treatments and biological determinants of psychopathology (Beijers et al., 2019; Cuijpers et al., 2019). Third, clinical judgment is prone to biases. As clinicians seldom rely on statistical rules only, they often apply cognitive heuristics. 
Although this might save time and effort, heuristics can lead to biases. In clinical judgment multiple types of biases are observed, including confirmation bias (bias through existing beliefs, expectations or hypotheses), anchoring bias (bias by initial information), availability bias (bias by easily available information) and representative biases (bias by assigning high probabilities to detailed prototypical combinations, i.e., the law of small numbers) (Bell and Mellor, 2009; Furnham and Boo, 2011; Nickerson, 1998; Richards et al., 2015). A fourth source of error is overreliance on unstructured clinical interviews that may lower the reliability and validity of clinical judgment. Explanations for this effect include confirmation bias as clinicians are more susceptible to focus on information that confirms their hypothesis and pay less attention to conflicting evidence (Bell and Mellor, 2009). A fifth source of error concerns the limited information processing abilities of humans. Even when biases are avoided, it is humanly impossible to differentially weight and combine large amounts of information (e.g., multiple conflicting predictors) (Bell and Mellor, 2009; Meehl, 1986). A final source of error is the lack of adequate and systematic feedback that clinicians can use to change inaccurate clinical judgment behavior (Grove et al., 2000).

One way to overcome the errors of human judgment is to use statistical or actuarial predictions that are based on reproducible algorithms. The accuracy of these predictions, relative to predictions based on clinical judgment, has been a topic of debate since the mid-50's of the last century (Meehl, 1954, 1986). Meehl (1954) indicated that in 19 out of 20 studies statistical predictions were as accurate as or more accurate than predictions based on clinical judgment. Since then, research has consistently shown that statistical prediction works at least as well as predictions based on clinical judgment, with on average $10 \%$ to $13 \%$ more accuracy for statistical predictions (/Egisdóttir et al., 2006; Grove et al., 2000).

Despite the evidence that statistical prediction is at least as accurate as predictions based on clinical judgment, the use of statistical predictions in clinical practice is still limited (Bell and Mellor, 2009). Several potential reasons have been put forward to explain this phenomenon (Garb, 2000; Grove and Meehl, 1996; Katsikopoulos et al., 2008; Meehl, 1986). One explanation is the (expected) high demands of statistical prediction on the clinician's time. Another potential reason are ethical objections, for instance the viewpoint that ignoring a strong personal preference that conflicts with statistical prediction is not right. These ethical objections also come into play in the fear of dehumanization, that is the fear that statistical prediction reduces individuals to inanimate objects (numbers). Another explanation for the limited use of statistical predictions may be the clinician's clinging to theory, resulting in a reluctance to abandon theory-mediated predictions. In addition, the theoretical orientation of clinicians may be closely tied to their professional identity; poor performance of clinical judgement based on theoretical orientation may be perceived as a threat to this professional identity (this is how I do it and that is who I am). Fear of unemployment due to automated procedures is 
another potential reason for the limited use of statistical predictions in clinical practice. Finally, lack of education could be an important factor as well, by poor training in scientific techniques and human biases, and by role models involved in clinical training that ignore or disregard scientific reasoning and/or evidence.

However, the potential of novel statistical approaches (e.g., machine learning) and the availability of big data (e.g., electronic medical records and smartphone data) are leading us to an renewed attention for and rethink the importance of statistical predictions to personalize treatment recommendations (Delgadillo and Lutz, 2020; DeRubeis, 2019; Perna et al., 2018). In the context of treatment selection for MDD, this change is demonstrated by the development of multivariable models that promise to generate powerful predictions (Cohen and DeRubeis, 2018). One approach that combines information of multiple variables is the Personalized Advantage Index (PAI) that provides individual treatment recommendations by predicting the advantage of an indicated treatment over a non-indicated treatment (DeRubeis et al., 2014).

With the recent technological advances in mind, the aim of the current study was to compare the accuracy of clinical judgment versus statistical (PAI) predictions in the context of treatment selection of two frequently used psychotherapies (cognitive therapy [CT] and interpersonal psychotherapy [IPT]) for MDD. It was hypothesized that statistical prediction outperforms clinical judgment and that clinical judgment has only a very modest added value to statistical prediction in treatment selection for MDD.

\section{Methods}

\subsection{Design and participants}

Participants were recruited from the mood disorders unit of the Maastricht Outpatient Mental Health Centre (the Netherlands) and data was collected in the context of a randomized trial on the effectiveness of CT and IPT for MDD (for a detailed description: Lemmens et al., 2015; Lemmens et al., 2011). Participants had to have a primary diagnosis of MDD, internet access, an email address, and sufficient knowledge of the Dutch language. Exclusion criteria were bipolar or highly chronic depression (episode duration $>5$ years), high acute suicide risk, concomitant pharmacological or psychological treatment, drugs and alcohol problems, and an IQ lower than 80. A total of 182 depressed outpatients were randomly assigned to CT $(n=76)$, IPT $(n=75)$, or a 2-month waiting-list control condition followed by treatment of participants' choice $(n=31)$. In this study we focused on data of individuals randomized to CT and IPT. Participants received 16-20 weekly sessions of 45 minutes ( $M=17.0, S D=2.9)$, using CT and IPT protocols (Beck et al., 1979; Klerman et al., 1984). 
Treatments were provided by ten therapists (licensed psychologist, psychotherapists, and psychiatrists) with on average 9.1 years of experience ( $S D=5.4$, range 4-21 years). There was no significant effect of individual therapists on treatment outcomes (Lemmens et al., 2015). Participating therapists delivered either CT or IPT to avoid contamination of treatment conditions. Quality of treatment was rated good to excellent with significant differences in therapy-specific behavior between treatments. Written informed consent was obtained and the study was approved by the Medical Ethics Committee of Maastricht University. The study is registered at the Netherlands Trial Register, part of the Dutch Cochrane Centre (ISRCTN67561918).

\subsection{Outcome variables}

Primary outcome was depression severity, measured with the Beck Depression Inventory II (BDI-II) during treatment (month 0-7) and follow-up (month 7-24, Beck et al., 1996). For the current study, we focused on post-treatment BDI-II scores (month 7) and follow-up BDI-II scores (month 7, 8, 9, 10, 11, 12 and 24, aggregated into an Area Under the Curve, see van Bronswijk et al., 2019). Treatment dropout (discontinuation of the treatment intervention by participants) was examined as a secondary outcome.

\subsection{Therapists' treatment recommendations}

Therapists' treatment recommendations (CT, IPT or no specific recommendation) were based on case presentations by clinicians who carried out the diagnostic workup during regular multidisciplinary team meetings at the mood disorders unit and were formulated after group-discussion. Team meetings were attended by a total of 4-5 clinicians (psychologists, psychotherapists and psychiatrists) that had allegiance to both CT and IPT. The specific composition of the attendees depended on the involvement in the diagnostic work-up procedure and on scheduling matters. Part of the attendees of the team meetings were involved as therapists in the study as well. Recommendations were formulated before randomization of the participants. After randomization, participants were coded as having received the recommended or nonrecommended treatment through random allocation (CT or IPT). Treatment recommendations were administered for a subset of the participants $(n=110, C T=55$, IPT=52, no preference for CT or IPT=3). For the current analyses we limited our sample to those that had a recorded treatment recommendation for either CT or IPT ( $n=107$, $\mathrm{CT}=55$, IPT=52).

\subsection{Statistical predictions}

Statistical treatment predictions were based on algorithms generating the PAI, a measure of the predicted advantage of the indicated therapy (CT or IPT) as compared 
to the other non-indicated therapy. In this study, two types of PAI scores were used: one that was focused on post-treatment BDI-II predictions (post-treatment PAI, Huibers et al., 2015) and one that was based on follow-up BDI-Il predictions (longterm PAI, van Bronswijk et al., 2019). Variables included in the PAl-models were predictors (prognostic factors that predict outcome irrespective of the received treatment type) and moderators (prescriptive factors that predict outcome as a function of treatment type) measured before treatment and randomization. For the post-treatment PAI, predictors and moderators were identified using a modified domain approach that involves a series of linear regression models for different predictor domains (e.g., history of illness or demographics; Fournier et al., 2008). Predictors and moderators were then combined in a linear regression model from which individual predictions were computed using a leave-one-out cross-validation approach (Picard and Cook, 1984). For each individual, a separate prediction for CT and for IPT was computed. The difference between these two predictions was a positive or negative score indicating the PAI-recommended treatment: CT or IPT (DeRubeis et al., 2014). For the long-term treatment PAl model, a two-step machine learning approach was used to identify predictors and moderators: the application of a random forest algorithm (Garge et al., 2013) followed by a backward elimination approach using multiple bootstrapped samples (Austin and Tu, 2004; Rizopoulos and Rizopoulos, 2009). The selected variables were combined in a regression model, and long-term outcomes were predicted for CT and IPT using a fivefold cross-validation (Picard and Cook, 1984) for each individual, and with these predictions, individual PAI scores were computed (DeRubeis et al., 2014). The post-treatment PAl scores were calculated for individuals that had a post-treatment BDI-II score ( $n=134, C T=69$, IPT=65; Huibers et al., 2015), while for the long-term PAl all individuals were included by applying an imputation technique for missing data based on non-parametric random forest approach (Stekhoven and Bühlmann, 2012; van Bronswijk et al., 2019) ( $n=151, C T=76, I P T=75)$. The two PAI models were developed after study termination, and therapists were therefore blind to these estimates. Participants were coded as having received a PAI-indicated or non-indicated treatment according to the posttreatment and long-term PAI models. More detailed information about the development of the PAI models can be found elsewhere (Huibers et al., 2015; van Bronswijk et al., 2019).

\subsection{Statistical analyses}

First, pre-treatment characteristics were compared between individuals that did not have a recorded therapists' recommendation $(n=41)$ and individuals that had a recorded therapists' recommendation for CT or IPT and were included in the analyses $(n=107)$. For these comparisons, $t$-tests and $\chi^{2}$-tests were applied for continuous and categorical variables respectively. Second, pre-treatment characteristics were 
examined between the participants that had a CT versus an IPT recommendation according to clinical judgment using $t$-tests and $\chi^{2}$-tests where appropriate. Significant differences in pre-treatment characteristics between CT versus IPT recommendations ( $p<0.10$ in the t-tests or $\chi^{2}$-tests) were further examined using a logistic regression model. In this logistic regression model, therapist preference was the dependent variable and the pre-treatment variables were the independent variables. Then, the overlap between these pre-treatment variables with the pre-treatment variables that are part of the algorithms of the PAI models was evaluated. Third, the percentage of agreement and kappa coefficients were calculated to examine the level of agreement between PAI recommendations and therapists' recommendations. Fourth, average post-treatment and follow-up depression severity (BDI-II scores) of patients receiving a therapists' recommended versus a non-recommended treatment were compared using t-tests. The results of these t-tests were then compared to the results of previously conducted t-tests on post-treatment and follow-up depression severity of individuals receiving PAI indicated versus PAI non-indicated treatment (Huibers et al., 2015; van Bronswijk et al., 2019). The comparisons between therapists' recommendations and post-treatment PAI recommendations were applied to a subset of the individuals $(n=95, C T=51, I P T=44)$, since 12 individuals with a therapist recommendation did not have a post-treatment PAl recommendation. For the comparisons between therapists' recommendations and long-term PAI recommendations, all individuals with a therapist recommendation had a long-term PAI recommendation and were therefore included in the analyses ( $n=107, C T=55$, IPT=52). Then, for the purpose of comparison between clinical judgment and statistical predictions, three separate regression models were constructed for both post-treatment BDI-II scores and long-term BDI-II scores as dependent variables. For each model, different predictors were included: model 1 included the statistical PAI recommendation for the optimal treatment (the statistical model), model 2 included the therapists' recommendation for the optimal treatment (the clinical judgment model) and model 3 included both the statistical PAI recommendation and therapist recommendation (the combined model). All six models were corrected for depression severity at baseline since there was a small but non-significant difference between CT and IPT at baseline (Lemmens et al., 2015). Finally, treatment dropout rates were compared between individuals receiving a therapists' recommended versus a nonrecommended treatment, and between individuals receiving a PAI indicated versus a PAI non-indicated treatment using $\chi^{2}$-tests. 


\section{3. $\quad$ Results}

\subsection{Pre-treatment characteristics of recorded vs. non recorded therapists' recommendation}

First, pre-treatment characteristics were compared between individuals that did not have a recorded therapists' recommendation $(n=41)$ with those that had a recorded therapists' recommendation for CT or IPT and were included in the analyses $(n=107)$. Results indicated that individuals with a recorded therapists' recommendation were younger $(M=39.6, S D=12.3$ vs. $M=45.7, S D=10.5, t=-2.80, p=0.01)$, had lower functional impairment (Work and Social Adjustment Scale; $M=23.6, S D=7.6$ vs. $\mathrm{M}=20.4, \mathrm{SD}=6.7, \mathrm{t}=2.38, \mathrm{p}=0.02$ ), had less cognitive complaints (Brief Symptom Inventory subscale; $\mathrm{M}=11.9, \mathrm{SD}=4.6$ vs. $\mathrm{M}=9.6, \mathrm{SD}=5.61, \mathrm{t}=2.57, \mathrm{p}=0.01$ ), and $\mathrm{h}$ and were more likely to have a recurrent depression $\left(63.4 \%\right.$ vs. $44.9 \%, \chi^{2}(1, n=107)=4.08$, $\mathrm{p}=0.04)$.

\subsection{Pre-treatment characteristics associated with therapists' and PAI- based recommendations}

In Table 10.1, comparisons between pre-treatment characteristics between the participants that had a CT versus an IPT recommendation by therapists are shown. The proportion of individuals with a comorbid personality disorder was higher for individuals with a CT recommendation versus those with an IPT recommendation according to clinical judgment $\left(\chi^{2}(1)=3.60, p=0.058\right)$. This was not in accordance with the pre-treatment characteristics of the PAI models: personality disorder status appeared only as a predictor (not a moderator) for the post-treatment PAI model, indicating that this variable predicted outcome irrespective of the received treatment (Huibers et al., 2015). In addition, personality disorder status was not part of the longterm outcome PAI algorithm. The level of pre-treatment somatic complaints was significantly higher for individuals with a CT recommendation compared to those with an IPT recommendation by therapists $(t=2.05, d f=105, p=0.043)$. This was in accordance with the post-treatment PAI model. In the post-treatment PAI model, somatic complaints were related to a better response to CT as compared to IPT (Huibers et al., 2015). The number of life events in the past year was significantly higher in individuals with an IPT recommendation versus a CT recommendation based on clinical judgment $(t=-2.18, d f=105, p=0.03)$. This is in contrast to the posttreatment PAI model and the long-term PAI model. In both models, more life events were associated with a better response in CT as compared to IPT (Huibers et al., 2015; van Bronswijk et al., 2019). The presence of a comorbid personality disorder, the level of pre-treatment somatic complaints and the number of life events were then combined in one logistic regression model with therapist recommendations as the 
outcome variable. Results of the model indicated that the presence of a comorbid personality disorder was associated with a CT recommendation $(b=-.75, p=0.104$; trend significant). In addition, the level of pre-treatment somatic complaints was significantly associated with a CT recommendation ( $6=-0.10, p=0.025)$, while the number of life events in the past year was significantly associated with an IPT recommendation $(6=0.33, p=0.041)$.

Table 10.1: Predictors and moderators of the post-treatment and long-term PAI models and comparisons of pre-treatment characteristics between individuals with CT versus IPT therapist recommendations.

\begin{tabular}{|c|c|c|}
\hline \multicolumn{3}{|l|}{ Post-treatment PAI model } \\
\hline Predictors & \multicolumn{2}{|c|}{$\begin{array}{l}\text { Baseline depression severity (BDI-II), gender, } \\
\text { employment status, anxiety symptoms (BSI), } \\
\text { personality disorder (SCID-II), quality of life utility } \\
\text { score (EQ-5D) }\end{array}$} \\
\hline Moderators & \multicolumn{2}{|c|}{$\begin{array}{l}\text { Somatic complaints (BSI), cognitive problems (BSI), } \\
\text { paranoid symptoms (BSI), self-sacrificing (IIP), } \\
\text { attributional style on achievement (ASQ), number of } \\
\text { life events in past year }\end{array}$} \\
\hline \multicolumn{3}{|l|}{ Long-term PAI model } \\
\hline Predictors & \multicolumn{2}{|l|}{ Parental alcohol abuse } \\
\hline Moderators & \multicolumn{2}{|c|}{$\begin{array}{l}\text { Number of life events in past year, number of } \\
\text { childhood trauma events }\end{array}$} \\
\hline \multicolumn{3}{|l|}{ Therapist recommendations } \\
\hline & $\mathrm{CT}$ recommendation & IPT recommendation \\
\hline \multicolumn{3}{|l|}{ Demographics } \\
\hline Age, years, mean (SD) & $39.0(12.7)$ & $40.2(12.1)$ \\
\hline Female, n (\%) & $36(67.9 \%)$ & $34(63.0)$ \\
\hline Partner, n (\%) & $33(62.3 \%)$ & $32(59.3 \%)$ \\
\hline Employed, n (\%) & $34(64.2 \%)$ & $33(61.1 \%)$ \\
\hline \multicolumn{3}{|l|}{ Education level } \\
\hline - Low, n (\%) & $9(17.0 \%)$ & $13(24.1 \%)$ \\
\hline - Intermediate, n (\%) & $35(66.0 \%)$ & 31 (57.4\%) \\
\hline - High, n (\%) & $9(17.0 \%)$ & $10(18.5 \%)$ \\
\hline \multicolumn{3}{|l|}{ Clinical features } \\
\hline BDI-II baseline score, mean (SD) & $30.8(6.6)$ & $29.1(9.4)$ \\
\hline Recurrent depression, $\mathrm{n}(\%)$ & $26(49.1 \%)$ & $22(40.7 \%)$ \\
\hline Comorbid personality disorders (SCID-II), n (\%) & $21(40.4)^{*}$ & $12(23.1)^{*}$ \\
\hline Other comorbid disorders (SCID-I), n (\%) & $24(45.3)$ & $31(57.4)$ \\
\hline Psychological distress (BSI), mean (SD) & $73.7(23.4)$ & $67.6(31.1)$ \\
\hline Anxiety symptoms (BSI), mean (SD) & $8.6(4.6)$ & $8.1(4.5)$ \\
\hline Somatic complaints (BSI), mean (SD) & $8.8(5.3)^{* *}$ & $6.9(4.2)^{* *}$ \\
\hline Cognitive problems (BSI), mean (SD) & $12.2(4.5)$ & $11.7(4.8)$ \\
\hline Paranoid symptoms (BSI), mean (SD) & $7.2(3.3)$ & $6.9(4.9)$ \\
\hline Attributional style on achievement (ASQ), mean (SD) & $5.1(0.8)$ & $4.8(1.0)$ \\
\hline Self-sacrificing (IIP), mean (SD) & $13.5(5.2)$ & $14.6(6.2)$ \\
\hline
\end{tabular}


Table 10.1: (continued)

\begin{tabular}{lcc}
\hline Functionality & & \\
WSAS, mean (SD) & $24.7(6.7)$ & $22.5(8.3)$ \\
EQ-5D, mean (SD) & $0.6(0.2)$ & $0.6(0.2)$ \\
RAND-36, mean (SD) & $14.1(2.8)$ & $14.2(3.1)$ \\
Life and family history & & $2.0(1.4)^{* * *}$ \\
Number of life events last year, mean (SD) & $1.5(1.4)^{* * *}$ & $0.9(1.2)$ \\
Number of childhood trauma events, mean (SD) & $0.9(1.1)$ & $6(11.1)$ \\
Parental alcohol abuse, yes (\%) & $12(22.6)$ & \\
\hline
\end{tabular}

${ }^{*} \mathrm{p}=0.058 ;{ }^{* *} \mathrm{p}=0.043 ;{ }^{* * *} \mathrm{p}=0.031$. BDI-II, Beck Depression Inventory, second edition; BSI, Brief Symptom Inventory; SCID-II, Structured Clinical Interview for DSM-IV Axis II disorders; EQ-5D, EuroQol 5D; IIP, Inventory of Interpersonal Problems; ASQ, Attributional Style Questionnaire; SCID-I, Structured Clinical Interview for DSM-IV Axis I disorders; WSAS, Work and Social Adjustment.

Predictors: prognostic factors that predict outcome irrespective of the received treatment type.

Moderators: prescriptive factors that predict outcome as a function of treatment type.

\subsection{Comparisons between therapists' and PAI-based recommendations}

There was no agreement between therapists' recommendations and post-treatment PAI recommendations (47.4\% agreement, kappa coefficient=-0.07). In addition, there was no agreement between therapists' recommendations and long-term PAI recommendations (43.0\% agreement, kappa coefficient=-0.14). As illustrated in Figure 10.1a, actual post-treatment BDI-II scores were (significantly) lower for individuals randomized to their PAl-indicated treatment as compared to individuals allocated to their PAI non-indicated treatment for both the CT and IPT condition (for a more detailed description, see Huibers et al., 2015). The opposite pattern was found for the treatment recommendations based on clinical judgment: the post-treatment BDI-II scores were higher for individuals who received the therapist recommended treatment as compared to those who received their non-recommended treatment (recommended treatment: $n=46, M=17.1$, non-recommended treatment: $n=49$, $\mathrm{M}=12.6, \mathrm{t}=-1.92, \mathrm{df}=93, \mathrm{p}=0.058, \mathrm{~d}=-0.39(95 \% \mathrm{Cl}=-0.80$ to 0.01$))$. In the $\mathrm{CT}$ condition this difference was not significant (recommended treatment: $n=23, M=15.9$, nonrecommended treatment: $n=21, M=13.8, t=0.67, d f=42, p=0.508, d=0.20(95 \% \mathrm{Cl}=-0.39$ to 0.79), Figure 10.1b). In IPT, this difference was borderline significant (recommended treatment: $n=23, M=18.3$, non-recommended treatment: $n=28$, $\mathrm{M}=11.7, \mathrm{t}=-1.89, \mathrm{df}=49, \mathrm{p}=0.064, \mathrm{~d}=-0.53$ (95\%Cl=-1.09 to 0.03$)$, Figure $10.1 \mathrm{~b}$ ). As shown in Figure 10.2a, follow-up BDI-II scores were significantly lower for individuals allocated to their PAI-indicated treatment as compared to individuals allocated to their PAI non-indicated treatment in the CT condition, but not in the IPT condition (for a more detailed description, see van Bronswijk et al., 2019). Again, for the recommendation based on clinical judgment opposite patterns were found with non- 
significant higher BDI-II follow-up scores for individuals who received their recommended treatment as compared to those that got their non-recommended treatment (recommended treatment: $n=51, M=16.3$, non-recommended treatment: $\mathrm{n}=56, \mathrm{M}=14.3, \mathrm{t}=-0.95, \mathrm{df}=105, \mathrm{p}=0.347, \mathrm{~d}=-0.18(95 \% \mathrm{Cl}=-0.56$ to 0.20$)$ ).
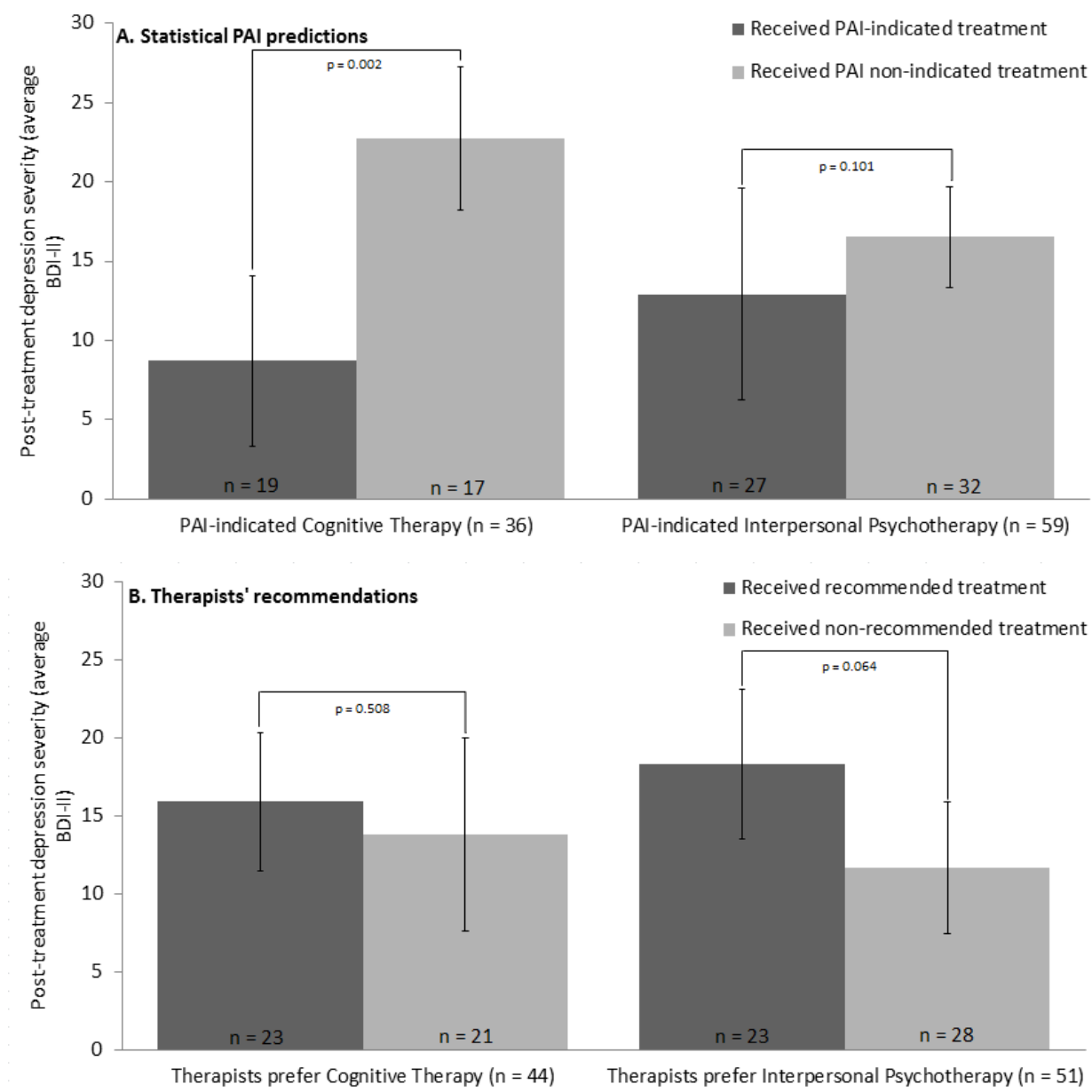

Figure 10.1: Comparison of the observed post-treatment BDI-II scores for individuals randomly assigned to their PAI-indicated optimal treatment versus their PAl-indicated non-optimal treatment (A) and for individuals randomly assigned to their recommended treatment versus non-recommended treatment by the therapists (B). Based on the availability of therapists' recommendations and statistical predictions, a subset of 95 individuals was used to compare post-treatment outcomes (CT=51, IPT=44). PAI predictions of individuals that completed the pre- and posttreatment assessment of the BDI-II $(n=134)$ can be found elsewhere (Huibers et al., 2015). 
This difference was not significant in the CT condition (recommended treatment: $\mathrm{n}=26, \mathrm{M}=14.2$, non-recommended treatment: $\mathrm{n}=27, \mathrm{M}=15.8, \mathrm{t}=-0.57, \mathrm{df}=51, \mathrm{p}=0.574$, $\mathrm{d}=-0.16(95 \% \mathrm{Cl}=-0.69$ to 0.38$)$, Figure $10.2 \mathrm{~b})$. In IPT, individuals with an IPT recommendation had borderline significantly higher follow-up BDI-II scores compared to those with a CT recommendation (recommended treatment: $n=25, M=18.5$, nonrecommended treatment: $n=29, M=12.8, t=-1.77, d f=55, p=0.082, d=-0.48(95 \% C l=$ -1.02 to 0.06$)$, Figure $10.2 b)$.
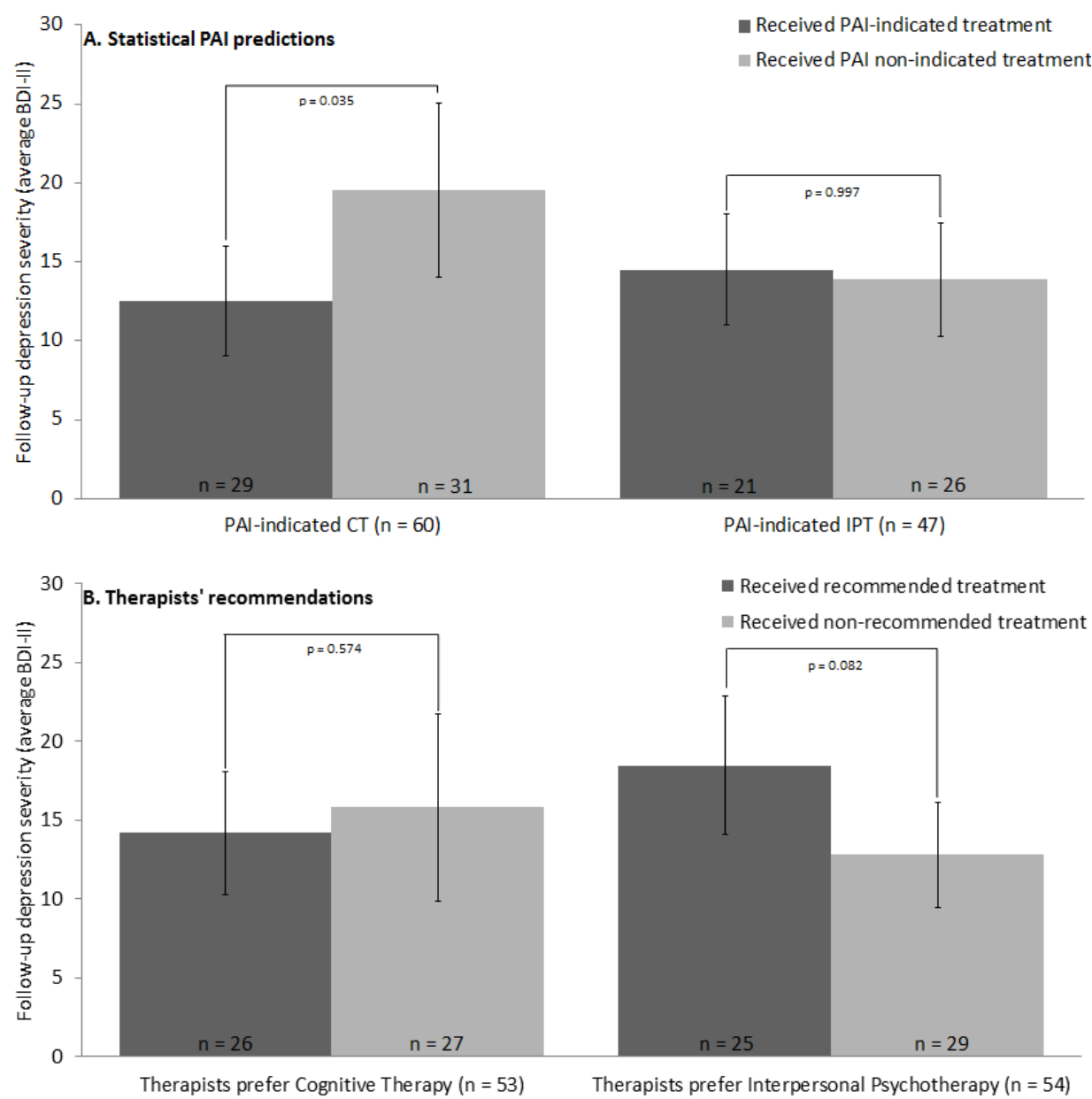

Figure 10.2: Comparison of the observed follow-up BDI-II scores for individuals randomly assigned to their PAl-indicated optimal treatment versus their PAl-indicated non-optimal treatment $(\mathrm{A})$ and for individuals randomly assigned to their recommended treatment versus non-recommended treatment by the therapists (B). Based on the availability of therapists' recommendations and statistical predictions, a subset of 107 individuals was used to compare follow-up outcomes $(C T=55, I P T=52)$. PAl predictions of the total sample ( $n=151$ ) can be found elsewhere (van Bronswijk et al., 2019). 
Model comparisons of statistical predictions versus therapists' recommendations are shown in Table 10.2. The statistical models indicate that the PAI recommendation (PAI-indicated vs. PAI non-indicated treatment) was (borderline) significantly associated with post-treatment and follow-up BDI-II scores, with lower depression severity for individuals receiving a PAl-indicated treatment. In contrast, the therapists' recommendations in the clinical judgment model were not significantly related to post-treatment and follow-up depression severity. When both the PAI recommendations and the therapists' recommendations variables were included in the combined model, only the statistical prediction was associated with posttreatment and follow-up BDI-II scores. In addition, the therapists' recommendation failed to add predictive value to the models in terms of explaining the variance (adjusted $\mathrm{R}^{2}$ ).

Table 10.2: Comparison of models with different predictors: statistical prediction (model 1, "statistical"), therapists' recommendation (model 2, "clinical") and both statistical prediction and therapists' recommendations (model 3, "combined").

\begin{tabular}{|c|c|c|c|c|c|c|c|c|c|}
\hline \multirow{2}{*}{$\begin{array}{l}\text { Outcome: post-treatment BDI-II } \\
\text { Variable }\end{array}$} & \multicolumn{3}{|c|}{ Statistical model } & \multicolumn{3}{|c|}{ Clinical judgment model } & \multicolumn{3}{|c|}{ Combined model } \\
\hline & Coef. & SE & $\mathrm{p}$ & Coef. & SE & $\mathrm{p}$ & Coef. & SE & $\mathrm{p}$ \\
\hline Intercept & 17.49 & 1.27 & $<0.0001$ & 13.20 & 1.52 & $<0.0001$ & 16.31 & 1.80 & $<0.0001$ \\
\hline BDI-II baseline & 5.40 & 0.91 & $<0.0001$ & 5.24 & 1.22 & $<0.0001$ & 4.64 & 1.19 & $<0.0001$ \\
\hline $\begin{array}{l}\text { Statistical prediction } \\
\text { (optimal or non-optimal) }\end{array}$ & -5.38 & 1.82 & 0.004 & & & & -6.23 & 2.12 & 0.004 \\
\hline $\begin{array}{l}\text { Therapists' recommendation } \\
\text { (optimal or non-optimal) }\end{array}$ & & & & 3.04 & 2.20 & 0.171 & 2.88 & 2.12 & 0.178 \\
\hline Adjusted $\mathrm{R}^{2}$ & 0.25 & & & 0.18 & & & 0.24 & & \\
\hline Outcome: follow-up BDI-II & \multicolumn{3}{|c|}{ Statistical model } & \multicolumn{3}{|c|}{ Clinical judgment model } & \multicolumn{3}{|c|}{ Combined model } \\
\hline Variable & Coef. & SE & $\mathrm{p}$ & Coef. & SE & $\mathrm{p}$ & Coef. & SE & $\mathrm{p}$ \\
\hline Intercept & 17.48 & 1.21 & $<0.0001$ & 15.01 & 1.36 & $<0.0001$ & 17.22 & 1.71 & $<0.0001$ \\
\hline BDI-II baseline & 5.32 & 0.87 & $<0.0001$ & 5.28 & 1.10 & $<0.0001$ & 5.46 & 1.09 & $<0.0001$ \\
\hline $\begin{array}{l}\text { Statistical prediction } \\
\text { (optimal or non-optimal) }\end{array}$ & -3.27 & 1.73 & 0.061 & & & & -4.07 & 1.96 & 0.040 \\
\hline $\begin{array}{l}\text { Therapists' recommendation } \\
\text { (optimal or non-optimal) }\end{array}$ & & & & 0.26 & 2.00 & 0.896 & -0.38 & 1.99 & 0.847 \\
\hline Adjusted $\mathrm{R}^{2}$ & 0.20 & & & 0.17 & & & 0.20 & & \\
\hline
\end{tabular}

$\mathrm{SE}$, standard error; BDI-II, Beck Depression Inventory, second edition.

As shown in Table 10.3, the proportion of treatment dropouts was not significantly different in individuals randomized to their PAl-indicated treatment as compared to individuals allocated to their PAI non-indicated treatment in both the post-treatment $\left(\chi^{2}(1)=0.12, p=0.730\right)$ and long-term PAI model $\left(\chi^{2}(1)=1.51, p=0.22\right)$. In addition, there were no differences in dropout rates between individuals that received their recommended versus their non-recommended treatment according to clinical judgment $\left(\chi^{2}(1)=0.25, p=0.62\right)$. 
Table 10.3: Comparison of treatment dropout rates for individuals randomly assigned to their PAI-indicated optimal treatment versus their PAI-indicated non-optimal treatment, and for individuals randomly assigned to their recommended treatment versus non-recommended treatment by the therapists.

\begin{tabular}{lcccc}
\hline & \multicolumn{2}{c}{ CT recommendation } & \multicolumn{2}{c}{ IPT recommendation } \\
Received CT & Received IPT & Received IPT & Received CT \\
$\begin{array}{c}\text { Post-treatment PAI model } \\
\text { Treatment dropouts, } \mathrm{n}(\%)\end{array}$ & $6(31.6)$ & $5(29.4)$ & $3(11.1)$ & $6(18.8)$ \\
$\begin{array}{c}\text { Long-term PAl model } \\
\text { Treatment dropouts, } \mathrm{n}(\%)\end{array}$ & $6(20.1)$ & $7(22.6)$ & $3(14.3)$ & $9(34.6)$ \\
$\begin{array}{c}\text { Therapist recommendations } \\
\text { Treatment dropouts, } \mathrm{n}(\%)\end{array}$ & $6(23.1)$ & $3(11.1)$ & $7(28.0)$ & $9(31.0)$ \\
\hline
\end{tabular}

*Note: Based on the availability of therapists' recommendations and statistical predictions, a subset of 95 individuals was used to compare dropout rates for the post-treatment PAI model (CT=51, IPT=44), and a subset of 107 individuals was used for the long-term PAI model and the therapist recommendations (CT=55, IPT=52).

\section{Discussion}

The current study compared the accuracy of clinical recommendations versus statistical PAI predictions in the context of treatment selection of CT versus IPT for MDD. There was limited overlap between pre-treatment variables associated with therapists' recommendations and pre-treatment variables included in the PAI algorithms. Overall, therapists were more likely to recommend CT to individuals that had a comorbid personality disorder (trend significant) and a higher level of somatic complaints, while an IPT recommendation was more common in individuals with a recent life event. For somatic complaints, the IPT recommendation was in accordance with the post-treatment PAI model. However, comorbid personality disorder was not a moderator in the PAI models and the presence of current life events was, in contrast to the therapist recommendation, identified as a moderator for worse outcomes in IPT in both PAI models. As we reported before, participants that received their indicated treatment according to their PAI score had lower post-treatment and followup depression severity as compared to those that received their PAI non-indicated treatment (for follow-up depression severity, this was only the case for the CT condition; Huibers et al., 2015; van Bronswijk et al., 2019). Interestingly, a reverse pattern was found for the therapists' IPT recommendations: participants that received the therapist recommended treatment had higher post-treatment and follow-up depression severity as compared to those receiving their non-recommended treatment. For individuals with a therapists' recommendation for CT, post-treatment and follow-up depression severity did not differ between those who received the recommended versus the non- recommended treatment. In addition, model comparisons showed a significant association between statistical prediction and 
depression severity, both at post-treatment and follow-up, and demonstrated that therapists' recommendations had no added value to statistical prediction for treatment selection in MDD. Finally, both statistical predictions and therapist recommendations were not associated with treatment dropout; there was no significant difference in treatment dropout rates between individuals assigned to a PAl-indicated or recommended treatment versus those randomized to a PAI nonindicated or non-recommended treatment.

In general, the results indicate that individuals that received a CT recommendation by therapists had more internal difficulties (personality disorder and somatic complaints), while the individuals that were given an IPT recommendation suffered more from external stressors. There are several possible explanations for these different patterns of treatment recommendation. The finding that a comorbid personality disorder was associated with a CT recommendation by therapists could be explained by awareness of therapists of (inconsistent) research findings of CT versus IPT head-to-head studies that suggest that personality disorder features are treatment moderators (Barber and Muenz, 1996; Carter et al., 2011; Joyce et al., 2007; Ryder et al., 2010). Other explanations could be that therapists perceived the more structured nature of CT a better match for individuals with a comorbid PD or that it was thought to be too difficult to find a specific interpersonal focus for IPT since complex interpersonal problems are common in depressed individuals with a comorbid personality disorder (Markowitz et al., 2007). The finding that a higher level of somatic complaints was associated with a CT recommendation by therapists, could be explained by knowledge of therapists on the role of CT in treatment of somatization (Kroenke, 2007). Another explanation could be that therapists thought that the focus on exposure in CT through in vivo homework assignments was a better match for individuals with physical problems. The finding that life events in the past year was associated with an IPT recommendation was in contrast to both PAI models, however not surprising given that three of the four treatment foci of IPT relate to recent life events (complicated bereavement, role dispute, and role transition, Klerman et al., 1984). An explanation for the IPT recommendation could therefore be that therapists aim to target (multiple) recent life events by assigning individuals to IPT.

The results of this study confirmed the finding that statistical prediction is at least as accurate as or more accurate than clinical recommendation (ÆEgisdóttir et al., 2006; Grove et al., 2000). The results are also in line with a recent study that demonstrated that prognostic information based on machine learning techniques outperforms clinical judgment in predicting social functioning in individuals with recent-onset MDD and psychosis (Koutsouleris et al., 2018). In addition to the finding that statistical prediction was more accurate then clinical judgment, our study indicated that participants receiving IPT as recommended by the therapists had higher posttreatment and follow-up depression severity compared to those receiving their non- 
recommended treatment. Although this finding was only trend significant and needs to be replicated, it points to potential iatrogenic effects of current clinical judgement in treatment selection for MDD. Based on previous studies, a few elements could explain the therapists' low predictive accuracy in the process of treatment selection for MDD. First, therapists involved in the clinical judgment came from the same setting as the study participants, which is associated with less accuracy compared to clinicians that make predictions for another setting (ÆEgisdóttir et al., 2006). Second, clinicians based their recommendations mainly on a clinical interview, which is known to negatively affect the prediction of outcome (Grove et al., 2000). Third, the statistical prediction was based on an algorithm, which has been proven to be more accurate then logically constructed rules (/Egisdóttir et al., 2006). Finally, the statistical prediction is based on state-of-the-art PAI methods including cross-validation, and model-based recursive partitioning (Huibers et al., 2015; van Bronswijk et al., 2019).

The final finding of this study was that both statistical prediction and therapist recommendations were not associated with different rates of treatment dropout. The absence of an association between statistical prediction and treatment dropout was not surprising, since treatment dropout is related to other variables than treatment outcome (Swift et al., 2017), and involves different types of PAI models (Keefe et al., 2018; Zilcha-Mano et al., 2016). However, this finding is in contrast to what one might expect from clinical judgment since treatment allocation in clinical practice is not only based on clinical predictions of treatment outcome, but also on clinical predictions of treatment tolerance and engagement.

This study has some limitations. First, therapists' treatment recommendations were based on case presentations by the interviewers and formulated after discussion during regular team meetings. Although this setting is representative for clinical practice, one could argue that the clinicians had access to less data compared to the variables included in the statistical algorithms. However, previous research has shown that increasing the amount of information decreases the clinicians' judgment accuracy, and that access to all variables included in statistical formulas does not improve the clinicians' accuracy (ÆEgisdóttir et al., 2006). Second, since treatment recommendations were formulated during regular interdisciplinary team meetings, the level of expertise of the attendees varied, which could have influenced the accuracy of the clinical predictions (AEgisdóttir et al., 2006). However, this remains unclear as there is conflicting evidence that the level of training, general experience, and task-related experience has impact on the correctness of clinical judgment (Grove et al., 2000). Third, although we link pre-treatment variables to the therapist recommendations, information on the clinical reasoning behind this recommendation was not collected. In addition, detailed information on the therapists involved in the team meetings was not available either. Fourth, for a substantial subset of all study participants no therapists' recommendation was available. This subset had significantly different pre-treatment characteristics (i.e., younger age, less cognitive 
problems, less functioning impairment and a higher percentage of recurrent depression) compared to the participants with a recorded treatment recommendation. However, these missing assessments were related to time constraints that are common in daily clinical practice, and are therefore more likely to be random (instead of related to the specific participant). Finally, although crossvalidation techniques were applied to minimize the risk of overfitting, the model was not tested in an independent dataset which is a limitation of most existing prediction models in mental health (Cohen and DeRubeis, 2018; DeRubeis, 2019). Without external validation and prospective testing, it remains unknown how well these models generalize to clinical practice.

The implementation of statistical prediction for treatment selection in MDD has great potential, particularly in the context of new statistical approaches and data availability (Cohen and DeRubeis, 2018; Perna et al., 2018). Moreover, one might argue that it is unethical to not implement these new approaches (Dawes, 2005). However, as history shows us, scientific findings on superiority of statistical prediction relative to clinical judgment are not sufficient to change mental health care practice. How can we advance the use of these empirical supported predictions in routine clinical practice? One suggestion is by changing clinical training. Education on effective clinical-decision making should be a key part of clinical training, including adequate statistics, probability theory, and education on human judgment errors (/Egisdóttir et al., 2006). Another suggestion is to provide feedback on treatment decision regularly and systematically (Knaup et al., 2009). Clinicians with proper training should collect and evaluate this feedback locally, and adjust existing prediction models accordingly to fit their population and setting (Spengler et al., 1995). Finally, these prediction models should be converted to easy-to-use computerized clinical decision support tools that can be used to fuel the dialogue between the clinician and the patient (Katsikopoulos et al., 2008; Roshanov et al., 2013). In this way, it turns statistical prediction into an empowering platform for ongoing shared decision making. 


\section{References}

ÆEgisdóttir, S., White, M.J., Spengler, P.M., Maugherman, A.S., Anderson, L.A., Cook, R.S., Nichols, C.N., Lampropoulos, G.K., Walker, B.S., Cohen, G., 2006. The meta-analysis of clinical judgment project: Fifty-six years of accumulated research on clinical versus statistical prediction. The Counseling Psychologist 34, 341-382.

Austin, P.C., Tu, J.V., 2004. Bootstrap methods for developing predictive models. The American Statistician 58, 131-137.

Barber, J.P., Muenz, L.R., 1996. The role of avoidance and obsessiveness in matching patients to cognitive and interpersonal psychotherapy: empirical findings from the treatment for depression collaborative research program. J Consult Clin Psychol 64, 951-958.

Beck, A.T., Rush, A.J., Shaw, B.F., Emery, G., 1979. Cognitive therapy of depression. Guilford Press, New York.

Beck, A.T., Steer, R., Brown, G.K., 1996. Beck Depression Inventory II: Manual. Harcourt Brace, Boston.

Beijers, L., Wardenaar, K.J., van Loo, H.M., Schoevers, R.A., 2019. Data-driven biological subtypes of depression: systematic review of biological approaches to depression subtyping. Molecular psychiatry 24, 888-900.

Bell, I., Mellor, D., 2009. Clinical judgements: Research and practice. Australian Psychologist 44, 112-121.

Carter, J.D., Luty, S.E., McKenzie, J.M., Mulder, R.T., Frampton, C.M., Joyce, P.R., 2011. Patient predictors of response to cognitive behaviour therapy and interpersonal psychotherapy in a randomised clinical trial for depression. J Affect Disord 128, 252-261.

Cohen, Z.D., DeRubeis, R.J., 2018. Treatment selection in depression. Annual review of clinical psychology 14, 209-236.

Cooper, A.A., Conklin, L.R., 2015. Dropout from individual psychotherapy for major depression: A metaanalysis of randomized clinical trials. Clinical Psychology Review 40, 57-65.

Cuijpers, P., Andersson, G., Donker, T., van Straten, A., 2011. Psychological treatment of depression: results of a series of meta-analyses. Nordic journal of psychiatry 65, 354-364.

Cuijpers, P., Noma, H., Karyotaki, E., Vinkers, C.H., Cipriani, A., Furukawa, T.A., 2020a. A network meta-analysis of the effects of psychotherapies, pharmacotherapies and their combination in the treatment of adult depression. World Psychiatry 19, 92-107.

Cuijpers, P., Reijnders, M., Huibers, M.J., 2019. The role of common factors in psychotherapy outcomes. Annual Review of Clinical Psychology 15, 207-231.

Cuijpers, P., Stringaris, A., Wolpert, M., 2020b. Treatment outcomes for depression: challenges and opportunities. The Lancet Psychiatry.

Dawes, R.M., 2005. The ethical implications of Paul Meehl's work on comparing clinical versus actuarial prediction methods. Journal of clinical psychology 61, 1245-1255.

Delgadillo, J., Gellatly, J., Stephenson-Bellwood, S., 2015. Decision making in stepped care: how do therapists decide whether to prolong treatment or not? Behavioural and cognitive psychotherapy 43, 328-341.

Delgadillo, J., Lutz, W., 2020. A Development Pathway Towards Precision Mental Health Care. JAMA Psychiatry.

DeRubeis, R.J., 2019. The history, current status, and possible future of precision mental health. Behaviour research and therapy $123,103506$.

DeRubeis, R.J., Cohen, Z.D., Forand, N.R., Fournier, J.C., Gelfand, L.A., Lorenzo-Luaces, L., 2014. The Personalized Advantage Index: translating research on prediction into individualized treatment recommendations. A demonstration. PloS one 9, e83875.

Fournier, J.C., DeRubeis, R.J., Shelton, R.C., Gallop, R., Amsterdam, J.D., Hollon, S.D., 2008. Antidepressant medications v. cognitive therapy in people with depression with or without personality disorder. The British Journal of Psychiatry 192, 124-129.

Furnham, A., Boo, H.C., 2011. A literature review of the anchoring effect. The journal of socio-economics 40, 35-42. 
Garb, H.N., 2000. Computers will become increasingly important for psychological assessment: Not that there's anything wrong with that. Psychological Assessment 12, 31.

Garb, H.N., 2005. Clinical judgment and decision making. Annu. Rev. Clin. Psychol. 2005 1, 67-89.

Garge, N.R., Bobashev, G., Eggleston, B., 2013. Random forest methodology for model-based recursive partitioning: the mobForest package for R. BMC bioinformatics 14, 1-8.

Grove, W.M., Meehl, P.E., 1996. Comparative efficiency of informal (subjective, impressionistic) and formal (mechanical, algorithmic) prediction procedures: The clinical-statistical controversy. Psychology, public policy, and law 2, 293.

Grove, W.M., Zald, D.H., Lebow, B.S., Snitz, B.E., Nelson, C., 2000. Clinical versus mechanical prediction: a meta-analysis. Psychological assessment 12, 19.

Huibers, M.J., Cohen, Z.D., Lemmens, L.H., Arntz, A., Peeters, F.P., Cuijpers, P., DeRubeis, R.J., 2015. Predicting Optimal Outcomes in Cognitive Therapy or Interpersonal Psychotherapy for Depressed Individuals Using the Personalized Advantage Index Approach. PLoS One 10, e0140771.

Joyce, P.R., McKenzie, J.M., Carter, J.D., Rae, A.M., Luty, S.E., Frampton, C.M., Mulder, R.T., 2007. Temperament, character and personality disorders as predictors of response to interpersonal psychotherapy and cognitive-behavioural therapy for depression. Br J Psychiatry 190, 503-508.

Katsikopoulos, K.V., Pachur, T., Machery, E., Wallin, A., 2008. From Meehl to Fast and Frugal Heuristics (and Back) New Insights into How to Bridge the Clinical-Actuarial Divide. Theory \& Psychology 18, 443464.

Keefe, J.R., Wiltsey Stirman, S., Cohen, Z.D., DeRubeis, R.J., Smith, B.N., Resick, P.A., 2018. In rape trauma PTSD, patient characteristics indicate which trauma-focused treatment they are most likely to complete. Depression and anxiety 35, 330-338.

Klerman, G.L., Weissman, M.M., Rounsaville, B.J., Chevron, E.S., 1984. Interpersonal psychotherapy for depression. Basis Books, New York.

Knaup, C., Koesters, M., Schoefer, D., Becker, T., Puschner, B., 2009. Effect of feedback of treatment outcome in specialist mental healthcare: meta-analysis. The British Journal of Psychiatry 195, 15-22.

Koutsouleris, N., Kambeitz-llankovic, L., Ruhrmann, S., Rosen, M., Ruef, A., Dwyer, D.B., Paolini, M., Chisholm, K., Kambeitz, J., Haidl, T., 2018. Prediction models of functional outcomes for individuals in the clinical high-risk state for psychosis or with recent-onset depression: a multimodal, multisite machine learning analysis. JAMA psychiatry 75, 1156-1172.

Kroenke, K., 2007. Efficacy of treatment for somatoform disorders: a review of randomized controlled trials. Psychosomatic medicine 69, 881-888.

Lemmens, L., Arntz, A., Peeters, F., Hollon, S., Roefs, A., Huibers, M., 2015. Clinical effectiveness of cognitive therapy $v$. interpersonal psychotherapy for depression: results of a randomized controlled trial. Psychological Medicine 45, 2095-2110.

Lemmens, L.H., Arntz, A., Peeters, F.P., Hollon, S.D., Roefs, A., Huibers, M.J., 2011. Effectiveness, relapse prevention and mechanisms of change of cognitive therapy vs. interpersonal therapy for depression: study protocol for a randomised controlled trial. Trials 12, 150.

Lorenzo-Luaces, L., DeRubeis, R.J., Bennett, I.M., 2015. Primary care physicians' selection of low-intensity treatments for patients with depression. Fam Med 47, 511-516.

Markowitz, J.C., Skodol, A.E., Petkova, E., Cheng, J., Sanislow, C.A., Grilo, C.M., Gunderson, J.G., McGlashan, T.H., 2007. Longitudinal effects of personality disorders on psychosocial functioning of patients with major depressive disorder. Journal of Clinical Psychiatry 68, 186.

Meehl, P.E., 1954. Clinical versus statistical prediction: A theoretical analysis and a review of the evidence.

Meehl, P.E., 1986. Causes and effects of my disturbing little book. Journal of personality assessment 50, 370-375.

Nickerson, R.S., 1998. Confirmation bias: A ubiquitous phenomenon in many guises. Review of general psychology 2, 175-220.

Papakostas, G.I., Fava, M., 2010. Pharmacotherapy for depression and treatment-resistant depression. World Scientific.

Perna, G., Grassi, M., Caldirola, D., Nemeroff, C., 2018. The revolution of personalized psychiatry: will technology make it happen sooner? Psychological medicine 48, 705-713. 
Picard, R.R., Cook, R.D., 1984. Cross-validation of regression models. Journal of the American Statistical Association 79, 575-583.

Richards, P.M., Geiger, J.A., Tussey, C.M., 2015. The dirty dozen: 12 sources of bias in forensic neuropsychology with ways to mitigate. Psychological injury and law 8, 265-280.

Rizopoulos, D., Rizopoulos, M.D., 2009. Package 'bootStepAIC'.

Roshanov, P.S., Fernandes, N., Wilczynski, J.M., Hemens, B.J., You, J.J., Handler, S.M., Nieuwlaat, R., Souza, N.M., Beyene, J., Van Spall, H.G., 2013. Features of effective computerised clinical decision support systems: meta-regression of 162 randomised trials. Bmj 346, f657.

Rush, A.J., Trivedi, M.H., Wisniewski, S.R., Nierenberg, A.A., Stewart, J.W., Warden, D., Niederehe, G., Thase, M.E., Lavori, P.W., Lebowitz, B.D., 2006. Acute and longer-term outcomes in depressed outpatients requiring one or several treatment steps: a STAR* D report. American Journal of Psychiatry 163, 19051917.

Ryder, A.G., Quilty, L.C., Vachon, D.D., Bagby, R.M., 2010. Depressive personality and treatment outcome in major depressive disorder. J Pers Disord 24, 392-404.

Simon, G.E., Perlis, R.H., 2010. Personalized medicine for depression: can we match patients with treatments? Am J Psychiatry 167, 1445-1455.

Spengler, P.M., Strohmer, D.C., Dixon, D.N., Shivy, V.A., 1995. A scientist-practitioner model of psychological assessment: Implications for training, practice and research. The Counseling Psychologist 23, 506-534.

Stekhoven, D.J., Bühlmann, P., 2012. MissForest-non-parametric missing value imputation for mixed-type data. Bioinformatics 28, 112-118.

Swift, J.K., Greenberg, R.P., Tompkins, K.A., Parkin, S.R., 2017. Treatment refusal and premature termination in psychotherapy, pharmacotherapy, and their combination: A meta-analysis of head-to-head comparisons. Psychotherapy 54, 47.

van Bronswijk, S.C., DeRubeis, R.J., Lemmens, L.H., Peeters, F.P., Keefe, J.R., Cohen, Z.D., Huibers, M.J., 2019. Precision medicine for long-term depression outcomes using the Personalized Advantage Index approach: cognitive therapy or interpersonal psychotherapy? Psychological medicine, 1-11.

Warden, D., Trivedi, M.H., Carmody, T., Toups, M., Zisook, S., Lesser, I., Myers, A., Kurian, K.R.B., Morris, D., Rush, A.J., 2014. Adherence to antidepressant combinations and monotherapy for major depressive disorder: a CO-MED report of measurement-based care. Journal of Psychiatric Practice ${ }^{\circledR} 20,118-132$.

Zilcha-Mano, S., Keefe, J.R., Chui, H., Rubin, A., Barrett, M.S., Barber, J.P., 2016. Reducing Dropout in Treatment for Depression: Translating Dropout Predictors Into Individualized Treatment Recommendations. The Journal of clinical psychiatry 77, e1584-e1590. 
S. van Bronswijk, S. Bruijniks, L. Lorenzo-Luaces, R. DeRubeis, L. Lemmens, F. Peeters, M. Huibers - Psychotherapy Research 2020

\section{Chapter}

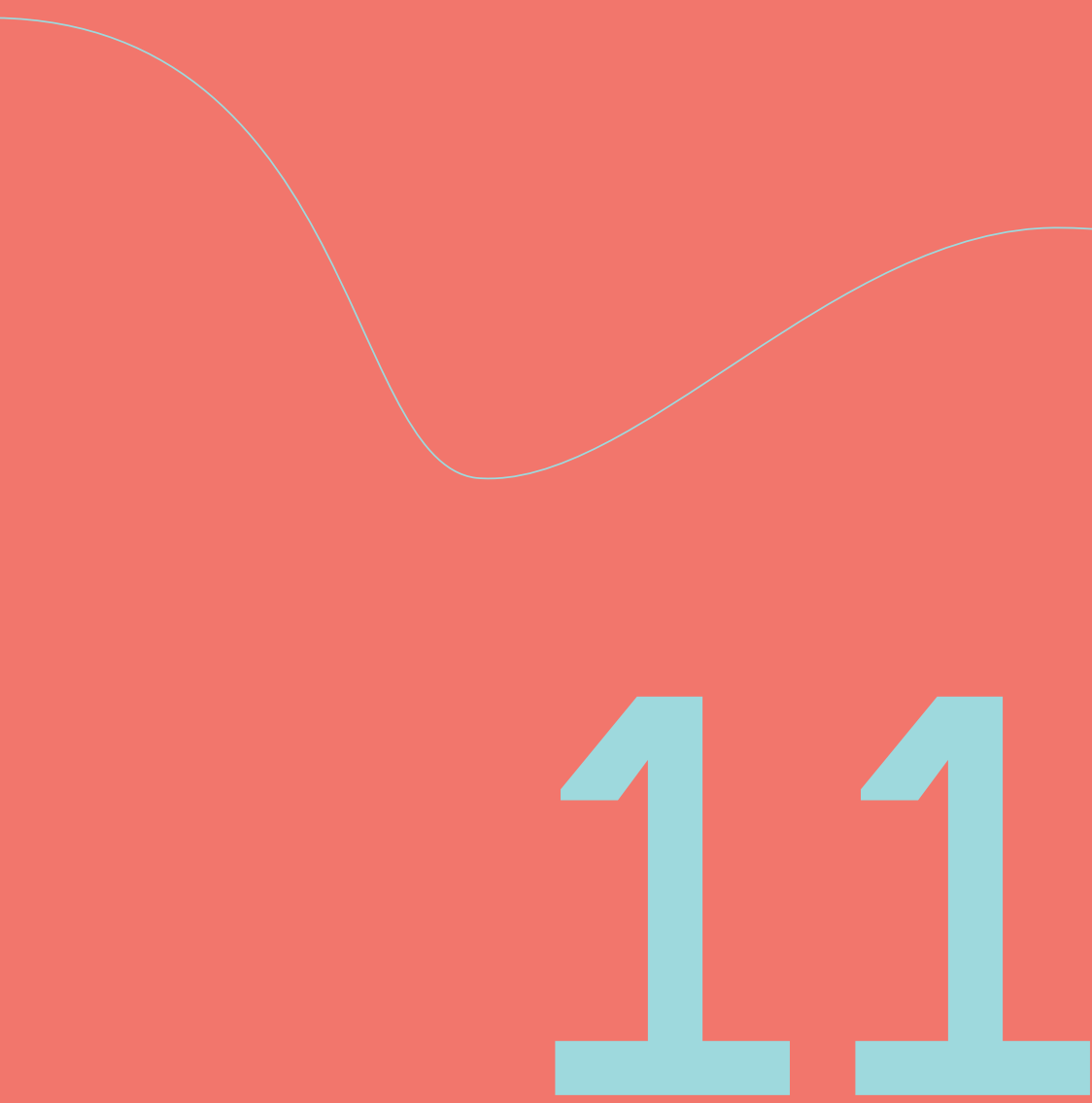


CROSS-TRIAL
PREDICTION IN

PSYCHOTHERAPY:

EXTERNAL

VALIDATION OF

THE PERSONALIZED

ADVANTAGE INDEX

USING MACHINE

LEARNING IN TWO

DUTCH RANDOMIZED

TRIALS COMPARING

COGNITIVE

BEHAVIORAL

THERAPY VERSUS

INTERPERSONAL

PSYCHOTHERAPY

FOR DEPRESSION 


\section{Abstract}

\section{Background}

Optimizing treatment selection may improve treatment outcomes in depression. A promising approach is the Personalized Advantage Index (PAI), which predicts the optimal treatment for a given individual. To determine the generalizability of the PAI, models needs to be externally validated, which has rarely been done.

Methods

Using machine learning techniques, PAI models were developed within each of two independent trials comparing CBT and IPT for depression (STEPd: $n=151$ and FreqMech: $n=200$ ). Subsequently, both PAI models were tested in the other dataset.

\section{Results}

In the STEPd study, post-treatment depression was significantly different between individuals assigned to their PAl-indicated treatment versus those assigned to their non-indicated treatment $(\mathrm{d}=.57)$. In the FreqMech study, post-treatment depression was not significantly different between patients receiving their indicated treatment versus those receiving their nonindicated treatment $(\mathrm{d}=.20)$. Cross-trial predictions indicated that post-treatment depression was not significantly different between those receiving their indicated treatment and those receiving their non-indicated treatment ( $d=.16$ and $d=.27)$. Sensitivity analyses indicated that cross-trial prediction based on only overlapping variables didn't improve the results.

\section{Conclusions}

External validation of the PAI has modest results and demonstrates and emphasizes betweenstudy differences and many other challenges. 


\section{Introduction}

Although there are multiple treatments available for major depressive disorder (MDD), only about half of the individuals recover after treatment (National Health Service, 2018). Current guidelines prescribe antidepressants, cognitive behavioral therapy (CBT) or interpersonal psychotherapy (IPT) among the first choices of treatment for depression, and this choice is often based on the severity of the depression and the number and effects of earlier treatments (American Psychiatric Association, 2009; Lorenzo-Luaces, DeRubeis, \& Bennett, 2015; National Collaborating Centre for Mental Health, 2010). However, individuals may respond differentially to different treatments, and these responses are difficult to predict. Matching individuals to their optimal treatment, also referred to as personalized or precision medicine, seems to be a promising way to improve treatment outcomes for depression (Cohen \& DeRubeis, 2018).

In order to match patients to their optimal treatment, it is necessary to predict which treatment works best for whom. DeRubeis et al. (2014) introduced and demonstrated the Personalized Advantage Index (PAI) which generates actionable individual treatment recommendations. The PAI represents, for a certain individual, the difference in predicted outcomes between two or more treatments. In order to compute this difference, DeRubeis et al. built a multivariable regression model based on pre-treatment variables that were found to be prognostic (i.e., predicting treatment outcome irrespective of treatment, also known as predictors) or prescriptive (i.e., predicting differential treatment outcome, also known as moderators). In that study, which compared CBT with antidepressants, $60 \%$ of the patients had a statistically significant and clinically relevant difference between their predicted optimal treatment and their predicted non-optimal treatment. Similar studies have been conducted since, investigating individual advantages in CBT versus IPT for MDD (Huibers et al., 2015), CBT versus psychodynamic treatment for MDD (Cohen, Kim, Van, Dekker, \& Driessen, 2019), and CBT versus CBT with integrated exposure and emotion-focused elements for MDD (Friedl, Berger, Krieger, Caspar, \& Grosse Holtforth, 2019). The PAI has also been studied in the contexts of supportiveexpressive therapy, antidepressants and placebo for MDD (Zilcha-Mano et al., 2016), eye movement desensitization and reprocessing versus cognitive therapy for posttraumatic stress disorder (PTSD; Deisenhofer et al., 2018), prolonged exposure versus cognitive processing therapy for PTSD (Keefe et al., 2018), antidepressants versus placebo for MDD (Webb et al., 2019), and CBT versus a positive psychology intervention for MDD (Lopez-Gomez et al., 2019). Overall, these studies indicated that different treatments may have different clinically relevant effects for a given individual and that use of the PAI may improve outcomes by optimizing treatment selection. 
However, to determine the generalizability of PAI models in actual clinical practice, the predictive accuracy of these models needs to be externally validated (Bleeker et al., 2003; Cohen \& DeRubeis, 2018; Gillan \& Whelan, 2017). External validation is considered a second phase in multivariable prognostic research, following model development and preceding impact studies (Moons, Royston, Vergouwe, Grobbee, \& Altman, 2009). Although it is widely acknowledged that the performance of prediction models should be examined by external validation studies, this has been done very infrequently in medicine in general and mental health care specifically (Siontis, Tzoulaki, Castaldi, \& loannidis, 2015). Most published studies examining PAI predictions that have focused on validation, focus on the issue of internal validation using techniques such as bootstrapping (random sampling with replacement), crossvalidation, and split-sampling (Efron, 1983; Efron \& Tibshirani, 1997; Efron \& Tibshirani, 1993). Internal validation methods, in particular bootstrapping (Steyerberg et al., 2001), can provide bias-corrected estimates of model performance. However, models tend to have better results in data on which the model was developed than in new data, which is often referred to as "overfitting" (i.e., high variance in the biasvariance trade-off: models perform well on training data but have high error rates across testing dataset; Bleeker et al., 2003). Internal validation alone is unlikely to be sufficient for PAI models which have typically been based on relatively small datasets, and are considered to be prone to the risk of overfitting (Cohen \& DeRubeis, 2018; Luedtke, Sadikova, \& Kessler, 2019). One PAl study that used an external validation sample successfully was recently presented by Delgadillo and colleagues (2019). In this study, individual advantages in CBT versus person centered counselling were investigated for depressed individuals in primary care. In order to develop a generalizable PAI model, the data was split into a training sample and a test sample. The training sample was used to develop two prognostic models to predict outcome, one model for each treatment. The test sample was subsequently used to test the accuracy of these models. Interestingly, the prognostic models that were developed in the training sample showed significant accuracy in the test sample, indicating proof for generalizability. As expected, the patients who received their indicated treatment showed better response compared to the patients who received their non-indicated treatment.

The present study used a machine learning approach to compute PAI models in data from two Dutch randomized trials comparing CBT and IPT for MDD. Subsequently, the PAI models that were developed and tested within each sample were externally validated in the other sample to determine the generalizability of these models to future groups of patients. To our knowledge, this study is the first that attempts to externally validate the generalizability of PAI models between two randomized trials. 


\section{Methods}

\subsection{Study design and clinical trial data}

PAI models were developed within each dataset of two Dutch randomized trials comparing acute-phase CBT and IPT for MDD. The first trial is called the STEPd trial and was conducted between February 2007 and April 2012, randomizing depressed patients into CBT ( $n=76)$, IPT ( $n=75)$ or a waitlist condition followed by a treatment of choice $(n=31)$. Main results were reported in 2015 and 2019, indicating no significant acute and long-term outcome difference between CT and IPT on average (Lemmens et al., 2015; Lemmens et al., 2019). The second trial is the FreqMech trial $(n=200)$, which was conducted between November 2014 and January 2018, randomizing depressed patients into different session frequencies of CBT and IPT (i.e., CBT weekly $(n=49)$, CBT twice weekly ( $n=49)$, IPT weekly $(n=55)$, IPT twice weekly $(n=47))$. A higher session frequency led to more reduction of depression, but there were no average group differences between CBT and IPT (Bruijniks et al., 2020). Although the studies used the same treatment protocols for CBT and IPT (i.e., only the session frequency differed), the studies differed in a number of other important aspects. First, while the STEPd trial was a single center study conducted in one specific area in the Netherlands (Maastricht), the FreqMech trial was a multi-center study conducted in multiple areas in the Netherlands (Maastricht, Amsterdam, The Hague, Leiden, Utrecht, Nijmegen, Oss, Haarlem). Second, the two studies had a different number of therapists (10 in the STEPd trial and 76 in the FreqMech trial). Third, the average levels of pre- and posttreatment depression differed: patients in the FreqMech trial started with higher levels of depression compared to patients in the STEPd trial (average Beck Depression Inventory - version II (BDI-II) scores of 29.8 versus 34.7 for STEPd and FreqMech respectively). After treatment, participants in the FreqMech study ended up with levels of moderate depression (average BDI-II scores between 20.0-24.2) that were still higher compared to the patients in the STEPd trial who showed mild levels of depression (average BDI-II scores between 13.3-15.8). This difference could be explained by different inclusion criteria: for the STEPd study a minimum BDI-II score of 10 was required, whereas for FreqMech this was a minimum of 20 . Fourth, as described below, in- and exclusion criteria were slightly different. Details about the protocols of both trials can be found in Lemmens et al. (2011) and Bruijniks et al. (2015).

\subsection{Participants}

Participants were adult outpatients (18-65 years) of the Academic Community Mental Health Center Maastricht (both FreqMech and STEPd), and for FreqMech of PsyQ (The Hague, Leiden, Amsterdam or Haarlem), Altrecht GGZ (Utrecht), GGZ inGeest 
(Amsterdam), GGZ Oost-Brabant (Oss), and Pro Persona (Nijmegen). Participants had a primary diagnosis of MDD, confirmed by the Structured Clinical Interview for DSM-IV Axis I disorders (SCID-I; First, Spitzer, Gibbon, \& Williams, 1995) or Mini International Neuropsychiatric Interview-Plus (MINI-Plus; Van Vliet, Leroy, \& Van Megen, 2000). Joint inclusion criteria were internet access and sufficient knowledge of the Dutch language. Joint exclusion criteria were a high suicide risk, or a diagnosis of abuse disorders (according to DSM-IV or V), concomitant psychological treatment, or mental retardation $(\mathrm{IQ}<80)$. Inclusion and exclusion criteria between trials differed in that STEPd excluded bipolar or highly chronic (current episode $>5$ years) depression, and current use of antidepressant medication, while in the FreqMech trial patients were only excluded when they started antidepressants or changed the dosage in the past three months. The FreqMech trial additionally only included patients with a pretreatment score $\geq 20$ on the Beck Depression Inventory II (BDI-II) and excluded presence of a diagnosis of a cluster A or B personality disorders (according to DSM-IV or $\mathrm{V}$ ). In addition, patients that had received more than five sessions of adequate CBT or IPT in the previous year were excluded. The Medical Ethics Committee (MEC) approved both trial protocols (i.e., MEC of Maastricht University for the STEPd trial, MEC of VU Medical Center for the FreqMech trial), and all participants provided written informed consent. The trials are registered at the Netherlands Trial Register, part of the Dutch Cochrane Center (NTR838 and NTR4856).

\subsection{Treatments}

The same treatment protocols were used for both studies: CBT was based on the manual by Beck and colleagues (Beck, Rush, Shaw, \& Emery, 1979) and IPT was based on the manual by Klerman and colleagues (Klerman, Weissman, Rounsaville, \& Chevron, 1984). In both trials, patients received 12 up to 20 individual sessions of 45 minutes, but session frequency differed per trial: in the FreqMech trial half of the patients received 16 weekly sessions and the other half received 16 twice weekly sessions. The last four sessions were scheduled weekly for both conditions. In the STEPd trial patients received weekly sessions with some flexibility to schedule appointments less often then weekly. Treatment competence was rated good to excellent in $83-90 \%$ of the videotapes from the STEPd trial (Lemmens et al., 2015) and poor to excellent in the FreqMech trial (only $12-16 \%$ of the video-tapes were rated good-excellent; Bruijniks et al., 2020) using the Cognitive Therapy Scale for CBT (Dobson, Shaw, \& Vallis, 1985), and the short version of the IPT Adherence and Quality Scale for IPT (Stuart, 2011) ${ }^{1}$. In both trials, there were significant differences in

\footnotetext{
${ }^{1}$ Questions regarding treatment competence had different Likert scales in the two studies. In STEPd, good to excellent competence was defined as a score of 4 or more on a 6 point Likert scale (for CBT) and as a score of 3 or more on a 5 point Likert scale (for IPT). In the Freqmech study, good to excellent competence was defined as a score of 5 or more on a 7 point Likert scale.
} 
therapy-specific behaviour, with higher CBT-specific behaviour in CBT as compared to IPT, and higher IPT-specific behaviour in IPT as compared to CBT (rated with the Collaborative Study Psychotherapy Rating Scale version 6; Hollon et al., 1988).

\subsection{Outcome}

In both trials, the BDI-II (Beck, Steer, \& Brown, 1996) was used as the primary outcome measure of post-treatment depression symptom severity. The BDI-II has 21 items with higher scores indicating higher severity. Reliability and validity of the BDI-II has been established (Wang \& Gorenstein, 2013). The post-treatment time point was 7 months and 6 months after the start of therapy in the STEPd study and the FreqMech study respectively.

\subsection{Variables}

Pre-treatment variables covered the following domains: demographics (11 variables), depression and other symptoms (18 variables), present and previous health care use (7 variables), general functioning (14 variables), psychological processes (14 variables) and life and family history ( 7 variables). An overview of the available pre-treatment variables can be found in Supplement 1.

\subsection{Statistical analyses}

\subsubsection{Data preparation}

\subsubsection{Pre-selection of pre-treatment variables}

Pre-treatment variables were pre-selected for each dataset by examining their correlation matrices to remove highly correlated and redundant variables. This was done in a step-wise approach by first removing the predictors with the most correlated relationship (Kuhn \& Johnson, 2013). In this way, a minimum number of variables was removed below the threshold of .7 corrected for attenuation. A few exceptions to this rule were made when variables were considered to relate to different underlying information. These decisions were achieved via consensus between the study authors. An overview of the included pre-treatment variables can be found in Supplement 1.

\subsubsection{Transformation of pre-treatment variables}

Pre-treatment variables were prepared in the following ways: 0. Categorical variables with limited observations in some of the categories were merged, since previous research recommends at least $10 \%$ of the sample in each category (Kuhn \& Johnson, 
2013), 0. Continuous predictors were checked on normality and were, in case of nonnormality, log transformed (rightly skewed distributions) or squared (left skewed distributions) to obtain a normal distribution, 0. Normal distributed continuous variables were standardized, and dichotomous and discrete variables were centered by recoding the variables to prevent potential errors in statistical inference (Kraemer \& Blasey, 2004). The applied transformation for each pre-treatment variable is described in Supplement 1.

\subsubsection{Transformation of the outcome variable}

Because residuals of the outcome variable in both datasets were not homoscedastic, outcomes were transformed using a squared root transformation. The transformed outcome variable had residuals that were not significantly different from a normal distribution.

\subsubsection{Data imputation}

Missing values of the pre-treatment and outcome variables were imputed using the "MissForest" package in R (Stekhoven, 2011; Stekhoven \& Bühlmann, 2011; Tang \& Ishwaran, 2017). The following information was used as input for the imputation procedure: 1 ) change scores from baseline of all non-missing post-treatment BDI-II outcomes (at 3 and 7 months for the STEPd trial, at 6 months for the FreqMech trial; Moons, Donders, Stijnen, \& Harrell, 2006); 2) all values of non-missing pre-treatment variables; 3 ) the received treatment (CBT versus IPT; twice weekly versus once weekly). The accuracy of the imputation method was tested by comparing imputed values with actual values using artificially produced missing data in the non-missing dataset. These comparisons were done by calculating the normalized root mean squared error (NRMSE) for continuous data and the proportion of falsely classified entries (PFC) for categorical data.

\subsubsection{Building the Personalized Advantage Index model}

Following (Keefe et al., 2018; van Bronswijk, DeRubeis, et al., 2019; van Bronswijk, Lemmens, et al., 2019), two machine learning techniques were used to select pretreatment variables in each dataset to build a treatment-modality interaction PAI model. Although the STEPd dataset was used before to develop a PAI model (Huibers et al., 2015), in that study less advanced methods were applied to build a prediction model (i.e., no imputation of missing data, stepwise variable selection approach and leave-one-out cross validation). Therefore, in the present study, we decided to build a PAI model for the STEPd dataset and the FreqMech dataset using state-of-the-art machine learning techniques. In the first variable selection step, a random forest algorithm was applied with the package "mobforest" in R (Garge, Bobashev, \& Eggleston, 2013). In this algorithm, a predetermined model is used, and multiple trees 
are created by splitting bootstrapped samples into subgroups based on pre-treatment variables that lead to significantly different model behaviour on either side of the split. The predetermined model for the current analyses was a regression model with post-treatment BDI-II scores as the dependent variable and treatment as the independent variable ( $y=$ treatment). A split variable therefore indicates a variable within which there are treatment differences (i.e., a potential moderator. At each split a random subset of variables was selected, to prevent that variables with smaller effects are dominated by the stronger variables (Strobl, Boulesteix, Kneib, Augustin, \& Zeileis, 2008). Variables were subsequently selected if the difference between predictive accuracy of a variable versus the predictive accuracy of a randomly permuted variable on the outcome (i.e., the variable importance score) was higher than the absolute value of the lowest ranking predictor. Parameters were set as follows: 10,000 trees were computed with a minimum level of 0.10 for splits and a minimum node size for splitting of 15 individuals. In the second variable selection step, the variables that resulted from random forest analyses were tested using backwards elimination on 1000 bootstrapped samples using the package 'bootstepAIC' in R (Rizopoulos \& Rizopoulos, 2009). A regression model with posttreatment BDI-II as the dependent variable and the selected variables with their interaction with treatment as independent variables was fitted and tested. Predictors and moderators that were included in at least $60 \%$ of the bootstrapped samples were included in the final model (Austin \& Tu, 2004).

Earlier studies have shown that building two separate prognostic models (one for the patients who received CBT and one for the patients who received IPT) might lead to a better PAI model compared to a treatment-modality interaction model (Delgadillo \& Gonzalez Salas Duhne, 2019). Therefore two prognostic models using elastic net regression were developed as well (with R-package glmnet; Friedman, Hastie, \& Tibshirani, 2010; Zou \& Hastie, 2005). In the elastic net regression, the following parameters were estimated in subsequent order: (1) alpha indicating the balance between Lasso (alpha=1) and Ridge regression (alpha=0), (2) lambda, reflecting the amount of shrinkage for the chosen alpha. The optimal alpha was chosen by estimating the cross-validated error for each potential .05 alpha between 0 and 1 (i.e., 0, .05, .10 etc.). For each possible alpha 10-fold cross-validation was conducted 25 times (i.e., 25 iterations) and the cross-validated error was computed. The alpha with the minimal mean cross- validated error (mcve) was chosen. Subsequently, the lambda's with the lowest mean cross- validated error was computed using 10-fold cross validation with the optimal alpha included in the model and repeating this process a 1000 times (i.e., 1000 iterations). For both treatmentmodality interaction models and the prognostic models, predictions were computed using a 5-fold cross-validation. For each fold, BDI-II post-treatment was predicted using the weights of the individuals from the remaining four folds. Model performance was compared using the R-squared (i.e., the explained variance 
corrected for the number of included pre-treatment variables, the higher the better) and the root mean squared error (RMSE, i.e., the root of the sum of the squared residuals, which are the observed values minus the model predictions, the lower the better). All treatment-modality interaction models had superior or comparable model performance results as compared to the prognostic models. It was therefore decided to use the treatment-modality interaction models to compute all PAI scores. In the remainder of this paper, only the results of the treatment-modality interaction models are reported.

\subsubsection{Computing the Personalized Advantage Index scores}

For each dataset separately, PAl scores were computed by combining all final predictors and moderators in a multivariable regression model with post-treatment BDI-II as the outcome using 5-fold cross validation. For each fold, BDI-II posttreatment was predicted as if this individual would have received CBT (or IPT) using the weights of the individuals from the remaining four folds. For each individual, PAI scores were calculated as the difference between his or her predicted outcomes in CBT and IPT. Differences in observed BDI-Il post-treatment scores between patients that received versus not received their PAI-indicated treatment were tested using a two-sample t-test for the total sample, and for CBT and IPT separately. Following DeRubeis et al. (2014), we also compared the observed post-treatment BDI-Il scores of individuals with the highest $60 \%$ PAl-scores (absolute values). The effect sizes (Cohen's d) for the difference in post-treatment scores between patients that were randomized versus not randomized to their optimal treatment were reported.

\subsubsection{Cross-trial prediction}

First, predictors and moderators from the PAI models of each independent dataset were compared to determine whether these predictors and moderators were also present in the other dataset. Second, PAI scores were generated in the other dataset using the PAI model and predictors and moderators' weights of the original dataset. To facilitate this, similar transformations of predictors and moderators in the original datasets were applied to the other variables of the other dataset. Differences in observed BDI-II post-treatment scores between patients that received versus not received their optimal treatment were presented for both datasets.

\subsubsection{Sensitivity analysis}

The aim of the present paper was to investigate how independent PAI models would translate to other study samples. Another way to investigate the potential of crosstrial prediction is to start with only overlapping variables from each dataset, develop the PAI models and investigate whether these models can be externally validated. This analysis would avoid the problem of differences in measurements between studies 
and provide insight on how PAI models would externally validate if, in a hypothetical situation, they would have been developed and tested in mental health care centers with similar data collection procedures. To test this, we conducted a sensitivity analyses were we only included variables that were overlapping between the datasets. An overview of the overlapping variables between the datasets and the applied transformations can be found in Supplement 2.

\section{Results}

\subsection{Variable description and missing data imputation}

Table 11.1 shows the sample description of the STEPd and FreqMech trial. Variables with missing data in the STEPd trial had missing values between 0.007 and 0.03 percent of the cases. A total of 17 individuals had a missing value on the posttreatment BDI-II (11.3\%). Variables that had missing data in the FreqMech dataset had between .5 and $32.5 \%$ missing values. A total of 55 values were missing for the posttreatment BDI-II scores (27.5\%). In both datasets, imputation appeared to be accurate when applied to the complete (non-missing) data with artificially produced missing data with estimated NRMSE's of 0.008 (STEPd) and 0.20 (FreqMech), and estimated PFC's of 0.10 and 0.32 (as compared to previous study examples; Stekhoven \& Bühlmann, 2011; Waljee et al., 2013).

\subsection{The Personalized Advantage Index in STEPd}

\subsubsection{Selection of predictors and moderators}

Nine variables were selected using the mobforest algorithm. Of these variables, three predictors and five moderators were selected in at least $60 \%$ of the bootstrap samples using the backwards elimination technique. Having a job, less anxiety symptoms and dysfunctional attitudes predicted lower post-treatment BDI-II scores irrespective of the type of treatment received. Paranoid symptoms, pre-treatment depression severity, and the number of life events in the past year was related to higher BDI-II scores post-treatment in IPT compared to CBT. Cognitive problems and hopelessness were related to higher BDI-II scores post-treatment in CBT compared to IPT. 
Table 11.1: Sample description for CBT and IPT per trial dataset.

\begin{tabular}{|c|c|c|c|c|}
\hline & STEPd & & FreqMech & \\
\hline & CBT $(n=76)$ & IPT $(n=75)$ & CBT $(n=98)$ & IPT $(n=102)$ \\
\hline \multicolumn{5}{|l|}{ Demographics } \\
\hline Female, n (\%) & $54(71.1)$ & $46(61.3)$ & $62(63.3)$ & $61(59.8)$ \\
\hline Age, M (SD) & $41.2(12.4)$ & $41.31(11.8)$ & $38.18(12.4)$ & $37.53(12.1)$ \\
\hline \multicolumn{5}{|l|}{ Highest completed education } \\
\hline $16(21.1)$ & $13(17.3)$ & $9(9.2)$ & $12(11.8)$ & $13(17.3)$ \\
\hline $48(63.2)$ & $41(54.7)$ & $50(51)$ & $52(51)$ & $41(54.7)$ \\
\hline $12(15.8)$ & $21(28.0)$ & $39(39.8)$ & $38(37.3)$ & $21(28.0)$ \\
\hline Partner, yes, n (\%) & $43(56.6)$ & $51(68.0)$ & $34(34.7)$ & $38(37.3)$ \\
\hline Current job, yes, n (\%) & $43(56.6)$ & $47(62.7)$ & $69(50.7)$ & $67(49.3)$ \\
\hline Born in the Netherlands, yes, $\mathrm{n}(\%)$ & $73(96.1)$ & $6(90.8)$ & $77(78.6)$ & $82(80.4)$ \\
\hline \multicolumn{5}{|l|}{ Depression } \\
\hline BDI-II baseline, M (SD) & $28.4(9)$ & $31.2(8.9)$ & $35.83(9)$ & $33.61(10.7)$ \\
\hline BDI-II post-treatment, $\mathrm{M}$ (SD) & $13.8(10.7)$ & $16.0(13.4)$ & $22.75(14)$ & $21.36(15.4)$ \\
\hline Recurrent depression, n (\%) & $38(50)$ & $36(48)$ & $35(35.7)$ & 39 (17.5) \\
\hline \multicolumn{5}{|l|}{ Clinical variables } \\
\hline Number of comorbid Axis I disorders, M (SD) & $0.6(0.8)$ & $0.7(0.7)$ & $1.01(1.1)$ & $1.07(1.3)$ \\
\hline $\begin{array}{l}\text { Treatment expectation, } M(S D) \\
(0=\text { not successful }-10=\text { very successful) }\end{array}$ & $6.8(1.2)$ & $6.5(1.3)$ & $6.59(1.5)$ & $6.63(1.6)$ \\
\hline RAND-36, physical functioning, M (SD) & $74(23)$ & $74.1(20.5)$ & $70.51(26.9)$ & $70.63(25.6)$ \\
\hline RAND-36, social functioning, M (SD) & $42.3(20.2)$ & $40.8(20.2)$ & $30.99(21.3)$ & $35.90(22.7)$ \\
\hline $\begin{array}{l}\text { RAND-36, role limitations (physical problems), } \\
\text { M (SD) }\end{array}$ & $37.8(41.3)$ & $33.7(38.4)$ & $33.41(40.6)$ & $37.50(39.3)$ \\
\hline $\begin{array}{l}\text { RAND-36, role limitations (emotional } \\
\text { problems), M (SD) }\end{array}$ & $18.8(33.2)$ & $13.3(25.1)$ & $9.86(20.4)$ & $10.78(21.5)$ \\
\hline RAND-36, general health experience, $M(S D)$ & $46.7(16.5)$ & $43.8(14.1)$ & $43.41(19.4)$ & $43.33(17.5)$ \\
\hline $\begin{array}{l}\text { RAND-36, perceived health change during } \\
\text { past year, } \mathrm{M}(\mathrm{SD})\end{array}$ & $32.2(27.0)$ & $26(24.8)$ & $33.92(25.7)$ & $31.61(23.8)$ \\
\hline
\end{tabular}

BDI-II=Beck Depression Inventory II; $M=$ mean; SD=standard deviation; RAND-36=Rand 36 Health Survey. None of the shown pre-treatment variables are transformed. In the STEPd study there was 1 missing on current job (in CBT), 17 missings in the BDI-Il post-treatment scores (7 in CBT; 10 in IPT), 5 missing on recurrent depression ( 3 in CBT; 2 in IPT), 1 missing on the RAND-36 (in CBT). In the FreqMech dataset there was 1 missing on treatment expectation (in CBT), 55 missing on the BDI-Il post-treatment scores (26 in CBT; 29 in IPT), 29 missing on the number of previous episodes (15 in CBT; 14 in IPT), 13 missing on the number of comorbid Axis I disorders (6 in CBT; 7 in IPT).

\subsubsection{Computing the Personalized Advantage Index scores}

A total of 67 patients (44.4\%) received their PAI-indicated treatment $(22.5 \%$ CBT and 21.9\% IPT). Post-treatment BDI-II scores were significantly lower for individuals that received their $\mathrm{PAl}$-indicated treatment $(\mathrm{M}=11.64, \mathrm{SD}=10.11)$ compared to those that received their PAI non-indicated treatment $(M=18.17, S D=12.27, t(149)=3.51$, $p<0.001$, effect size Cohen's $d=.57$ ). As shown in Figure 11.1a, for individuals who had a PAI indicating CBT, post-treatment BDI-II scores were significantly lower for individuals that were actually randomized to $C B T(M=11.19, S D=9.79)$ compared to those randomized to IPT $(M=19.85, S D=13.69, t(74)=-3.10, p=0.003$, effect size 
Cohen's $d=.71$ ). For individuals who had a PAI indicating IPT, post-treatment BDI-II was non-significantly different for those receiving IPT $(M=12.09, S D=10.56)$ and $C B T$ $(\mathrm{M}=16.48, \mathrm{SD}=10.55, \mathrm{t}(73)=1.79, \mathrm{p}=0.08$, effect size Cohen's $\mathrm{d}=.18)$. Among individuals with the highest $60 \%$ PAI scores, mean post-treatment BDI-II scores differed significantly between these individuals that received the PAl-indicated treatment versus those that received the PAI non-indicated treatment (indicated treatment: $M=10.43$, non-indicated treatment: $M=18.5, t(89)=3.35, p=0.001)$, with a Cohen's $d$ effect size estimate of 0.71 .

\subsection{The Personalized Advantage Index in the FreqMech study}

\subsubsection{Building the Personalized Advantage Index model}

A total of 15 variables were selected using the mobforest algorithm. Of these 15 variables, eight predictors and one moderator were selected in at least $60 \%$ of the bootstrap samples using the backwards elimination technique. A higher baseline depression, having received more previous treatments, higher levels of dysfunctional thinking, fewer physical problems, worse physical functioning, lower quality of life, worse emotional problems and less vitality were related to higher scores on the BDI-II post-treatment, irrespective of the received treatment. In addition, being female was related to higher BDI-II scores post-treatment in IPT compared to CBT.

\subsubsection{Computing the Personalized Advantage Index scores}

A total of 103 patients (51.5\%) received their PAl-indicated treatment (31\% CBT and $20.5 \%$ IPT). Post-treatment BDI-II scores were lower for individuals who received their PAl-indicated treatment $(M=20.87, S D=13.23)$ compared to those that received their PAI non-indicated treatment, but this result was not significant $(M=23.68, S D=13.65$, $t(198)=1.47, p=.14$, effect size Cohen's $d=.20$ ). As shown in Figure $11.1 b$, for individuals who had a PAI indicating CBT, post-treatment BDI-II scores were lower for individuals that were actually randomized to $\mathrm{CBT}(\mathrm{M}=23.86, \mathrm{SD}=13.86)$ compared to those randomized to IPT ( $M=25.17, S D=14.71)$, but this difference was not significant, $\mathrm{t}(121)=-.50, \mathrm{p}=.61$, effect size Cohen's $d=.09$ ). For individuals who had a PAl indicating IPT, the post-treatment BDI-II was lower but not significantly different for those receiving IPT $(M=16.35, S D=10.89)$ and $C B T(M=21.15, S D=11.40, t(75)=1.88, p=.06$, effect size Cohen's $d=.43$ ). Among those with the highest $60 \%$ PAl scores, mean posttreatment $\mathrm{BDI}-\mathrm{Il}$ scores were lower between these individuals that received the PAIindicated treatment versus those that received the PAI non-indicated treatment (indicated treatment=19.92 ( $S D=12.72)$, non-indicated treatment=22.19 ( $S D=12.99)$, $t(118)=.96, p=.33$ ), but this difference was not significant (Cohen's d effect size of .17). 
A more detailed description of the selection process of the pre-treatment variables in both datasets can be found in Supplement 3.
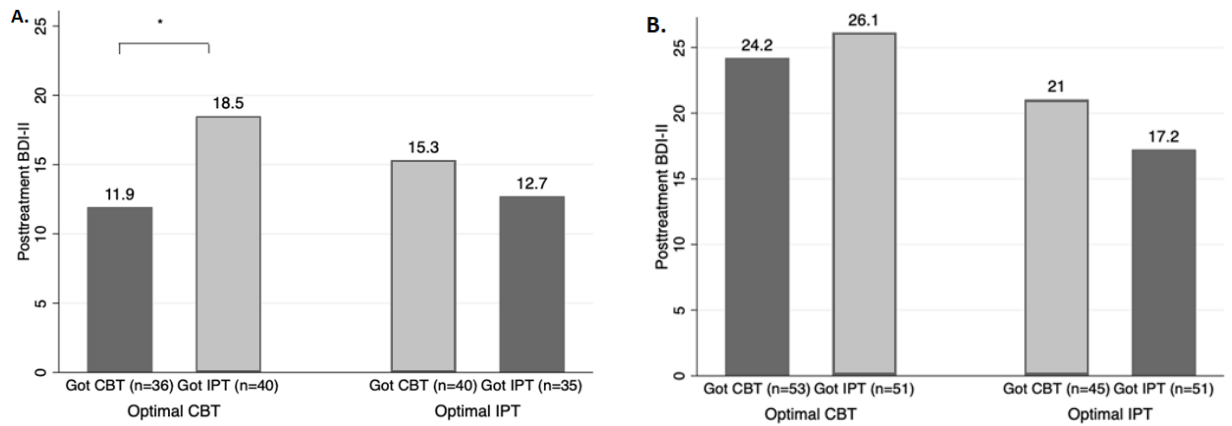

Figure 11.1: Comparisons of observed BDI-II post-treatment scores for patients randomly assigned to their PAI-indicated treatment versus those assigned to their PAI non-indicated treatment, by psychotherapy type in the STEPd study (A) and the FreqMech study (B). CBT=cognitive behavioral therapy; IPT=interpersonal psychotherapy; BDI-II=Beck Depression Inventory, second edition. *indicates a significant difference between observed BDI-II scores.

\subsection{Cross-trial prediction}

\subsubsection{STEPd to FreqMech}

Part of the STEPd PAI model could be tested in the FreqMech dataset, since we could not use all predictors and moderator because of differences in data collection. The following predictors and moderators were available in the FreqMech dataset: employment status (predictor), anxiety symptoms (Remission of Depression Questionnaire subscale; predictor), overall social, occupational, and psychological functioning (Global Assessment of Functioning DSM-IV; moderator), and pretreatment depression severity (BDI-II; moderator). Results of the STEPd PAI model in the FreqMech dataset indicated that post-treatment BDI-II scores were nonsignificantly lower for individuals that received their PAl-indicated treatment $(n=112)$ $(\mathrm{M}=21.28, \mathrm{SD}=13.07)$ compared to those that received their PAI non-indicated treatment $(n=88)(M=23.38, S D=13.60, t(198)=1.11, p=.27$, effect size Cohen's $d=.16)$. For those that had a PAI indicating CBT, post-treatment BDI-II scores were nonsignificantly lower for individuals that received to CBT $(M=25.07, S D=13.55)$, as compared to individuals that received to IPT $(M=26.59, S D=14.59, t(112)=-0.58$, $\mathrm{p}=.57$ ). For individuals who had a PAI indicating IPT, post-treatment BDI-II scores were non-significantly lower for those randomized to IPT $(M=16.58, S D=10.85)$ compared to those randomized to $\mathrm{CBT}(\mathrm{M}=18.75, \mathrm{SD}=10.60, \mathrm{t}(84)=0.92, \mathrm{p}=.36)$. 


\subsubsection{FreqMech to STEPd}

It was possible to test a part of the FreqMech PAI model in the STEPd dataset, however because of differences in data collection we could not use all predictors and moderators. The following predictors and moderators were available in the STEPd dataset: baseline depression (BDI-II; predictor), physical problems (RAND-36; predictor), emotional problems (RAND-36; predictor), physical functioning (RAND-36; predictor), vitality (RAND-36; predictor), gender (moderator) and quality of life utility score (EQ5D; moderator). Using the FreqMech PAI model, in the STEPd dataset posttreatment BDI-II scores were lower for individuals that received their PAI-indicated treatment $(n=83, M=13.81, S D=11.26)$ compared to those that received their PAI nonindicated treatment $(n=68, M=17.06, S D=12.55, t(149)=1.67, p=.09$, effect size Cohen's $d=.27)$, however this difference was not significant. For those who had a PAI indicating $\mathrm{CBT}$, post-treatment $\mathrm{BDI}-\mathrm{Il}$ scores were non-significantly lower for individuals that were randomized to $C B T(M=14.36, S D=11.06)$, as compared to individuals randomized to IPT $(\mathrm{M}=18.81, \mathrm{SD}=13.56, \mathrm{t}(98)=-1.80, \mathrm{p}=.07)$. For individuals who had a PAI indicating IPT, there were no significant differences between those receiving IPT $(M=12.79, S D=11.75)$ and for those receiving $C B T(M=13.39, S D=9.36$, $\mathrm{t}(49)=.19, \mathrm{p}=.84)$.

\subsection{Sensitivity analyses}

We conducted a sensitivity analyses were we included only the 20 variables that were overlapping between the two datasets (see Supplement 2).

\subsubsection{STEPd}

\subsubsection{PAI model in the STEPd dataset}

Seven variables were selected using the mobforest algorithm. Of these variables, four predictors and one moderator were selected in at least $60 \%$ of the bootstrap samples using the backwards elimination technique. Lower BDI-II baseline scores, fewer social problems, fewer anxiety symptoms and having a job predicted lower BDI-II posttreatment scores irrespective of the received type of treatment. Childhood trauma was related to higher post-treatment BDI-II scores in IPT compared to CBT. There were no significant differences between individuals who received their PAI-indicated treatment and those that received their PAI non-indicated treatment $(M=14.92$, $S D=12.06$ versus $M=15.64, S D=11.87, t(149)=.37, p=.71$, effect size Cohen's $d=.06)$.

\subsubsection{Cross trial prediction to the FreqMech dataset}

Using the STEPd PAI model in the FreqMech dataset, post-treatment BDI-II scores were non-significantly lower for individuals that received their PAI-indicated 
treatment $(n=83)(M=21.69, S D=12.87)$ compared to those that received their PAI non-indicated treatment $(n=117)(M=22.88, S D=13.99, t(198)=0.61, p=.54$, effect size Cohen's d=.09).

\subsubsection{FreqMech}

\subsubsection{PAI model in FreqMech dataset}

Five variables were selected using the mobforest algorithm. Of these variables, three predictors and one moderator were selected in at least $60 \%$ of the bootstrap samples using the backwards elimination technique. Higher BDI-II baseline scores, more physical problems and less physical functioning predicted higher BDI-II scores posttreatment irrespective of the received type of treatment. Being female related to higher BDI-II scores post-treatment in IPT compared to CBT. Post-treatment BDI-II scores were lower for individuals who received their PAl-indicated treatment $(\mathrm{M}=20.90, \mathrm{SD}=13.06)$ compared to those that received their PAI non-indicated treatment, however this difference was not significant $(M=23.96, S D=13.87$, $t(198)=1.60, p=.11$, effect size Cohen's $d=.22$ ).

\subsubsection{Cross trial prediction to the STEPd dataset}

Using the FreqMech PAI model in the STEPd dataset, post-treatment BDI-II scores were non-significantly lower for individuals that received their PAl-indicated treatment $(n=83)(M=13.81, S D=11.26)$ compared to those that received their PAI non-indicated treatment $(\mathrm{n}=68)(\mathrm{M}=17.06, \mathrm{SD}=12.55, \mathrm{t}(149)=1.67, \mathrm{p}=.09$, effect size Cohen's d=.27).

\section{Discussion}

The overall aim of the current study was to determine the generalizability of the PAI to clinical practice by externally validating PAI models using data from two independent Dutch randomized trials comparing CBT and IPT for MDD (i.e., the STEPd trial and the FreqMech trial). In the STEPd dataset, two predictors and five moderators were found. The STEPd PAI resulted in a significant difference in observed post-treatment depression severity when comparing individuals assigned to their PAIindicated treatment versus those assigned to their PAI non-indicated treatment (Cohen's $d=.57$ ). This mean difference was more pronounced for individuals with the top 60\% PAl scores (Cohen's $d=.71$ ), and for individuals that had a CBT recommendation (Cohen's $d=.71$ ). In the FreqMech study one moderator and eight predictors were found. Here, the PAl showed a modest effect size (Cohen's $d=.20$ ) and resulted in modest and non-significant differences in observed post-treatment BDI-II 
scores between patients who received their indicated versus patients who received their non-indicated treatment. This difference in observed post-treatment BDI-II scores was not more pronounced for the highest 60\% PAl scores (Cohen's $d=.17$ ). In order to externally validate the PAI models, cross-trial predictions from the STEPd model to the FreqMech dataset, and from the FreqMech model to the STEPd dataset were conducted. Cross-prediction from STEPd to FreqMech was limited because three of the five moderators from the STEPd model were not available in the FreqMech dataset. The resulting PAI recommendations of the STEPd model in the FreqMech dataset showed a small effect size (Cohen's $d=.16$ ) with non-significant differences between individuals that received their indicated versus their non-indicated treatment. For the cross-trial prediction of the FreqMech PAI model to the STEPd dataset, two FreqMech predictors were not available in the STEPd dataset. The resulting treatment recommendations of the FreqMech model in the STEPd dataset had a moderate effect size (Cohen's $d=.27$ ), and the differences between the individuals who were randomized to their indicated versus those who were randomized to their non-indicated treatment were not statistically significant. In addition, a sensitivity analysis was conducted using only the overlapping 20 variables from each dataset for cross-trial prediction. Treatment recommendations of the STEPd model resulted in poor effect sizes in the STEPd dataset (Cohen's $d=.06$ ), and in the FreqMech dataset (Cohen's $d=.09$ ). Results of the FreqMech PAI model indicated small to moderate effect sizes of treatment recommendation in the FreqMech dataset (Cohen's $d=.22$ ) as well as in the STEPd dataset (Cohen's $d=.27$ ).

This study demonstrates that the effects of the PAI in the context of cross-trial prediction are rather modest. In the STEPd study, within-study PAI scores seemed very promising with a high effect size, however the effect size of this PAl decreased substantially in the cross-trial prediction. This decrease of effect size might be indicative for overfitting of the within-study PAI model, which could be explained by double-dipping when applying the variable selection procedure in the complete sample (Fiedler, 2011). In the FreqMech study, within-study PAI scores showed small to moderate effect sizes that were maintained during cross-trial prediction. These results indicate that effect sizes seemed more stable across analyses in the study with a larger sample size ( $n=200$ vs. $n=151$ in the STEPd study) and more heterogeneous data from multiple sites (instead of one treatment center in the STEPd study). The effect sizes of the FreqMech study are also in line with the out-of-sample predictions of previous PAl study of Delgadillo and colleagues (2019, Hedges' $g=.26$ ) that is based on a larger dataset from multiple study sites. Overall, the results of our study emphasize the many challenges of external validation of PAl models, which is a necessary step for implementation in clinical practice.

One major challenge for external validation of PAI models is that the concept of external validation is not as straightforward as one might expect. Validation studies may include temporal validation (same location, but later in time), geographical 
validation (different locations), validation in different settings (e.g., primary and secondary settings) and validation in different domains (e.g., in adults and children; Debray et al., 2015). One way to understand these different types of validation is by distinguishing between model reproducibility (accuracy across samples from the same target population) and model transportability (accuracy across samples from different but related populations; i.e., external validation) (Debray et al., 2015; Justice, Covinsky, \& Berlin, 1999). However, the optimal balance between different and related data is unclear. In the context of our paper, we could debate that the STEPd and FreqMech study samples were too different and therefore lead to different moderators and predictors, despite the fact that these studies were both from the Netherlands and conducted by some members of the same research group. Differences between the STEPd and the FreqMech study involved the single-center vs. multi-center design, a low vs. high number of therapists, medium vs. high pre- and post-treatment depression severity, low vs. medium dropout rates, differences in inand exclusion criteria, and differences in quality of the performed treatments. In addition to these differences, when we consider the population with a diagnosis of MDD, this categorization alone already involves a high level of heterogeneity. Possibly, depressed individuals within one study already belong to multiple distinctive populations (Nandi, Beard, \& Galea, 2009).

Another major obstacle for external validation of PAI models are the different types of data collection and statistical methods used in the different studies. The problem of dissimilar data collection is illustrated by our own findings, since we could only partially validate the STEPd and the FreqMech model in the other dataset. Moreover, restricting the number of variables to the degree of overlap between the datasets (sensitivity analyses) negatively affected the PAI recommendations of the STEPd model. Development of generalizable PAI models requires unambiguous definitions of outcome, potential predictors, and potential moderators. In addition, these variables need to be based on reproducible measurements that are potentially feasible for application in clinical practice. Since the development of PAI models is typically a secondary analysis of data of effectiveness trials, thorough pre-trial evaluation on what variables to include for a reproducible and generalizable PAI model is needed. In addition to the data collection problem, there are also statistical difficulties. For example, one possible reason why we did not find significant crosstrial prediction effects might be because of the (relatively) small sample sizes of our studies, in particular the STEPd study (Luedtke et al., 2019). Besides the problem of small samples sizes, there is also a striking heterogeneity in statistical methods used for missing data imputation and model building (Cohen \& DeRubeis, 2018; Cohen et al., 2019). This is of importance since there is evidence that different methods of data imputation and model building may lead to differences in clinical conclusions (Cohen et al., 2019; Stavseth, Clausen, \& Røislien, 2019; Webb et al., 2020). However, comparisons between the STEPd PAI model built in 2015 (Huibers et al., 2015) and the 
PAI model in the present paper that used different methods on the same dataset, indicate significant overlap between selected pre-treatment variables and similar effect sizes. A better understanding of how differences in statistical methods affect predictive accuracy is warranted when comparing and externally validating different PAl models.

The final major challenge for external validation of PAl models is the generalizability of the contrasts between treatments. One possible explanation for finding different moderators in the STEPd versus the FreqMech trial is that the contrasts between CBT and IPT were different for each study. Despite the fact that therapists were instructed to follow the same CBT and IPT protocols in both studies, the overall quality of therapy was considerably lower in the FreqMech trial compared to the STEPd trial. Treatments in the FreqMech study were delivered by a high number of therapists from multiple treatment centers of different mental health organizations located at different parts of the Netherlands. In the STEPd study this was a small number of experienced therapists all working in the same clinical setting. It is possible that these differences have led to differences in the performance of the CBT and IPT protocols and that the better and more consistent therapy quality in the STEPd dataset is responsible for finding a stronger PAI model within the STEPd dataset compared to the FreqMech dataset. Even holding quality and adherence constant, between-therapist differences as well as differences in locations or organizations may make external validation of prescriptive models much more complex than external validation of prognostic models. The results of various PAl studies so far seem to support these hypotheses. The finding that the effects of the FreqMech PAI were modest and non-significant contrasts earlier findings (DeRubeis et al., 2014; Huibers et al., 2015; Webb et al., 2019; Zilcha-Mano et al., 2016), but is in line with some, most notably newer studies (Cohen et al., 2019; Delgadillo \& Gonzalez Salas Duhne, 2019; Eskildsen et al., 2020; Friedl et al., 2019; Lopez-Gomez et al., 2019). Like the FreqMech dataset, the studies that had modest and non-significant findings included a high number of treatment centers and/or therapists. Therefore, one explanation for these modest and non-significant findings is that large heterogeneity in the sample and treatment might make it difficult to find large PAls compared to studies with less variation. However, this explanation contrasts with findings of Webb and colleagues (2019), who included multiple centers and therapists as well, and found a PAI with a large effect size. A possible explanation for this could be that Webb and colleagues (2019) focused on treatment with antidepressants instead of psychotherapy, in which heterogeneity in therapists may play a smaller role.

Although the many challenges in the external validation of the PAI in psychotherapy are not easy to solve, they need to be addressed in order to make findings in precision medicine relevant for clinical practice. How do we make progress from here? Previous authors have proposed to start with building prescriptive algorithms on large (combined) observational datasets followed by pragmatic trials 
that randomize clinicians to receive or not receive information from these algorithms (Kessler, 2018; Luedtke et al., 2019). We think that this approach is promising, especially for less complex prognostic models. For the more fine-grained prescriptive models, such as the PAI models, we suggest a more "zoomed in" or local method that occurs parallel to the approach that Kessler and colleagues propose. Since the heterogeneity of study populations and treatments is very high, we first need to establish to what extent external validation is realistic; maybe some treatment selection decisions are local decisions. With clear agreements on data collection and data analyses, we could first start with the development of local prescriptive models. In this context "local" means within the same geographical area, mental health care organization, and with a low number of therapists that have joint supervision. From there, we could focus on temporal validation (same location, different time), and then work towards external validation by expanding the location in terms of other geographical locations, other mental health care organizations and by increasing the number of therapists. Model updating should be a central part of these "zooming out" steps, enhancing the performance of models in other samples using methods such as recalibration (adjustment of intercepts and the regression coefficients using the calibration intercept and calibration slope) or model revision (re-estimation of the intercept and the regression coefficients using the combined datasets; Janssen, Moons, Kalkman, Grobbee, \& Vergouwe, 2008). In addition, Bayesian inference, that inherently updates probabilities with new data, could be integrated in these models, especially in the context of small study samples (Depaoli, Rus, Clifton, van de Schoot, \& Tiemensma, 2017). Besides externally validating PAI models, simulation studies are needed to find out how different choices in statistical methods and parameters (for example: differences in imputing missing data, differences between different forms (and combinations) of machine learning can lead to different (clinical) conclusions.

The present study investigated the generalizability of PAI models, by building PAI models using data from two independent Dutch randomized trials and externally validated each model in the other sample. In one dataset (STEPd), post-treatment BDIII scores were significantly lower for individuals that received their PAI-indicated treatment compared to those that received their PAI non-indicated treatment, however treatment recommendations based on cross-trial predictions had low effect sizes. In the other dataset (FreqMech), small to moderate effect sizes and nonsignificant post-treatment differences were found and these outcomes could be maintained during cross-trial prediction in the STEPd dataset. Before implementing the use of PAI models into clinical practice, studies that address external validation of the PAI are highly necessary.

Supplementary data associated with this article can be found online. 


\section{References}

American Psychiatric Association. (2009). Practice guideline for the treatment of patients with major depressive disorder (3rd). http://psychiatryonline. org/guidelines. aspx.

Austin, P. C., \& Tu, J. V. (2004). Bootstrap methods for developing predictive models. The American Statistician, 58(2), 131-137.

Beck, A. T., Rush, A. J., Shaw, B. F., \& Emery, G. (1979). Cognitive therapy of depression. New York: Guilford Press.

Beck, A. T., Steer, R., \& Brown, G. K. (1996). Beck Depression Inventory II: Manual. Boston: Harcourt Brace.

Bleeker, S., Moll, H., Steyerberg, E., Donders, A., Derksen-Lubsen, G., Grobbee, D., \& Moons, K. (2003). External validation is necessary in prediction research:: A clinical example. Journal of clinical epidemiology, 56(9), 826-832.

Bruijniks, S., Lemmens, L., Hollon, S., Peeters, F., Cuijpers, P., Arntz, A., \& Huibers, M. (2020). Seeing depressed patients twice weekly improves outcomes: results from an RCT comparing cognitive behavior therapy and interpersonal psychotherapy. British Journal of Psychiatry - in Press.

Bruijniks, S. J., Bosmans, J., Peeters, F. P., Hollon, S. D., van Oppen, P., van den Boogaard, M., ... Franx, G. (2015). Frequency and change mechanisms of psychotherapy among depressed patients: study protocol for a multicenter randomized trial comparing twice-weekly versus once-weekly sessions of CBT and IPT. BMC psychiatry, 15(1), 137.

Cohen, Z. D., \& DeRubeis, R. J. (2018). Treatment selection in depression. Annual Review of Clinical Psychology, 14, 209-236.

Cohen, Z. D., Kim, T. T., Van, H. L., Dekker, J. J., \& Driessen, E. (2019). A demonstration of a multi-method variable selection approach for treatment selection: Recommending cognitive-behavioral versus psychodynamic therapy for mild to moderate adult depression. Psychotherapy Research, 1-14.

Debray, T. P., Vergouwe, Y., Koffijberg, H., Nieboer, D., Steyerberg, E. W., \& Moons, K. G. (2015). A new framework to enhance the interpretation of external validation studies of clinical prediction models. Journal of clinical epidemiology, 68(3), 279-289.

Deisenhofer, A. K., Delgadillo, J., Rubel, J. A., Böhnke, J. R., Zimmermann, D., Schwartz, B., \& Lutz, W. (2018). Individual treatment selection for patients with posttraumatic stress disorder. Depression and anxiety, 35(6), 541-550.

Delgadillo, J., \& Gonzalez Salas Duhne, P. (2019). Targeted prescription of cognitive behavioral therapy vs. person-centered counseling for depression using a machine learning approach. Journal of Consulting and Clinical Psychology.

Depaoli, S., Rus, H. M., Clifton, J. P., van de Schoot, R., \& Tiemensma, J. (2017). An introduction to Bayesian statistics in health psychology. Health Psychology Review, 11(3), 248-264.

DeRubeis, R. J., Cohen, Z. D., Forand, N. R., Fournier, J. C., Gelfand, L. A., \& Lorenzo-Luaces, L. (2014). The Personalized Advantage Index: translating research on prediction into individualized treatment recommendations. A demonstration. PloS one, 9(1), e83875.

Dobson, K. S., Shaw, B. F., \& Vallis, T. M. (1985). Reliability of a measure of the quality of cognitive therapy. British Journal of Clinical Psychology, 24(4), 295-300.

Efron, B. (1983). Estimating the error rate of a prediction rule: improvement on cross-validation. Journal of the American statistical association, 78(382), 316-331.

Efron, B., \& Tibshirani, R. (1997). Improvements on cross-validation: the 632+ bootstrap method. Journal of the American statistical association, 92(438), 548-560.

Efron, B., \& Tibshirani, R. J. (1993). An Introduction to the Bootstrap (Monograph on Statistics and Applied Probability) New York: Chapman \& Hall View Article.

Eskildsen, A., Reinholt, N., van Bronswijk, S., Brund, R. B., Christensen, A. B., Hvenegaard, M., . . Rosenberg, N. K. (2020). Personalized Psychotherapy for Outpatients with Major Depression and Anxiety Disorders: Transdiagnostic Versus Diagnosis-Specific Group Cognitive Behavioural Therapy.

Fiedler, K. (2011). Voodoo correlations are everywhere-not only in neuroscience. Perspectives on psychological science, 6(2), 163-171. 
First, M. B., Spitzer, R. L., Gibbon, M., \& Williams, J. B. W. (1995). Structured Clinical Interview for DSM-IV Axis I Disorders (SCID-I). New York: Biometrics Research Department New York State Psychiatric Institute.

Friedl, N., Berger, T., Krieger, T., Caspar, F., \& Grosse Holtforth, M. (2019). Using the Personalized Advantage Index for individual treatment allocation to cognitive behavioral therapy (CBT) or a CBT with integrated exposure and emotion-focused elements (CBT-EE). Psychotherapy Research, 1-13.

Friedman, J., Hastie, T., \& Tibshirani, R. (2010). Regularization paths for generalized linear models via coordinate descent. Journal of statistical software, 33(1), 1.

Garge, N. R., Bobashev, G., \& Eggleston, B. (2013). Random forest methodology for model-based recursive partitioning: the mobForest package for R. BMC bioinformatics, 14(1), 125.

Gillan, C. M., \& Whelan, R. (2017). What big data can do for treatment in psychiatry. Current opinion in behavioral sciences, 18, 34-42.

Hollon, S., Evans, M., Auerbach, A., DeRubeis, R., Elkin, I., Lowery, A., . . Piasecki, J. (1988). Development of a system for rating therapies for depression: Differentiating cognitive therapy, interpersonal psychotherapy, and clinical management pharmacotherapy. Unpublished manuscript.

Huibers, M. J., Cohen, Z. D., Lemmens, L. H., Arntz, A., Peeters, F. P., Cuijpers, P., \& DeRubeis, R. J. (2015). Predicting Optimal Outcomes in Cognitive Therapy or Interpersonal Psychotherapy for Depressed Individuals Using the Personalized Advantage Index Approach. PloS one, 10(11), e0140771. doi:10.1371/journal.pone.0140771

Janssen, K., Moons, K., Kalkman, C., Grobbee, D., \& Vergouwe, Y. (2008). Updating methods improved the performance of a clinical prediction model in new patients. Journal of clinical epidemiology, 61(1), 7686.

Justice, A. C., Covinsky, K. E., \& Berlin, J. A. (1999). Assessing the generalizability of prognostic information. Annals of internal medicine, 130(6), 515-524.

Keefe, J. R., Wiltsey Stirman, S., Cohen, Z. D., DeRubeis, R. J., Smith, B. N., \& Resick, P. A. (2018). In rape trauma PTSD, patient characteristics indicate which trauma-focused treatment they are most likely to complete. Depression and anxiety, 35(4), 330-338.

Kessler, R. C. (2018). The potential of predictive analytics to provide clinical decision support in depression treatment planning. Current opinion in psychiatry, 31(1), 32-39.

Klerman, G. L., Weissman, M. M., Rounsaville, B. J., \& Chevron, E. S. (1984). Interpersonal psychotherapy for depression. New York: Basis Books.

Kraemer, H. C., \& Blasey, C. M. (2004). Centring in regression analyses: a strategy to prevent errors in statistical inference. International journal of methods in psychiatric research, 13(3), 141-151.

Kuhn, M., \& Johnson, K. (2013). Applied predictive modeling (Vol. 26): Springer.

Lemmens, L., Arntz, A., Peeters, F., Hollon, S., Roefs, A., \& Huibers, M. (2015). Clinical effectiveness of cognitive therapy $v$. interpersonal psychotherapy for depression: results of a randomized controlled trial. Psychological medicine, 45(10), 2095-2110.

Lemmens, L., Van Bronswijk, S., Peeters, F., Arntz, A., Hollon, S., \& Huibers, M. (2019). Long-term outcomes of acute treatment with cognitive therapy $v$. interpersonal psychotherapy for adult depression: follow-up of a randomized controlled trial. Psychological medicine, 49(3), 465-473.

Lemmens, L. H., Arntz, A., Peeters, F. P., Hollon, S. D., Roefs, A., \& Huibers, M. J. (2011). Effectiveness, relapse prevention and mechanisms of change of cognitive therapy vs. interpersonal therapy for depression: Study protocol for a randomised controlled trial. Trials, 12(1), 150.

Lopez-Gomez, I., Lorenzo-Luaces, L., Chaves, C., Hervas, G., DeRubeis, R. J., \& Vázquez, C. (2019). Predicting optimal interventions for clinical depression: Moderators of outcomes in a positive psychological intervention vs. cognitive-behavioral therapy. General Hospital Psychiatry, 61, 104-110.

Lorenzo-Luaces, L., DeRubeis, R. J., \& Bennett, I. M. (2015). Primary care physicians' selection of lowintensity treatments for patients with depression. Fam Med, 47(7), 511-516.

Luedtke, A., Sadikova, E., \& Kessler, R. C. (2019). Sample size requirements for multivariate models to predict between-patient differences in best treatments of major depressive disorder. Clinical Psychological Science, 7(3), 445-461.

Moons, K. G., Donders, R. A., Stijnen, T., \& Harrell, J. F. E. (2006). Using the outcome for imputation of missing predictor values was preferred. Journal of clinical epidemiology, 59(10), 1092-1101. 
Moons, K. G., Royston, P., Vergouwe, Y., Grobbee, D. E., \& Altman, D. G. (2009). Prognosis and prognostic research: what, why, and how? Bmj, 338, b375.

Nandi, A., Beard, J. R., \& Galea, S. (2009). Epidemiologic heterogeneity of common mood and anxiety disorders over the lifecourse in the general population: a systematic review. BMC psychiatry, 9(1), 31.

National Collaborating Centre for Mental Health. (2010). Depression: the treatment and management of depression in adults (updated edition).

National Health Service. (2018). Psychological Therapies, Annual report on the use of IAPT services England, 2017-18. Retrieved from https://digital.nhs.uk/data-and-information/publications/ statistical/psychological-therapies-annual-reports-on-the-use-of-iapt-services/annual-report-2017--18

Rizopoulos, D., \& Rizopoulos, M. D. (2009). Package 'bootStepAIC'.

Siontis, G. C., Tzoulaki, I., Castaldi, P. J., \& Ioannidis, J. P. (2015). External validation of new risk prediction models is infrequent and reveals worse prognostic discrimination. Journal of clinical epidemiology, 68(1), 25-34.

Stavseth, M. R., Clausen, T., \& Røislien, J. (2019). How handling missing data may impact conclusions: A comparison of six different imputation methods for categorical questionnaire data. SAGE open medicine, 7, 2050312118822912.

Stekhoven, D. J. (2011). Using the missForest package. R package, 1-11.

Stekhoven, D. J., \& Bühlmann, P. (2011). MissForest-non-parametric missing value imputation for mixedtype data. Bioinformatics, 28(1), 112-118.

Steyerberg, E. W., Harrell Jr, F. E., Borsboom, G. J., Eijkemans, M., Vergouwe, Y., \& Habbema, J. D. F. (2001). Internal validation of predictive models: efficiency of some procedures for logistic regression analysis. Journal of clinical epidemiology, 54(8), 774-781.

Strobl, C., Boulesteix, A.-L., Kneib, T., Augustin, T., \& Zeileis, A. (2008). Conditional variable importance for random forests. BMC bioinformatics, 9(1), 307.

Stuart, S. (2011). IPT Adherence and Quality Scale. Interpersonal Psychotherapy Institute, lowa (unpublished manuscript).

Tang, F., \& Ishwaran, H. (2017). Random forest missing data algorithms. Statistical Analysis and Data Mining: The ASA Data Science Journal, 10(6), 363-377.

van Bronswijk, S. C., DeRubeis, R. J., Lemmens, L. H., Peeters, F. P., Keefe, J. R., Cohen, Z. D., \& Huibers, M. J. (2019). Precision medicine for long-term depression outcomes using the Personalized Advantage Index approach: cognitive therapy or interpersonal psychotherapy? Psychological medicine, 1-11.

van Bronswijk, S. C., Lemmens, L. H., Keefe, J. R., Huibers, M. J., DeRubeis, R. J., \& Peeters, F. P. (2019). A prognostic index for long-term outcome after successful acute phase cognitive therapy and interpersonal psychotherapy for major depressive disorder. Depression and anxiety, 36(3), 252-261.

Van Vliet, I., Leroy, H., \& Van Megen, H. (2000). De MINI-Internationaal neuropsychiatrisch interview: een kort gestructureerd diagnostisch interview voor DSM-IV en ICD-10 psychiatrische stoornissen. Leiden: LUMC.

Waljee, A. K., Mukherjee, A., Singal, A. G., Zhang, Y., Warren, J., Balis, U., . . . Higgins, P. D. (2013). Comparison of imputation methods for missing laboratory data in medicine. BMJ open, 3(8), e002847.

Wang, Y.-P., \& Gorenstein, C. (2013). Psychometric properties of the Beck Depression Inventory-II: a comprehensive review. Brazilian Journal of Psychiatry, 35(4), 416-431.

Webb, C. A., Cohen, Z. D., Beard, C., Forgeard, M., Peckham, A. D., \& Björgvinsson, T. (2020). Personalized prognostic prediction of treatment outcome for depressed patients in a naturalistic psychiatric hospital setting: A comparison of machine learning approaches. Journal of Consulting and Clinical Psychology, 88(1), 25.

Webb, C. A., Trivedi, M. H., Cohen, Z. D., Dillon, D. G., Fournier, J. C., Goer, F., . . . Parsey, R. (2019). Personalized prediction of antidepressant v. placebo response: evidence from the EMBARC study. Psychological medicine, 1-10.

Zilcha-Mano, S., Keefe, J. R., Chui, H., Rubin, A., Barrett, M. S., \& Barber, J. P. (2016). Reducing Dropout in Treatment for Depression: Translating Dropout Predictors Into Individualized Treatment Recommendations. The Journal of clinical psychiatry, 77(12), e1584-e1590. 
Chapter 11

Zou, H., \& Hastie, T. (2005). Regularization and variable selection via the elastic net. Journal of the royal statistical society: series B (statistical methodology), 67(2), 301-320. 

S. van Bronswijk, N. Baştürk, L. Lemmens, M. Huibers, F. Peeters - Submitted

Chapter

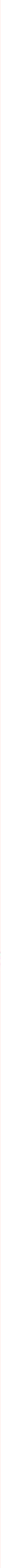




\section{BAYESIAN}

ESTIMATION

OF TREATM

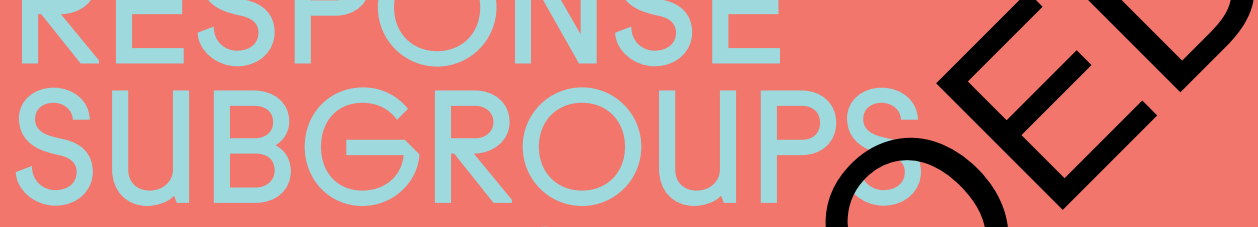

IN

DEPRESQO

EN

DISO
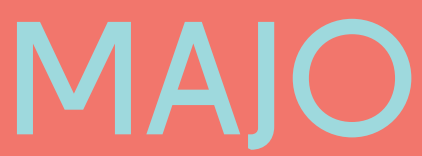

R

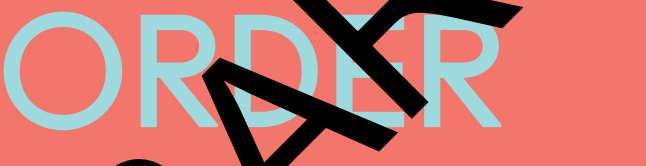


Chapter

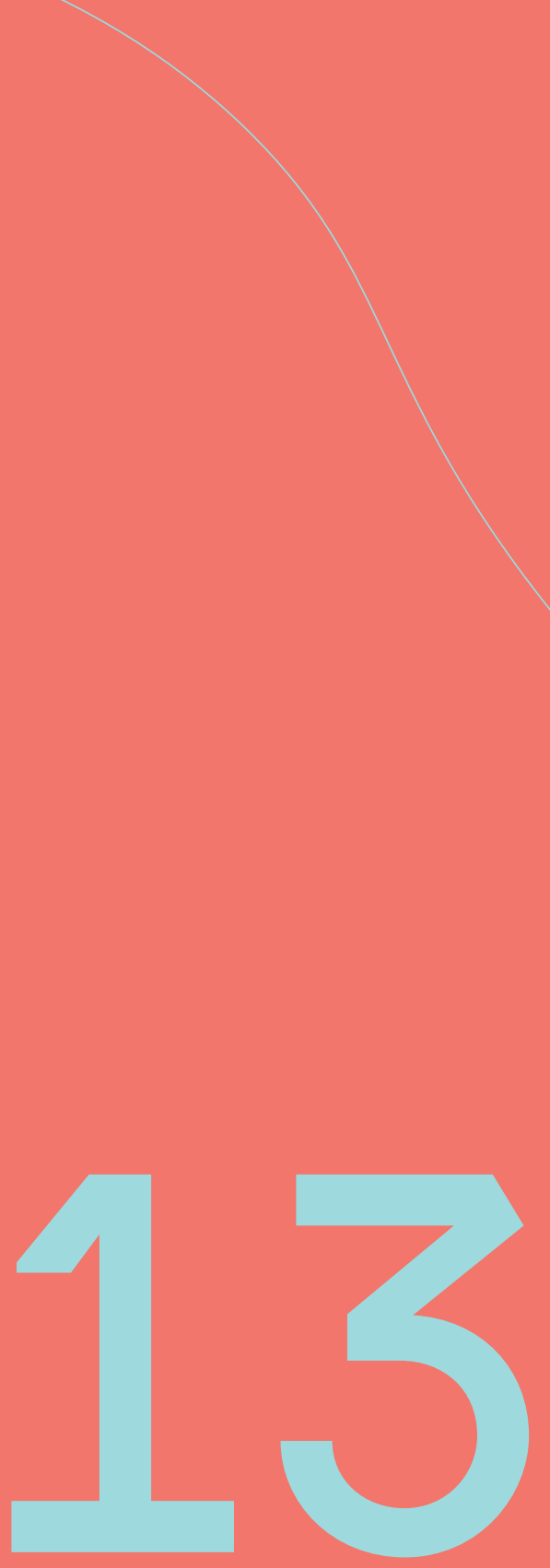




\section{GENERAL DISCUSSION}



The aim of this thesis was to investigate ways to personalize treatment strategies for major depressive disorder (MDD). In the first part (Chapter 1-5), the impact of comorbid anxiety and personality pathology on acute and long-term treatment outcome in MDD was examined, as well as their impact on treatment planning in MDD. In the second part (Chapter 6-9), potential strategies for treatment planning were investigated to achieve better long-term treatment outcomes for MDD. In the third and final part (Chapter 10-12), future personalized treatment strategies for MDD were discussed by entering the "clinical judgment vs. statistical prediction" debate, and by exploring external validation of prediction models and the possibilities of Bayesian subgroup modelling. In this general discussion, the findings of these studies are summarized and evaluated, and future directions for personalized treatment strategies for depression are discussed in more detail.

\section{Short summary of the main findings}

\subsection{Depression treatment and comorbidity (part one)}

Chapter $\mathbf{2}$ described the findings of an empirical study that examined the (differential) effects of comorbid anxiety (anxiety disorders and anxiety symptoms) on the effectiveness and treatment continuation of cognitive therapy (CT) and interpersonal psychotherapy (IPT) for MDD during treatment and throughout five months of followup. Comorbid anxiety disorders and anxiety symptoms were associated with less favorable depression change in IPT as compared to CT during treatment, however these differences did not sustain throughout the follow-up phase. Individuals with a comorbid anxiety disorder had significantly higher treatment dropout in both treatments as compared to individuals without a comorbid anxiety disorder. Chapter 3 reported the results of the same empirical study aimed at investigating the impact of personality pathology (personality disorders and personality features) on the effectiveness and treatment continuation of CT and IPT for MDD during treatment and five months of follow-up. Results indicated no negative effect of a personality disorder (PD) diagnosis on treatment dropout and treatment effectiveness. Cluster $A$ personality features moderated treatment outcome: individuals with high cluster A features had greater depression severity reductions over time when treated with CT as compared to IPT. In Chapter 4, a meta-analysis and meta-regression on the impact of comorbid PDs on acute-phase treatment outcome for MDD was presented. Results indicated that there was no significant difference in depression severity change between individuals with and without comorbid PDs during treatment (psychotherapy and pharmacotherapy). Chapter $\mathbf{5}$ described the results of an observational study on the impact of comorbid personality pathology on the amount and type of received treatment for MDD. Results indicated that depressed individuals with comorbid 
personality pathology receive on average more psychotherapy sessions compared to those without comorbid personality pathology, which conflicts with current treatment guidelines.

\subsection{Treatment planning for depression (part two)}

Chapter 6 reported the results of an empirical study into the enduring effects of acute-phase CT and IPT for MDD throughout 17 months of follow-up. Findings of this study indicated that individuals who responded to IPT were no more likely to relapse following treatment termination than patients who responded to CT. In Chapter 7, a study on a prognostic model (i.e., a Prognostic Index) for long-term outcome after successful acute phase CT and IPT for MDD was presented. Results of this study showed that long-term predictions of MDD are multifactorial and promising. Chapter $\mathbf{8}$ described a study of a prescriptive model, the Personalized Advantage Index, that creates individualized treatment recommendations for CT or IPT prior to treatment, based on long-term outcome predictions. This study provided evidence that treatment recommendations based on the Personalized Advantage Index could enhance precision medicine by selecting the optimal treatment for a given depressed individual. Chapter 9 reported a meta-analysis and a meta-regression on the effectiveness of psychotherapy for treatment-resistant depression. Results of this study indicated that the addition of psychotherapy to treatment as usual could benefit treatment outcomes in this difficult-to-treat population.

\subsection{Future strategies (part three)}

In Chapter 10, a direct comparison of clinical judgment from therapists and statistical prediction (with the Personalized Advantage Index) for treatment selection for MDD was presented. Findings of this study indicated that statistical prediction outperforms clinical judgment in treatment selection of MDD. Chapter 11 described the results from cross-trial predictions with the Personalized Advantage Index, using data of two Dutch randomized trials comparing CT and IPT for MDD. Cross-validation results were modest, and therefore demonstrated and emphasized the challenges in the external validation of prediction models in MDD. Chapter 12 introduced an innovative Bayesian methodology to estimate treatment response subgroups in MDD. Results gave valuable insights into the different depression severity subgroups that exist during treatment, and provided promising directions for longitudinal subgroup modeling. 


\section{Traditional approaches to personalize treatment strategies for depression}

\subsection{Why comorbidity alone should not impact treatment strategies}

The most important findings of our analyses on the impact of comorbid anxiety (Chapter 2) were that, during treatment, anxiety disorders and anxiety symptoms were associated with better depression change in CT as compared to IPT, and that individuals with anxiety disorders (but not anxiety symptoms) were more likely to dropout from both treatments. From these findings one could formulate the following clinical implications: in the presence of co-morbid anxiety 1) a preference to CT over IPT is justifiable (at least for the acute phase), 2) if IPT is the first choice of treatment for other reasons, clinicians could also consider using a modified version of IPT with cognitive-behavioral strategies to target anxiety (Cyranowski et al., 2005), and 3) adherence to treatment should be carefully monitored. However, when placing these findings in the context of previous studies, there has been little agreement on tailored treatment recommendations. A key explanation for these inconsistent results is that anxiety has been characterized in different ways (syndromal and dimensional definitions) that have limited overlap (Ionescu, Niciu, Henter, \& Zarate, 2013; Rao, 2009; van der Veen, Comijs, van Zelst, Schoevers, \& Oude Voshaar, 2014; Zimmerman, Kerr, Kiefer, Balling, \& Dalrymple, 2019). Moreover, even the recently introduced "anxious distress specifier" in DSM-5 has only a modest association between the different definitions of anxious depression (American Psychiatric Association, 2013; Zimmerman, Martin, et al., 2019). With this lack of consensus on how to conceptualize comorbid anxiety, formulating clear and straightforward clinical implications remains problematic.

In our empirical study and meta-analysis on the impact of comorbid personality pathology (Chapter 3 \& Chapter 4) we found no significant difference between individuals with and without a comorbid personality disorder (PD) in depression severity change during acute phase treatments. These findings did not concur with results from two previous meta-analyses that indicated negative effects of comorbid PD on depression outcome (Newton-Howes, Tyrer, \& Johnson, 2006; Newton-Howes et al., 2014). However, these meta-analyses included multiple studies with unstructured PD and MDD assessments, naturalistic treatments, no control for significant confounders (e.g., earlier onset, childhood adversity, and episode duration), and no consideration of baseline depression severity differences between individuals with and without comorbid PD (Mulder, 2002). In addition, in both metaanalyses misinterpretations of odds ratios occurred, which likely lead to an overestimation of the relative risks between individuals with and without PD (Newton-Howes et al., 2006; Newton-Howes et al., 2014; Sheldrick, Chung, \& 
Jacobson, 2017) ${ }^{1,2}$. Despite their troubling methodological issues, the results of these meta-analyses are often used as proof of why comorbid PD should be an important element of our conceptualization and clinical management of MDD (Berk et al., 2018; Van \& Kool, 2018). This point of view might also explain the results of our observational study of naturalistic MDD treatment, where we found that, in contrast to clinical practice guidelines, individuals with personality pathology received significantly more psychotherapy sessions as compared to individuals without personality pathology (Chapter 5).

One can conclude that, although frequently advocated in the literature and in daily clinical practice, inclusion of information on comorbid anxiety and personality pathology in treatment planning does not lead to more effective, person-tailored treatment strategies for MDD. However, even if both comorbidities resulted in straightforward clinical implications, examining single pre-treatment variables in isolation can be considered a reductionist strategy (DeRubeis et al., 2014). Since predictions, and therefore recommendations, on treatment outcome have been proven to be multifactorial (Cohen \& DeRubeis, 2018), a clinician is left with the challenge to aggregate predictive information from multiple pre-treatment variables that match the depressed individuals' profile. For example, one individual could have several factors that indicate a successful outcome in CT, while having a similar number of factors related to a perfect match for IPT. If the goal is to move beyond the "one size fits all" approach towards personalized treatment strategies for MDD, more holistic methods are warranted.

\subsection{Why clinical judgment is not sufficient to personalize treatment strategies}

An often-practiced holistic method to integrate various predictive information is clinical judgment. Clinical judgment is based on the clinicians' experience with similar pathology, the clinicians' training background, the patient's preference and the clinicians' beliefs, theoretical perspective, and knowledge of empirical literature and (inter)national guidelines (Delgadillo, Gellatly, \& Stephenson-Bellwood, 2015; Lorenzo-Luaces, DeRubeis, \& Bennett, 2015). Advocates of clinical judgment describe this as a set of sophisticated strategies that are well-matched to the complexity of clinical situations in MDD treatment. In addition, they argue that this approach goes beyond the limitations of the current classification systems that ignore clinical

\footnotetext{
${ }^{1}$ Example of the misinterpretation of odds ratios in Newton-Howes et al. 2006 (from Abstract): “Comorbid personality disorder with depression was associated with a doubling of the risk of a poor outcome for depression compared with no personality disorder (OR=2.18, $95 \% \mathrm{Cl}=1.70$ to 2.80$)$ "

2 Example of the misinterpretation of odds ratios in Newton-Howes et al. 2014 (from paragraph Conclusions): "Presence of a comorbid personality disorder doubles the likelihood of nonresponse to treatment for depressive disorders (OR=2.16, $95 \% \mathrm{Cl}=1.83$ to 2.56$)^{\prime \prime}$.
} 
heterogeneity and uniqueness of the individual. Moreover, clinical judgment transcends the myopic focus on mean outcomes in RCTs' and the negative influence of pharmaceutical industries in developing evidence based treatments (Fava, 2013; Fava, Rafanelli, \& Tomba, 2012; Tomba \& Fava, 2012). This critical stance could explain the gap between clinical science and daily clinical work, which is reflected in the low adherence to evidence based treatment guidelines for MDD (Herzog et al., 2017; Schneider et al., 2004). Although expert knowledge could be a valuable source of information (Drescher et al., 2013), the direct predictions derived from clinical judgment are far from robust. Over the years, research has consistently replicated the finding of overall superiority of statistical predictions over predictions based on clinical judgment (Chapter 10, AEgisdóttir et al., 2006; Grove, Zald, Lebow, Snitz, \& Nelson, 2000; Meehl, 1954, 1986). This modest predictive power of clinical judgment has several explanations. First, the sample sizes an individual clinician is exposed to are skewed, unrepresentative, and too small to support stable inferences that connect, for example, treatment procedures to their effects (i.e., representative biases). Second, the contribution of their preferred theory to inference is strong for a majority of clinicians, even if firm empirical grounds of these theories are lacking (i.e., confirmation bias). Third, in comparison to statistical inference, clinicians have limited information processing abilities in selecting predictive variables and combining these variables in a meaningful way. Fourth, clinicians rarely seek and receive corrective feedback to test and adjust their inferences for new cases (Dawes, 2005; Meehl, 1954).

\subsection{Why these strategies are still common practice}

Despite the above mentioned disadvantages of using comorbidity and clinical judgment to guide personalized treatment strategies for MDD, these approaches have not been abandoned in clinical practice. In particular, personality pathology and clinical judgment are still important pillars in treatment selection for MDD.

A potential explanation for the strong focus on comorbid PD diagnoses in MDD treatment is that it taps into our conceptualization of mental problems. With the introduction of Axis 1 disorders and Axis 2 disorders in the DSM-III, there is a traditional distinction between current illness ('state') and tentatively stable personality ('trait') (American Psychiatric Association, 1980). Although this distinction may provide clarity at first sight, this does not fully capture how MDD and PD relate. Previous research has proposed multiple models of the relationship between MDD and PD: (a) PD and MDD have common causes; (b) PD and MDD are on a continuous spectrum; (c) PD is a precursor of MDD; (d) PD predisposes to developing MDD; (e) PD has pathoplastic effects on MDD; (f) PD features are state-dependent concomitants of MDD episodes; and (g) PD features are consequences (or scars) of MDD (Klein, Kotov, \& Bufferd, 2011). Despite the elimination of the Axis 1 and Axis 2 distinction in DSM-5 
(American Psychiatric Association, 2013), it is not unlikely that clinicians still make this traditional state-trait division, and have their own specific models on how these two relate. As illustrated with Quote 1 (Textbox 13.1), these conceptualizations on mental problems may shape clinical decision making accordingly.

Regarding clinical judgment, one would expect clinicians to be informed and conscious of the well-known errors of clinical prediction, and eager to investigate new approaches for decision making; or as Meehl stated "We, of all people, ought to be highly suspicious of ourselves" (Meehl, 1954, p.27). Even though one might argue that it is unethical to rely on clinical judgment (Dawes, 2005), as illustrated with Quote 2 and Quote 3 (Textbox 13.1), using clinical judgment instead of statistical prediction to guide treatment decisions is still highly common in clinical practice (Bell \& Mellor, 2009). Among the reasons that contribute to this status quo have been put forward are; the clinician's poor education on statistics and philosophy of science, fear of unemployment, the clinician's professional identity, attachment to theory, fear of dehumanizing patients, and ethical objections (e.g., trying to make the "right" decision by ignoring a statistical prediction that disagrees with a strong personal preference) (Garb, 2000; Grove \& Meehl, 1996; Katsikopoulos, Pachur, Machery, \& Wallin, 2008).

Textbox 13.1: Quotes from clinicians (anonymous)

1) "Cognitive therapy is a quick fix for depressive symptoms, not a sustaining solution to treat the underlying personality pathology, which is the cause of all depressive symptoms"

2) "This type of patient will not respond to antidepressants"

3) “I don't believe in self-report instruments for depression: It's the psychiatrist who decides whether this patient's depression is in remission or not"

\section{Reflections about prediction models to personalize treatment strategies for depression}

\subsection{True is what works: black boxes}

The primary goal of prediction models (or algorithms) is to generate accurate predictions. A secondary interest may be to interpret the model and gain understanding on why the models works (e.g., understanding the relationship between the predictors and the outcome). However, there is a trade-off between prediction and interpretation; achieving high accuracy often involves complex models that are difficult to interpret. These complex models are a departure from more conventional statistics that facilitates explanations and tests of theory. One could argue that when it comes to prediction in the medical field, it is unethical to constrain predictive accuracy by the requirement of interpretability (Kuhn \& Johnson, 2013). 
This point of view aligns with a pragmatist's perspective that focuses on the practical consequences of ideas in real life (James, 1909).

When prediction models become difficult to interpret, they are referred to as "black boxes". According to philosopher, anthropologist and sociologist Bruno Latour, "blackboxing" is an almost inescapable result of the success and efficiency of scientific and technical work: "When a machine runs efficiently, when a matter of fact is settled, one need focus only on its inputs and outputs and not on its internal complexity. Thus, paradoxically, the more science and technology succeed, the more opaque and obscure they become" (Latour, 1999, p.304). An example of a medical black box in this context is the use of Computerized Tomography (CT) scans: when the CT-scan indicates a broken wrist, doctors will normally not evaluate the working mechanisms of CT scans, but start directly with a treatment plan. With prediction models that aim to personalize treatment strategies for depression we are far from "blackboxing" yet, since the development of prediction models is still work in progress.

\subsection{Work in progress: challenges in the development of prediction models}

Although the accuracy of prediction models in MDD is potentially promising (Chapter 7 \& 8, Cohen \& DeRubeis, 2018), development of these models is still in its infancy since they have mainly been applied in research contexts rather than being tested and implemented in real-world settings. To determine the generalizability of Personalized Advantage Index (PAI) models in actual clinical practice, the predictive accuracy of these models needs to be externally validated (Cohen \& DeRubeis, 2018; Gillan \& Whelan, 2017). External validation is considered a second phase in research into prediction models, following model development and preceding impact studies (Moons, Royston, Vergouwe, Grobbee, \& Altman, 2009). Although it is widely acknowledged that this external validation is an essential step, it has been done very infrequently in medicine in general and mental health care specifically (Siontis, Tzoulaki, Castaldi, \& loannidis, 2015). Moreover, the modest effects of our crossvalidation study (Chapter 11) in particular emphasize the challenges of external validation. As described in this chapter, external validation is impeded by several factors. First, in external validation studies, the predictive accuracy of a prediction model is tested in different samples from the same target populations (Debray et al., 2015). Although this may seem plausible at first sight, because of its' highly heterogeneous nature, defining a different sample within the same target population is not as evident in MDD. Second, there is a lack of consensus and a striking amount of heterogeneity in types of data collection and statistical methods. Third, contrasts between treatments are difficult to generalize since treatment delivery and quality varies per therapist and/or location. 


\subsection{Your robot doctor will see you now: rise of the machine learning?}

"The development of full artificial intelligence could spell the end of the human race" (Hawking, 2014).

Artificial intelligence (Al), a term originally coined by computer scientist John Carthy in 1956, was defined as "the science and engineering of making intelligent machines" (McCarthy, 1989). Decades later, Al is omnipresent in our lives facilitating our modern-day conveniences (e.g., social media, Netflix). In mental health care, however, we are still far from routine application of Al (Graham et al., 2019; Tiffin \& Paton, 2018). Since the stakes are high, patients, clinicians, policy makers, health insurance companies and researchers should use this momentum to contemplate on the consequences of creating algorithms that can surpass human judgment (Rigby, 2019).

Machine learning (ML) is an important branch of $\mathrm{Al}$, and involves various methods (e.g., random forest algorithms from Chapter 7 \& 8) that aim to improve performance by mechanizing the acquisition of knowledge from experience. Acquisition of knowledge, or learning, can be either "supervised" or "unsupervised". In supervised ML there is a labeled set of training data, while in the unsupervised version of $\mathrm{ML}$ hidden structures are identified in unlabeled data (Alanazi, Abdullah, \& Qureshi, 2017). In Chapter 7 and $\mathbf{8}$ of this thesis, examples of supervised ML are given with labeled candidate predictors and moderators, and an a-priori defined outcome measure. In $\mathrm{ML}$, and in particular in the unsupervised variant, systems gradually gain more flexibility and independence with the accumulative input of new data.

Once systems become more independent and autonomous, the question regarding responsibility, which naturally lies with its controllers, becomes more complicated (Luxton, 2014a). Although ML-based prediction models have the potential to optimize clinical decision making, predictions are never flawless. As illustrated by the public outrage on the pedestrian death due to a driverless car error (Wakabayashi, 2018), society sets particularly high standards to the safety of Al applications (Maddox, Rumsfeld, \& Payne, 2019). Mistakes by Al application seem more difficult to accept in comparison to mistakes by humans. Researchers and stakeholders should proactively identify and minimize potential incorrect predictions. Another concern stems from the presence of potential data biases. Quality of MLbased predictions depends on the quality of training data, and an underrepresentation of minorities in these data can lead to the amplifying of existing biases (e.g., biases by sex, race, ethnicity). Efforts to avoid inequality by acquiring comprehensive data are therefore important (Caplan \& Friesen, 2017; Chen, Szolovits, \& Ghassemi, 2019; Maddox et al., 2019). However, this increasing demand on data collection and storage also raises the risk of oversharing personal information. Managing and persevering privacy should therefore be a priority in research (Russell, Dewey, \& Tegmark, 2015). 
Some fear that these new technologies will make clinicians obsolete and will subsequently lead to job loss (Luxton, 2014a). In particular with the development of AI care providers, new technologies could reshape therapeutic relationships (Luxton, 2014b). In response to these somewhat apocalyptic concerns ("your robot doctor will see you now"), opportunities that humanize Al should be explored, for example by answering the question whether Al could help clinicians deliver more humanistic care (Israni \& Verghese, 2019). In this context, one could imagine that the accuracy of ML predictions encourages clinicians to depart from the paternalistic role of an allknowing expert (Textbox 13.1), and provide a starting point for a more balanced dialogue between the patient and the clinicians on treatment strategies.

\section{Future directions}

\subsection{Bridging the gap between scientific research and clinical practice: a global network of local models}

In order to translate prediction models into ready-to-use clinical instruments, both research and clinical practice will have to undergo cultural changes. A stronger cooperation between mental health care institutions and academic research is warranted. On the one hand, scientists should consider the translatability of their findings, and explore the possibilities of converting these findings into technology. Researchers should actively involve end-users (i.e., clinicians and patients) in all phases including model development, validation and implementation: if end-users do not embrace these new strategies from the start, innovation is impossible and unwanted (Perna, Grassi, Caldirola, \& Nemeroff, 2018). In turn, clinicians should receive clinical training and systematic feedback that may help them to be more aware of their errors in clinical judgement and stimulate modesty about their clinical predictions (Ægisdóttir et al., 2006; Knaup, Koesters, Schoefer, Becker, \& Puschner, 2009). Examples of a modest therapeutic stance are already part of current treatment manuals, such as collaborative empiricism in CT (i.e., therapist and patient work as a team to investigate the validity of the patient's thoughts; Beck \& Beck, 1995), and the non-directive and supportive description of the therapists' role in IPT (Blom, 2015).

With these cultural challenges, and the aforementioned "work in progress" (paragraph 3.2) in mind, how do we depart from here? As mentioned in Chapter 11, previous researchers have proposed to start with building prediction models on large (combined) observational datasets followed by pragmatic trials that randomize clinicians to receive or not receive information from these algorithms (Delgadillo \& Gonzalez Salas Duhne, 2019; Kessler, 2018; Luedtke, Sadikova, \& Kessler, 2019). Parallel to these developments, we should also think about the development of "more local" prediction models. Local in this context refers to models in the same 
geographical locations within the same mental health care organization and with a low number of therapists that have joint supervision. With clear agreements on what data to collect and on how to analyze these data, we could focus on temporal validation (same location, different time period) first, and then work towards external validation by expanding the location in terms of other geographical locations, other mental health care organizations and by increasing the number of therapists. Model updating should be a central part of these steps, enhancing the performance of models in other samples using methods such as recalibration (adjustment of intercepts and the regression coefficients using the calibration intercept and calibration slope) and model revision (re-estimation of the intercept and the regression coefficients using the combined datasets; Janssen, Moons, Kalkman, Grobbee, \& Vergouwe, 2008).

"Starting small" with local models has several advantages. First, since the heterogeneity of patient populations and treatments is very high, it is unclear to what extent one overarching prediction model is realistic. One could argue that some treatment strategies are predominantly depended on clinical and population context. There could be a trade-off between generalizability and precision: the more generalizable the model, the less specific the treatment recommendations (e.g., psychotherapy or pharmacotherapy versus CT or sertraline). Second, the above mentioned collaboration between scientists and clinicians could be strengthened by local model building: with scientists being part of a mental health care institution, feedback on model development and decision making could be more easily interchanged between researchers and clinicians.

\subsection{Setting treatment goals by integrating multiple outcomes}

In this thesis, optimal treatment was defined as a treatment that results in large depressive symptom reduction in the acute phase and sustained depressive symptom reduction in the long term. Although this is an important outcome, one could argue that depressive symptom reduction is not the only indicator of a successful treatment strategy. A somewhat neglected outcome variable that diminishes treatment effectiveness in routine daily practice is treatment drop-out, defined as a situation in which, without clinical improvement, a patient prematurely discontinuous engagement in the chosen treatment. Drop-out is estimated to occur in about $20 \%$ of psychotherapeutic treatments (Cooper \& Conklin, 2015) and between 10-35\% in pharmacological treatments (Warden et al., 2014). Timely prediction of treatment dropout can be expected to increase treatment effectiveness since, in general, treatment will be of little benefit if individuals are unwilling to engage in it. Most studies show that predictors of dropout only partially overlap with predictors of good treatment response (Cooper \& Conklin, 2015) and, as illustrated in Chapter 10, there 
is a need for different models when it comes to predicting treatment dropout (Keefe et al., 2018; Zilcha-Mano et al., 2016).

In addition to treatment dropout, other outcomes regarding positive emotions and positive mental health should be explored as outputs of prediction models to personalize treatment strategies for MDD (Huber et al., 2011). In contrast to clinicians, depressed individuals perceive depressive symptom reduction as only one factor in "being cured from MDD" (Zimmerman et al., 2012; Zimmerman et al., 2013). Factors of positive health such as regaining optimism, self-confidence and positive affect, and returning to one's usual self and usual level of functioning are considered of importance, and are unaddressed in our current definitions of remission from MDD (Demyttenaere et al., 2015; Zimmerman et al., 2006). Addressing these positive mental health factors in prediction models would better support the patients' perspective, and has also shown to be of importance in predicting MDD outcomes (Geschwind et al., 2011; Höhn et al., 2013; Khazanov \& Ruscio, 2016; Wichers, Lothmann, Simons, Nicolson, \& Peeters, 2012). This potential is illustrated as well by

Chapter 7, in which hope and self-esteem are indicated as important post-treatment predictors for long-term outcome in MDD.

\subsection{Identifying relevant information as input for prediction models}

Although current prediction models are based on data-driven methods, choices on data collection in clinical trials are by definition not data-driven. Financial costs and time limitations have forced researchers to select a limited amount of study data. In addition, most research on prediction models in MDD is based on secondary analyses of trials with different primary research questions. These study designs result in limited overlap in data collection between trials (as illustrated in Chapter 11), and enhance the likelihood that valuable information was not recorded and therefore was not included in a prediction model.

In the process of identifying relevant information as input for prediction models, factors such as cost, feasibility, and patient burden should be taken into account. As recommended by Kessler (2018), data collection should involve an easy to collect number of inexpensive measures including clinical variables, sociodemographic information, life and family history, psychological variables, and current level of functioning. With the development and implementation of electronic medical records (EMR), data that meets these criteria are now more easily available for clinical researchers. However, EMRs often include missing, messy and unstandardized information that impedes the accuracy of prediction models ("garbage in, garbage out" principle). Efforts should be made to address these issues and to align information collected in EMRs between all clinical centers (Hallgren, Bauer, \& Atkins, 2017; Perna et al., 2018). In addition, the application of language processing techniques that extract and code information from unstandardized clinical notes are 
promising (Castro et al., 2015; Perlis et al., 2012). Wearable devices (e.g., smartphones) also provide opportunities to collect data on a large scale. In particular, the experience sampling methodology (ESM), a structured self-report diary technique, can provide ecologically valid data by measuring experiences within, and in interaction with, real-world contexts (Myin-Germeys et al., 2018).

The potential of machine learning and prediction models has also been acknowledged in biological research of psychiatry (Iniesta, Stahl, \& McGuffin, 2016). Although inclusion of indicators of physical health that are known to relate to depression (e.g., Scott et al., 2007) should be considered, a critical position towards the inclusion of biomarkers (e.g., genotyping, pharmacogenetics, metabolic and inflammatory parameters) is justifiable for several reasons. First, the process of collecting biomarkers is too complex and too expensive for widespread use in depressed patients that, due to the high prevalence of MDD, are being treated in a wide variety of care contexts (e.g., private practice, outpatient departments from hospitals and mental health institutions). Second, in contrast to other areas of medicine, the intense search for a biological basis for psychiatric disorders during the last decades has not resulted in a significant improvement of our understanding of psychopathology (Beijers, Wardenaar, van Loo, \& Schoevers, 2019; Cristea, Karyotaki, Hollon, Cuijpers, \& Gentili, 2019). Likewise, and again in contrast with other areas of medicine (e.g., oncology), recent results of research into the use of biomarkers for prediction or moderation of course and treatment response do not support overt enthusiasm. For example, this is illustrated in a recent report of the NESDA study (large naturalistic cohort study examining the long-term course of depressive and anxiety disorders (Beijers, Wardenaar, Bosker, et al., 2019). The authors conclude after examination of 36 biomarkers as potential explanatory variables for heterogeneity that there was no association with course or diagnoses at 2- and 6-year follow-up and "that biomarker heterogeneity among depressive and/or anxiety patients mostly reflects variations in somatic health that extend into the part of the population without mental health problems" (p. 623).

\subsection{Treatment selection and adaptation tools: updating Bayesian models}

This thesis focused on strategies that aim to find the optimal depression treatment for a specific individual at a specific point in time. In addition to these treatment selection strategies, another approach to optimize depression treatments is enriching ongoing therapies with personalized treatment adaptations. While treatment selection is based on personalized predictions prior to treatment, treatment adaptation can be seen as a strategy in which ongoing treatments are tailored to the individual's treatment response. Previous studies have predominantly focused on routine outcome monitoring and feedback tools to adapt individual treatments. In these 
studies, findings indicated that feedback to therapists on the patient's progress resulted in better therapy outcomes, in particular for patients that were "not on track" (Kendrick et al., 2016; Knaup et al., 2009; Lambert \& Shimokawa, 2011; Poston \& Hanson, 2010; Shimokawa, Lambert, \& Smart, 2010). In addition, feedback was associated with a longer treatment duration for the "not on track" patients, and a shorter treatment duration for patients that were "on track" (Lambert et al., 2001; Whipple et al., 2003). These differential treatment durations indicate that personalized treatment adaptations do not only improve treatment results, but also potentially prevent under- and overtreatment by directing a higher level of care to those that need it the most. In addition to routine outcome monitoring and feedback tools, several studies have investigated the use of clinical support tools as well, as an add-on to feedback regarding the patient's progress. Clinical support tools aim to identify potential factors responsible for outcome deterioration and provide clinical suggestions to improve treatments. These clinical suggestions are based on measures of therapeutic alliance, patient motivation, and social support. Using clinical support tools in addition to feedback increases the odds of clinically significant improvement (Shimokawa et al., 2010).

So far, these treatment selection and adaptation strategies have been studied independently. However, to maximize the potential for personalized treatment strategies, we need to combine these methods into one comprehensive model (Lutz, Zimmermann, Müller, Deisenhofer, \& Rubel, 2017). In addition, personalized adaptions should be improved by basing feedback on ongoing predictions instead of on ongoing measurements. Once an individual is "not on track" according routine outcome monitoring, one could argue that we actually "lag behind events". As with personalized prediction for treatment selection, providing information on predicted outcomes helps us anticipate, rather than react upon, treatment failures and successes. Computing predictions to optimize treatment selection and adaptation involves complex models that update over time. Bayesian inference, that inherently updates prior probabilities with new data, could be integrated in these models, especially in the context of small study samples (Depaoli, Rus, Clifton, van de Schoot, \& Tiemensma, 2017).

To paint a picture on how these updating prediction and adaptation models could change clinical decision making, illustrations are provided in Figure 13.1 and Figure 13.2. In Figure 13.1, predictions prior to treatment for a fictional patient are listed. Based on this example cognitive behavioral therapy (CBT) is predicted to have the greatest symptom reduction (declining line) and lowest risk of dropout (dropout icon with a light color). Figure 13.2 illustrates several ongoing predictions and adaptations during treatment for the same fictional patient. In this figure, the lightly colored area is the initial prediction of symptom reduction based on the prediction prior to treatment (95\% confidence intervals). The bold line represents the actual symptom change during treatment. During treatment, predictions indicate an 
elevated risk of dropout (prediction 1) and an elevated risk of symptom increase (prediction 2), for which two adaptations (motivational interviewing and behavior activation) are applied successfully.
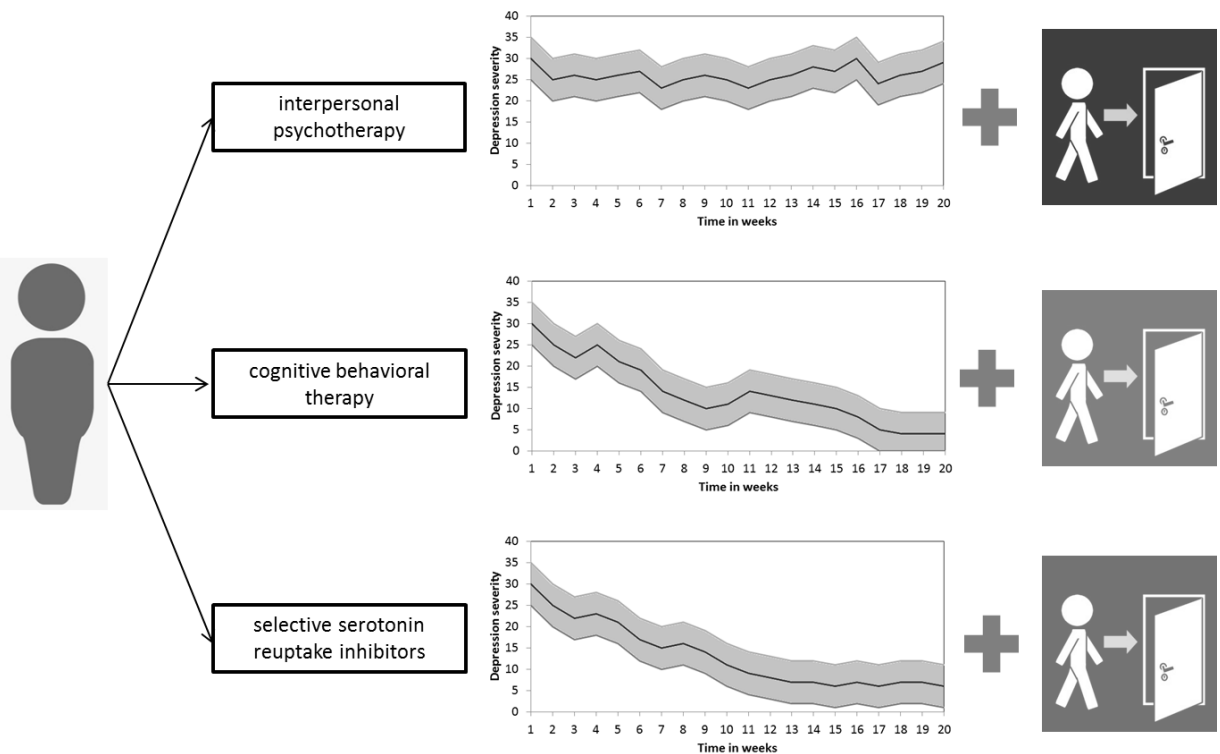

Figure 13.1: Illustration of predictions of symptom reduction and dropout prior to treatment to guide the selection of different types of depression treatments for a fictional patient.

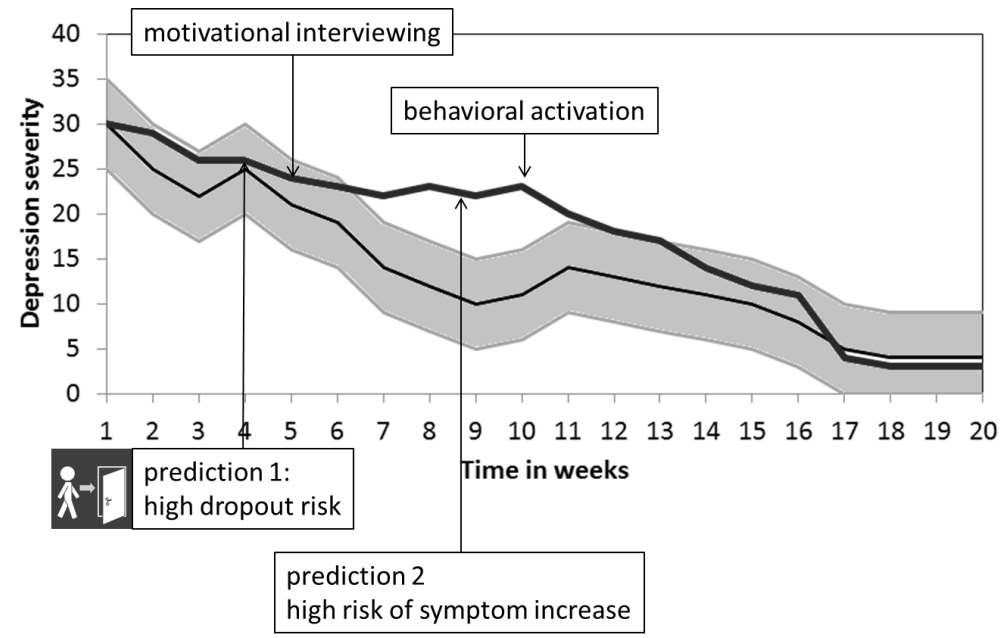

Figure 13.2: Illustration of predictions of symptom reduction and dropout during treatment to guide treatment adaptations for a fictional patient. 


\section{In conclusion}

Traditionally, comorbidity and clinical judgment are used to guide personalized treatment strategies for MDD. These traditional approaches are frequently advocated in the literature, and common in clinical practice, however this is supported by limited and non-robust evidence. With the development of novel statistical approaches and the availability of "big" data, prediction models that guide clinical decision making are gaining ground. Although results are promising, the development of these prediction models is still a work in progress, and steps towards external validation are impeded by the striking heterogeneity of data collection, statistical methods, study populations and the execution of treatments. In addition, concerns on the implementation of these models have been raised regarding interpretability (black boxes), responsibilities, incorrect predictions, biases, privacy and the dehumanization of treatments. In order to develop and implement prediction models, efforts to bridge the gap between clinical practice and research are warranted, in which the involvement of end-users (patients and clinicians) should be a central part. Starting small with the development of local prediction models in mental health care institutions could serve this purpose, and could facilitate external validation and model updating between centers as part of a global network. To target meaningful treatment goals with these prediction models, multiple indicators of successful treatment should be investigated, focusing on outcomes other than depressive symptom reduction including treatment dropout and positive mental health. In addition to defining indicators of successful treatment, efforts to identify relevant data (sources) to inform prediction models are needed, in which factors such as cost, feasibility, and patient burden should be taken into account. Personalizing treatment strategies should not be based on a single "snap shot" prediction. Instead, models should provide prediction updates over the course of treatments, leading to optimal treatment selection and optimal treatment adaptation. Implementing prediction models in a patient and therapist oriented manner could change depression treatments drastically. Ultimately, it could provide a modern platform for ongoing shared decision making between clinicians and patients leading towards personalized treatment strategies for depression. 


\section{References}

Egisdóttir, S., White, M. J., Spengler, P. M., Maugherman, A. S., Anderson, L. A., Cook, R. S., . . Cohen, G. (2006). The meta-analysis of clinical judgment project: Fifty-six years of accumulated research on clinical versus statistical prediction. The Counseling Psychologist, 34(3), 341-382.

Alanazi, H. O., Abdullah, A. H., \& Qureshi, K. N. (2017). A critical review for developing accurate and dynamic predictive models using machine learning methods in medicine and health care. Journal of medical systems, 41(4), 69.

American Psychiatric Association. (1980). Diagnostic and statistical manual of mental disorders (3rd ed.). Washington DC.

American Psychiatric Association. (2013). Diagnostic and statistical manual of mental disorders (5th ed.). Washington DC.

Beck, J. S., \& Beck, A. T. (1995). Cognitive therapy: Basics and beyond: Guilford press New York.

Beijers, L., Wardenaar, K. J., Bosker, F. J., Lamers, F., van Grootheest, G., de Boer, M. K., . . . Schoevers, R. A. (2019). Biomarker-based subtyping of depression and anxiety disorders using Latent Class Analysis. A NESDA study. Psychological medicine, 49(4), 617-627.

Beijers, L., Wardenaar, K. J., van Loo, H. M., \& Schoevers, R. A. (2019). Data-driven biological subtypes of depression: systematic review of biological approaches to depression subtyping. Molecular psychiatry.

Bell, I., \& Mellor, D. (2009). Clinical judgements: Research and practice. Australian Psychologist, 44(2), 112121.

Berk, M., Boyce, P., Hamilton, A., Morris, G., Outhred, T., Das, P., . . . Mulder, R. (2018). Personality: distraction or driver in the diagnosis of depression. Personality and mental health, 12(2), 126-130.

Blom, M. (2015). Leerboek interpersoonlijke psychotherapie: Bohn Stafleu van Loghum.

Caplan, A., \& Friesen, P. (2017). Health disparities and clinical trial recruitment: Is there a duty to tweet? PLoS biology, 15(3), e2002040.

Castro, V. M., Minnier, J., Murphy, S. N., Kohane, I., Churchill, S. E., Gainer, V., . . . Block, S. (2015). Validation of electronic health record phenotyping of bipolar disorder cases and controls. American Journal of Psychiatry, 172(4), 363-372.

Chen, I. Y., Szolovits, P., \& Ghassemi, M. (2019). Can Al Help Reduce Disparities in General Medical and Mental Health Care? AMA Journal of Ethics, 21(2), 167-179.

Cohen, Z. D., \& DeRubeis, R. J. (2018). Treatment selection in depression. Annual review of clinical psychology, 14, 209-236.

Cooper, A. A., \& Conklin, L. R. (2015). Dropout from individual psychotherapy for major depression: A metaanalysis of randomized clinical trials. Clinical Psychology Review, 40, 57-65.

Cristea, I. A., Karyotaki, E., Hollon, S. D., Cuijpers, P., \& Gentili, C. (2019). Biological markers evaluated in randomized trials of psychological treatments for depression: a systematic review and meta-analysis. Neuroscience \& Biobehavioral Reviews.

Cyranowski, J. M., Frank, E., Shear, M. K., Swartz, H., Fagiolini, A., Scott, J., \& Kupfer, D. J. (2005). Interpersonal psychotherapy for depression with panic spectrum symptoms: a pilot study. Depression and anxiety, 21(3), 140-142.

Dawes, R. M. (2005). The ethical implications of Paul Meehl's work on comparing clinical versus actuarial prediction methods. Journal of clinical psychology, 61(10), 1245-1255.

Debray, T. P., Vergouwe, Y., Koffijberg, H., Nieboer, D., Steyerberg, E. W., \& Moons, K. G. (2015). A new framework to enhance the interpretation of external validation studies of clinical prediction models. Journal of clinical epidemiology, 68(3), 279-289.

Delgadillo, J., Gellatly, J., \& Stephenson-Bellwood, S. (2015). Decision making in stepped care: how do therapists decide whether to prolong treatment or not? Behavioural and cognitive psychotherapy, 43(3), 328-341.

Delgadillo, J., \& Gonzalez Salas Duhne, P. (2019). Targeted prescription of cognitive behavioral therapy vs. person-centered counseling for depression using a machine learning approach. Journal of Consulting and Clinical Psychology. 
Demyttenaere, K., Donneau, A.-F., Albert, A., Ansseau, M., Constant, E., \& Van Heeringen, K. (2015). What is important in being cured from depression? Discordance between physicians and patients (1). Journal of Affective Disorders, 174, 390-396.

Depaoli, S., Rus, H. M., Clifton, J. P., van de Schoot, R., \& Tiemensma, J. (2017). An introduction to Bayesian statistics in health psychology. Health Psychology Review, 11(3), 248-264.

DeRubeis, R. J., Cohen, Z. D., Forand, N. R., Fournier, J. C., Gelfand, L. A., \& Lorenzo-Luaces, L. (2014). The Personalized Advantage Index: translating research on prediction into individualized treatment recommendations. A demonstration. PloS one, 9(1), e83875.

Drescher, M., Perera, A., Johnson, C., Buse, L., Drew, C., \& Burgman, M. (2013). Toward rigorous use of expert knowledge in ecological research. Ecosphere, 4(7), 1-26.

Fava, G. A. (2013). Clinical judgment in psychiatry. Requiem or reveille? Nordic journal of psychiatry, 67(1), 1-10.

Fava, G. A., Rafanelli, C., \& Tomba, E. (2012). The clinical process in psychiatry: a clinimetric approach. The Journal of clinical psychiatry, 73(2), 177-184.

Garb, H. N. (2000). Computers will become increasingly important for psychological assessment: Not that there's anything wrong with that. Psychological assessment, 12(1), 31.

Geschwind, N., Nicolson, N. A., Peeters, F., van Os, J., Barge-Schaapveld, D., \& Wichers, M. (2011). Early improvement in positive rather than negative emotion predicts remission from depression after pharmacotherapy. European Neuropsychopharmacology, 21(3), 241-247.

Gillan, C. M., \& Whelan, R. (2017). What big data can do for treatment in psychiatry. Current opinion in behavioral sciences, 18, 34-42.

Graham, S., Depp, C., Lee, E. E., Nebeker, C., Tu, X., Kim, H.-C., \& Jeste, D. V. (2019). Artificial intelligence for mental health and mental illnesses: an overview. Current psychiatry reports, 21(11), 116.

Grove, W. M., \& Meehl, P. E. (1996). Comparative efficiency of informal (subjective, impressionistic) and formal (mechanical, algorithmic) prediction procedures: The clinical-statistical controversy. Psychology, public policy, and law, 2(2), 293.

Grove, W. M., Zald, D. H., Lebow, B. S., Snitz, B. E., \& Nelson, C. (2000). Clinical versus mechanical prediction: a meta-analysis. Psychological assessment, 12(1), 19.

Hallgren, K. A., Bauer, A. M., \& Atkins, D. C. (2017). Digital technology and clinical decision making in depression treatment: Current findings and future opportunities. Depression and anxiety, 34(6), 494501.

Herzog, D. P., Wagner, S., Ruckes, C., Tadic, A., Roll, S. C., Härter, M., \& Lieb, K. (2017). Guideline adherence of antidepressant treatment in outpatients with major depressive disorder: a naturalistic study. European archives of psychiatry and clinical neuroscience, 267(8), 711-721.

Höhn, P., Menne-Lothmann, C., Peeters, F., Nicolson, N. A., Jacobs, N., Derom, C., . . Wichers, M. (2013). Moment-to-moment transfer of positive emotions in daily life predicts future course of depression in both general population and patient samples. PloS one, 8(9), e75655.

Huber, M., KNOTTNERUS, J. A., GREEN, L., VAN DER HORST, H., JADAD, A. R., KROMHOUT, D., ... VAN DER MEER, J. W. (2011). Health how should we define it? Bmj, 343(7817), 235-237.

Iniesta, R., Stahl, D., \& McGuffin, P. (2016). Machine learning, statistical learning and the future of biological research in psychiatry. Psychological medicine, 46(12), 2455-2465.

Ionescu, D. F., Niciu, M. J., Henter, I. D., \& Zarate, C. A. (2013). Defining anxious depression: a review of the literature. CNS spectrums, 18(5), 252-260.

Israni, S. T., \& Verghese, A. (2019). Humanizing artificial intelligence. Jama, 321(1), 29-30.

James, W. (1909). The Meaning of Truth.

Janssen, K., Moons, K., Kalkman, C., Grobbee, D., \& Vergouwe, Y. (2008). Updating methods improved the performance of a clinical prediction model in new patients. Journal of clinical epidemiology, 61(1), 7686.

Katsikopoulos, K. V., Pachur, T., Machery, E., \& Wallin, A. (2008). From Meehl to Fast and Frugal Heuristics (and Back) New Insights into How to Bridge the Clinical-Actuarial Divide. Theory \& Psychology, 18(4), 443-464 
Keefe, J. R., Wiltsey Stirman, S., Cohen, Z. D., DeRubeis, R. J., Smith, B. N., \& Resick, P. A. (2018). In rape trauma PTSD, patient characteristics indicate which trauma-focused treatment they are most likely to complete. Depression and anxiety, 35(4), 330-338.

Kendrick, T., El-Gohary, M., Stuart, B., Gilbody, S., Churchill, R., Aiken, L., . . . de Jong, K. (2016). Routine use of patient reported outcome measures (PROMs) for improving treatment of common mental health disorders in adults. Cochrane Database of Systematic Reviews(7).

Kessler, R. C. (2018). The potential of predictive analytics to provide clinical decision support in depression treatment planning. Current opinion in psychiatry, 31(1), 32-39.

Khazanov, G. K., \& Ruscio, A. M. (2016). Is low positive emotionality a specific risk factor for depression? A meta-analysis of longitudinal studies. Psychological Bulletin, 142(9), 991.

Klein, D. N., Kotov, R., \& Bufferd, S. J. (2011). Personality and depression: explanatory models and review of the evidence. Annual review of clinical psychology, 7, 269-295.

Knaup, C., Koesters, M., Schoefer, D., Becker, T., \& Puschner, B. (2009). Effect of feedback of treatment outcome in specialist mental healthcare: meta-analysis. The British Journal of Psychiatry, 195(1), 1522.

Kuhn, M., \& Johnson, K. (2013). Applied predictive modeling (Vol. 26): Springer.

Lambert, M. J., \& Shimokawa, K. (2011). Collecting client feedback. Psychotherapy, 48(1), 72.

Lambert, M. J., Whipple, J. L., Smart, D. W., Vermeersch, D. A., Nielsen, S. L., \& Hawkins, E. J. (2001). The effects of providing therapists with feedback on patient progress during psychotherapy: Are outcomes enhanced? Psychotherapy research, 11(1), 49-68.

Latour, B. (1999). Pandora's hope: essays on the reality of science studies: Harvard university press.

Lorenzo-Luaces, L., DeRubeis, R. J., \& Bennett, I. M. (2015). Primary care physicians' selection of lowintensity treatments for patients with depression. Fam Med, 47(7), 511-516.

Luedtke, A., Sadikova, E., \& Kessler, R. C. (2019). Sample size requirements for multivariate models to predict between-patient differences in best treatments of major depressive disorder. Clinical Psychological Science, 7(3), 445-461.

Lutz, W., Zimmermann, D., Müller, V. N., Deisenhofer, A.-K., \& Rubel, J. A. (2017). Randomized controlled trial to evaluate the effects of personalized prediction and adaptation tools on treatment outcome in outpatient psychotherapy: study protocol. BMC psychiatry, 17(1), 306.

Luxton, D. D. (2014a). Artificial intelligence in psychological practice: Current and future applications and implications. Professional Psychology: Research and Practice, 45(5), 332.

Luxton, D. D. (2014b). Recommendations for the ethical use and design of artificial intelligent care providers. Artificial intelligence in medicine, 62(1), 1-10.

Maddox, T. M., Rumsfeld, J. S., \& Payne, P. R. (2019). Questions for artificial intelligence in health care. Jama, 321(1), 31-32.

McCarthy, J. (1989). Artificial intelligence, logic and formalizing common sense. In Philosophical logic and artificial intelligence (pp. 161-190): Springer.

Meehl, P. E. (1954). Clinical versus statistical prediction: A theoretical analysis and a review of the evidence.

Meehl, P. E. (1986). Causes and effects of my disturbing little book. Journal of personality assessment, 50(3), 370-375.

Moons, K. G., Royston, P., Vergouwe, Y., Grobbee, D. E., \& Altman, D. G. (2009). Prognosis and prognostic research: what, why, and how? Bmj, 338, b375.

Mulder, R. T. (2002). Personality pathology and treatment outcome in major depression: a review. American Journal of Psychiatry, 159(3), 359-371.

Myin-Germeys, I., Kasanova, Z., Vaessen, T., Vachon, H., Kirtley, O., Viechtbauer, W., \& Reininghaus, U. (2018). Experience sampling methodology in mental health research: new insights and technical developments. World Psychiatry, 17(2), 123-132.

Newton-Howes, G., Tyrer, P., \& Johnson, T. (2006). Personality disorder and the outcome of depression: meta-analysis of published studies. The British Journal of Psychiatry, 188(1), 13-20.

Newton-Howes, G., Tyrer, P., Johnson, T., Mulder, R., Kool, S., Dekker, J., \& Schoevers, R. (2014). Influence of personality on the outcome of treatment in depression: systematic review and meta-analysis. Journal of Personality Disorders, 28(4), 577-593. 
Perlis, R., losifescu, D., Castro, V., Murphy, S., Gainer, V., Minnier, J., . . . Gallagher, P. (2012). Using electronic medical records to enable large-scale studies in psychiatry: treatment resistant depression as a model. Psychological medicine, 42(1), 41-50.

Perna, G., Grassi, M., Caldirola, D., \& Nemeroff, C. (2018). The revolution of personalized psychiatry: will technology make it happen sooner? Psychological medicine, 48(5), 705-713.

Poston, J. M., \& Hanson, W. E. (2010). Meta-analysis of psychological assessment as a therapeutic intervention. Psychological assessment, 22(2), 203.

R., C.-J. (Producer). (2014). Stephen Hawking warns artificial intelligence could end mankind. BBC News

Rao, S. (2009). Anxious depression: clinical features and treatment. Current psychiatry reports, 11(6), 429436.

Rigby, M. J. (2019). Ethical dimensions of using artificial intelligence in health care. AMA Journal of Ethics, 21(2), 121-124.

Russell, S., Dewey, D., \& Tegmark, M. (2015). Research priorities for robust and beneficial artificial intelligence. Ai Magazine, 36(4), 105-114.

Schneider, F., Kratz, S., Bermejo, I., Menke, R., Mulert, C., Hegerl, U., . . . Härter, M. (2004). Insufficient depression treatment in outpatient settings. German medical science: GMS e-journal, 2.

Scott, K. M., Bruffaerts, R., Tsang, A., Ormel, J., Alonso, J., Angermeyer, M. C., . . . De Graaf, R. (2007). Depression-anxiety relationships with chronic physical conditions: results from the World Mental Health Surveys. Journal of Affective Disorders, 103(1-3), 113-120.

Sheldrick, R. C., Chung, P. J., \& Jacobson, R. M. (2017). Math matters: how misinterpretation of odds ratios and risk ratios may influence conclusions. Academic pediatrics, 17(1), 1-3.

Shimokawa, K., Lambert, M. J., \& Smart, D. W. (2010). Enhancing treatment outcome of patients at risk of treatment failure: meta-analytic and mega-analytic review of a psychotherapy quality assurance system. Journal of Consulting and Clinical Psychology, 78(3), 298.

Siontis, G. C., Tzoulaki, I., Castaldi, P. J., \& Ioannidis, J. P. (2015). External validation of new risk prediction models is infrequent and reveals worse prognostic discrimination. Journal of clinical epidemiology, 68(1), 25-34.

Tiffin, P. A., \& Paton, L. W. (2018). Rise of the machines? Machine learning approaches and mental health: opportunities and challenges. The British Journal of Psychiatry, 213(3), 509-510.

Tomba, E., \& Fava, G. A. (2012). Treatment selection in depression: the role of clinical judgment. Psychiatric Clinics, 35(1), 87-98.

van der Veen, D. C., Comijs, H. C., van Zelst, W. H., Schoevers, R. A., \& Oude Voshaar, R. C. (2014). Defining anxious depression in later life: a scaring heterogeneity in results. The American Journal of Geriatric Psychiatry, 22(11), 1375-1378. doi:10.1016/j.jagp.2014.02.012

Van, H. L., \& Kool, M. (2018). What we do, do not, and need to know about comorbid depression and personality disorders. The Lancet Psychiatry, 5(10), 776-778.

Wakabayashi, D. (2018). Self-Driving Uber Car Kills Pedestrian in Arizona, Where Robots Roam. Retrieved from https://www.nytimes.com/2018/03/19/technology/uber-driverless-fatality.html

Warden, D., Trivedi, M. H., Carmody, T., Toups, M., Zisook, S., Lesser, I., . . Rush, A. J. (2014). Adherence to antidepressant combinations and monotherapy for major depressive disorder: a CO-MED report of measurement-based care. Journal of Psychiatric Practice ${ }^{\circledR}, 20(2), 118-132$.

Whipple, J. L., Lambert, M. J., Vermeersch, D. A., Smart, D. W., Nielsen, S. L., \& Hawkins, E. J. (2003). Improving the effects of psychotherapy: the use of early identification of treatment and problemsolving strategies in routine practice. Journal of Counseling Psychology, 50(1), 59.

Wichers, M., Lothmann, C., Simons, C. J., Nicolson, N. A., \& Peeters, F. (2012). The dynamic interplay between negative and positive emotions in daily life predicts response to treatment in depression: a momentary assessment study. British Journal of Clinical Psychology, 51(2), 206-222.

Zilcha-Mano, S., Keefe, J. R., Chui, H., Rubin, A., Barrett, M. S., \& Barber, J. P. (2016). Reducing Dropout in Treatment for Depression: Translating Dropout Predictors Into Individualized Treatment Recommendations. The Journal of clinical psychiatry, 77(12), e1584-e1590.

Zimmerman, M., Kerr, S., Kiefer, R., Balling, C., \& Dalrymple, K. (2019). What is anxious depression? Overlap and agreement between different definitions. Journal of psychiatric research, 109, 133-138. 
Zimmerman, M., Martin, J., McGonigal, P., Harris, L., Kerr, S., Balling, C., . . . Dalrymple, K. (2019). Validity of the DSM-5 anxious distress specifier for major depressive disorder. Depression and anxiety, 36(1), 3138.

Zimmerman, M., Martinez, J. A., Attiullah, N., Friedman, M., Toba, C., Boerescu, D. A., \& Rahgeb, M. (2012). Why do some depressed outpatients who are in remission according to the Hamilton Depression Rating Scale not consider themselves to be in remission? Journal of Clinical Psychiatry, 73(6), 790.

Zimmerman, M., Martinez, J. H., Attiullah, N., Friedman, M., Toba, C., Boerescu, D. A., \& Ragheb, M. (2013). A new type of scale for determining remission from depression: the remission from depression questionnaire. Journal of psychiatric research, 47(1), 78-82.

Zimmerman, M., McGlinchey, J. B., Posternak, M. A., Friedman, M., Attiullah, N., \& Boerescu, D. (2006). How should remission from depression be defined? The depressed patient's perspective. American Journal of Psychiatry, 163(1), 148-150. 


ADDENDUM

Summary

Samenvatting

Valorization

Dankwoord /

Acknowledgements

Curriculum vitae 



\section{Summary}

Major depressive disorder (MDD) is highly prevalent, and a DSM-5 diagnosis of MDD involves many different symptom profiles, courses and prognoses. Current clinical practice guidelines recommend antidepressant medication, psychotherapy, and their combination, as the first treatment choices for MDD. Over the years, research consistently indicated that these different types of treatment are equally effective on average, a phenomenon that is referred to as the "dodo bird verdict". However, individual responses to MDD treatments vary widely and are highly unpredictable. Therefore, different types of treatment are not equally effective for each specific individual. Since it is unclear what works best for a specific individual, in clinical practice, consecutive treatments are often being offered to find the optimal regimen, a strategy that can be best described as a "trial and error approach". An alternative to this approach is optimizing treatment selection by focusing on the question "what works for whom". Treatment strategies can be personalized by finding the optimal depression treatment for a specific individual at a specific point in time. Traditional approaches to personalize treatment strategies strongly focused on comorbidity, and on predictions of treatment responses using clinical judgment. These traditional approaches are frequently advocated in the literature, and common in clinical practice, however this is supported by limited and non-robust evidence. An alternative approach is the use of statistical prediction. Recently, novel prediction models (or prediction algorithms) have been developed. These models show promising results in optimizing treatment selection, although the development of these models is still in its infancy. The aim of this thesis was to examine ways to personalize treatment strategies for MDD. First, the impact of comorbid anxiety and personality pathology on acute and long-term treatment outcome in MDD was examined (Chapter 1-5). Second, potential strategies that lead to better treatment outcomes for MDD in the long term were investigated (Chapter 6-9). Third, possibilities and challenges of future personalized treatment strategies for MDD were examined (Chapter 10-13). Aims, methods and results of each chapter are summarized below.

Chapter 1 provides a general introduction. First, the criteria for, and prevalence and heterogeneity of, a depression diagnosis are described. After that, current treatments for depression are introduced, with an emphasis on the two most used psychotherapies for MDD, cognitive therapy (CT) and interpersonal psychotherapy (IPT), which are also the main treatments examined in this thesis. Then, the problem of equal effectiveness of treatment for MDD ("the dodo bird verdict") is outlined, and the subsequent "trial and error approach" with its negative consequences is described. After that, a summary of approaches to optimize treatment selection in MDD is provided, including the more traditional focus on comorbid diagnoses and symptoms and clinical prediction, as well as statistical prediction models. The chapter 
ends with a description of the aims of this thesis, that is divided into three parts that correspond to three objectives. The first objective is to examine the impact of comorbid anxiety and personality pathology on treatment outcomes of MDD. The second objective is to investigate potential ways of treatment planning that lead to better treatment outcomes for MDD in the long term. The third objective is to provide insight into possibilities and challenges of future personalized treatment strategies for MDD.

Chapters 2, 3, 6, 7, 8, 10, 11 and $\mathbf{1 2}$ of this dissertation present findings obtained in the context of a randomized trial, the STEPd study, that evaluated the clinical effectiveness of CT and IPT. A total of 182 individuals with a primary MDD diagnosis as confirmed with the Structured Clinical Interview for DSM-IV Axis I disorders (SCID-I) were recruited from the mood disorders unit of the Maastricht Outpatient Mental Health Center (RIAGG Maastricht). After baseline assessment, individuals were randomized to CT $(n=76)$, IPT $(n=75)$, or a 2-month waiting-list control condition followed by treatment of choice $(n=31)$. CT and IPT were carried out using guidelines by Beck et al. (1979) for CT, and guidelines by Klerman et al. (1984) for IPT. Treatment consisted of 16-20 individual sessions of $45 \mathrm{~min}$. Primary outcome was depression severity measured with the Beck Depression Inventory (BDI-II) during treatment (0-7 months) and throughout 17 months of follow-up (7-24 months).

\section{Part 1: Depression treatment and comorbidity}

In Chapter 2, findings on the influence of comorbid anxiety on the acute and longterm effectiveness of CT and IPT are presented. Comorbid anxiety in MDD can be defined by both syndromal (anxiety disorder) and dimensional (anxiety symptoms) criteria. Although both comorbid anxiety disorders and symptoms have been associated with a more severe clinical picture at the start of treatment, previous studies show inconsistent results regarding the impact of comorbid anxiety on the treatment outcome and completion. The aim of the study presented in this chapter was to examine the influence of comorbid anxiety disorders and symptoms on outcomes and treatment dropout in CT and IPT for MDD. Data came from the STEPd study, and we limited the sample to data of individuals randomized to the active conditions CT $(n=76)$ and IPT $(n=75)$. Primary outcome was depression severity measured with the BDI-II at the start of each therapy session, at post-treatment, and monthly throughout a five-month follow-up period. Anxiety disorders were diagnosed using the SCID-I, and anxiety symptoms were measured with the Brief Symptom Inventory subscales. The impact of comorbid anxiety on acute and long-term outcomes and dropout was examined using mixed-effect models and logistic regression models respectively. Results indicated that individuals with comorbid anxiety disorders and symptoms had less favorable depression severity change in IPT compared to CT during treatment. However, this difference did not remain in the 
follow-up phase. Treatment dropout was more prevalent in individuals with comorbid anxiety disorders. Based on these results one can conclude that a preference of CT could be justifiable when comorbid anxiety is present although replication of these findings is needed.

Chapter 3 describes findings on the impact of comorbid personality disorders (PD) and PD features on the acute and long-term effectiveness of CT and IPT for MDD. As with comorbid anxiety, previous studies indicate that comorbid PD (features) are associated with a more severe clinical picture at the start of treatment. However, results regarding the impact of comorbid PD on the treatment outcome and dropout are inconsistent, and there is no broad consensus on how comorbid PD (features) affect treatments for MDD. The aim of this empirical study was to evaluate the effects of PD (features) on outcomes and treatment dropout of CT and IPT for MDD. Data came from a subsample of 146 depressed outpatients of the STEPd study that were randomized to CT $(n=72)$ and IPT $(n=74)$. Depression severity, the primary outcome, was measured with BDI-II at the start of each therapy session, at post-treatment, and monthly during five months of follow-up period. PD (features) were assessed using the SCID-II. The impact of comorbid PD (features) on depression severity, remission rates, and dropout was examined using mixed-effect models and Cox regression models. Findings of this study indicated no impact of PD on depression severity change and remission rates during treatment and follow-up, irrespective of the treatment received. Regarding PD features, higher dependent PD features predicted overall lower depression severity scores during treatment, however this effect did not sustain through follow-up. In addition, individuals with high cluster A PD features had a more favorable depression severity change in CT as compared to IPT during treatment and follow-up. PD and PD features did not affect dropout. In conclusion, PD did not affect treatment outcome and dropout in CT and IPT. In addition, more subtle effects were found regarding specific PD features.

In Chapter 4 a meta-analysis and meta-regression on the impact of comorbid PD on acute phase treatment outcomes for MDD is presented. Findings of previous metaanalyses have shown an adverse effect of comorbid PD on acute phase treatment outcome for MDD. Although these analyses included a broad variety of studies, the results are likely to be biased due to inclusion of studies with naturalistic designs, the use of non-structured instruments to assess PD and MDD, and the presence of (noncontrolled) effects of baseline differences between individuals with and without PD (e.g., depression severity). The aim of this meta-analysis and meta-regression was therefore to examine the impact of comorbid PD on acute phase treatment outcome for MDD, using only data from well-designed controlled trials. A database search (up to April 3th, 2017) was conducted in Pubmed, PsycINFO and Embase, using terms regarding $M D D, P D$ and antidepressant treatments (psychotherapy, antidepressants and other biological treatments). Reference lists of the selected research articles, and reference lists of earlier reviews and meta-analyses were searched as well. Inclusion 
criteria were a primary diagnosis of MDD, acute phase treatment for MDD, controlled treatment(s) and random treatment assignment for studies with more than one treatment, MDD and PD diagnoses based on a structured interview conducted prior to treatment, and the measurement of change of depression severity scores from baseline to post-treatment assessed with a validated instrument. Data were extracted from published records and all corresponding authors were contacted to check these data and provide missing information. Effect sizes were estimated with randomeffects models. Study-level variables were examined using meta-regression. Included studies were evaluated with the Risk of Bias tool, and publication bias was assessed using funnel plots and Egger's test. A total of six studies with 942 individuals (447 with PD) were included. Findings indicated no effect of comorbid PD on acute phase treatment outcome for MDD. The meta-regression did not indicate relevant variables. Heterogeneity and risk of bias were low, and there was no indication for publication bias. This synthesis of high-quality and unbiased evidence indicated no negative impact of comorbid PD on the effectiveness of acute phase treatment for MDD. It is therefore highly recommended to follow evidence-based treatment guidelines for MDD regardless of a comorbid PD diagnosis.

Chapter 5 describes the findings of an observational study on the impact of comorbid personality pathology on the amount and type of received treatment for MDD. As mentioned above, previous and frequently cited meta-analyses have shown an adverse effect of comorbid PD on treatment outcome for MDD. However, the evidence for these negative effects stems partially from studies with uncontrolled treatment designs. Since depressed individuals with a comorbid PD tend to receive suboptimal treatment within such contexts, the negative effects on treatment outcome may be incorrectly attributed to the comorbidity itself instead of the insufficiently provided MDD treatment. The aim of this observational study was to examine whether and to what extend the presence of comorbid personality pathology was associated with the type and the amount of received treatment sessions in a naturalistic setting of outpatient MDD care. Data of 1455 depressed individuals from seven outpatient departments of a secondary mental healthcare center were retrospectively extracted from electronic patient records. Personality pathology was measured with the Standardized Assessment of Personality-Abbreviated Scale. The associations between personality pathology and type and number of treatment sessions were analyzed using regression models. Findings indicated that depressed individuals with comorbid personality pathology received more psychotherapy sessions then individuals without comorbid personality pathology, irrespective of depression severity at the start of treatment. The number of pharmacotherapy sessions, supportive visits, and crisis visits did not differ between individuals with and without comorbid personality pathology. The fact that depressed individuals with comorbid personality pathology received more psychotherapy sessions compared to 
those without comorbid personality pathology is not in line with current treatment guidelines, which may be indicative of overtreatment of this large group of patients.

\section{Part two: Treatment planning for depression}

In Chapter 6, findings on the enduring effects of acute-phase CT and IPT for MDD throughout 17 months of follow-up are presented. Even when treated effectively in the acute phase, MDD has an unfavorable prognosis with high recurrence rates. Although CT and IPT have shown to be effective treatments in the acute phase, little is known on how they compare in the long run. CT has consistently demonstrated an enduring effect that lasts beyond the end of treatment, research on long-term effects of IPT are less extensive. The aim of this study was to examine long-term outcomes of CT versus IPT for MDD. A total of 134 depressed individuals from the STEPd study were examined after randomization and treatment with CT $(n=69)$ or IPT $(n=65)$. Primary outcome was depression severity measured with the BDI-II at post-treatment, and throughout 17 months of follow-up. Additional secondary outcomes for the subsample of treatment-responders $(n=85)$, were relapse and sustained response measured with the BDI-II and the clinician-rated Longitudinal Interval Follow-up Evaluation (LIFE). Depression severity over time and (differential) treatment effects were examined using mixed-effect models. Comparisons of relapse and sustained response rates between treatments were done using Cox regression and $\chi^{2}$ tests of independence. Findings indicated that, on average, the symptom reduction achieved with CT and IPT was maintained across 17 months follow-up, and that two-third of the treatment responders did not relapse. No differential treatment effects were found on depression severity over time, relapse rates and sustained response rates. Based on these findings one can conclude that in this study CT and IPT are equally effective in the long run. In addition, since there is evidence that CT has prophylactic effect following successful treatment, our findings suggested that IPT might have enduring effects as well.

In Chapter 7, an empirical study on a prognostic model for long-term outcome after successful acute phase CT and IPT for MDD is presented. Since MDD has a highly recurrent nature, it is clinically relevant to identify individuals who are at risk of an unfavorable long-term course even after a successful treatment. Despite extensive research, there is no consensus yet on the clinically relevant predictors of long-term outcome in MDD. In addition, the development of prediction algorithms that combine these predictors into a quantified estimate of the individual's prognosis (i.e., a Prognostic Index) are still in their infancy and the current evidence base is mostly based on naturalistic datasets. The aim of this study was to create a Prognostic Index (PI) to estimate long-term depression severity after successful and high quality psychotherapy for MDD. A total of 85 responders to CT $(n=45)$ and IPT $(n=40)$ from the STEPd study were included. Primary outcome was depression severity, assessed with 
the BDI-II, measured at post-treatment and throughout 17-months of follow-up. First, a total of 29 post-treatment variables were examined, including clinical measures, demographics, and information about psychological distress, general functioning, psychological processes, and life and family history. Of these 29-post-treatment variables, predictors were selected using a model-based recursive partitioning method followed by bootstrap resampling in conjunction with backwards elimination. The selected predictors were then combined into one model and individual PI scores were estimated using a 5-fold cross-validation approach. A total of three post-treatment predictors were selected: depression severity, hopelessness and self-esteem. The individual PI scores had a strong correlation $(r=0.60)$ with actual follow-up depression severity scores. Based on these findings, one can conclude that long-term predictions of MDD are multifactorial, involving a combination of variables that each have a small prognostic effect. The PI has the potential to predict follow-up depression severity for each individual after successful treatment, however replication and (external) validation are needed.

Chapter $\mathbf{8}$ describes an empirical study of a prescriptive model, the Personalized Advantage Index (PAI), a multivariate approach that computes individualized treatment recommendations. In the past years, the PAI has shown promise as a means of producing powerful predictions. The PAI delivers individual treatment recommendations, and provides a quantitative estimate of the predicted advantage of the indicated treatment over the non-indicated treatment(s). These recommendations are based on the difference between predicted outcomes of two or more treatments using a model that includes multiple predictors and moderators. The aim of the current study was to extend the PAI approach to treatment selection based on long-term depression outcomes (i.e., the "long-term PAI"). Data came from a subsample of the STEPd study, in which 151 depressed individuals were randomized to CT $(n=75)$ and IPT $(n=76)$. Primary outcome was depression severity, as assessed with the BDI-II, during 17-months of follow-up after acute phase treatment. First, a total of 38 post-treatment variables were examined, including clinical measures, demographics, and information about psychological distress, general functioning, psychological processes, and life and family history. Of these 38-post-treatment variables, predictors and moderators were selected using a model-based recursive partitioning method followed by bootstrap resampling in conjunction with backwards elimination. After that, predictors and moderators were combined into a final model, from which long-term PAI predictions were estimated using 5-fold cross-validation. Long-term PAI predictions were then compared to actual follow-up outcomes and with PAI predictions from a previous study based on part of the same dataset that focused on the prediction of BDI-II scores at post-treatment. One predictor (parental alcohol abuse) and two moderators (recent life events and childhood maltreatment) were selected for the PAI model, and individual PAI scores were calculated. Individuals randomized to their PAI-indicated treatment had lower follow-up depression severity 
compared to those assigned to their PAI-non-indicated treatment. This difference was significant for those whose PAI score was in the upper $60 \%$ (i.e., with the highest predicted advantage), and for those whose PAI indicated CT. Long-term predictions did not overlap substantially with predictions for acute benefit (post-treatment PAI predictions). In conclusion, findings indicated that long-term PAl predictions are also promising and could enhance precision medicine by selecting the optimal treatment for a given depressed individual. However, replication and (external) validation are needed.

Chapter 9 reports a meta-analysis and a meta-regression on the effectiveness of psychotherapy for treatment-resistant depression (TRD). MDD that is treatment refractory, mostly described as TRD, is characterized by marked functional impairment, a large burden of disease and high societal costs. Traditionally, clinical practice and research has focused on pharmacotherapy and other somatic treatments as treatment options for TRD. However, over the years, there is a growing number of controlled studies of psychotherapy for TRD. In addition, a majority of the depressed individuals prefer psychotherapy over pharmacotherapy. Therefore, the aim of this meta-analysis and meta-regression was to examine the effectiveness of psychotherapy for MDD as either a substitute for or add-on to treatment as usual (TAU; routine treatments such as clinical management and/or the continuation, optimization or next step pharmacotherapy). A database search (up to December $19^{\text {th }}$, 2016) was conducted in Pubmed, PsycINFO and Embase, combining terms regarding psychotherapy, TRD and chronic MDD. Reference lists of the selected research articles, and reference lists of earlier reviews and meta-analyses were searched as well. Included studies were randomized controlled trials that examined the effectiveness of psychotherapy for adults ( $\geq 18$ years of age) with TRD by comparing psychotherapy versus TAU or add-on psychotherapy and TAU versus TAU only. In line with previous reviews, TRD was defined as an individual's failure to respond to at least one adequate trial of antidepressants for the current episode irrespective of duration of the current episode. Studies on chronic MDD were included if the majority of the sample fulfilled this definition for TRD. Data were extracted from published records and all corresponding authors were contacted to check this data and provide missing information. Effect sizes of depression severity change during treatment were estimated using random-effects models. Study-level variables were examined using meta-regression. Included studies were evaluated with the Risk of Bias tool, and publication bias was assessed using funnel plots and Egger's test. A total of 21 studies (25 comparisons) with 3539 individuals (293 in psychotherapy only, 1588 in add-on psychotherapy plus TAU and 1638 in TAU) were included. Findings indicated that there was no significant benefit of psychotherapy as compared to TAU ( 3 comparisons), and that there was a moderate effect size of depression severity change in favor of psychotherapy plus TAU in the 22 add-on psychotherapy plus TAU versus TAU only comparisons. Findings of the meta-regression indicated a positive 
association between baseline severity as well as group v. individual therapy format with the treatment effect. Risk of bias was low, and there was no indication for publication bias. In conclusion, this study provided evidence that the addition of psychotherapy to TAU could result in better outcomes for individuals with TRD. Inclusion of add-on psychotherapy to the treatment guidelines of TRD is therefore justified.

\section{Part three: Future strategies}

In Chapter 10, a direct comparison of clinical judgment from therapists and statistical prediction (with the PAl approach) for treatment selection for MDD is presented. Although research has consistently shown that statistical prediction is as accurate - or even more accurate - than predictions based on clinical judgment, treatment selection still heavily depends on clinical judgment in clinical practice. With the development of state-of-the-art prediction models, the aim of this empirical study was to compare the accuracy of clinical judgment versus statistical predictions in selecting CT and IPT for MDD. Data came from the STEPd study, and we limited the sample to data of individuals randomized to the active conditions CT $(n=76)$ and IPT $(n=75)$. Prior to randomization, therapists' recommendations were formulated during multidisciplinary staff meetings. Statistical predictions were based on the PAI approach. Primary outcome was depression severity measured post-treatment and throughout 17-months of follow-up using the BDI-II. Secondary outcome was treatment dropout. Findings on statistical prediction indicated that individuals that were allocated to their PAl-indicated treatment (statistical prediction) were less depressed at post-treatment and follow-up compared to those receiving their predicted non-indicated treatment. Results on clinical prediction indicated that this advantage was not found for recommended versus non-recommended treatments based on clinical judgment. Moreover, for individuals with an IPT recommendation by therapists, higher post-treatment and follow-up depression severity were found for those that were actually allocated to IPT compared to those receiving CT. Recommendations based on statistical prediction and clinical judgment were not associated with differences in treatment dropout. In conclusion, statistical prediction (PAI approach) outperforms clinical judgment in treatment selection for MDD.

Chapter 11 describes the results from cross-trial predictions with the PAI approach for treatment selection of CT and IPT for MDD. To determine the generalizability of the PAI in clinical practice, models need to be externally validated, which has rarely been done in previous studies. The aim of this empirical study was to develop and externally validate PAI models in two independent study samples. Data came from two randomized studies comparing CT and IPT for MDD (FreqMech: $n=200$ and STEPd: $n=151)$. Results of the FreqMech study indicated that post-treatment depression was not significantly different between patients receiving their PAI- 
indicated treatment versus those receiving their PAI non-indicated treatment. In the STEPd study, post-treatment depression was significantly lower for individuals allocated to their PAl-indicated treatment versus those assigned to their nonindicated treatment. Cross-trial predictions were modest, indicating that posttreatment depression was not significantly different between those receiving their indicated treatment and those receiving their non-indicated treatment. In conclusion, external validation of the PAI approach has modest results, and demonstrated challenges in this process, such as the heterogeneity of data collection, statistical methods, study populations and treatments.

Chapter 12 introduces and demonstrates an innovative Bayesian methodology to estimate treatment response subgroups in MDD. Traditionally, studies have relied on a priori defined categories for treatment response based on cut-offs on depression severity scales. More recent studies applied a data driven approach to study depression severity subgroups using Latent Variable Mixture Models. Although these models are promising, determining the right number of subgroups is challenging, and the robustness of the identified subgroups can be questioned. The aim of this study was to introduce and demonstrate a recently developed method, a mixture of shifted Poisson distributions with Bayesian inference, to identify latent subgroups of depression severity over the course of treatment. This method focused on modes of the depression severity distribution as a straightforward and robust indicator of subgroups. In addition, a count data model was applied to better match the nonnormal and discrete data distributions of depression severity sum scores. In the first step, multimodal density features were investigated using an infinite mixture model with shifted Poisson densities. Then, the number and location of the modes were examined using a Bayesian inference method (Reversible Jump Markov Chain Monte Carlo). After that, mode membership, the probability of an individual to belong to a mode (subgroup), was determined. An application of this method was presented with data of ten subsequent therapy sessions from subsample of the STEPd study comparing the effectiveness of CT $(n=53)$ and IPT $(n=49)$ for MDD. Findings of this application indicated that there were three to four distinct subgroups of treatment response to MDD. In addition, the number of latent subgroups increased when therapy progresses, particularly in the final two therapy sessions. Subgroup memberships indicated potential prognostic relevance when focusing on a "good prognosis" and a "bad prognosis" subgroup. In conclusion, results of the application give valuable insights into subgroups of treatment response, and provide promising directions for longitudinal modeling.

Chapter 13 provides a short summary of the studies presented in this thesis, followed by a discussion of these findings from a broader perspective and future directions. The main message of this chapter - and of the thesis as a whole - is that the development of personalizing treatment strategies for depression is an exciting work in progress. Traditional approaches focused on comorbidity and clinical judgment, and 
have a limited and non-robust evidence base. More recently introduced prediction models are promising, however development of these models is still in its infancy and external validation efforts are needed to determine their significance in clinical practice. As exciting and promising as they are, these new prediction models also raise ethical and legal concerns, including low interpretability, responsibilities, the impact of incorrect predictions and biases, the protection of privacy and the fear of the dehumanization of treatments. Efforts to bridge the gap between clinical practice and research are key to the development and implementation of prediction models, and starting small with the development of local prediction models in mental health care institutions could be an appropriate starting point. To target meaningful treatment goals with these prediction models, we should identify indicators of successful treatment, including measures of treatment dropout and positive mental health. Efforts to identify relevant data (sources) that feed these prediction models need to be evaluated, in which factors such as cost, feasibility, and patient burden should be taken into account. Prediction models should deliver predictions that update during treatment, and adapt treatments accordingly. Together, these future directions could help us understand how to personalize treatment strategies for depression effectively. 


\section{Samenvatting}

Depressie is een veelvoorkomend probleem met een verscheidenheid aan symptoomprofielen, beloopsvormen en prognoses. Huidige klinische behandelrichtlijnen voor depressie adviseren antidepressieve medicatie, psychotherapie of een combinatie van beide als effectieve behandelopties voor depressie. Wetenschappelijk heeft onderzoek consequent aangetoond dat deze verschillende soorten behandelingen (als monotherapie) gemiddeld gezien even effectief zijn, een fenomeen dat ook wel "het dodo bird verdict" wordt genoemd. Echter, de effecten van deze depressie behandelingen verschillen sterk per persoon en zijn moeilijk te voorspellen. Het is daarom ook niet vanzelfsprekend dat de verschillende typen behandelingen even effectief zijn voor elk specifiek individu. Aangezien het onduidelijk is welke behandeling het beste werkt voor wie, worden er in de klinische praktijk vaak opeenvolgende behandelingen aangeboden totdat de juiste optie gevonden is. Deze strategie kan het beste worden omschreven als een "vallen en opstaan" aanpak. Een alternatief voor deze aanpak is het optimaliseren van de selectie uit de verschillende depressiebehandelingen door te focussen op de vraag "wat werkt voor wie?". Behandelstrategieën kunnen dan worden gepersonaliseerd door de optimale depressiebehandeling voor een specifiek individu op een specifiek moment te selecteren. Traditionele benaderingen van deze gepersonaliseerde behandelstrategieën zijn gericht op verschillende vormen van co-morbiditeit (bijvoorbeeld met depressie gepaard gaande angstklachten) en op het voorspellen van behandeleffecten op grond van een klinische inschatting. Deze traditionele methoden worden regelmatig aanbevolen in de literatuur en worden vaak toegepast in de klinische praktijk. Robuuste wetenschappelijke onderbouwing hiervoor ontbreekt echter. Een alternatieve benadering voor gepersonaliseerde behandelstrategieën is het toepassen van statistische voorspellingen. De afgelopen jaren is er steeds meer aandacht gekomen voor de ontwikkeling van predictiemodellen (of predictiealgoritmen) om behandeluitkomsten te voorspellen. Deze modellen laten veelbelovende resultaten zien in het voorspellen van individuele behandeluitkomsten, en daarmee het personaliseren van behandelstrategieën. De ontwikkeling van predictiemodellen staat echter nog in haar kinderschoenen, en er zijn tot nu toe nog geen toepassingen van predictiemodellen in de klinische praktijk. Het doel van dit proefschrift is om verschillende manieren te onderzoeken waarmee behandelstrategieën voor depressie gepersonaliseerd kunnen worden. Allereerst wordt er gekeken naar de impact van co-morbide angst- en persoonlijkheidspathologie op acute- en langetermijneffecten van depressiebehandelingen (hoofdstuk $1 \mathrm{t} / \mathrm{m} 5$ ). Vervolgens worden potentiële behandelstrategieën onderzocht die op de lange termijn bijdragen aan betere behandelingsresultaten voor depressie (hoofdstuk $6 \mathrm{t} / \mathrm{m}$ 9). Ten slotte worden de mogelijkheden en uitdagingen van toekomstige gepersonaliseerde behandelstrategieën voor depressie onderzocht (hoofdstuk $10 \mathrm{t} / \mathrm{m}$ 
13). Doelstellingen, methoden en resultaten van elk hoofdstuk worden hieronder samengevat.

Hoofdstuk 1 omvat een algemene introductie van dit proefschrift. Eerst worden de criteria voor, en de prevalentie en heterogeniteit van, een depressiediagnose beschreven. Daarna worden de huidige behandelingen voor depressie beschreven, met de nadruk op de twee meest toegepaste psychotherapieën voor depressie, Cognitieve Therapie (CT) en Interpersoonlijke Psychotherapie (IPT). CT en IPT zijn ook de behandelingen die het meeste zijn onderzocht in dit proefschrift. Vervolgens wordt het "dodo bird verdict", het probleem van gelijke effectiviteit van depressiebehandelingen, geïntroduceerd en wordt de daaruit volgende "vallen en opstaan" aanpak beschreven. Daarna wordt een samenvatting gegeven van strategieën om de selectie voor depressiebehandelingen te optimaliseren, inclusief de traditionele benaderingen (focus op co-morbiditeit en klinische inschattingen), en de statistische voorspellingen met predictiemodellen. Het hoofdstuk eindigt met een beschrijving van de drie doelstellingen van dit proefschrift die overeenkomen met drie delen van dit proefschrift. De eerste doelstelling is het onderzoeken van de impact van comorbide angst- en persoonlijkheidspathologie op de resultaten van depressiebehandelingen. De tweede doelstelling is meer inzicht te verkrijgen in verschillende potentiële behandelstrategieën die tot doel hebben om betere lange termijn behandelresultaten voor depressie te bewerkstelligen. De derde en laatste doelstelling is het onderzoeken van de mogelijkheden en uitdagingen van toekomstige gepersonaliseerde behandelstrategieën voor depressie.

Hoofdstukken 2, 3, 6, 7, 8, 10, 11 en 12 van dit proefschrift presenteren bevindingen van een gerandomiseerde behandelstudie, de STEPd-studie, naar de (differentiële) effecten van CT en IPT voor depressie. In deze studie werden 182 deelnemers geworven tijdens reguliere intakes bij de RIAGG Maastricht. Bij alle deelnemers was een depressie vastgesteld als primaire diagnose met een gestructureerd klinisch interview (SCID-I). Vervolgens werden deelnemers door middel van loting of randomisatie toegewezen aan één van drie groepen: CT $(n=76)$, IPT $(n=75)$ of een wachtlijstcontroleconditie van 2 maanden gevolgd door behandeling naar keuze $(n=31)$. Behandelingen werden uitgevoerd volgens standaardprotocollen. Het CT-protocol was gebaseerd op het protocol van Beck e.a. (1979). Het IPT-protocol volgde de richtlijnen van Klerman e.a. (1984). De behandeling bestond - afhankelijk van de vorderingen van individuele deelnemers - uit 16 tot 20 individuele sessies van 45 minuten. Primaire uitkomstmaat was de ernst van de depressie, vastgesteld met de Beck Depressie Vragenlijst-II (BDI-II) tijdens de behandeling (0-7 maanden) en gedurende 17 maanden follow-up (7-24 maanden). 


\section{Deel 1: Depressiebehandeling en co-morbiditeit}

In hoofdstuk 2 worden bevindingen over de impact van co-morbide angst op de korteen langetermijneffecten van CT en IPT gepresenteerd. Co-morbide angst bij depressie kan worden geclassificeerd met zowel syndromale (angststoornis) als dimensionale (angstsymptomen) criteria. De aanwezigheid van co-morbide angststoornissen en comorbide angstsymptomen aan het begin van een depressiebehandeling is geassocieerd met een ernstiger klinisch beeld. De impact van co-morbide angst op de uiteindelijke behandelresultaten van een depressiebehandeling is echter onduidelijk; eerdere studies vertonen inconsistente resultaten. Het doel van de studie die gepresenteerd wordt in dit hoofdstuk was om de invloed van co-morbide angststoornissen en angstsymptomen op behandeluitkomsten van CT en IPT voor depressie te onderzoeken. Ook bekeken we de impact van co-morbide angst op het voortijdig stoppen van behandeling (behandeluitval). Data waren afkomstig van de STEPd-studie, waarbij we ons beperkten tot het deel van de proefpersonen $(n=151)$ dat werd gerandomiseerd naar CT $(n=76)$ en IPT $(n=75)$. De primaire uitkomstmaat was de ernst van de depressie gemeten met de BDI-Il aan het begin van elke therapiesessie, na afloop van de behandeling en maandelijks gedurende een follow-up periode van vijf maanden. Angststoornissen werden gediagnosticeerd met behulp van een gestructureerd klinisch interview (SCID-I) en angstsymptomen werden gemeten met een zelfrapportagelijst (subschaal van de Brief Symptom Inventory). De impact van co-morbide angst op korte en lange termijn behandelresultaten werd onderzocht met multilevel analyses. De impact van co-morbide angst op behandeluitval werd geanalyseerd met behulp van logistische regressiemodellen. De multilevel analyses lieten zien dat individuen met co-morbide angststoornissen en angstsymptomen die met IPT behandeld werden aan het einde van de behandeling minder verbetering in depressieve klachten lieten zien, vergeleken met individuen die behandeld waren met CT. Dit verschil verdween tijdens de vijf maanden durende follow-up fase. De logistische regressieanalyses lieten zien dat behandeluitval vaker voorkwam bij individuen met co-morbide angststoornissen. Op basis van deze resultaten kan worden geconcludeerd dat een voorkeur voor CT voor depressie gerechtvaardigd kan zijn wanneer co-morbide angst aanwezig is. Replicatie van deze bevindingen is echter gewenst.

Hoofdstuk 3 beschrijft een studie naar de impact van co-morbide persoonlijkheidsstoornissen (PS) en kenmerken van PS op de korte- en langetermijneffecten van CT en IPT voor depressie. Net als bij co-morbide angst wijzen eerdere onderzoeken uit dat co-morbide PS (-kenmerken) geassocieerd zijn met een ernstiger klinisch beeld aan het begin van een depressiebehandeling. Het is echter onduidelijk wat de invloed van co-morbide PS (-kenmerken) is op de uiteindelijke behandelresultaten. Studieresultaten met betrekking tot deze vraag zijn inconsistent en er ontbreekt consensus over hoe co-morbide PS (-kenmerken) depressie- 
behandelingen beïnvloeden. Het doel van deze studie was daarom om de effecten van PS (-kenmerken) op de korte en lange termijn uitkomsten van CT en IPT voor depressie te onderzoeken. Daarnaast werd de impact van co-morbide PS (kenmerken) op het voortijdig stoppen van behandeling (behandeluitval) onderzocht. Data waren afkomstig van de STEPd-studie, waarbij we ons beperkten tot een deel van de proefpersonen, dat werd gerandomiseerd naar CT en IPT en waarbij een gestructureerd persoonlijkheidsinterview was afgenomen $(n=146, C T: n=72$, IPT: $\mathrm{n}=74$ ). De primaire uitkomstmaat was de ernst van de depressie, gemeten met de BDIII aan het begin van elke therapiesessie, na afloop van de behandeling en maandelijks gedurende een follow-up periode van vijf maanden. PS (kenmerken) werden gediagnosticeerd met behulp van een gestructureerd klinisch interview (SCID-II). De impact van co-morbide PS (-kenmerken) op de ernst van de depressie werd onderzocht met multilevel analyses. De impact van co-morbide PS (-kenmerken) op remissiepercentages en behandeluitval werd onderzocht met behulp van Coxregressiemodellen. De analyses lieten zien dat co-morbide PS geen invloed hebben op de verandering van depressie-ernst en remissiepercentages tijdens behandeling en de follow-up fase, zowel voor CT als voor IPT. Wat betreft de PS-kenmerken werden er bij individuen met hogere afhankelijke PS-kenmerken, lagere scores voor depressie-ernst gevonden tijdens de behandeling, maar dit effect hield niet aan tijdens de follow-up. Individuen met hogere cluster A PS-kenmerken lieten meer verbetering in depressieernst zien in de CT-groep ten opzichte van de IPT-groep. Dit verschil was zowel in de behandelfase als tijdens de follow-up fase significant. PS en PS-kenmerken hadden geen invloed op behandeluitval. Concluderend kan men zeggen dat co-morbide PS geen invloed hebben op de behandelresultaten en behandeluitval van CT en IPT. Wel worden er meer subtiele differentiële behandeleffecten gevonden met betrekking tot specifieke PS-kenmerken. Replicatie van deze bevindingen is echter gewenst.

In Hoofdstuk 4 wordt een meta-analyse en meta-regressie naar de impact van co-morbide PS op de uitkomsten van acute fasebehandeling voor depressie gepresenteerd. Bevindingen van eerdere meta-analyses impliceren een nadelig effect van co-morbide PS op de uitkomst van acute fasebehandelingen voor depressie. Hoewel deze conclusies gebaseerd zijn op een groot aantal studies, is er waarschijnlijk sprake van een vertekend beeld door methodologische tekortkomingen van deze studies. Zo zijn er studies geïncludeerd met naturalistische designs en met nietgestructureerde meetinstrumenten voor het vaststellen van PS en depressie. Ook is er in de eerdere meta-analyses niet gecorrigeerd op vooraf bestaande verschillen tussen individuen met en zonder PS, zoals voor verschillen in depressie-ernst. Het doel van deze meta-analyse en metaregressie was daarom om de impact van co-morbide PS op de uitkomst van acute fasebehandeling voor depressie te onderzoeken, wanneer alleen gegevens van onderzoeken met een hoge methodologische kwaliteit werden meegenomen. Studies werden gezocht in Pubmed, PsycINFO en Embase (tot 3 april 2017) op basis van zoektermen met betrekking tot depressie, PS en depressie- 
behandelingen (psychotherapie, antidepressieve medicatie en andere biologische behandelingen). Ook werd in referentielijsten van de geselecteerde artikelen en referentielijsten van eerdere reviews en meta-analyses gezocht naar geschikte studies. Inclusie van studies werd bepaald op basis van verschillende criteria. Zo moesten proefpersonen depressie als primaire diagnose hebben, en moesten depressie en PS op basis van een gestructureerd interview voorafgaand aan de behandeling zijn vastgesteld. De depressiebehandeling in de studies moest een acute fasebehandeling zijn, waarbij de behandeling op gecontroleerde wijze werd uitgevoerd. Voor studies waarin naar meer dan één behandeling gekeken werd, moest er sprake zijn van een gerandomiseerde behandeltoewijzing. Ook moest er sprake zijn van een gemeten verandering in depressie-ernst tijdens de behandeling met een gevalideerd meetinstrument. Na selectie van de artikelen, werden relevante gegevens uit de artikelen verzameld en werd er contact opgenomen met alle corresponderende auteurs met de vraag om deze gegevens te controleren en ontbrekende informatie te verstrekken. De effecten van de impact van PS op acute fasebehandeling werd geanalyseerd met behulp van de meta-analyse. De impact van variabelen (gemeten op studieniveau) op deze effecten werd onderzocht met behulp van een meta-regressie. De kwaliteit van de studies werd vervolgens geëvalueerd met de "Risk of Bias" tool, en de eventuele aanwezigheid van publicatiebias werd bepaald met behulp van een "funnel-plot" en de Egger's test. Er werden in totaal zes onderzoeken met 942 personen (waarvan 447 met PS) in deze meta-analyse geïncludeerd. De resultaten van de meta-analyse wezen uit dat co-morbide PS geen effect hadden op de uitkomsten van acute fase depressiebehandelingen. Resultaten van de meta-regressie toonden geen relevant verband met studie-variabelen aan. De heterogeniteit van de studies en het risico op bias was laag, en er werd geen indicatie voor publicatiebias gevonden. Op basis van de resultaten van deze studie kan men concluderen dat comorbide PS geen negatieve invloed heeft op de effectiviteit van acute fasebehandelingen voor depressie. Het wordt daarom sterk aanbevolen om de behandelrichtlijnen voor depressie te volgen, ongeacht de aanwezigheid van comorbide PS.

Hoofdstuk 5 beschrijft de bevindingen van een observationele studie naar de impact van co-morbide PS op de hoeveelheid en soorten ontvangen depressiebehandelingen in de klinische praktijk. Zoals hierboven vermeld, concluderen eerdere en vaak geciteerde meta-analyses dat co-morbide PS nadelige effecten hebben op behandelresultaten van depressie.. Het bewijs voor deze negatieve effecten komt echter gedeeltelijk voort uit onderzoeken met ongecontroleerde studie designs, waarbij de behandeltoewijzing niet wordt gecontroleerd. Er zijn aanwijzingen dat depressieve individuen met een co-morbide PS in dergelijke contexten suboptimale behandeling ontvangen. De negatieve effecten van co-morbiditeit op de behandelresultaten kunnen daarom ten onrechte worden toegeschreven aan de co-morbiditeit zelf, in plaats van aan de onvoldoende 
verstrekte depressie behandeling. Het doel van deze observationele studie was daarom om te onderzoeken of, en in hoeverre, de aanwezigheid van co-morbide PS geassocieerd is met de hoeveelheid en soorten ontvangen depressiebehandelingen in een naturalistische ambulante setting. Gegevens van 1455 depressieve individuen van zeven poliklinieken van een tweedelijns GGZ-instelling werden retrospectief uit elektronische patiëntendossiers verzameld. Persoonlijkheidspathologie werd gemeten met een kort gestructureerd klinisch interview (SAPAS). Associaties tussen PS en hoeveelheid en soorten ontvangen behandelingen werden geanalyseerd met behulp van regressiemodellen. De resultaten toonden aan dat depressieve individuen met comorbide PS meer psychotherapie sessies ontvingen dan personen zonder co-morbide PS, ongeacht de ernst van de depressie aan het begin van de behandeling. Het aantal sessies farmacotherapie, ondersteunende contacten en crisiscontacten verschilden niet tussen individuen met en zonder co-morbide PS. Het feit dat depressieve individuen met co-morbide PS meer psychotherapiesessies ontvingen in vergelijking met mensen zonder co-morbide PS, strookt niet met de huidige behandelrichtlijnen. Deze bevinding kan daarom indicatief zijn voor de aanwezigheid van overbehandeling van deze grote subgroep.

\section{Deel 2: Behandelplannen voor depressie}

In hoofdstuk 6 worden bevindingen gepresenteerd van een studie naar de langetermijneffecten van CT en IPT voor depressie gedurende 17 maanden follow-up. CT en IPT zijn bewezen effectieve depressiebehandelingen in de acute fase, en zijn gemiddeld gezien even effectief. Er zijn echter geen vergelijkingen beschikbaar van deze therapieën met betrekking tot de langetermijneffecten. Onderzoek heeft meermaals aangetoond dat CT een blijvend positief effect heeft na het afsluiten van de behandeling, maar het onderzoek naar de langetermijneffecten van IPT is minder uitgebreid. Het doel van deze studie was om de langetermijnresultaten van CT versus IPT voor depressie te onderzoeken. Gegevens van 134 depressieve individuen uit de STEPd-studie werden onderzocht na randomisatie en behandeling met CT $(n=69)$ en IPT ( $n=65)$. De primaire uitkomstmaat was de ernst van de depressie gemeten met de BDI-II na afloop van de behandeling en gedurende 17 maanden follow-up. Voor de groep van proefpersonen met een behandelrespons waren de terugvalpercentages en de percentages van een aanhoudende respons de secundaire uitkomstmaten. Deze werden bepaald met behulp van de BDI-II en met een klinisch interview (LIFE). De (differentiële) behandeleffecten op de primaire uitkomstmaat werden onderzocht met een multilevel analyse. De recidiefpercentages en de percentages van een aanhoudende respons werden vergeleken tussen behandelingen met Coxregressiemodellen en $\chi 2$ toetsen. Resultaten toonden aan dat de vermindering van depressie-ernst tijdens de behandeling werd behouden tijdens 17 maanden van follow-up. Daarnaast werd bij tweederde van de individuen met een behandelrespons 
geen recidief vastgesteld. $\mathrm{Er}$ waren geen differentiële behandeleffecten voor vermindering van depressie-ernst, terugvalpercentages en percentages voor aanhoudende respons. Op basis van deze bevindingen kan men concluderen dat in deze studie CT en IPT op de lange termijn niet van elkaar verschillen. Aangezien er aanwijzingen zijn dat CT een profylactisch effect heeft na een succesvolle behandeling, suggereren onze bevindingen dat IPT mogelijk ook met blijvende langetermijneffecten gepaard gaat.

In hoofdstuk 7 worden onderzoeksresultaten gepresenteerd met betrekking tot een prognostisch model voor lange termijn uitkomsten na succesvolle acute fase depressiebehandeling met CT en IPT. Omdat terugval veel voorkomt bij depressie is het klinisch relevant om individuen te identificeren die een hoog risico lopen op een ongunstig langdurig beloop na een succesvolle acute fasebehandeling. Ondanks uitgebreid onderzoek is er geen consensus over klinisch relevante voorspellers voor lange termijn uitkomsten bij depressie. Bovendien staat de ontwikkeling van predictiemodellen (zoals een Prognostische Index) die deze voorspellers combineren nog in haar kinderschoenen, en zijn de meeste predictiemodellen voor lange termijn uitkomsten gebaseerd op studies met naturalistische designs. Het doel van deze studie was om een Prognostische Index (PI) te maken om depressie-ernst op de lange termijn te voorspellen na succesvolle en hoogkwalitatieve psychotherapie voor depressie voor een specifiek individu. Gegevens van 85 proefpersonen met een behandelrespons op CT $(n=45)$ en IPT $(n=40)$ uit de STEPd-studie werden geanalyseerd. De primaire uitkomstmaat was de ernst van de depressie, gemeten met de BDI-II na afloop van de behandeling en gedurende 17 maanden follow-up. Van deze proefpersonen werden 29 variabelen, gemeten aan het einde van de behandeling, onderzocht, waaronder klinische kenmerken, demografische gegevens en informatie over psychisch lijden, algemeen functioneren, psychologische processen en levens- en familiegeschiedenis. Uit deze 29 variabelen werden voorspellers geselecteerd met behulp van twee "machine learning" technieken. Deze geselecteerde voorspellers werden vervolgens gecombineerd in één predictiemodel waaruit individuele PI-scores werden berekend met behulp van een kruisvalidatiemethode. $\mathrm{Er}$ werden in totaal drie voorspellers voor lange termijn depressie geïdentificeerd: depressie-ernst, hopeloosheid en zelfvertrouwen, allen gemeten na afloop van de succesvolle behandeling. De individuele $\mathrm{PI}$-scores correleerden sterk $(r=0,60)$ met daadwerkelijke follow-up scores voor depressie-ernst. Op basis van deze bevindingen kan men concluderen dat lange termijn voorspellingen voor depressie multifactorieel bepaald zijn en opgebouwd zijn uit een combinatie van meerdere voorspellers met elk een klein prognostisch effect. De PI heeft de potentie om lange termijn depressie uitkomsten op individueel niveau te voorspellen na een succesvolle behandeling. Replicatie en (externe) validatie zijn echter gewenst.

In hoofdstuk 8 worden onderzoeksresultaten gepresenteerd van een studie over de Personalized Advantage Index (PAI). De PAI is gebaseerd op een prescriptief model, 
waaruit behandeladviezen geformuleerd kunnen worden. In de afgelopen jaren hebben onderzoeken naar de PAI veelbelovende resultaten laten zien. De voorspellingen van een PAI-model resulteren niet alleen in een behandeladvies, maar er wordt ook een kwantitatieve schatting gemaakt van het voorspelde voordeel van de geadviseerde behandeling ten opzichte van de niet-geadviseerde behandeling(en). Deze kwantitatieve schatting is gebaseerd op het verschil tussen de door het model voorspelde uitkomsten van twee of meer behandelingen. Het doel van de huidige studie was om de PAl-benadering toe te passen voor het selecteren van de behandeling met de beste langetermijneffecten voor een specifiek individu, de zogenaamde "langetermijneffecten PAl". Data waren afkomstig van de STEPd-studie, waarbij we ons beperkten tot het deel van de proefpersonen $(n=151)$ dat werd gerandomiseerd naar CT $(n=76)$ en IPT $(n=75)$. De primaire uitkomstmaat was de ernst van de depressie, gemeten met de BDI-II, gedurende 17 maanden follow-up na afloop van de behandeling. Van deze proefpersonen werden 38 variabelen, gemeten voor de behandeling, onderzocht, waaronder klinische kenmerken, demografische gegevens, informatie over psychisch lijden, algemeen functioneren, psychologische processen en levens- en familiegeschiedenis. Uit deze 38 variabelen werden voorspellers en moderatoren geselecteerd met behulp van twee "machine learning" technieken. Deze geselecteerde voorspellers en moderatoren werden vervolgens gecombineerd in één PAl-model, waaruit individuele PAl-scores werden berekend met behulp van een kruisvalidatiemethode. Deze PAI-voorspellingen van de langetermijneffecten werden vervolgens vergeleken met de werkelijke follow-upresultaten en met PAIvoorspellingen uit een eerdere studie op basis van een deel van dezelfde dataset die zich richtte op de voorspelling van depressie-ernst na afloop van de behandeling. Eén voorspeller (alcoholmisbruik door ouders) en twee moderatoren (recente gebeurtenissen in het leven en mishandeling in de kindertijd) werden geselecteerd voor het PAI-model. Daarna werden individuele PAI-scores berekend. Individuen gerandomiseerd naar de door de PAI geadviseerde behandeling hadden tijdens de follow-up periode lagere depressie ernstscores in vergelijking met de proefpersonen die waren toegewezen aan de door de PAI niet geadviseerde behandeling. Dit verschil was significant voor de individuen met een hoge PAl-score (d.w.z. de 60\% met het hoogste voorspelde voordeel), en voor de individuen waarvoor CT de door de PAI geadviseerde behandeling was. Langetermijnvoorspellingen overlapten niet substantieel met voorspellingen voor acuut voordeel (PAI-voorspellingen voor depressie-ernst na afloop van de behandeling). Concluderend waren de PAIvoorspellingen en behandeladviezen voor langetermijneffecten van depressiebehandeling veelbelovend. Ook zou een dergelijk predictiemodel bij kunnen dragen aan de ontwikkeling van "precisiegeneeskunde" door de optimale behandeling voor een specifiek individu te selecteren. Replicatie en (externe) validatie zijn echter gewenst. 
Hoofdstuk 9 presenteert de resultaten van een meta-analyse en een metaregressie naar de effectiviteit van psychotherapie voor therapieresistente depressie (TRD). Depressie met onvoldoende behandelrespons na verschillende depressiebehandelingen wordt vaak beschreven als TRD. TRD is geassocieerd met functionele beperkingen, een grote ziektelast en hoge maatschappelijke kosten. Van oudsher is de klinische praktijk in het wetenschappelijk onderzoek vooral gericht op farmacotherapie en andere somatische behandelingen voor TRD. In de loop der jaren is er echter een groeiend aantal studies naar psychotherapie voor TRD gedaan. Ook geeft de meerderheid van de depressieve individuen de voorkeur aan psychotherapie boven farmacotherapie. Het doel van deze meta-analyse en meta-regressie was daarom om de effectiviteit van psychotherapie voor TRD te onderzoeken als alternatief voor - of aanvulling op - de standaardbehandeling ("treatment as usual") voor TRD. Standaardbehandeling is hier gedefinieerd als klinisch management en/of voor voortzetting, optimalisatie of verandering van antidepressieve medicatie. Studies werden gezocht in Pubmed, PsycINFO en Embase (tot 19 december 2016) op basis van zoektermen met betrekking tot psychotherapie, TRD en chronische MDD. Ook werden referentielijsten van de geselecteerde artikelen en referentielijsten van eerdere reviews en meta-analyses doorzocht op geschikte studies. Inclusie van studies werd bepaald op basis van verschillende criteria. Meer specifiek werden gerandomiseerde en gecontroleerde behandelstudies geïncludeerd, waarin de effectiviteit van psychotherapie werd getoetst met een vergelijking tussen psychotherapie en standaardbehandeling, of met een vergelijking tussen standaardbehandeling met psychotherapie en standaardbehandeling zonder psychotherapie. Ook moesten proefpersonen een volwassen leeftijd hebben ( $\geq 18$ jaar) en aan de minimale criteria van TRD voldoen, namelijk het ontbreken van behandelrespons na ten minste één adequate behandeling met antidepressieve medicatie. Studies naar chronische depressie werden alleen geïncludeerd als de meerderheid van de proefpersonen voldeed aan de criteria voor TRD. Na selectie van de artikelen, werden relevante gegevens uit de artikelen verzameld en werd er contact opgenomen met alle corresponderende auteurs met de vraag om deze gegevens te controleren en ontbrekende informatie te verstrekken. De behandeleffecten van psychotherapie (als alternatief of als aanvulling op standaardbehandeling) werden geanalyseerd met behulp van een meta-analyse. De impact van variabelen (gemeten op studieniveau) op de behandeleffecten werd onderzocht met behulp van een meta-regressie. De kwaliteit van de studies werd vervolgens geëvalueerd met de "Risk of Bias" tool, en de eventuele aanwezigheid van publicatiebias werd bepaald met behulp van een "funnelplot" en de Egger's test. Er werden in totaal 21 studies met 25 vergelijkingen en 3539 proefpersonen geïncludeerd. Hiervan zaten 293 proefpersonen in drie psychotherapie vs. standaardbehandeling vergelijkingen, en 1588 proefpersonen in 22 vergelijkingen tussen standaardbehandeling met psychotherapie en standaardbehandeling zonder psychotherapie. De resultaten van de meta-analyse lieten zien dat er geen significant 
voordeel was voor psychotherapie ten opzichte van de standaardbehandeling. Er werd wel een matig groot effect gezien in de verandering van depressie-ernst ten gunste van psychotherapie als aanvulling op de standaardbehandeling (de standaardbehandeling met psychotherapie vs. standaardbehandeling zonder psychotherapie vergelijking). De bevindingen van de meta-regressie wezen op een positief verband tussen de depressie-ernst vóór de behandeling en de grootte van dit behandeleffect. Ook werd er een positief verband gevonden tussen groepstherapie (i.p.v. individuele therapie) en de grootte van het behandeleffect. Verder was het risico op bias laag, en werd er geen indicatie voor publicatiebias gevonden. Concluderend kan men zeggen dat de toevoeging van psychotherapie aan de standaardbehandeling kan leiden tot betere behandelresultaten voor individuen met TRD. Toevoeging van psychotherapie bij de behandeling van TRD is daarom op basis van deze studie gerechtvaardigd.

\section{Deel 3: Toekomstige strategieën}

In hoofdstuk 10 worden de resultaten van een directe vergelijking tussen statistische voorspellingen door predictiemodellen (de PAI) en de klinische inschatting van therapeuten voor het selecteren van depressiebehandelingen gepresenteerd. Hoewel onderzoek meermaals heeft aangetoond dat statistische voorspellingen even nauwkeurig - of zelfs nauwkeuriger - zijn dan voorspellingen op basis van klinische inschattingen, hangt de selectie van verschillende typen depressiebehandelingen in de klinische praktijk nog steeds sterk af van klinische inschattingen. Met de recente ontwikkelingen van innovatieve predictiemodellen was het doel van deze studie om de nauwkeurigheid van klinische inschattingen te vergelijken met statistische voorspellingen bij de keuze tussen CT en IPT voor depressie. Data waren afkomstig van de STEPd-studie, waarbij we ons beperkte tot het deel van de proefpersonen $(n=151)$ dat werd gerandomiseerd naar CT $(n=76)$ en IPT $(n=75)$. Voorafgaand aan de randomisatie procedure werden de aanbevelingen van therapeuten bepaald tijdens multidisciplinaire stafvergaderingen. Statistische voorspellingen werden gebaseerd op de eerder besproken PAI-modellen. De primaire uitkomstmaat was de ernst van de depressie, gemeten na afloop van de behandeling en gedurende 17 maanden followup met behulp van de BDI-II. De secundaire uitkomstmaat was vroegtijdige uitval tijdens de behandeling (behandeluitval). Uit de resultaten van de statistische voorspellingen bleek dat proefpersonen die waren toegewezen aan de door de PAIgeadviseerde behandeling, minder depressief waren na afloop van de behandeling en gedurende de follow-up, in vergelijking met proefpersonen die de door de PAI nietgeadviseerde behandelingen ontvingen. Uit de resultaten van de klinische voorspellingen bleek dat dit voordeel niet werd gevonden voor de aanbevolen versus de niet-aanbevolen behandelingen op basis van de klinische inschatting. Bovendien werden bij proefpersonen met een IPT-aanbeveling door therapeuten een hogere 
depressie-ernst na de behandeling en tijdens de follow-up gevonden bij degenen die daadwerkelijk IPT kregen (t.o.v. van degenen die CT kregen). Aanbevelingen op basis van statistische voorspellingen en klinische inschatting werden niet geassocieerd met verschillen in behandeluitval. Concluderend kan men stellen dat statistische voorspellingen (de PAI-benadering) de klinische inschatting overtreft in het selecteren van optimale depressiebehandelingen.

Hoofdstuk 11 beschrijft de resultaten van PAI-voorspellingen voor behandelingselectie van CT en IPT voor depressie tussen studies. Om de toepasbaarheid van PAImodellen in de klinische praktijk te bepalen, is het van groot belang om deze modellen extern te valideren. Externe validatie wordt echter zelden toegepast in onderzoek. Het doel van deze studie was dan ook om PAl-modellen te ontwikkelen en extern te valideren in datasets van twee onafhankelijke studies. Deze studies betroffen twee gerandomiseerde studies waarin de effectiviteit van CT en IPT voor depressie werden vergeleken (FreqMech-studie: $n=200$ en STEPd-studie: $n=151$ ). Resultaten van de FreqMech-studie toonden dat depressie-ernst na afloop van behandeling niet significant verschilde tussen proefpersonen die de door de PAI geadviseerde behandeling kregen versus proefpersonen die de door de PAI niet geadviseerde behandeling ontvingen. In de STEPd-studie was de depressie-ernst na de behandeling significant lager voor personen die waren toegewezen aan de door de PAI geadviseerde behandeling vergeleken met degenen die waren toegewezen aan de door de PAI niet geadviseerde behandeling. Er waren bescheiden voorspellende effecten van de PAI-modellen tussen de studies, wat betekent dat de depressie-ernst na behandeling niet significant verschilde tussen degenen die de geadviseerde behandeling kregen versus degenen die de niet geadviseerde behandeling hadden ontvangen. Concluderend kan men zeggen dat externe validatie van PAI-modellen bescheiden resultaten heeft opgeleverd. Ook komen in deze studie de uitdagingen van externe validatie naar voren, zoals de heterogeniteit van dataverzameling, statistische methoden, onderzoekspopulaties en behandelingen.

Hoofdstuk 12 introduceert en demonstreert een innovatieve Bayesiaanse methodologie die bedoeld is om subgroepen van behandelrespons te identificeren op basis van depressie-ernst scores. Van oudsher zijn depressiestudies gebaseerd op a priori gedefinieerde categorieën voor behandelingsrespons waarbij afkappunten op depressieschalen worden gehanteerd. Meer recente studies passen een "data gestuurde" benadering toe om verschillende subgroepen van behandelrespons te analyseren. Hierbij wordt gebruik gemaakt van zogenaamde "Latent Variable Mixture Models". Hoewel deze modellen veelbelovende resultaten laten zien, blijft het een uitdaging om het juiste aantal subgroepen te bepalen en kan men twijfelen aan de juistheid van deze subgroepen. Het doel van deze studie was om een recent ontwikkelde methode te introduceren en te demonstreren. Deze methode, "a mixture of shifted Poisson distributions", heeft als doel latente subgroepen van behandelrespons te identificeren tijdens depressiebehandelingen. Een belangrijke 
eigenschap van deze methode is de focus op zogenaamde modi van de verdelingen van depressie-ernst scores. Modi (of pieken) worden als een potentieel robuuste indicator voor subgroepen gezien. Naast de focus op modi, is het model zo opgebouwd dat het beter overeenkomt met de niet-normale verdeling van depressieernst scores. Met het model worden allereerst de verdeling van depressie-ernst scores geschat. Vervolgens worden het aantal en de locatie van de modi bepaald met een Bayesiaanse inferentiemethode (Reversible Jump Markov Chain Monte Carlo). Ten slotte wordt er per modus (of potentiele subgroep) gekeken hoe groot de kans is dat proefpersonen tot deze modus behoren (subgroep lidmaatschap). Na uitleg van het model, werd de toepassing van het model gedemonstreerd met data van de STEPd-studie. Er werden data van 102 proefpersonen gebruikt, waarbij een deel was gerandomiseerd naar CT $(n=53)$, en een deel was gerandomiseerd naar IPT $(n=49)$. De toepassing van het model op deze data liet zien dat er drie tot vier verschillende subgroepen van behandelrespons te onderscheiden zijn. Ook werd er gevonden dat het aantal subgroepen toenam naarmate de therapie vorderde, met name in de laatste twee therapiesessies. De kans voor een proefpersoon om tot een bepaalde subgroep te behoren (subgroep lidmaatschap) had potentiele prognostische relevantie. Concluderend kan men zeggen dat deze nieuwe ontwikkelde methode een veelbelovende methode is voor het identificeren van subgroepen van behandelrespons in depressiebehandelingen. Resultaten van de toepassing op de STEPd-studie data geven namelijk interessante inzichten in subgroepen en bieden veelbelovende aanwijzingen voor de ontwikkeling van longitudinale prognostische modellen.

Hoofdstuk 13 geeft een korte samenvatting van de studies in dit proefschrift, gevolgd door een bespreking van de bevindingen vanuit een breder perspectief met daaruit volgende suggesties voor toekomstig onderzoek en beleid. De hoofdboodschap van dit hoofdstuk - en van het proefschrift als geheel - is dat de ontwikkeling van gepersonaliseerde behandelstrategieën voor depressie een geweldige uitdaging is. Traditionele benaderingen gericht op co-morbiditeit en klinisch inschatting zijn gebaseerd op beperkt en niet robuust wetenschappelijk bewijs. Innovatieve predictiemodellen zijn veelbelovend, hoewel de ontwikkeling van deze modellen nog in haar kinderschoenen staat en de toepasbaarheid van deze modellen in de klinische praktijk op basis van externe validatie nog getoetst moet worden. Ook brengt de ontwikkeling van deze nieuwe statistische methode en technologie ethische en juridische vraagstukken met zich mee. Hierbij kan men denken aan de inherente complexiteit van de modellen ("black box"), de verantwoordelijkheden voor de output van modellen, de impact van onjuiste voorspellingen en bias, privacybescherming, en de angst voor het verdwijnen van de "menselijkheid" in de behandelcontext. Inspanningen om de kloof tussen de klinische praktijk en het wetenschappelijk onderzoek te verkleinen zijn van cruciaal belang voor de ontwikkeling en implementatie van predictiemodellen. Dicht bij huis beginnen met de ontwikkeling van lokale predictiemodellen in GGZ-instellingen zou een goede start kunnen zijn. Om 
met predictiemodellen zinvolle behandeldoelen te bereiken, dient men stil te staan bij de indicatoren van een succesvolle behandeling. Hierbij kan men naast de vermindering van depressie symptomen ook denken aan het voorkomen van behandeluitval en het bevorderen van positieve geestelijke gezondheid. Het vinden van relevante gegevens en bronnen om predictiemodellen te informeren moet worden geëvalueerd, waarbij factoren zoals kosten, haalbaarheid en de belasting voor het individu in overweging moet worden genomen. Toekomstige predictiemodellen zouden niet alleen voorspellingen moeten geven om behandelselectie te ondersteunen, maar zouden ook bijgewerkte voorspellingen moeten geven die sturing geven aan aanpassing van de behandelingen. De genoemde suggesties hebben als uiteindelijke doel om behandelstrategieën voor depressie in de klinische praktijk op effectieve wijze te personaliseren. 



\section{Valorization addendum}

In the present thesis, both traditional and more innovative ways to personalize treatment strategies for major depressive disorder (MDD) are studied. In this chapter, the valorization addendum, the usefulness of this research to society will be reviewed. First, the social and economic relevance of the findings will be addressed, and an overview of potential target groups for whom the research results might be of interest will be given. After that, possible activities and products as well as innovative aspects of the findings will be discussed. Finally, planning and implementation of the results and future projects will be outlined.

\section{Relevance}

MDD is a health care problem affecting over 300 million people annually and leading to over 50 million of Years Lived with Disability (YLD) worldwide. On a global level, MDD is the largest contributor to non-fatal health loss (7.5\% of all YLD; WHO, 2017). Additionally, economic costs are large; total costs in the Netherlands are about 3 billion Euros each year (de Graaf, Tuithof, van Dorsselaer, \& ten Have, 2011; Slobbe, Smit, Groen, Poos, \& Kommer, 2011). Efficacious treatments for MDD are available, showing comparable effect-sizes (Cipriani et al., 2009; Cuijpers, Andersson, Donker, \& van Straten, 2011; Gartlehner et al., 2008). However, individual responses to these treatments vary widely and are largely unpredictable (Kravitz, Duan, \& Braslow, 2004; Simon \& Perlis, 2010). In clinical practice, this results in a trial and error approach (most typical for secondary and tertiary care settings) in which consecutive treatments are being offered to determine what works best for the given individual (Rush et al., 2006). This trial and error method has many negative consequences including long treatment trajectories, chronicity, demoralization, decreased psychosocial functioning, high societal costs and treatment dropout. In other words, this approach and its consequences all add to the known over- and under treatment of MDD (Lorenzo-Luaces, 2015; Thornicroft et al., 2017).

Considering the impact of over- and under treatment of MDD on an individual and societal level, it is of high importance to improve treatment strategies, finding the optimal depression treatment for a specific individual at a specific point in time (Cohen \& DeRubeis, 2018). To this end, we investigated both traditional and innovative methods. Findings indicated that traditional approaches are supported by limited and non-robust evidence, and that innovative approaches (prediction models) are promising, however still in development. These findings contribute to the growing knowledge on possibilities and limitations of personalized medicine in MDD, and provide future directions on how to improve treatment strategies, leading to higher (cost) effectiveness. 


\section{Target groups}

The research findings of this thesis could be of interest for a broader public outside the academic circles as well. First of all, these results might be relevant for individuals with an MDD diagnosis, and their friends and family members. The current trial and error approach in MDD treatment leads to potentially long treatment trajectories, chronicity, demoralization, and decreased psychosocial functioning, that are all affecting this group directly. In addition, the application of prediction models as an alternative to clinical judgment to guide treatment selection might improve shared decision making. Although shared decision is considered key in patient-centered care (Weston, 2001), it has not yet been widely implemented in clinical practice (Couët et al., 2015). Information provided by prediction models could facilitate patient involvement by changing the dynamic between patient and clinician, moving from paternalism to a dialogue between two more equally engaged and informed partners. Secondly, since the results suggest various directions for improvement in clinical decision making (e.g., less focus on comorbidity and clinical judgment), they might be of interest to mental health care professionals. In addition, investigating ways to reduce the negative consequences of the trial and error approach can be beneficial for professionals as well, since these consequences can potentially lead to demoralization and professional dissatisfaction. Finally, since the results of this thesis contribute to future development of more efficient treatment strategies for MDD, they might be relevant to policy makers and health insurance companies as well.

\section{Activities \& products}

The results of this dissertation could contribute to the development of various activities and products. First, findings of this thesis point towards potential adjustment of multidisciplinary guidelines for MDD. Results indicated that the traditional focus on comorbidity and clinical judgment to guide treatment strategies for MDD is supported by inconsistent, non-robust and limited evidence (Chapter 2, 3, 4, 10). However, this focus has not been abandoned in clinical practice. In particular, personality pathology and clinical judgment are still important pillars in treatment selection for MDD. This is illustrated by Chapter 5, where we found that, in contrast to clinical practice guidelines, individuals with personality pathology received significantly more psychotherapy sessions as compared to individuals without personality pathology. Although multidisciplinary guidelines for MDD do not support the more traditional approaches, the potential negative effects of relying on these approaches could be emphasized more strongly. In addition, future research is needed to better understand how current clinical decision making is impacting individual treatment outcomes. Once we gain this understanding, directions on how to relate to the current state of practice could be added to the multidisciplinary guidelines. In addition to the results on traditional methods, findings regarding long- 
term effects of cognitive therapy (CT) and interpersonal psychotherapy (IPT), and findings on the role of psychotherapy in treatment resistant depression (TRD) could contribute to potential adjustment of guidelines. In the current multidisciplinary guidelines for MDD, CT and IPT are the two time-limited psychotherapies of choice for MDD. The finding that CT and IPT are equally effective in the long run (Chapter 6), should strengthen the guideline's stance that these are both valid treatment options. A final potential contribution of this thesis's findings to guidelines is the meta-analytic evidence that the addition of psychotherapy to treatment as usual can be beneficial for individuals with TRD (Chapter 9).

Second, findings of this thesis could contribute to the potential adjustment of clinical training programs of mental health care professionals. As mentioned in Chapter 10 and Chapter 13, despite the evidence of the superiority of statistical prediction over clinical judgement, the role of clinical judgement in decision making is still strong in daily practice. To change this, mental health care professionals should receive ongoing clinical training and feedback on known errors in clinical judgement. In addition, education on statistics and probability theory should be a significant part of the curriculum, to better understand and evaluate new technological advances in mental health. Furthermore, the finding that different individuals could benefit differently from CT and IPT (Chapter 8), highlights the importance of a varied range of psychotherapeutic modalities in treatment programs for MDD. In current clinical programs the emphasis on CT is strong, while IPT is often not a required part of training (Markowitz \& Weissman, 2012). To preserve a varied range of psychotherapeutic options, clinical programs should offer training of different types of time-limited psychotherapy for MDD.

Third, findings of this thesis could provide future directions on the development of prediction models and decision tools that can guide treatment strategies for MDD. As illustrated in Chapter 7 and Chapter 8, prediction models show promising results in optimizing treatment planning and selection. However, current studies are predominantly "proof of concepts", since prediction models have mainly been applied in research contexts rather than being tested and implemented in real-world settings. Important next steps involve improvement of statistical techniques (Chapter 12), external validation (Chapter 11) and prospective testing. In addition, models that optimize both treatment selection and adaptation need to be explored to maximize the effectiveness for personalized treatment strategies (Chapter 13). Once prediction models are proven effective based on prospective testing, efforts should be made to develop decision tools that translate predictions and recommendations of these models in an evident and engaging way. In order to achieve this, involvement of endusers (patients and mental health care professionals) should be a central part to bridge the gap between clinical practice and research (Chapter 13). 


\section{Innovation}

The studies in this thesis have various innovative aspects. First of all, the overall aim of this thesis is innovative, by moving from the "one size fits all" approach towards a more personalized perspective on treatment. This perspective is in line with the personalized or precision medicine movement, that has attracted a great deal of attention in the field of medicine (Katsnelson, 2013). Second, both traditional and innovative methods to personalize treatment strategies for MDD have been investigated. Examining the "status quo" of treatment strategies enables understanding of potential opportunities for innovation. Since we found that the more traditional treatment strategies are problematic, the need for more innovative solutions, such as prediction models, becomes more apparent. Third, the statistical techniques that were used in the chapters of this dissertation may be considered scientifically innovative. In Chapter 7, 8, and $\mathbf{1 1}$ supervised machine learning techniques were applied to build prediction models, and in Chapter 12 a recently developed Bayesian methodology was introduced to model subgroups of depression treatment response. Fourth, the designs and results of the studies have innovative aspects as well. In Chapter $\mathbf{3}$ and $\mathbf{4}$, no impact of personality pathology on MDD treatment outcome was found, which is contrary to previous meta-analyses. In Chapter 6 enduring effects of acute phase CT and IPT were studied head-to-head for the first time. In Chapter 11, efforts to externally validate the Personalized Advantage Index (prediction model) are presented, which has not been done before in the context of two randomized trials. Fifth, findings reported in Chapter 7, 8, 11, 12 and 13 of this dissertation highlight the challenges associated with the development of prediction models and decision tools and could therefore potentially serve as starting points for future innovation. Finally, findings of this dissertation include interdisciplinary research that could lead to surprising and innovate insights. In Chapter 12, joint efforts of researchers and clinicians from the fields of psychiatry, clinical psychology and econometrics are presented, resulting in valuable insights on subgroups of treatment response in MDD. Given the methodological challenges associated with the development of prediction models (Chapter 13), one could argue that these joint forces are crucial to solve complex clinical and statistical problems.

\section{Planning \& implementation}

The chapters of this thesis were published in or submitted to international peerreviewed journals, and are therefore accessible to researchers and mental health care professionals. To maximize the accessibility of the findings, part of the studies were published Open Access, and results of the STEPd study were published in clinical journals that specifically targets mental health care professionals who are not working in a research setting (Tijdschrift voor Psychiatrie / Tijdschrift voor Psychotherapie). In addition, findings have been presented to researchers and clinicians on various (inter) 
national conferences (e.g., conferences of VGCT, NVVP, ISIPT, ABCT, SPR and NedKad) and at mental health care centers (e.g., Zuiderland GGZ Sittard, METggz Roermond, MUMC+). Efforts to disseminate these results to a broader audience have been undertaken as well, by discussing findings in public debates (i.e., P.C. Kuiperdebat, Studium Generale), and by outlining the need for personalized treatment strategies in a publication on an online platform (i.e., dejongepsychiater.nl). In addition, we are planning to report the results of the STEPd study in an article of Dagblad de Limburger. Furthermore, this thesis represents a solid infrastructure for knowledge dissemination, since it is a result of an interfaculty collaboration, and based on an (inter)national network of other research groups (e.g., VU Amsterdam, PsyQ, Radboud University, University of Pennsylvania).

In addition to the knowledge dissemination efforts for the findings of this thesis, plans for further valorization of innovative methods to personalize treatment strategies are being put in place. In the next years, we plan to develop easy-to-use decision tools to guide treatment strategies for MDD using complex statistics based on interdisciplinary teamwork. During this process, the methods and techniques that are developed will be presented and made available continuously, by means of (inter)national publications and presentations. In addition, we intend to make statistical models available to researchers, mental health care professionals and their organizations by offering free CRAN (Comprehensive R Archive Network) packages for the models using open source software. If successful, this project will result in improvement of the effectiveness of treatments for MDD in secondary and tertiary mental health settings leading to a reduction of disease burden and related costs. The new models developed in this interdisciplinary context might have potential application areas in other academic fields as well, such as social sciences, medicine, and economics. We plan to facilitate this with inter-disciplinary seminars. In the development of prediction models and decision tools we aim to 1) extent our global network, and 2) intensify end-user involvement (patients and mental health care professionals), that will contribute to the knowledge utilization. As mentioned in Chapter 13, a strong global network of "academic workplaces" is key to the development and validation of prediction models. Efforts to broaden our international network have been undertaking, including plans of work visits and joint grant applications. End-user involvement will be a central part of the development of prediction models and decision tools. For the prediction model development, "expert elicitation methods" will be investigated, in which expert opinion (and its uncertainty) of mental health care professionals and patients can be translated to prediction models. Part of this plan has been recently awarded with the Data Science Research Competition 2020 award of Maastricht University. In addition to model development, we will involve end-users in the creation of user-friendly interfaces of the decision tools. 


\section{References}

Cipriani, A., Furukawa, T. A., Salanti, G., Geddes, J. R., Higgins, J. P., Churchill, R., . . McGuire, H. (2009). Comparative efficacy and acceptability of 12 new-generation antidepressants: a multiple-treatments meta-analysis. The lancet, 373(9665), 746-758.

Cohen, Z. D., \& DeRubeis, R. J. (2018). Treatment selection in depression. Annual review of clinical psychology, 14, 209-236.

Couët, N., Desroches, S., Robitaille, H., Vaillancourt, H., Leblanc, A., Turcotte, S., . . . Légaré, F. (2015). Assessments of the extent to which health-care providers involve patients in decision making: a systematic review of studies using the OPTION instrument. Health Expectations, 18(4), 542-561.

Cuijpers, P., Andersson, G., Donker, T., \& van Straten, A. (2011). Psychological treatment of depression: results of a series of meta-analyses. Nordic journal of psychiatry, 65(6), 354-364.

de Graaf, R., Tuithof, M., van Dorsselaer, S., \& ten Have, M. (2011). Verzuim door psychische en somatische aandoeningen bij werkenden. Resultaten van de 'Netherlands Mental Health Survey and Incidence Study-2'(NEMESIS-2)[Absenteeism due to psychological or somatic disorders in workers. Results of the 'Netherlands Mental Health Survey and Incidence Study-2'(NEMESIS-2)]. Utrecht: Trimbos-Instituut.

Gartlehner, G., Gaynes, B. N., Hansen, R. A., Thieda, P., DeVeaugh-Geiss, A., Krebs, E. E., . . . Lohr, K. N. (2008). Comparative benefits and harms of second-generation antidepressants: background paper for the American College of Physicians. Annals of internal medicine, 149(10), 734-750.

Katsnelson, A. (2013). Momentum grows to make'personalized'medicine more'precise'. In: Nature Publishing Group.

Kravitz, R. L., Duan, N., \& Braslow, J. (2004). Evidence-based medicine, heterogeneity of treatment effects, and the trouble with averages. The Milbank Quarterly, 82(4), 661-687.

Lorenzo-Luaces, L. (2015). Heterogeneity in the prognosis of major depression: from the common cold to a highly debilitating and recurrent illness. Epidemiology and Psychiatric Sciences, 24(6), 466-472.

Markowitz, J. C., \& Weissman, M. M. (2012). Interpersonal psychotherapy: past, present and future. Clinical psychology \& psychotherapy, 19(2), 99-105.

Rush, A. J., Trivedi, M. H., Wisniewski, S. R., Nierenberg, A. A., Stewart, J. W., Warden, D., . . Lebowitz, B. D. (2006). Acute and longer-term outcomes in depressed outpatients requiring one or several treatment steps: a STAR* D report. American Journal of Psychiatry, 163(11), 1905-1917.

Simon, G. E., \& Perlis, R. H. (2010). Personalized medicine for depression: can we match patients with treatments? American Journal of Psychiatry, 167(12), 1445-1455.

Slobbe, L., Smit, J., Groen, J., Poos, M., \& Kommer, G. (2011). Kosten van ziekten in Nederland 2007: Trends in de Nederlandse zorguitgaven 1999-2010.

Thornicroft, G., Chatterji, S., Evans-Lacko, S., Gruber, M., Sampson, N., Aguilar-Gaxiola, S., . . Borges, G. (2017). Undertreatment of people with major depressive disorder in 21 countries. The British Journal of Psychiatry, 210(2), 119-124.

Weston, W. W. (2001). Informed and shared decision-making: the crux of patient-centered care. CMAJ: Canadian Medical Association journal= journal de l'Association medicale canadienne, 165(4), 438-439.

WHO. (2017). Other Common Mental Disorders: Global Health Estimates. Geneva: World Health Organization, 1-24. 


\section{Dankwoord / Acknowledgements}

Een promotietraject is een avontuur. De term avontuur heeft verschillende betekenissen, die ieder van toepassing zijn op de totstandkoming van dit proefschrift. Het beleven van een avontuur kan gezien worden als een situatie waarin je ongewone, onverwachte en zonderlinge dingen overkomen. Ook kan het opgevat worden als een riskante onderneming. Het avontuur beproeven betekent hetzelfde als het geluk beproeven, en men kan op avontuur uit gaan door op goed geluk, zonder bepaalde bestemming, op pad te gaan. Ik wil in dit hoofdstuk graag iedereen bedanken die dit avontuur, mijn promotietraject, mogelijk heeft gemaakt. Het is dankzij jullie dat ik daadwerkelijk de bestemming van het avontuur, een voltooid proefschrift, heb bereikt.

Graag zou ik beginnen met het bedanken van alle deelnemers aan de studies. In het bijzonder de deelnemers van de STEPd studie (hoofdstuk 2, 3, 6, 7, 8, 10, 11 en 12), de deelnemers van verschillende studies uit de meta-analyses (hoofdstuk 4 en 9) en de deelnemers van de observationele studie bij psyQ (hoofdstuk 5). Hartelijk dank voor jullie cruciale inzet en medewerking.

Mijn promotieteam, Frenk, Marcus en Lotte, wil ik hartelijk bedanken. Ik had me geen beter team kunnen voorstellen. Ik vind het erg jammer om deze periode met jullie af te sluiten, maar ik hoop, en ben van plan, nog veel met jullie samen te werken de komende jaren. Frenk, jouw mentorschap is een rode draad in mijn opleiding tot psychiater en mijn promotietraject, bedankt voor alles wat je me hebt geleerd. Mijn enthousiasme voor de behandeling van stemmingsstoornissen is begonnen bij jou op de poli tijdens het coschap psychiatrie. Dank voor je onuitputtelijke inhoudelijke kennis: het is indrukwekkend hoe jij moeiteloos in- en uitzoomt in alle ingewikkelde materie. Ik zal dan ook nooit vergeten hoe je tijdens onze eerste afspraken uit je hoofd het ene na het andere artikel citeerde: "dit is een levende bibliotheek" dacht ik toen. Later kwam ik erachter dat je ook iets weg hebt van een levend woordenboek, iets waar ik op "tentatieve wijze" van heb kunnen leren. Dank voor je goede zorgen, geduld en vertrouwen. Waar ik soms het overzicht, mijn geduld of mijn koffer verloor, kon jij rustig naar mijn problemen luisteren om vervolgens iets te zeggen waardoor de storm weer ging liggen. Bedankt dat je me naast die goede zorgen ook altijd de vrijheid en aanmoediging hebt gegeven om onderwerpen die ik interessant vond te onderzoeken, ook als dit niets meer met het originele promotieplan te maken had. Dit maakte het promotietraject een waar avontuur, en het woensdagochtendoverleg was daar een belangrijk onderdeel van. Ik kijk uit naar een toekomst met nog veel woensdagochtenden. Marcus, je hebt me de afgelopen jaren geïnspireerd. Dankzij jou ben ik uitdagingen aangegaan die ik anders als onmogelijk had gezien. Zo adviseerde je mij tijdens de eerste STEPd 2.0 vergadering om het lab van Rob DeRubeis aan de Universiteit van Philadelphia te bezoeken. Deze ervaring heeft me als wetenschapper gevormd en als mens verrijkt. Doordat jij als vanzelfsprekend ervanuit ging dat 
dergelijke uitdagingen haalbaar waren, kreeg ik er zowaar ook vertrouwen in. Dank ook voor je constructieve feedback en oplossingsgerichte input. Je was altijd beschikbaar voor overleg, en kwam regelmatig naar het Zuiden afgereisd. Andersom, was ik, of een anonieme knoflookstreng, altijd welkom in Amsterdam en Utrecht. Dank voor je aanstekelijk enthousiasme, ik kijk ernaar uit om de zoektocht naar predictiemodellen met jou voort te zetten. Lotte, jij bent mijn rolmodel, zowel als wetenschapper als persoon. Dankjewel hiervoor! Bij moeilijke zaken denk ik regelmatig "Hoe zou Lotte dit aanpakken?". Ik ben zeer onder de indruk van hoe jij in alles wat je doet, altijd bij jezelf blijft: AWESOME. Ik heb veel geleerd van zowel je kennis over het uitvoeren van onderzoek, als je inhoudelijke kennis over psychotherapie en depressie. Dankzij jou heb ik met een prachtig geordende dataset mogen werken. Ook heb je me, met veel geduld, veel geleerd over het wetenschappelijke schrijven. Wat ik hierin erg waardeerde was dat je altijd positieve feedback in mijn manuscripten verwerkte. Dankjewel voor je oprechte betrokkenheid. Je was de afgelopen jaren altijd beschikbaar voor elk probleem, groot of klein. Steun gaf je door te luisteren en met hele goede adviezen en konijn-gerelateerde oplossingen te komen. Het is een waar genoegen om je collega te zijn. Dank dat je mijn partner-in-crime bent als het gaat om dansroutines (met of zonder dino's), je eigen pizza versieren, verdwalen in de Trierse bossen en toekomstige avonturen (StP).

Graag wil ik de beoordelingscommissie bedanken voor het beoordelen van mijn proefschrift. Dank voor jullie tijd en inspanning. Daarnaast zou ik graag alle coauteurs willen bedanken die betrokken waren bij de totstandkoming van de manuscripten van dit proefschrift. Arnoud, dank voor je inhoudelijk en methodologische expertise. Dank dat je altijd de tijd nam om je constructieve feedback persoonlijk toe te lichten. Wolfgang, thank you for your statistical advice and funny cat pictures while explaining statistics. Dyllis, het is een genoegen om met jou samen te werken, dank voor je enthousiasme en teamspirit. Je hebt me laten zien hoe je als psychiater op een zeer krachtige wijze kwetsbaarheid kan tonen, dank daarvoor. Michael, dank voor de inspirerende samenwerking met het schrijven van papers en beursaanvragen, en het presenteren van symposia op congressen. Jan en Mathijs, dank voor jullie samenwerking bij de observationele studie. Eric, dankzij jou ben ik wegwijs geraakt in de methodologie van een meta-analyse. Ik heb veel van jouw doortastende aanpak in het onderzoek geleerd. Eva, dank je vasthoudendheid en zorgvuldigheid tijdens onze literatuurzoektochten. Neha, thank you for your collaboration on the TRD metaanalysis. Rob, thank you for mentoring me during my stays at your wonderful lab. Thank you for introducing me to the world of personalized treatment selection, and thank you for our brainstorming sessions on all types of modeling issues, including pizza dough model solutions. Zach, thank you for your expertise on the Personalized Advantage Index, and predictive modelling in general. Thank you for your constructive feedback and input. Jack, thank you for introducing me to the magical random forest. It is a pleasure to work with you!! You inspire me in both research and clinical 
practice. I am very grateful for your friendship, please consider moving to the Netherlands as soon as possible. Lorenzo, thank you for your valuable feedback and input. Thank you for always asking the right questions that help me improve my work. Sanne! Ik kan met zekerheid zeggen dat het uitvoeren van externe validatie ons dichter bij elkaar heeft gebracht (inclusief crazy eyes selfies). Het is geweldig om met zo'n getalenteerde onderzoeker als jij samen te werken. Ik stel voor dat we alle toekomstige predictiemodellen altijd eerst valideren tussen Maastricht en Freiburg. Nalan, thank you for introducing me to the beautiful world of econometric models and Bayesian inference. Thank you for thoughtful advices and kindness, you always brighten my day. I look forward to working with you on various $B M I$ projects in the future.

Graag wil ik mijn collega's op de onderzoeksafdeling Psychiatrie en Neuropsychologie bedanken. Deze afdeling heeft mij de kans gegeven om van onderzoekstagiaire te groeien naar een echte dokter. Simone, dank voor je enthousiasme, adviezen, lunchherinneringen en steun. Jij stond altijd aan mijn kant, met een kopje koffie. Het wordt tijd dat we samen een project gaan starten. Boris, zullen we het ooit met elkaar eens worden? Dank voor je kritische tegengeluid, goeddoordachte adviezen en verloren weddenschap. Nicole, waar statistiekcursussen niet goed voor zijn. Dank voor je vriendschap en aanstekelijk positieve levenshouding. Sanne, ook al woon je nu in Italië, je bent nog niet van mij af, eens jouw stagiaire, altijd jouw stagiaire. Tim, oud-kamergenootje, bedankt voor de gezelligheid en gezamenlijke zorg voor Ben. Naomi, als plan B kunnen we altijd nog de acteursopleiding overwegen? Marjan, dank voor je (statistische) adviezen en lunchgesprekken over konijnen en paarden. Maarten, dank voor je oprechte verontwaardiging toen ik naar een andere afdeling ging, ik kijk uit naar gezamenlijk overgewicht (in de meta-analyse dan). Patrick, ik hoop dat we in de toekomst nog eens samen kunnen brainstormen over klinisch onderwijs. Koen, dank dat ik altijd bij jou terecht kon voor advies, zowel werkgerelateerd, als restaurantgerelateerd. Machteld, dank voor je supervisie tijdens mijn allereerste onderzoeksstages, vanuit deze stages is mijn interesse voor onderzoek begonnen. Thérèse, dank voor de inspirerende gesprekken tijdens afdelingsetentjes. Truda, dank dat je deur altijd open staat, bedankt voor je praktische adviezen en betrokkenheid. Nele, dank voor je oprechte belangstelling. Karel, bedankt voor je hulp bij het grote filmproject. Daphne, dank voor de gezelligheid tijdens de lunches en op de flexplek toen ik stagiaire was. Trees, Jolanda en Ine, dank dat ik altijd bij jullie terecht kan voor ondersteuning. Jo en Ron dank voor jullie digitale ondersteuning. Verder wil ik alle overige collega's op de afdeling bedanken voor de prettige werksfeer en de gezellige gesprekken.

Graag wil ik mijn collega's van mijn werkplekken bij het MUMC+, MET ggz, Zuyderland GGZ en Mondriaan bedanken. Jullie hebben mij tijdens mijn opleiding tot psychiater niet alleen het vak geleerd, maar jullie hebben mij ook geleerd hoe ik een goede collega moet zijn. Een aantal collega's wil ik in het bijzonder noemen. 
Jacqueline, dank dat je mij de kansen en de ruimte hebt gegeven om me wetenschappelijk te ontwikkelen. Het is dankzij jou dat ik dit promotietraject binnen de opleiding ben gestart en heb voortgezet. Lieve, dank voor je luisterend oor, betrokkenheid, en gedeelde verontwaardiging over vastgeroeste GGZ-patronen bij een kopje koffie verkeerd. Ina, psychotherapie supervisie van jou was een van de beste leermomenten van mijn opleiding. Dankjewel dat je me de kans gaf om zo veel mee te kijken (modelleren +++). Jim en Arthur, bedankt voor jullie betrokken supervisie tijdens mijn werkzaamheden op de poli persoonlijkheidsstoornissen bij Zuyderland GGZ. Jolien, dankjewel voor alles wat je me hebt geleerd in het FACT team, en dank ook voor je aandacht voor persoonlijke ontwikkeling. Harm, Heidi, en Maddy het was geweldig om onderdeel van jullie team te zijn. Baer, Bart, Carsten, Marijntje, Servé, Frans, Jan, Wiesette en Albert, dank voor alle leermomenten tijdens mijn MUMC+ stages. Mandy, dank voor je oprechte betrokkenheid, zullen we snel weer een kopje thee drinken? Resi en Lidwine, dank dat ik altijd bij jullie terecht kan, en dank voor jullie steun in mindere tijden. Dankjewel alle AIOSen, samen in hetzelfde schuitje was helemaal niet verkeerd. Lieve Jaimie, Kirsten en Amber, ik kijk uit naar nog veel ontbijtjes en cocktails in Aachen. Beste Jill, Hannah, Frank K, Frank S, Gabriel, Julie, Michiel, Roger en Wemke, dank voor jullie gezelligheid tijdens de eindeloze onderwijsdagen. Mashood, Danique, Emma, Sven, Joris, Jory, Geert, Hussam en Jill, dank voor jullie collegialiteit in het allerlaatste AIOS jaartje. Dondeyne, dank voor de verdiepende gesprekken op de door ons ingerichte kamer op B1. Pim, dank voor je vertrouwen om mij heel veel vragen voor te leggen. Jur, dank dat je altijd voor mij klaar staat, ook in de Albert Heijn. Anne (wijs wijs!) dank voor je zorgzaamheid en je strijdlust. Eva, ondanks je valse huisdier ben ik superblij dat ik je heb leren kennen, dank voor je vriendschap. Machteld, dank voor je betrokkenheid, ook bij al mijn geklaag op de maandagochtend. Wim, dank voor de samenwerking in de onderwijscommissie en je adviezen bij ethische dilemma's.

Graag wil ik ook mijn collega's bij de vakgroep Clinical Psychological Science bedanken voor de hartelijke ontvangst op mijn nieuwe werkplek. Wat leuk om samen te werken met collega's met affiniteit voor onderzoek, onderwijs, klinische werk en dansjes. Nicole, bedankt voor je gezonde invloed (kikkersprongen!). Cor, dank dat je me wegwijs hebt gemaakt in de historie en gang van zaken op de afdeling. Natalja, Stefanie, Reinier en Chantal, wat supercool om met jullie samen te werken aan het klinisch onderwijs, dank hiervoor! Anke, Brechje, David, Eline, Jeffrey, Jill, Lorraine, Maarten, Marisol, Martijn, Marleen, Pauline, Peter, Dalena, Sanne en Vanessa, ook jullie bedankt voor de samenwerking in de klinische sectie.

Daarnaast wil ik graag anderen bedanken die de afgelopen jaren betrokken zijn geweest bij mijn werkzaamheden. Jael, dank voor de gezelligheid tijdens congressen, en dank voor de verdieping in suïcidale klachten binnen de resultaten van de STEPd studie. Anita, thank you for your collaboration, I enjoyed working together even if all analyses failed to convert. Birgitte, dank voor je aanstekelijke UM sport 
enthousiasme. Annemiek en Nienke, bedankt dat jullie mij wegwijs hebben gemaakt in de wereld van de IPT. Marjolein, dank voor je gastvrijheid, steun en geduld met die eindeloze (wetenschappelijke) discussies van Frenk en mij.

Graag wil ik tenslotte de VIPs bedanken, mijn vrienden en familie. Bedankt voor jullie interesse en steun, maar vooral ook bedankt voor de afleiding. Het combineren van een promotietraject met een opleiding tot psychiater maakte mij niet altijd de meest toegankelijke persoon. Dank daarom ook voor jullie begrip bij de zoveelste deadline of weekenddienst. Graag zou ik een aantal VIPs in het bijzonder willen noemen. Ilse, dank voor je niet aflatende interesse in mijn promotietraject, ook als ik helemaal niet over de door jou bijgehouden lijst van artikelen wilde praten. Ik geniet altijd erg van onze discussies, je helpt me om zaken van verschillende kanten te bekijken. Dank dat je oprecht met mij meeleeft, maar ook dank voor je relativering (bijvoorbeeld bij ontplofte fietsen). En Jos, ook jij bedankt voor je steun en bedankt dat ik me altijd heel welkom voel als ik bij jullie ben. Marion, ik denk dat jij helemaal begrijpt hoe je volledig op kan gaan in de wetenschap. Bedankt voor je adviezen als medeonderzoeker, maar ook bedankt voor alle wandelingen, skype afspraken, en pizza of sushi dates. Ik vind het heel mooi om te zien hoe jij je eigen geluk altijd belangeloos met de mensen om je heen deelt. Ook wil ik Robin bedanken, ik vind het altijd supergezellig om bij jullie op bezoek te komen. Lotte, dankjewel voor je steun tijdens dit promotietraject. Samen lunchen of film kijken was een welkome afleiding. Je bent niet alleen een super stoere mama en dokter, maar ook een van de meest zorgzame mensen die ik ken. Ik ben nog steeds heel erg blij dat je reis rond de wereld de eindbestemming Maastricht had. Grant, thank you for your support and your price winning cappuccinos. Anja en Bregje, ik ben zo blij dat ik jullie tijdens onze studententijd heb leren kennen. Ik denk dan ook met veel plezier terug aan de zwemtrainingen, wedstrijdweekenden en (scoren)feestjes. Ik hoop dat we nog op veel (soortgelijke) weekendjes en uitjes gaan de komende jaren. Anne en Matthijs, dank voor jullie vriendschap de afgelopen jaren. De kinderfeestjes en uitjes waren een welkome afleiding van mijn promotietraject. Martine, dank voor de gezellige afspraken en high teas. En Kirsten, we moeten echt weer bijpraten hè, wat fijn dat we elkaar altijd weer terugvinden tussen alle drukke levens door. Dan wil ik ook graag de enige echte PASOA-groep bedanken, dankjewel Anne, Devi, Ingrid, Lys, Marjolijn, Romee, Rosita, Sufia en Violet! Ondanks dat we niet bij elkaar om de hoek wonen, voelen jullie altijd heel dichtbij. Het Sinterklaasweekend is dan ook HET event van het jaar (tenminste in de regio Drenthe). Dankjewel dat ik elk jaar weer een weekendje onbezorgd en een beetje onverstandig kan zijn. Marianne, dank dat ik altijd bij jou terecht kan, dank ook de gezelligheid voor en tijdens carnaval. Henk, bedankt voor je interesse in mijn werkzaamheden, en voor onze discussies over de GGZ. Floor en Tim dank voor jullie steun en interesse, wat fijn dat ik altijd bij jullie een kopje koffie mag komen drinken. En natuurlijk ook Kaat, zullen we snel weer een keertje gaan zwemmen? En Marie, ik kijk ernaar uit om je stapje voor stapje beter te leren kennen. 
Marcel en Margriet, jullie staan aan de basis van dit alles, en in het bijzonder van mijn interesse voor klinische psychologie en onderwijs. Papa, dankjewel voor je betrokkenheid en je nieuwgierigheid. Je bent altijd bereikbaar voor een gesprek, een dilemma of een random vraag. Voor jou bestaan er geen obstakels om er voor mij te zijn, ook als het moeilijk is, zit je in het publiek bij mijn presentatie. Mama, jou praktische en oplossingsgerichte manier van denken is voor mij een groot voorbeeld, dat ik hoop mee te nemen in mijn wetenschappelijke en klinische werk. Dankjewel dat je zo strijdvaardig met mij meeleeft, ook als debatteren niet zo je ding is. Rosa, mijn kleine zusje, dankjewel voor je enthousiaste steun. Ik zal nooit vergeten dat jij tijdens een presentatie als enige in de zaal om mijn grapje over de "dodo bird" moest lachen. Dankjewel dat je mij er altijd aan herinnert om van het leven te genieten, en om te focussen op wat echt belangrijk is. Nadia, grote zus, ons contact bekent veel voor me. Jouw creatieve denkwijze is voor mij een inspiratiebron. Praten met jou leert mij om "outside the box" te denken, dankjewel daarvoor. Ramses, grote broer, dankjewel dat je altijd geïnteresseerd bent in waar ik mee bezig ben, en dank voor alle afleiding met gemberthee, videogames, series en films. Suzanne en Olaf, ook bedank voor jullie steun, wat fijn dat jullie bij ons zijn. En tenslotte, mijn meest favoriete persoon, Matthijs. Over het plan om te promoveren, zei je "Dat moet je doen want ik denk dat het je gelukkig maakt". Dank voor je onvoorwaardelijk steun en rotsvaste vertrouwen, je had mij geen beter advies kunnen geven. Je inspireert me, in jouw gezelschap gaat schrijven twee keer zo snel, en zijn nieuwe ideeën grenzeloos. Ik vind het ongelofelijk hoe je me altijd aan het lachen kan maken. Dank voor het meedenken, je geduld en ondersteuning met de espressootjes, gezond eten en verrassingen. Het mooiste van het avontuur van dit proefschrift was elke dag thuiskomen bij jou. 


\section{Curriculum vitae}

Suzanne van Bronswijk was born on July $11^{\text {th }} 1988$ in Nijmegen, the Netherlands. After graduating from secondary school (Nijmeegse Scholengemeenschap Groenewoud), she started studying Medicine at Maastricht University in 2006. She obtained her bachelor's degree in Medicine in 2009. After that, she received a first year's degree in Philosophy at the Radboud University Nijmegen in 2010, and became a research intern at the Department of Psychiatry and Neuropsychology at Maastricht University. During this internship she collected and analyzed structural imaging data in a project on pituitary volume, stress reactivity and genetic risk for psychotic disorders. In January 2014, Suzanne obtained her Master's degree in Medicine. Within this master's program, she undertook a facultative research internship, where she spent two months analyzing functional imaging data under the supervision of Prof. dr. J. Suckling at the Department of Psychiatry at the University of Cambridge. This internship resulted in a published master's thesis on cognitive correlates, resting state frontoparietal network connectivity and genetic risk for psychotic disorders. In addition to the master's curriculum, she completed several PhD courses in statistical methods in 2013.

After obtaining her master's degree, Suzanne worked briefly as junior doctor in child and adolescent psychiatry at the Maastricht University Medical Center+ (MUMC+). During this clinical job, she wrote a case report on SSRIs for adolescents with medically unexplained symptoms. In April 2014, she enrolled in the psychiatry residency of the Universitaire Opleiding Psychiatrie Zuid-Limburg. Within this program she worked at both in- and outpatient settings at the MUMC+, MET ggz, Zuyderland GGZ and Mondriaan. From April 2015 onwards, she combined this work with a PhDprogram under the supervision of Prof. dr. F. Peeters, Prof. dr. M. Huibers and dr. L. Lemmens at the Department of Psychiatry and Neuropsychology of Maastricht University. The results of this PhD-program are presented in this thesis. Suzanne received a travel grant from Stichting de Drie Lichten to conduct part of her research at the University of Pennsylvania (Philadelphia, USA), under the supervision of Prof. dr. R. DeRubeis. She was the recipient of the European Student Travel Award to present her research at the 2017 annual meeting of the international Society for Psychotherapy Research in Toronto (Canada). Furthermore, she received the Beck Institute Graduate Student Scholarship to attend an early career workshop about depression and suicidality at the Beck Institute for cognitive therapy (Philadelphia, USA). In addition to her clinical and scientific work, Suzanne was involved in clinical skills teaching of medical students, and she was a member of the committee on education of her residency program. This committee was awarded with the European Federation of Psychiatric Trainees Award for Excellence for organizing extra-curricular activities. In September 2019, Suzanne graduated from her residency program. 
Suzanne currently works as an assistant professor at the department of Clinical Psychological Science at Maastricht University, where she is further developing prediction models to guide treatment strategies for depression in an interdisciplinary team of researchers. In March 2020, this team received the Data Science Research Competition award of Maastricht University to kick-start their future plans.

\section{List of publications}

van Bronswijk, S. C., Lemmens, L. H. J. M., Huibers, M. J. H., Peeters, F. P. M. L. (2020). Selecting the optimal treatment for a depressed individual: clinical judgment or statistical prediction? Journal of Affective Disorders

van Bronswijk, S. C. \& Bruijniks, S. J. E., Lorenzo-Luaces, L., DeRubeis, R. J., Lemmens, L. H. J. M., Peeters, F. P. M. L., Huibers, M. J. H (2020). Cross-trial prediction in psychotherapy: external validation of the Personalized Advantage Index using machine learning in two Dutch randomized trials comparing cognitive behavioral therapy versus interpersonal psychotherapy for depression. Psychotherapy Research.

Rauwenhoff, J. C. C., van Bronswijk, S. C., Peeters, F. P. M. L., Bol, Y., Geurts, A. C. H., van Heugten, C. M. (2020). Personalized predictions of treatment outcome in patients with post-stroke depressive symptoms. Journal of Rehabilitation Medicine.

Eskildsen, A., Reinholt, N., van Bronswijk, S.C., Brund, R. B. K., Christensen, A. B., Hvenegaard, M., Arendt, M., Alro, A., Poulsen, S., Rosenberg, N. K., Huibers, M. J. H., Arnfred, S. M. (2020). Personalized psychotherapy for outpatients with major depression and anxiety disorders: transdiagnostic versus diagnosis-specific group cognitive behavioral therapy. Cognitive Therapy and Research.

Lemmens, L. H. J. M., van Bronswijk, S. C., Peeters, F. P. M. L., Arntz, A., Roefs, A., Hollon, S. D., DeRubeis, R. J., \& Huibers, M. J. H. (2020). Interpersonal psychotherapy versus cognitive therapy for depression: how they work, how long, and for whomkey findings from an RCT. The American Journal of Psychotherapy, 73(1), 8-14

van Bronswijk, S. C., DeRubeis, R. J., Lemmens, L. H., Peeters, F. P., Keefe, J. R., Cohen, Z. D., \& Huibers, M. J. H. (2019). Precision medicine for long-term depression outcomes using the Personalized Advantage Index approach: cognitive therapy or interpersonal psychotherapy? Psychological Medicine, 1-11. 
van Bronswijk, S. C., Köster, E. M., \& Peeters, F. P. (2019). Effectiveness of acutephase treatment of depression is not influenced by comorbid personality disorders: results from a meta-analysis and meta-regression. Psychotherapy and Psychosomatics, $1-2$.

Lemmens, L. H. J. M., van Bronswijk, S. C., Peeters, F. P. M. L., Arntz, A., Roefs, A., Hollon, S. D., DeRubeis, R. J., \& Huibers, M. J. H. (2019). Cognitieve therapie en interpersoonlijke psychotherapie voor depressie: hoe werken ze, hoe lang en voor wie? Tijdschrift voor Psychiatrie, 61, 710-719.

van Bronswijk, S. C., Lemmens, L. H. J. M., Keefe, J. R., Huibers, M. J. H., DeRubeis, R. J., \& Peeters, F. P. M. L. (2019). A prognostic index for long-term outcome after successful acute phase cognitive therapy and interpersonal psychotherapy for major depressive disorder. Depression and Anxiety, 36(3), 252-261.

van Bronswijk, S. C., Moopen, N., Beijers, L., Ruhe, H.G., \& Peeters, F. P. M. L. (2019). Effectiveness of psychotherapy for treatment-resistant depression: a meta-analysis and meta-regression. Psychological Medicine, 49(3), 366-379.

Lemmens, L. H. J. M. , van Bronswijk, S. C., Peeters, F. P. M. L, Arntz, A., Hollon, S. D., \& Huibers, M. J. H. (2019). Long-term outcomes of acute treatment with cognitive therapy v. interpersonal psychotherapy for adult depression: follow-up of a randomized controlled trial. Psychological Medicine, 49(3), 465-473.

van Bronswijk, S. C., Lemmens, L. H. J. M., Huibers, M. J. H., Arntz, A., \& Peeters, F. P. M. L. (2018). The influence of comorbid anxiety on the effectiveness of cognitive therapy and interpersonal psychotherapy for major depressive disorder. Journal of Affective Disorders, 232, 52-60.

van Bronswijk, S. C., Lemmens, L. H. J. M., Viechtbauer, W., Huibers, M. J. H., Arntz, A., \& Peeters, F. P. M. L. (2018). The impact of personality disorder pathology on the effectiveness of cognitive therapy and interpersonal psychotherapy for major depressive disorder. Journal of Affective Disorders, 225, 530-538.

van Bronswijk, S. C., Lionarons, J. M., Stupp, A., Schieveld, J. N. (2016). SSRIs for adolescents with medically unexplained symptoms. Nederlands Tijdschrift voor Geneeskunde, 160, 9380 
Peeters, S. C. T., van Bronswijk, S. C., van de Ven, V., Gronenschild, E. H. B. M., Goebel, R., van Os, J., \& Marcelis, M. (2015). Cognitive correlates of frontoparietal network connectivity 'at rest' in individuals with differential risk for psychotic disorder. European Neuropsychopharmacology, 25(11), 1922-1932.

van de Riet, E. H., van Bronswijk, S. C., Schieveld, J. N. (2015). Movement disorders in anti-N-NMDA receptor encephalitis. Tijdschrift voor Psychiatrie, 57(2), 109-13.

Habets, P., Collip, D., Myin-Germeys, I., Gronenschild, E., van Bronswijk, S. C., Hofman, P., Lataster, T., Lardinois, M., Nicolson, N. A., van Os, J., \& Marcelis, M. (2012). Pituitary volume, stress reactivity and genetic risk for psychotic disorder. Psychological Medicine, 42(7), 1523-1533. 